\title{
Amphidinolides F and C2: An Odyssey in Total Synthesis.
}

Laurent Ferrié, ${ }^{*[\mathrm{a}]}$ Ismaila Ciss, ${ }^{[\mathrm{a}, \mathrm{b}]}$ Johan Fenneteau, ${ }^{[\mathrm{a}]}$ Sara Vallerotto, ${ }^{\mathrm{a}]}$ Matar Seck $^{[\mathrm{b}]}$ and Bruno Figadère ${ }^{[a]}$

${ }^{[a]}$ BioCIS, Université Paris-Saclay, CNRS, 92290, Châtenay-Malabry, France.

${ }^{[b]}$ Laboratoire de Chimie Organique et Chimie Thérapeutique, FMPO-UCAD, Université Cheikh Anta-diop, 5199, Dakar-Fann, Senegal. 


\section{Table des matières}

I-General

II-Experimental Procedures and Compounds Characterization .................................................... 4

Siloxyfuran 13

Glyceraldehyde 12

Adduct 17 and 17'

Tri-TBS ether 18

Acetylated lactol 19

Oxazolidinethione $7 \mathrm{~b}$

THF 20

Alcohol 21

Acetylene 24.

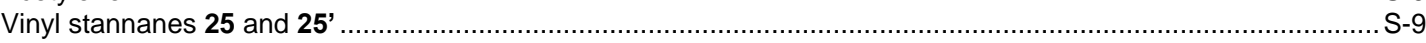

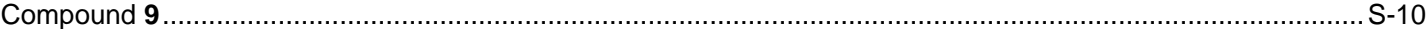

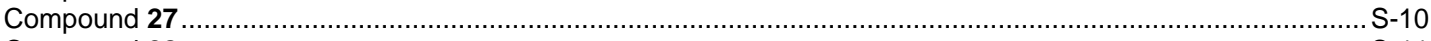

Compound 28

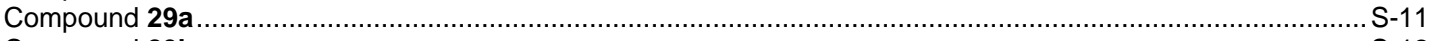

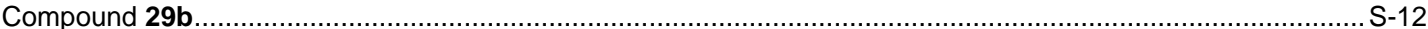

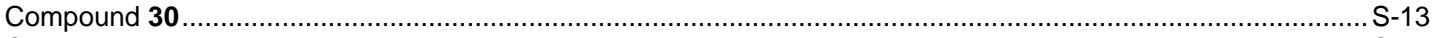

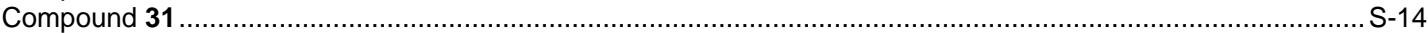

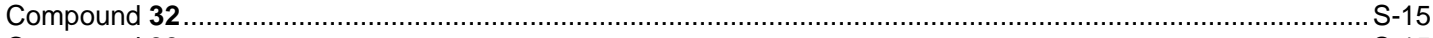

Compound 33

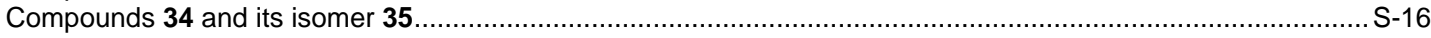

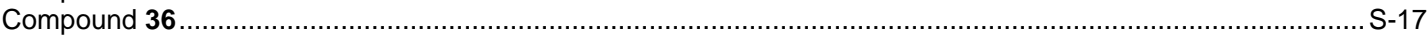

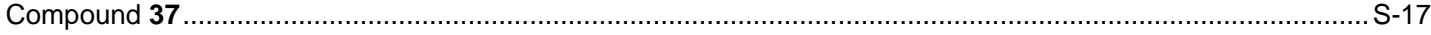

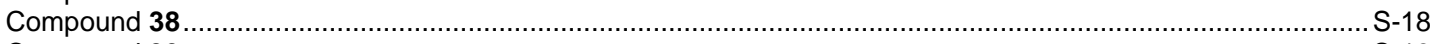

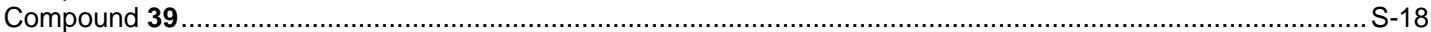

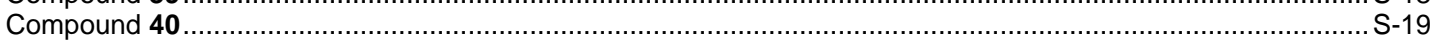

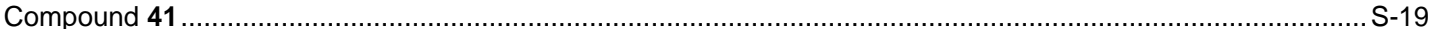

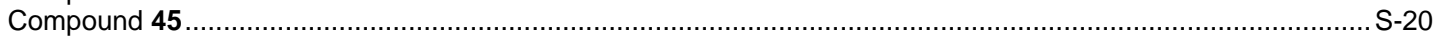

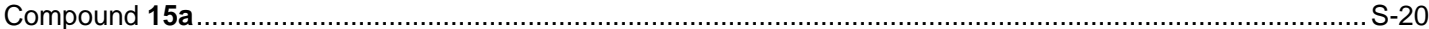

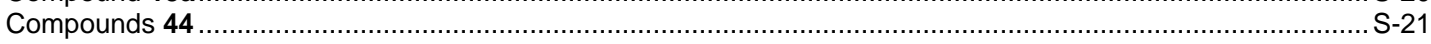

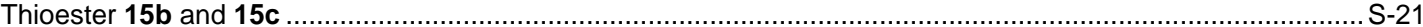

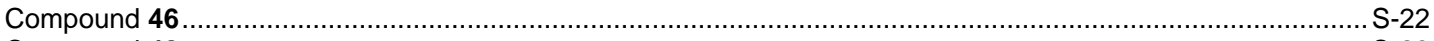

Compound 48

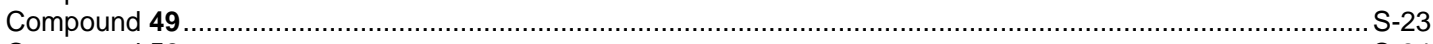

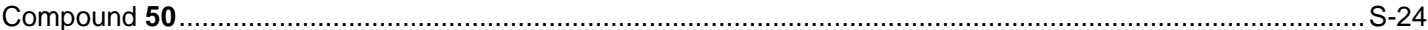

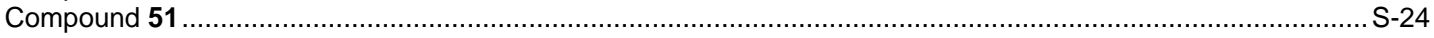

Compound 52

Compound 42 …

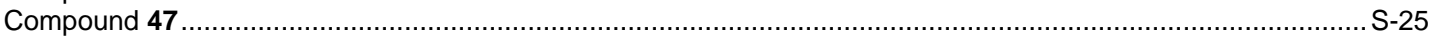

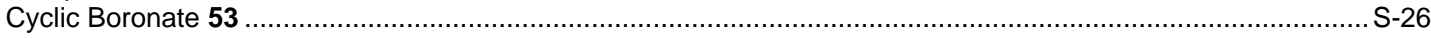

lodide 54

Compound 55

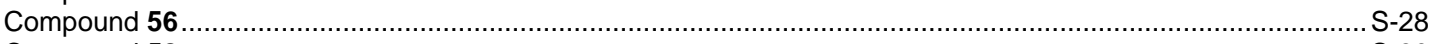

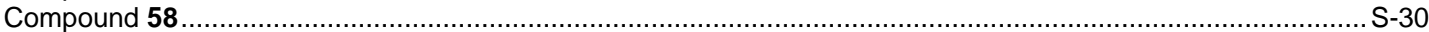

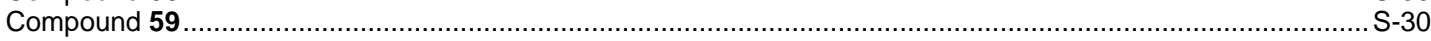

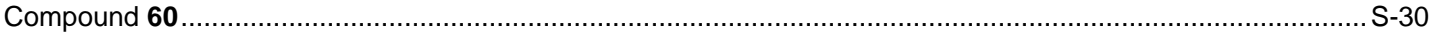

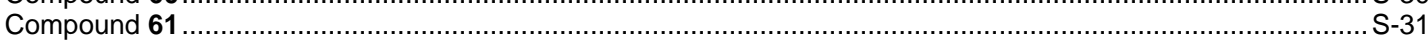

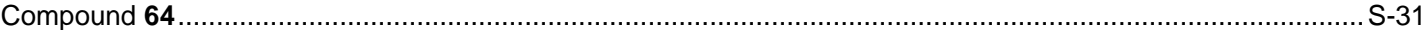

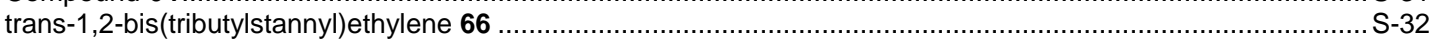

Compound 69

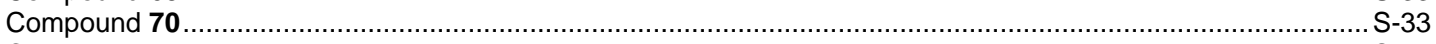

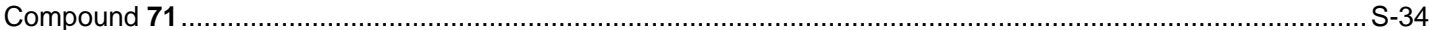

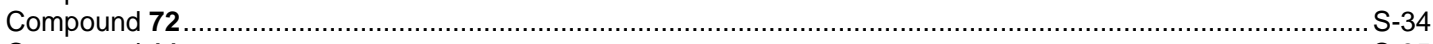

Compound 11a

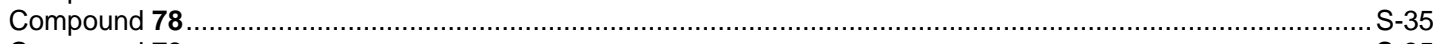

Compound 79

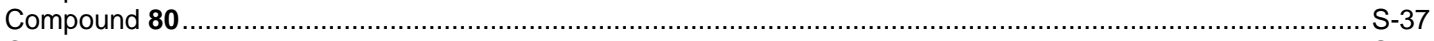

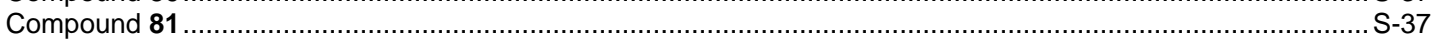

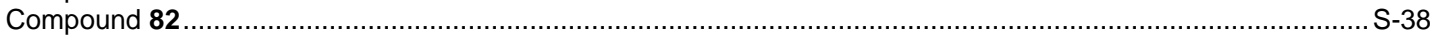

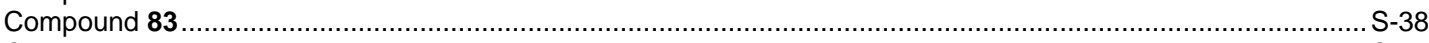

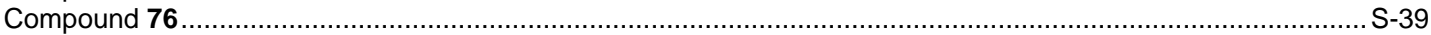

Compound 84

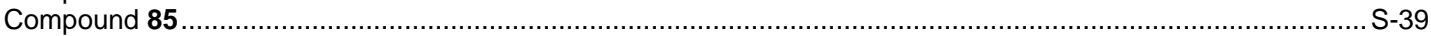




\section{SUPPORTING INFORMATION}

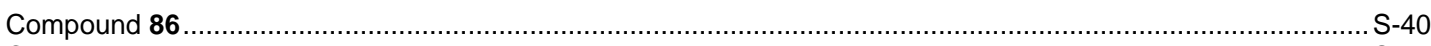

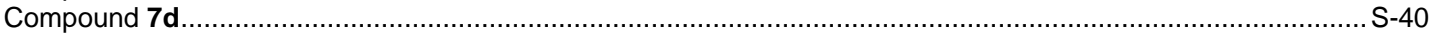

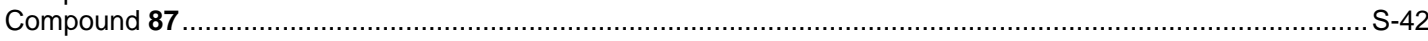

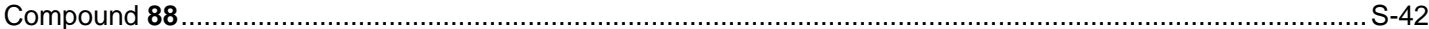

Compound 89

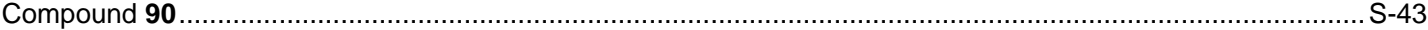

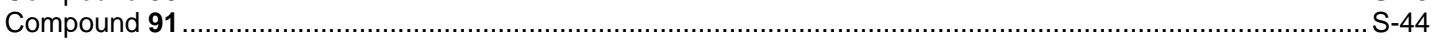

Compound 92

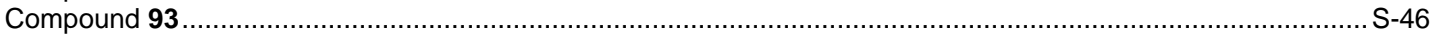

Compound 77a

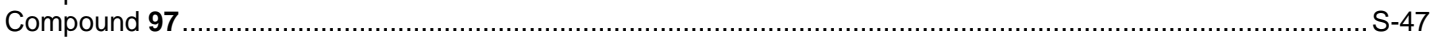

Compound 98

Compound 99

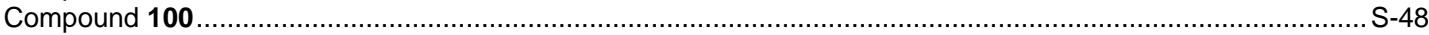

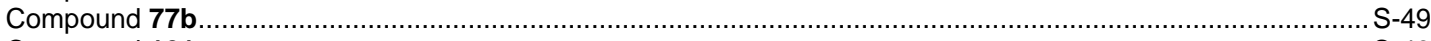

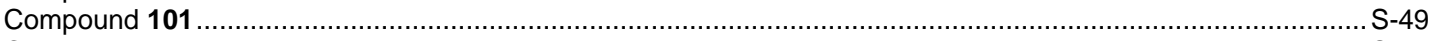

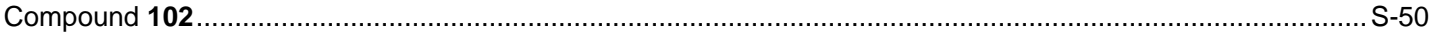

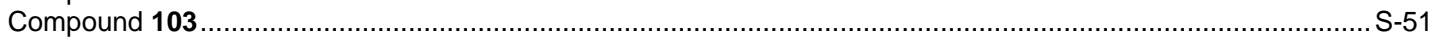

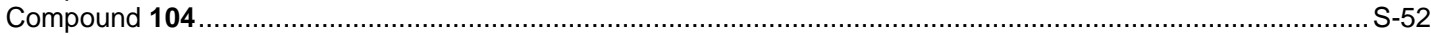

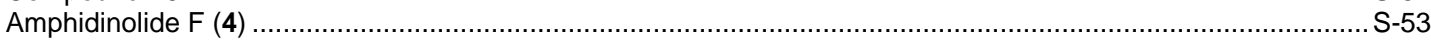

Table S1. Comparison of ${ }^{1} \mathrm{H}$ NMR spectra[a] of our synthetic sample of amphidinolide F.................................... S-54

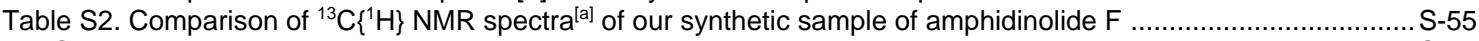

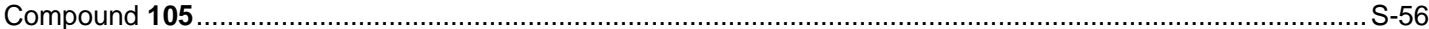

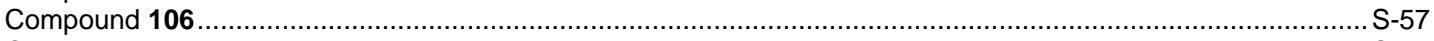

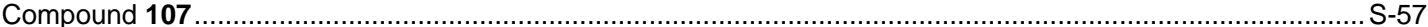

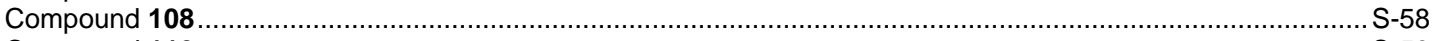

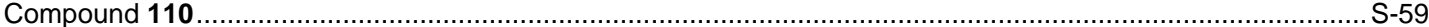

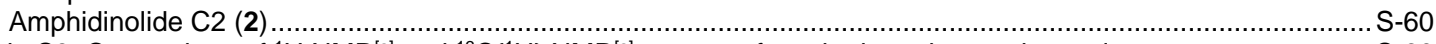

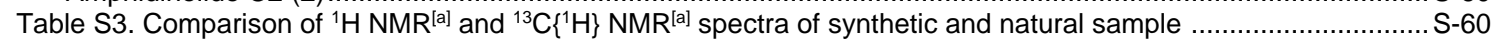

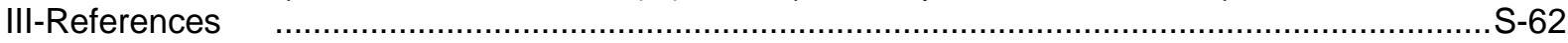

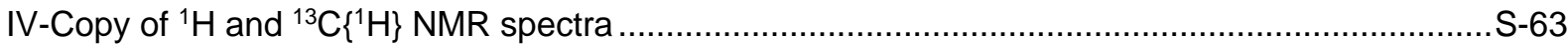




\section{SUPPORTING INFORMATION}

\section{I-General}

All the reactions were performed under an inert atmosphere $\left(\mathrm{N}_{2}\right.$ or $\left.\mathrm{Ar}\right)$. THF was distilled over sodium/benzophenone mixture. $\mathrm{Et}_{2} \mathrm{O}$ and $\mathrm{CH}_{2} \mathrm{Cl}_{2}$ were purified by filtration over activated molecular sieves. $\mathrm{MeOH}$ was purified by filtration over activated alumina. DMF was purchased as anhydrous grade from Acros Organics and used as received. Analytical thin-layer chromatography (TLC) was performed on silica gel 60 $\mathrm{F}_{254}(0.25 \mathrm{~mm})$ plates purchased from Merck. Compounds were visualized by exposure to a UV lamp $(\lambda=$ 254 and $365 \mathrm{~nm}$ ), an aqueous solution of $\mathrm{KMnO}_{4} / \mathrm{K}_{2} \mathrm{CO}_{3}$, or an acidic solution of vanillin in EtOH and followed by gentle heating. Flash chromatographies were performed using Merck (230-400 mesh) silica gel.

${ }^{1} \mathrm{H}$ and ${ }^{13} \mathbf{C}\left\{{ }^{1} \mathbf{H}\right\}$ NMR spectra were recorded by using a Brucker Advance $300(300 \mathrm{MHz})$ or a Brucker Advance $400(400 \mathrm{MHz})$ spectrometers in the solvent indicated. Amphidinolide F spectra were recorded on $800 \mathrm{MHz}$ cryocool spectrometer from ICSN at Gif-sur-Yvette, France. NMR spectra of Amphidinolide C2 were recorded on a $600 \mathrm{MHz}$ and $700 \mathrm{MHz}$ cryocool spectrometers for ${ }^{1} \mathrm{H} \& 2 \mathrm{D}$ experiments and ${ }^{13} \mathrm{C}\left\{{ }^{1} \mathrm{H}\right\}$ NMR experiment, respectively, from ICSN at Gif-sur-Yvette, France. Chemical shifts $(\delta)$ are given in ppm and the coupling constants $(\mathcal{J})$ in $\mathrm{Hz}$. The solvent signals were used as reference $\left(\mathrm{CDCl}_{3}: \delta_{\mathrm{C}}=77.16 \mathrm{ppm}\right.$ unless notified, residual $\mathrm{CHCl}_{3}$ in $\mathrm{CDCl}_{3}: \delta_{\mathrm{H}}=7.26 \mathrm{ppm} ; \mathrm{C}_{6} \mathrm{D}_{6}: \delta_{\mathrm{C}}=128.06 \mathrm{ppm}$ unless notified, residual $\mathrm{C}_{6} \mathrm{HD}_{5}$ in $\mathrm{C}_{6} \mathrm{D}_{6}: \delta_{\mathrm{H}}=7.16 \mathrm{ppm}$. Multiplicities are described by the following abbreviations: $\mathrm{s}=$ singlet, $\mathrm{d}=$ doublet, $\mathrm{t}=$ triplet, $\mathrm{q}=$ quartet, quint. $=$ quintet, sext. $=$ sextet, sept. $=$ septet, $\mathrm{m}=$ multiplet, $\mathrm{br}=$ broad. An optimized sequence for $1 \mathrm{D}{ }^{13} \mathrm{C}\left\{{ }^{1} \mathrm{H}\right\}$ spectra, called UDEFT, ${ }^{[1]}$ was used for most compounds.

Melting points were performed on a Buchi melting point B-540. Infrared spectra were recorded by using a Brucker IRTF Vector 22 spectrometer, and wavenumbers $(v)$ were given in $\mathrm{cm}^{-1}$. Mass spectra were obtained on a Waters LCT Premier (ESI-TOF) spectrometer, Agilent QTOF 6530, or Agilent QTOF 6546 in BioCIS, at Université Paris-Saclay. Some HRMS were also performed on Waters GCT (LIFDI) at University of California, Riverside. Optical rotations $\left([\alpha]_{D}\right)$ were measured with an Optical Activity polAAr 32 polarimeter. 


\section{II-Experimental Procedures and Compounds Characterization}

\section{Siloxyfuran 13}

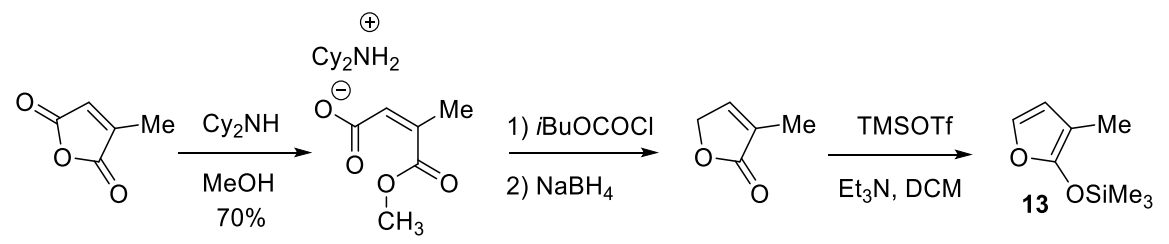

Prepared according to reported experimental procedure:[1,2]

To dry $\mathrm{MeOH}(400 \mathrm{~mL})$ at $-20^{\circ} \mathrm{C}$ was added citraconic anhydride $(56 \mathrm{~g}, 45 \mathrm{~mL}, 0.5 \mathrm{~mol}, 1$ equiv) followed by dicyclohexylamine ( $99 \mathrm{~mL}, 0.55 \mathrm{mmol}, 1.1$ equiv) over $5 \mathrm{~min}$. After $30 \mathrm{~min}$ at rt, the solution was concentrated on rotavapor, with a heating bath below $40^{\circ} \mathrm{C}$. AcOEt was added to the residue, and the white precipitate was then filtered on a glass sinter and washed with AcOEt. Drying the solid under vacuum afforded carboxylate salt as a white powder (114 g, $70 \%)$. Characterization data were in agreement with the literature. ${ }^{[1]}$

${ }^{1} \mathrm{H}$ NMR $(300 \mathrm{MHz}$, DMSO-d6) $\delta 10.00-6.50$ (brs, $1 \mathrm{H}), 5.79(\mathrm{~s}, 1 \mathrm{H}), 3.58(\mathrm{~s}, 3 \mathrm{H}), 2.95-2.69(\mathrm{~m}, 2 \mathrm{H}), 2.00$ $-1.84(\mathrm{~m}, 4 \mathrm{H}), 1.82(\mathrm{~s}, 3 \mathrm{H}), 1.76-1.64(\mathrm{~m}, 4 \mathrm{H}), 1.58(\mathrm{~d}, J=11.6 \mathrm{~Hz}, 2 \mathrm{H}), 1.31-1.01(\mathrm{~m}, 10 \mathrm{H})$.

To a solution of above carboxylate salt $\left(114 \mathrm{~g}, 350 \mathrm{mmol}, 1\right.$ equiv) in DCM $(290 \mathrm{~mL})$ at $-10^{\circ} \mathrm{C}$ (cooled with a ice/salt mixture), was added iso-butyl chloroformate $(50.0 \mathrm{~mL}, 385 \mathrm{mmol}, 1.1$ equiv) over $5 \mathrm{~min}$. After $1 \mathrm{~h}$ at this temperature, a precipitate of ammonium hydrochloride salt formed, and after a further $2 \mathrm{~h}$, dry THF $(290 \mathrm{~mL})$ was added. The reaction mixture was filtered, and solids were washed with more THF $(290 \mathrm{~mL})$. At $0{ }^{\circ} \mathrm{C}$, a fresh cold solution of $\mathrm{NaBH}_{4}$ in $\mathrm{H}_{2} \mathrm{O}(26.5 \mathrm{~g}, 700 \mathrm{mmol}, 2$ equiv) was added dropwise under vigorous stirring (exotherm!). After two more hours at $0{ }^{\circ} \mathrm{C}$, Celite was added, and the solid was filtered. The filtrate was concentrated, dissolved in $\mathrm{Et}_{2} \mathrm{O}$, dried over $\mathrm{MgSO}_{4}$ to be concentrated again. The residue was distilled under vacuum $\left(90^{\circ} \mathrm{C}, 15-17 \mathrm{mmHg}\right.$ ), to afford 2-methyl-2,3-dihydro-y-lactone as a colorless oil (23 $\mathrm{g}, 67 \%)$. Characterization data were in agreement with the literature. ${ }^{[1]}$

${ }^{1} \mathrm{H}$ NMR $(200 \mathrm{MHz}$, Chloroform- $d) \delta 7.13(\mathrm{~h}, J=1.7 \mathrm{~Hz}, 1 \mathrm{H}), 4.80-4.65(\mathrm{~m}, 2 \mathrm{H}), 1.98-1.84(\mathrm{~m}, 3 \mathrm{H})$.

To a solution of above lactone $\left(7.97 \mathrm{~g}, 81 \mathrm{mmol}\right.$, 1equiv) in $\mathrm{DCM}(145 \mathrm{~mL})$ was added $\mathrm{Et}_{3} \mathrm{~N}(16.9 \mathrm{~mL}, 122$ $\mathrm{mmol}, 16.9 \mathrm{~mL}$ ), and the solution was cooled to $-20^{\circ} \mathrm{C}$ (cooled with dry ice/EtOH) to further add TMSOTf (15.2 $\mathrm{mL}, 83.7 \mathrm{mmol}, 1.03$ equiv) dropwise. After $45 \mathrm{~min}$ the solution was concentrated carefully and the residue was distilled under vacuum $\left(52-53{ }^{\circ} \mathrm{C}, 15 \mathrm{mmHg}\right)$ to give siloxyfuran 13 (10.9 $\left.\mathrm{g}, 79 \%\right)$. Characterization data were in agreement with the literature. ${ }^{[2]}$

${ }^{1} \mathrm{H}$ NMR $(200 \mathrm{MHz}$, Chloroform- $d) \delta 6.76(\mathrm{~d}, J=2.2 \mathrm{~Hz}, 1 \mathrm{H}), 6.10$ (d, J = 2.2 Hz, 1H), $1.82(\mathrm{~s}, 3 \mathrm{H}), 0.27$ (s, $9 \mathrm{H})$. 


\section{Glyceraldehyde 12}

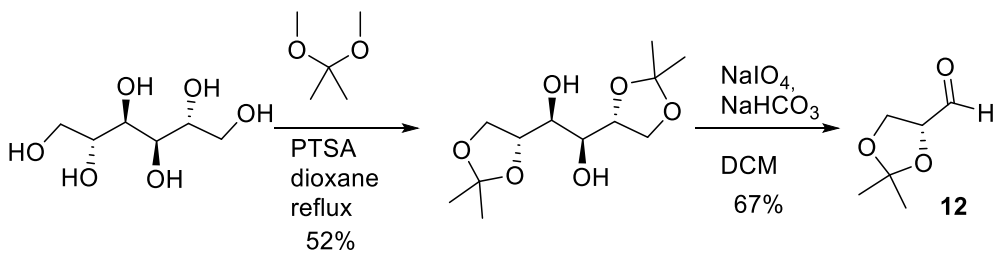

Prepared according to a reported experimental procedure: ${ }^{[3]}$

To a suspension of D-mannitol (100 g, $549 \mathrm{mmol}, 1$ equiv) in 2,2 dimethoxypropane (100 mL, $1.30 \mathrm{~mol}$, 2.36 equiv) and dioxane $(240 \mathrm{~mL})$ was added PTSA $(1 \mathrm{~g})$, and the mixture was refluxed with the help of an oil bath until complete dissolution of $\mathrm{D}$-mannitol. The solution was cooled down to $\mathrm{rt}$, and the reaction mixture was quenched with saturated $\mathrm{NaHCO}_{3}(50 \mathrm{~mL})$. After concentration under vacuum, the so obtained white solid was redissolved in $\mathrm{DCM}$ washed with $\mathrm{NaHCO}_{3}$, followed by $\mathrm{H}_{2} \mathrm{O}$, dried over $\mathrm{Na}_{2} \mathrm{SO}_{4}$, filtered, and concentrated. The white solid was recrystallized with boiling toluene: heptane mixture (150 mL: $200 \mathrm{~mL})$ to afford 1,2:5,6-diisopropylidene-D-mannitol as pure white crystals (75 g, $52 \%)$. Characterization data were in agreement with the literature. ${ }^{[3]}$

${ }^{1} \mathrm{H}$ NMR $\left(300 \mathrm{MHz}, \mathrm{CDCl}_{3}\right): \delta 4.25-4.04(\mathrm{~m}, 4 \mathrm{H}), 3.97(\mathrm{dd}, \mathrm{J}=8.2,5.5 \mathrm{~Hz}, 2 \mathrm{H}), 3.74(\mathrm{~d}, \mathrm{~J}=6.2 \mathrm{~Hz}, 2 \mathrm{H})$, 2.60 (brs, 2H), $1.41(\mathrm{~s}, 6 \mathrm{H}), 1.35(\mathrm{~s}, 6 \mathrm{H})$.

To a solution of 1,2:5,6-diisopropylidene-D-mannitol $(6.5 \mathrm{~g}, 25.1 \mathrm{mmol}, 1$ equiv) in a three-neck bottom-flask equipped with a condenser, was added $\mathrm{NaHCO}_{3}(2.6 \mathrm{~mL})$, then $\mathrm{NalO}_{4}(10.7 \mathrm{~g}, 50.2 \mathrm{mmol}, 2$ equiv) in five portions over $15 \mathrm{~min}$. After $1.25 \mathrm{~h}$, Celite $(10 \mathrm{~g}), \mathrm{MgSO}_{4}(4 \mathrm{~g})$ were added, and the reaction mixture was stirred for further $30 \mathrm{~min}$. The reaction mixture was filtered, concentrated and the residue was distilled with a heat gun, at atmospheric pressure and argon atmosphere at $110-140{ }^{\circ} \mathrm{C}$, to yield glyceraldehyde acetonide 12 as a colorless oil (4.33 g, 67\%). Immediate utilization of 12 is advised to avoid polymer contamination. Long cold storage of $\mathbf{1 2}$ can be done, but it requires redistillation process with a heat gun to crack polymers. Characterization data were in agreement with the literature..$^{[3]}$

${ }^{1} \mathrm{H}$ NMR $\left(300 \mathrm{MHz}, \mathrm{CDCl}_{3}\right): \delta 9.78(\mathrm{~d}, \mathrm{~J}=1.9 \mathrm{~Hz}, 1 \mathrm{H}), 4.45(\mathrm{~m}, 1 \mathrm{H}), 4.30-4.0(\mathrm{~m}, 2 \mathrm{H}), 1.54(\mathrm{~s}, 3 \mathrm{H}), 1.46(\mathrm{~s}$, $3 \mathrm{H})$.

\section{Adduct 17 and 17'}

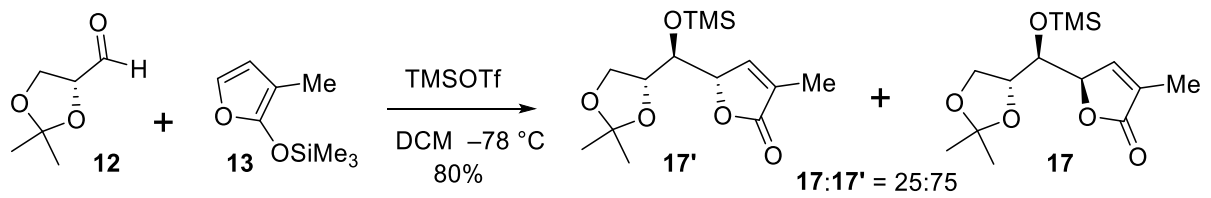

To a solution of freshly distilled 1-trimethylsilyloxy-2-furan $13(1.98 \mathrm{~g}, 11.67 \mathrm{mmol}, 1$ equiv) in $\mathrm{DCM}(25 \mathrm{~mL})$ at $-78^{\circ} \mathrm{C}$ was added freshly distilled $(R)-(+)-2,2$,-dimethyl-1,3-dioxolane-4-carboxaldehyde $12(1.82 \mathrm{~g}, 14.0$ mmol, 1.2 equiv) followed by TMSOTf $\left(212 \mu \mathrm{L}, 1.17 \mathrm{mmol}, 0.1\right.$ equiv) dropwise. After $1 \mathrm{~h}$ at $-78{ }^{\circ} \mathrm{C}$, the reaction mixture was quenched with 2,6-lutidine $(3.25 \mathrm{~mL}, 28 \mathrm{mmol}, 2.2$ equiv) and 15 min later by a saturated solution of $\mathrm{NaHCO}_{3}$. After the reaction mixture rose to room temperature, the reaction mixture was extracted with DCM (x2), washed with a $50: 50 \mathrm{HCl} 1 \mathrm{~N}$ : $\mathrm{NH}_{4} \mathrm{Cl}$ solution mixture, washed with brine, dried over $\mathrm{MgSO}_{4}$, filtered and concentrated. The residue was purified by two silica gel chromatography columns (85:15, Petroleum Ether, AcOEt) to afford in the order of elution adduct 17' (1.78 g, 20\%) and adduct 17 $(5.68 \mathrm{~g}, 61 \%)$ as oils. 


\section{SUPPORTING INFORMATION}

Isomer syn 17, $[\alpha]^{20} \mathrm{D}=+56.4\left(c 0.82, \mathrm{CHCl}_{3}\right)$. IR (neat): $v=2959,1758,1372,1252,1076,840,753 \mathrm{~cm}^{-1}$. ${ }^{1} \mathrm{H}$ NMR $\left(300 \mathrm{MHz}, \mathrm{CDCl}_{3}\right): \delta 7.00$ (quint, $J=1.7 \mathrm{~Hz}, 1 \mathrm{H}$ ), 4.97 (dquint, $J=3.6,1.7 \mathrm{~Hz}, 1 \mathrm{H}$ ), 4.15 (q, $J=6.3$ $\mathrm{Hz}, 1 \mathrm{H}), 4.03(\mathrm{dd}, J=8.4,6.3 \mathrm{~Hz}, 1 \mathrm{H}), 3.81(\mathrm{dd}, J=8.4,6.2 \mathrm{~Hz}, 1 \mathrm{H}), 3.74(\mathrm{dd}, J=6.9,3.6 \mathrm{~Hz}, 1 \mathrm{H}), 1.93(\mathrm{t}$, $J=1.7 \mathrm{~Hz}, 3 \mathrm{H}), 1.40(\mathrm{~s}, 3 \mathrm{H}), 1.35(\mathrm{~s}, 3 \mathrm{H}), 0.09(\mathrm{~s}, 9 \mathrm{H}) \cdot{ }^{13} \mathrm{C}\left\{{ }^{1} \mathrm{H}\right\}$ NMR $\left(75 \mathrm{MHz}, \mathrm{CDCl}_{3}\right): \delta 173.8(\mathrm{~s}), 145.7$ (d), 131.2 (s), 109.5 (s), 81.8 (d), 76.1 (d), 73.4 (d), 66.8 (t), 26.7 (q), 25.3 (q), 10.8 (q), 0.4 (q). HRMS (ESI) calcd for $\mathrm{C}_{14} \mathrm{H}_{24} \mathrm{O}_{5} \mathrm{Si}[\mathrm{M}+\mathrm{Na}]^{+}:$323.1285, found 323.1294.

Isomer anti 17', [a] ${ }^{20} \mathrm{D}=-64.9\left(c 2.605, \mathrm{CHCl}_{3}\right) .{ }^{1} \mathrm{H}$ NMR $\left(300 \mathrm{MHz}, \mathrm{CDCl}_{3}\right): \delta 7.07$ (quint, $\left.J=1.7 \mathrm{~Hz}, 1 \mathrm{H}\right)$, $5.05(\mathrm{~m}, 1 \mathrm{H}), 4.10-3.92(\mathrm{~m}, 3 \mathrm{H}), 3.81(\mathrm{dd}, J=7.7,4.5 \mathrm{~Hz}, 1 \mathrm{H}), 1.90(\mathrm{t}, J=1.7 \mathrm{~Hz}, 3 \mathrm{H}), 1.41(\mathrm{~s}, 3 \mathrm{H}), 1.33$ (s, 3H), 0.07 (s, 9H). ${ }^{13} \mathrm{C}\left\{{ }^{1} \mathrm{H}\right\}$ NMR $(75 \mathrm{MHz}, \mathrm{CDCl} 3): \delta 173.9$ (s), 144.8 (d), 131.5 (s), 110.0 (s), 81.6 (d), 76.1 (d), 73.4 (d), 67.2 (t), 26.7 (q), 25.2 (q), 10.7 (q), 0.1 (q).

\section{Tri-TBS ether 18}

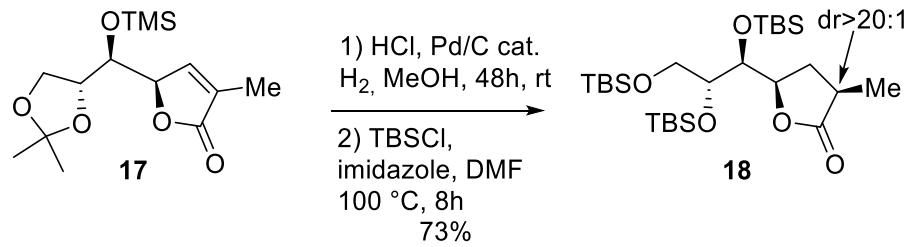

To a solution of adduct 17 (11.28 g, $37.6 \mathrm{mmol}, 1$ equiv) in normal grade $\mathrm{MeOH}(120 \mathrm{~mL})$ was added $\mathrm{Pd} / \mathrm{C} 10 \%$ (600 mg, $1.5 \mathrm{~mol} \%)$ and $\mathrm{HCl}(10 \mathrm{~mL}, 12.5 \mathrm{mmol}, 0.33 \mathrm{mmol}, 1.25 \mathrm{M}$ in $\mathrm{MeOH})$. The reaction mixture was stirred vigorously at rt under atmospheric pressure of $\mathrm{H}_{2}$ until complete hydrogenation of the double bond (NMR sample analysis), ca. $48 \mathrm{~h}$. The reaction mixture was filtered over celite and concentrated under a vacuum to give a very viscous gum. To remove residual $\mathrm{MeOH}$ or $\mathrm{H}_{2} \mathrm{O}$ jailed into the product, the gum was dissolved in a minimum amount of $\mathrm{MeOH}(20 \mathrm{~mL})$ and was diluted by toluene $(100 \mathrm{~mL})$. The mixture was concentrated to give the dry crude triol. It was used for the next step without further purification.

To a solution of this crude triol in dry DMF $(120 \mathrm{~mL})$, was added imidazole $(26.0 \mathrm{~g}, 376 \mathrm{mmol}$, 10 equiv) and TBSCI (28.0 g, $188 \mathrm{mmol}, 5$ equiv), and the reaction mixture was stirred $1 \mathrm{~h}$ at rt then heated at $100{ }^{\circ} \mathrm{C}$ with the help of an oil bath. After $8 \mathrm{~h}$ at this temperature, the reaction mixture was allowed to cool at $\mathrm{rt}$. It was quenched with $\mathrm{NH}_{4} \mathrm{Cl}$, extracted with $\mathrm{Et}_{2} \mathrm{O}(\mathrm{x} 3)$, washed with brine $(\mathrm{x} 3)$, and concentrated. The residue was purified by chromatography on silica gel (Petroleum Ether:Et2O, 90:10) to afford tri-TBS ether 18 (14.65 $\mathrm{g}, 73 \%)$ as a colorless oil.

$[\alpha]^{20} \mathrm{D}=-13.2\left(c\right.$ 2.28, $\left.\mathrm{CHCl}_{3}\right)$. IR (neat): $v=2929,2857,1779,1742,1253,1089,831,775 \mathrm{~cm}^{-1} .{ }^{1} \mathbf{H}$ NMR $\left(300 \mathrm{MHz}, \mathrm{CDCl}_{3}\right): \delta 4.46$ (ddd, $\left.J=11.2,7.6,5.4 \mathrm{~Hz}, 1 \mathrm{H}\right), 3.83-3.69(\mathrm{~m}, 3 \mathrm{H}), 3.43(\mathrm{dd}, J=8.1,3.5 \mathrm{~Hz}, 1 \mathrm{H})$, $2.59(\mathrm{~m}, 1 \mathrm{H}), 2.41$ (ddd, $J=12.3,8.2,5.4 \mathrm{~Hz}, 1 \mathrm{H}), 1.51$ (qapp, $J=11.7 \mathrm{~Hz}, 1 \mathrm{H}), 1.26(\mathrm{~d}, J=7.0 \mathrm{~Hz}, 3 \mathrm{H}$ ), 0.89 (s, 18H), 0.91 (s, 9H), 0.13 (s, 3H), 0.09 (s, 6H), 0.07 (s, 3H), 0.06 (s, 6H). ${ }^{13} \mathrm{C}\left\{{ }^{1} \mathrm{H}\right\}$ NMR $(75 \mathrm{MHz}$, $\left.\mathrm{CDCl}_{3}\right): \delta 178.9(\mathrm{~s}), 80.2(\mathrm{~d}), 78.1(\mathrm{~d}), 74.1(\mathrm{~d}), 63.3(\mathrm{t}), 35.9(\mathrm{~d}), 34.0(\mathrm{t}), 25.9(\mathrm{q}), 18.3(\mathrm{~s}), 18.2(\mathrm{~s}), 14.9$ (q), -4.6 (q), -4.7 (q), -5.5 (q). HRMS (ESI) calcd for $\mathrm{C}_{26} \mathrm{H}_{56} \mathrm{O}_{5} \mathrm{Si}_{3}[\mathrm{M}+\mathrm{Na}]^{+}: 555.3328$, found 555.3317. 


\section{$\underline{\text { Acetylated lactol } 19}$}
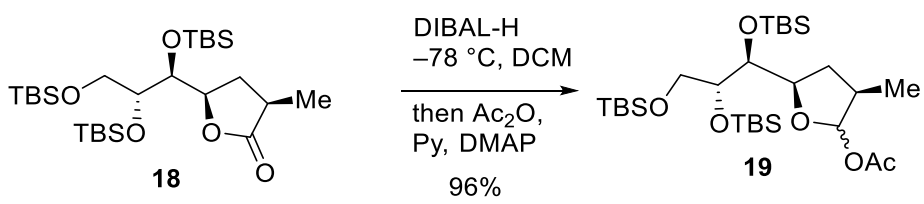

To a solution of tri-TBS ether $18(3.143 \mathrm{~g}, 5.91 \mathrm{mmol}, 1$ equiv) in DCM $(50 \mathrm{~mL})$ at $-78{ }^{\circ} \mathrm{C}$ was added DIBAL-H (13.0 mL, $13.0 \mathrm{mmol}, 2.2$ equiv, $1.0 \mathrm{M}$ in hexanes). After $30 \mathrm{~min}$ at $-78{ }^{\circ} \mathrm{C}$, pyridine (2.38 mL, $29.6 \mathrm{mmol}, 5$ equiv), $\mathrm{Ac}_{2} \mathrm{O}$ ( $3.0 \mathrm{~mL}, 29.6 \mathrm{mmol}, 5$ equiv) and DMAP $(2.15 \mathrm{~g}, 17.7 \mathrm{mmol}$, 3 equiv) were added in this order, and the reaction mixture was allowed to warm slowly until $-20^{\circ} \mathrm{C}$. The reaction mixture was then quenched with a saturated solution of potassium sodium tartrate, stirred $30 \mathrm{~min}$ at rt, diluted with water, extracted with $\mathrm{Et}_{2} \mathrm{O}(\mathrm{x} 3)$, washed with a diluted solution of $\mathrm{NaHCO}_{3}$, and washed with a saturated solution of $\mathrm{CuSO}_{4}(\mathrm{x} 2)$ and finally with a brine solution. The organic layer was then dried over $\mathrm{MgSO}_{4}$, filtered and concentrated, and the residue was filtered over a small plug of silica gel eluting with $\mathrm{Et}_{2} \mathrm{O}$ to afford after concentration acetylated lactol $19(3.275 \mathrm{~g}, 96 \%)$ as a 1:1 mixture of anomers.

IR (neat): $v=2929,1748,1472,1252,1083,831,775 \mathrm{~cm}^{-1} .{ }^{1} \mathbf{H}$ NMR $\left(300 \mathrm{MHz}, \mathrm{CDCl}_{3}\right): \delta 6.18(, J=4.2$ $\mathrm{Hz}, 0.5 \mathrm{H}), 5.86(\mathrm{~d}, J=2.5 \mathrm{~Hz}, 0.5 \mathrm{H}), 4.17(\mathrm{~m}, 1 \mathrm{H}), 3.88-3.60(\mathrm{~m}, 3 \mathrm{H}), 4.50(\mathrm{~m}, 1 \mathrm{H}), 2.30(\mathrm{~m}, 1 \mathrm{H}), 2.25-$ $2.05(\mathrm{~m}, 1 \mathrm{H}), 2.03(\mathrm{~s}, 1.5 \mathrm{H}), 2.01(\mathrm{~s}, 1.5 \mathrm{H}), 1.41(\mathrm{td}, J=12.1,10.8 \mathrm{~Hz}, 0.5 \mathrm{H}), 1.15$ (ddd, $J=12.1,9.6,7.6$ $\mathrm{Hz}, 0.5 \mathrm{H}), 1.11(\mathrm{~d}, J=7.2 \mathrm{~Hz}, 1.5 \mathrm{H}), 1.02(\mathrm{~d}, J=6.5 \mathrm{~Hz}, 1.5 \mathrm{H}), 0.88(\mathrm{~s}, 18 \mathrm{H}), 0.80(\mathrm{~s}, 9 \mathrm{H}), 0.09(\mathrm{~s}, 1.5 \mathrm{H})$, $0.07(\mathrm{~s}, 6 \mathrm{H}), 0.06(\mathrm{~s}, 4.5 \mathrm{H}), 0.05(\mathrm{~s}, 4.5 \mathrm{H}), 0.04(\mathrm{~s}, 1.5 \mathrm{H}) .{ }^{13} \mathrm{C}\left\{{ }^{1} \mathrm{H}\right\} \mathrm{NMR}\left(75 \mathrm{MHz}, \mathrm{CDCl}_{3}\right): \delta 170.4(\mathrm{~s}), 170.2$ (s), 103.7 (d), 99.0 (d), 83.0 (d), 81.9 (d), 80.3 (d), 79.1 (d), 75.1 (d), 75.0 (d), 63.8 (t), 63.7 (t), 40.1 (d), 38.9 (d), $35.5(\mathrm{t}), 33.3(\mathrm{t}), 26.0(\mathrm{q}), 21.3(\mathrm{q}), 21.2(\mathrm{q}), 18.4(\mathrm{~s}), 18.4(\mathrm{~s}), 18.3(\mathrm{~s}), 18.2(\mathrm{~s}), 18.2(\mathrm{~s}), 18.1(\mathrm{q}), 12.5$ (q), -4.5 (q), -4.6 (q), -4.8 (q), -4.9 (q), -5.5 (q), -5.5 (q). HRMS (ESI) calcd for $\mathrm{C}_{28} \mathrm{H}_{60} \mathrm{O}_{6} \mathrm{Si}_{3}\left[\mathrm{M}+\mathrm{Na}^{+}\right.$: 599.3590, found 599.3605 .

\section{Oxazolidinethione $7 b$}
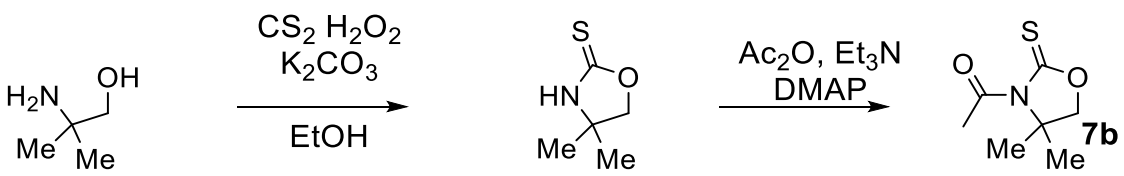

To a solution of 2-methyl-2-aminopropanol $(21.4 \mathrm{~mL}, 225 \mathrm{mmol}, 1$ equiv) in EtOH (200 mL) was added $\mathrm{K}_{2} \mathrm{CO}_{3}\left(15.5 \mathrm{~g}, 112 \mathrm{mmol}, 0.5\right.$ equiv) and $\mathrm{CS}_{2}(27.1 \mathrm{~mL}, 450 \mathrm{mmol}, 2$ equiv). The reaction was heated to 50 ${ }^{\circ} \mathrm{C}$ with the help of an oil bath, and $\mathrm{H}_{2} \mathrm{O}_{2}\left(33.8 \mathrm{~mL}, 337.5 \mathrm{mmol}, 1.5\right.$ equiv, $30 \%$ in $\mathrm{H}_{2} \mathrm{O}$ ) was added slowly over $1 \mathrm{~h}$. After stirring $1 \mathrm{~h}$ more at $50^{\circ} \mathrm{C}$ the reaction mixture was cooled down to $\mathrm{rt}$ and was quenched with sat $\mathrm{NH}_{4} \mathrm{Cl}(100 \mathrm{~mL})$ and water $(100 \mathrm{~mL})$. The reaction mixture was then extracted with AcOEt (three times), and combined organic phases were dried over $\mathrm{MgSO}_{4}$, filtered, and concentrated under a vacuum. Chromatography on silica gel (Petroleum ether: AcOEt, 80:20), followed by trituration of the product with a small amount of $\mathrm{Et}_{2} \mathrm{O}$ and filtration, afforded 4,4-dimethyl-oxazolidine-2-thione (12.65 g, 43\%) as white needles.

Mp $=124-125^{\circ} \mathrm{C}$ (litt: $\left.123.5-125^{\circ} \mathrm{C}\right) \cdot .^{[4]}{ }^{1} \mathrm{H}$ NMR $(300 \mathrm{MHz}$, Chloroform-d) $\delta 8.63$ (brs, $1 \mathrm{H}), 4.27(\mathrm{~s}, 2 \mathrm{H}), 1.36$ $(\mathrm{s}, 6 \mathrm{H}) .{ }^{13} \mathrm{C}\left\{{ }^{1} \mathrm{H}\right\}$ NMR $\left(75 \mathrm{MHz}, \mathrm{CDCl}_{3}\right) \delta 188.44,81.80,60.27,26.80$.

To pure 4,4-dimethyl-oxazolidine-2-thione $(12.65 \mathrm{~g}, 33.0 \mathrm{mmol}, 1$ equiv) and DMAP (1.17 g, $9.66 \mathrm{mmol}$, 0.1 equiv) was added $\mathrm{Et}_{3} \mathrm{~N}$ ( $30.0 \mathrm{~mL}, 217.4 \mathrm{mmol}, 1.5$ equiv) followed by $\mathrm{Ac}_{2} \mathrm{O}$ ( $30 \mathrm{~mL}, 289.8 \mathrm{mmol}, 3$ equiv). The reaction mixture was stirred for $2 \mathrm{~h}$ at $\mathrm{rt}$, water was added $(30 \mathrm{~mL})$, and the reaction mixture was stirred for $30 \mathrm{~min}$ at $\mathrm{rt}$. The reaction mixture was extracted with $\mathrm{Et}_{2} \mathrm{O}(\mathrm{x} 3)$, and the combined ethereal 


\section{SUPPORTING INFORMATION}

layers were washed with water, saturated $\mathrm{NaHCO}_{3}(\mathrm{x} 2)$, dried over $\mathrm{MgSO}_{4}$, filtered, and concentrated. The residue was purified by silica gel chromatography and afforded $7 \mathbf{b}$ as white needles (12.9 $\mathrm{g}, 75 \%$ ). Characterization data were in agreement with the literature. ${ }^{[5]}$

${ }^{1} \mathrm{H}$ NMR $(200 \mathrm{MHz}, \mathrm{CDCl}): \delta=4.16(\mathrm{~s}, 2 \mathrm{H}), 2.77(\mathrm{~s}, 3 \mathrm{H}), 1.57(\mathrm{~s}, 6 \mathrm{H}) \mathrm{ppm} .{ }^{13} \mathrm{C}\left\{{ }^{1} \mathrm{H}\right\} \mathrm{NMR}(50 \mathrm{MHz}$, $\left.\mathrm{CDCl}_{3}\right): \delta=186.8,172.2,79.1,65.4,27.7,24.0 \mathrm{ppm}$.

\section{THF 20}

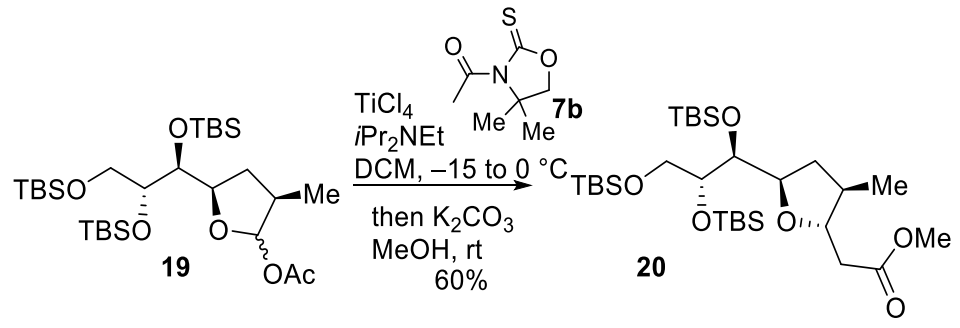

To a solution of $\mathrm{N}$-acetyl-oxazolidinethione $7 \mathrm{~b}\left(1.91 \mathrm{~g}, 11.02 \mathrm{mmol}, 2\right.$ equiv) in $\mathrm{DCM}(25 \mathrm{~mL})$ at $-20{ }^{\circ} \mathrm{C}$ was added $\mathrm{TiCl}_{4}\left(1.20 \mathrm{~mL}, 11.02 \mathrm{mmol}, 2\right.$ equiv) followed by a dropwise addition of $\operatorname{Pr}_{2} \mathrm{NEt}(1.86 \mathrm{~mL}, 11.02$ mmol, 2 equiv) and the reaction color turned from bright orange to dark red. After 15 min at $-20^{\circ} \mathrm{C}$, a solution of acetylated lactol $19(3.175 \mathrm{~g}, 5.51 \mathrm{mmol}, 1$ equiv) in DCM $(25 \mathrm{~mL})$ was added, and the reaction mixture was stirred $30 \mathrm{~min}$ at $-20^{\circ} \mathrm{C}$ followed by $60 \mathrm{~min}$ at $0{ }^{\circ} \mathrm{C} . \mathrm{K}_{2} \mathrm{CO}_{3}(15.2 \mathrm{~g}, 110.2 \mathrm{mmol}, 15 \mathrm{equiv})$ and dry $\mathrm{MeOH}(60 \mathrm{~mL})$ were added, and the reaction mixture was stirred for $2 \mathrm{~h}$ at $\mathrm{rt}$. The reaction mixture was diluted with water and extracted with $\mathrm{Et}_{2} \mathrm{O}(\mathrm{x} 3)$. After concentration, the residue was purified by silica gel chromatography (Et $2 \mathrm{O}$ :Petroleum Ether, 3:97 to 5:95) to afford THF 20 (1.95 g, 60\%) as a colorless oil.

$[\alpha]^{20} \mathrm{D}=-29.5$ (c 0.44, $\left.\mathrm{CHCl}_{3}\right)$. IR (neat): $v=2929,1746,1253,1085,831,775 \mathrm{~cm}^{-1} .{ }^{1} \mathbf{H}$ NMR $(300 \mathrm{MHz}$, CDCl3): $\delta 3.98$ (ddd, $J=13.5,7.7,5.5 \mathrm{~Hz}, 1 \mathrm{H}), 3.80(\mathrm{~m}, 1 \mathrm{H}), 3.76(\mathrm{dd}, J=10.0,6.7 \mathrm{~Hz}, 1 \mathrm{H}), 3.67(\mathrm{~s}, 3 \mathrm{H})$, 3.65 (m, 1H), 3.57 (brd, $J=7.5 \mathrm{~Hz}, 1 \mathrm{H}$ ), 3.43 (brdd, $J=10.0,6.0 \mathrm{~Hz}, 1 \mathrm{H}), 2.51$ (dd, $J=14.6,4.0 \mathrm{~Hz}, 1 \mathrm{H}$ ), $2.41(\mathrm{dd}, J=14.6,8.6 \mathrm{~Hz}, 1 \mathrm{H}), 2.10(\mathrm{dt}, J=12.0,6.4 \mathrm{~Hz}, 1 \mathrm{H}), 1.86(\mathrm{~m}, 1 \mathrm{H}), 1.25$ (qapp, $J=10.5 \mathrm{~Hz}, 1 \mathrm{H})$, $1.02(\mathrm{~d}, J=6.4 \mathrm{~Hz}, 3 \mathrm{H}), 0.88(\mathrm{~s}, 27 \mathrm{H}), 0.06(\mathrm{~s}, 3 \mathrm{H}), 0.05(\mathrm{~s}, 6 \mathrm{H}), 0.04(\mathrm{~s}, 9 \mathrm{H}) .{ }^{13} \mathrm{C}\left\{{ }^{1} \mathrm{H}\right\} \mathrm{NMR}(75 \mathrm{MHz}$, CDCl3): $\delta 172.0$ (s), 80.8 (d), 79.9 (d), 79.8 (d), 77.2 (d), 64.1 (t), 51.5 (q), 40.2 (d), 39.4 (t), 37.9 (t), 26.0 (q), 18.3 (s), 18.2 (s), 16.0 (q), -4.6(q), -5.1(q), -5.5 (q). HRMS (ESI) calcd for $\mathrm{C}_{29} \mathrm{H}_{62} \mathrm{O}_{6} \mathrm{Si}_{3}\left[\mathrm{M}+\mathrm{Na}^{+}\right.$: 613.3746, found 613.3757 .

\section{$\underline{\text { Alcohol } 21}$}

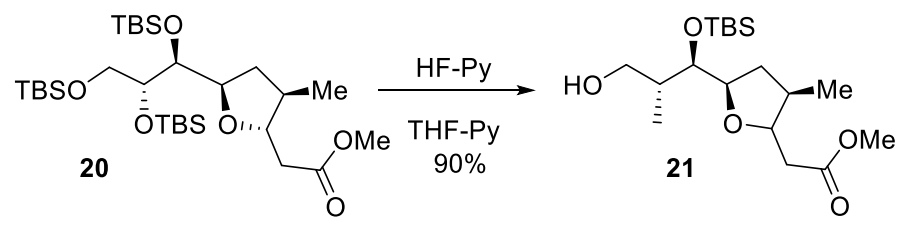

To a solution of THF 20 (470 mg, $0.816 \mathrm{mmol}, 1$ equiv) in THF $(9 \mathrm{~mL})$ at $0{ }^{\circ} \mathrm{C}$ was added HF-pyridine solution in THF [(5.44 mL, $24.5 \mathrm{mmol}, 30$ equiv, HF $4.5 \mathrm{M})$ prepared as follow: HF-pyridine $65 \%(1.0 \mathrm{~mL}$, $35.8 \mathrm{mmol})$, pyridine $(2.0 \mathrm{~mL}, 21.5 \mathrm{mmol})$ and THF $(5 \mathrm{~mL})]$. After $30 \mathrm{~min}$ at $0{ }^{\circ} \mathrm{C}$, the reaction was stirred for $4 \mathrm{~h}$ at $25^{\circ} \mathrm{C}$ until complete conversion by TLC monitoring. The reaction mixture was then quenched with a solution of $\mathrm{NaHCO}_{3}$. It was extracted with $\mathrm{Et}_{2} \mathrm{O}(\mathrm{x} 3)$, washed with a saturated solution of $\mathrm{CuSO}_{4}$, washed with brine, dried over $\mathrm{MgSO}_{4}$, filtered and concentrated. The residue was purified over a silica gel chromatography (Petroleum Ether: Et $2 \mathrm{O}, 85: 15$ to 80:20) to afford the primary alcohol 21 as an oil (340 mg, $90 \%)$. 


\section{SUPPORTING INFORMATION}

$[\alpha]^{20} \mathrm{D}=-43.0\left(c 0.395, \mathrm{CHCl}_{3}\right)$. IR (neat): $v=3500,2928,1744,1252,1040,834,777 \mathrm{~cm}^{-1} .{ }^{1} \mathbf{H}$ NMR $(300$ $\mathrm{MHz}, \mathrm{CDCl} 3$ ): $\delta 4.04$ (ddd, $J=8.0,6.0,4.0 \mathrm{~Hz}, 1 \mathrm{H}$ ), $3.86(\mathrm{td}, J=9.0,3.7 \mathrm{~Hz}, 1 \mathrm{H}), 3.81-3.69(\mathrm{~m}, 2 \mathrm{H}), 3.68$ (s, 3H), $3.65(\mathrm{dd}, J=4.0,1.6 \mathrm{~Hz}, 1 \mathrm{H}), 3.69(\mathrm{~m}, 1 \mathrm{H}), 3.13(\mathrm{~m}, 1 \mathrm{H}, \mathrm{OH}), 2.54(\mathrm{dd}, J=14.8,3.8 \mathrm{~Hz}, 1 \mathrm{H}), 2.41$ (dd, $J=14.8,8.6 \mathrm{~Hz}, 1 \mathrm{H}), 2.05(\mathrm{dt}, J=11.6,6.4 \mathrm{~Hz}, 1 \mathrm{H}), 1.91(\mathrm{~m}, 1 \mathrm{H}), 1.58(\mathrm{td}, J=11.6,10.3 \mathrm{~Hz}, 1 \mathrm{H}), 1.03$ $(\mathrm{d}, J=6.5 \mathrm{~Hz}, 3 \mathrm{H}), 0.92(\mathrm{~s}, 9 \mathrm{H}), 0.90(\mathrm{~s}, 9 \mathrm{H}), 0.12(\mathrm{~s}, 3 \mathrm{H}), 0.08(\mathrm{~s}, 3 \mathrm{H}), 0.07(\mathrm{~s}, 3 \mathrm{H}), 0.06(\mathrm{~s}, 3 \mathrm{H}) .{ }^{13} \mathrm{C}\left\{{ }^{1} \mathrm{H}\right\}$ NMR $(75 \mathrm{MHz}, \mathrm{CDCl}$ ): $\delta 171.7$ (s), 81.8 (d), 79.6 (d), 78.8 (d), 75.5 (d), 62.9 (t), 51.7 (t), 40.1 (d), $39.2(\mathrm{t})$, $37.2(\mathrm{t}), 26.1$ (q), 26.0 (q), 18.4 (s), 18.2 (s), 15.4 (q), -4.2 (q), -4.6 (q), -4.7 (q), -4.9 (q). HRMS (ESI) calcd for $\mathrm{C}_{23} \mathrm{H}_{48} \mathrm{O}_{6} \mathrm{Si}_{2}[\mathrm{M}+\mathrm{Na}]^{+}:$499.2882, found 499.2875 .

\section{$\underline{\text { Acetylene } 24}$}

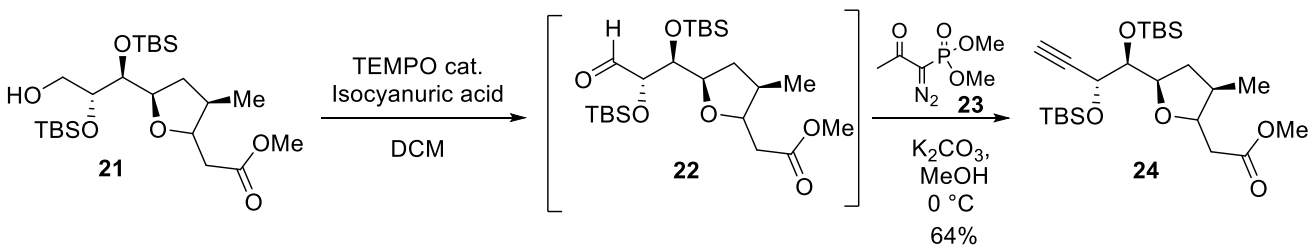

To a solution of alcohol 21 (238 mg, $0.515 \mathrm{mmol}, 1$ equiv) in DCM (1 mL) at $0{ }^{\circ} \mathrm{C}$ was added trichloroisocyanuric acid ( $131 \mathrm{mg}, 0.567 \mathrm{mmol}, 1.1$ equiv) followed by TEMPO (1.6 mg, $0.0103 \mathrm{mmol}, 0.02$ equiv). After 20 min at rt, the reaction mixture was filtered over a small pad of Celite, washed with $\mathrm{CH}_{2} \mathrm{Cl}_{2}$, and the organic solution was washed with a solution of $\mathrm{Na}_{2} \mathrm{CO}_{3}$, washed with $\mathrm{HCl} 1 \mathrm{~N}$, washed with brine, dried over $\mathrm{MgSO}_{4}$, filtered and concentrated to afford aldehyde 22. It was directly used in the next step without further purification. To a solution of crude aldehyde 22 and Bestmann-Ohira reagent 23 (178 mg, $0.927 \mathrm{mmol}, 1.8$ equiv) in $\mathrm{MeOH}(3 \mathrm{~mL})$ at $0{ }^{\circ} \mathrm{C}$ was added $\mathrm{K}_{2} \mathrm{CO}_{3}(142 \mathrm{mg}, 1.03 \mathrm{mmol}$, 2 equiv). After 3 to $5 \mathrm{~h}$ at $0{ }^{\circ} \mathrm{C}$ and until complete conversion by TLC, the reaction mixture was quenched with $\mathrm{HCl} 1 \mathrm{~N}$, extracted with $\mathrm{Et}_{2} \mathrm{O}(\mathrm{x} 3)$, washed with brine, dried over $\mathrm{MgSO}_{4}$, filtered and concentrated. The residue was purified by silica gel column chromatography (Petroleum Ether: $\left.\mathrm{Et}_{2} \mathrm{O}, 96: 4\right)$ to afford acetylene 24 (151 mg, 64\% over 2 steps).

$[\alpha]^{20} \mathrm{D}=-31.4\left(\mathrm{c} 0.925, \mathrm{CHCl}_{3}\right)$. IR (neat): $v=2928,2857,1743,1462,1252,1081,834,777$ $\mathrm{cm}^{-1}$. ${ }^{1} \mathrm{H}$ NMR $(400 \mathrm{MHz}, \mathrm{CDCl} 3): \delta 4.36$ (dd, $\left.J=3.5,2.3 \mathrm{~Hz}, 1 \mathrm{H}\right), 4.04(\mathrm{dt}, J=10.2,6.0 \mathrm{~Hz}, 1 \mathrm{H}$ ), 3.80 (td, $J=8.3,4.0 \mathrm{~Hz}, 1 \mathrm{H}$ ), $3.66(\mathrm{~s}, 3 \mathrm{H}), 3.60(\mathrm{dd}, J=6.2,3.6 \mathrm{~Hz}, 1 \mathrm{H}), 2.50(\mathrm{dd}, J=14.6,4.0 \mathrm{~Hz}, 1 \mathrm{H}$ ), 2.42 (dd, $J$ $=14.6,8.3 \mathrm{~Hz}, 1 \mathrm{H}), 2.36(\mathrm{~d}, J=2.3 \mathrm{~Hz}, 1 \mathrm{H}), 2.08(\mathrm{dt}, J=12.0,6.2 \mathrm{~Hz}, 1 \mathrm{H}), 1.91(\mathrm{~m}, 1 \mathrm{H}), 1.58(\mathrm{td}, J=12.0$, $10.2 \mathrm{~Hz}, 1 \mathrm{H}), 1.02(\mathrm{~d}, J=6.5 \mathrm{~Hz}, 3 \mathrm{H}), 0.89(\mathrm{~s}, 18 \mathrm{H}), 0.12(\mathrm{~s}, 3 \mathrm{H}), 0.10(\mathrm{~s}, 3 \mathrm{H}), 0.09(\mathrm{~s}, 3 \mathrm{H}), 0.06(\mathrm{~s}, 3 \mathrm{H})$. ${ }^{13} \mathrm{C}\left\{{ }^{1} \mathrm{H}\right\}$ NMR (75 MHz, CDCl3): $\delta 172.2$ (s), 83.9 (s), 81.1 (d), 79.0 (d), 78.8 (d), 73.9 (d), 65.5 (d), 51.5 (q), $40.3(\mathrm{~d}), 39.4(\mathrm{t}), 36.9(\mathrm{t}), 26.0$ (q), 25.8 (q), $18.3(\mathrm{~s}), 18.2(\mathrm{~s}), 15.8(\mathrm{q}),-4.3(\mathrm{q}),-4.4(\mathrm{q}),-4.8(\mathrm{q}),-5.1$ (q). HRMS (ESI) calcd for $\mathrm{C}_{24} \mathrm{H}_{46} \mathrm{O}_{5} \mathrm{Si}_{2}[\mathrm{M}+\mathrm{Na}]^{+}:$493.2776, found 493.2765.

\section{Vinyl stannanes 25 and 25}

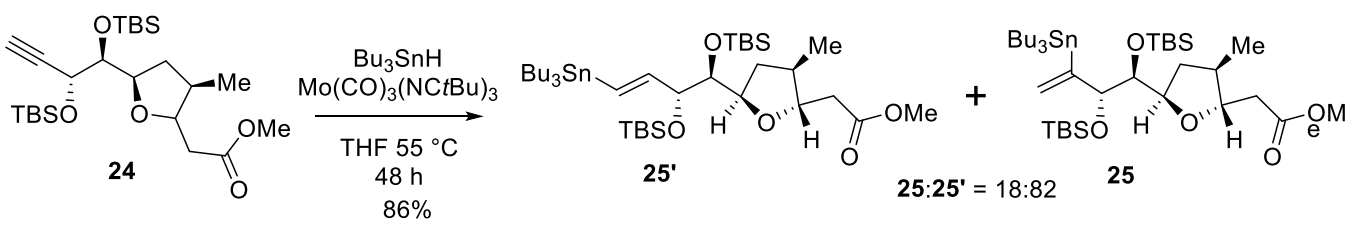

To a solution of acetylene 24 (110 mg, $0.234 \mathrm{mmol}, 1$ equiv), 2,6-tert-butyl-4-methylphenol $(5 \mathrm{mg})$ and $\mathrm{Mo}(\mathrm{CO})_{3}(\mathrm{NCtBu})_{3}{ }^{[6]}\left(5.0 \mathrm{mg}, 0.0117 \mathrm{mmol}, 0.05\right.$ equiv) in THF $(1 \mathrm{~mL})$ was added $n B u_{3} \mathrm{SnH}(185 \mu \mathrm{L}, 0.702$ $\mathrm{mmol}, 3$ equiv). After $24 \mathrm{~h}$ at $55^{\circ} \mathrm{C}$ with the help of an oil bath, additional $\mathrm{Mo}(\mathrm{CO})_{3}(\mathrm{NCtBu})_{3}(5.0 \mathrm{mg}, 0.0117$ mmol, 0.05 equiv) and $n \mathrm{Bu}_{3} \mathrm{SnH}(76 \mu \mathrm{L}, 0.351 \mathrm{mmol}, 1.5$ equiv) were added and the solution was continued to stir at $55{ }^{\circ} \mathrm{C}$ for $24 \mathrm{~h}$. After concentration, the reaction mixture was then directly loaded on a silica gel 


\section{SUPPORTING INFORMATION}

column chromatography (Petroleum Ether: $\mathrm{Et}_{2} \mathrm{O}, 100: 0$ to $97: 3$ ) to afford in the order of elution 1,1disubstituted vinylstannane 25 (125.9 mg, 71\%) and (E)-disubstituted vinylstannane 25' (27.7 mg, 16\%).

1,1-disubstituted vinylstannane 25, $[\alpha]^{20} \mathrm{D}=-16.2$ (c 3.715, $\left.\mathrm{CHCl}_{3}\right)$. IR (neat): $v=2927,1745,1250,1082$, 833, $776 \mathrm{~cm}^{-1} .{ }^{1} \mathrm{H}$ NMR $(300 \mathrm{MHz}, \mathrm{CDCl} 3): \delta 5.96$ (brs, $\left.{ }^{3} \mathrm{~J} \mathrm{SnH}=132 \mathrm{~Hz}, 1 \mathrm{H}\right), 5.23\left(\mathrm{brs},{ }^{3} \mathrm{JSnH}_{\mathrm{SH}}=64 \mathrm{~Hz}, 1 \mathrm{H}\right)$, $4.34\left(\mathrm{brs},{ }^{3} \mathrm{~J} \mathrm{SnH}=28 \mathrm{~Hz}, 1 \mathrm{H}\right), 3.97(\mathrm{~m}, 1 \mathrm{H}), 3.71(\mathrm{td}, J=8.7,4.0 \mathrm{~Hz}, 1 \mathrm{H}), 3.66(\mathrm{~s}, 3 \mathrm{H}), 3.56(\mathrm{brd}, J=7.7$ $\mathrm{Hz}, 1 \mathrm{H}), 2.48(\mathrm{dd}, J=14.6,4.0 \mathrm{~Hz}, 1 \mathrm{H}), 2.40(\mathrm{dd}, J=14.6,8.1 \mathrm{~Hz}, 1 \mathrm{H}), 1.96(\mathrm{dt}, J=12.4,6.0 \mathrm{~Hz}, 1 \mathrm{H}), 1.81$ $(\mathrm{m}, 1 \mathrm{H}), 1.60-1.40(\mathrm{~m}, 6 \mathrm{H}), 1.40-1.22(\mathrm{~m}, 6 \mathrm{H}), 1.13\left(\mathrm{q}_{\mathrm{app}}, J=11.4 \mathrm{~Hz}, 1 \mathrm{H}\right), 0.96(\mathrm{~d}, J=6.7 \mathrm{~Hz}, 3 \mathrm{H}), 0.95-$ $0.81(\mathrm{~m}, 33 \mathrm{H}), 0.07(\mathrm{~s}, 9 \mathrm{H}),-0.01(\mathrm{~s}, 3 \mathrm{H}) \cdot{ }^{13} \mathrm{C}\left\{{ }^{1} \mathrm{H}\right\}$ NMR $(75 \mathrm{MHz}, \mathrm{CDCl} 3): \delta 172.1(\mathrm{~s}), 153.5(\mathrm{~s}), 125.2(\mathrm{t}$, $\left.J_{S n C}=17 \mathrm{~Hz}\right), 82.5\left(\mathrm{~d}, J_{\mathrm{SnC}}=60 \mathrm{~Hz}\right), 80.3\left(\mathrm{~d}, J_{\mathrm{SnC}}=40 \mathrm{~Hz}, 2 \mathrm{C}\right), 79.3(\mathrm{~d}), 51.5(\mathrm{q}), 40.4(\mathrm{~d}), 39.4(\mathrm{t}), 39.0$

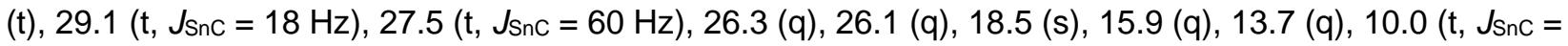
$330 \mathrm{~Hz}$ ), -4.2 (q), -4.5 (q). HRMS (ESI) calcd for $\mathrm{C}_{36} \mathrm{H}_{74} \mathrm{O}_{5} \mathrm{Si}_{2} \mathrm{Sn}[\mathrm{M}+\mathrm{Na}]^{+}:$785.3995, found 785.3971.

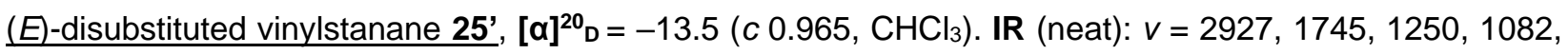
833, $776 \mathrm{~cm}^{-1} .{ }^{1} \mathrm{H}$ NMR $(300 \mathrm{MHz}, \mathrm{CDCl} 3): \delta 6.03\left(\mathrm{~m},{ }^{2} \mathrm{~J}_{\mathrm{SnH}}=40 \mathrm{~Hz}, 1 \mathrm{H}\right), 6.02\left(\mathrm{brs},{ }^{3} \mathrm{~J} \mathrm{snH}=71 \mathrm{~Hz}, 1 \mathrm{H}\right)$, 3.97 (brs, $1 \mathrm{H}), 3.88-3.73(\mathrm{~m}, 2 \mathrm{H}), 3.67(\mathrm{~s}, 3 \mathrm{H}), 3.52(\mathrm{dd}, J=7.4,1.4 \mathrm{~Hz}, 1 \mathrm{H}), 2.50(\mathrm{dd}, J=14.5,4.0 \mathrm{~Hz}$, $1 \mathrm{H}), 2.42(\mathrm{dd}, J=14.5,8.1 \mathrm{~Hz}, 1 \mathrm{H}), 2.03(\mathrm{dt}, J=12.1,6.5 \mathrm{~Hz}, 1 \mathrm{H}), 1.86(\mathrm{~m}, 1 \mathrm{H}), 1.55-1.40(\mathrm{~m}, 6 \mathrm{H}), 1.38-$ $1.20(\mathrm{~m}, 7 \mathrm{H}), 1.01(\mathrm{~d}, J=6.5 \mathrm{~Hz}, 3 \mathrm{H}), 0.96-0.74(\mathrm{~m}, 33 \mathrm{H}), 0.06(\mathrm{~s}, 3 \mathrm{H}), 0.04(\mathrm{~s}, 3 \mathrm{H}), 0.03(\mathrm{~s}, 3 \mathrm{H}), 0.00(\mathrm{~s}$, $3 \mathrm{H}) .{ }^{13} \mathrm{C}\left\{{ }^{1} \mathrm{H}\right\}$ NMR (75 MHz, CDCl3): $\delta 172.1$ (s), 148.6 (d), 129.2 (d), 80.8 (d), 80.3 (d), 79.8 (d), 78.8 (d), 51.6 (q), 40.3 (d), 39.4 (t), 37.7 (t), 29.1 (t, J Jnc $=20 \mathrm{~Hz}$ ), 27.3 (t, Jsnc = 54 Hz), 26.1 (q), 26.0 (q), 18.4 (s), $18.3(\mathrm{~s}), 15.9$ (q), 13.7 (q), $9.4\left(\mathrm{t}, \mathrm{JSnC}_{\mathrm{Sn}}=342 \mathrm{~Hz}\right),-4.3(\mathrm{q}),-4.45$ (q), -4.6 (q), -4.7 (q).

\section{Compound 9}

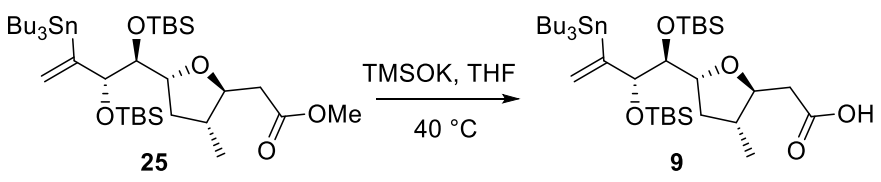

To a solution of stannane 25 (285 mg, $0.354 \mathrm{mmol}, 1$ equiv) in THF (2 mL), was added TMSOK (140 $\mathrm{mg}, 1.06 \mathrm{mmol}, 3$ equiv) and the reaction mixture was heated at $40^{\circ} \mathrm{C}$, with the help of an oil bath, for 90 min. The reaction mixture was quenched by saturated $\mathrm{NH}_{4} \mathrm{Cl}$, extracted with $\mathrm{Et}_{2} \mathrm{O}(\mathrm{x} 3)$, dried over $\mathrm{MgSO}_{4}$, filtered and concentrated under vacuum to afford carboxylic acid 9 as a clear oil (280 $\mathrm{mg}, 100 \%$ ).

$[\alpha]^{20} \mathrm{D}=-12.8\left(c 1.32, \mathrm{CHCl}_{3}\right)$. IR (neat): $v=3500-2300(\mathrm{br}), 2928,1714,1461,1251,1090,1069,834,777$, $660 \mathrm{~cm}^{-1} .{ }^{1} \mathrm{H}$ NMR $\left(300 \mathrm{MHz}, \mathrm{CDCl}_{3}\right) \delta 11.5-8.0(\mathrm{brs}, 1 \mathrm{H}, \mathrm{COOH}), 6.00\left(\mathrm{t}, J=2.0 \mathrm{~Hz},{ }^{3} \mathrm{Jsn}-\mathrm{H}=131.3 \mathrm{~Hz}, 1 \mathrm{H}\right)$, $5.26\left(\mathrm{t}, J=2.0 \mathrm{~Hz},{ }^{3} \mathrm{JSn}_{\mathrm{H}}=63.8 \mathrm{~Hz}, 1 \mathrm{H}\right), 4.37\left(\mathrm{t}, J=3.5,2.0 \mathrm{~Hz},{ }^{3} \mathrm{JSn}-\mathrm{H}=21.2 \mathrm{~Hz}, 1 \mathrm{H}\right), 4.11$ (dt, $J=10.8,6.0$ $\mathrm{Hz}, 1 \mathrm{H}$ ), 3.65 (ddd, $J=9.5,6.7,4.3 \mathrm{~Hz}, 1 \mathrm{H}), 3.61$ (dd, $J=7.1,1.5 \mathrm{~Hz}, 1 \mathrm{H}), 2.64$ (dd, $J=15.5,3.6 \mathrm{~Hz}, 1 \mathrm{H}$ ), 2.47 (dd, $J=15.5,6.8 \mathrm{~Hz}, 1 \mathrm{H}$ ), 2.00 (quint, $J=6.1 \mathrm{~Hz}, 1 \mathrm{H}$ ), $1.87(\mathrm{~m}, 1 \mathrm{H}), 1.60-1.40(\mathrm{~m}, 6 \mathrm{H}), 1.32$ (sext, $J$ $=7.3 \mathrm{~Hz}, 6 \mathrm{H}), 1.25(\mathrm{~m}, 1 \mathrm{H}), 1.07-0.82(\mathrm{~m}, 15 \mathrm{H}), 0.91(\mathrm{~s}, 9 \mathrm{H}), 0.90(\mathrm{~s}, 9 \mathrm{H}), 0.88(\mathrm{~d}, J \approx 5.5 \mathrm{~Hz}, 3 \mathrm{H}), 0.09$ $(\mathrm{s}, 6 \mathrm{H}), 0.08(\mathrm{~s}, 3 \mathrm{H}),-0.00(\mathrm{~s}, 3 \mathrm{H}) \cdot{ }^{13} \mathrm{C}\left\{{ }^{1} \mathrm{H}\right\}$ NMR $\left(75 \mathrm{MHz}, \mathrm{CDCl}_{3}\right) \delta 173.6,153.3,125.87,82.42\left({ }^{2} J_{\mathrm{Sn}-\mathrm{C}}=\right.$ $59.0 \mathrm{~Hz}), 80.50,80.14,78.51,40.11,38.99,38.15,29.25\left({ }^{3} \mathrm{JSn}_{\mathrm{S}-\mathrm{C}}=18.2 \mathrm{~Hz}, 3 \mathrm{C}\right), 27.6\left({ }^{2} \mathrm{~J} \mathrm{Sn}-\mathrm{C}=59.2 \mathrm{~Hz}, 3 \mathrm{C}\right)$, 26.4 (3C), $26.3(3 \mathrm{C}), 18.7,18.6,15.8,13.9(3 \mathrm{C}), 10.2\left({ }^{1} \mathrm{~J}_{\mathrm{Sn}-\mathrm{C}}=321.4,337.1 \mathrm{~Hz}, 3 \mathrm{C}\right),-3.7,-4.0,-4.3(2 \mathrm{C})$. HRMS (ESI) calcd for $\mathrm{C}_{35} \mathrm{H}_{72} \mathrm{O}_{5} \mathrm{Si}_{2} \mathrm{Na}[\mathrm{M}+\mathrm{Na}]^{+}:$771.3.3865, found 771.3844. 


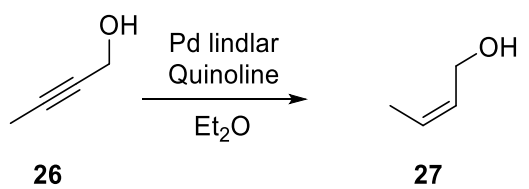

To a solution of 2-butyn-1-ol $26(14 \mathrm{~g}, 200 \mathrm{mmol})$ and quinoline $\left(0.23 \mathrm{~mL}, 2 \mathrm{mmol}, 0.01\right.$ equiv) in $\mathrm{Et}_{2} \mathrm{O}$ $(100 \mathrm{~mL})$ was added Pd Lindlar $(2.13 \mathrm{~g}, 1 \mathrm{mmol}, 0.005$ equiv, $5 \% \mathrm{Pd})$. Then $\mathrm{H}_{2}$ was bubbled into the solution for $36 \mathrm{~h}$ at $\mathrm{rt}$. After completion of the reaction (monitoring by ${ }^{1} \mathrm{H}$ NMR), the reaction was filtered over a Celite pad, and the solvent was carefully concentrated at atmospheric pressure. The resulting crude mixture was distilled at atmospheric pressure to afford (Z)-alcohol $27(11.8 \mathrm{~g}, 163 \mathrm{mmol}, 81 \%)$ as a pale yellow oil. Characterization data were in agreement with the literature. ${ }^{[7]}$

$\mathbf{B p}=119-120^{\circ} \mathrm{C}$ (literature: $\left.119-121^{\circ} \mathrm{C}\right) .{ }^{[7]}$ IR (neat): $v=3021,1025,968 \mathrm{~cm}^{-1} .{ }^{1} \mathbf{H}$ NMR $\left(300 \mathrm{MHz}, \mathrm{CDCl}_{3}\right)$ $\delta 5.68-5.61(\mathrm{~m}, 2 \mathrm{H}), 4.24(\mathrm{~m}, 2 \mathrm{H}), 1.69(\mathrm{~d}, J=5.2 \mathrm{~Hz}, 1 \mathrm{H}), 1.39(\mathrm{brs}, 1 \mathrm{H}, \mathrm{OH}) \mathrm{ppm} .{ }^{13} \mathrm{C}\left\{{ }^{1} \mathrm{H}\right\}$ NMR $(75$ $\left.\mathrm{MHz} \mathrm{CDCl}_{3}\right) \delta 129.3,127.5,58.5,13.2 . \mathrm{MS}$ (GC-El): $72.1\left(\mathrm{M}^{\circ+}, 35\right), 57.10\left(\mathrm{M}^{+}-\mathrm{CH}_{3}, 100\right), 55\left(\mathrm{M}^{+}-\mathrm{HO}, 71\right)$.

\section{Compound 28}

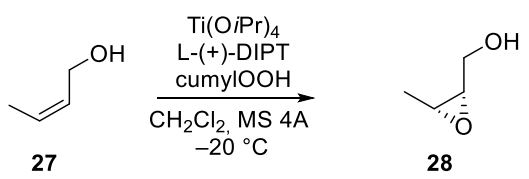

To a cooled $\left(-20^{\circ} \mathrm{C}\right)$ solution of $(Z)$-alcohol $27\left(5.12 \mathrm{~g}, 71.125 \mathrm{mmol}, 1\right.$ equiv) in dry $\mathrm{CH}_{2} \mathrm{Cl}_{2}(175 \mathrm{~mL})$ was added molecular sieves $(2.66 \mathrm{~g})$, L-(+)-DIPT $(2.08 \mathrm{~mL}, 9.95 \mathrm{mmol}, 0.14$ equiv) and Ti(OiPr) 4 (2.1 mL, $7.11 \mathrm{mmol}, 0.1$ equiv). The mixture was stirred at this temperature for 30 minutes. Then cumyl hydroperoxide (25.5 mL, $142.25 \mathrm{mmol}, 2$ equiv $80 \%)$ was added dropwise over 15 minutes. The reaction mixture was maintained at $-20^{\circ} \mathrm{C}$ for $48 \mathrm{~h}$ in a freezer. After this time, a solution of citric acid ( 1 eq in $\mathrm{Et}_{2} \mathrm{O}$ /acetone 1:1) was added, and the resulting mixture was allowed to warm at room temperature for 30 minutes. The crude mixture was then filtered on Celite, concentrated, and purified on silica gel ( $\mathrm{Et}_{2} \mathrm{O} /$ petroleum ether- $50 \%$ to $100 \%$ ) to furnish epoxide 28 (4.935 g, $56.07 \mathrm{mmol}, 79 \%)$. Enantiomeric excess was determined on sulfonyl derivative $\mathbf{2 9}$ a or $\mathbf{2 9} \mathbf{b}$. Characterization data were in agreement with the literature. ${ }^{[8,9]}$

$[\alpha]^{20} \mathrm{D}=-8.7\left(c=4.0, \mathrm{CHCl}_{3}\right)$. IR (neat): $v=3320,1033,987,938,782 \mathrm{~cm}^{-1} .{ }^{1} \mathbf{H} \mathbf{~ N M R}\left(300 \mathrm{MHz}, \mathrm{CDCl}_{3}\right) \delta$ 3.84 (dd, $J=12.2,4.0 \mathrm{~Hz}, 1 \mathrm{H}$ ), 3.68 (dd, $J=12.2,6.4 \mathrm{~Hz}, 1 \mathrm{H}$ ), $3.20-3.07(\mathrm{~m}, 2 \mathrm{H}), 2.07$ (brs, $1 \mathrm{H}, \mathrm{OH}$ ), $1.31(\mathrm{~d}, J=5.6 \mathrm{~Hz}, 3 \mathrm{H}) .{ }^{13} \mathrm{C}\left\{{ }^{1} \mathrm{H}\right\}$ NMR $\left(75 \mathrm{MHz}, \mathrm{CDCl}_{3}\right): \delta 60.8,56.9,52.9,13.5$.

\section{Compound 29a}

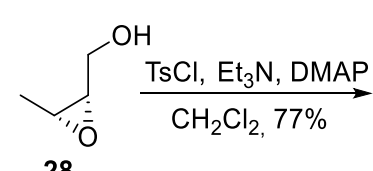

28

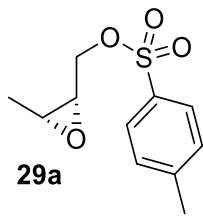

To a cooled $\left(0{ }^{\circ} \mathrm{C}\right)$ solution of epoxide $28\left(3.37 \mathrm{~g}, 38.31 \mathrm{mmol}, 1\right.$ equiv) in dry $\mathrm{CH}_{2} \mathrm{Cl}_{2}(150 \mathrm{~mL})$ was added triethylamine (11.7 mL, $84.12 \mathrm{mmol}, 1.5$ equiv), DMAP (3.39 g, $28.04 \mathrm{mmol}, 0.5$ equiv) and tosyl chloride (12.78 g, $67.30 \mathrm{mmol}, 1.2$ equiv). The solution was allowed to warm to rt, and after $1.5 \mathrm{~h}$ it was hydrolyzed with saturated $\mathrm{NH}_{4} \mathrm{Cl}$ and extracted with $\mathrm{Et}_{2} \mathrm{O}$. The combined organic phase was washed by saturated $\mathrm{NH}_{4} \mathrm{Cl}(50 \mathrm{~mL})$, water $(50 \mathrm{~mL})$, brine $(50 \mathrm{~mL})$, dried over $\mathrm{MgSO}_{4}$, filtered, and concentrated under reduced pressure. The crude material was purified on silica gel column chromatography $\left(\mathrm{Et}_{2} \mathrm{O} /\right.$ petroleum ether $30 \%$ to $40 \%)$ to yield tosylate $29(7.139 \mathrm{~g}, 29.5 \mathrm{mmol}, 77 \%)$. 


\section{SUPPORTING INFORMATION}

$[\alpha]^{20} \mathrm{D}=-18.2\left(c=6.6, \mathrm{CHCl}_{3}\right)$. IR (neat): $v=1598,1360$, 1174, 956, 784, $663 \mathrm{~cm}^{-1}$. ${ }^{1} \mathrm{H}$ NMR $\left(300 \mathrm{MHz}, \mathrm{CDCl}_{3}\right) \delta$ $7.80(\mathrm{~d}, J=8.4 \mathrm{~Hz}, 2 \mathrm{H}), 7.35$ (d, $J=8.4 \mathrm{~Hz}, 2 \mathrm{H}$ ), 4.16 (dd, $J=11.2,5.1 \mathrm{~Hz}, 1 \mathrm{H}), 4.09$ (dd, $J=11.2,6.0 \mathrm{~Hz}, 1 \mathrm{H}), 3.16$ - $3.05(\mathrm{~m}, 2 \mathrm{H}), 2.44(\mathrm{~s}, 3 \mathrm{H}), 1.22(\mathrm{~d}, J=5.6 \mathrm{~Hz}, 3 \mathrm{H})$. ${ }^{13} \mathrm{C}\left\{{ }^{1} \mathrm{H}\right\}$ NMR $\left(75 \mathrm{MHz}, \mathrm{CDCl}_{3}\right) \delta 145.2,132.9,130.1$, 128.1, 68.0, 53.1, 52.3, 21.8, 13.2. HRMS (ESI): $\mathrm{m} / \mathrm{z}$ : calcd for $\mathrm{C}_{11} \mathrm{H}_{14} \mathrm{O}_{4} \mathrm{SNa}[\mathrm{M}+\mathrm{Na}]^{+}$: 265.0505, found 265.0502 . HPLC (Chiralcel AD column 4.6 X $250 \mathrm{~mm}$, hexane/ $i-\mathrm{PrOH}$ $=90 / 10$, flow rate $=1.0 \mathrm{~mL} / \mathrm{min}, \mathrm{I}=254 \mathrm{~nm}) t_{R}=14.17 \mathrm{~min}$ (major), $17.74 \mathrm{~min}$ (minor)]: ee $=86 \%(\mathrm{er}=93: 7)$.

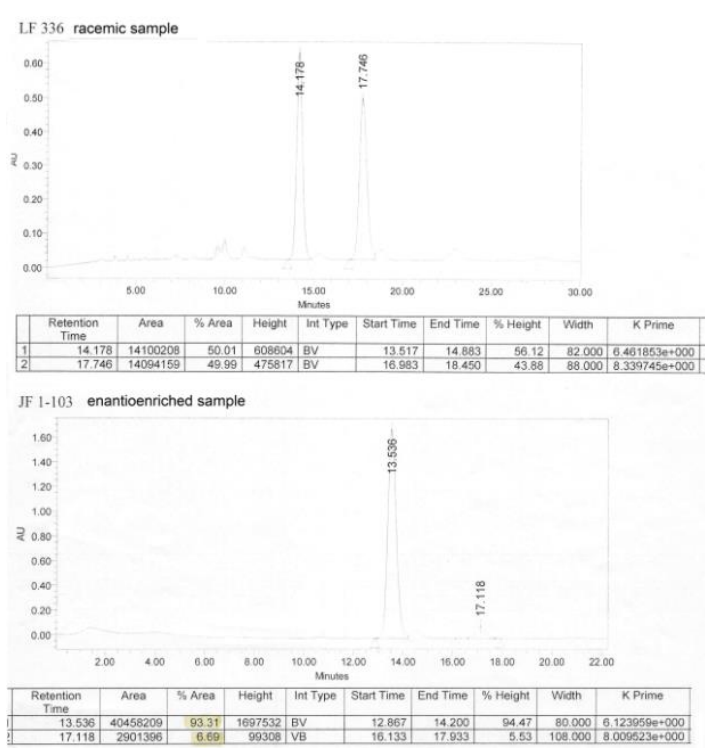

\section{Compound 29b}

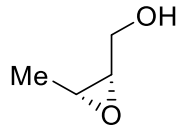

28

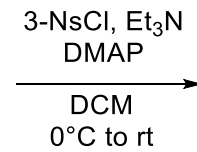

$0^{\circ} \mathrm{C}$ to rt

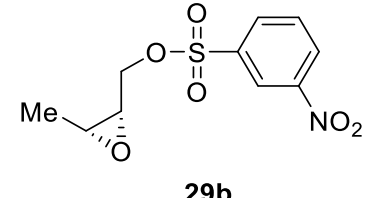

29b

To a cooled $\left(0{ }^{\circ} \mathrm{C}\right)$ solution of epoxide $28(849 \mathrm{mg}, 9.65 \mathrm{mmol}, 1$ equiv) in dry DCM (25 mL) was added triethylamine ( $2.01 \mathrm{~mL}, 14.47 \mathrm{mmol}, 1.5$ equiv), dimethyaminopyridine ( $583 \mathrm{mg}, 4.82 \mathrm{mmol}, 0.5$ equiv) and $m$-nosylchloride ( $2.35 \mathrm{~g}, 67.30 \mathrm{mmol}, 1.2$ equiv). The solution was allowed to warm at room temperature. After $1.5 \mathrm{~h}$ the reaction was hydrolyzed with saturated $\mathrm{NH}_{4} \mathrm{Cl}$ and extracted with $\mathrm{Et}_{2} \mathrm{O}$. The combined organic phases were washed by saturated $\mathrm{NH}_{4} \mathrm{Cl}(50 \mathrm{~mL})$, water $(50 \mathrm{~mL})$, brine $(50 \mathrm{~mL})$, dried over $\mathrm{MgSO}_{4}$, filtered, and concentrated under reduced pressure. The crude was purified on silica gel (AcOEt/PE $30 \%$ to $40 \%$ ) to yield sulfonate $29 \mathrm{~b}$ ( $1.52 \mathrm{~g}, 5.57 \mathrm{mmol}, 57 \%)$ as white needles.

$[\alpha]_{\mathrm{D}}=-15.8\left(c=1.2, \mathrm{CHCl}_{3}\right) . \mathbf{I R}$ (neat): $v=1535,1340,1188,951,883,812,734,660 \mathrm{~cm}^{-1} .{ }^{1} \mathbf{H}$ NMR $(300$ $\mathrm{MHz}, \mathrm{CDCl}_{3}$ ): $\delta=8.77$ (brt, $J=2.0 \mathrm{~Hz}, 1 \mathrm{H}$ ), 8.52 (ddd, $J=8.2,2.1,1.1 \mathrm{~Hz}, 1 \mathrm{H}$ ), 8.27 (ddd, $J=7.9,1.8,1.1$ $\mathrm{Hz}, 1 \mathrm{H}$ ), 7.82 (brt, $J=8.2 \mathrm{~Hz}, 1 \mathrm{H}), 4.39$ (dd, $J=11.4,4.3 \mathrm{~Hz}, 1 \mathrm{H}), 4.19$ (dd, $J=11.4,6.7 \mathrm{~Hz}, 1 \mathrm{H}), 3.23-$ $3.05(\mathrm{~m}, 2 \mathrm{H}), 1.26(\mathrm{~d}, J=5.5 \mathrm{~Hz}, 3 \mathrm{H}) \mathrm{ppm} .{ }^{13} \mathrm{C}\left\{{ }^{1} \mathrm{H}\right\}$ NMR $\left(75 \mathrm{MHz}, \mathrm{CDCl}_{3}\right): \delta=148.5,138.3,133.3,131.0$, 128.5, 123.4, 69.6, 53.0, 52.4, 13.3 ppm. MS (ESI): $\mathrm{m} / \mathrm{z}:[\mathrm{M}+\mathrm{Na}]^{+}:$296.1. HRMS (ESI): $\mathrm{m} / \mathrm{z}$ : calcd for $\mathrm{C}_{10} \mathrm{H}_{12} \mathrm{NO}_{6} \mathrm{~S}[\mathrm{M}+\mathrm{H}]^{+}:$274.0385, found: 274.0387. HPLC (Chiralcel AD column $4.6 \times 250 \mathrm{~mm}$, hexane/ $i-$ $\mathrm{PrOH}=90 / 10$, flow rate $=1.0 \mathrm{~mL} / \mathrm{min}, \mathrm{I}=254 \mathrm{~nm}) t_{\mathrm{R}}=26.26 \min$ (major), $27.47 \mathrm{~min}$ (minor): $1^{\text {st }}$ recrystallization from Hexanes : AcOEt, ee $=84 \% ; 2^{\text {nd }}$ recrystallization from Hexanes: $A c O E t$, ee $=80 \%$. 


\section{SUPPORTING INFORMATION}
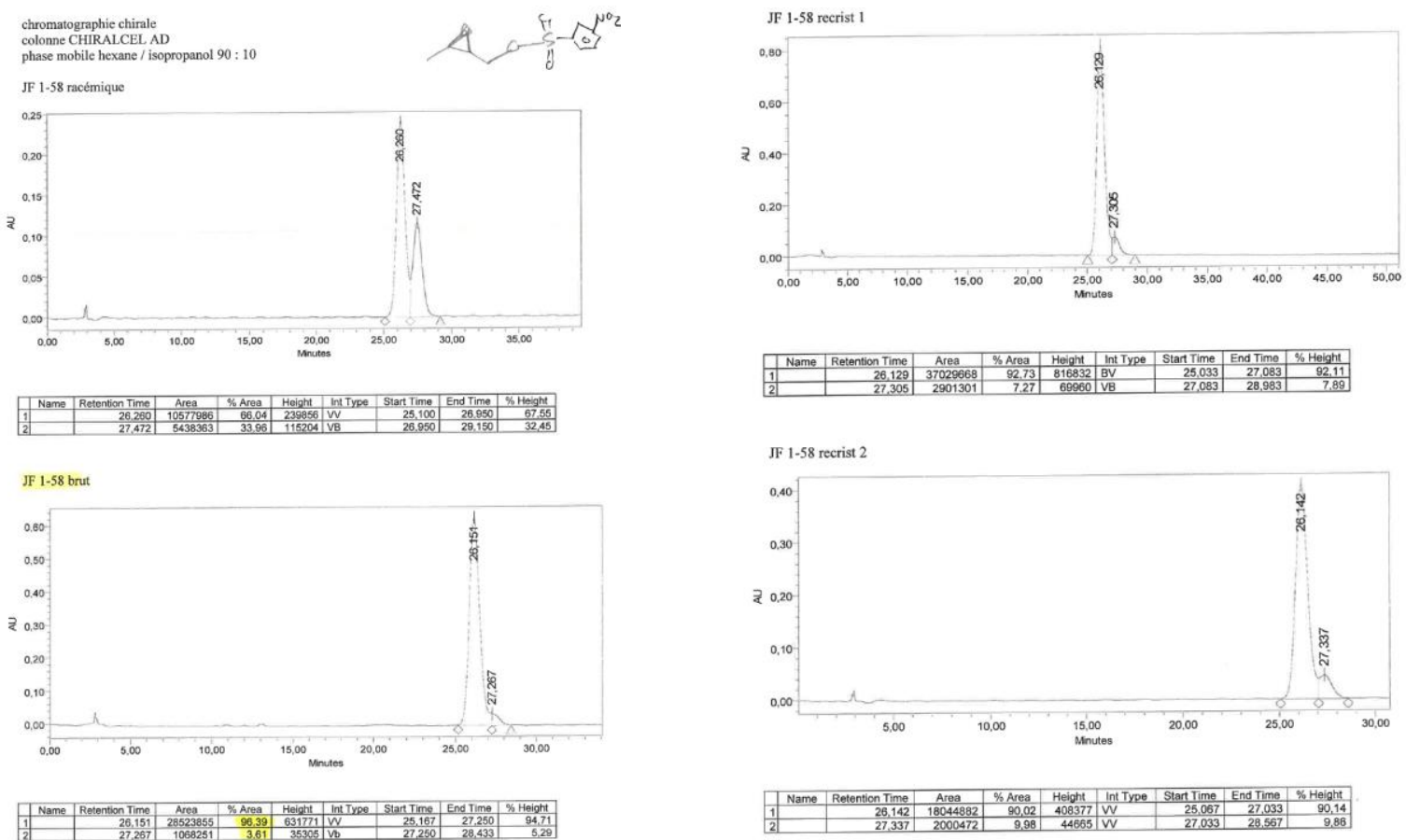

\section{Compound 30}
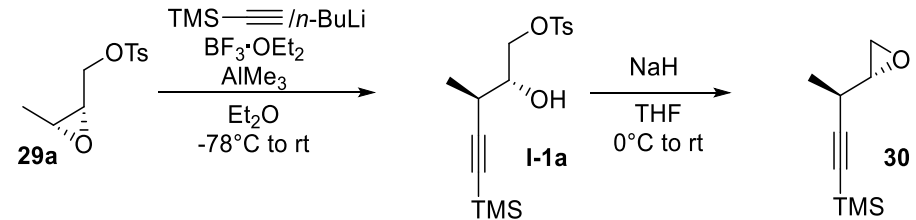

From 29a:

To a cooled $\left(-78^{\circ} \mathrm{C}\right)$ solution of trimethylacetylene $\left(8.36 \mathrm{~mL}, 58.75 \mathrm{mmol}\right.$, 2 equiv) in $\mathrm{Et}_{2} \mathrm{O}(150 \mathrm{~mL})$ was added dropwise $n$-BuLi ( $23 \mathrm{~mL}, 58.75 \mathrm{mmol}, 2$ equiv, $2.5 \mathrm{M}$ in hexanes), and the solution was allowed to warm at $0^{\circ} \mathrm{C}$ for 20 minutes. At $-78^{\circ} \mathrm{C}$ trimethylaluminium $(29.3 \mathrm{~mL}, 58.75 \mathrm{mmol}, 2$ equiv, $2 \mathrm{M}$ in hexane) was added dropwise, the mixture was allowed to warm at $0{ }^{\circ} \mathrm{C}$ for 20 minutes. At $-78^{\circ} \mathrm{C}$ a solution of epoxide 29a (7.109 g, $29.37 \mathrm{mmol}, 1$ equiv) in $\mathrm{Et}_{2} \mathrm{O}(25 \mathrm{~mL})$ was added via cannula, and boron trifluoride diethyl etherate $(7.25 \mathrm{~mL}, 58.75 \mathrm{mmol}, 2$ equiv) was then added dropwise. The mixture was allowed to warm at room temperature for $1.5 \mathrm{~h}$. The resulting mixture was poured into a cooled $\left(0^{\circ} \mathrm{C}\right)$ aqueous solution of sodium potassium tartrate $(150 \mathrm{~mL}, 10 \%)$, and the stirring was maintained for 20 minutes. The mixture was extracted with ethyl acetate, dried over $\mathrm{MgSO}_{4}$, filtered, and concentrated in vacuum to afford crude compound I-1a.

Crude I-1a was dissolved into anhydrous THF $(150 \mathrm{~mL})$ and cooled at $0{ }^{\circ} \mathrm{C}$. Sodium hydride $(2.35 \mathrm{~g}, 58.75$ mmol, 2 equiv, $60 \%$ in mineral oil) was added in portions, and the solution was allowed to warm to room temperature. After consumption of the starting material, indicated by TLC, the reaction was quenched with saturated $\mathrm{NH}_{4} \mathrm{Cl}$, extracted with $\mathrm{Et}_{2} \mathrm{O}$, dried over $\mathrm{MgSO}_{4}$, filtered, and concentrated at reduced pressure. The crude residue was then purified on silica gel ( $\mathrm{Et}_{2} \mathrm{O} /$ petroleum ether $0 \%$ to $\left.5 \%\right)$ to afford epoxide $30(4.83 \mathrm{~g}$, $28.75 \mathrm{mmol}, 98 \%$ over two steps) as a clear oil.

$[\alpha]^{20} \mathrm{D}=+12.2\left(c=5.0, \mathrm{CHCl}_{3}\right)$. IR (neat): $v=2170,1249,890,840,760 \mathrm{~cm}^{-1} .{ }^{1} \mathbf{H}$ NMR $\left(300 \mathrm{MHz}, \mathrm{CDCl}_{3}\right) \delta$ 2.99 (ddd, $J=4.9,3.8,2.7 \mathrm{~Hz}, 1 \mathrm{H}$ ), 2.75 (dd, $J=5.0,3.8 \mathrm{~Hz}, 1 \mathrm{H}$ ), 2.70 (dd, $J=5.0,2.7 \mathrm{~Hz}, 1 \mathrm{H}$ ), 2.64 (qd, $J$ $=7.1,5.0 \mathrm{~Hz}, 1 \mathrm{H}), 1.23(\mathrm{~d}, J=7.1 \mathrm{~Hz}, 3 \mathrm{H}), 0.15(\mathrm{~s}, 9 \mathrm{H}) .{ }^{13} \mathrm{C}\left\{{ }^{1} \mathrm{H}\right\} \mathbf{N M R}\left(75 \mathrm{MHz}, \mathrm{CDCl}_{3}\right): \delta 106.3,86.6,54.6$, 45.8, 29.2, 16.9, 0.2 ppm. HRMS (ESI): $\mathrm{m} / \mathrm{z}$ : calcd for $\mathrm{C}_{9} \mathrm{H}_{17} \mathrm{OSi}[\mathrm{M}+\mathrm{H}]^{+}:$169.1049, found 169.1043 . 
From 29b:
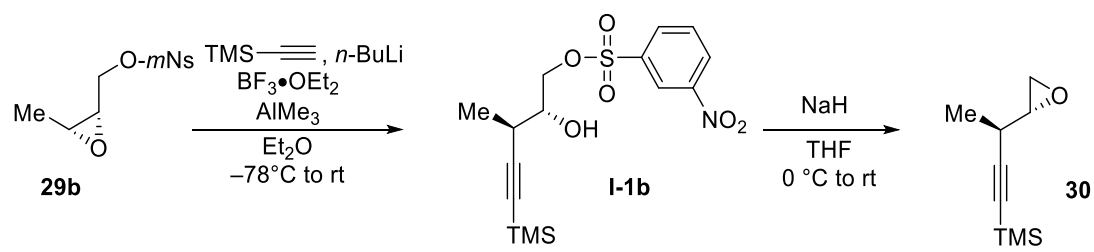

To a cooled $\left(-78{ }^{\circ} \mathrm{C}\right)$ solution of trimethylacetylene $\left(7.89 \mathrm{~mL}, 55.42 \mathrm{mmol}, 1.5\right.$ equiv) in $\mathrm{Et}_{2} \mathrm{O}(100 \mathrm{~mL})$ was added dropwise $n$-BuLi (19.2 mL, $55.42 \mathrm{mmol}, 1.5 \mathrm{eq}, 2.5 \mathrm{M}$ in hexanes), the solution was allowed to warm at $0{ }^{\circ} \mathrm{C}$ during 20 minutes. At $-78^{\circ} \mathrm{C}$ trimethylaluminium $(27.7 \mathrm{~mL}, 55.42 \mathrm{mmol}, 1.5 \mathrm{eq}, 2 \mathrm{M}$ in hexane) was added dropwise, and the mixture was allowed to warm at $0{ }^{\circ} \mathrm{C}$ for 20 minutes. At $-78{ }^{\circ} \mathrm{C}$ a solution of epoxide $29 \mathrm{~b}$ (10.09 g, $36.95 \mathrm{mmol}, 1$ equiv) in $\mathrm{Et}_{2} \mathrm{O}(25 \mathrm{~mL})$ was added via cannula, and boron trifluoride diethyl etherate $(9.12 \mathrm{~mL}, 73.90 \mathrm{mmol}, 2$ equiv) was then added dropwise. The mixture was allowed to warm at room temperature for $0.5 \mathrm{~h}$. The resulting mixture was poured into a cooled $\left(0^{\circ} \mathrm{C}\right)$ aqueous solution of $\mathrm{Na} / \mathrm{K}$ tartrate $(150 \mathrm{~mL}, 10 \%)$, and stirring was maintained for 20 minutes. The mixture was extracted with ethyl acetate, dried over $\mathrm{MgSO}_{4}$, filtered, and concentrated in vacuum. The residue was finally purified on silica gel (AcOEt/PE - 30\%) to afford expected sulfonate l-1b $(8.17 \mathrm{~g}, 22.02 \mathrm{mmol}, 60 \%)$ as a yellow oil.

$[\alpha]_{\mathrm{D}}=+6.0\left(c=3.8, \mathrm{CHCl}_{3}\right) . \mathbf{I R}$ (neat): $v=1607,1535,1366,1347,1170,1074,952,883,755,662 \mathrm{~cm}^{-1} .{ }^{1} \mathbf{H}$ NMR $\left(300 \mathrm{MHz}, \mathrm{CDCl}_{3}\right): \delta=8.79$ (ddd, $\left.J=2.3,1.8,0.5 \mathrm{~Hz}, 1 \mathrm{H}\right), 8.52(\mathrm{ddd}, J=8.3,2.3,1.1 \mathrm{~Hz}, 1 \mathrm{H}), 8.27$ (ddd, $J=7.9,1.8,1.1 \mathrm{~Hz}, 1 \mathrm{H}$ ), 7.80 (ddd, $J=8.3,7.9,0.5 \mathrm{~Hz}, 1 \mathrm{H}$ ), 4.24 (dd, $J=10.3,4.8 \mathrm{~Hz}, 1 \mathrm{H}$ ), 4.18 (dd, $J=10.3,6.2 \mathrm{~Hz}, 1 \mathrm{H}), 3.81-3.67(\mathrm{~m}, 1 \mathrm{H}), 2.73(\mathrm{qd}, J=7.1,4.5 \mathrm{~Hz}, 1 \mathrm{H}), 2.09(\mathrm{~d}, J=6.7 \mathrm{~Hz}, 1 \mathrm{H}, \mathrm{OH}), 1.23$ $(\mathrm{d}, J=7.1 \mathrm{~Hz}, 3 \mathrm{H}), 0.13(\mathrm{~s}, 9 \mathrm{H}) \mathrm{ppm} .{ }^{13} \mathrm{C}\left\{{ }^{1} \mathrm{H}\right\}$ NMR $\left(75 \mathrm{MHz}, \mathrm{CDCl}_{3}\right): \delta=148.5,138.3,133.5,130.9,128.5$, 123.4, 104.9, 89.3, 72.4, 71.5, 30.8, 17.1, 0.1 ppm. MS (ESI): $\mathrm{m} / \mathrm{z}:[\mathrm{M}+\mathrm{Na}]^{+}:$394.1. HRMS (ESI): $\mathrm{m} / \mathrm{z}$ : calcd for $\mathrm{C}_{15} \mathrm{H}_{22} \mathrm{NO}_{6} \mathrm{SiS}[\mathrm{M}+\mathrm{H}]^{+}: 372.0937$, found: 372.0938 .

To a cooled $\left(0^{\circ} \mathrm{C}\right)$ solution of 3-nosylate $\mathbf{l}-1 \mathbf{b}(8.17 \mathrm{~g}, 22.02 \mathrm{mmol}, 1$ equiv) in anhydrous THF (100 $\mathrm{mL})$ was added sodium hydride (1.05 g, $26.42 \mathrm{mmol}, 1.2 \mathrm{eq}, 60 \%$ in mineral oil) by portions and the solution was allowed to warm to room temperature. After completion of the reaction, judged by TLC monitoring, the reaction was quenched with saturated $\mathrm{NH}_{4} \mathrm{Cl}$, extracted with $\mathrm{Et}_{2} \mathrm{O}$, dried over $\mathrm{MgSO}_{4}$, filtered, and concentrated at reduced pressure. The crude material was then purified on silica gel (Et $2 \mathrm{O} / \mathrm{PE} 0 \%$ to $5 \%$ ) to afford epoxide 30 (3.61 g, $21.48 \mathrm{mmol}, 97 \%)$ as a clear oil. See above for characterization.

\section{Compound 31}

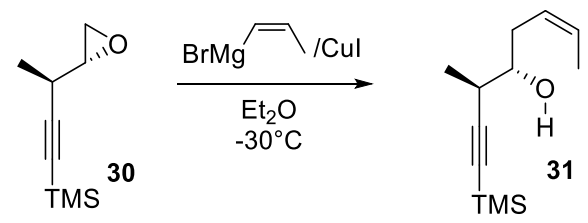

To a cooled $\left(-30^{\circ} \mathrm{C}\right.$, from a dry ice/acetone bath) solution of $\mathrm{Cul}$ (958 mg, $5.04 \mathrm{mmol}, 0.15$ equiv) in $\mathrm{Et}_{2} \mathrm{O}$ $(50 \mathrm{~mL})$ was added dropwise a solution of $(Z)$ - propenylmagnesium bromide $(45.6 \mathrm{~mL}, 50.44 \mathrm{mmol}, \mathrm{C}=1.10$ $M$ in THF, 1.5 equiv) [Prepared from Mg turning and (Z)-1-bromopropene in THF]. After 20 minutes at this temperature, epoxide 30 (5.64 g, $33.63 \mathrm{mmol}, 1$ equiv) in $\mathrm{Et}_{2} \mathrm{O}(25 \mathrm{~mL})$ was added via cannula. The reaction mixture was stirred for $1 \mathrm{~h}$ at $-30^{\circ} \mathrm{C}$, hydrolyzed by aqueous $\mathrm{NH}_{4} \mathrm{Cl} / \mathrm{NH}_{3}(2 / 1)$ solution, extracted with $\mathrm{Et}_{2} \mathrm{O}$, dried over $\mathrm{MgSO}_{4}$, filtered, and concentrated in vacuum. The crude residue was purified on silica gel $\left(\mathrm{Et}_{2} \mathrm{O} /\right.$ petroleum ether- 10\%) to yield alcohol $22(5.369 \mathrm{~g}, 25.56 \mathrm{mmol}, 76 \%)$ as a colorless oil. 
$[\alpha]^{20} \mathrm{D}=+23.3\left(c=1.3, \mathrm{CHCl}_{3}\right)$. IR (neat): $v=2163,1249,991,837,759,697 \mathrm{~cm}^{-1} .{ }^{1} \mathrm{H}$ NMR $(300 \mathrm{MHz}$, $\left.\mathrm{CDCl}_{3}\right) \delta 5.69-5.56(\mathrm{~m}, 1 \mathrm{H}), 5.51-5.39(\mathrm{~m}, 1 \mathrm{H}), 3.46(\mathrm{tdd}, J=7.0,6.0,4.4 \mathrm{~Hz}, 1 \mathrm{H}), 2.63(\mathrm{qd}, J=7.0,4.4$ $\mathrm{Hz}, 1 \mathrm{H}), 2.45-2.22(\mathrm{~m}, 2 \mathrm{H}), 1.84(\mathrm{~d}, J=6.9 \mathrm{~Hz}, 1 \mathrm{H}, \mathrm{OH}), 1.66(\mathrm{~d}, J=6.7 \mathrm{~Hz}, 3 \mathrm{H}), 1.22(\mathrm{~d}, J=7.0 \mathrm{~Hz}, 3 \mathrm{H})$, $0.16(\mathrm{~d}, J=0.5 \mathrm{~Hz}, 9 \mathrm{H}) .{ }^{13} \mathrm{C}\left\{{ }^{1} \mathrm{H}\right\}$ NMR $\left(75 \mathrm{MHz}, \mathrm{CDCl}_{3}\right) \delta 127.1,126.1,107.5,87.8,74.1,33.5,32.9,17.5$, 13.1, 0.3. HRMS (ESI): $\mathrm{m} / \mathrm{z}$ : calcd for $\mathrm{C}_{12} \mathrm{H}_{22} \mathrm{OSiNa}[\mathrm{M}+\mathrm{Na}]^{+}: 233.1332$, found 233.1329 .

\section{Compound 32}
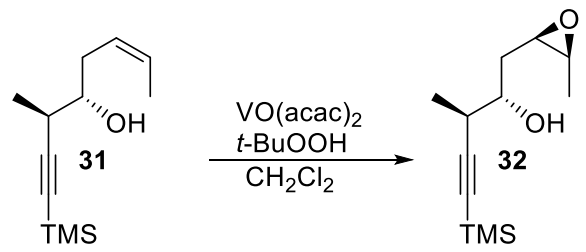

To a cooled $\left(0^{\circ} \mathrm{C}\right)$ solution of alcohol $31(5.368 \mathrm{~g}, 25.56 \mathrm{mmol}, 1$ equiv) in dry $\mathrm{CH}_{2} \mathrm{Cl}_{2}(225 \mathrm{~mL})$ was added $\mathrm{VO}(\mathrm{acac})_{2}(336 \mathrm{mg}, 1.27 \mathrm{mmol}$, 0.05 equiv) then $t$-BuOOH (13.1 mL, $51.12 \mathrm{mmol}, 2$ equiv $3.90 \mathrm{M}$ in isooctane). The reaction was stirred overnight, and an aqueous solution of $\mathrm{KI}(2 \%)$ and aqueous $10 \% \mathrm{Na}_{2} \mathrm{~S}_{2} \mathrm{O}_{3}$ were added. The mixture was stirred for further 15 minutes, and the layers were separated. The aqueous phase was extracted with AcOEt, and the combined organic layers were dried over $\mathrm{MgSO}_{4}$, filtered, and concentrated in a vacuum. The crude mixture was then purified on silica gel $\left(\mathrm{Et}_{2} \mathrm{O} /\right.$ petroleum ether- $30 \%$ to $50 \%)$ to afford epoxyalcohol $32(4.476 \mathrm{~g}, 19.80 \mathrm{mmol}, 77 \%)$ as a clear oil. The diastereoselectivity of the reaction was determined to be $43: 1$ by $\mathrm{GC}$ analysis on the crude material compared to a pseudo equimolar mixture of diastereomers obtained by epoxidation with mCPBA.

$[\alpha]^{20} \mathrm{D}=+20.4\left(c=0.88, \mathrm{CHCl}_{3}\right)$. IR (neat): $v=2168,1249,994,837$, $759,698,650 \mathrm{~cm}^{-1} .{ }^{1} \mathbf{H}$ NMR $\left(300 \mathrm{MHz}, \mathrm{CDCl}_{3}\right) \delta=\delta 3.72$ (ddt, $J=8.3$, $5.5,4.6 \mathrm{~Hz}, 1 \mathrm{H}), 3.17-3.11(\mathrm{~m}, 1 \mathrm{H}), 3.10-3.03(\mathrm{~m}, 1 \mathrm{H}), 2.68(\mathrm{qd}, J=$ 7.0, $4.8 \mathrm{~Hz}, 1 \mathrm{H}$ ), 2.24 (d, $J=5.6 \mathrm{~Hz}, 1 \mathrm{H}$ ), 1.85 (ddd, $J=14.5,5.0,4.4$

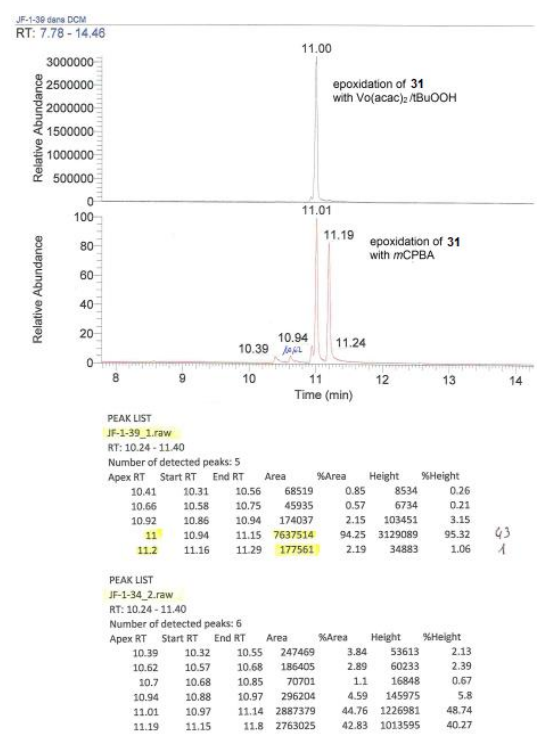
$\mathrm{Hz}, 1 \mathrm{H}$ ), 1.74 (ddd, $J=14.5,8.3,7.2 \mathrm{~Hz}, 1 \mathrm{H}), 1.30$ (d, $J=5.4 \mathrm{~Hz}, 3 \mathrm{H}), 1.23(\mathrm{~d}, J=7.0 \mathrm{~Hz}, 3 \mathrm{H}), 0.16(\mathrm{~s}, 9 \mathrm{H})$. ${ }^{13} \mathrm{C}\left\{{ }^{1} \mathrm{H}\right\}$ NMR $\left(75 \mathrm{MHz}, \mathrm{CDCl}_{3}\right) \delta 107.3,87.8,72.9,54.8,52.2,33.9,32.2,16.7,13.4,0.2$. HRMS (ESI): $\mathrm{m} / \mathrm{z}$ : calcd for $\mathrm{C}_{12} \mathrm{H}_{22} \mathrm{O}_{2} \mathrm{SiNa}[\mathrm{M}+\mathrm{Na}]^{+}:$249.1287, found 249.1282 .

\section{Compound 33}

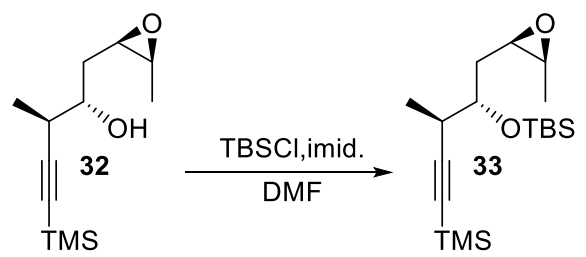

To a solution of epoxyalcohol 32 (2.772 g, $12.26 \mathrm{mmol}, 1$ equiv) in DMF (11 mL) was added TBSCl (2.214 $\mathrm{g}, 14.71 \mathrm{mmol}, 1.2$ equiv) and imidazole (1.66 g, $24.52 \mathrm{mmol}, 2$ equiv). The mixture was stirred overnight at $\mathrm{rt}$, and the reaction was quenched with water and extracted with $\mathrm{Et}_{2} \mathrm{O}$. The organic phase was then washed with brine, dried over $\mathrm{MgSO}_{4}$, filtered, and concentrated under reduced pressure. The residue was then purified on silica gel (Et $2 \mathrm{O} /$ petroleum ether: 0 to $5 \%)$ to yield protected alcohol $\mathbf{3 3}(3.391 \mathrm{~g}, 10.15 \mathrm{mmol}, 83 \%)$ as a colorless oil.

$[\alpha]^{20} \mathrm{D}=-1.8\left(c=3.7, \mathrm{CHCl}_{3}\right) .{ }^{1} \mathrm{H}$ NMR $\left(300 \mathrm{MHz} \mathrm{CDCl}_{3}\right) \delta=3.86(\mathrm{ddd}, J=7.3,4.9,4.1 \mathrm{~Hz}, 1 \mathrm{H}), 3.12-2.93$ $(\mathrm{m}, 2 \mathrm{H}), 2.67$ (qd, $J=7.0,4.1 \mathrm{~Hz}, 1 \mathrm{H}), 1.91-1.70(\mathrm{~m}, 2 \mathrm{H}), 1.30(\mathrm{~d}, J=5.5 \mathrm{~Hz}, 3 \mathrm{H}), 1.16(\mathrm{~d}, J=7.0 \mathrm{~Hz}, 3 \mathrm{H})$, 
$0.90(\mathrm{~s}, 9 \mathrm{H}), 0.12(\mathrm{~s}, 9 \mathrm{H}), 0.08(\mathrm{~s}, 3 \mathrm{H}), 0.07(\mathrm{~s}, 3 \mathrm{H}) .{ }^{13} \mathrm{C}\left\{{ }^{1} \mathrm{H}\right\}$ NMR $\left(75 \mathrm{MHz}, \mathrm{CDCl}_{3}\right): \delta=108.9,86.3,72.8$, 54.8, 52.6, 33.5, 31.3, 25.9 (3C), 18.2, 14.9, 13.4, 0.3, -4.3, -4.5. HRMS (ESI): $\mathrm{m} / \mathrm{z}$ : calcd for $\mathrm{C}_{18} \mathrm{H}_{37} \mathrm{O}_{2} \mathrm{Si}_{2}$ $[\mathrm{M}+\mathrm{H}]^{+}: 341.2332$, found 341.2333 .

\section{Compounds 34 and its isomer 35}

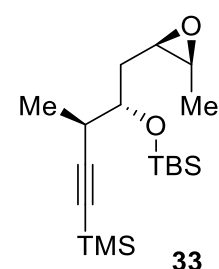

33
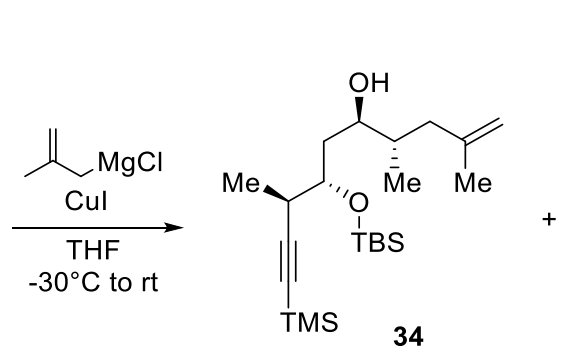

34

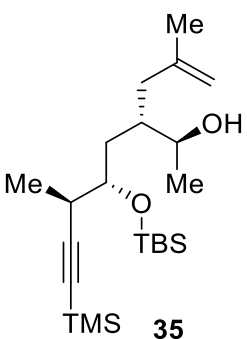

35

To a cooled $\left(-30{ }^{\circ} \mathrm{C}\right.$, from a dry ice/acetone bath) solution of Cul $(255 \mathrm{mg}, 1.347 \mathrm{mmol}, 0.6$ equiv) in anhydrous THF (15 mL) was added dropwise a solution of freshly prepared of 2-methyl-2-propenylmagnesium chloride (14 mL, $13.47 \mathrm{mmol}, \mathrm{C}=0.8 \mathrm{M}$ in THF, 6 equiv) [prepared form Mg turnings and 2-methyl-allyl chloride maintained at $10-15{ }^{\circ} \mathrm{C}$ by an ice bath in THF. After 10 minutes at this temperature, protected alcohol 33 (750 mg, $2.245 \mathrm{mmol}, 1$ equiv) in THF (15 mL) was added via cannula, and the mixture was allowed to warm to room temperature for 30 minutes. After consumption of the starting material, the reaction was hydrolyzed with aqueous $\mathrm{NH}_{4} \mathrm{Cl} / \mathrm{NH}_{3}(2 / 1)$ solution, extracted with $\mathrm{Et}_{2} \mathrm{O}$, dried over $\mathrm{MgSO}_{4}$, filtered, and concentrated in vacuum to yield alcohol 34 (684 $\mathrm{mg}, 1.72 \mathrm{mmol}, 77 \%)$ as a clear oil. Further elution afforded the regioisomer 35 (106 mg, $0.269 \mathrm{mmol}, 12 \%)$ as a clear oil.

\section{Isomer 34:}

$[\alpha]_{\mathrm{D}}=-6.9\left(c=2.0, \mathrm{CHCl}_{3}\right)$. IR (neat): $v=2958,2167,1249,1067,1025,836,776,758,646 \mathrm{~cm}^{-1} .{ }^{1} \mathbf{H}$ NMR $\left(300 \mathrm{MHz}, \mathrm{CDCl}_{3}\right): \delta=4.76$ (brs, $\left.1 \mathrm{H}\right), 4.69$ (brs, $\left.1 \mathrm{H}\right), 4.01$ (dt, $\left.J=9.2,3.9 \mathrm{~Hz}, 1 \mathrm{H}\right), 3.66-3.60(\mathrm{~m}, 1 \mathrm{H}), 2.83$ $(\mathrm{d}, J=2.0 \mathrm{~Hz}, 1 \mathrm{H}, \mathrm{OH}$ ), 2.68 (qd, $J=7.0,3.9 \mathrm{~Hz}, 1 \mathrm{H}), 2.22$ (bq, $J=9.2 \mathrm{~Hz}, 1 \mathrm{H}$ ), 1.94 (ddd, $J=14.3,3.9,1.9$ $\mathrm{Hz}, 1 \mathrm{H}), 1.88-1.76(\mathrm{~m}, 2 \mathrm{H}), 1.72(\mathrm{~s}, 3 \mathrm{H}), 1.50$ (dt, $J=14.3,9.2 \mathrm{~Hz}, 1 \mathrm{H}), 1.15(\mathrm{~d}, J=7.0 \mathrm{~Hz}, 3 \mathrm{H}), 0.91$ (s, $9 \mathrm{H}), 0.87(\mathrm{~d}, J=6.4 \mathrm{~Hz}, 3 \mathrm{H}), 0.14(\mathrm{~s}, 12 \mathrm{H}), 0.10(\mathrm{~s}, 3 \mathrm{H}) \mathrm{ppm} .{ }^{13} \mathrm{C}\left\{{ }^{1} \mathrm{H}\right\} \mathbf{N M R}\left(75 \mathrm{MHz}, \mathrm{CDCl}_{3}\right): \delta=144.5$, 111.8, 108.7, 86.6, 75.1, 74.6, 41.3, 36.5, 34.5, 33.0, 25.9, 22.2, 18.1, 14.7, 14.0, 0.2, -4.1, -4.6 ppm. MS (ESI): $m / z:[M+H]^{+}:$397.4. HRMS (ESI): $m / z$ : calcd for $\mathrm{C}_{22} \mathrm{H}_{45} \mathrm{O}_{2} \mathrm{Si}_{2}[\mathrm{M}+\mathrm{H}]^{+}: 397.2958$, found: 397.2961 .

\section{Isomer 35:}

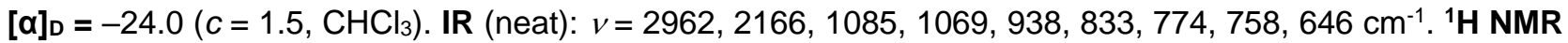
$\left(300 \mathrm{MHz}, \mathrm{CDCl}_{3}\right): \delta=4.76$ (brs, $\left.1 \mathrm{H}\right), 4.72(\mathrm{brs}, 1 \mathrm{H}), 3.90-3.76(\mathrm{~m}, 2 \mathrm{H}), 2.64(\mathrm{qd}, J=7.0,3.9 \mathrm{~Hz}, 1 \mathrm{H}), 2.03$ (dd, $J=14.1,7.2 \mathrm{~Hz}, 1 \mathrm{H}), 1.95(\mathrm{dd}, J=14.1,7.2 \mathrm{~Hz}, 1 \mathrm{H}), 1.82(\mathrm{~m}, 2 \mathrm{H}, 1 \mathrm{OH}), 1.73(\mathrm{~s}, 3 \mathrm{H}), 1.57-1.50(\mathrm{~m}$, 2H), $1.10(\mathrm{~d}, J=7.0 \mathrm{~Hz}, 3 \mathrm{H}), 1.08(\mathrm{~d}, J=6.5 \mathrm{~Hz}, 3 \mathrm{H}), 0.87(\mathrm{~s}, 9 \mathrm{H}), 0.11(\mathrm{~s}, 9 \mathrm{H}), 0.07(\mathrm{~s}, 3 \mathrm{H}), 0.05(\mathrm{~s}, 3 \mathrm{H})$ ppm. ${ }^{13} \mathrm{C}\left\{{ }^{1} \mathrm{H}\right\}$ NMR $\left(75 \mathrm{MHz}, \mathrm{CDCl}_{3}\right): \delta=144.3,112.5,109.2,86.3,73.4,69.5,40.3,39.1,33.4,32.0,26.1$, 22.6, 18.4, 18.2, 14.1, 0.3, -4.1, -4.2 ppm. MS (ESI): $\mathrm{m} / \mathrm{z}:[\mathrm{M}+\mathrm{H}]^{+}:$397.4, [M+Na] $]^{+}:$419.4. HRMS (ESI): $\mathrm{m} / \mathrm{z}$ : calcd for $\mathrm{C}_{22} \mathrm{H}_{45} \mathrm{O}_{2} \mathrm{Si}_{2}[\mathrm{M}+\mathrm{H}]^{+}:$397.2958, found: 397.2959. 


\section{Compound 36}
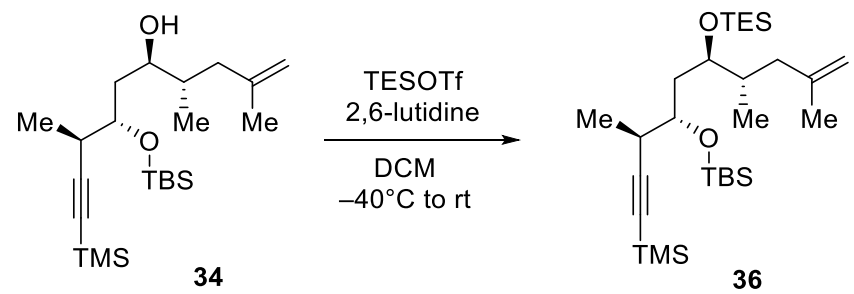

To a cooled $\left(-40^{\circ} \mathrm{C}\right.$, from a dry ice/acetone bath) solution of alcohol 34 (618 $\left.\mathrm{mg}, 1.56 \mathrm{mmol}, 1 \mathrm{equiv}\right)$ and 2,6-Iutidine ( $435 \mu \mathrm{L}, 3.744 \mathrm{mmol}, 2.4$ equiv) in DCM $(40 \mathrm{~mL})$ was added dropwise TESOTf (422 $\mu \mathrm{L}, 1.872$ mmol, 1.2 equiv). After 15 minutes, the mixture was allowed to warm to room temperature for $1 \mathrm{~h}$. Water was then added, the reaction medium was extracted with $\mathrm{Et}_{2} \mathrm{O}$, dried over $\mathrm{MgSO}_{4}$, filtered, and concentrated under reduced pressure. The crude mixture was purified on silica gel $\left(\mathrm{Et}_{2} \mathrm{O} / \mathrm{PE}-1.5 \%\right)$ to furnish TES-ether 36 (757 $\mathrm{mg}, 1.48 \mathrm{mmol}, 95 \%)$ as a colorless oil.

$[\alpha]_{\mathrm{D}}=+7.8\left(c=1.9, \mathrm{CHCl}_{3}\right)$. IR (neat): $v=2957,2933,2877,2172,1460,1375,1250,1111,1004,837,774$, $742 \mathrm{~cm}^{-1} .{ }^{1} \mathrm{H}$ NMR $\left(300 \mathrm{MHz}, \mathrm{CDCl}_{3}\right): \delta=4.75$ (brs, $\left.1 \mathrm{H}\right), 4.72(\mathrm{brs}, 1 \mathrm{H}), 3.75(\mathrm{td}, J=6.5,3.1 \mathrm{~Hz}, 1 \mathrm{H}), 3.73$ (td, $J=6.6,2.2 \mathrm{~Hz}, 1 \mathrm{H}), 2.61(\mathrm{qd}, J=7.0,3.2 \mathrm{~Hz}, 1 \mathrm{H}), 2.10(\mathrm{~m}, 1 \mathrm{H}), 1.89(\mathrm{dt}, J=13.8,6.1 \mathrm{~Hz}, 1 \mathrm{H}), 1.86-$ $1.77(\mathrm{~m}, 2 \mathrm{H}), 1.71(\mathrm{~s}, 3 \mathrm{H}), 1.61-1.47(\mathrm{~m}, 1 \mathrm{H}), 1.15(\mathrm{~d}, J=7.0 \mathrm{~Hz}, 3 \mathrm{H}), 0.96(\mathrm{t}, J=7.8 \mathrm{~Hz}, 9 \mathrm{H}), 0.90(\mathrm{~s}, 9 \mathrm{H})$, $0.86(\mathrm{~d}, J=6.3 \mathrm{~Hz}, 3 \mathrm{H}) 0.58(\mathrm{q}, J=7.8 \mathrm{~Hz}, 6 \mathrm{H}), 0.12(\mathrm{~s}, 9 \mathrm{H}), 0.06(\mathrm{~s}, 3 \mathrm{H}), 0.05(\mathrm{~s}, 3 \mathrm{H}) \mathrm{ppm} .{ }^{13} \mathrm{C}\left\{{ }^{1} \mathrm{H}\right\}$ NMR $\left(75 \mathrm{MHz}, \mathrm{CDCl}_{3}\right): \delta=144.5,111.8,108.9,86.0,72.9,71.7,39.9,37.2,35.4,32.8,26.0,22.4,18.2,15.9$, 15.0, 7.1, 5.4, 0.3, $-4.2,-4.4 \mathrm{ppm}$. MS (ESI): $\mathrm{m} / \mathrm{z}:[\mathrm{M}+\mathrm{H}]^{+}: 551.4,[\mathrm{M}+\mathrm{Na}]^{+}: 533.4$. HRMS (ESI): $\mathrm{m} / \mathrm{z}$ : calcd for $\mathrm{C}_{28} \mathrm{H}_{59} \mathrm{O}_{2} \mathrm{Si}_{3}[\mathrm{M}+\mathrm{H}]^{+}: 511.3823$, found: 511.3821 .

\section{Compound 37}

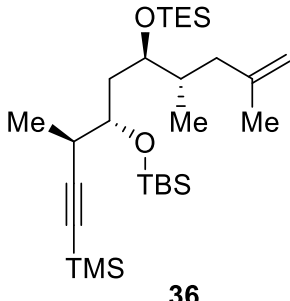

36

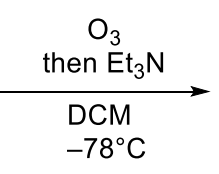

To a cooled $\left(-78{ }^{\circ} \mathrm{C}\right)$ solution of TES-ether $36(70 \mathrm{mg}, 0.137 \mathrm{mmol}, 1$ equiv) in DCM $(1.5 \mathrm{~mL})$ was added a cooled $\left(-78{ }^{\circ} \mathrm{C}\right)$ saturated solution of $\mathrm{O}_{3}$ in $\mathrm{DCM}(5.5 \mathrm{~mL}, 0.137 \mathrm{mmol}, 1 \mathrm{eq}, \mathrm{C}=0.025 \mathrm{M}$, titrated by iodometry) in one portion. ${ }^{[10]}$ After 15 minutes, the reaction was quenched with $\mathrm{Et}_{3} \mathrm{~N}(90 \mu \mathrm{L}, 0.635$ mmol, 5 equiv), and the resulting mixture was allowed to warm to room temperature. The hydrolysis was then continued by adding $2 \%$ aqueous $\mathrm{KI}$ and saturated $\mathrm{Na}_{2} \mathrm{~S}_{2} \mathrm{O}_{3}$, and the mixture was stirred for 3 hours. Then the layers were separated, the aqueous phase was extracted with $\mathrm{Et}_{2} \mathrm{O}$, dried over $\mathrm{MgSO}_{4}$, filtered, and concentrated in a vacuum. The crude was then purified on silica gel ( $\left.\mathrm{Et} \mathrm{t}_{2} \mathrm{O} / \mathrm{PE}-3 \%\right)$ to afford methylketone 37 (44 mg, $0.0859 \mathrm{mmol}, 63 \%)$ as a colorless oil.

$[\alpha]_{D}=-6.1\left(c=2.8, \mathrm{CHCl}_{3}\right)$. IR (neat): $v=2957,2878,2164,1719,1461,1249,1066,835,774,742,649$ $\mathrm{cm}^{-1} .{ }^{1} \mathrm{H}$ NMR $\left(300 \mathrm{MHz}, \mathrm{CDCl}_{3}\right): \delta=3.73(\mathrm{~m}, 2 \mathrm{H}), 2.59(\mathrm{qd}, J=7.1,3.6 \mathrm{~Hz}, 1 \mathrm{H}), 2.49(\mathrm{dd}, J=16.3,3.4 \mathrm{~Hz}$, $1 \mathrm{H}), 2.37-2.21(\mathrm{~m}, 2 \mathrm{H}), 2.14(\mathrm{~s}, 3 \mathrm{H}), 1.78(\mathrm{ddd}, J=13.6,7.7,4.3 \mathrm{~Hz}, 1 \mathrm{H}), 1.57$ (ddd, $J=13.6,8.7,5.8 \mathrm{~Hz}$, $1 \mathrm{H}), 1.14(\mathrm{~d}, J=7.1 \mathrm{~Hz}, 3 \mathrm{H}), 0.96(\mathrm{t}, J=7.8 \mathrm{~Hz}, 9 \mathrm{H}), 0.89(\mathrm{~s}, 12 \mathrm{H}), 0.60(\mathrm{q}, J=8.0 \mathrm{~Hz}, 6 \mathrm{H}), 0.12(\mathrm{~s}, 9 \mathrm{H})$, $0.06(\mathrm{~s}, 3 \mathrm{H}), 0.06(\mathrm{~s}, 3 \mathrm{H}) \mathrm{ppm} .{ }^{13} \mathrm{C}\left\{{ }^{1} \mathrm{H}\right\} \mathrm{NMR}\left(75 \mathrm{MHz}, \mathrm{CDCl}_{3}\right): \delta=208.7,108.8,86.1,72.7,71.2,44.9,37.6$, $33.1,32.9,30.8,25.9,18.1,16.6,15.1,7.1,5.3,0.3,-4.3,-4.4 \mathrm{ppm}$. MS (ESI): $\mathrm{m} / z:[\mathrm{M}+\mathrm{H}]^{+}: 513.3,[\mathrm{M}+\mathrm{Na}]^{+}$: 535.3. HRMS (ESI): $\mathrm{m} / z$ : calcd for $\mathrm{C}_{27} \mathrm{H}_{57} \mathrm{O}_{3} \mathrm{Si}_{3}[\mathrm{M}+\mathrm{H}]^{+}: 513.3616$, found: 513.3610 . 


\section{Compound 38}
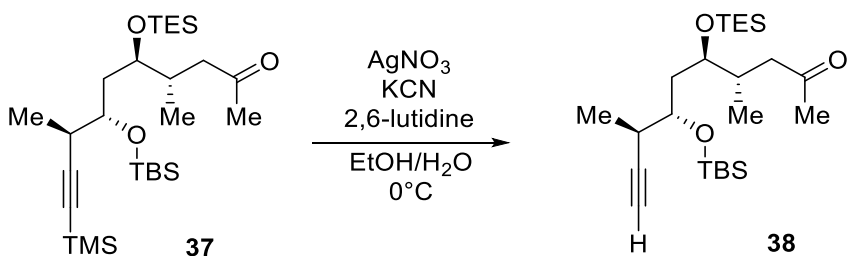

To a cooled $\left(0^{\circ} \mathrm{C}\right)$ solution of methylketone $37\left(215 \mathrm{mg}, 0.419 \mathrm{mmol}, 1\right.$ equiv) in $\mathrm{EtOH} / \mathrm{H}_{2} \mathrm{O}(5.5 \mathrm{~mL}, 9: 1)$ was added 2,6-lutidine $(3 \mathrm{~mL})$ followed by $\mathrm{AgNO}_{3}(284 \mathrm{mg}, 1.676 \mathrm{mmol}, 4$ equiv) in one portion and the flask was covered with aluminum foil to protect from light. After 20 minutes, a solution of KCN $(408 \mathrm{mg}, 6.28 \mathrm{mmol}$, 15 equiv) in $\mathrm{H}_{2} \mathrm{O}(1 \mathrm{~mL})$ was added. After consumption of the starting material, the reaction mixture was hydrolyzed with saturated $\mathrm{NH}_{4} \mathrm{Cl}$, extracted with $\mathrm{Et}_{2} \mathrm{O}$, dried over $\mathrm{MgSO}_{4}$, filtered, and concentrated under reduced pressure. The crude mixture was then purified on silica gel (Et $2 \mathrm{O} / \mathrm{PE}-7 \%)$ to yield alkyne 38 (175 $\mathrm{mg}, 0.397 \mathrm{mmol}, 95 \%)$ as a colorless oil.

$[\alpha]_{D}=-7.3\left(c=2.4, \mathrm{CHCl}_{3}\right)$. IR (neat): $v=3277,2957,2878,2162,1718,1461,1254,1080,1005,835,774$, $743 \mathrm{~cm}^{-1} .{ }^{1} \mathrm{H}$ NMR $\left(300 \mathrm{MHz}, \mathrm{CDCl}_{3}\right): \delta=3.77$ (ddd, $\left.J=7.5,5.0,3.7 \mathrm{~Hz}, 1 \mathrm{H}\right), 3.71(\mathrm{td}, J=6.2,2.2 \mathrm{~Hz}, 1 \mathrm{H})$, $2.64-2.47(\mathrm{~m}, 2 \mathrm{H}), 2.30-2.17(\mathrm{~m}, 2 \mathrm{H}), 2.12(\mathrm{~s}, 3 \mathrm{H}), 2.01(\mathrm{~d}, J=2.4 \mathrm{~Hz}, 1 \mathrm{H}), 1.77$ (ddd, $J=13.9,7.2,5.0$ $\mathrm{Hz}, 1 \mathrm{H}), 1.57$ (ddd, $J=13.9,7.5,6.3 \mathrm{~Hz}, 1 \mathrm{H}), 1.15(\mathrm{~d}, J=7.0 \mathrm{~Hz}, 3 \mathrm{H}), 0.96(\mathrm{t}, J=7.8 \mathrm{~Hz}, 9 \mathrm{H}), 0.92(\mathrm{~d}, J=$ $6.5 \mathrm{~Hz}, 3 \mathrm{H}), 0.89(\mathrm{~s}, 9 \mathrm{H}), 0.59(\mathrm{q}, J=7.8 \mathrm{~Hz}, 6 \mathrm{H}), 0.06(\mathrm{~s}, 3 \mathrm{H}), 0.06(\mathrm{~s}, 3 \mathrm{H}) \mathrm{ppm} .{ }^{13} \mathrm{C}\left\{{ }^{1} \mathrm{H}\right\} \mathrm{NMR}(75 \mathrm{MHz}$, $\left.\mathrm{CDCl}_{3}\right): \delta=208.8,86.0,72.6,71.1,70.1,45.1,37.6,33.0,31.8,30.7,26.0,18.2,16.5,15.1,7.1,5.3,-4.3$, $-4.4 \mathrm{ppm}$. MS (ESI): $\mathrm{m} / \mathrm{z}:[\mathrm{M}+\mathrm{H}]^{+}: 441.3,[\mathrm{M}+\mathrm{Na}]^{+}:$463.3. HRMS (ESI): $\mathrm{m} / \mathrm{z}$ : calcd for $\mathrm{C}_{24} \mathrm{H}_{49} \mathrm{O}_{3} \mathrm{Si}_{2}[\mathrm{M}+\mathrm{H}]^{+}$: 441.3220, found: 441.3227 .

\section{Compound 39}
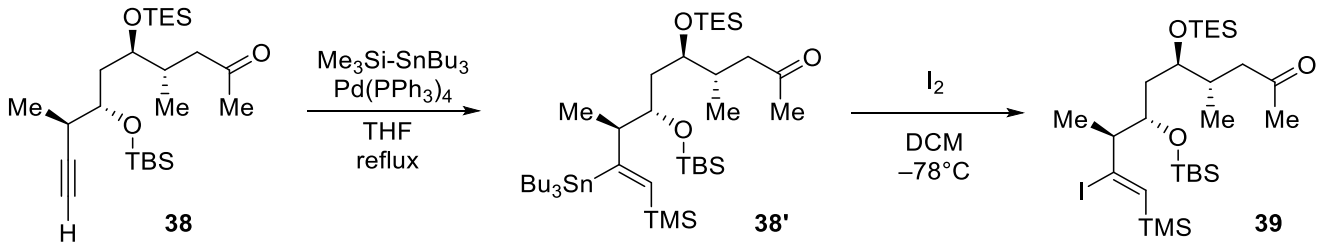

To a solution of alkyne 38 (175 mg, $0.397 \mathrm{mmol}, 1$ equiv) in THF (2.5 mL) was added trimethylsilyltributylstannane (216 mg, $0.595 \mathrm{mmol}, 1.5$ equiv) followed by $\mathrm{Pd}\left(\mathrm{PPh}_{3}\right)_{4}(27 \mathrm{mg}, 23.8 \mu \mathrm{mol}, 0.06$ equiv) and the reaction mixture was heated to reflux during $12 \mathrm{~h}$, with the help of an oil bath. After this, the reaction was cooled to room temperature, then filtered on a silica plug $(3 \mathrm{~cm})$ and eluted with $\mathrm{Et}_{2} \mathrm{O}$. The filtrate was then concentrated and purified on silica gel (Et $2 \mathrm{O} / \mathrm{PE}-1 \%)$ to obtain the corresponding vinylstannane 38' (213 $\mathrm{mg}, 0.265 \mathrm{mmol}, 67 \%)$ as a colorless oil.

$[\alpha]_{D}=-25.0\left(c=1.6, \mathrm{CHCl}_{3}\right)$. IR (neat): $v=2956,1719,1461,1247,1003,860,833,773,743,722,664 \mathrm{~cm}^{-}$ 1. ${ }^{1} \mathrm{H}$ NMR $\left(300 \mathrm{MHz}, \mathrm{CDCl}_{3}\right): \delta=6.66(\mathrm{~s}, J \mathrm{Sn}-\mathrm{H}=185.4 \mathrm{~Hz}, 1 \mathrm{H}), 3.79(\mathrm{ddd}, J=7.5,5.0,4.0 \mathrm{~Hz}, 1 \mathrm{H}), 3.73$ (ddd, $J=7.7,6.4,2.0 \mathrm{~Hz}, 1 \mathrm{H}$ ), 2.59 (qd, $J=7.0,4.0 \mathrm{~Hz}, 1 \mathrm{H}), 2.26(\mathrm{~d}, J=6.5 \mathrm{~Hz}, 1 \mathrm{H}), 2.19-2.07(\mathrm{~m}, 1 \mathrm{H}$ ), 2.10 (brs, 3H), $1.53-1.17(\mathrm{~m}, 14 \mathrm{H}), 0.98(\mathrm{t}, J=7.9 \mathrm{~Hz}, 9 \mathrm{H}), 0.95-0.86(\mathrm{~m}, 30 \mathrm{H}), 0.59(\mathrm{q}, J=7.9 \mathrm{~Hz}, 6 \mathrm{H})$, $0.09(\mathrm{~s}, 15 \mathrm{H}) \mathrm{ppm} .{ }^{13} \mathrm{C}\left\{{ }^{1} \mathrm{H}\right\}$ NMR $\left(75 \mathrm{MHz}, \mathrm{CDCl}_{3}\right): \delta=208.5,167.2,144.7,73.3,72.1,50.4,44.5,37.3,32.5$, $30.9,29.4,27.7,26.1,18.3,17.1,15.2,13.8,11.9,7.1,5.4,0.5,-3.7,-4.2 \mathrm{ppm}$. MS (ESI): $\mathrm{m} / \mathrm{z}:\left[\mathrm{M}^{120} \mathrm{Sn}+\mathrm{H}\right]^{+}$: 805.5, $\left[\mathrm{M}^{120} \mathrm{Sn}+\mathrm{Na}\right]^{+}:$827.4. HRMS (ESI): $\mathrm{m} / \mathrm{z}$ : calcd for $\mathrm{C}_{39} \mathrm{H}_{85} \mathrm{O}_{3} \mathrm{Si}_{3}{ }^{120} \mathrm{Sn}[\mathrm{M}+\mathrm{H}]^{+}:$805.4829, found: 805.4838 .

To a cooled $\left(-78^{\circ} \mathrm{C}\right)$ solution of above vinylstannane $38^{\prime}$ ( $213 \mathrm{mg}, 0.265 \mathrm{mmol}, 1$ equiv) in $\mathrm{DCM}(5 \mathrm{~mL})$ was added a solution of iodine $(213 \mu \mathrm{L}, 1 \mathrm{M}$ in DCM) dropwise. After a persistent coloration of the solution 
and consumption of the starting material by TLC monitoring, the reaction mixture was quenched by saturated $\mathrm{Na}_{2} \mathrm{~S}_{2} \mathrm{O}_{3}$. After separating the phases, the aqueous layer was extracted with $\mathrm{Et}_{2} \mathrm{O}$; the organic phases were combined, dried over $\mathrm{MgSO}_{4}$, filtered, and concentrated. The residue was then purified on silica gel $\left(\mathrm{Et} \mathrm{t}_{2} \mathrm{O} / \mathrm{PE}\right.$ $-4 \%$ ) to afford vinyl iodide 39 (157 $\mathrm{mg}, 0.245 \mathrm{mmol}, 92 \%)$ as pale yellow oil.

$[\alpha]_{\mathrm{D}}=-21.4\left(c=2.1, \mathrm{CHCl}_{3}\right)$. IR (neat): $v=2956,1719,1248,1109,1005,834,774,743,723 \mathrm{~cm}^{-1} .{ }^{1} \mathbf{H}$ NMR $\left(300 \mathrm{MHz}, \mathrm{CDCl}_{3}\right): \delta=6.56(\mathrm{~d}, J=1.0 \mathrm{~Hz}, 1 \mathrm{H}), 4.11(\mathrm{td}, J=8.1,4.0 \mathrm{~Hz}, 1 \mathrm{H}), 3.79(\mathrm{td}, J=6.9,1.6 \mathrm{~Hz}, 1 \mathrm{H})$, 2.70 (qdd, $J=6.9,4.0,1.0 \mathrm{~Hz}, 1 \mathrm{H}), 2.41(\mathrm{~d}, J=13.6 \mathrm{~Hz}, 1 \mathrm{H}), 2.33-2.18(\mathrm{~m}, 2 \mathrm{H}), 2.14(\mathrm{~s}, 3 \mathrm{H}), 1.44(\mathrm{~m}, 2 \mathrm{H})$, $1.08(\mathrm{~d}, J=6.9 \mathrm{~Hz}, 3 \mathrm{H}), 0.96(\mathrm{t}, J=7.9 \mathrm{~Hz}, 9 \mathrm{H}), 0.94(\mathrm{~d}, J=6.3 \mathrm{~Hz}, 3 \mathrm{H}), 0.90(\mathrm{~s}, 9 \mathrm{H}), 0.61(\mathrm{q}, J=7.9 \mathrm{~Hz}$, $6 \mathrm{H}), 0.19(\mathrm{~s}, 9 \mathrm{H}), 0.12(\mathrm{~s}, 3 \mathrm{H}), 0.11(\mathrm{~s}, 3 \mathrm{H}) \mathrm{ppm} .{ }^{13} \mathrm{C}\left\{{ }^{1} \mathrm{H}\right\}$ NMR $\left(75 \mathrm{MHz}, \mathrm{CDCl}_{3}\right): \delta=208.7,137.5,125.1$, 73.0, 71.0, 56.6, 44.4, 35.7, 32.3, 30.7, 26.0, 18.1, 17.3, 13.7, 7.1, 5.3, -0.9, -4.1, -4.2 ppm. MS (ESI): $\mathrm{m} / \mathrm{z}$ : $[\mathrm{M}+\mathrm{H}]^{+}:$641.3, $[\mathrm{M}+\mathrm{Na}]^{+}:$663.3. HRMS (ESI): $\mathrm{m} / \mathrm{z}$ : calcd for $\mathrm{C}_{27} \mathrm{H}_{58} \mathrm{O}_{3} \mathrm{Si}_{3} \mathrm{l}[\mathrm{M}+\mathrm{H}]^{+}:$: 641.2739, found: 641.2736.

\section{Compound 40}
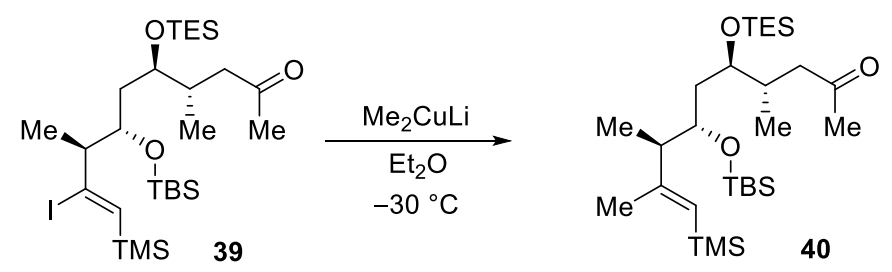

To a cooled $\left(-30^{\circ} \mathrm{C}\right.$, from a dry ice/acetone bath) suspension of Cul ( $\left.243 \mathrm{mg}, 1.280 \mathrm{mmol}, 5.5 \mathrm{equiv}\right)$ in

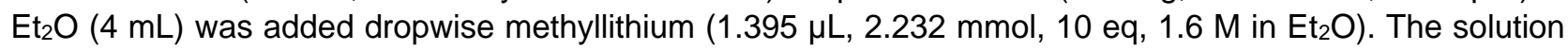
turned yellow, and Cul dissolved upon the addition of MeLi. After $5 \mathrm{~min}$, a solution of vinyl iodide 39 (149 mg, $0.232 \mathrm{mmol}, 1$ equiv) in $\mathrm{Et}_{2} \mathrm{O}(2 \mathrm{~mL})$ was added. After $20 \mathrm{~min}$ at this temperature and completion of the starting material, aqueous $\mathrm{NH}_{4} \mathrm{Cl} / \mathrm{NH}_{3}(2 / 1)$ was poured into the mixture. The medium was then extracted with $\mathrm{Et}_{2} \mathrm{O}$, dried over $\mathrm{MgSO}_{4}$, filtered, and concentrated under vacuum. The crude mixture was then purified on silica gel (Et $\left.t_{2} \mathrm{O} / \mathrm{PE}-1.5 \%\right)$ to give olefin $40(114 \mathrm{mg}, 0.216 \mathrm{mmol}, 93 \%)$ as a colorless oil.

$[\alpha]_{D}=-16.8\left(c=1.9, \mathrm{CHCl}_{3}\right) . \mathbf{I R}$ (neat): $v=2955,1719,1611,1461,1248,1041,1005,833,773,745,664$ $\mathrm{cm}^{-1} .{ }^{1} \mathrm{H}$ NMR $\left(300 \mathrm{MHz}, \mathrm{CDCl}_{3}\right): \delta=5.29-5.11(\mathrm{~s}, 1 \mathrm{H}), 3.81$ (ddd, $J=8.1,4.8,3.5 \mathrm{~Hz}, 1 \mathrm{H}$ ), 3.71 (ddd, $J=$ 8.1, 6.0, $2.3 \mathrm{~Hz}, 1 \mathrm{H}), 2.36-2.19(\mathrm{~m}, 3 \mathrm{H}), 2.20-2.11(\mathrm{~m}, 1 \mathrm{H}), 2.10(\mathrm{~s}, 3 \mathrm{H}), 1.78(\mathrm{~s}, 3 \mathrm{H}), 1.45$ (ddd, $J=13.8$, 7.9, $6.0 \mathrm{~Hz}, 1 \mathrm{H}$ ), 1.34 (ddd, $J=13.8,7.6,4.8 \mathrm{~Hz}, 1 \mathrm{H}), 1.01$ (d, $J=7.0 \mathrm{~Hz}, 3 \mathrm{H}), 0.96(\mathrm{t}, J=7.9 \mathrm{~Hz}, 9 \mathrm{H}), 0.90$ $(\mathrm{s}, 9 \mathrm{H}), 0.89$ (d, $J=6.4 \mathrm{~Hz}, 3 \mathrm{H}), 0.60(\mathrm{q}, J=7.9 \mathrm{~Hz}, 6 \mathrm{H}), 0.08(\mathrm{~s}, 12 \mathrm{H}), 0.07(\mathrm{~s}, 3 \mathrm{H}) \mathrm{ppm} .{ }^{13} \mathrm{C}\left\{{ }^{1} \mathrm{H}\right\}$ NMR $(75$ $\left.\mathrm{MHz}, \mathrm{CDCl}_{3}\right): \delta=208.6,156.7,124.6,73.1,71.1,49.8,44.9,36.8,32.9,30.8,26.0,21.6,18.2,16.8,13.3$, 7.1, 5.3, 0.2, -4.2, -4.3 ppm. MS (ESI): $\mathrm{m} / \mathrm{z}:[\mathrm{M}+\mathrm{Na}]^{+}: 551.4$. HRMS (ESI): $\mathrm{m} / \mathrm{z}$ : calcd for $\mathrm{C}_{28} \mathrm{H}_{60} \mathrm{O}_{3} \mathrm{Si}_{3} \mathrm{Na}$ $[\mathrm{M}+\mathrm{Na}]^{+}: 551.3748$, found: 551.3743 .

\section{Compound 41}
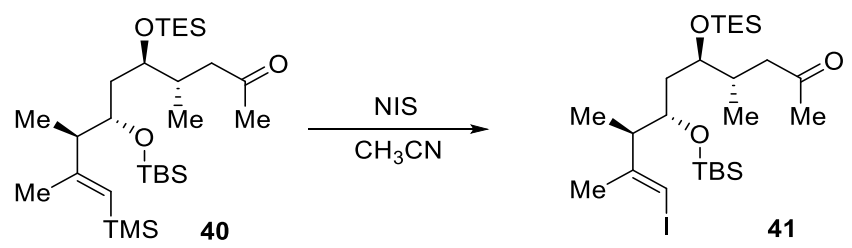

Olefin 40 (34 mg, $62.4 \mu \mathrm{mol}, 1$ equiv) was dissolved into $\mathrm{CH}_{3} \mathrm{CN}(1.5 \mathrm{~mL})$ at $0{ }^{\circ} \mathrm{C}$, and the flask was protected from light by aluminum foil. Then, NIS ( $22 \mathrm{mg}, 96.4 \mu \mathrm{mol}, 1.5$ equiv) was added to the mixture. The reaction mixture was allowed to slowly warm to rt over $2.5 \mathrm{~h}$; the reaction was quenched with a saturated solution of $\mathrm{Na}_{2} \mathrm{~S}_{2} \mathrm{O}_{3}$ and then extracted with $\mathrm{Et}_{2} \mathrm{O}$, dried over $\mathrm{MgSO}_{4}$, filtered, and concentrated in a vacuum. 


\section{SUPPORTING INFORMATION}

The residue was then purified on silica gel ( $\mathrm{Et}{ }_{2} \mathrm{O} / \mathrm{PE}-2 \%$ ) to yield the vinyliodide 41 ( $27 \mathrm{mg}, 46.3 \mu \mathrm{mol}, 74 \%$ ) as pale yellow oil.

$[\alpha]^{20} \mathrm{D}=-18.3\left(c=1.8, \mathrm{CHCl}_{3}\right)$. IR (neat): $v=2956,2930,2877,2856,1718,1609,1249,1068,1042,834$, $773,744 \mathrm{~cm}^{-1} .{ }^{1} \mathrm{H}$ NMR $\left(300 \mathrm{MHz}, \mathrm{CDCl}_{3}\right): \delta=5.88(\mathrm{~s}, 1 \mathrm{H}), 3.78(\mathrm{td}, J=6.4,3.4 \mathrm{~Hz}, 1 \mathrm{H}), 3.67$ (ddd, $J=8.1$, 5.5, $2.5 \mathrm{~Hz}, 1 \mathrm{H}$ ), 2.52 (qd, $J=7.0,3.4 \mathrm{~Hz}, 1 \mathrm{H}), 2.37-2.10(\mathrm{~m}, 3 \mathrm{H}), 2.15(\mathrm{~s}, 3 \mathrm{H}), 1.83(\mathrm{~s}, 3 \mathrm{H}), 1.52-1.20$ $(\mathrm{m}, 2 \mathrm{H}), 1.07(\mathrm{~d}, J=7.0 \mathrm{~Hz}, 3 \mathrm{H}), 0.97(\mathrm{t}, J=7.9 \mathrm{~Hz}, 9 \mathrm{H}), 0.90(\mathrm{~s}, 9 \mathrm{H}), 0.88(\mathrm{~d}, J=6.3 \mathrm{~Hz}, 3 \mathrm{H}), 0.60(\mathrm{q}, J=$ $7.9 \mathrm{~Hz}, 6 \mathrm{H}), 0.06(\mathrm{~s}, 6 \mathrm{H}) \mathrm{ppm} .{ }^{13} \mathrm{C}\left\{{ }^{1} \mathrm{H}\right\}$ NMR $\left(75 \mathrm{MHz}, \mathrm{CDCl}_{3}\right): \delta=208.3,150.0,77.1,72.2,71.3,47.6,45.8$, $37.1,33.3,30.9,26.0,23.5,18.1,15.5,14.8,7.1,5.3,-4.2,-4.5 \mathrm{ppm}$. MS (ESI): $\mathrm{m} / \mathrm{z}:[\mathrm{M}+\mathrm{H}]^{+}: 583.2,[\mathrm{M}+\mathrm{Na}]^{+}$: 605.2. HRMS (ESI): $\mathrm{m} / \mathrm{z}$ : calcd for $\mathrm{C}_{25} \mathrm{H}_{52} \mathrm{O}_{3} \mathrm{Si}_{2} \mathrm{l}[\mathrm{M}+\mathrm{H}]^{+}:$583.2500, found: 583.2508.

\section{Compound 45}

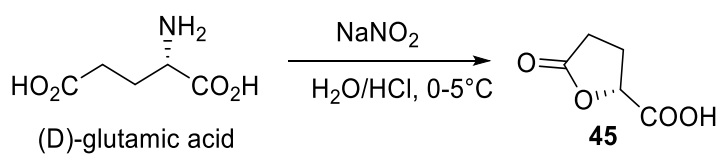

To a cooled $\left(0^{\circ} \mathrm{C}\right)$ solution of (D)-glutamic acid $(10 \mathrm{~g}, 68 \mathrm{mmol}, 1$ equiv) in a mixture of water and hydrochloric acid $35 \%$ (35 mL, 3:2) was added a solution of sodium nitrite $(9.36 \mathrm{~g}, 136 \mathrm{mmol}, 2$ equiv) in water (26 mL) via a dropping funnel over $2 \mathrm{~h}$. During this operation, the temperature must not exceed $5{ }^{\circ} \mathrm{C}$. After completion of the addition, the reaction was allowed to warm to room temperature and stirred overnight. The mixture was then concentrated, the solid residue was triturated with AcOEt and filtered. The filtrate was then dried over $\mathrm{MgSO}_{4}$, filtered, and concentrated. The crude acid was then azeotropically dried with toluene ( 3 times), and finally dried overnight on $\mathrm{P}_{2} \mathrm{O}_{5}$ in a high vacuum. This compound was used in the next step without further purification. Characterization data were in agreement with the literature. ${ }^{[11]}$

$[\alpha]_{D}=-14.4(c=2.0, \mathrm{EtOH}) . \mathbf{I R}$ (neat): $v=3540,2380,1795,1740,1465,1140,1425,1350,1270,1245$, $1180,1010 \mathrm{~cm}^{-1} .{ }^{1} \mathrm{H}$ NMR $\left(300 \mathrm{MHz}, \mathrm{CDCl}_{3}\right): \delta=10.65(\mathrm{~s}, 1 \mathrm{H}), 5.01(\mathrm{dd}, J=8.4,4.4 \mathrm{~Hz}, 1 \mathrm{H}), 2.72-2.53$ $(\mathrm{m}, 3 \mathrm{H}), 2.48-2.32(\mathrm{~m}, 1 \mathrm{H}) \mathrm{ppm} .{ }^{13} \mathrm{C}\left\{{ }^{1} \mathrm{H}\right\}$ NMR $\left(75 \mathrm{MHz}, \mathrm{CDCl}_{3}\right): \delta=178.1,174.5,75.2,26.7,25.8 \mathrm{ppm}$.

\section{Compound 15a}

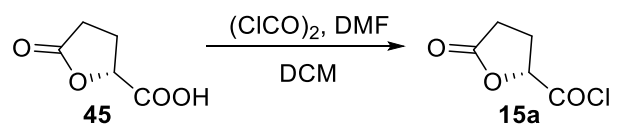

The crude acid was dissolved into DCM $(25 \mathrm{~mL})$, and oxalyl chloride $(8.75 \mathrm{~mL}, 102 \mathrm{mmol}, 1.5$ equiv) was added in one portion. Tree drops of DMF were then added to the solution. After $1 \mathrm{~h}$, the crude mixture was then distilled under reduced pressure $\left(\mathrm{bp}=90^{\circ} \mathrm{C}, 0.05 \mathrm{mmHg}\right)$ to afford acid chloride $15 \mathrm{a}(7.3 \mathrm{~g}, 49 \mathrm{mmol}$, $72 \%$ on two steps) as a colorless oil. Characterization data were in agreement with the literature. ${ }^{[12]}$

$\mathbf{B p}=90^{\circ} \mathrm{C}, 0.05 \mathrm{mmHg}$. IR (neat): $v=1780,1145,920 \mathrm{~cm}^{-1} .{ }^{1} \mathbf{H} \mathbf{N M R}\left(300 \mathrm{MHz}, \mathrm{CDCl}_{3}\right): \delta=5.15$ (dd, $J=$ 8.7, $4.6 \mathrm{~Hz}, 1 \mathrm{H}), 2.76-2.58(\mathrm{~m}, 3 \mathrm{H}), 2.56-2.45(\mathrm{~m}, 1 \mathrm{H}) \mathrm{ppm} .{ }^{13} \mathrm{C}\left\{{ }^{1} \mathrm{H}\right\} \mathbf{N M R}\left(75 \mathrm{MHz}, \mathrm{CDCl}_{3}\right): \delta=176.2$, $171.9,79.9,25.9,24.9 \mathrm{ppm}$. 


\section{Compounds 44}

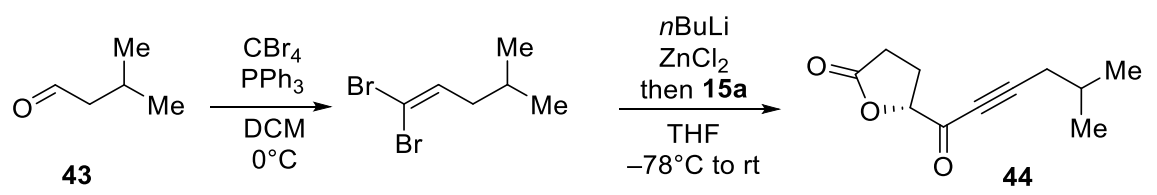

To a cooled $\left(0^{\circ} \mathrm{C}\right)$ solution of $\mathrm{CBr}_{4}(6.72 \mathrm{~g}, 20 \mathrm{mmol}, 3$ equiv) in $\mathrm{DCM}(20 \mathrm{~mL})$ was added a solution of $\mathrm{PPh}_{3}(10.48 \mathrm{~g}, 40 \mathrm{mmol}, 6$ equiv) in DCM $(20 \mathrm{~mL})$ via canula. The solution turned orange, and after $15 \mathrm{~min}$, iso-valeraldehyde (43) $(0.358 \mathrm{~mL}, 10 \mathrm{mmol}, 1.5$ equiv) was added. After $1.5 \mathrm{~h}$, the reaction mixture was precipitated by the addition of pentane $(150 \mathrm{~mL})$, the suspension was filtered on a silica pad, eluted with pentane, and concentrated to afford crude 1,1-dibromo-pent-1-ene as pale yellow oil. This product was immediately engaged in the next step without further purification.

IR (neat): $v=2957,1465,780 \mathrm{~cm}^{-1} .{ }^{1} \mathrm{H}$ NMR $\left(300 \mathrm{MHz}, \mathrm{CDCl}_{3}\right): \delta=6.40(\mathrm{t}, J=7.3 \mathrm{~Hz}, 1 \mathrm{H}), 2.00(\mathrm{t}, J=7.1$ $\mathrm{Hz}, 2 \mathrm{H}), 1.75$ (nonuplet, $J=6.6 \mathrm{~Hz}, 1 \mathrm{H}), 0.95(\mathrm{~d}, J=6.7 \mathrm{~Hz}, 6 \mathrm{H}) \mathrm{ppm} .{ }^{13} \mathbf{C}\left\{{ }^{1} \mathrm{H}\right\} \mathbf{N M R}\left(75 \mathrm{MHz}, \mathrm{CDCl}_{3}\right): \delta=$ 137.8, 88.9, 41.8, 27.7, 22.2 ppm. HRMS (ESI) calculated for $\mathrm{C}_{6} \mathrm{H}_{9}{ }^{79} \mathrm{Br}_{2}$, [M-H] $]^{+}:$238.9066, found 238.9057.

To a cooled $\left(-78^{\circ} \mathrm{C}\right)$ solution of above gem-dibromoolefine in THF $(20 \mathrm{~mL})$ was added dropwise $n \mathrm{BuLi}(8$ $\mathrm{mL}, 20 \mathrm{mmol}$, 2 equiv $2.5 \mathrm{M}$ in hexanes), the reaction was maintained at this temperature for 30 min and slowly warmed to $-50^{\circ} \mathrm{C}$ during $30 \mathrm{~min}$. At $-50^{\circ} \mathrm{C}$, a freshly prepared solution of $\mathrm{ZnCl}_{2}(16 \mathrm{~mL}, 16 \mathrm{mmol}, 2.4$ equiv $1 \mathrm{M}$ in THF) was added dropwise, and the reaction mixture was warmed to $0{ }^{\circ} \mathrm{C}$. After $20 \mathrm{~min}$ at $0{ }^{\circ} \mathrm{C}$, the mixture was cooled to $-30^{\circ} \mathrm{C}$, and a solution of acyl chloride $15 \mathrm{a}$ (989 mg, $6.66 \mathrm{mmol}, 1$ equiv) in THF $(10 \mathrm{~mL})$ was added via canula, the reaction was allowed to warm to room temperature. After $1.5 \mathrm{~h}$, saturated $\mathrm{NH}_{4} \mathrm{Cl}$ was added, the reaction medium was extracted with $\mathrm{Et}_{2} \mathrm{O}$, dried with $\mathrm{MgSO}_{4}$, filtered and concentrated under reduced pressure. The crude residue was purified on silica gel ( $\mathrm{Et} 2 \mathrm{O} / \mathrm{PE}-50 \%)$ to give propargylic ketone 44 (904 mg, $4.66 \mathrm{mmol}, 70 \%)$ as a clear yellow oil.

$[\alpha]_{\mathrm{D}}=-4.3(c=1.4, \mathrm{DCM}) . \mathbf{I R}$ (neat): $v=2961,2210,1789,1678,1173,1066 \mathrm{~cm}^{-1} .{ }^{1} \mathrm{H}$ NMR $(300 \mathrm{MHz}$, $\left.\mathrm{CDCl}_{3}\right) \delta=4.93(\mathrm{~m}, 1 \mathrm{H}), 2.55(\mathrm{~m}, 3 \mathrm{H}), 2.36(\mathrm{~m}, 3 \mathrm{H}), 1.95$ (hept, $\left.J=6.6 \mathrm{~Hz}, 1 \mathrm{H}\right), 1.04(\mathrm{~s}, 3 \mathrm{H}), 1.02(\mathrm{~s}, 3 \mathrm{H})$. ${ }^{13} \mathrm{C}\left\{{ }^{1} \mathrm{H}\right\}$ NMR $\left(75 \mathrm{MHz}, \mathrm{CDCl}_{3}\right): \delta=184.0,176.0,100.7,82.1,79.0,28.4,27.7,26.9,25.3,22.1 \mathrm{ppm}$. HRMS (ESI): $\mathrm{m} / \mathrm{z}$ : calcd for $\mathrm{C}_{11} \mathrm{H}_{14} \mathrm{O}_{3} \mathrm{Na}[\mathrm{M}+\mathrm{Na}]^{+}: 217.0841$, found: 217.0834 .

\section{$\underline{\text { Thioester } 15 b \text { and } 15 c}$}

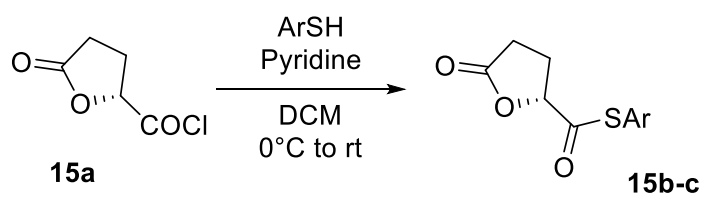

To a cooled $\left(0^{\circ} \mathrm{C}\right)$ solution of acid chloride $15 \mathrm{a}$ (1.1 equiv) in DCM $(0.75 \mathrm{~mL} / \mathrm{mmol})$ was added pyridine $(0.15 \mathrm{~mL})$ and corresponding thiophenol (1 equiv). The solution was warmed to room temperature and stirred for $1 \mathrm{~h}$. The mixture was then quenched with water. The organic layer was extracted with DCM; the combined organic phases were then washed with $\mathrm{HCl}(1 \mathrm{~N})$, saturated solution of $\mathrm{NaHCO}_{3}$, dried over $\mathrm{MgSO}_{4}$ and filtered.

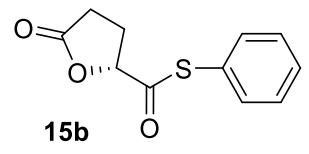

Purification of the crude mixture on silica gel (AcOEt/PE - 30\%) led to thioester $15 \mathrm{~b}(\mathrm{Ar}=\mathrm{Ph})(5.91 \mathrm{~g}$, $26.62 \mathrm{mmol}, 87 \%$ ) as a beige semi-solid. 
$[\alpha]_{D}=+151.9(c=0.5, D C M)$. IR (neat): $v=1776,1689,1143,1053,912,745,688 \mathrm{~cm}^{-1} .{ }^{1} \mathbf{H ~ N M R}(300 \mathrm{MHz}$, $\left.\mathrm{CDCl}_{3}\right): \delta=7.56-7.28(\mathrm{~m}, 5 \mathrm{H}), 5.05(\mathrm{~m}, 1 \mathrm{H}), 2.72-2.53(\mathrm{~m}, 3 \mathrm{H}), 2.42(\mathrm{~m}, 1 \mathrm{H}) \mathrm{ppm} .{ }^{13} \mathrm{C}\left\{{ }^{1} \mathrm{H}\right\} \mathrm{NMR}(75 \mathrm{MHz}$, $\left.\mathrm{CDCl}_{3}\right): \delta=197.7,175.5,134.7,130.0,129.5,125.8,81.8,26.7,26.3 \mathrm{ppm}$. HRMS (ESI): $\mathrm{m} / \mathrm{z}$ : calcd for $\mathrm{C}_{11} \mathrm{H}_{10} \mathrm{O}_{3} \mathrm{SNa}[\mathrm{M}+\mathrm{Na}]^{+}:$245.0248, found: 245.0244 .

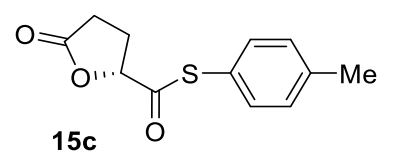

Purification of the crude mixture on silica gel (AcOEt/PE - 30\%) led to thioester 15c $(\mathrm{Ar}=\mathrm{Tol})(3.71 \mathrm{~g}$, $13.89 \mathrm{mmol}, 78 \%$ ) as a white solid.

$\operatorname{mp~} 102-103^{\circ} \mathrm{C} .[\alpha]_{\mathrm{D}}=+125.0(c=0.5, \mathrm{DCM})$. IR (neat): $v=1786,1688,1494,1460,1145,1078,1056$, 1008, 913, 813, $712 \mathrm{~cm}^{-1} .{ }^{1} \mathrm{H}$ NMR $\left(300 \mathrm{MHz}, \mathrm{CDCl}_{3}\right): \delta=7.30-7.19(\mathrm{~m}, 4 \mathrm{H}), 5.02(\mathrm{~m}, 1 \mathrm{H}), 2.68-2.52(\mathrm{~m}$, $3 \mathrm{H}), 2.35-2.45(\mathrm{~m}, 1 \mathrm{H}), 2.37(\mathrm{~s}, 3 \mathrm{H}) \mathrm{ppm} .{ }^{13} \mathrm{C}\left\{{ }^{1} \mathrm{H}\right\}$ NMR $\left(75 \mathrm{MHz}, \mathrm{CDCl}_{3}\right): \delta=198.2,175.6,140.4,134.6$, 130.4, 122.1, 81.9, 26.8, 26.3, 21.5 ppm. HRMS (ESI): $\mathrm{m} / \mathrm{z}$ : calcd for $\mathrm{C}_{12} \mathrm{H}_{12} \mathrm{O}_{3} \mathrm{SNa}[\mathrm{M}+\mathrm{Na}]^{+}: 259.0405$, found: 259.0399 .

\section{Compound 46}

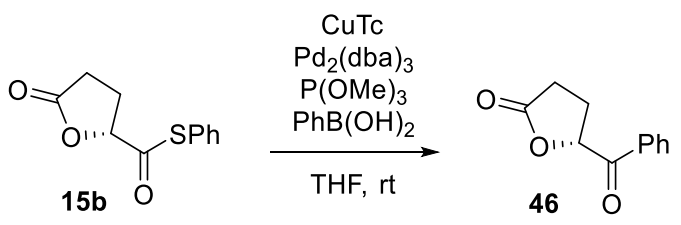

To a solution of thioester $\mathbf{1 5 b}$ ( $222 \mathrm{mg}, 1 \mathrm{mmol}, 1$ equiv) in THF, was added simultaneously all the solids: Copper (I) thiophene carboxylate (CuTc) ${ }^{[13]}(228 \mathrm{mg}, 1.5 \mathrm{mmol}, 1.5$ equiv), phenylboronic acid $8 \mathrm{a}$ ( $182.9 \mathrm{mg}$, $1.5 \mathrm{mmol}, 1.5$ equiv), $\mathrm{Pd}_{2}(\mathrm{dba})_{3}$ ( $23 \mathrm{mg}, 0.025 \mathrm{mmol}, 0.025$ equiv) followed by $\mathrm{P}(\mathrm{OMe})_{3}(34 \mu \mathrm{L}, 0.2 \mathrm{mmol}, 0.2$ equiv). The reaction mixture was stirred for $4 \mathrm{~h}$ at $\mathrm{rt}$, the reaction suspension color passing from red to green. The reaction mixture was quenched by aqueous $\mathrm{K}_{2} \mathrm{CO}_{3}(10 \%)$, filtered over Celite and eluted with $\mathrm{Et}_{2} \mathrm{O}$. The aqueous layer was extracted with $\mathrm{Et}_{2} \mathrm{O}$; the combined organic phases were washed with aqueous $\mathrm{K}_{2} \mathrm{CO}_{3}$ $(10 \%)$, dried over $\mathrm{MgSO}_{4}$, filtered, and concentrated under reduced pressure. The resulting residue was purified on silica gel (AcOEt/PE - 30 to $40 \%$ ) to afford starting material ( $22 \mathrm{mg}, 0.1 \mathrm{mmol}, 10 \%)$ followed by ketone 46 (92 mg, 52\%).

$\mathbf{M p}=95-96{ }^{\circ} \mathrm{C} .[\alpha]_{\mathrm{D}}=-23.7(c=0.8, \mathrm{DCM}) . \mathbf{I R}$ (neat): $v=2950,1766,1695,1228,1192,1177,1065,974$, $693 \mathrm{~cm}^{-1} .{ }^{1} \mathrm{H}$ NMR $\left(300 \mathrm{MHz}, \mathrm{CDCl}_{3}\right): \delta=7.98(\mathrm{~m}, 1 \mathrm{H}), 7.64(\mathrm{tt}, J=7.5,1.5 \mathrm{~Hz}, 1 \mathrm{H}), 7.51(\mathrm{~m}, 1 \mathrm{H}), 5.81(\mathrm{~m}$, $1 \mathrm{H}), 2.70-2.35(\mathrm{~m}, 2 \mathrm{H}) \mathrm{ppm} .{ }^{13} \mathrm{C}\left\{{ }^{1} \mathrm{H}\right\} \mathbf{N M R}\left(75 \mathrm{MHz}, \mathrm{CDCl}_{3}\right): \delta=194.4,176.3,134.4,133.7,129.1,128.9$, 78.4, 77.2, 26.9, $25.1 \mathrm{ppm}$. HRMS (ESI): $\mathrm{m} / \mathrm{z}$ : calcd for $\mathrm{C}_{11} \mathrm{H}_{10} \mathrm{O}_{3} \mathrm{Na}[\mathrm{M}+\mathrm{Na}]^{+}:$213.0528, found: 213.0531 . 


\section{Compound 48}

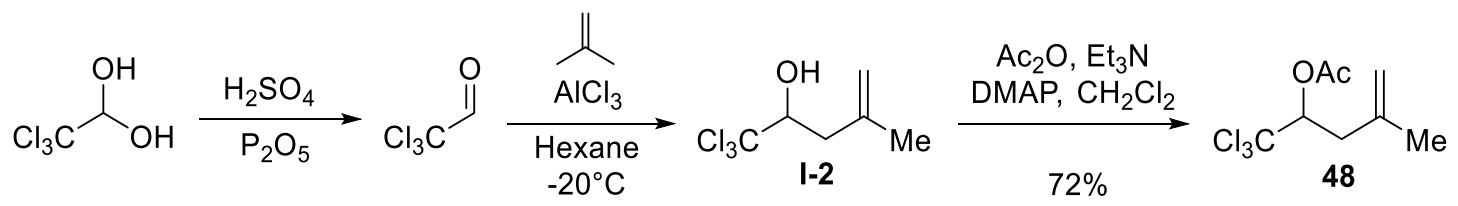

The experimental protocol was adapted from the literature ${ }^{[14]}$

To a mixture of chloral hydrate $(97.5 \mathrm{~g}, 589 \mathrm{mmol})$ and $\mathrm{P}_{2} \mathrm{O}_{5}(29 \mathrm{~g}, 204 \mathrm{mmol}, 0.35$ equiv) was added concentrated $\mathrm{H}_{2} \mathrm{SO}_{4}(117 \mathrm{~mL}, 603 \mathrm{mmol}, 1.02$ equiv) in one portion. The reaction mixture was distillated at atmospheric pressure $\left(\mathrm{bp}=95{ }^{\circ} \mathrm{C}\right)$ to afford chloral $(86.0 \mathrm{~g}, 585 \mathrm{mmol}, 99 \%)$ as a colorless oil. Characterization data were in agreement with literature. ${ }^{[14]}$

${ }^{1} \mathbf{H}$ NMR $\left(300 \mathrm{MHz}, \mathrm{CDCl}_{3}\right): \delta=9.05(\mathrm{~s}, 1 \mathrm{H}) \mathrm{ppm} .{ }^{13} \mathrm{C}\left\{{ }^{1} \mathrm{H}\right\}$ NMR $\left(75 \mathrm{MHz}, \mathrm{CDCl}_{3}\right): \delta=176.8,94.2 \mathrm{ppm}$.

To a cooled $\left(-20^{\circ} \mathrm{C}\right.$, cooled from a salt/ ice bath) solution of iso-butylene (100 g, $1171 \mathrm{mmol}, 2$ equiv) and freshly prepared chloral $\left(86.0 \mathrm{~g}, 585 \mathrm{mmol}, 1\right.$ equiv) in hexane $(500 \mathrm{~mL})$ was added $\mathrm{AlCl}_{3}(7.78 \mathrm{~g}, 58.5 \mathrm{mmol}$, 0.1 equiv) by portions over $1.5 \mathrm{~h}$. After complete addition of $\mathrm{AlCl}_{3}$, the flask was placed in an ice bath and stirred for an additional hour. The mixture was quenched with water, extracted with hexanes, dried over $\mathrm{MgSO}_{4}$, filtered and concentrated under reduced pressure to afford 1,1,1-trichloro-4-methyl-4-penten-2-ol I-2 $(114.0 \mathrm{~g}, 551 \mathrm{mmol}, 93 \%)$ as a colorless oil. Characterization data were in agreement with literature. ${ }^{[14]}$

${ }^{1} \mathrm{H}$ NMR $\left(300 \mathrm{MHz}, \mathrm{CDCl}_{3}\right): \delta=4.93(\mathrm{brs}, 1 \mathrm{H}), 4.89$ (brs, $\left.1 \mathrm{H}\right), 4.18(\mathrm{dd}, J=9.9,2.1 \mathrm{~Hz}, 1 \mathrm{H}),, 2.90(\mathrm{brs}, 1 \mathrm{H},-$ $\mathrm{OH}), 2.78$ (brd, $J=14.4 \mathrm{~Hz}, 1 \mathrm{H}), 2.34$ (dd, $J=14.4,9.9 \mathrm{~Hz}, 1 \mathrm{H}), 1.82(\mathrm{~s}, 3 \mathrm{H}) \mathrm{ppm} .{ }^{13} \mathrm{C}\left\{{ }^{1} \mathrm{H}\right\} \mathbf{N M R}(75 \mathrm{MHz}$, $\left.\mathrm{CDCl}_{3}\right): \delta=140.9,114.6,103.7,80.9,40.3,22.5 \mathrm{ppm}$.

To a cooled $\left(0^{\circ} \mathrm{C}\right)$ solution of $\mathbf{I - 2}\left(114.0 \mathrm{~g}, 551 \mathrm{mmol}, 1\right.$ equiv) and $\mathrm{Ac}_{2} \mathrm{O}(77.8 \mathrm{~mL}, 826 \mathrm{mmol}, 1.5$ equiv) in DCM $(200 \mathrm{~mL})$ was added $\mathrm{Et}_{3} \mathrm{~N}(76.7 \mathrm{~mL}, 551 \mathrm{mmol}, 1$ equiv) and DMAP (3.5 g, $28.9 \mathrm{mmol}, 0.05$ equiv) and warmed to room temperature. After $1.5 \mathrm{~h}$, the reaction medium was quenched with saturated $\mathrm{NH}_{4} \mathrm{Cl}$ and extracted with DCM. The combined organic phases were washed with aqueous $\mathrm{HCl}(1 \mathrm{~N})$, saturated $\mathrm{NaHCO}_{3}$, brine, dried over $\mathrm{MgSO}_{4}$, filtered, and finally concentrated in vacuo. The crude residue was purified by distillation under reduced pressure $\left(P=0.7 \mathrm{mmHg}, \mathrm{bp}=75-80^{\circ} \mathrm{C}\right)$ to yield 1,1,1-trichloro-2-acetyl-4-methyl4-penten-2-ol 48 (114.4 g, $466 \mathrm{mmol}, 84 \%)$ as a colorless oil. Characterization data were in agreement with the literature. ${ }^{[14]}$

$\mathrm{Bp}=75-80{ }^{\circ} \mathrm{C}, 0.7 \mathrm{mmHg} .{ }^{1} \mathrm{H}$ NMR $\left(300 \mathrm{MHz}, \mathrm{CDCl}_{3}\right): \delta=5.63(\mathrm{dd}, J=10.3,2.0 \mathrm{~Hz}, 1 \mathrm{H}), 4.86(\mathrm{brs}, 1 \mathrm{H})$, 4.83 (brs, $1 \mathrm{H}$ ), 2.79 (brd, $J=14.0 \mathrm{~Hz}, 1 \mathrm{H}), 2.53$ (dd, $J=14.0,10.3 \mathrm{~Hz}, 1 \mathrm{H}), 2.13(\mathrm{~s}, 3 \mathrm{H}), 1.80(\mathrm{~s}, 3 \mathrm{H}) \mathrm{ppm}$. ${ }^{13} \mathrm{C}\left\{{ }^{1} \mathrm{H}\right\}$ NMR $\left(75 \mathrm{MHz}, \mathrm{CDCl}_{3}\right): \delta=169.4,139.6,115.2,100.0,78.8,39.3,22.2,20.6 \mathrm{ppm}$.

\section{Compound 49}

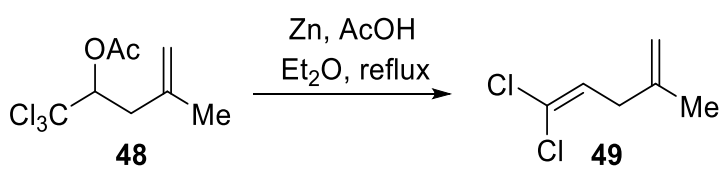

The experimental protocol was adapted from the literature ${ }^{[14]}$

To a suspension of zinc dust (117 g, $1864 \mathrm{mmol}, 4$ equiv) and AcOH (112 mL, $1864 \mathrm{mmol}, 4$ equiv) in $\mathrm{Et}_{2} \mathrm{O}(200 \mathrm{~mL})$ at rt was added, via a dropping funnel, a solution of acetate 48 (114.4 g, $466 \mathrm{mmol}, 1$ equiv) in $\mathrm{Et}_{2} \mathrm{O}(300 \mathrm{~mL})$ over $2 \mathrm{~h}$. After completion of the addition, the reaction mixture was heated to reflux for an additional $2 \mathrm{~h}$ with the help of an oil bath. The cooled reaction mixture was filtered, and the organic phase was washed with water (3 times), aqueous $\mathrm{NaOH}(3 \mathrm{~N})$, dried over $\mathrm{MgSO}_{4}$, filtered, and concentrated under reduced pressure. The crude material was distilled under reduced pressure $\left(P=80 \mathrm{mmHg}, \mathrm{bp}=75-80{ }^{\circ} \mathrm{C}\right)$ 
to obtain 1,1-dichloro-4 methyl-1,4-diene $49(59.0 \mathrm{~g}, 391 \mathrm{mmol}, 84 \%)$ as a clear oil. Characterization data were in agreement with the literature. ${ }^{[14]}$

$\mathrm{Bp}=75-80^{\circ} \mathrm{C}, 80 \mathrm{mmHg} .{ }^{1} \mathrm{H}$ NMR $\left(300 \mathrm{MHz}, \mathrm{CDCl}_{3}\right): \delta=5.91(\mathrm{t}, J=7.6 \mathrm{~Hz}, 1 \mathrm{H}), 4.78(\mathrm{brs}, 1 \mathrm{H}), 4.74(\mathrm{brs}, 1 \mathrm{H})$, $2.87(\mathrm{~d}, J=7.6 \mathrm{~Hz}, 2 \mathrm{H}), 1.75(\mathrm{~s}, 3 \mathrm{H}) \mathrm{ppm} .{ }^{13} \mathrm{C}\left\{{ }^{1} \mathrm{H}\right\}$ NMR $\left(75 \mathrm{MHz}, \mathrm{CDCl}_{3}\right): \delta=142.0,127.6,121.3,111.8$, 37.9, $22.6 \mathrm{ppm}$.

\section{Compound 50}

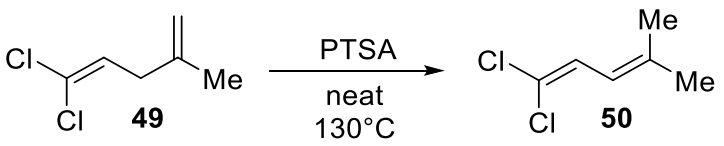

The experimental protocol was adapted from the literature ${ }^{[14]}$

To pure 1,1-dichloro-4 methyl-1,4-diene 49 (59.0 g, $391 \mathrm{mmol}, 1$ equiv) was added PTSA (550 mg, 2.6 mmol, 0.006 equiv) the mixture was heated at $130^{\circ} \mathrm{C}$ for $2 \mathrm{~h}$, with the help of an oil bath. The cooled residue was distilled under reduced pressure $\left(P=13 \mathrm{mmHg}, b p=62-65^{\circ} \mathrm{C}\right)$ to yield conjugated 1,1-dichloro-4 methyl1,3-diene 50 (55.3 g, $366 \mathrm{mmol}, 94 \%)$ as a colorless oil. Characterization data were in agreement with the literature. ${ }^{[14]}$

$\mathrm{Bp}=62-65^{\circ} \mathrm{C}, 13 \mathrm{mmHg} .{ }^{1} \mathrm{H}$ NMR $\left(300 \mathrm{MHz}, \mathrm{CDCl}_{3}\right): \delta=6.58(\mathrm{~d}, J=10.8 \mathrm{~Hz}, 1 \mathrm{H}), 5.95$ (dsept, $J=10.8$, $1.4 \mathrm{~Hz}, 1 \mathrm{H},), 1.83(\mathrm{~s}, 3 \mathrm{H}), 1.76(\mathrm{~s}, 3 \mathrm{H}) \mathrm{ppm} .{ }^{13} \mathrm{C}\left\{{ }^{1} \mathrm{H}\right\}$ NMR $\left(75 \mathrm{MHz}, \mathrm{CDCl}_{3}\right): \delta=140.2,125.6,119.7,119.0$, 26.4, $19.1 \mathrm{ppm}$.

\section{Compound 51}

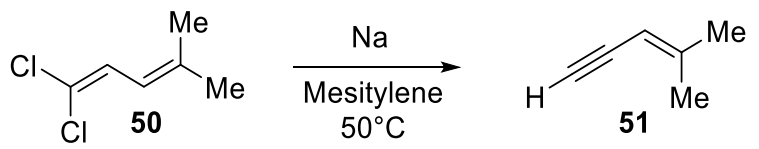

The experimental protocol was adapted from the literature ${ }^{[15]}$

To a hot $\left(50^{\circ} \mathrm{C}\right.$, heating with an oil bath) suspension of sodium turning $(26.7 \mathrm{~g}, 1164 \mathrm{mmol}, 3$ equiv) in mesitylene $(120 \mathrm{~mL})$ under Ar atmosphere was added dropwise 1,1-dichloro-4 methyl-1,3-diene 50 (58.65 g, $388 \mathrm{mmol}, 1$ equiv) over $2 \mathrm{~h}$. After $2 \mathrm{~h}$ at this temperature, the reaction medium was cooled to $0{ }^{\circ} \mathrm{C}$, and $\mathrm{MeOH}(35 \mathrm{~mL})$ was carefully added, followed by $\mathrm{H}_{2} \mathrm{O}(25 \mathrm{~mL})$ (All this operation was performed under $\mathrm{Ar}$ bubbling). The reaction mixture was finally extracted with mesitylene, dried with $\mathrm{MgSO}_{4}$, and filtered. The organic phase was atmospherically distilled $\left(T<140^{\circ} \mathrm{C}\right)$ to obtain a contaminated fraction of 4-methyl-pent3-enyne 51. This fraction was carefully distilled on $\mathrm{CaH}_{2}\left(\mathrm{P}=760 \mathrm{mmHg}, \mathrm{bp}=75-80^{\circ} \mathrm{C}\right)$ to obtain analytically pure 4-methyl-pent-3-enyne (14.71 g, $183 \mathrm{mmol}, 47 \%)$ as pale yellow oil. Characterization data were in agreement with the literature. ${ }^{[15]}$

$\mathbf{B p}=75-80^{\circ} \mathrm{C}, 760 \mathrm{mmHg}$. IR (neat): $v=3311,2975,2937,2912,2102,1629,1437,1380,1201,1083,819$ $\mathrm{cm}^{-1} .{ }^{1} \mathrm{H}$ NMR $\left(300 \mathrm{MHz}, \mathrm{CDCl}_{3}\right): \delta=5.25$ (dsept, $J=2.2,1.3 \mathrm{~Hz}, 1 \mathrm{H}$ ), 2.98 (dsept, $\left.J=2.2,0.5 \mathrm{~Hz}, 1 \mathrm{H}\right), 1.92$ (tt, $J=1.1,0.5 \mathrm{~Hz}, 3 \mathrm{H}), 1.81$ (dquint, $J=1.5,0.5 \mathrm{~Hz}, 3 \mathrm{H}) \mathrm{ppm} .{ }^{13} \mathrm{C}\left\{{ }^{1} \mathrm{H}\right\} \mathbf{N M R}\left(75 \mathrm{MHz}, \mathrm{CDCl}_{3}\right): \delta=150.1$, 104.4, 81.6, 79.1, 24.5, $20.6 \mathrm{ppm}$. 


\section{Compound 52}

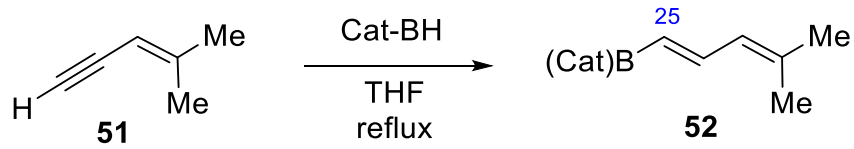

To a solution of catechol borane (2.4 mL, $2.4 \mathrm{mmol}, 1.2$ equiv $1 \mathrm{M}$ in THF) was added 4-methyl-pent-3enyne (217 mg, $2 \mathrm{mmol}, 1$ equiv), the mixture was then warmed to reflux during $12 \mathrm{~h}$, with the help of an oil bath. Then, the reaction mixture was cooled to room temperature, hydrolyzed with water, and extracted with AcOEt. The organic phase was then washed with saturated $\mathrm{NaHCO}_{3}$, dried over $\mathrm{MgSO}_{4}$, filtered, and concentrated into rotavapor. The resulting catechol boronate 52 , contaminated with free catechol $(\approx 50 \%)$, was then used in the next step without further purification.

${ }^{1} \mathbf{H}$ NMR $\left(300 \mathrm{MHz}, \mathrm{CDCl}_{3}\right): \delta=7.64(\mathrm{dd}, J=17.5,11.1 \mathrm{~Hz}, 1 \mathrm{H}), 7.22(\mathrm{dd}, J=5.8,3.4 \mathrm{~Hz}, 2 \mathrm{H}), 7.07(\mathrm{dd}, J=$ 5.8, 3.4 Hz, 2H), $6.08(\mathrm{~d}, J=11.1 \mathrm{~Hz}, 1 \mathrm{H}), 5.73(\mathrm{~d}, J=17.5 \mathrm{~Hz}, 1 \mathrm{H}), 1.93(\mathrm{~s}, 3 \mathrm{H}), 1.88(\mathrm{~s}, 3 \mathrm{H}) \mathrm{ppm} .{ }^{11} \mathrm{~B}$ NMR $\left(400 \mathrm{MHz}, \mathrm{CDCl}_{3}\right): \delta=31.5 \mathrm{ppm} .{ }^{13} \mathrm{C}\left\{{ }^{1} \mathrm{H}\right\} \mathrm{NMR}\left(75 \mathrm{MHz}, \mathrm{CDCl}_{3}\right): \delta=148.5,143.8,143.1,127.8,122.6,112.4$, 26.5, 19.0 ppm. $C_{25}$ was not detected due to quadripolar relaxation.

\section{Compound 42}

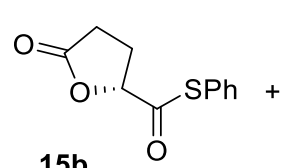

$15 b$

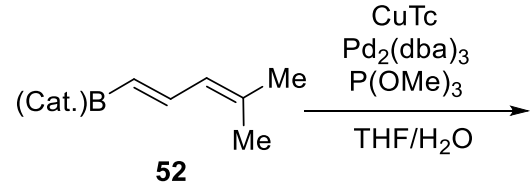

52

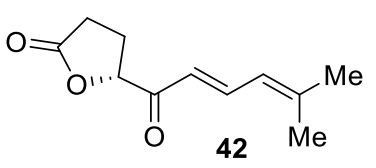

$42 \mathrm{Me}$

To a solution of thioester $15 \mathrm{~b}$ (295 mg, $1.33 \mathrm{mmol}, 1$ equiv) in THF $(13 \mathrm{~mL})$ at rt was added crude catechol boronate 52 (2 mmol, 1.5 equiv), CuTC ${ }^{[13]}$ ( $357 \mathrm{mg}, 2 \mathrm{mmol}, 1.5$ equiv), $\mathrm{Pd}_{2}(\mathrm{dba})_{3}$ (30 mg, $33 \mu \mathrm{mol}, 0.025$ equiv) and $\mathrm{P}(\mathrm{OMe})_{3}\left(31 \mu \mathrm{L}, 0.266 \mathrm{mmol}, 0.2\right.$ equiv) and $\mathrm{H}_{2} \mathrm{O}(1.3 \mathrm{~mL})$. The solution turned from dark red to bright green. After consumption of the starting material, as indicated by TLC monitoring, the reaction was filtered on celite, and aqueous $\mathrm{NaOH}(1 \mathrm{~N})$ was added. The resulting mixture was extracted with AcOEt; the organic phase was then washed with aqueous $\mathrm{NaOH}(1 \mathrm{~N})$, dried over $\mathrm{MgSO}_{4}$, filtered, and concentrated in vacuo. The crude residue was then purified on silica gel (AcOEt/PE - 30\%) to afford dienic ketone $42(144$ $\mathrm{mg}, 0.73 \mathrm{mmol}, 55 \%)$ as a brown oil.

$[\alpha]_{\mathrm{D}}=-51.7\left(c=0.9, \mathrm{CH}_{2} \mathrm{Cl}_{2}\right)$. IR (neat): $v=2985,1765,1690,1624,1592,1372,1269,1176,1043,1029$, $885 \mathrm{~cm}^{-1}{ }^{1}{ }^{1} \mathrm{H}$ NMR $\left(300 \mathrm{MHz}, \mathrm{CDCl}_{3}\right): \delta=7.66(\mathrm{dd}, J=15.1,11.7 \mathrm{~Hz}, 1 \mathrm{H}), 6.33(\mathrm{~d}, J=15.1 \mathrm{~Hz}, 1 \mathrm{H}), 6.03$ (brd, $J=11.7 \mathrm{~Hz}, 1 \mathrm{H}), 5.00(\mathrm{~m}, 1 \mathrm{H}), 2.60-2.44(\mathrm{~m}, 3 \mathrm{H}), 2.30(\mathrm{~m}, 1 \mathrm{H}), 1.91(\mathrm{~s}, 3 \mathrm{H}), 1.90(\mathrm{~s}, 3 \mathrm{H}) \mathrm{ppm} .{ }^{13} \mathrm{C}\left\{{ }^{1} \mathrm{H}\right\}$ NMR $\left(75 \mathrm{MHz}, \mathrm{CDCl}_{3}\right): \delta=196.0,176.4,151.3,142.4,124.5,121.0,81.2,27.4,27.0,25.3,19.4$ ppm. HRMS (ESI): $\mathrm{m} / \mathrm{z}$ : calcd for $\mathrm{C}_{11} \mathrm{H}_{14} \mathrm{O}_{3} \mathrm{Na}[\mathrm{M}+\mathrm{Na}]^{+}: 217.0841$, found: 217.0840 .

\section{$\underline{\text { Compound } 47}$}

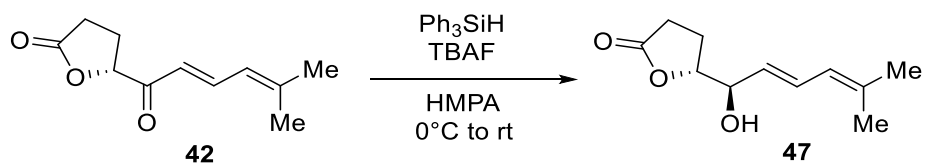

To a cooled $\left(0^{\circ} \mathrm{C}\right)$ solution of ketone $42(98 \mathrm{mg}, 0.500 \mathrm{mmol}, 1$ equiv) in HMPA (1.5 mL) was added triphenylsilane (156 mg, $0.6 \mathrm{mmol}, 1.2$ equiv), TBAF $(75 \mu \mathrm{L}, 75 \mu \mathrm{mol}, 0.15$ equiv $1 \mathrm{M}$ in THF) was added dropwise and the solution was warmed to rt. After consumption of the starting material, visualized by TLC monitoring, water was added to the reaction. The reaction was extracted with $\mathrm{Et}_{2} \mathrm{O}$; the organic layer was 
then washed with brine, dried over $\mathrm{MgSO}_{4}$, filtered, and concentrated on reduced pressure. Purification of the crude mixture on silica gel (AcOEt/PE - 40\%) yielded alcohol 47 (67 mg, $0.338 \mathrm{mmol}, 67 \%$ ) as a colorless oil.

$[\alpha]_{\mathrm{D}}=-12.0\left(c=0.8, \mathrm{CH}_{2} \mathrm{Cl}_{2}\right)$. IR (neat): $v=3374,2937,1775,1180,987,871 \mathrm{~cm}^{-1} .{ }^{1} \mathbf{H}$ NMR $(300 \mathrm{MHz}$, $\left.\mathrm{CDCl}_{3}\right): \delta=6.55$ (ddd, $\left.J=15.2,11.0,1.1 \mathrm{~Hz}, 1 \mathrm{H}\right), 5.81(\mathrm{brd}, J=11.0 \mathrm{~Hz}, 1 \mathrm{H}), 5.52(\mathrm{dd}, J=15.2,7.1 \mathrm{~Hz}, 1 \mathrm{H})$, 4.45 (td, $J=7.2,5.5 \mathrm{~Hz}, 1 \mathrm{H}$ ), 4.18 (ddd, $J=7.1,5.5,1.1 \mathrm{~Hz}, 1 \mathrm{H}$ ), 2.58 (ddd, $J=17.7,9.9,6.0 \mathrm{~Hz}, 1 \mathrm{H}$ ), 2.49 (ddd, $J=17.7,9.2,8.3 \mathrm{~Hz}, 1 \mathrm{H}$ ), 2.32 (brs, $1 \mathrm{H},-\mathrm{OH}$ ), 2.22 (dddd, $J=13.2,9.2,7.2,6.0 \mathrm{~Hz}, 1 \mathrm{H}$ ), 2.08 (dddd, $J=13.2,9.9,8.3,7.1 \mathrm{~Hz}, 1 \mathrm{H}), 1.78(\mathrm{~s}, 1 \mathrm{H}), 1.76(\mathrm{~s}, 3 \mathrm{H}) \mathrm{ppm} .{ }^{13} \mathrm{C}\left\{{ }^{1} \mathrm{H}\right\}$ NMR $\left(75 \mathrm{MHz}, \mathrm{CDCl}_{3}\right): \delta=177.3$, 138.0, 130.7, 126.2, 124.1, 82.9, 75.0, 28.6, 26.1, 23.9, 18.5 ppm. HRMS (ESI): $\mathrm{m} / z$ : calcd for $\mathrm{C}_{11} \mathrm{H}_{16} \mathrm{O}_{3} \mathrm{Na}$ $[\mathrm{M}+\mathrm{Na}]^{+}:$219.0997, found: 219.0997. HPLC (Xbridge column $4.6 \times 150 \mathrm{~mm}, \mathrm{MeCN} / \mathrm{H}_{2} \mathrm{O}$ gradient from $5 \%$ to $100 \%$ over $20 \mathrm{~min}$, flow rate $=1.0 \mathrm{~mL} / \mathrm{min}, \mathrm{I}=254 \mathrm{~nm}) t_{\mathrm{R}}=13.82 \mathrm{~min}($ syn), $14.18 \mathrm{~min}($ anti) $: \mathrm{dr}>95: 5$

reduction with $\mathrm{MnCl}_{2} / \mathrm{NaBH}_{4}$

$\begin{array}{ll}\text { column description : XBRIDGE 2.1 } & \text { column diam. : } 2.1 \mathrm{~mm} \\ \text { column serial \# : } 013739317136 & \text { column length : } 150 \mathrm{~mm}\end{array}$

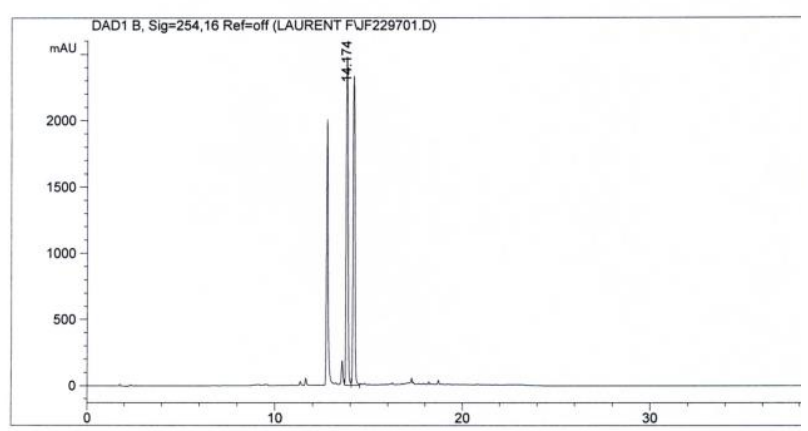

DAD1 B, Sig=254,16 Ref=off

\begin{tabular}{ccccccc}
$\#$ & Ret. Time & Area & Area 8 & Height Height 8 & Width Sym \\
\hline 1 & 13.805 & 17966.19 & 52.31 & 2475.45 & 51.52 & 0.12 \\
2 & 14.174 & 16378.24 & 47.69 & 2329.80 & 48.48 & 0.11 \\
\hline
\end{tabular}

\section{reduction with $\mathrm{Ph}_{3} \mathrm{SiH} / \mathrm{TBAF}$ in HMPA}

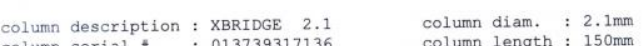

\section{Cyclic Boronate 53}

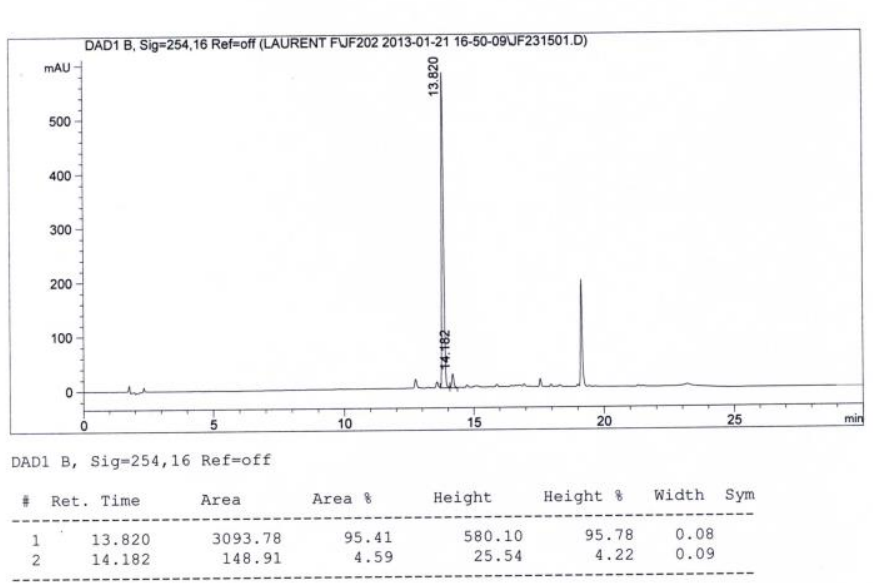

The experimental procedure was adapted from the literature. ${ }^{[16]}$

To magnesium turning (10.8 g, $450 \mathrm{mmol}, 3$ equiv) under argon, in a three-neck round bottom flask equipped with a condenser, was added THF $(5 \mathrm{~mL})$, followed by a crystal of iodine. Reflux was conducted with a heat gun until color vanished, and $0.5 \mathrm{~mL}$ of $n \mathrm{BuBr}$ was added. Reflux was renewed until full activation of magnesium, i.e. sign of sparkling and grey color of the solution. The rest of THF was added ( $115 \mathrm{~mL})$, and the rest of $n B u B r$ [ $36.7 \mathrm{~mL}$ ( $37.2 \mathrm{~mL}$ in total), $345 \mathrm{mmol}, 2.3$ equiv] was then added dropwise over $1 \mathrm{~h}$ in order that the temperature does not reach $45-50^{\circ} \mathrm{C}$. After addition, the solution was stirred at reflux 30 more min with the help of an oil bath. The Grignard reagent $(C \sim 2.5 \mathrm{M})$ was allowed to cool down and must be used within the next $24 \mathrm{~h}$. 
To a suspension of Cul (2.86 g, $15 \mathrm{mmol}, 0.1$ equiv) in THF $(140 \mathrm{~mL})$ at $-20{ }^{\circ} \mathrm{C}$ was added propargyl alcohol ( $8.77 \mathrm{~mL}, 150 \mathrm{mmol}, 1$ equiv), followed by the above $n B u M g B r$ solution (2.2-2.3 equiv). The solution was allowed to stir at $\mathrm{rt}$, and the reaction mixture was stirred for $2 \mathrm{~h}$. The reaction mixture was then cooled down at $-78^{\circ} \mathrm{C}$ and $\mathrm{B}(\mathrm{OMe})_{3}(20.1 \mathrm{~mL}, 180 \mathrm{mmol}, 1.2$ equiv) was added. The reaction mixture was then stirred by slowly warming it from $-78^{\circ} \mathrm{C}$ to rt overnight. The reaction mixture was quenched with $\mathrm{HCl} 10 \%$, extracted with $\mathrm{Et}_{2} \mathrm{O}$, dried over $\mathrm{MgSO}_{4}$, filtered and concentrated. The residue was dissolved in $\mathrm{Et}_{2} \mathrm{O}$, and $\mathrm{NaOH} 8 \mathrm{~N}(20 \mathrm{~mL})$ was added, causing precipitation of sodium boronate as viscous gum. The whole mixture was filtered over Celite, washed with $\mathrm{Et}_{2} \mathrm{O}(\times 3)$, and all filtrates were discarded. All remaining sodium boronate gum was treated and washed with $\mathrm{HCl} 10 \%$ and $\mathrm{Et}_{2} \mathrm{O}$ until complete dissolution. The acidic aqueous layer was extracted with $\mathrm{Et}_{2} \mathrm{O}$, and combined organic layers were dried over $\mathrm{MgSO}_{4}$, filtered, and concentrated to give cyclic boronate 53 as a viscous, yellowish oil (8.7 g, 41\%). Spectral data were in agreement with the literature. ${ }^{[16]}$ Boronate might exist partially under the form of dimer [anhydride], NMR characterization was then performed with the addition of $\mathrm{D}_{2} \mathrm{O}$ to $\mathrm{CDCl}_{3}$.

${ }^{1} \mathrm{H}$ NMR $\left(300 \mathrm{MHz}, \mathrm{CDCl}_{3}-\mathrm{D}_{2} \mathrm{O}\right) \delta 5.50(\mathrm{~s}, 1 \mathrm{H}), 4.44(\mathrm{~s}, 2 \mathrm{H}), 2.25(\mathrm{t}, J=7.6 \mathrm{~Hz}, 2 \mathrm{H}), 1.51(\mathrm{p}, J=7.2 \mathrm{~Hz}, 2 \mathrm{H})$, $1.34(\mathrm{~h}, J=7.1 \mathrm{~Hz}, 3 \mathrm{H}), 0.91$ (t, $J=7.2 \mathrm{~Hz}, 4 \mathrm{H}) .{ }^{13} \mathrm{C}\left\{{ }^{1} \mathrm{H}\right\} \mathrm{NMR}\left(75 \mathrm{MHz}, \mathrm{CDCl}_{3}-\mathrm{D}_{2} \mathrm{O}\right) \delta 173.4,116.9$ (br), $74.5,30.6,30.0,22.5,13.9$.

lodide 54

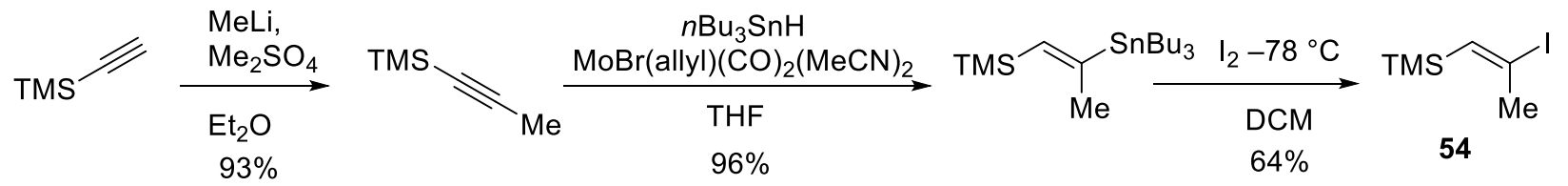

The experimental procedure was adapted from the literature. ${ }^{[17]}$

To a solution of TMS-acetylene $(21 \mathrm{~mL}, 150 \mathrm{mmol})$ in dry $\mathrm{Et}_{2} \mathrm{O}(15 \mathrm{~mL})$ under argon atmosphere and inside a $100 \mathrm{~mL}$ round bottom flask equipped with a septum, was added MeLi (103 mL, $165 \mathrm{mmol}, 1.1$ equiv, $1.60 \mathrm{M}$ ) dropwise at $-78^{\circ} \mathrm{C}$. After $40 \mathrm{~min}$ at this temperature, dimethylsulfate $(22.7 \mathrm{~mL}, 180 \mathrm{mmol}, 1.2$ equiv) was added, and a condenser was placed between the flask and the septum, and a water bath was used to warm the reaction mixture until $15-20^{\circ} \mathrm{C}$. At this point, the alkylation reaction started with an exotherm, resulting in the self-reflux of the reaction mixture. The reflux rate was controlled with the use of an external water bath. After the stop of the self-reflux, a distillation apparatus was installed in place of the condenser. $\mathrm{Et}_{2} \mathrm{O}$ was carefully removed at atmospheric pressure, and TMS-propyne was collected in a fraction between $70-100{ }^{\circ} \mathrm{C}(\mathrm{m}=15.65 \mathrm{~g}, 93 \%)$ as a colorless liquid. Its purity was evaluated being about $92 \%$ by NMR (contaminated with $8 \%$ of $\mathrm{Et}_{2} \mathrm{O}$ ). Note: Mel appeared to be an inefficient methylating agent under these conditions.

$\mathrm{Bp}=98^{\circ} \mathrm{C}$, Patm (litt. 98-99 $\left.{ }^{\circ} \mathrm{C}\right) .{ }^{[18]}{ }^{1} \mathrm{H}$ NMR $(300 \mathrm{MHz}$, Chloroform- $d) \delta 1.87(\mathrm{~s}, 1 \mathrm{H}), 0.14(\mathrm{~s}, 3 \mathrm{H})$.

To a solution of of TMS-propyne (15.6 g, $139 \mathrm{mmol}, 1$ equiv) and $\operatorname{MoBr}\left(\right.$ allyl) $(\mathrm{CO})_{2}(\mathrm{MeCN})_{2}{ }^{[19]}(2.4 \mathrm{~g}(6.4$ mmol, 0.046 equiv) in THF (133 mL), was added freshly prepared Bu3 $\mathrm{SnH}(37 \mathrm{~mL}, 135 \mathrm{mmol}, 0.97$ equiv) [from reaction between neat $\left(\mathrm{Bu}_{3} \mathrm{Sn}\right)_{2} \mathrm{O}$ and $\mathrm{PMHS}$ followed by distillation at $0.5 \mathrm{mmHg}, 90{ }^{\circ} \mathrm{C}$. ${ }^{[20]}$ The rreaction mixture was stirred $4 \mathrm{~h}$ at $\mathrm{rt}$ and solvent was removed by evaporation. The residue was then purified on $\mathrm{SiO}_{2}$ (pure petroleum ether) to give adduct (E)-1-TMS-2-SnBu3-prop-1-ene (52g, 96\%) as a brownish oil. Characterization data were in agreement with literature. ${ }^{[17]}$

${ }^{1} \mathbf{H}$ NMR $(200 \mathrm{MHz}$, Chloroform- $d) \delta 5.82\left(\mathrm{q}, J=1.5 \mathrm{~Hz},{ }^{3} \mathrm{~J}_{\mathrm{Sn}-\mathrm{H}}=99 \mathrm{~Hz}, 1 \mathrm{H}\right), 2.02\left(\mathrm{~d}, J=1.5 \mathrm{~Hz},{ }^{3} \mathrm{~J}_{\mathrm{Sn}-\mathrm{H}}=46\right.$ $\mathrm{Hz}, 3 \mathrm{H}), 1.72-1.42(\mathrm{~m}, 6 \mathrm{H}), 1.33(\mathrm{dt}, J=14.7,7.0 \mathrm{~Hz}, 6 \mathrm{H}), 0.88(\mathrm{td}, J=7.5,3.4 \mathrm{~Hz}, 15 \mathrm{H}), 0.12(\mathrm{~s}, 9 \mathrm{H})$. 
To a solution of (E)-1-TMS-2-SnBu3-prop-1-ene $(52 \mathrm{~g}, 129 \mathrm{mmol}, 1$ equiv) in DCM ( $350 \mathrm{~mL})$ at $0^{\circ} \mathrm{C}$ was added a solution of $\mathrm{I}_{2}$ ( $39.3 \mathrm{~g}, 154 \mathrm{mmol}, 1.2$ equiv) in $\mathrm{Et}_{2} \mathrm{O}(350 \mathrm{~mL})$ over $10 \mathrm{~min}$. The dark solution was then stirred for ten more minutes and was quenched with $\mathrm{Na}_{2} \mathrm{SO}_{4}$ until discoloration to a light brown color. The aqueous layer was extracted with ether, and the combined organic phases were dried over $\mathrm{MgSO}_{4}$, filtered, and concentrated. Purification from $\mathrm{Bu}_{3} \mathrm{Sn}$-byproducts was performed by distillation under vacuum $\left(40{ }^{\circ} \mathrm{C}, 0.5\right.$ $\mathrm{mmHg}$ ) to give vinyl iodide 54 as a colorless liquid $(19.68 \mathrm{~g}, 64 \%)$. Characterization data were in agreement with the literature. ${ }^{[17]}$

$\mathrm{Bp}=40^{\circ} \mathrm{C}, 0.5 \mathrm{mmHg} .{ }^{1} \mathrm{H}$ NMR $(300 \mathrm{MHz}$, Chloroform- $d) \delta 6.29(\mathrm{~s}, 1 \mathrm{H}), 2.54(\mathrm{~s}, 3 \mathrm{H}), 0.14(\mathrm{~s}, 9 \mathrm{H})$.

\section{$\underline{\text { Compound } 55}$}

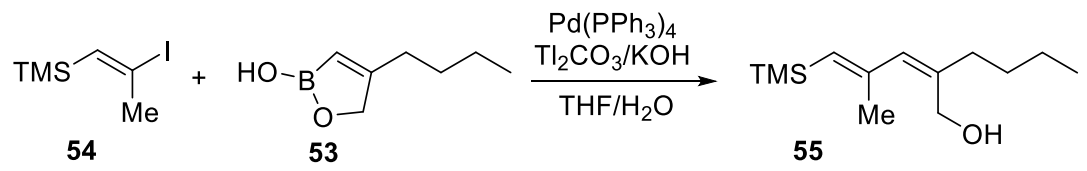

To a degassed solution of vinyl iodide 54 (3.28 g, $7.31 \mathrm{mmol}, 1$ equiv) and boronate $53(1.12 \mathrm{~g}, 8.05 \mathrm{mmol}$, 1.1 equiv) in $\mathrm{THF} / \mathrm{H}_{2} \mathrm{O}(16 \mathrm{~mL}, 3: 1)$ at rt, was added $\mathrm{Pd}\left(\mathrm{PPh}_{3}\right)_{4}$ (335 mg, $0.307 \mathrm{mmol}, 0.04$ equiv), $\mathrm{Tl}_{2} \mathrm{CO}_{3}$ (2.04 g, $4.37 \mathrm{mmol}, 0.6$ equiv) and a pellet of $\mathrm{KOH}(50 \mathrm{mg})$. After $1 \mathrm{~h}$, the reaction mixture was filtered on celite, diluted with $\mathrm{Et}_{2} \mathrm{O}$, and washed with saturated $\mathrm{NaCl}$. The organic layer was dried over $\mathrm{MgSO}_{4}$, filtered, and concentrated in vacuo. The crude residue was purified on silica gel ( $\mathrm{Et}_{2} \mathrm{O} / \mathrm{PE}-10$ to $15 \%$ ) to afford coupling product $55(1.43 \mathrm{~g}, 6.33 \mathrm{mmol}, 86 \%)$ as a colorless oil.

IR (neat): $v=3300,2955,2929,2858,1598,1467,1273,1031,853,690 \mathrm{~cm}^{-1} .{ }^{1} \mathbf{H}$ NMR $\left(300 \mathrm{MHz}, \mathrm{CDCl}_{3}\right): \delta$ $=5.87$ (brs, $1 \mathrm{H}), 5.25$ (quint, $J=1.0 \mathrm{~Hz}, 1 \mathrm{H}), 4.26(\mathrm{~s}, 2 \mathrm{H}), 2.16(\mathrm{td}, J=8.0,1.0 \mathrm{~Hz}, 2 \mathrm{H}), 1.86(\mathrm{~s}, 3 \mathrm{H}), 1.45$ $(\mathrm{m}, 2 \mathrm{H}), 1.32(\mathrm{~m}, 2 \mathrm{H}), 1.25(\mathrm{~s}, 1 \mathrm{H},-\mathrm{OH}), 0.91(\mathrm{t}, J=7.2 \mathrm{~Hz}, 3 \mathrm{H}), 0.13(\mathrm{~s}, 9 \mathrm{H}) \mathrm{ppm} .{ }^{13} \mathrm{C}\left\{{ }^{1} \mathrm{H}\right\} \mathrm{NMR}(75 \mathrm{MHz}$, $\left.\mathrm{CDCl}_{3}\right): \delta=150.4,139.9,133.6,129.2,61.4,34.9,30.7,22.7,22.6,14.1,0.1 \mathrm{ppm}$. HRMS (APCl): $\mathrm{m} / \mathrm{z}$ : calcd for $\mathrm{C}_{13} \mathrm{H}_{25} \mathrm{OSi}[\mathrm{M}-\mathrm{H}]^{-}: 225.1680$, found: 225.1674 .

\section{Compound 56}

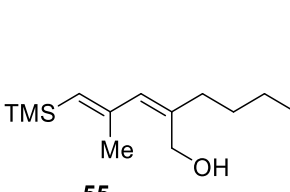

55

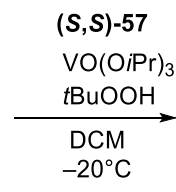

$\left(200^{\circ}\right.$

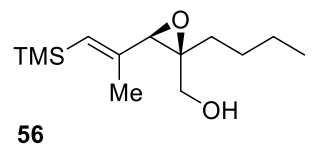

56

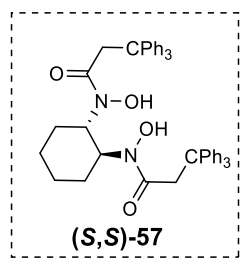

To a solution of ligand $(S, S)-57^{[8,9]}(76 \mathrm{mg}, 107 \mu \mathrm{mol}, 0.017$ equiv) in DCM $(5 \mathrm{~mL})$ at room temperature was added $\mathrm{VO}(\mathrm{O} i-\operatorname{Pr})_{3}(22 \mu \mathrm{L}, 95.2 \mu \mathrm{mol}, 0.015$ equiv). The solution turned red, and the stirring was maintained for $1 \mathrm{~h}$. The above solution of catalyst was then added to a cooled $\left(-20^{\circ} \mathrm{C}\right.$, cooled with a dry ice/ acetone bath) solution of allylic alcohol $55(1.43 \mathrm{~g}, 6.34 \mathrm{mmol}, 1$ equiv) in DCM $(15 \mathrm{~mL})$ and tertbutylhydroperoxide $(2.3 \mathrm{~mL}, 12.70 \mathrm{mmol}, 2$ equiv $5.5 \mathrm{M}$ in nonane) was added. The reaction medium was stored in the freezer $\left(-18^{\circ} \mathrm{C}\right)$ without agitation for 5 days. The mixture was then quenched with saturated $\mathrm{Na}_{2} \mathrm{~S}_{2} \mathrm{O}_{3}$ and $2 \%$ aqueous $\mathrm{KI}$ and stirred for 15 minutes at rt. The layers were separated, and the aqueous phase was extracted with $\mathrm{Et}_{2} \mathrm{O}$. The combined organic phases were dried over $\mathrm{MgSO}_{4}$, filtered, concentrated, and finally purified by silica gel chromatography ( $E t_{2} \mathrm{O} / \mathrm{PE}-10$ to $\left.20 \%\right)$ to afford the epoxy alcohol $56(1.42 \mathrm{~g}$, $5.87 \mathrm{mmol}, 92 \%$ ) as a pale yellow oil. 


\section{SUPPORTING INFORMATION}

$[\alpha]_{D}=-33.5\left(c=1.64, \mathrm{CH}_{2} \mathrm{Cl}_{2}\right)$. IR (neat): $v=3300,2957,1627,1248,1042,854,690 \mathrm{~cm}^{-1} .{ }^{1} \mathbf{H}$ NMR $(300$ $\mathrm{MHz}, \mathrm{CDCl}_{3}$ ): $\delta=5.49$ (quint, $J=1.1 \mathrm{~Hz}, 1 \mathrm{H}$ ), $3.62(\mathrm{~d}, J=11.8 \mathrm{~Hz}, 1 \mathrm{H}), 3.45(\mathrm{~d}, J=11.8 \mathrm{~Hz}, 1 \mathrm{H}), 3.29(\mathrm{~s}$, $1 \mathrm{H}), 1.97(\mathrm{~m}, 2 \mathrm{H}), 1.81(\mathrm{~s}, 3 \mathrm{H}), 1.61-1.26(\mathrm{~m}, 5 \mathrm{H}), 0.92(\mathrm{t}, J=7.0 \mathrm{~Hz}, 3 \mathrm{H}), 0.12(\mathrm{~s}, 9 \mathrm{H}) \mathrm{ppm} .{ }^{13} \mathrm{C}\left\{{ }^{1} \mathrm{H}\right\}$ NMR $\left(75 \mathrm{MHz}, \mathrm{CDCl}_{3}\right): \delta=146.6,124.9,66.6,65.6,61.5,33.1,27.3,23.0,19.1,14.1,0.1$ ppm. HRMS (APCI): $\mathrm{m} / \mathrm{z}$ : calcd for $\mathrm{C}_{13} \mathrm{H}_{25} \mathrm{O}_{2} \mathrm{Si}[\mathrm{M}-\mathrm{H}]^{-}: 241.1629$, found: 241.1630 .

Determination of enantiomeric excess of 56: 4-Nitrobenzoyl ester derivatization I-3
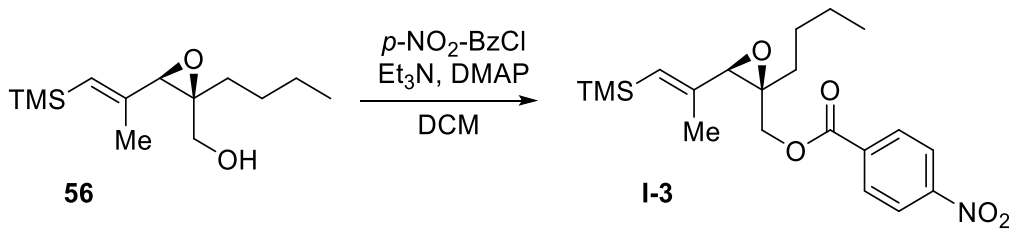

To a solution of epoxide 56 (20 mg, $82.5 \mu \mathrm{mol}, 1$ equiv) in dry DCM (1 mL) was added triethylamine (23 $\mu \mathrm{L}, 0.165 \mathrm{mmol}, 2$ equiv), $p-\mathrm{NO}_{2}-\mathrm{BzCl}$ (46 mg, $0.247 \mathrm{mmol}, 1.2$ equiv) and DMAP (1 mg, $8.2 \mu \mathrm{mol}, 0.1$ equiv). The solution was stirred for $1 \mathrm{~h}$ and hydrolyzed with saturated $\mathrm{NH}_{4} \mathrm{Cl}$. The reaction mixture was extracted with $\mathrm{Et}_{2} \mathrm{O}$, dried over $\mathrm{MgSO}_{4}$, filtered, and concentrated under reduced pressure. The crude material was purified on silica gel ( $\left.\mathrm{Et}_{2} \mathrm{O} / \mathrm{PE}-10 \%\right)$ to yield the corresponding $p-\mathrm{NO}_{2}$-benzoate $\mathrm{I}-3(25 \mathrm{mg}, 63.9 \mu \mathrm{mol}, 75 \%)$ as a yellow oil.

$[\alpha]_{D}=+7.7\left(c=1.04, \mathrm{CH}_{2} \mathrm{Cl}_{2}\right)$. IR (neat): $v=2957,1729,1608,1528,1345,1265,1118,1015,873,718 \mathrm{~cm}^{-}$ 1. ${ }^{1} \mathrm{H}$ NMR $\left(300 \mathrm{MHz}, \mathrm{CDCl}_{3}\right): \delta=8.31$ (brdt, $\left.J=9.0,2.0 \mathrm{~Hz}, 2 \mathrm{H}\right), 8.22$ (brdt, $J=9.0,2.0 \mathrm{~Hz}, 2 \mathrm{H}$ ), 5.57 (quint, $J=1.0 \mathrm{~Hz}, 1 \mathrm{H}), 4.44(\mathrm{~d}, J=11.8 \mathrm{~Hz}, 1 \mathrm{H}), 4.19(\mathrm{~d}, J=11.8 \mathrm{~Hz}, 1 \mathrm{H}), 3.34$ (brs, $1 \mathrm{H}), 1.94$ (ddd, $J=13.9,9.4$, $5.4 \mathrm{~Hz}, 1 \mathrm{H}), 1.85(\mathrm{~s}, 3 \mathrm{H}), 1.64$ (ddd, $J=13.9,9.1,6.3 \mathrm{~Hz}, 1 \mathrm{H}), 1.52-1.20(\mathrm{~m}, 4 \mathrm{H}), 0.92(\mathrm{t}, J=7.1 \mathrm{~Hz}, 3 \mathrm{H})$, $0.11(\mathrm{~s}, 9 \mathrm{H}) \mathrm{ppm} .{ }^{13} \mathrm{C}\left\{{ }^{1} \mathrm{H}\right\}$ NMR $\left(75 \mathrm{MHz}, \mathrm{CDCl}_{3}\right): \delta=164.4,150.8,145.6,135.4,130.9,125.7,123.8,65.6$, 64.8, 62.8, 33.3, 27.1, 22.9, 19.2, 14.1, 0.0 ppm. HRMS (ESI): $\mathrm{m} / \mathrm{z}$ : calcd for $\mathrm{C}_{20} \mathrm{H}_{30} \mathrm{NO}_{5} \mathrm{Si}[\mathrm{M}+\mathrm{H}]^{+}:$:392,1893, found: 392,1900. HPLC (Chiralcel AD column $4.6 \times 250 \mathrm{~mm}$, hexane/ $i-\mathrm{PrOH}=99 / 1$, flow rate $=1.0 \mathrm{~mL} / \mathrm{min}$, $\mathrm{I}=254 \mathrm{~nm}$ ) $t_{\mathrm{R}}=7.28 \mathrm{~min}$ (major), $8.74 \mathrm{~min}$ (minor)]: ee $=94 \%$.
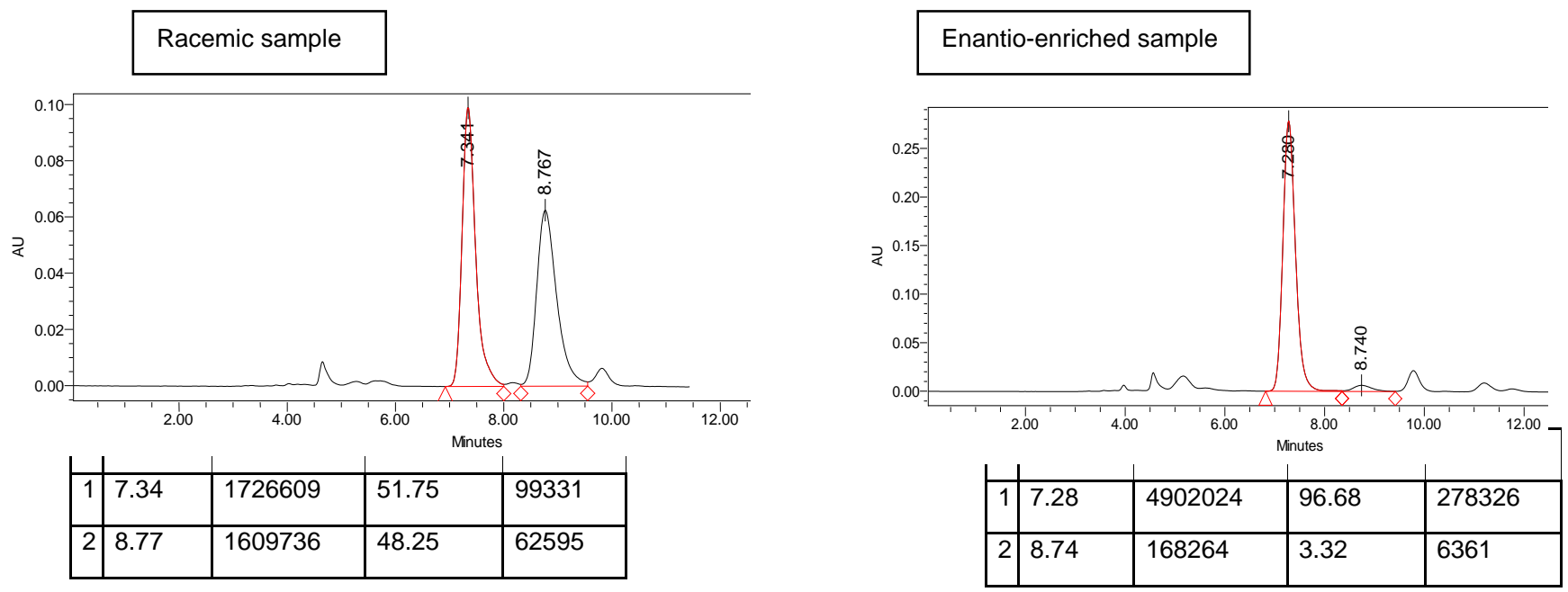


\section{$\underline{\text { Compound } 58}$}

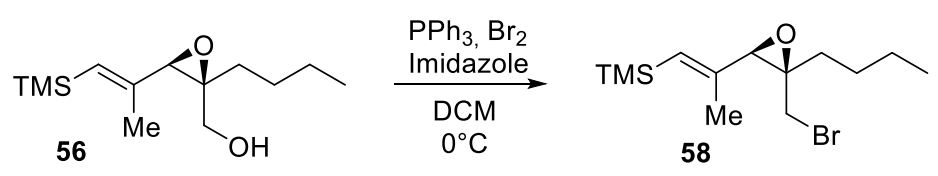

To a cooled $\left(0^{\circ} \mathrm{C}\right)$ solution of $\mathrm{Br}_{2}\left(1.49 \mathrm{~mL}, 28.92 \mathrm{mmol}, 5\right.$ equiv) in $\mathrm{DCM}(20 \mathrm{~mL})$ was added $\mathrm{PPh}_{3}(7.57$ $\mathrm{g}, 28.92 \mathrm{mmol}, 5$ equiv) in DCM $(20 \mathrm{~mL})$ stirring was maintained for 15 minutes at this temperature before imidazole ( $3.93 \mathrm{~g}, 57.85 \mathrm{mmol}, 10$ equiv) was added to the mixture. The above mixture was then added to a cooled $\left(0^{\circ} \mathrm{C}\right)$ solution of alcohol $56(1.40 \mathrm{~g}, 5.78 \mathrm{mmol}, 1$ equiv) in $\mathrm{DCM}(20 \mathrm{~mL})$, and the temperature was slowly warmed to room temperature. After $30 \mathrm{~min}$, the mixture was poured into pentane $(500 \mathrm{~mL})$, filtered over alumina $(5 \mathrm{~cm})$, and eluted with $\mathrm{Et}_{2} \mathrm{O}$. Flash chromatography on silica gel $\left(\mathrm{Et}_{2} \mathrm{O} / \mathrm{PE}-3 \%\right)$ afforded corresponding brominated compound $58(1.21 \mathrm{~g}, 3.97 \mathrm{mmol}, 68 \%)$ as a colorless oil.

$[\alpha]_{\mathrm{D}}=-6.6(c=1.7, \mathrm{DCM})$. IR (neat): $v=2957,2931,2860,1625,1432,1378,1248,1218,836,771 \mathrm{~cm}^{-1}$. ${ }^{1} \mathrm{H}$ NMR $\left(300 \mathrm{MHz}, \mathrm{CDCl}_{3}\right.$ ): $\delta=5.52$ (quint, $J=1.0 \mathrm{~Hz}, 1 \mathrm{H}$ ), $3.36(\mathrm{~d}, J=10.2 \mathrm{~Hz}, 1 \mathrm{H}), 3.34,(\mathrm{~s}, 1 \mathrm{H}), 3.25$ (d, $J=10.2 \mathrm{~Hz}, 1 \mathrm{H}), 1.88(\mathrm{~s}, 3 \mathrm{H}), 1.83(\mathrm{~m}, 2 \mathrm{H}), 1.47-1.24(\mathrm{~m}, 4 \mathrm{H}), 0.93(\mathrm{t}, J=7.1 \mathrm{~Hz}, 3 \mathrm{H}), 0.13(\mathrm{~s}, 9 \mathrm{H}) \mathrm{ppm}$. ${ }^{13} \mathrm{C}\left\{{ }^{1} \mathrm{H}\right\}$ NMR $\left(75 \mathrm{MHz} \mathrm{CDCl}_{3}\right): \delta=146.0,125.3,67.7,64.2,33.0,31.8,26.9,22.8,19.5,14.1,0.0 \mathrm{ppm}$. HRMS (LIFDI): $\mathrm{m} / \mathrm{z}$ : calcd for $\mathrm{C}_{13} \mathrm{H}_{25} \mathrm{OSi}^{79} \mathrm{Br}[\mathrm{M}]^{+*}: 304.0853$, found: 304.0856 .

\section{Compound 59}
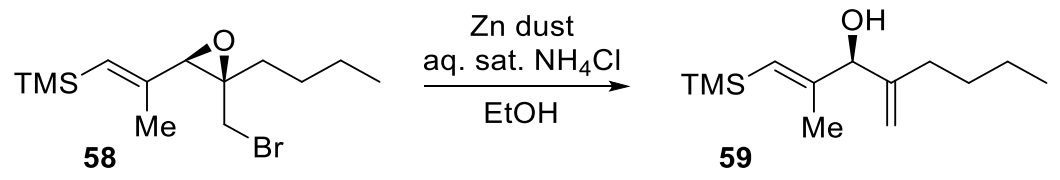

To a solution of bromide 58 (1.20 g, $3.93 \mathrm{mmol}, 1$ equiv) in EtOH (16 mL) was added zinc dust (767 mg, $11.80 \mathrm{mmol}, 3$ equiv). The reaction mixture was stirred vigorously, and saturated $\mathrm{NH}_{4} \mathrm{Cl}$ was added in portions of $500 \mu \mathrm{L}$ until total consumption of the starting material by TLC monitoring. The suspension was then filtered on Celite and eluted with $\mathrm{Et}_{2} \mathrm{O}$. The filtrate was acidified with aqueous $\mathrm{HCl}(0.1 \mathrm{~N})$ and extracted with $\mathrm{Et}_{2} \mathrm{O}$. The organic phase was washed with brine, dried over $\mathrm{MgSO}_{4}$, filtered, and concentrated in vacuo. The crude material was purified on silica gel $\left(\mathrm{Et}_{2} \mathrm{O} / \mathrm{PE}-10 \%\right)$ to obtain alcohol $59(846 \mathrm{mg}, 3.74 \mathrm{mmol}, 95 \%)$ as a pale yellow oil.

$[\alpha]_{D}=-29.3(c=1.4, \mathrm{DCM}) . \mathbf{I R}$ (neat): $v=3400,2956,2930,2859,1619,1378,1248,1041,900,861,690$ $\mathrm{cm}^{-1} .{ }^{1} \mathrm{H}$ NMR $\left(300 \mathrm{MHz}, \mathrm{CDCl}_{3}\right): \delta=5.61$ (quint, $\left.J=1.0 \mathrm{~Hz}, 1 \mathrm{H}\right), 5.10(\mathrm{~s}, 1 \mathrm{H}), 4.93(\mathrm{q}, J=1.8 \mathrm{~Hz}, 1 \mathrm{H}), 4.44$ $(\mathrm{s}, 1 \mathrm{H}), 1.97(\mathrm{dt}, J=15.5,7.7 \mathrm{~Hz}, 1 \mathrm{H}), 1.90(\mathrm{dt}, J=15.5,7.7 \mathrm{~Hz}, 1 \mathrm{H}), 1.68(\mathrm{~d}, J=0.9 \mathrm{~Hz}, 3 \mathrm{H}), 1.48-1.25$ $(\mathrm{m}, 4 \mathrm{H}), 0.89(\mathrm{t}, J=7.2 \mathrm{~Hz}, 3 \mathrm{H}), 0.13(\mathrm{~s}, 9 \mathrm{H}) \mathrm{ppm} .{ }^{13} \mathbf{C}\left\{{ }^{1} \mathrm{H}\right\} \mathbf{N M R}\left(75 \mathrm{MHz}, \mathrm{CDCl}_{3}\right): \delta=153.5,149.6,124.7$, 110.7, 81.8, 31.3, 30.4, 22.7, 17.2, 14.1, 0.0 ppm. HRMS (LIFDI): $\mathrm{m} / \mathrm{z}$ : calcd for $\mathrm{C}_{13} \mathrm{H}_{26} \mathrm{OSi}[\mathrm{M}]^{+*}: 226.1747$, found: 226.1757.

\section{Compound 60}

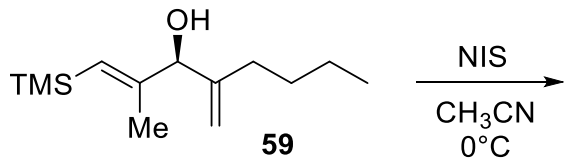<smiles>C=C(CCCC)C(O)/C(C)=C/I</smiles>

To a cooled $\left(0^{\circ} \mathrm{C}\right)$ solution of alcohol $59\left(817 \mathrm{mg}, 3.61 \mathrm{mmol}, 1\right.$ equiv) in $\mathrm{CH}_{3} \mathrm{CN}(18 \mathrm{~mL})$ was added NIS (917 mg, $0.612 \mathrm{mmol}, 1.1$ equiv) in one portion. After $0.5 \mathrm{~h}$, TBAF (3.61 mL, $3.61 \mathrm{mmol}, 1$ equiv $1 \mathrm{M}$ in THF was added, stirring was maintained for an additional $10 \mathrm{~min}$, and the reaction mixture was quenched with saturated $\mathrm{Na}_{2} \mathrm{~S}_{2} \mathrm{O}_{3}$, extracted with $\mathrm{Et}_{2} \mathrm{O}$, dried over $\mathrm{MgSO}_{4}$, filtered, and concentrated under reduced pressure. The crude residue was purified on silica gel ( $\left.\mathrm{Et}_{2} \mathrm{O} / \mathrm{PE}-15 \%\right)$ to yield vinyl iodide 60 (890 mg, 3.18 $\mathrm{mmol}, 88 \%$ ) as a pale yellow oil. 
$[\alpha]_{\mathrm{D}}=-21.1(c=1.75, \mathrm{DCM})$. IR (neat): $v=3400,2957,2928,2872,2857,1689,1456,1378,1270,906$, $781,698 \mathrm{~cm}^{-1} .{ }^{1} \mathrm{H}$ NMR $\left(300 \mathrm{MHz}, \mathrm{CDCl}_{3}\right): \delta=6.39$ (quint, $J=1.1 \mathrm{~Hz}, 1 \mathrm{H}$ ), 5.14 (quint, $J=0.9 \mathrm{~Hz}, 1 \mathrm{H}$ ), 4.98 (qd, $J=1.3,0.8 \mathrm{~Hz}, 1 \mathrm{H}), 4.61(\mathrm{~s}, 1 \mathrm{H}), 1.96(\mathrm{td}, J=15.5,7.7 \mathrm{~Hz}, 1 \mathrm{H}), 1.86(\mathrm{dt}, J=15.5,7.7 \mathrm{~Hz}, 1 \mathrm{H}), 1.78$ (brs, $1 \mathrm{H},-\mathrm{OH}), 1.73(\mathrm{~d}, J=1.3 \mathrm{~Hz}, 3 \mathrm{H}), 1.48-1.23(\mathrm{~m}, 4 \mathrm{H}), 0.90(\mathrm{t}, J=7.1 \mathrm{~Hz}, 3 \mathrm{H}) \mathrm{ppm} .{ }^{13} \mathrm{C}\left\{{ }^{1} \mathrm{H}\right\}$ NMR $(75$ $\left.\mathrm{MHz}, \mathrm{CDCl}_{3}\right): \delta=148.6,147.8,111.1,79.5,79.2,31.3,30.2,22.6,20.2,14.1 \mathrm{ppm}$. HRMS (LIFDI): $\mathrm{m} / \mathrm{z}: \mathrm{calcd}$ for $\mathrm{C}_{10} \mathrm{H}_{17} \mathrm{Ol}[\mathrm{M}]^{+*}: 280.0319$, found: 280.0306 .

\section{Compound 61}
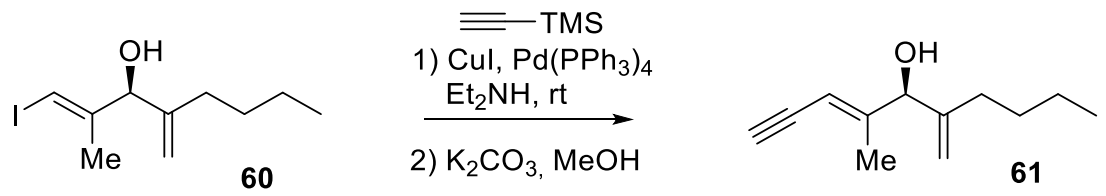

To a degassed solution of vinyliodide 60 (50 mg, $0.178 \mathrm{mmol}, 1$ equiv) in $\mathrm{Et}_{2} \mathrm{NH}$ (1.5 mL) was added Cul ( $3 \mathrm{mg}, 0.018 \mathrm{mmol}, 0.1$ equiv) and the solution turned clear brown. After $5 \mathrm{~min}, \mathrm{Pd}\left(\mathrm{PPh}_{3}\right)_{4}(4 \mathrm{mg}, 3.5 \mu \mathrm{mol}$, 0.02 equiv) was added, followed by trimethylsilylacetylene ( $33 \mu \mathrm{L}, 0.233 \mathrm{mmol}, 1.3$ equiv), the solution turned bright yellow, and the reaction mixture was stirred for additional $2 \mathrm{~h}$. The reaction medium was diluted with $\mathrm{Et}_{2} \mathrm{O}$ and poured into saturated $\mathrm{NH}_{4} \mathrm{Cl}(10 \mathrm{~mL})$. The crude mixture was extracted with $\mathrm{Et}_{2} \mathrm{O}$, dried over $\mathrm{MgSO}_{4}$, filtered and concentrated under reduced pressure.

The crude residue was dissolved into dry $\mathrm{MeOH}(2 \mathrm{~mL})$, and $\mathrm{K}_{2} \mathrm{CO}_{3}(74 \mathrm{mg}, 0.534 \mathrm{mmol}, 3$ equiv) was added in one portion. The mixture was stirred at room temperature for $2 \mathrm{~h}$. The reaction was quenched with saturated aqueous $\mathrm{NH}_{4} \mathrm{Cl}$ and extracted with $\mathrm{Et}_{2} \mathrm{O}$. The combined organics layers were washed with brine, dried over $\mathrm{MgSO}_{4}$, filtered and concentrated under reduced pressure. Flash chromatography on silica gel $\left(\mathrm{Et}_{2} \mathrm{O} / \mathrm{PE}-15 \%\right)$ yielded the terminal alkyne $61(31 \mathrm{mg}, 0.174 \mathrm{mmol}, 98 \%)$ as a colorless oil.

$[\alpha]_{D}=-57.0\left(c=0.3, \mathrm{CHCl}_{3}\right)$. IR (neat): $v=3340,3313,2958,2930,2859,1648,1467,1433,1380,1024$, 904, $633 \mathrm{~cm}^{-1} .{ }^{1} \mathrm{H}$ NMR $\left(300 \mathrm{MHz}, \mathrm{CDCl}_{3}\right.$ ): $\delta 5.67$ (dquint, $J=2.3,1.3 \mathrm{~Hz}, 1 \mathrm{H}$ ), 5.13 (quint, $J=1.0 \mathrm{~Hz}, 1 \mathrm{H}$ ), $4.98(\mathrm{qd}, J=1.3,0.5 \mathrm{~Hz}, 1 \mathrm{H}), 4.54(\mathrm{~s}, 1 \mathrm{H}), 3.10(\mathrm{~d}, J=2.3 \mathrm{~Hz}, 1 \mathrm{H}), 2.00-1.60$ (brs, $1 \mathrm{H},-\mathrm{OH}), 1.98$ (dt, $J=$ 15.5, 7.7 Hz, $1 \mathrm{H}), 1.90(\mathrm{dt}, J=15.5,7.7 \mathrm{~Hz}, 1 \mathrm{H}), 1.81(\mathrm{dt}, J=1.2,0.6 \mathrm{~Hz}, 3 \mathrm{H}), 1.53-1.16(\mathrm{~m}, 4 \mathrm{H}), 0.90(\mathrm{t}$, $J=7.2 \mathrm{~Hz}, 3 \mathrm{H}) \mathrm{ppm} .{ }^{13} \mathrm{C}\left\{{ }^{1} \mathrm{H}\right\}$ NMR $\left(75 \mathrm{MHz}, \mathrm{CDCl}_{3}\right): \delta=152.8,148.9,111.5,105.6,81.5,81.2,79.1,31.2$, 30.2, 22.7, 15.5, $14.1 \mathrm{ppm}$. HRMS (ESI): $\mathrm{m} / \mathrm{z}$ : calcd for $\mathrm{C}_{12} \mathrm{H}_{19} \mathrm{O}[\mathrm{M}+\mathrm{H}]^{+}:$179.1436, found: 179.1433 .

\section{Compound 64}

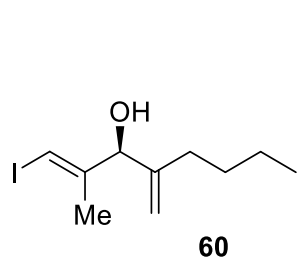

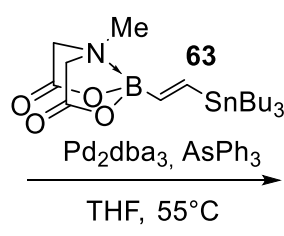

THF, $55^{\circ} \mathrm{C}$

To a degassed solution of vinyl iodide 60 (19 mg, $67.9 \mu \mathrm{mol}, 1$ equiv) and MIDA boronate $63^{[21]}$ (48 $\mathrm{mg}$, $0.102 \mathrm{mmol}, 1.5$ equiv) in THF ( $1 \mathrm{~mL}$ ) was added $\mathrm{AsPPh}_{3}\left(8 \mathrm{mg}, 27 \mu \mathrm{mol}, 0.4\right.$ equiv) and $\mathrm{Pd}_{2} \mathrm{dba}_{3}(6 \mathrm{mg}$, $6.79 \mu \mathrm{mol}, 0.1$ equiv), the reaction mixture was heated to $55^{\circ} \mathrm{C}$ for $1 \mathrm{~h}$ with the help of an oil bath. The solvent was evaporated, and the crude residue was purified on silica gel (acetone/PE - 50 to $70 \%$ ) to afford MIDA boronate 64 (18.6 mg, $55.5 \mu \mathrm{mol}, 82 \%)$ as a clear oil.

$[\alpha]_{\mathrm{D}}=-35.4\left(c=0.5, \mathrm{CH}_{2} \mathrm{Cl}_{2}\right)$. IR (neat): $v=3450,2957,2929,1766,1664,1460,1301,1111,1026,988$ $\mathrm{cm}^{-1} .{ }^{1} \mathrm{H}$ NMR $\left(300 \mathrm{MHz}, \mathrm{CDCl}_{3}\right): \delta=6.92(\mathrm{dd}, J=17.2,10.5 \mathrm{~Hz}, 1 \mathrm{H}), 6.23(\mathrm{dq}, J=10.5,0.9 \mathrm{~Hz}, 1 \mathrm{H}), 5.59$ 
(d, J = 17.2 Hz, 1H), $5.13(\mathrm{~s}, 1 \mathrm{H}), 4.96(\mathrm{~s}, 1 \mathrm{H}), 4.49(\mathrm{~s}, 1 \mathrm{H}), 3.93(\mathrm{~d}, J=16.5 \mathrm{~Hz}, 2 \mathrm{H}), 3.72(\mathrm{dd}, J=16.5,2.1$ $\mathrm{Hz}, 2 \mathrm{H}), 2.85(\mathrm{~s}, 3 \mathrm{H}), 1.95(\mathrm{dt}, J=15.5,7.7 \mathrm{~Hz}, 1 \mathrm{H}), 1.86(\mathrm{dt}, J=15.5,7.7 \mathrm{~Hz}, 1 \mathrm{H}), 1.75$ (brs, $1 \mathrm{H},-\mathrm{OH}), 1.69$ $(\mathrm{s}, 3 \mathrm{H}), 1.46-1.26(\mathrm{~m}, 4 \mathrm{H}), 0.89(\mathrm{t}, J=6.8 \mathrm{~Hz}, 3 \mathrm{H}) \mathrm{ppm} .{ }^{13} \mathrm{C}\left\{{ }^{1} \mathrm{H}\right\}$ NMR $\left(75 \mathrm{MHz}, \mathrm{CDCl}_{3}\right): \delta=167.6,149.4$, $140.5,140.2,128.0,110.5,79.9,61.6,46.7,31.6,30.2,22.7,14.1,12.9 \mathrm{ppm}$. $C_{25}$ was not detected due to quadripolar relaxation. ${ }^{11} \mathbf{B}$ NMR $\left(400 \mathrm{MHz}, \mathrm{CDCl}_{3}\right): \delta=11.1 \mathrm{ppm}$. HRMS (ESI): $\mathrm{m} / \mathrm{z}$ : calcd for $\mathrm{C}_{34} \mathrm{H}_{52} \mathrm{~B}_{2} \mathrm{~N}_{2} \mathrm{O}_{10} \mathrm{Na}[2 \mathrm{M}+\mathrm{Na}]^{+}: 693.3706$, found: 693.3707 .

trans-1,2-bis(tributylstannyl)ethylene 66

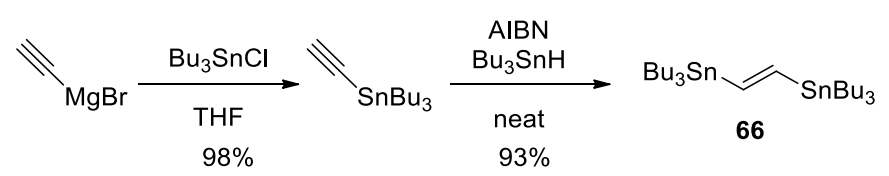

To a solution of ethynylmagnesium bromide $\left(416 \mathrm{~mL}, 200 \mathrm{mmol}, 1\right.$ equiv, $0.48 \mathrm{M}$ in THF) at $0{ }^{\circ} \mathrm{C}$ was added $\mathrm{Bu}_{3} \mathrm{SnCl}(54.25 \mathrm{~mL}, 200 \mathrm{mmol}, 1$ equiv) over $30 \mathrm{~min}$ and the reaction mixture was allowed to stir at rt. After $2 \mathrm{~h}$, the reaction mixture was quenched with $\mathrm{NH}_{4} \mathrm{Cl}$, extracted with $\mathrm{Et}_{2} \mathrm{O}$, dried over $\mathrm{MgSO}_{4}$, filtered, and concentrated on rotavapor. The residue was purified by distillation $\left(100{ }^{\circ} \mathrm{C}, 0.04 \mathrm{mmHg}\right)$ to give $n$-tributyltin acetylene $(62.47 \mathrm{~g}, 98 \%)$.

$\mathbf{B p}=100{ }^{\circ} \mathrm{C}, 0.04 \mathrm{mmHg}$ (litt.: $\left.100-105^{\circ} \mathrm{C}, 0.01 \mathrm{mmHg}\right) .{ }^{[22]}{ }^{1} \mathrm{H}$ NMR $(200 \mathrm{MHz}$, Chloroform- $d) \delta 2.20\left(\mathrm{~s},{ }^{3} \mathrm{~J}_{\mathrm{Sn}-}\right.$ $H=25 \mathrm{~Hz}, 1 \mathrm{H}), 1.58(\mathrm{~m}, 6 \mathrm{H}), 1.34$ (sext, $J=7.2 \mathrm{~Hz}, 6 \mathrm{H}), 1.02(\mathrm{t}, J=7.9 \mathrm{~Hz}, 6 \mathrm{H}), 0.91$ (t, $J=7.2 \mathrm{~Hz}, 9 \mathrm{H}$ ).

To $n$-tributyltin acetylene $(62.04 \mathrm{~g}, 198 \mathrm{mmol}, 1$ equiv) and $n$-Bu3 $\mathrm{SnH}(69.2 \mathrm{~g}, 64 \mathrm{~mL}, 217.8 \mathrm{mmol}, 1.1$ equiv) [freshly prepared from reaction between neat $(\mathrm{Bu} 3 \mathrm{Sn})_{2} \mathrm{O}$ and $\mathrm{PMHS}$ followed by distillation at 0.5 $\mathrm{mmHg}, 90^{\circ} \mathrm{C}$ ] was added AIBN (812 mg, $4.95 \mathrm{mmol}, 0.025$ equiv). The mixture was stirred at $90^{\circ} \mathrm{C}$ for $2 \mathrm{~h}$ with the help of an oil bath, and the whole mixture was directly purified by distillation $\left(160-170{ }^{\circ} \mathrm{C}, 0.05 \mathrm{mmHg}\right)$ to give trans-1,2-bis(tributylstannyl)ethylene 66 (112 g, 93\%). Spectral data were in agreement with the literature. ${ }^{[22]}$

$\mathbf{B p}=160-170{ }^{\circ} \mathrm{C}, 0.05 \mathrm{mmHg}$ (litt.: 175-195 $\left.{ }^{\circ} \mathrm{C}, 0.07 \mathrm{mmHg}\right) .{ }^{[22]}{ }^{1} \mathbf{H}$ NMR $(300 \mathrm{MHz}$, Chloroform-d) $\delta 6.90$ (s, ${ }^{3} J_{S n-H}=104.7,109.8 \mathrm{~Hz}, 1 \mathrm{H}$ ), 1.53 (quint, $J=7.6 \mathrm{~Hz}, 6 \mathrm{H}$ ), 1.33 (sext, $J=7.3 \mathrm{~Hz}, 7 \mathrm{H}$ ), 0.89 (t, $J=7.2 \mathrm{~Hz} ;{ }^{2} J_{S n-}$ $H=49 \mathrm{~Hz}, 15 \mathrm{H})$.

\section{Compound 68}
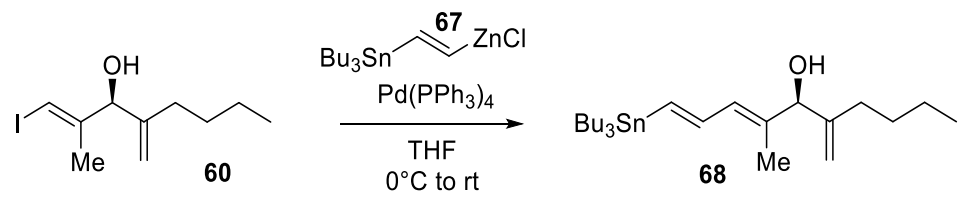

To a cooled $\left(-78^{\circ} \mathrm{C}\right)$ solution of trans-1,2-bis(tributylstannyl)ethylene $66(1.29 \mathrm{~g}, 2.14 \mathrm{mmol}, 6$ equiv) in THF $(3 \mathrm{~mL})$ was added dropwise $n$-BuLi $(0.43 \mathrm{~mL}, 1.07 \mathrm{mmol}, 3$ equiv) and the solution turned pale yellow. After 20 min a freshly prepared solution of $\mathrm{ZnCl}_{2}(1.11 \mathrm{~mL}, 1.11 \mathrm{mmol}, 3.1$ equiv $1 \mathrm{M}$ in THF) was added, and the reaction medium was allowed to warm to $-20^{\circ} \mathrm{C}$. After $15 \mathrm{~min}$, the resulting solution of 67 was transferred to a cooled $\left(0{ }^{\circ} \mathrm{C}\right)$ solution of vinyliodide 60 (100 mg, $0.357 \mathrm{mmol}, 1$ equiv) and $\mathrm{Pd}\left(\mathrm{PPh}_{3}\right)_{4}(20 \mathrm{mg}, 17.8 \mu \mathrm{mol}$, 0.05 equiv) in THF ( $3 \mathrm{~mL}$ ). After 10 min the solution was allowed to warm to room temperature, and stirring was maintained for an additional $2 \mathrm{~h}$. The reaction medium was quenched by saturated $\mathrm{NH}_{4} \mathrm{Cl}$, extracted with $\mathrm{Et}_{2} \mathrm{O}$, dried over $\mathrm{MgSO}_{4}$, filtered, and concentrated under reduced pressure. The crude residue was purified by flash chromatography on silica gel $\left(\mathrm{Et}_{2} \mathrm{O} / \mathrm{PE}-15 \%\right)$ to obtain stannylated diene 68 (110 mg, $0.234 \mathrm{mmol}$, $65 \%)$ as a clear oil. 
$[\alpha]_{D}=-19.2\left(c=2.0, \mathrm{CH}_{2} \mathrm{Cl}_{2}\right)$. IR (neat): $v=2956,2928,2872,2855,1464,1377,1071,986 \mathrm{~cm}^{-1} .{ }^{1} \mathbf{H}$ NMR $\left(300 \mathrm{MHz}, \mathrm{CDCl}_{3}\right): \delta=6.77\left(\mathrm{dd}, J=18.6,10.3 \mathrm{~Hz}, J_{\mathrm{sn}-\mathrm{H}}=66 \mathrm{~Hz}, 1 \mathrm{H}\right), 6.28(\mathrm{~d}, J=18.6 \mathrm{~Hz}, J \mathrm{sn}-\mathrm{H}=66 \mathrm{~Hz}, 1 \mathrm{H})$, $6.13(\mathrm{dm}, J=10.3 \mathrm{~Hz}, 1 \mathrm{H}), 5.15(\mathrm{brs}, 1 \mathrm{H}), 4.96$ (brs, $1 \mathrm{H}), 4.49$ (brd, $J=3.3 \mathrm{~Hz}, 1 \mathrm{H}), 1.92(\mathrm{~m}, 2 \mathrm{H}), 1.70(\mathrm{~d}, J$ $=1.3 \mathrm{~Hz}, 3 \mathrm{H}), 1.59(\mathrm{~d}, J=3.3 \mathrm{~Hz}, 1 \mathrm{H}, \mathrm{OH}), 1.55-1.26(\mathrm{~m}, 16 \mathrm{H}), 0.94-0.85(\mathrm{~m}, 3 \mathrm{H}), 0.89(\mathrm{t}, J=7.3 \mathrm{~Hz}$, 9H) ppm. ${ }^{13} \mathrm{C}\left\{{ }^{1} \mathrm{H}\right\}$ NMR $\left(75 \mathrm{MHz}, \mathrm{CDCl}_{3}\right): \delta=149.5,142.6\left(\mathrm{JSn}_{\mathrm{S}-\mathrm{C}}=10.0 \mathrm{~Hz}\right), 136.4,134.9,130.0,109.9,80.1$, 31.8, 30.2, $29.3\left(\mathrm{JSn}_{\mathrm{S}} \mathrm{C}=20.3 \mathrm{~Hz}\right), 27.4\left(\mathrm{~J}_{\mathrm{Sn}-\mathrm{C}}=27.4 \mathrm{~Hz}\right), 22.7,14.1,13.8,12.4,9.7\left(\mathrm{JSn}_{\mathrm{S}} \mathrm{C}=328,344 \mathrm{~Hz}\right) \mathrm{ppm}$. HRMS $\left(\mathrm{APCl}^{-}\right): \mathrm{m} / \mathrm{z}$ : calcd for $\mathrm{C}_{24} \mathrm{H}_{45} \mathrm{O}^{120} \mathrm{Sn}[\mathrm{M}-\mathrm{H}]^{-}: 469.2502$, found: 469.2493 .

\section{Compound 69}

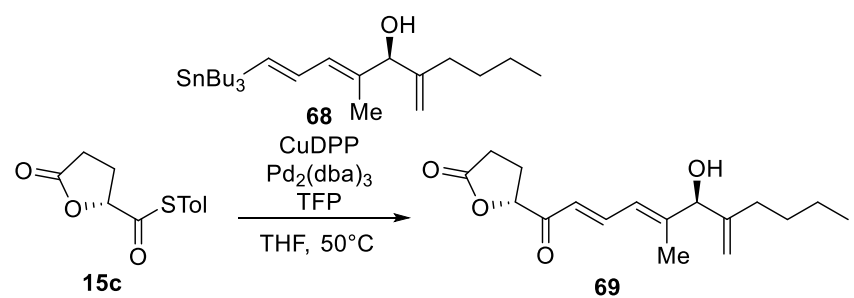

To a degassed solution of thioester $15 \mathrm{c}(50 \mathrm{mg}, 0.21 \mathrm{mmol}, 1$ equiv), stannane 68 (110 mg, $0.23 \mathrm{mmol}$, 1.1 equiv) and tri-ortho-furylphosphine (10 mg, $0.04 \mathrm{mmol}, 0.2$ equiv) in THF (2 mL) was added copper diphenylphosphinate (CuDPP) ${ }^{[23]}$ (71 mg, $0.25 \mathrm{mmol}, 1.2$ equiv), $\mathrm{Pd}_{2}(\mathrm{dba})_{3}(5 \mathrm{mg}, 5.30 \mu \mathrm{mol}, 0.025$ equiv) and the reaction flask was heated to $50^{\circ} \mathrm{C}$ for $2 \mathrm{~h}$ with the help of an oil bath. The reaction was cooled to rt, diluted with $\mathrm{Et}_{2} \mathrm{O}$, and filtered over Celite. The filtrate was concentrated under reduced pressure and purified on silica gel (AcOEt/PE - 50\%) to afford butyrolactone 69 (53 mg, $0.18 \mathrm{mmol}, 85 \%)$ as a yellow oil.

$[\alpha]_{D}=+55.0\left(c=0.8, \mathrm{CH}_{2} \mathrm{Cl}_{2}\right)$. IR (neat): $v=2958,2929,2858,1787,1694,1626,1587,1465,1180,1042$, 983, $903 \mathrm{~cm}^{-1} .{ }^{1} \mathrm{H}$ NMR $\left(300 \mathrm{MHz}, \mathrm{CDCl}_{3}\right): \delta=7.72(\mathrm{dd}, J=15.1,11.7 \mathrm{~Hz}, 1 \mathrm{H}), 6.52(\mathrm{~d}, J=15.1 \mathrm{~Hz}, 1 \mathrm{H})$, $6.42(\mathrm{brd}, J=11.7 \mathrm{~Hz}, 1 \mathrm{H}), 5.15(\mathrm{~s}, 1 \mathrm{H}), 5.04-4.96(\mathrm{~m}, 2 \mathrm{H}), 4.59(\mathrm{~s}, 1 \mathrm{H}), 2.60-2.46(\mathrm{~m}, 3 \mathrm{H}), 2.34(\mathrm{~m}, 1 \mathrm{H})$, $2.05-1.77(\mathrm{~m}, 2 \mathrm{H}), 1.84(\mathrm{~s}, 3 \mathrm{H}), 1.70(\mathrm{brs}, 1 \mathrm{H}, \mathrm{OH}), 1.47-1.25(\mathrm{~m}, 4 \mathrm{H}), 0.89(\mathrm{t}, J=7.2 \mathrm{~Hz}, 3 \mathrm{H}) \mathrm{ppm}$. ${ }^{13} \mathrm{C}\left\{{ }^{1} \mathrm{H}\right\}$ NMR $\left(75 \mathrm{MHz}, \mathrm{CDCl}_{3}\right): \delta=196.1,176.4,152.4,148.8,141.6,123.9,123.3,111.9,81.3,79.8,31.1$, 30.1, 27.5, 25.2, 22.6, 14.1, 14.0 ppm. HRMS (ESI): $\mathrm{m} / \mathrm{z}$ : calcd for $\mathrm{C}_{17} \mathrm{H}_{24} \mathrm{O}_{4} \mathrm{Na}[\mathrm{M}+\mathrm{Na}]^{+}: 315.1572$, found: 315.1562 .

\section{Compound 70}

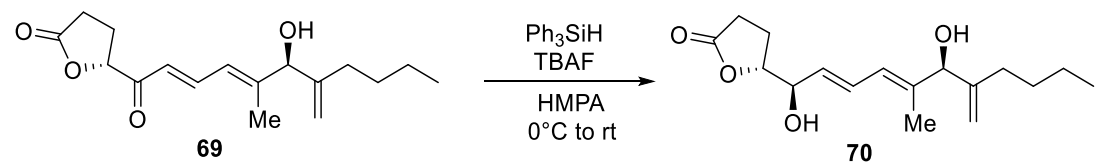

To a cooled $\left(0^{\circ} \mathrm{C}\right)$ solution of butyrolactone $69(25 \mathrm{mg}, 85.6 \mu \mathrm{mol}, 1$ equiv) in HMPA ( $525 \mu \mathrm{L})$ was added $\mathrm{Ph}_{3} \mathrm{SiH}$ (44 mg, $0.171 \mathrm{mmol}, 2$ equiv) and TBAF (13 $\mu \mathrm{L}, 12.8 \mu \mathrm{mol}, 0.15$ equiv). The reaction mixture was stirred at $0{ }^{\circ} \mathrm{C}$ for $10 \mathrm{~min}$ and warmed to room temperature. The reaction was forced by the subsequent addition of TBAF ( $13 \mu \mathrm{L}, 12.8 \mu \mathrm{mol}, 0.15$ equiv) until complete consumption of the starting material, as observed by TLC monitoring. The reaction was diluted with $\mathrm{Et}_{2} \mathrm{O}$, quenched with $\mathrm{H}_{2} \mathrm{O}$, and extracted with $\mathrm{Et}_{2} \mathrm{O}$. The combined organic phases were washed with brine, dried over $\mathrm{MgSO}_{4}$, filtered, and concentrated in a vacuum. The crude residue was purified on silica gel (AcOEt/PE - 60\%) to obtain 70 (16 mg, $54.4 \mu \mathrm{mol}$, $63 \%)$ as a colorless oil.

$[\alpha]_{D}=-6.6\left(c=0.6, \mathrm{CHCl}_{3}\right) . \mathbf{I R}$ (neat): $v=2957,2929,2857,1753,1647,1461,1186,1106,1039,970,900$ $\mathrm{cm}^{-1}$. ${ }^{1} \mathrm{H}$ NMR $\left(300 \mathrm{MHz}, \mathrm{CDCl}_{3}\right): \delta=6.62(\mathrm{ddd}, J=15.2,11.0,1.0 \mathrm{~Hz}, 1 \mathrm{H}), 6.16(\mathrm{~d}, J=11.0 \mathrm{~Hz}, 1 \mathrm{H}), 5.71$ $(\mathrm{dd}, J=15.2,6.9 \mathrm{~Hz}, 1 \mathrm{H}), 5.13(\mathrm{~s}, 1 \mathrm{H}), 4.96(\mathrm{~s}, 1 \mathrm{H}), 4.50(\mathrm{~s}, 1 \mathrm{H}), 4.47(\mathrm{td}, J=7.2,5.9 \mathrm{~Hz}, 1 \mathrm{H}), 4.23(\mathrm{brt}, J=$ 
$5.9 \mathrm{~Hz}, 1 \mathrm{H}$ ), 2.62 (ddd, $J=17.9,10.0,5.9 . \mathrm{Hz}, 1 \mathrm{H}), 2.53$ (ddd, $J=17.9,9.2,8.5 \mathrm{~Hz}$ ), $2.33-2.06(\mathrm{~m}, 2 \mathrm{H}), 2.27$ (brs, $1 \mathrm{H}, \mathrm{OH}), 1.90(\mathrm{~m}, 2 \mathrm{H}), 1.73(\mathrm{brs}, 1 \mathrm{H}, \mathrm{OH}), 1.67(\mathrm{~s}, 3 \mathrm{H}), 1.48-1.20(\mathrm{~m}, 4 \mathrm{H}), 0.89$ (t, J = 7.2 Hz, 3H) ppm. ${ }^{13} \mathrm{C}\left\{{ }^{1} \mathrm{H}\right\}$ NMR $\left(75 \mathrm{MHz}, \mathrm{CDCl}_{3}\right): \delta=177.1,149.3,140.1,129.8,129.2,125.0,110.6,82.7,79.9,74.9$, $31.5,30.2,28.6,23.9,22.7,14.1,12.8 \mathrm{ppm}$. HRMS (ESI): $\mathrm{m} / \mathrm{z}$ : calcd for $\mathrm{C}_{17} \mathrm{H}_{26} \mathrm{O}_{4} \mathrm{Na}[\mathrm{M}+\mathrm{Na}]^{+}: 317,1729$, found: 317,1732 .

\section{Compound 71}

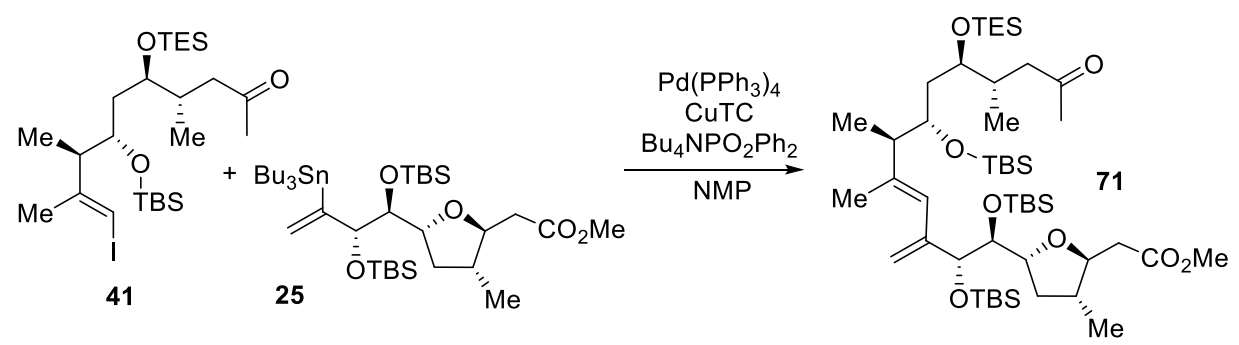

To a degassed solution of stannane 25 ( $22 \mathrm{mg}, 29.0 \mu \mathrm{mol}, 1$ equiv), vinyliodide 41 (17 mg, $29.0 \mu \mathrm{mol}, 1$ equiv) and tetrabutylammonium diphenylphosphinate ( $16 \mathrm{mg}, 35.5 \mu \mathrm{mol}, 1.2$ equiv) in NMP $(750 \mu \mathrm{L})$ at rt was added CuTC ${ }^{[13]}\left(7 \mathrm{mg}, 35.5 \mu \mathrm{mol}, 1.2\right.$ equiv) and $\mathrm{Pd}\left(\mathrm{PPh}_{3}\right)_{4}(10 \mathrm{mg}, 8.7 \mu \mathrm{mol}, 0.3$ equiv). After $2 \mathrm{~h}$ at $\mathrm{rt}$, the reaction medium was diluted with $\mathrm{Et}_{2} \mathrm{O}$ and aqueous $\mathrm{NH}_{4} \mathrm{Cl} / \mathrm{NH}_{3}(2 / 1)$ was added. The aqueous phase was extracted with $\mathrm{Et}_{2} \mathrm{O}$, and the combined organic phases were washed with brine, dried over $\mathrm{MgSO}_{4}$, filtered, and concentrated under reduced pressure. The crude residue was purified on silica gel $\left(\mathrm{Et}_{2} \mathrm{O} / \mathrm{EP}-10 \%\right)$ to yield coupled product 71 (13 mg, $13.6 \mu \mathrm{mol}, 47 \%$ ) as a colorless oil.

$[\alpha]_{\mathrm{D}}=+21.2\left(c=0.85, \mathrm{CHCl}_{3}\right)$. IR (neat): $v=2955,2929,2879,2856,746,1718,1461,1387,1253,1071$, 1042, $833 \mathrm{~cm}^{-1} .{ }^{1} \mathrm{H}$ NMR $\left(400 \mathrm{MHz}, \mathrm{CDCl}_{3}\right): \delta=5.60(\mathrm{~s}, 1 \mathrm{H}), 5.30$ (brs, $\left.1 \mathrm{H}\right), 4.90$ (brs, $\left.1 \mathrm{H}\right), 4.06$ (brs, $\left.1 \mathrm{H}\right)$, $3.87(\mathrm{~m}, 2 \mathrm{H}), 3.70(\mathrm{~m}, 2 \mathrm{H}), 3.65(\mathrm{~s}, 3 \mathrm{H}), 3.52(\mathrm{dd}, J=7.9,2.0 \mathrm{~Hz}, 1 \mathrm{H}), 2.48(\mathrm{dd}, J=14.6,3.9 \mathrm{~Hz}, 1 \mathrm{H}), 2.40$ $(\mathrm{dd}, J=14.6,8.5 \mathrm{~Hz}, 1 \mathrm{H}), 2.33(\mathrm{~m}, 1 \mathrm{H}), 2.26(\mathrm{~m}, 1 \mathrm{H}), 2.16(\mathrm{~m}, 1 \mathrm{H}), 2.13(\mathrm{~m}, 1 \mathrm{H}), 2.06(\mathrm{~s}, 3 \mathrm{H}), 2.06(\mathrm{~m}, 1 \mathrm{H})$, $1.83(\mathrm{~m}, 1 \mathrm{H}), 1.77(\mathrm{~s}, 3 \mathrm{H}), 1.48(\mathrm{~m}, 1 \mathrm{H}), 1.39-1.21(\mathrm{~m}, 1 \mathrm{H}), 1.07(\mathrm{~d}, J=7.0 \mathrm{~Hz}, 3 \mathrm{H}), 0.98(\mathrm{~d}, J=7.5 \mathrm{~Hz}$, $3 \mathrm{H}), 0.97(\mathrm{t}, J=8.0 \mathrm{~Hz}, 9 \mathrm{H}), 0.91(\mathrm{~s}, 9 \mathrm{H}), 0.90(\mathrm{~s}, 9 \mathrm{H}), 0.89-0.85(\mathrm{~m}, 3 \mathrm{H}), 0.87(\mathrm{~s}, 9 \mathrm{H}), 0.60(\mathrm{q}, J=7.9 \mathrm{~Hz}$, $6 \mathrm{H}), 0.08(\mathrm{~s}, 3 \mathrm{H}), 0.07(\mathrm{~s}, 6 \mathrm{H}), 0.04(\mathrm{~s}, 3 \mathrm{H}), 0.02(\mathrm{~s}, 3 \mathrm{H}),-0.01(\mathrm{~s}, 3 \mathrm{H}) \mathrm{ppm} .{ }^{13} \mathrm{C}\left\{{ }^{1} \mathrm{H}\right\} \mathrm{NMR}\left(101 \mathrm{MHz}, \mathrm{CDCl}_{3}\right)$ ठ 208.5, 172.2, 145.6, 141.5, 125.6, 114.7, 80.6, 80.0, 79.6, 78.8, 72.7, 72.0, 51.7, 47.6, 45.7, 40.5, 39.5, $38.1,37.4,33.5,30.7,26.3,26.1,26.1,18.6,18.5,18.2,17.2,16.1,15.7,14.4,7.1,5.3$. HRMS (ESI): $\mathrm{m} / \mathrm{z}$ : calcd for $\mathrm{C}_{49} \mathrm{H}_{98} \mathrm{O}_{8} \mathrm{Si}_{4} \mathrm{Na}[\mathrm{M}+\mathrm{Na}]^{+}:$949.6237, found: 949.6250 .

\section{Compound 72}

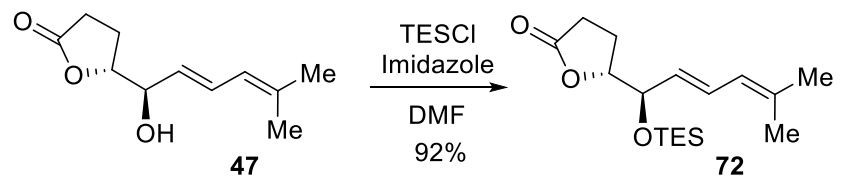

To a solution of compound 47 (324 mg, $1.636 \mathrm{mmol}, 1$ equiv) in DMF (2 mL) at rt was added imidazole (222 mg, $3.272 \mathrm{mmol}, 2$ equiv) and TESCl (327 $\mu \mathrm{L}, 1.963 \mathrm{mmol}, 1.2$ equiv). After stirring $16 \mathrm{~h}$ at $\mathrm{rt}$, the reaction mixture was quenched with aqueous $\mathrm{NH}_{4} \mathrm{Cl}$ and extracted with $\mathrm{Et}_{2} \mathrm{O}$. The combined organic layers were washed with brine, dried over $\mathrm{MgSO}_{4}$, filtered, and concentrated under reduced pressure. Flash chromatography on silica gel (AcOEt/PE - 5\%) yielded compound 72 (472 mg, 92\%) as a colorless oil.

$[\alpha]_{D}=-8.1(c=1.1, D C M) .{ }^{1} \mathrm{H}$ NMR $(300 \mathrm{MHz}$, Chloroform- $d) \delta 6.47(\mathrm{dd}, J=15.2,11.0 \mathrm{~Hz}, 1 \mathrm{H}), 5.81(\mathrm{~d}, J$ $=11.0 \mathrm{~Hz}, 1 \mathrm{H}), 5.52(\mathrm{dd}, J=15.2,6.9 \mathrm{~Hz}, 1 \mathrm{H}), 4.44(\mathrm{ddd}, J=7.7,5.6,4.4 \mathrm{~Hz}, 1 \mathrm{H}), 4.27(\mathrm{dd}, J=6.9,4.4 \mathrm{~Hz}$, $1 \mathrm{H}$ ), 2.50 (ddd, $J=17.6,10.0,7.4 \mathrm{~Hz}, 1 \mathrm{H}$ ), 2.41 (ddd, $J=17.6,9.2,7.2 \mathrm{~Hz}, 1 \mathrm{H}$ ), 2.18 (ddt, $J=13.1,9.2,7.5$ $\mathrm{Hz}, 1 \mathrm{H}$ ), 2.08 (dddd, $J=13.1,10.0,7.2,5.7 \mathrm{~Hz}, 1 \mathrm{H}$ ), 1.78 (s, 3H), $1.76(\mathrm{~s}, 3 \mathrm{H}), 0.95$ (t, $J=7.9 \mathrm{~Hz}, 9 \mathrm{H}), 0.60$ 
(q, $J=8.0 \mathrm{~Hz}, 6 \mathrm{H}) .{ }^{13} \mathrm{C}\left\{{ }^{1} \mathrm{H}\right\} \mathrm{NMR}\left(75 \mathrm{MHz}, \mathrm{CDCl}_{3}\right) \delta 177.6,137.0,129.4,127.5,124.3,83.0,75.0,28.5,26.1$, 23.3, 18.4, 6.9, 5.1. HRMS (ESI): $\mathrm{m} / \mathrm{z}$ : calcd for $\mathrm{C}_{17} \mathrm{H}_{30} \mathrm{O}_{3} \mathrm{SiNa}[\mathrm{M}+\mathrm{Na}]^{+}: 333.1862$, found: 333.1855 .

\section{Compound 11a}
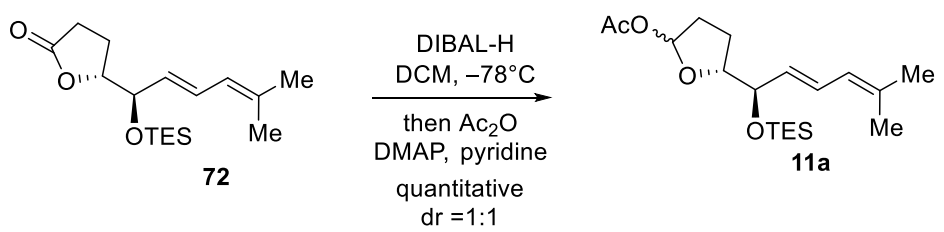

To a solution of compound 72 (130 mg, $0.416 \mathrm{mmol}, 1$ equiv) in $\mathrm{CH}_{2} \mathrm{Cl}_{2}(2 \mathrm{~mL})$ at $-78{ }^{\circ} \mathrm{C}$ was added DIBALH (378 $\mu \mathrm{L}, 0.416 \mathrm{mmol}, 1.0$ equiv, $1.1 \mathrm{M}$ in hexanes). After $30 \mathrm{~min}$ at $-78{ }^{\circ} \mathrm{C}, \mathrm{Ac}_{2} \mathrm{O}(196 \mu \mathrm{L}, 2.08 \mathrm{mmol}, 5$ equiv), pyridine ( $167 \mu \mathrm{L}, 2.08 \mathrm{mmol}, 5$ equiv) and DMAP (151 mg, $1.248 \mathrm{mmol}, 3$ equiv) were added in this order, and the reaction mixture was allowed to warm slowly to $0^{\circ} \mathrm{C}$ and was stirred then for 30 min at this temperature. The reaction mixture was then poured slowly into a stirred saturated solution of potassium sodium tartrate, stirred 30 min at rt, diluted with water, extracted with $\mathrm{Et}_{2} \mathrm{O}(\times 3)$, washed with a diluted solution of $\mathrm{NaHCO}_{3}$, and finally with a brine solution. The organic layer was then dried over solid $\mathrm{NaHCO}_{3}$, filtered and concentrated, and the residue was filtered over a small plug of silica gel eluting with $\mathrm{Et}_{2} \mathrm{O}$ to afford unstable lactol acetate 11a (149.0 mg, quantitative) as a 1:1 mixture of anomers.

${ }^{1} \mathrm{H}$ NMR (300 MHz, Chloroform-d, in about 50:50 mixture of anomers) $\delta 6.47$ (dd, $J=15.3,11.0 \mathrm{~Hz}, 0.5 \mathrm{H}$ ), $6.45(\mathrm{dd}, J=15.3,11.0 \mathrm{~Hz}, 0.5 \mathrm{H}), 6.29(\mathrm{~d}, J=3.8 \mathrm{~Hz}, 0.5 \mathrm{H}), 6.26(\mathrm{t}, J=3.0,1.5 \mathrm{~Hz}, 0.5 \mathrm{H}), 5.81(\mathrm{~d}, J=11.0$ $\mathrm{Hz}, 1 \mathrm{H}), 5.51(\mathrm{dd}, J=15.3,5.4 \mathrm{~Hz}, 1 \mathrm{H}), 4.31-4.17(\mathrm{~m}, 1.5 \mathrm{H}), 4.01(\mathrm{dt}, J=9.9,6.0 \mathrm{~Hz}, 0.5 \mathrm{H}), 2.02(\mathrm{~s}, 1.5 \mathrm{H})$, $2.01(\mathrm{~s}, 1.5 \mathrm{H}), 2.06-1.78(\mathrm{~m}, 4 \mathrm{H}), 1.80-1.73(\mathrm{~m}, 6 \mathrm{H}), 0.95(\mathrm{t}, J=7.9 \mathrm{~Hz}, 4.5 \mathrm{H}), 0.94(\mathrm{t}, J=7.9 \mathrm{~Hz}, 4.5 \mathrm{H})$, $0.60(\mathrm{q}, J=7.9 \mathrm{~Hz}, 3 \mathrm{H}), 0.59(\mathrm{q}, J=7.9 \mathrm{~Hz}, 3 \mathrm{H})$. HRMS (ESI): $\mathrm{m} / z$ : calcd for $\mathrm{C}_{19} \mathrm{H}_{34} \mathrm{O}_{4} \mathrm{SiNa}[\mathrm{M}+\mathrm{Na}]^{+}$: 377.2124, found: 377.2133 .

\section{Compound 78}

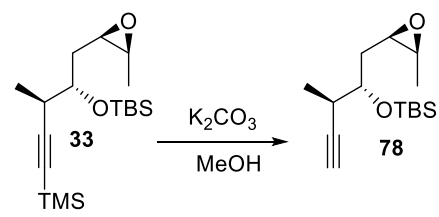

Protected alkyne 33 (137 mg, $0.402 \mathrm{mmol}, 1$ equiv) was dissolved into dry $\mathrm{MeOH}(4 \mathrm{~mL})$, and $\mathrm{K}_{2} \mathrm{CO}_{3}(277$ $\mathrm{mg}, 2.01 \mathrm{mmol}, 5$ equiv) was added in one portion at $0{ }^{\circ} \mathrm{C}$. The mixture was stirred at room temperature for $2 \mathrm{~h}$. The reaction was quenched with water, extracted with $\mathrm{Et}_{2} \mathrm{O}$. The combined organic layers were washed with brine, dried over $\mathrm{MgSO}_{4}$, filtered, and concentrated under reduced pressure. Chromatography on silica gel (Et $t_{2} \mathrm{O} /$ petroleum ether: $5 \%$ ) yielded the terminal alkyne 78 (103 mg, $\left.0.386 \mathrm{mmol}, 96 \%\right)$ as a colorless oil.

$[\alpha]^{20} \mathrm{D}=-7.5\left(c=5.8, \mathrm{CHCl}_{3}\right)$. IR (neat): $v=3313,2957,2930,2857,1473,1376,1253,1132,1101,833$, $774,634 \mathrm{~cm}^{-1} .{ }^{1} \mathbf{H}$ NMR $\left(300 \mathrm{MHz}, \mathrm{CDCl}_{3}\right) \delta=3.86$ (ddd, $\left.J=7.0,5.0,4.2 \mathrm{~Hz}, 1 \mathrm{H}\right), 3.08-2.95(\mathrm{~m}, 2 \mathrm{H}), 2.66$ (qdd, $J=7.2,4.2,2.5 \mathrm{~Hz}), 2.04(\mathrm{~d}, J=2.5 \mathrm{~Hz}, 1 \mathrm{H}), 1.90-1.69(\mathrm{~m}, 2 \mathrm{H}), 1.27(\mathrm{~d}, J=5.7 \mathrm{~Hz}, 3 \mathrm{H}), 1.18(\mathrm{~d}, J=$ $7.2 \mathrm{~Hz}, 3 \mathrm{H}), 0.89(\mathrm{~s}, 9 \mathrm{H}), 0.07(\mathrm{~s}, 3 \mathrm{H}), 0.06(\mathrm{~s}, 3 \mathrm{H}) .{ }^{13} \mathrm{C}\left\{{ }^{1} \mathrm{H}\right\} \mathrm{NMR}\left(75 \mathrm{MHz}, \mathrm{CDCl}_{3}\right) \delta 86.1,72.7,70.2,54.6$, 52.4, 32.2, 31.5, 25.9 (3C), 18.2, 15.1, 13.4, -4.4, -4.5. HRMS (ESI): $\mathrm{m} / \mathrm{z}$ : calcd for $\mathrm{C}_{15} \mathrm{H}_{28} \mathrm{O}_{2} \mathrm{SiNa}[\mathrm{M}+\mathrm{Na}]^{+}$: 291.1756 , found 291.1749 .

\section{Compound 79}




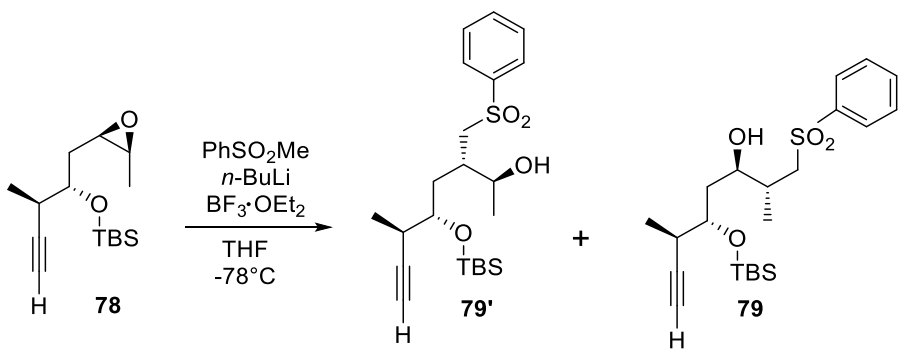

To a cooled $\left(-78^{\circ} \mathrm{C}\right)$ solution of methylphenylsulfone $(3.18 \mathrm{~g}, 20.36 \mathrm{mmol}, 2$ equiv) in dry THF $(100 \mathrm{~mL})$, was added dropwise $n \mathrm{BuLi}(8.15 \mathrm{~mL}, 20.36 \mathrm{mmol}, 2$ equiv $2.5 \mathrm{M}$ in hexanes). After 15 minutes at this temperature, a solution of epoxide $78(2.75 \mathrm{~g}, 10.18 \mathrm{mmol}, 1$ equiv) in THF (20 mL) was added, followed by $\mathrm{BF}_{3} \cdot \mathrm{OEt}_{2}(1.29 \mathrm{~mL}, 10.18 \mathrm{mmol}, 1$ equiv). After $30 \mathrm{~min}$, the mixture was quenched with a saturated solution of $\mathrm{NaHCO}_{3}$, then extracted with $\mathrm{Et}_{2} \mathrm{O}$, dried over $\mathrm{MgSO}_{4}$, filtered, and concentrated on reduced pressure. Pentane was added to the crude mixture, and an excess of unreacted methylphenylsulfone was removed by filtration. Successive purification on silica gel (AcOEt/petroleum ether-20\%) afforded desired alcohol 79 (3.08 g, $7.23 \mathrm{mmol}, 71 \%)$, and its regioisomer 79' (1.27 g, $2.98 \mathrm{mmol}, 29 \%)$ as colorless oils.

Compound 79: $[\alpha]^{20} \mathrm{D}=+4.6\left(c=3.6, \mathrm{CHCl}_{3}\right)$. IR (neat): $v=3400,2976,2930,1447,1303,1148,1085,741$, $666,623 \mathrm{~cm}^{-1} .{ }^{1} \mathrm{H}$ NMR $\left(300 \mathrm{MHz}, \mathrm{CDCl}_{3}\right) \delta=7.92(\mathrm{~m}, 2 \mathrm{H}), 7.67-7.60(\mathrm{~m}, 1 \mathrm{H}), 7.55(\mathrm{~m}, 2 \mathrm{H}), 3.93(\mathrm{~m}, 1 \mathrm{H})$, 3.87 (ddd, $J=8.6,7.4,3.7 \mathrm{~Hz}, 1 \mathrm{H}$ ), 3.28 (dd, $J=14.5,5.6 \mathrm{~Hz}, 1 \mathrm{H}$ ), 3.16 (dd, $J=14.5,5.2 \mathrm{~Hz}, 1 \mathrm{H}$ ), 2.59 (qdd, $J=7.0,3.7,2.5 \mathrm{~Hz}, 1 \mathrm{H}), 2.41(\mathrm{~s}, 1 \mathrm{H}, \mathrm{OH}), 2.21(\mathrm{dqd}, J=7.7,5.3,4.7 \mathrm{~Hz}, 1 \mathrm{H}), 2.05(\mathrm{~d}, J=2.5 \mathrm{~Hz}, 1 \mathrm{H}), 1.80$ (ddd, $J=14.3,7.7,3.3 \mathrm{~Hz}, 1 \mathrm{H}$ ), 1.67 (ddd, $J=14.3,8.6,4.7 \mathrm{~Hz}, 1 \mathrm{H}), 1.14(\mathrm{~d}, J=6.3 \mathrm{~Hz}, 3 \mathrm{H}), 1.11(\mathrm{~d}, J=$ $7.0 \mathrm{~Hz}, 3 \mathrm{H}), 0.85(\mathrm{~s}, 9 \mathrm{H}), 0.07(\mathrm{~s}, 3 \mathrm{H}), 0.06(\mathrm{~s}, 3 \mathrm{H}) .{ }^{13} \mathrm{C}\left\{{ }^{1} \mathrm{H}\right\} \mathbf{N M R}\left(75 \mathrm{MHz}, \mathrm{CDCl}_{3}\right) \delta 140.19,133.76,129.41$ (2C), 127.89 (2C), 86.03, 72.52, 70.55, 68.79, 57.48, 37.93, 33.94, 32.18, 25.91 (3C), 20.36, 18.08, 13.91, 4.37. HRMS (ESI): $\mathrm{m} / \mathrm{z}$ : calcd for $\mathrm{C}_{22} \mathrm{H}_{36} \mathrm{O}_{4} \mathrm{SiSNa}[\mathrm{M}+\mathrm{Na}]^{+}: 447.2001$, found 447.1996.

Compound 79': $[\alpha]^{20} \mathrm{D}=-19.6\left(c=2.9, \mathrm{CHCl}_{3}\right)$. IR (neat): $v=3400,2976,2930,1447,1303,1148,1085$, $741,666,623 \mathrm{~cm}^{-1} .{ }^{1} \mathrm{H}$ NMR $\left(300 \mathrm{MHz}, \mathrm{CDCl}_{3}\right) \delta=7.96-7.89(\mathrm{~m}, 2 \mathrm{H}), 7.68-7.60(\mathrm{~m}, 1 \mathrm{H}), 7.59-7.52(\mathrm{~m}$, 2H), 3.99 (dt, $J=9.1,3.7 \mathrm{~Hz}, 1 \mathrm{H}$ ), 3.56 (ddd, $J=9.4,5.4,1.7 \mathrm{~Hz}, 1 \mathrm{H}), 3.43(\mathrm{dd}, J=14.3,2.8 \mathrm{~Hz}, 1 \mathrm{H}$ ), 3.02 (bs, 0.6H, OH), 2.93 (dd, $J=14.3,9.1 \mathrm{~Hz}, 1 \mathrm{H}$ ), 2.64 (qdd, $J=7.0,3.7,2.5 \mathrm{~Hz}, 1 \mathrm{H}$ ), 2.12 (dqdd, $J=9.3,7.2$, 5.4, $2.8 \mathrm{~Hz}, 1 \mathrm{H}$ ), 2.07 (d, J =2.5 Hz, 1H), 1.83 (ddd, $J=14.3,3.8,2.0 \mathrm{~Hz}, 1 \mathrm{H}$ ), 1.66 (brs, 0.4H, OH), 1.46 (dt, $J=14.3,9.5 \mathrm{~Hz}, 1 \mathrm{H}), 1.16(\mathrm{~d}, J=7.0 \mathrm{~Hz}, 3 \mathrm{H}), 1.13(\mathrm{~d}, J=7.2 \mathrm{~Hz}, 3 \mathrm{H}), 0.87(\mathrm{~s}, 9 \mathrm{H}), 0.09(\mathrm{~s}, 3 \mathrm{H}), 0.09(\mathrm{~s}$, 3H). ${ }^{13} \mathrm{C}\left\{{ }^{1} \mathrm{H}\right\}$ NMR $\left(75 \mathrm{MHz}, \mathrm{CDCl}_{3}\right) \delta 140.3,133.7,129.4(2 \mathrm{C}), 128.0(2 \mathrm{C}), 85.6,74.7,74.3,70.8,58.4,35.5$, 35.0, 31.8, $25.9(3 \mathrm{C}), 18.0,17.0,13.8,-4.2,-4.7$. HRMS (ESI): $\mathrm{m} / \mathrm{z}$ : calcd for $\mathrm{C}_{22} \mathrm{H}_{36} \mathrm{O}_{4} \mathrm{SSiNa}[\mathrm{M}+\mathrm{Na}]^{+}$: 447.2001 , found 447.1996 . 


\section{Compound 80}

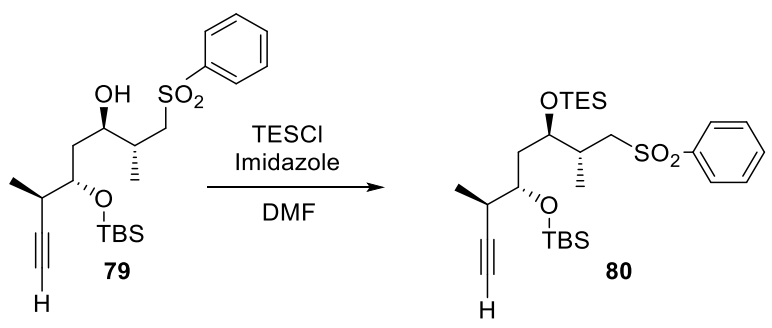

Alcohol 79 (3.03 g, $7.113 \mathrm{mmol}, 1$ equiv) was dissolved into dry DMF (20 mL), and TESCl (1.6 g, 10.7 mmol, 1.5 equiv) was added, followed by imidazole $(1.93 \mathrm{~g}, 28.4 \mathrm{mmol}, 4$ equiv) at rt. After consumption of the starting material as visualized by TLC monitoring, the mixture was hydrolyzed with water and extracted with $\mathrm{Et}_{2} \mathrm{O}$. The combined organic phases were washed with brine, dried over $\mathrm{MgSO}_{4}$, filtered then concentrated in a vacuum. Purification on silica gel ( $\mathrm{Et}_{2} \mathrm{O} /$ petroleum ether: $\left.10 \%\right)$ yielded TES ether $80(3.68$ $\mathrm{g}, 6.81 \mathrm{mmol}, 96 \%)$ as a colorless oil.

$[\alpha]^{20} \mathrm{D}=-29.7\left(c=1.6, \mathrm{CHCl}_{3}\right)$. IR (neat): $v=2956,2878,1447,1305,1256,1149,1110,1084,1004,955$, 833, $774 \mathrm{~cm}^{-1} .{ }^{1} \mathrm{H}$ NMR $\left(300 \mathrm{MHz}, \mathrm{CDCl}_{3}\right) \delta=7.95-7.90(\mathrm{~m}, 2 \mathrm{H}), 7.68-7.61(\mathrm{~m}, 1 \mathrm{H}), 7.60-7.52(\mathrm{~m}, 2 \mathrm{H})$, 3.74 (ddd, $J=8.2,5.7,2.4 \mathrm{~Hz}, 1 \mathrm{H}$ ), 3.57 (dt, $J=8.1,3.7 \mathrm{~Hz}, 1 \mathrm{H}$ ), 3.23 (dd, $J=14.4,2.5 \mathrm{~Hz}, 1 \mathrm{H}$ ), 2.92 (dd, $J$ $=14.4,9.7 \mathrm{~Hz}, 1 \mathrm{H}$ ), 2.53 (qdd, $J=6.8,3.7,2.4 \mathrm{~Hz}, 1 \mathrm{H}), 2.14(\mathrm{~m}, 1 \mathrm{H}), 2.05(\mathrm{~d}, J=2.4 \mathrm{~Hz}, 1 \mathrm{H}), 1.69$ (ddd, $J$ $=13.9,8.1,4.0 \mathrm{~Hz}, 1 \mathrm{H}), 1.58(\mathrm{ddd}, J=13.9,8.2,5.7 \mathrm{~Hz}, 1 \mathrm{H}), 1.17(\mathrm{~d}, J=6.8 \mathrm{~Hz}, 3 \mathrm{H}), 1.12(\mathrm{~d}, J=7.0 \mathrm{~Hz}$, $3 \mathrm{H}), 0.92(\mathrm{t}, J=7.9 \mathrm{~Hz}, 9 \mathrm{H}), 0.86(\mathrm{~s}, 9 \mathrm{H}), 0.54(\mathrm{q}, J=7.9 \mathrm{~Hz}, 6 \mathrm{H}), 0.00(\mathrm{~s}, 3 \mathrm{H}),-0.03(\mathrm{~s}, 3 \mathrm{H}) \mathrm{ppm} .{ }^{13} \mathrm{C}\left\{{ }^{1} \mathrm{H}\right\}$ NMR $\left(75 \mathrm{MHz}_{1} \mathrm{CDCl}_{3}\right) \delta 140.2,133.6,129.4(2 \mathrm{C}), 128.1$ (2C), 85.9, 73.0, 70.6, 70.5, 57.5, 37.1, 32.1, 31.89, 25.9 (3C), 18.1, 17.0, 14.5, 7.0 (3C), 5.2 (3C), -4.3, -4.5. HRMS (ESI): $\mathrm{m} / \mathrm{z}$ : calcd for $\mathrm{C}_{28} \mathrm{H}_{50} \mathrm{O}_{4} \mathrm{Si}_{2} \mathrm{SNa}[\mathrm{M}+\mathrm{Na}]^{+}$: 561.2866 , found 561.2859 .

\section{$\underline{\text { Compound } 81}$}

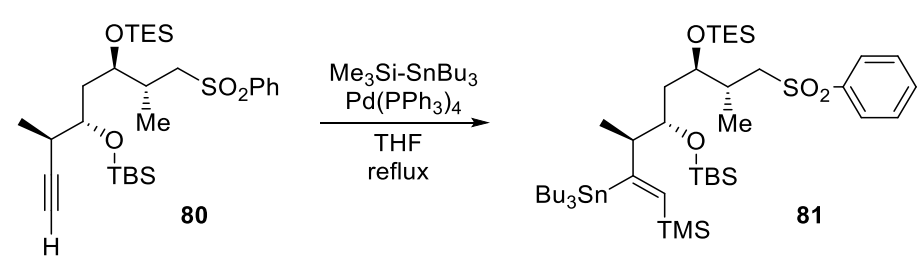

To a solution of alkyne 80 (3.60 g, $6.607 \mathrm{mmol}, 1$ equiv) in THF (20 mL) was added trimetylsilyltributylstannane $\left(2.80 \mathrm{~mL}, 8.00 \mathrm{mmol}, 1.2\right.$ equiv) followed by $\mathrm{Pd}\left(\mathrm{PPh}_{3}\right)_{4}(455 \mathrm{mg}, 1.0 \mathrm{mmol}, 0.15$ equiv) and warmed to reflux during $12 \mathrm{~h}$ with the help of an oil bath. After this, the reaction was cooled to room temperature, concentrated, and purified on silica gel ( $\mathrm{Et}_{2} \mathrm{O} /$ petroleum ether: 5 to $8 \%$ ) to obtain desired vinylstannane 81 (4.7 g, $5.20 \mathrm{mmol}, 78 \%)$ as a colorless oil.

$[\alpha]^{20} \mathrm{D}=-43.6\left(\mathrm{c}=1.4, \mathrm{CHCl}_{3}\right)$. IR (neat): $v=2955,2929,2855,1461,1306,1247,1150,1084,1002,832$, 773, $744 \mathrm{~cm}^{-1}$. ${ }^{1} \mathbf{H}$ NMR $\left(300 \mathrm{MHz}, \mathrm{CDCl}_{3}\right) \delta=7.93-7.86(\mathrm{~m}, 2 \mathrm{H}), 7.68-7.60(\mathrm{~m}, 1 \mathrm{H}), 7.55(\mathrm{~m}, 2 \mathrm{H}), 6.75$ (s, Jn- $=187 \mathrm{~Hz}, 1 \mathrm{H}), 3.76-3.61(\mathrm{~m}, 2 \mathrm{H}), 3.03(\mathrm{dd}, J=14.4,1.5 \mathrm{~Hz}, 1 \mathrm{H}), 2.84(\mathrm{dd}, J=14.4,9.6 \mathrm{~Hz}, 1 \mathrm{H})$, $2.42(\mathrm{qd}, J=6.8,3.8, \mathrm{~Hz}, 1 \mathrm{H}), 2.13(\mathrm{~m}, 1 \mathrm{H}), 1.60(\mathrm{~m}, 1 \mathrm{H}), 1.53-1.40(\mathrm{~m}, 6 \mathrm{H}), 1.40-1.24(\mathrm{~m}, 7 \mathrm{H}), 1.12(\mathrm{~d}$, $J=6.8 \mathrm{~Hz}, 3 \mathrm{H}), 1.00-0.75(\mathrm{~m}, 36 \mathrm{H}), 0.53(\mathrm{q}, J=7.9 \mathrm{~Hz}, 6 \mathrm{H}), 0.06(\mathrm{~s}, 9 \mathrm{H}), 0.05(\mathrm{~s}, 3 \mathrm{H}), 0.02(\mathrm{~s}, 3 \mathrm{H}) \mathrm{ppm}$. ${ }^{13} \mathrm{C}\left\{{ }^{1} \mathrm{H}\right\}$ NMR $\left(75 \mathrm{MHz}, \mathrm{CDCl}_{3}\right) \delta 167.0,145.3,140.4,133.6,129.4(2 \mathrm{C}), 128.0(2 \mathrm{C}), 73.3,72.4,57.9,49.9$, 38.7, 32.4, $29.4\left({ }^{3} \mathrm{JSn}_{\mathrm{S}-\mathrm{C}}=19.3 \mathrm{~Hz}, 3 \mathrm{C}\right), 27.7\left({ }^{2} \mathrm{~J}_{\mathrm{Sn}-\mathrm{C}}=71 \mathrm{~Hz}, 3 \mathrm{C}\right), 26.2(3 \mathrm{C}), 18.3,17.5,17.2,13.8(3 \mathrm{C}), 11.9$ (3C), 7.1 (3C), $5.3(3 \mathrm{C}), 0.5(3 \mathrm{C}),-3.6,-4.0$. HRMS (ESI): $\mathrm{m} / \mathrm{z}$ : calcd for $\mathrm{C}_{43} \mathrm{H}_{86} \mathrm{O}_{4} \mathrm{Si}_{3} \mathrm{~S}^{120} \mathrm{SnNa}[\mathrm{M}+\mathrm{Na}]^{+}$: 925.4474, found 925.4479 . 


\section{Compound 82}

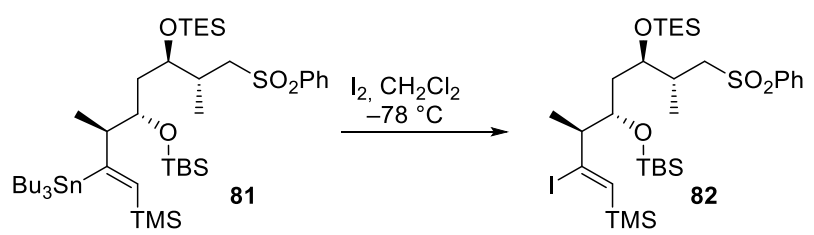

To a cooled $\left(-78^{\circ} \mathrm{C}\right)$ solution of vinylstannane 81 (4.61 g, $5.19 \mathrm{mmol}$, 1 equiv) in $\mathrm{CH}_{2} \mathrm{Cl}_{2}(5 \mathrm{~mL})$ was added dropwise a solution of iodine $\left(5.66 \mathrm{~mL}, 5.66 \mathrm{mmol}, 1.1\right.$ equiv $1 \mathrm{M}$ in $\mathrm{CH}_{2} \mathrm{Cl}_{2}$ ). The brown coloration of the solution persisted, meaning the total conversion of the starting material; the mixture was then quenched with a solution of $10 \% \mathrm{Na}_{2} \mathrm{~S}_{2} \mathrm{O}_{3}$. After separating the phases, the aqueous layer was extracted with $\mathrm{Et}_{2} \mathrm{O}$; the organic phases were combined, dried over $\mathrm{MgSO}_{4}$, filtered, and concentrated. The residue was then purified on a silica gel column chromatography ( $\mathrm{Et}_{2} \mathrm{O} /$ petroleum ether-10\%) to afford vinyl iodine 82 (3.64 g, 4.94 $\mathrm{mmol}, 96 \%$ ) as a pale yellow oil.

$[\alpha]^{20} \mathrm{D}=-37.1\left(c=1.7, \mathrm{CHCl}_{3}\right) . \mathbf{I R}$ (neat): $v=2954,1587,1147,1304,1247,1147,1084,1004,833,775$, $745 \mathrm{~cm}^{-1} .{ }^{1} \mathrm{H}$ NMR $\left(300 \mathrm{MHz}, \mathrm{CDCl}_{3}\right): \delta=7.95(\mathrm{~m}, 2 \mathrm{H}), 7.67-7.60(\mathrm{~m}, 1 \mathrm{H}), 7.54(\mathrm{~m}, 2 \mathrm{H}), 6.55(\mathrm{~s}, 1 \mathrm{H}), 4.03$ (dt, $J=9.1,4.1 \mathrm{~Hz}, 1 \mathrm{H}$ ), 3.79 (ddd, $J=8.1,5.6,2.3 \mathrm{~Hz}, 1 \mathrm{H}$ ), 3.09 (dd, $J=14.1,2.0 \mathrm{~Hz}, 1 \mathrm{H}$ ), 2.94 (dd, $J=$ 14.1, $10.4 \mathrm{~Hz}, 1 \mathrm{H}$ ), 2.65 (qd, $J=6.8,4.1 \mathrm{~Hz}, 1 \mathrm{H}), 2.29(\mathrm{~m}, 1 \mathrm{H}), 1.47$ (ddd, $J=14.1,9.1,5.6 \mathrm{~Hz}, 1 \mathrm{H}$ ), 1.31 (ddd, $J=14.1,8.1,4.1 \mathrm{~Hz}, 1 \mathrm{H}), 1.22(\mathrm{~d}, J=6.8 \mathrm{~Hz}, 3 \mathrm{H}), 1.05(\mathrm{~d}, J=6.8 \mathrm{~Hz}, 3 \mathrm{H}), 0.93(\mathrm{t}, J=7.9 \mathrm{~Hz}, 9 \mathrm{H})$, $0.88(\mathrm{~s}, 9 \mathrm{H}), 0.54(\mathrm{q}, J=7.9 \mathrm{~Hz}, 6 \mathrm{H}), 0.13(\mathrm{~s}, 9 \mathrm{H}), 0.09(\mathrm{~s}, 3 \mathrm{H}), 0.06(\mathrm{~s}, 3 \mathrm{H}) \mathrm{ppm} .{ }^{13} \mathrm{C}\left\{{ }^{1} \mathrm{H}\right\} \mathrm{NMR}(75 \mathrm{MHz}$, $\left.\mathrm{CDCl}_{3}\right) \delta 140.7,138.0,133.5,129.4(2 \mathrm{C}), 128.1(2 \mathrm{C}), 124.2,73.3,71.0,57.7,56.2,35.6,32.3,25.99(3 \mathrm{C})$, 18.1, 17.0, 14.6, $7.1(3 \mathrm{C}), 5.2(3 \mathrm{C}),-0.9(3 \mathrm{C}),-4.0,-4.3$. HRMS (ESI): $\mathrm{m} / \mathrm{z}$ : calcd for $\mathrm{C}_{31} \mathrm{H}_{59} \mathrm{O}_{4} \mathrm{SSi}_{3} \mathrm{INa}[\mathrm{M}+\mathrm{Na}]^{+}$: 761.2384 , found 761.2382 .

\section{Compound 83}

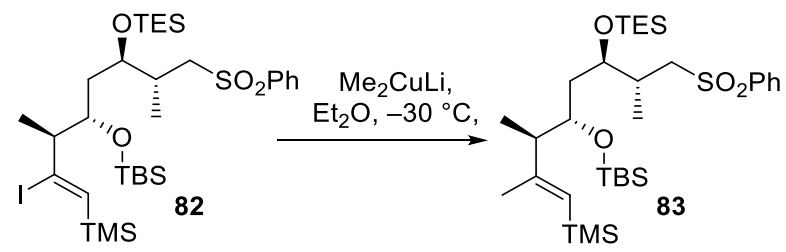

To a cooled $\left(-30^{\circ} \mathrm{C}\right)$ suspension of Cul $\left(3.89 \mathrm{~g}, 20.47 \mathrm{mmol}, 4.2\right.$ equiv) in $\mathrm{Et}_{2} \mathrm{O}(40 \mathrm{~mL})$ was added dropwise MeLi (24.3 mL, $38.9 \mathrm{mmol}, 8$ equiv $1.6 \mathrm{M}$ in $\mathrm{Et}_{2} \mathrm{O}$ ) over 15 minutes. Cul was dissolving while MeLi was added, and the solution turned colorless. After $5 \mathrm{~min}$, a solution of vinyl iodine $82(3.60 \mathrm{~g}, 4.86 \mathrm{mmol}, 1$ equiv) in $\mathrm{Et}_{2} \mathrm{O}\left(15 \mathrm{~mL}\right.$ ) was added. After 20 min, an aqueous mixture of saturated $\mathrm{NH}_{4} \mathrm{Cl}$ : concentrated $\mathrm{NH}_{3}$ (2:1) was added to the reaction. The reaction medium was then extracted with $\mathrm{Et}_{2} \mathrm{O}$, dried over $\mathrm{MgSO}_{4}$, filtered, and concentrated in a vacuum. The crude mixture was then purified on silica gel $\left(\mathrm{Et}_{2} \mathrm{O} / \mathrm{petroleum}\right.$ ether-10\%) to give olefin $83(2.95 \mathrm{~g}, 4.69 \mathrm{mmol}, 97 \%)$ as a colorless oil.

$[\alpha]^{20} \mathrm{D}=-33.3\left(c=0.9, \mathrm{CHCl}_{3}\right)$. IR (neat): $v=2955,2878,2856,1611,1412,1306,1248,1150,1085,1043$, 953, $746 \mathrm{~cm}^{-1} .{ }^{1} \mathbf{H}$ NMR $\left(300 \mathrm{MHz}, \mathrm{CDCl}_{3}\right) \delta=7.92-7.87(\mathrm{~m}, 2 \mathrm{H}), 7.68-7.61(\mathrm{~m}, 1 \mathrm{H}), 7.59-7.52(\mathrm{~m}, 2 \mathrm{H})$, 5.17 (s, 1H), 3.80 (ddd, $J=8.1,5.0,3.8 \mathrm{~Hz}, 1 \mathrm{H}$ ), 3.71 (ddd, $J=7.4,6.4,2.1 \mathrm{~Hz}, 1 \mathrm{H}$ ), 2.97 (dd, $J=14.4,2.1$ $\mathrm{Hz}, 1 \mathrm{H}), 2.83(\mathrm{dd}, J=14.4,9.7 \mathrm{~Hz}, 1 \mathrm{H}), 2.29(\mathrm{qd}, J=7.0,3.8 \mathrm{~Hz}, 1 \mathrm{H}), 2.20(\mathrm{~m}, 1 \mathrm{H}), 1.81(\mathrm{~s}, 3 \mathrm{H}), 1.47$ (ddd, $J=15.7,8.1,6.4 \mathrm{~Hz}, 1 \mathrm{H}), 1.24(\mathrm{~m}, 1 \mathrm{H}), 1.13(\mathrm{~d}, J=6.8 \mathrm{~Hz}, 3 \mathrm{H}), 1.00(\mathrm{~d}, J=7.0 \mathrm{~Hz}, 3 \mathrm{H}), 0.91(\mathrm{t}, J=8.0 \mathrm{~Hz}$, $9 \mathrm{H}), 0.89(\mathrm{~s}, 9 \mathrm{H}), 0.52(\mathrm{q}, J=8.0 \mathrm{~Hz}, 6 \mathrm{H}), 0.07(\mathrm{~s}, 12 \mathrm{H}), 0.04(\mathrm{~s}, 3 \mathrm{H}) \mathrm{ppm} .{ }^{13} \mathrm{C}\left\{{ }^{1} \mathrm{H}\right\} \mathrm{NMR}\left(75 \mathrm{MHz}, \mathrm{CDCl}_{3}\right) \delta$ 156.5, 140.4, 133.6, 129.4 (2C), 128.0 (2C), 124.8, 73.3, 70.7, 58.1, 49.7, 36.6, 32.4, 26.0 (3C), 21.4, 18.2, 17.0, 13.7, $7.0(3 \mathrm{C}), 5.2(3 \mathrm{C}), 0.3(3 \mathrm{C}),-4.2,-4.3$. HRMS (ESI): $m / z$ : calcd for $\mathrm{C}_{32} \mathrm{H}_{62} \mathrm{O}_{4} \mathrm{Si}_{3} \mathrm{SNa}[\mathrm{M}+\mathrm{Na}]^{+}$: 649.3574 , found 645.3566 . 


\section{Compound 76}

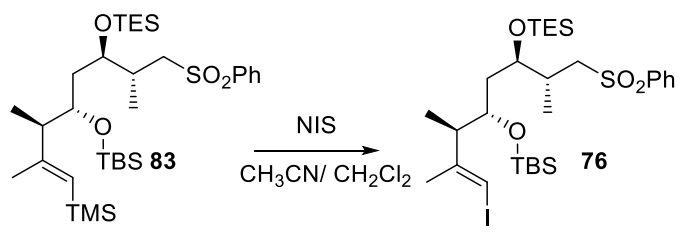

To a solution of compound 83 (1.137 g, $1.82 \mathrm{mmol}, 1.0$ equiv) in a mixture of $\mathrm{MeCN} / \mathrm{CH}_{2} \mathrm{Cl}_{2}(15 / 5 \mathrm{~mL})$ at $0{ }^{\circ} \mathrm{C}$ was added $\mathrm{N}$-iodosuccinimide $(613 \mathrm{mg}, 2.73 \mathrm{mmol}, 1.5$ equiv) and the reaction mixture was stirred $2 \mathrm{~h}$ at rt. The reaction mixture was then quenched with a $10 \% \mathrm{Na}_{2} \mathrm{~S}_{2} \mathrm{O}_{3}$ solution, extracted with $\mathrm{Et}_{2} \mathrm{O}(\mathrm{x} 2)$, dried over $\mathrm{MgSO}_{4}$, and purified on a silica gel column chromatography (5-8\% gradient $\mathrm{Et}_{2} \mathrm{O}$ in petroleum ether) to give vinyl iodide 76 as colorless oil $(1.21 \mathrm{~g}, 98 \%)$.

$[\alpha]^{20} \mathrm{D}=-35.3\left(c 1.6, \mathrm{CHCl}_{3}\right)$. IR (neat): $v=2956,1461,1305,1254,1150,1069,1043,1004,834,775,742$, $720,688 \mathrm{~cm}^{-1} .{ }^{1} \mathrm{H}$ NMR $\left(300 \mathrm{MHz}, \mathrm{CDCl}_{3}\right) \delta 8.01-7.91(\mathrm{~m}, 2 \mathrm{H}), 7.70-7.51(\mathrm{~m}, 3 \mathrm{H}), 5.95(\mathrm{brs}, 1 \mathrm{H}), 3.79(\mathrm{td}$, $J=7.3,5.6,3.6 \mathrm{~Hz}, 1 \mathrm{H}$ ), $3.74(\mathrm{dd}, J=6.9,2.5 \mathrm{~Hz}, 1 \mathrm{H}), 2.94(\mathrm{dd}, J=14.2,3.0 \mathrm{~Hz}, 1 \mathrm{H}), 2.78$ (dd, $J=14.2,8.6$ $\mathrm{Hz}, 1 \mathrm{H}$ ), 2.51 (qd, $J=6.8,3.6 \mathrm{~Hz}, 1 \mathrm{H}), 2.31$ (dqdd, $J=8.6,7.0,3.0,2.5 \mathrm{~Hz}, 1 \mathrm{H}$ ), $1.88(\mathrm{~d}, J=0.5 \mathrm{~Hz}, 3 \mathrm{H}$ ), $1.47(\mathrm{dt}, J=14.0,7.0 \mathrm{~Hz}, 1 \mathrm{H}), 1.22(\mathrm{dd}, J=14.0,7.4 \mathrm{~Hz}, 1 \mathrm{H}), 1.16(\mathrm{~d}, J=6.8 \mathrm{~Hz}, 3 \mathrm{H}), 1.05(\mathrm{~d}, J=7.0 \mathrm{~Hz}$, $3 \mathrm{H}), 0.92(\mathrm{t}, J=8.0 \mathrm{~Hz}, 9 \mathrm{H}), 0.89(\mathrm{~s}, 9 \mathrm{H}), 0.55(\mathrm{q}, J=8.0 \mathrm{~Hz}, 6 \mathrm{H}), 0.07(\mathrm{~s}, 3 \mathrm{H}), 0.05(\mathrm{~s}, 3 \mathrm{H}) .{ }^{13} \mathrm{C}\left\{{ }^{1} \mathrm{H}\right\}$ NMR (75 MHz, $\left.\mathrm{CDCl}_{3}\right) \delta 149.7,140.3,133.7,129.5$ (2C), 128.1 (2C), 77.4, 72.6, 70.6, 58.2, 47.94, 36.9, 32.4, 26.0 (3C), 23.8, 18.1, 16.8, 14.1, 7.0 (3C), $5.2(3 \mathrm{C}),-4.2,-4.3$. HRMS (ESI) calcd for $\mathrm{C}_{29} \mathrm{H}_{53} \mathrm{O}_{4} \mathrm{Si}_{2} \mathrm{SINa}[\mathrm{M}+\mathrm{Na}]^{+}$: 703.2146, found 703.2159.

\section{Compound 84}

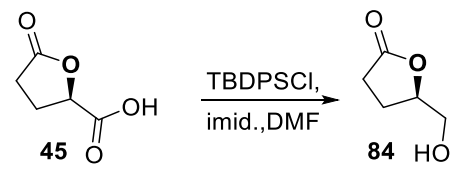

To a solution of acid $45\left(7.6 \mathrm{~g}, 58.7 \mathrm{mmol}, 1\right.$ equiv) in THF $(65 \mathrm{~mL})$ at $-15{ }^{\circ} \mathrm{C}$ was added dropwise $\mathrm{BH}_{3} \cdot$ DMS $(7.11 \mathrm{~mL}, 70.4 \mathrm{mmol}, 1.2 \mathrm{mmol})$. Intense bubbling of $\mathrm{H}_{2}$ was observed, and the addition rate of borane was adjusted according to the gas release. After adding borane, the reaction mixture was warmed to rt and stirred overnight. The reaction mixture was quenched at $0{ }^{\circ} \mathrm{C}$ by dropwise addition of $\mathrm{MeOH}(50 \mathrm{~mL})$, was then stirred $30 \mathrm{~min}$ at rt, concentrated, and purified on a silica gel column chromatography ( $50 \%$ Acetone in petroleum ether) to give compound 84 as a viscous oil $(6.05 \mathrm{~g}, 89 \%)$. Characterization data were in agreement with the literature. ${ }^{[11]}$

${ }^{1} \mathrm{H}$ NMR (300 MHz, Chloroform-d) $\delta 4.62$ (qd, $J=7.3,4.6,2.6 \mathrm{~Hz}, 1 \mathrm{H}$ ), 3.89 (dd, $J=12.5,2.6 \mathrm{~Hz}, 1 \mathrm{H}$ ), 3.64 (dd, $J=12.5,4.5 \mathrm{~Hz}, 1 \mathrm{H}), 2.83(\mathrm{~s}, 1 \mathrm{H}), 2.59(\mathrm{dd}, J=10.0,6.3 \mathrm{~Hz}, 1 \mathrm{H}), 2.52(\mathrm{~d}, J=9.0 \mathrm{~Hz}, 1 \mathrm{H}), 2.36-2.04$ $(\mathrm{m}, 2 \mathrm{H})$.

\section{Compound 85}

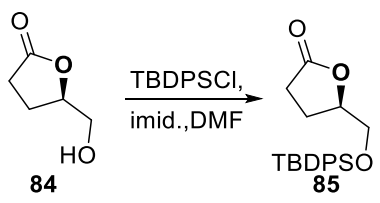

To a solution of compound 84 (5.87 g, $50.6 \mathrm{mmol}, 1$ equiv) in DMF (30 mL) at rt, was added imidazole (7.22 g, $106.2 \mathrm{mmol}, 2$ equiv), followed by TBDPSCl (14.6 g, $53.1 \mathrm{mmol}, 1.05$ equiv). After $16 \mathrm{~h}$ at $\mathrm{rt}$, the 
reaction mixture was quenched with saturated $\mathrm{NH}_{4} \mathrm{Cl}$, extracted with $\mathrm{Et}_{2} \mathrm{O}(\mathrm{x} 2)$, washed with brine (x3), dried over $\mathrm{MgSO}_{4}$, filtered, and concentrated under reduced pressure. The residue was purified on a silica gel column chromatography (15\% AcOEt in petroleum ether) to afford compound $85(13.21 \mathrm{~g}, 74 \%)$ as white crystals.

$\mathbf{M p}=71-73^{\circ} \mathrm{C} .[\mathbf{\alpha}]^{20} \mathrm{D}=-27,\left(c 2.93, \mathrm{CHCl}_{3}\right) . \mathbf{I R}$ (neat): $v=2960,1775,1174,111,746,703 \mathrm{~cm}^{-1} .{ }^{1} \mathbf{H}$ NMR $\left(300 \mathrm{MHz}, \mathrm{CDCl}_{3}\right) \delta 7.74-7.63(\mathrm{~m}, 4 \mathrm{H}), 7.50-7.34(\mathrm{~m}, 6 \mathrm{H}), 4.60(\mathrm{ddt}, J=7.8,5.6,3.3 \mathrm{~Hz}, 1 \mathrm{H}), 3.88(\mathrm{dd}, J$ $=11.3,3.3 \mathrm{~Hz}, 1 \mathrm{H}$ ), 3.69 (dd, $J=11.3,3.4 \mathrm{~Hz}, 1 \mathrm{H}$ ), 2.68 (ddd, $J=17.8,9.7,6.8 \mathrm{~Hz}, 1 \mathrm{H}$ ), 2.51 (ddd, $J=17.8$, 9.7, $6.8 \mathrm{~Hz}, 1 \mathrm{H}$ ), 2.29 (dddd, $J=12.0,9.7,7.5,6.8 \mathrm{~Hz}, 2 \mathrm{H}$ ), 2.21 (dddd, $J=12.8,9.7,6.8,5.6 \mathrm{~Hz}, 2 \mathrm{H}$ ), 1.07 $(\mathrm{s}, 9 \mathrm{H}) .{ }^{13} \mathrm{C}\left\{{ }^{1} \mathrm{H}\right\}$ NMR $\left(75 \mathrm{MHz}, \mathrm{CDCl}_{3}\right) \delta 177.5,135.8(2 \mathrm{C}), 135.7(2 \mathrm{C}), 133.1,132.7,130.1(2 \mathrm{C}), 128.0(4 \mathrm{C})$, 80.1, 65.6, 28.7, 26.9 (3C), 23.8, 19.3. HRMS (ESI) calcd for $\mathrm{C}_{21} \mathrm{H}_{26} \mathrm{O}_{3} \mathrm{SiNa}[\mathrm{M}+\mathrm{Na}]^{+}:$377.1549, found 377.1555 .

\section{Compound 86}

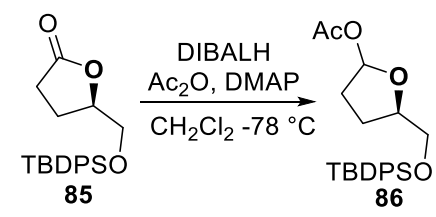

To a solution of compound $85\left(8.14 \mathrm{~g}, 23.0 \mathrm{mmol}, 1\right.$ equiv) in $\mathrm{CH}_{2} \mathrm{Cl}_{2}(120 \mathrm{~mL})$ at $-78{ }^{\circ} \mathrm{C}$ was added DIBALH (41.4 mL, $41.4 \mathrm{mmol}, 1.8$ equiv, $1.0 \mathrm{M}$ in hexanes). After $30 \mathrm{~min}$ at $-78{ }^{\circ} \mathrm{C}, \mathrm{Ac}_{2} \mathrm{O}(11.7 \mathrm{~mL}, 115$ mmol, 5 equiv) and DMAP ( $8.35 \mathrm{~g}, 69 \mathrm{mmol}, 3$ equiv) were added in this order, and the reaction mixture was allowed to warm slowly to $0^{\circ} \mathrm{C}$ and was stirred then for $30 \mathrm{~min}$ at this temperature. The reaction mixture was then poured slowly into a stirred saturated solution of potassium sodium tartrate, stirred $30 \mathrm{~min}$ at rt, diluted with water, extracted with $\mathrm{Et}_{2} \mathrm{O}(\times 3)$, washed with a diluted solution of $\mathrm{NaHCO}_{3}$, and finally with a brine solution. The organic layer was then dried over solid $\mathrm{NaHCO}_{3}$, filtered and concentrated, and the residue was filtered over a small plug of silica gel eluting with $\mathrm{Et}_{2} \mathrm{O}$ to afford after concentration acetylated lactol 86 (9.16 $\mathrm{g}, 100 \%)$ as a colorless oil in a 1:1 mixture of anomers.

IR (neat): $v=2930,1748,1235,1112,1090,704 \mathrm{~cm}^{-1} .{ }^{1} \mathbf{H}$ NMR $\left(300 \mathrm{MHz}, \mathrm{CDCl}_{3}, 1: 1\right.$ mixture of anomers) $\delta$ $7.77-7.62(\mathrm{~m}, 4 \mathrm{H}), 7.48-7.32(\mathrm{~m}, 6 \mathrm{H}), 6.34(\mathrm{~d}, J=4.1 \mathrm{~Hz}, 0.5 \mathrm{H}, 1$ diastereomer), $6.28(\mathrm{t}, J=1.9 \mathrm{~Hz}, 0.5 \mathrm{H}, 1$ diastereomer), 4.37 (dq, $J=7.4,4.3 \mathrm{~Hz}, 0.5 \mathrm{H}, 1$ diastereomer), 4.24 (ddt, $J=8.5,6.0,5.0 \mathrm{~Hz}, 0.5 \mathrm{H}, 1$ diastereomer), 3.77 (dd, $J=10.8,5.0 \mathrm{~Hz}, 0.5 \mathrm{H}, 1$ diastereomer), 3.71 (dd, $J=10.8,5.3 \mathrm{~Hz}, 0.5 \mathrm{H}, 1$ diastereomer), 3.68 (dd, $J=11.2,4.3 \mathrm{~Hz}, 0.5 \mathrm{H}, 1$ diastereomer), 3.65 (dd, $J=11.2,4.3 \mathrm{~Hz}, 0.5 \mathrm{H}, 1$ diastereomer), 2.25-1.85 (m, $4 \mathrm{H}), 2.04(\mathrm{~s}, 1.5 \mathrm{H}, 1$ diastereomer), $1.91(\mathrm{~s}, 1.5 \mathrm{H}, 1$ diastereomer), 1.07 (s, $4.5 \mathrm{H}, 1$ diastereomer), $1.05\left(\mathrm{~s}, 4.5 \mathrm{H}, 1\right.$ diastereomer). ${ }^{13} \mathrm{C}\left\{{ }^{1} \mathrm{H}\right\} \mathbf{N M R}\left(75 \mathrm{MHz}, \mathrm{CDCl}_{3}, 1: 1\right.$ mixture of anomers) $\delta$ 170.7, 170.7, 135.8, 133.6, 133.4, 129.8, 127.8, 99.7, 99.0, 82.3, 80.7, 66.6, 65.8, 32.7, 32.0, 27.0, 25.3, 24.9, 21.6, 19.4. HRMS (ESI) calcd for $\mathrm{C}_{23} \mathrm{H}_{30} \mathrm{O}_{4} \mathrm{SiNa}[\mathrm{M}+\mathrm{Na}]^{+}: 421.1811$, found 421.1807.

\section{Compound 7d}

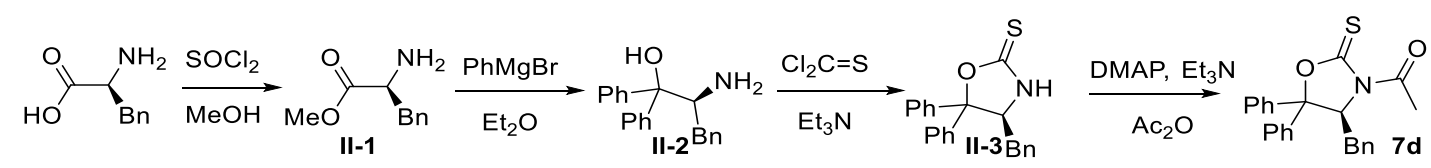

To a solution of $(S)$-phenylalanine $\left(33.0 \mathrm{~g}, 200 \mathrm{mmol}, 1\right.$ equiv) in $\mathrm{MeOH}(250 \mathrm{~mL})$ at $0{ }^{\circ} \mathrm{C}$ in a reaction flask equipped with a bubble outlet was added dropwise $\mathrm{SOCl}_{2}(30 \mathrm{~mL}, 413 \mathrm{mmol}, 2.06$ equiv). After stirring overnight at rt, the reaction mixture was concentrated to dryness. To the residue was added $\mathrm{CH}_{2} \mathrm{Cl}_{2}(200 \mathrm{~mL})$ and slowly $30 \%$ aqueous $\mathrm{K}_{2} \mathrm{CO}_{3}(200 \mathrm{~mL})$ at $0{ }^{\circ} \mathrm{C}$. The mixture was stirred for about $10 \mathrm{~min}$ until the residue 
was fully dissolved. The aqueous layer was extracted with $\mathrm{CH}_{2} \mathrm{Cl}_{2}(2 \mathrm{x})$, and the combined organic phases were dried over $\mathrm{NaHCO}_{3} / \mathrm{K}_{2} \mathrm{CO}_{3}$ mixture, filtered, and concentrated to give crude $(S)$-phenylalanine methyl ester II-1.

To a solution of freshly prepared $\mathrm{PhMgBr}\left(400 \mathrm{~mL}, \approx 2 \mathrm{mmol}, \approx 4\right.$ equiv, $\approx 2.0 \mathrm{M}$ in $\left.\mathrm{Et}_{2} \mathrm{O}\right)$ in a 3-neck flask equipped with a condenser was added dropwise a solution of above crude ( $S$ )-phenylalanine methyl ester II1 in $\mathrm{Et}_{2} \mathrm{O}(150 \mathrm{~mL})$ over $1 \mathrm{~h}$ and the reaction mixture was refluxed overnight with the help of an oil bath. The reaction mixture was poured slowly into saturated $\mathrm{NH}_{4} \mathrm{Cl}(300 \mathrm{~mL})$ with ice. And the whole mixture was filtered onto celite and washed with AcOEt. The aqueous layer was washed with AcOEt (x3), and the combined organic layers were dried over $\mathrm{MgSO}_{4}$, filtered, and concentrated. The residue was dissolved in a small amount of AcOEt, and petroleum ether was added to precipitate the amino-alcool II-2. The beige solid was collected by filtration (36.4 g, 55\%, 2 steps). Characterization data were in agreement with the literature.[24]

${ }^{1} \mathbf{H}$ NMR $\left(300 \mathrm{MHz}, \mathrm{CDCl}_{3}\right) \delta 7.66(\mathrm{~d}, J=8.0 \mathrm{~Hz}, 2 \mathrm{H}), 7.61(\mathrm{~d}, J=8.1 \mathrm{~Hz}, 2 \mathrm{H}), 7.37-7.27(\mathrm{~m}, 6 \mathrm{H}), 7.23(\mathrm{td}, J$ $=6.7,1.2 \mathrm{~Hz}, 1 \mathrm{H}), 7.20(\mathrm{t}, J=7.2 \mathrm{~Hz}, 4 \mathrm{H}), 4.49$ (brs, $1 \mathrm{H}, \mathrm{OH}), 4.19$ (dd, $J=10.8,2.4 \mathrm{~Hz}, 1 \mathrm{H}), 2.66(\mathrm{dd}, J=$ 13.8, $2.4 \mathrm{~Hz}, 1 \mathrm{H}), 2.45(\mathrm{dd}, J=13.8,10.8 \mathrm{~Hz}, 1 \mathrm{H})$.

To a solution of compound II-2 (36.4 g, $120 \mathrm{mmol}, 1$ equiv) and $\mathrm{Et}_{3} \mathrm{~N}$ (42 mL, $300 \mathrm{mmol}, 2.5$ equiv) in $\mathrm{CH}_{2} \mathrm{Cl}_{2}(150 \mathrm{~mL})$ was added dropwise at $0{ }^{\circ} \mathrm{C}$ thiophosgene $(10.1 \mathrm{~mL}, 132 \mathrm{mmol}, 1.1$ equiv). After $1 \mathrm{~h}$ at 0 ${ }^{\circ} \mathrm{C}$, the reaction mixture was warmed to rt, hydrolyzed with saturated $\mathrm{NH}_{4} \mathrm{Cl}$, extracted with $\mathrm{AcOEt}$, dried over $\mathrm{MgSO}_{4}$, filtered, and concentrated. The residue was roughly purified on a large and short silica gel column (25-30\% gradient AcOEt in petroleum ether). The solid residue was then triturated in a small amount of $\mathrm{Et}_{2} \mathrm{O}$ to precipitate pure oxazolidinethione II-3, filtered, and collected as a white solid (37.8 $\mathrm{g}, 90 \%)$. Characterization data were in agreement with the literature. ${ }^{[25]}$

$\mathrm{Mp}=128-130{ }^{\circ} \mathrm{C} .[\alpha]^{20} \mathrm{D}=-384\left(c 0.331, \mathrm{CHCl}_{3}\right)$. IR (neat): $v=3200(\mathrm{br}), 1493,1162,1147,972,697,686$ cm-1. ${ }^{1} \mathrm{H}$ NMR $\left(300 \mathrm{MHz}, \mathrm{CDCl}_{3}\right) \delta 7.54(\mathrm{dd}, J=8.0,1.4 \mathrm{~Hz}, 2 \mathrm{H}), 7.48-7.30(\mathrm{~m}, 11 \mathrm{H}), 7.14(\mathrm{dd}, J=7.2,1.0$ $\mathrm{Hz}, 2 \mathrm{H}$ ), 4.95 ( brs, 1H, NH), 4.85 (dd, $J=11.5,3.3 \mathrm{~Hz}, 1 \mathrm{H}), 2.68$ (dd, $J=13.8,3.3 \mathrm{~Hz}, 1 \mathrm{H}$ ), 2.21 (dd, $J=$ $13.8,11.5 \mathrm{~Hz}, 1 \mathrm{H}) .{ }^{13} \mathrm{C}\left\{{ }^{1} \mathrm{H}\right\}$ NMR $\left(50 \mathrm{MHz}, \mathrm{CDCl}_{3}\right) \delta 187.9,141.0,138.2,136.1,129.2,128.8,128.7,128.4$, 127.5, 126.6, 126.0, 95.5, 65.4, 38.9. HRMS (ESI) calcd for $\mathrm{C}_{22} \mathrm{H}_{20} \mathrm{NOS}[\mathrm{M}+\mathrm{H}]^{+}: 346.1268$, found 346.1266.

To pure oxazolidinethione II-3 (11.40 g, $33.0 \mathrm{mmol}, 1$ equiv) and DMAP (200 mg, $1.65 \mathrm{mmol}, 0.05$ equiv) was added $\mathrm{Et}_{3} \mathrm{~N}$ (13.8 mL, $99.0 \mathrm{mmol}, 3$ equiv) followed by $\mathrm{Ac}_{2} \mathrm{O}(10 \mathrm{~mL}, 99.0 \mathrm{mmol}, 3$ equiv). The reaction mixture was stirred for $2 \mathrm{~h}$ at $\mathrm{rt}$, water was added $(30 \mathrm{~mL})$, and the reaction mixture was stirred for $30 \mathrm{~min}$ at rt. The reaction mixture was extracted with $\mathrm{Et}_{2} \mathrm{O}(\mathrm{x} 3)$, and the combined ethereal layers were washed with water, saturated $\mathrm{NaHCO}_{3}(\mathrm{x} 2)$, dried over $\mathrm{MgSO}_{4}$, filtered on a pad of silica gel $\left(3 \mathrm{~cm}\right.$, washing with $\left.\mathrm{Et}_{2} \mathrm{O}\right)$ to give compound 7d as a sticky and vitreous colorless solid (12.8 g, 100\%). Characterization data were in agreement with the literature. ${ }^{[25]}$

$[\alpha]^{20} \mathrm{D}=-306\left(c 0.225, \mathrm{CHCl}_{3}\right)$. IR (neat): $v=1757,1376,1355,1324,1227,1204,1165,1155,962,763$, 732, $698 \mathrm{~cm}^{-1}$. ${ }^{1} \mathbf{H}$ NMR $\left(300 \mathrm{MHz}, \mathrm{CDCl}_{3}\right) \delta 7.45(\mathrm{dd}, J=8.2,1.6 \mathrm{~Hz}, 2 \mathrm{H}), 7.40-7.30(\mathrm{~m}, 3 \mathrm{H}), 7.30-7.19$ $(\mathrm{m}, 5 \mathrm{H}), 7.11(\mathrm{dd}, J=4.9,1.8 \mathrm{~Hz}, 3 \mathrm{H}), 6.74(\mathrm{~m}, 2 \mathrm{H}), 5.62(\mathrm{dd}, J=7.7,5.2 \mathrm{~Hz}, 1 \mathrm{H}), 2.87(\mathrm{dd}, J=14.1,5.2$ $\mathrm{Hz}, 1 \mathrm{H}), 2.74(\mathrm{dd}, J=14.1,7.7 \mathrm{~Hz}, 1 \mathrm{H}), 2.39(\mathrm{~s}, 3 \mathrm{H}) .{ }^{13} \mathrm{C}\left\{{ }^{1} \mathrm{H}\right\} \mathbf{N M R}\left(50 \mathrm{MHz}, \mathrm{CDCl}_{3}\right) \delta 184.1,170.5,140.7$, 137.0, 136.2, 129.1 (2C), 129.0 (3C), 128.5 (2C), 128.4, 128.3 (2C), 126.7, 126.6 (2C), 125.9 (2C), 92.7, 77.2, 65.2, 36.5, 26.0. HRMS (ESI) calcd for $\mathrm{C}_{24} \mathrm{H}_{21} \mathrm{NO}_{2} \mathrm{SNa}[\mathrm{M}+\mathrm{Na}]^{+}: 410.1191$, found 410.1196 . 


\section{Compound 87}

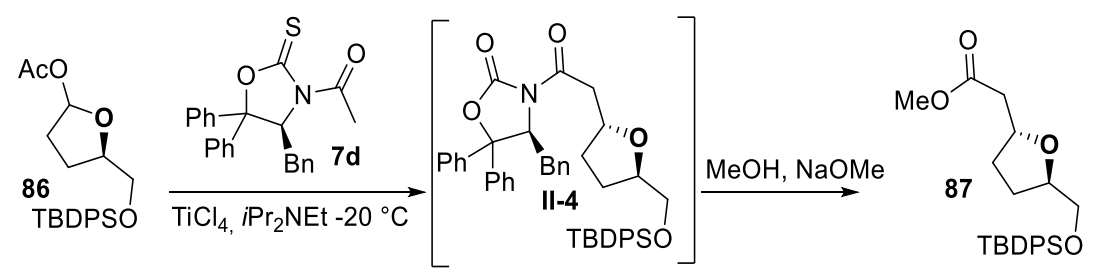

To a solution of oxazolidinethione $7 \mathbf{d}\left(3.1 \mathrm{~g}, 8.0 \mathrm{mmol}, 1.59\right.$ equiv) in dry $\mathrm{CH}_{2} \mathrm{Cl}_{2}(31 \mathrm{~mL})$ at $0{ }^{\circ} \mathrm{C}$ was added $\mathrm{TiCl}_{4}\left(880 \mu \mathrm{L}, 8.0 \mathrm{mmol}, 1.59\right.$ equiv) over $1 \mathrm{~min}$. The solution turned bright orange, and $\operatorname{Pr}_{2} \mathrm{NEt}(1.39$ $\mathrm{mL}, 8.0 \mathrm{mmol}, 1.59$ equiv) was dropwise added over $10 \mathrm{~min}$. The dark red titanium enolate was then cooled down to $-20{ }^{\circ} \mathrm{C}$, and a solution of lactol acetate $86\left(2.0 \mathrm{~g}, 5.02 \mathrm{mmol}\right.$, 1 equiv) in $\mathrm{CH}_{2} \mathrm{Cl}_{2}(10 \mathrm{~mL})$ was added. After $1 \mathrm{~h}$ at this temperature, the reaction mixture was poured carefully onto a $10 \% \mathrm{Na}_{2} \mathrm{CO}_{3}$ solution $(100$ $\mathrm{mL}$ ). The mixture was then filtered over Celite to remove titanium oxide and washed with ether. The organic layer was collected, the aqueous layer was extracted with ether, and the combined organic phases were washed with saturated $\mathrm{NH}_{4} \mathrm{Cl}$, dried over $\mathrm{MgSO}_{4}$, filtered, and concentrated to give a crude oil containing II4.

The crude mixture was then dissolved in $\mathrm{CH}_{2} \mathrm{Cl}_{2}(5 \mathrm{~mL}), \mathrm{MeOH}(30 \mathrm{~mL})$, and $\mathrm{MeONa}(1.83 \mathrm{~mL}, 8.0 \mathrm{mmol}$, $25 \%$ in $\mathrm{MeOH}$ ) were then added at $0{ }^{\circ} \mathrm{C}$. After $30 \mathrm{~min}$ the reaction mixture was quenched with saturated $\mathrm{NH}_{4} \mathrm{Cl}(50 \mathrm{~mL})$ and diluted with AcOEt $(60 \mathrm{~mL})$. The organic phases were separated; the aqueous layer was extracted with AcOEt, and the combined organic layers were washed twice with brine. The organic phase was then dried over $\mathrm{MgSO}_{4}$, filtered, and concentrated. The residue was triturated with $\mathrm{Et}_{2} \mathrm{O}$ / petroleum ether to precipitate deacylated oxazolidethione II-3. The solid was then filtered on a small sintered glass and washed with some small amounts of ether $(2.15 \mathrm{~g}, 78 \%$ of recovered oxazolidinethione II-3). The filtrate was concentrated and purified on a silica gel column chromatography (AcOEt:Petroleum ether, from 8:92 to 15:85) to afford THF 87 as a colorless oil (1.78 g, 86\%, dr $\geq 95: 5$ as judged by ${ }^{1} \mathrm{H}$ NMR).

$[\alpha]^{20} \mathrm{D}=-6.7$ (c 2.67, $\left.\mathrm{CHCl}_{3}\right) . \mathbf{I R}$ (neat): $v=2930,1740,1428,1112,1076,703 \mathrm{~cm}^{-1} .{ }^{1} \mathbf{H} \mathbf{~ N M R}(300 \mathrm{MHz}$, $\left.\mathrm{CDCl}_{3}\right) \delta 7.77-7.57(\mathrm{~m}, 4 \mathrm{H}), 7.49-7.29(\mathrm{~m}, 6 \mathrm{H}), 4.38(\mathrm{tt}, J=7.0,6.6 \mathrm{~Hz}, 1 \mathrm{H}), 4.16(\mathrm{tt}, J=6.7,4.8 \mathrm{~Hz}, 1 \mathrm{H})$, 3.69 (s, 3H), $3.63(\mathrm{~d}, J=4.8 \mathrm{~Hz}, 2 \mathrm{H}), 2.61$ (dd, $J=15.0,7.1 \mathrm{~Hz}, 1 \mathrm{H}), 2.46$ (dd, $J=15.0,6.1 \mathrm{~Hz}, 1 \mathrm{H}), 2.22-$ $2.08(\mathrm{~m}, 1 \mathrm{H}), 2.08-1.97(\mathrm{~m}, 1 \mathrm{H}), 1.97-1.81(\mathrm{~m}, 1 \mathrm{H}), 1.59(\mathrm{dq}, J=11.7,8.1 \mathrm{~Hz}, 2 \mathrm{H}), 1.06(\mathrm{~s}, 9 \mathrm{H}) .{ }^{13} \mathrm{C}\left\{{ }^{1} \mathrm{H}\right\}$ NMR $\left(75 \mathrm{MHz}, \mathrm{CDCl}_{3}\right) \delta 171.3,77.2,77.1,76.6,52.0,40.0,31.0,30.0$. HRMS (ESI) calcd for $\mathrm{C}_{24} \mathrm{H}_{32} \mathrm{O}_{4} \mathrm{SiNa}$ $[\mathrm{M}+\mathrm{Na}]^{+}:$435.1968, found 435.1971 .

\section{Compound 88}

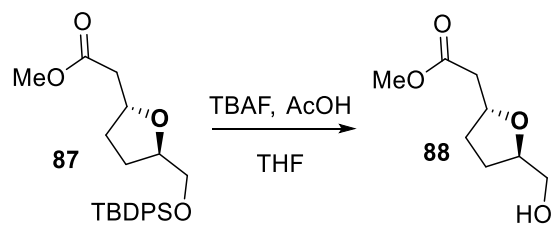

To a solution of THF $87(7.1 \mathrm{~g}, 17.3 \mathrm{mmol}, 1$ equiv) in THF $(100 \mathrm{~mL})$ was added glacial acetic acid $(2.6 \mathrm{~mL}$, $44.8 \mathrm{mmol}, 2.6$ equiv) followed by TBAF (22.5 mL, $22.5 \mathrm{mmol}, 1.3$ equiv, $1.0 \mathrm{M}$ in THF). After $16 \mathrm{~h}$ stirring at $\mathrm{rt}$, the reaction mixture was quenched with saturated $\mathrm{NaHCO}_{3}$, extracted with AcOEt, dried over $\mathrm{MgSO}_{4}$, filtered, and concentrated. The residue was purified on a silica gel column chromatography (AcOEt: Petroleum ether, from 50:50 to 100:0) to give alcohol 88 as a colorless oil (3.16 g, 100\%).

$[\alpha]^{20} \mathrm{D}=-150.0\left(c 0.72, \mathrm{CHCl}_{3}\right)$. IR (neat): $v=3400,2917,1738,1439,1203,1066 \mathrm{~cm}^{-1} .{ }^{1} \mathbf{H} \mathbf{~ N M R}(300 \mathrm{MHz}$, $\mathrm{CDCl}_{3}$ ) $\delta 4.35$ (quint, $J=7.1 \mathrm{~Hz}, 1 \mathrm{H}$ ), 4.11 (tdd, $J=7.1,6.0,3.3 \mathrm{~Hz}, 1 \mathrm{H}$ ), $3.67(\mathrm{~s}, 3 \mathrm{H}), 3.62(\mathrm{dd}, J=11.6,3.3$ $\mathrm{Hz}, 1 \mathrm{H}), 3.47(\mathrm{dd}, J=11.6,6.0 \mathrm{~Hz}, 1 \mathrm{H}), 2.60(\mathrm{dd}, J=15.4,7.3 \mathrm{~Hz}, 1 \mathrm{H}), 2.46(\mathrm{dd}, J=15.4,5.9 \mathrm{~Hz}, 1 \mathrm{H}), 2.33$ 
(brs, $1 \mathrm{H}, \mathrm{OH}), 2.21-2.05(\mathrm{~m}, 1 \mathrm{H}), 2.04-1.90(\mathrm{~m}, 1 \mathrm{H}), 1.80-1.53(\mathrm{~m}, 2 \mathrm{H}) .{ }^{13} \mathrm{C}\left\{{ }^{1} \mathrm{H}\right\} \mathrm{NMR}\left(75 \mathrm{MHz}, \mathrm{CDCl}_{3}\right)$ $\delta 171.80,79.50,75.51,64.89,51.79,40.49,31.99,27.38$. HRMS (ESI) calcd for $\mathrm{C}_{8} \mathrm{H}_{14} \mathrm{O}_{4} \mathrm{Na}[\mathrm{M}+\mathrm{Na}]^{+}$: 197.0790, found 197.0796 .

\section{Compound 89}

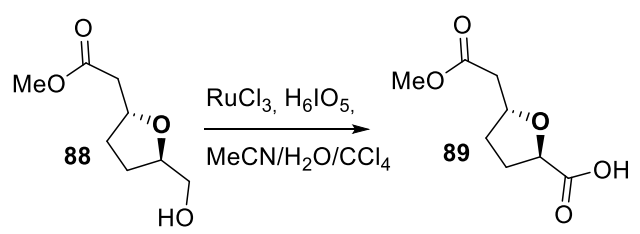

To a solution of alcohol 88 ( $3.0 \mathrm{~g}, 17.3 \mathrm{mmol}, 1$ equiv) in a mixture of $\mathrm{H}_{2} \mathrm{O} / \mathrm{CCl} / \mathrm{MeCN}(50 / 30 / 30 \mathrm{~mL})$ was added $\mathrm{RuCl}_{3}$ hydrate ( $35 \mathrm{mg}, 0.17 \mathrm{mmol}, 0.01$ equiv) followed by $\mathrm{H}_{5} \mathrm{IO}_{6}(9.9 \mathrm{~g}, 43.3 \mathrm{mmol}, 2.5$ equiv) in five portions over $30 \mathrm{~min}$. after vigorous stirring for $4 \mathrm{~h}$ at rt, the reaction mixture was partitioned by dilution with a mixture of $\mathrm{CH}_{2} \mathrm{Cl}_{2}$ : $\mathrm{PrOH}(80: 20,40 \mathrm{~mL})$ and extracted 4 times with this mixture of solvents. Combined organic layers were washed with water $(10 \mathrm{~mL})$, with a sodium thiosulfate solution $(10 \mathrm{~mL})$, and they were dried over $\mathrm{MgSO}_{4}$, filtered, concentrated to give crude carboxylic acid 89 (2.91 g, 89\%) as a tan viscous oil. The residue was sufficiently pure and was used directly in the next step without further purification.

$[\alpha]^{20} \mathrm{D}=+3.7\left(c 1.08, \mathrm{CHCl}_{3}\right)$. IR (neat): $v=3700-2300(\mathrm{br}), 2950,1734,1439,1202,1069 \mathrm{~cm}^{-1} .{ }^{1} \mathbf{H}$ NMR (300 $\left.\mathrm{MHz}, \mathrm{CDCl}_{3}\right) \delta 8.94(\mathrm{brs}, 1 \mathrm{H}), 4.69-4.47(\mathrm{~m}, 2 \mathrm{H}), 3.69(\mathrm{~s}, 3 \mathrm{H}), 2.68$ (dd, $\left.J=15.6,6.9 \mathrm{~Hz}, 1 \mathrm{H}\right), 2.51$ (dd, $J=$ 15.6, $6.2 \mathrm{~Hz}, 1 \mathrm{H}), 2.46-2.28(\mathrm{~m}, 1 \mathrm{H}), 2.28-1.98(\mathrm{~m}, 2 \mathrm{H}), 1.82-1.55(\mathrm{~m}, 1 \mathrm{H}) .{ }^{13} \mathrm{C}\left\{{ }^{1} \mathrm{H}\right\} \mathrm{NMR}(75 \mathrm{MHz}$, $\left.\mathrm{CDCl}_{3}\right) \delta 171.3,77.1,76.6,52.0,40.0,31.0,30.0$. HRMS (ESI) calcd for $\mathrm{C}_{8} \mathrm{H}_{12} \mathrm{O}_{5} \mathrm{Na}[\mathrm{M}+\mathrm{Na}]^{+}: 211.0582$, found 211.0582 .

\section{Compound 90}

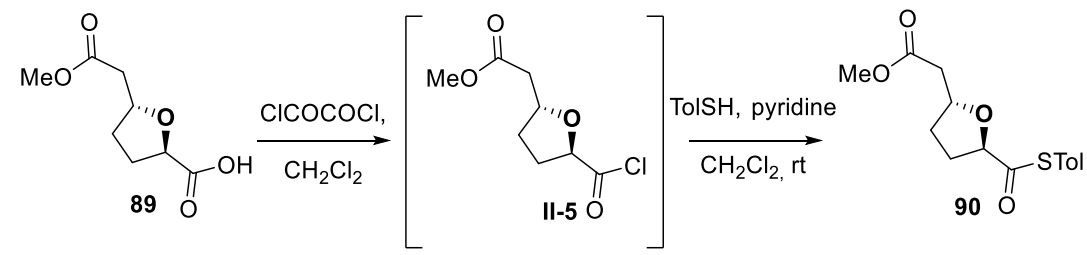

To a solution of crude acid 89 (2.91 g, $15.4 \mathrm{mmol}, 1$ equiv) in $\mathrm{CH}_{2} \mathrm{Cl}_{2}(30 \mathrm{~mL})$ in a reaction flask equipped with a bubbler outlet, was added oxalyl chloride $(2.64 \mathrm{~mL}, 30.8 \mathrm{mmol}, 2$ equiv) followed by 2 drops of DMF. Some gas evolution was observed, and the reaction mixture was stirred for $90 \mathrm{~min}$ at rt. Some dry toluene was added $(10 \mathrm{~mL})$, and the reaction mixture was concentrated to dryness to give crude acyl chloride II-5. The crude residue was diluted in a $\mathrm{CH}_{2} \mathrm{Cl}_{2}(30 \mathrm{~mL})$, and a mixture of $p$-toluenethiol $(2.9 \mathrm{~g}, 23.1 \mathrm{mmol}, 1.5$ equiv) and anhydrous pyridine (3.7 mL, $46.2 \mathrm{mmol}$, 3 equiv) in $\mathrm{CH}_{2} \mathrm{Cl}_{2}(5 \mathrm{~mL})$ was added via syringe at $0{ }^{\circ} \mathrm{C}$. After 30 min at rt, the reaction mixture was quenched with $\mathrm{HCl} 1 \mathrm{~N}$, extracted 3 times with $\mathrm{Et}_{2} \mathrm{O}$, washed with brine, and the combined ethereal phases were dried over $\mathrm{MgSO}_{4}$, filtered, and concentrated. The residue was ultimately purified by column chromatography on silica gel (from $15 \%$ to $20 \% \mathrm{Et}_{2} \mathrm{O}$ in Petroleum Ether) to afford thioester 90 as an oil (3.90 g, 86\%).

$[\alpha]^{20} \mathrm{D}=+93.8\left(c\right.$ 1.95, $\left.\mathrm{CHCl}_{3}\right) . \mathbf{I R}$ (neat): $v=2950,1736,1695,1436,1059,903,809 \mathrm{~cm}^{-1} .{ }^{1} \mathbf{H}$ NMR (300 $\left.\mathrm{MHz} \mathrm{CDCl}_{3}\right) \delta 7.27(\mathrm{~d}, J=8.0 \mathrm{~Hz}, 2 \mathrm{H}), 7.20(\mathrm{~d}, J=8.0 \mathrm{~Hz}, 2 \mathrm{H}), 4.71(\mathrm{~d}, J=6.6 \mathrm{~Hz}, 1 \mathrm{H}), 4.64(\mathrm{dd}, J=8.0$, $5.5 \mathrm{~Hz}, 1 \mathrm{H}$ ), $3.72(\mathrm{~s}, 3 \mathrm{H}), 2.73(\mathrm{dd}, J=15.5,6.7 \mathrm{~Hz}, 1 \mathrm{H}), 2.55(\mathrm{dd}, J=15.5,6.5 \mathrm{~Hz}, 1 \mathrm{H}), 2.44-2.31(\mathrm{~m}, 1 \mathrm{H})$, $2.36(\mathrm{~s}, 3 \mathrm{H}), 2.30-2.05(\mathrm{~m}, 2 \mathrm{H}), 1.70(\mathrm{dq}, J=11.3,7.3 \mathrm{~Hz}, 1 \mathrm{H}) .{ }^{13} \mathrm{C}\left\{{ }^{1} \mathrm{H}\right\} \mathbf{N M R}\left(75 \mathrm{MHz}, \mathrm{CDCl}_{3}\right) \delta 202.7$, 171.2, 139.6, 134.7, 130.1, 123.9, 83.8, 77.6, 51.9, 40.2, 30.9, 30.6, 21.4. HRMS (ESI) calcd for $\mathrm{C}_{15} \mathrm{H}_{18} \mathrm{O}_{4} \mathrm{SNa}$ $[\mathrm{M}+\mathrm{Na}]^{+}:$317.0823, found 317.0818. 


\section{Compound 91}

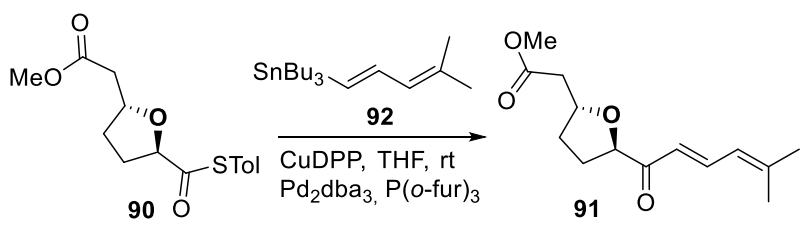

To a solution of thioester 90 ( $3.7 \mathrm{~g}, 12.6 \mathrm{mmol}, 1$ equiv) and stannane 92 (5.65 g, $16.38 \mathrm{mmol}, 1.3$ equiv) in THF $(50 \mathrm{~mL})$ was added in one portion copper diphenylphosphinate (CuDPP) ${ }^{[23]}(5.3 \mathrm{~g}, 18.9 \mathrm{mmol}, 1.5$ equiv), $\mathrm{Pd}_{2} \mathrm{dba}_{3}$ ( $230 \mathrm{mg}, 0.126 \mathrm{mmol}, 0.02$ equiv), trifurylphosphine ( $350.8 \mathrm{mg}, 0.756 \mathrm{mmol}, 0.12$ equiv). The reaction mixture was then heated for $2 \mathrm{~h}$ in a preheated oil bath at $50{ }^{\circ} \mathrm{C}$. The reaction mixture turned dark brown and was cooled down to rt and diluted with petroleum ether $(50 \mathrm{~mL})$. The reaction mixture was then filtered on a pad of silica gel, and the filter cake was washed copiously with $\mathrm{Et}_{2} \mathrm{O}$. The filtrate was concentrated, and the residue was triturated with small amounts of $\mathrm{Et}_{2} \mathrm{O}$. After filtration on cotton, the filtrate was again concentrated. The residue was finally purified on a silica gel column chromatography (from 10 to $15 \%$ AcOEt in petroleum ether) to afford dienone 91 (2.22 g, 70\%). Unreacted thioester 90 was also recovered (232 mg, 6\%).

$[\alpha]^{20} \mathrm{D}=+58.1\left(c 1.17, \mathrm{CHCl}_{3}\right)$. IR (neat): $v=2950,1736,1679,1628,1581,1437,1354,1312,1281,1199$, 1058, 994, $884 \mathrm{~cm}^{-1} .{ }^{1} \mathrm{H}$ NMR $\left(300 \mathrm{MHz}, \mathrm{CDCl}_{3}\right) \delta 7.60(\mathrm{dd}, J=15.1,11.7 \mathrm{~Hz}, 1 \mathrm{H}), 6.40(\mathrm{~d}, J=15.1 \mathrm{~Hz}, 1 \mathrm{H})$, $6.02(\mathrm{~d}, J=11.7 \mathrm{~Hz}, 1 \mathrm{H}$ ), $4.53(\mathrm{t}, J=7.5 \mathrm{~Hz}, 1 \mathrm{H}$ ), 4.46 (quint, $J=6.9 \mathrm{~Hz}, 1 \mathrm{H}$ ), 3.68 (s, 3H), 2.68 (dd, $J=15.4$, $6.9 \mathrm{~Hz}, 1 \mathrm{H}), 2.50(\mathrm{dd}, J=15.4,6.2 \mathrm{~Hz}, 1 \mathrm{H}), 2.26$ (dtd, $J=12.5,8.0,4.2 \mathrm{~Hz}, 2 \mathrm{H}), 2.17-2.05(\mathrm{~m}, 1 \mathrm{H}), 1.96$ (dtd, $J=12.4,7.9,6.8 \mathrm{~Hz}, 1 \mathrm{H}), 1.89(\mathrm{~s}, 3 \mathrm{H}), 1.87(\mathrm{~s}, 3 \mathrm{H}), 1.62(\mathrm{dq}, J=11.9,8.0 \mathrm{~Hz}, 1 \mathrm{H}) .{ }^{13} \mathrm{C}\left\{{ }^{1} \mathrm{H}\right\}$ NMR $(75$ $\left.\mathrm{MHz}, \mathrm{CDCl}_{3}\right) \delta 201.3,171.5,149.2,140.7,124.7,121.8,82.9,76.7,51.8,40.3,31.3,29.6,26.9,19.3$. HRMS (ESI) calcd for $\mathrm{C}_{14} \mathrm{H}_{21} \mathrm{O}_{4}[\mathrm{M}+\mathrm{H}]^{+}:$253.1440, found 253.1433 .

\section{Compound 92}

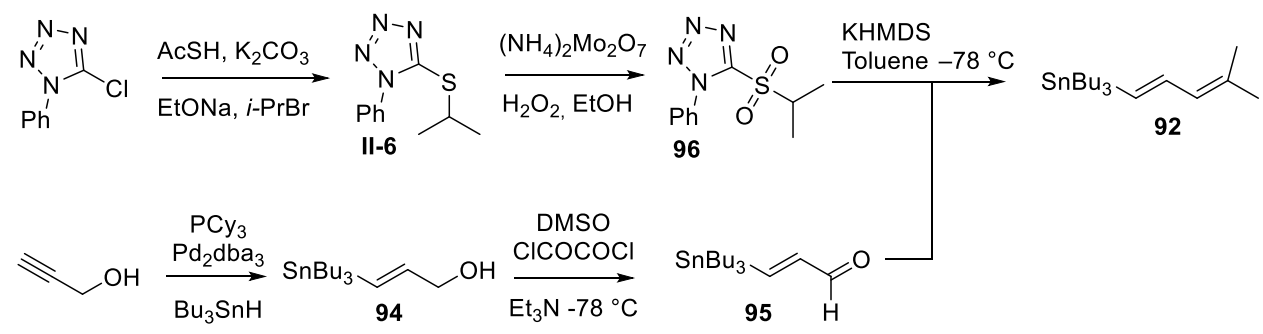

To a solution of $\mathrm{Pd}_{2} \mathrm{dba}_{3}\left(120 \mathrm{mg}, 130 \mu \mathrm{mol}, 0.0025\right.$ equiv, $0.5 \mathrm{~mol} \%$ [Pd]) in $\mathrm{CH}_{2} \mathrm{Cl}_{2}(120 \mathrm{~mL}$ ) was added $\mathrm{PCy}_{3}\left(1.5 \mathrm{~mL}, 1.04 \mathrm{mmol}, 0.02\right.$ equiv, $20 \%$ in toluene). The reaction mixture was stirred for $15 \mathrm{~min}$ at $0{ }^{\circ} \mathrm{C}$, then propargyl alcohol ( $3.04 \mathrm{~mL}, 52.17 \mathrm{mmol}, 1$ equiv) was added followed by tributyltin hydride ( $16.2 \mathrm{~mL}, 60$ mmol, 1.15 equiv) and the stirring was continued for $3 \mathrm{~h}$ at $0{ }^{\circ} \mathrm{C}$. After concentration the residue was purified on a silica gel column (gradient 8 to $15 \% \mathrm{Et}_{2} \mathrm{O}$ in petroleum ether) to give by order of elution 2(tributylstannyl)prop-2-en-1-ol (4.35 g, $12.5 \mathrm{mmol}, 24 \%)$ and $(E)$-3-(tributylstannyl)prop-2-en-1-ol 94 (11.55 g, $33.3 \mathrm{mmol}, 64 \%)$ as coloroless oils. Spectral data of (E)-3-(tributylstannyl)prop-2-en-1-ol 94 was in accordance with the literature. ${ }^{[26]}$

${ }^{1} \mathbf{H}$ NMR $\left(300 \mathrm{MHz}, \mathrm{CDCl}_{3}\right) \delta 6.26-6.13\left(\mathrm{~m},{ }^{3} \mathrm{Jnn}-\mathrm{H}=68.5 \mathrm{~Hz},{ }^{2} \mathrm{JSn}_{\mathrm{H}-\mathrm{H}}=38.2 \mathrm{~Hz}, 2 \mathrm{H}\right), 4.19(\mathrm{~m}, 2 \mathrm{H}), 1.65-1.50(\mathrm{~m}$, $1 \mathrm{H}, \mathrm{OH}$ ), 1.52 (quint, $J=7.5 \mathrm{~Hz}, 6 \mathrm{H}$ ), 1.33 (sext, $J=7.3 \mathrm{~Hz}, 6 \mathrm{H}), 1.00-0.80(\mathrm{~m}, 15 \mathrm{H})$.

To a solution of DMSO (2.84 mL, $40.0 \mathrm{mmol}$, 2 equiv) in $\mathrm{CH}_{2} \mathrm{Cl}_{2}(100 \mathrm{~mL})$ was added oxalyl chloride (2.23 $\mathrm{mL}, 26 \mathrm{mmol}, 1.3$ equiv) dropwise at $-78^{\circ} \mathrm{C}$. After $30 \mathrm{~min}$ at this temperature, $(E)$-3-(tributylstannyl)prop-2- 
en-1-ol 94 (6.94 g, $10 \mathrm{mmol})$ in $\mathrm{CH}_{2} \mathrm{Cl}_{2}(10 \mathrm{~mL})$ was added, followed by $\mathrm{Et}_{3} \mathrm{~N}$ (11.1 mL, $80 \mathrm{mmol}, 4$ equiv). The reaction mixture was allowed to warm slowly to $0{ }^{\circ} \mathrm{C}$ and was then quenched by saturated $\mathrm{NH}_{4} \mathrm{Cl}$. The reaction mixture was then extracted with $\mathrm{Et}_{2} \mathrm{O}$, dried over $\mathrm{MgSO}_{4}$, filtered, and concentrated. The residue was then purified on a silica gel column chromatography $\left(2 \% \mathrm{Et}_{2} \mathrm{O}\right.$ in petroleum ether) to afford $(E)-3-$ (tributylstannyl)acrylaldehyde $95(5.42 \mathrm{~g}, 79 \%)$. Characterization data were in agreement with the literature. ${ }^{[27]}$

IR (neat): $v=2957,1690,1463,1071,665 \mathrm{~cm}^{-1} .{ }^{1} \mathbf{H}$ NMR $\left(300 \mathrm{MHz}, \mathrm{CDCl}_{3}\right) \delta 9.40(\mathrm{~d}, J=7.6 \mathrm{~Hz}, 1 \mathrm{H}), 7.79$ $\left(\mathrm{d}, J=19.2 \mathrm{~Hz},{ }^{2} J_{\mathrm{Sn}-\mathrm{H}}=52.9 \mathrm{~Hz}, 1 \mathrm{H}\right), 6.62\left(\mathrm{dd}, J=19.2,7.6 \mathrm{~Hz},{ }^{3} \mathrm{Jnn}_{\mathrm{S}-\mathrm{H}}=48.5 \mathrm{~Hz}, 1 \mathrm{H}\right.$ ), 1.52 (quint, $J=7.5 \mathrm{~Hz}$, $\left.{ }^{3} J_{\mathrm{Sn}-\mathrm{H}}=53.0 \mathrm{~Hz}, 6 \mathrm{H}\right), 1.32(\mathrm{sext}, J=7.2 \mathrm{~Hz}, 6 \mathrm{H}) 1.00\left(\mathrm{t}, J=8.0 \mathrm{~Hz},{ }^{2} J_{\mathrm{Sn}-\mathrm{H}}=51.4 \mathrm{~Hz}, 6 \mathrm{H}\right), 0.91(\mathrm{t}, J=7.3 \mathrm{~Hz}$, 9H). ${ }^{13} \mathrm{C}\left\{{ }^{1} \mathrm{H}\right\}$ NMR $\left(75 \mathrm{MHz}, \mathrm{CDCl}_{3}\right) \delta 193.8\left({ }^{4} \mathrm{JSn}_{\mathrm{S}-\mathrm{H}}=70.1 \mathrm{~Hz}\right), 163.4,147.76,29.2\left({ }^{3} \mathrm{JSn}_{\mathrm{C}} \mathrm{C}=21.3 \mathrm{~Hz}\right), 27.3$ $\left({ }^{2} J_{S n-C}=55.5 \mathrm{~Hz}\right), 13.8,10.0\left({ }^{1} \mathrm{JSn}_{\mathrm{C}}=336 \mathrm{~Hz}\right)$.

To a solution of $\mathrm{AcSH}\left(5.9 \mathrm{~mL}, 83.1 \mathrm{mmol}, 1.5\right.$ equiv) in DMF $(50 \mathrm{~mL})$ was added $\mathrm{K}_{2} \mathrm{CO}_{3}(11.5 \mathrm{~g}, 83.1$ mmol, 1.5 equiv) followed by $i-\operatorname{PrBr}(8.32 \mathrm{~mL}, 88.6 \mathrm{mmol}, 1.6$ equiv) and the reaction mixture was heated at $100{ }^{\circ} \mathrm{C}$ for $30 \mathrm{~min}$ with the help of an oil bath. The reaction mixture was slightly cooled down, and EtONa (5.64 g, $83.1 \mathrm{mmol}, 1.5$ equiv) was added. After $30 \mathrm{~min}$ at $100^{\circ} \mathrm{C}$, the reaction mixture was slightly cooled down, and 2-chlorophenyltetrazole (10 g, $55.4 \mathrm{mmol}, 1.0$ equiv) was added. After $1 \mathrm{~h}$ at $100^{\circ} \mathrm{C}$, the reaction mixture was quenched with $\mathrm{H}_{2} \mathrm{O}$, extracted with $\mathrm{Et}_{2} \mathrm{O}(\mathrm{x} 3)$, washed with brine $(\mathrm{x} 3)$, dried over $\mathrm{MgSO}_{4}$, filtered, and concentrated to give crude thioether II-6. The residue was dissolved in $\mathrm{EtOH}(200 \mathrm{~mL})$, and ammonium dimolybdate (1.1 g, $3.24 \mathrm{mmol}, 0.06$ equiv) was added, followed by $\mathrm{H}_{2} \mathrm{O}_{2}$ ( $19.2 \mathrm{~mL}, 220 \mathrm{mmol}, 4$ equiv). After $16 \mathrm{~h}$ stirring at $40{ }^{\circ} \mathrm{C}$, the reaction mixture was diluted with water $(200 \mathrm{~mL})$ and extracted with a 50:50 $\mathrm{AcOEt} / \mathrm{Et}_{2} \mathrm{O}$ mixture $(\mathrm{x} 3)$. The combined organic layers were washed with water $(2 \mathrm{x})$, washed with thiosulfate solution, dried over $\mathrm{MgSO}_{4}$, filtered, and concentrated. The residue was recrystallized from EtOH to afford white crystals of sulfone 96 (13.4 g, 95\%). Spectral data were in agreement with those reported. ${ }^{[28,29]}$

$\mathbf{M p}=66-68{ }^{\circ} \mathrm{C}$. IR (neat): $v=1592,1496,1460,1334,1263,1173,1145,1057,1042,763,733,714,692$, $674 \mathrm{~cm}^{-1} .{ }^{1} \mathrm{H}$ NMR $\left(300 \mathrm{MHz}, \mathrm{CDCl}_{3}\right) \delta 7.77-7.49(\mathrm{~m}, 5 \mathrm{H}), 4.00(\mathrm{sept}, J=6.9 \mathrm{~Hz}, 1 \mathrm{H}), 1.50(\mathrm{~d}, J=6.9 \mathrm{~Hz}$, $6 \mathrm{H}) .{ }^{13} \mathrm{C}\left\{{ }^{1} \mathrm{H}\right\}$ NMR $\left(75 \mathrm{MHz}, \mathrm{CDCl}_{3}\right) \delta 152.7,133.2,131.5,129.7,125.5,56.9,15.1$. HRMS (ESI) calcd for $\mathrm{C}_{10} \mathrm{H}_{13} \mathrm{O}_{4} \mathrm{~N}_{4} \mathrm{O}_{2} \mathrm{~S}[\mathrm{M}+\mathrm{H}]^{+}:$253.0759, found 253.0756 .

To a solution of sulfone 96 (5.06 g, $20.1 \mathrm{mmol}, 1.05$ equiv) and aldehyde 95 (6.6 g, $19.1 \mathrm{mmol}, 1.0$ equiv) in toluene $(100 \mathrm{~mL})$ at $-78^{\circ} \mathrm{C}$ was added dropwise KHMDS $(40.2 \mathrm{~mL}, 20.1 \mathrm{mmol}, 1.05$ equiv, $0.5 \mathrm{M}$ in toluene) over $20 \mathrm{~min}$. After $20 \mathrm{~min}$ at $-78^{\circ} \mathrm{C}$, the reaction mixture was allowed to warm at rt, quenched by saturated $\mathrm{NH}_{4} \mathrm{Cl}$, extracted with $\mathrm{Et}_{2} \mathrm{O}$, dried over $\mathrm{MgSO}_{4}$, filtered, and concentrated. The residue was further purified on a silica gel column chromatography (100\% petroleum ether) to afford stannane 92 as a clear oil (4.0 g, 61\%).

IR (neat): $v=2926,1463,1377,1341,991,868,691,664,639 \mathrm{~cm}^{-1} .{ }^{1} \mathbf{H}$ NMR $\left(300 \mathrm{MHz}, \mathrm{CDCl}_{3}\right) \delta 6.74$ (dd, $\left.J=18.6,10.3 \mathrm{~Hz},{ }^{3} J_{\mathrm{Sn}-\mathrm{H}}=60.6 \mathrm{~Hz}, 1 \mathrm{H}\right), 6.06\left(\mathrm{~d}, J=18.6 \mathrm{~Hz},{ }^{2} J_{\mathrm{Sn}-\mathrm{H}}=74 \mathrm{~Hz}, 1 \mathrm{H}\right), 5.85(\mathrm{~d}, J=10.3 \mathrm{~Hz}, 1 \mathrm{H})$, $1.80(\mathrm{~s}, 3 \mathrm{H}), 1.78(\mathrm{~s}, 3 \mathrm{H}), 1.61-1.43(\mathrm{~m}, 6 \mathrm{H}), 1.32(\mathrm{sext}, J=7.2 \mathrm{~Hz}, 6 \mathrm{H}), 0.89(\mathrm{t}, J=7.3 \mathrm{~Hz}, 15 \mathrm{H}) .{ }^{13} \mathrm{C}\left\{{ }^{1} \mathrm{H}\right\}$ NMR $\left(75 \mathrm{MHz}, \mathrm{CDCl}_{3}\right) \delta 143.5,134.5,130.3,128.9,29.3\left({ }^{3} J_{\mathrm{Sn}-\mathrm{C}}=19.7 \mathrm{~Hz}\right), 27.5\left({ }^{2} \mathrm{~J}_{\mathrm{Sn}-\mathrm{C}}=53.4 \mathrm{~Hz}\right), 26.1,18.5$, 13.9, $9.7\left({ }^{1} \mathrm{JSn}_{\mathrm{C}}=328,343 \mathrm{~Hz}\right)$. HRMS $(\mathrm{ESI})$ calcd for $\mathrm{C}_{15} \mathrm{H}_{31} \mathrm{OSn}[\mathrm{M}-\mathrm{Bu}+\mathrm{MeOH}]^{+}: 347.1397$, found 347.1377 


\section{Compound 93}

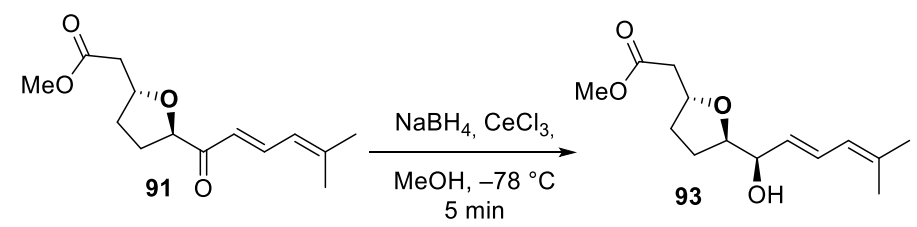

To a solution of dienone 91 (2.22 g, $8.81 \mathrm{mmol}, 1$ equiv) in $\mathrm{MeOH}(50 \mathrm{~mL})$ at $-78{ }^{\circ} \mathrm{C}$ was added $\mathrm{CeCl}_{3}$ [the hydrate was flame-dried under vaccum] $(2.2 \mathrm{~g}, 8.81 \mathrm{mmol}, 1.0$ equiv) in $\mathrm{MeOH}(20 \mathrm{~mL})$. After $15 \mathrm{~min}$ at $-78{ }^{\circ} \mathrm{C}, \mathrm{NaBH}_{4}(670 \mathrm{mg}, 17.62 \mathrm{mmol}, 1$ equiv) was added, and the reaction mixture was quenched after 5 min (a longer reaction time provided ester reduction side reaction) with saturated $\mathrm{NH}_{4} \mathrm{Cl}$. The reaction mixture was extracted with a petroleum ether: $\mathrm{Et}_{2} \mathrm{O}$ mixture (50:50, x2), washed with brine, dried over $\mathrm{MgSO}_{4}$, filtered, and concentrated. The residue was purified on a silica gel chromatography to give alcohol $\mathbf{9 3}$ as a colorless oil (1.60 g, 72\%, dr $\geq 90: 10$ as judged on ${ }^{1} \mathrm{H} N M R$ ).

$[\alpha]^{18} \mathrm{D}=+20.0\left(c 0.9, \mathrm{CHCl}_{3}\right)$. IR (neat): $v=3400$ (br), 2969, 1738, 1660, 1438, 1058, 989, $961 \mathrm{~cm}^{-1} .{ }^{1} \mathbf{H}$ NMR $\left(300 \mathrm{MHz}, \mathrm{CDCl}_{3}\right) \delta 6.51(\mathrm{dd}, J=15.1,11.0 \mathrm{~Hz}, 1 \mathrm{H}), 5.80(\mathrm{~d}, J=11.0 \mathrm{~Hz}, 1 \mathrm{H}), 5.44(\mathrm{dd}, J=15.1,6.7 \mathrm{~Hz}$, $1 \mathrm{H}$ ), 4.37 (quint, $J=6.5 \mathrm{~Hz}, 1 \mathrm{H}$ ), $3.95(\mathrm{t}, J=7.0 \mathrm{~Hz}, 1 \mathrm{H}), 3.88(\mathrm{q}, J=6.7 \mathrm{~Hz}, 1 \mathrm{H}), 3.68(\mathrm{~s}, 3 \mathrm{H}), 2.64(\mathrm{dd}, J=$ 15.4, $6.9 \mathrm{~Hz}, 1 \mathrm{H}), 2.56$ (brs, $1 \mathrm{H}, \mathrm{OH}), 2.46(\mathrm{dd}, J=15.4,6.3 \mathrm{~Hz}, 1 \mathrm{H}), 2.21-2.07(\mathrm{~m}, 1 \mathrm{H}), 2.04-1.90(\mathrm{~m}$, $1 \mathrm{H}), 1.76(\mathrm{~s}, 3 \mathrm{H}), 1.75(\mathrm{~s}, 3 \mathrm{H}), 1.74-1.44(\mathrm{~m}, 2 \mathrm{H}) .{ }^{13} \mathrm{C}\left\{{ }^{1} \mathrm{H}\right\} \mathrm{NMR}\left(75 \mathrm{MHz}, \mathrm{CDCl}_{3}\right) \delta$ 171.7, 136.7, 129.5, 128.1, 124.5, 82.4, 75.6, 75.5, 51.8, 40.4, 32.1, 28.0, 26.1, 18.5. HRMS (ESI) calcd for $\mathrm{C}_{14} \mathrm{H}_{22} \mathrm{O}_{4} \mathrm{Na}[\mathrm{M}+\mathrm{Na}]^{+}$: 377.1416 , found 377.1409 .

\section{Compound 77a}

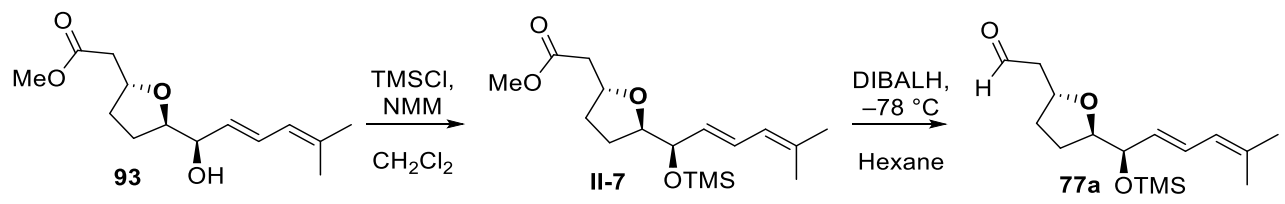

To a solution of alcohol $93(1.6 \mathrm{~g}, 6.3 \mathrm{mmol})$ in $\mathrm{CH}_{2} \mathrm{Cl}_{2}(20 \mathrm{~mL})$ at $0{ }^{\circ} \mathrm{C}$ was added $\mathrm{N}$-methyl morpholine (1.04 mL, $9.45 \mathrm{mmol}, 1.5$ equiv) followed by TMSCl (1.193 mL, $9.45 \mathrm{mmol}, 1.5$ equiv). The reaction mixture was stirred for $30 \mathrm{~min}$ at $\mathrm{rt}$ and was then quenched by water and diluted with pentane $(30 \mathrm{~mL})$. The two phases were separated, and the aqueous layer was extracted with $\mathrm{Et}_{2} \mathrm{O}(\mathrm{x} 2)$. The combined organic layers were washed with brine, dried over $\mathrm{MgSO}_{4}$, filtered, and concentrated. The residue was no further purified, and silyl ether II-7 was collected as a colorless oil ( $2.03 \mathrm{~g}, 99 \%)$.

$[\alpha]^{30} \mathrm{D}=+16.3\left(c 1.47, \mathrm{CHCl}_{3}\right)$. IR (neat): $v=2954,1740,1659,1437,1249,1060,990,960,839,751 \mathrm{~cm}^{-1}$. ${ }^{1} \mathrm{H}$ NMR $\left(300 \mathrm{MHz}, \mathrm{CDCl}_{3}\right) \delta 6.42(\mathrm{t}, J=15.0,11.2 \mathrm{~Hz}, 1 \mathrm{H}), 5.81(\mathrm{~d}, J=11.2 \mathrm{~Hz}, 1 \mathrm{H}), 5.50$ (dd, $J=15.0,6.2$ $\mathrm{Hz}, 1 \mathrm{H}$ ), 4.30 (quint, $J=6.5 \mathrm{~Hz}, 1 \mathrm{H}$ ), $4.12(\mathrm{t}, J=5.8 \mathrm{~Hz}, 1 \mathrm{H}$ ), 3.94 (q, $J=6.9 \mathrm{~Hz}, 1 \mathrm{H}$ ), $3.66(\mathrm{~s}, 3 \mathrm{H}), 2.62$ (dd, $J=15.1,6.8 \mathrm{~Hz}, 1 \mathrm{H}), 2.42(\mathrm{dd}, J=15.0,6.5 \mathrm{~Hz}, 1 \mathrm{H}), 2.14-1.98(\mathrm{~m}, 1 \mathrm{H}), 1.88(\mathrm{ddd}, J=11.5,7.8,3.8 \mathrm{~Hz}$, $1 \mathrm{H}), 1.76(\mathrm{~s}, 3 \mathrm{H}), 1.75(\mathrm{~s}, 3 \mathrm{H}), 1.76-1.60(\mathrm{~m}, 1 \mathrm{H}), 1.52(\mathrm{dq}, J=11.8,8.3 \mathrm{~Hz}, 1 \mathrm{H}), 0.10(\mathrm{~s}, 9 \mathrm{H}) .{ }^{13} \mathrm{C}\left\{{ }^{1} \mathrm{H}\right\} \mathrm{NMR}$ $\left(75 \mathrm{MHz}, \mathrm{CDCl}_{3}\right) \delta 171.9,135.4,129.6,127.9,124.8,82.5,75.7$ (2C), 51.7, 40.7, 32.1, 27.5, 26.1, 18.4, 0.4 (3C). HRMS (ESI) calcd for $\mathrm{C}_{17} \mathrm{H}_{30} \mathrm{O}_{4} \mathrm{NaSi}[\mathrm{M}+\mathrm{Na}]^{+}: 349.1811$, found 349.1804.

To a solution of methyl ester II-7 (1.99 g, $6.10 \mathrm{mmol}, 1$ equiv) in hexanes $(60 \mathrm{~mL})$ at $-78{ }^{\circ} \mathrm{C}$ was added dropwise DIBAL-H (6.85 mL, $6.85 \mathrm{mmol}, 1.1$ equiv, $1.0 \mathrm{M}$ in hexanes). After $1 \mathrm{~h}$ at $-78^{\circ} \mathrm{C}$, the reaction mixture was poured into a $25 \%$ solution of potassium sodium tartrate $(30 \mathrm{~mL})$ under stirring. After $30 \mathrm{~min}$ stirring, the two layers were separated, the organic phase was extracted with $\mathrm{Et}_{2} \mathrm{O}(\mathrm{x} 3)$. The combined organic layers were washed with a $5 \% \mathrm{NaHCO}_{3}$ solution (x2). The ethereal solution was dried over $\mathrm{MgSO}_{4}$, filtered, and concentrated. The residue was purified on a silica gel column chromatography $\left(15-20 \% \mathrm{Et}_{2} \mathrm{O}\right.$ gradient in petroleum ether) to give aldehyde $77 \mathrm{a}$ as a colorless oil ( $1.558 \mathrm{~g}, 86 \%)$. 
$[\alpha]^{20} \mathrm{D}=+6.67\left(c 2.70, \mathrm{CHCl}_{3}\right)$. IR (neat): $v=2958,1726,1659,1380,1249,1118,1080,988,968,839,751$ $\mathrm{cm}^{-1} .{ }^{1} \mathrm{H}$ NMR $\left(300 \mathrm{MHz}, \mathrm{CDCl}_{3}\right) \delta 9.79(\mathrm{t}, J=2.3,1.8 \mathrm{~Hz}, 1 \mathrm{H}), 6.42(\mathrm{dd}, J=15.1,10.9 \mathrm{~Hz}, 1 \mathrm{H}), 5.81(\mathrm{~d}, J=$ $10.9 \mathrm{~Hz}, 1 \mathrm{H}$ ), $5.51(\mathrm{dd}, J=15.1,6.2 \mathrm{~Hz}, 1 \mathrm{H}), 4.36$ (quint, $J=8.2,7.4,5.8 \mathrm{~Hz}, 1 \mathrm{H}$ ), $4.12(\mathrm{t}, J=5.8 \mathrm{~Hz}, 1 \mathrm{H}$ ), $3.96(\mathrm{q}, J=6.9 \mathrm{~Hz}, 1 \mathrm{H}$ ), 2.67 (ddd, $J=16.2,7.2,2.3 \mathrm{~Hz}, 1 \mathrm{H}$ ), 2.53 (ddd, $J=16.2,5.4,1.8 \mathrm{~Hz}, 1 \mathrm{H}$ ), 2.11 (dddd, $J=11.8,8.0,5.7,3.4 \mathrm{~Hz}, 1 \mathrm{H}), 2.00-1.84(\mathrm{~m}, 1 \mathrm{H}), 1.76(\mathrm{~s}, 3 \mathrm{H}), 1.75(\mathrm{~s}, 3 \mathrm{H}), 1.77-1.63(\mathrm{~m}, 1 \mathrm{H})$, $1.51(\mathrm{dq}, J=11.4,8.3 \mathrm{~Hz}, 1 \mathrm{H}), 0.11(\mathrm{~s}, 9 \mathrm{H}) .{ }^{13} \mathrm{C}\left\{{ }^{1} \mathrm{H}\right\}$ NMR $\left(75 \mathrm{MHz}, \mathrm{CDCl}_{3}\right) \delta 201.5,135.7,129.6,128.1$, 124.7, 82.6, 75.8, 74.4, 49.6, 32.4, 27.6, 26.1, 18.4, 0.5 (3C). HRMS (ESI) calcd for $\mathrm{C}_{17} \mathrm{H}_{32} \mathrm{O}_{4} \mathrm{NaSi}$ $[\mathrm{M}+\mathrm{MeOH}+\mathrm{Na}]^{+}: 351.1968$, found 351.1960 .

\section{Compound 97}

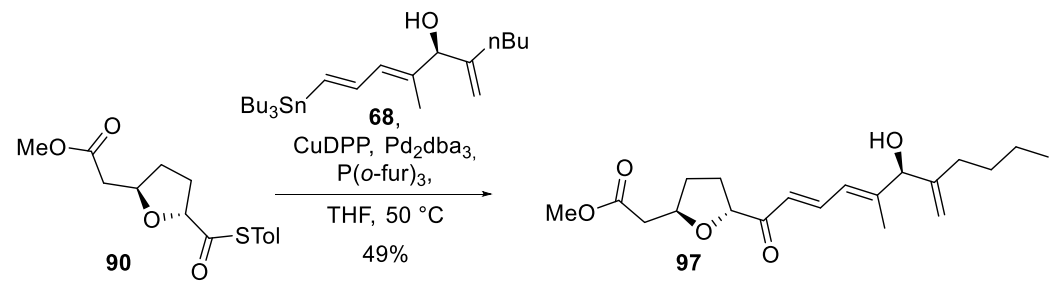

To a solution of thioester 90 ( $2.98 \mathrm{~g}, 10.1 \mathrm{mmol}, 1$ equiv) and stannane 68 (5.3 g, $11.3 \mathrm{mmol}, 1.1$ equiv) in THF : hexanes $(30 \mathrm{~mL}: 60 \mathrm{~mL})$ was added in one portion copper diphenylphosphinate (CuDPP) ${ }^{[23]}(4.24$ g, $15.15 \mathrm{mmol}, 1.5$ equiv), $\mathrm{Pd}_{2} \mathrm{dba}_{3}$ (290.4 mg, $0.505 \mathrm{mmol}, 0.05$ equiv), trifurylphosphine (468 mg, $0.2 \mathrm{mmol}$, 2.02 equiv). The reaction mixture was then heated for $2 \mathrm{~h}$ in a preheated oil bath at $50{ }^{\circ} \mathrm{C}$. The reaction mixture turned dark brown and was cooled down to rt and diluted with petroleum ether $(50 \mathrm{~mL})$. The reaction mixture was then filtered on a pad of silica gel, and the filter cake was washed copiously with $\mathrm{Et}_{2} \mathrm{O}$. The filtrate was concentrated, and the residue was triturated with small amounts of $\mathrm{Et}_{2} \mathrm{O}$. After filtration on cotton, the filtrate was concentrated again. Finally, the residue was purified on a silica gel column chromatography (from 10 to $15 \%$ AcOEt in petroleum ether) to afford dienone 91 as light orange oil (1.74 g, 49\%).

$[\alpha]^{20} \mathrm{D}=+6.4$ (c 2.65, $\left.\mathrm{CHCl}_{3}\right)$. IR (neat): $v=3400,2930,1739,1680,1624,1583,1437,1383,1281,1201$, 1139, 1047, 986, $901 \mathrm{~cm}^{-1} .{ }^{1} \mathrm{H}$ NMR (300 MHz, Chloroform- $\left.d\right) \delta 7.63(\mathrm{dd}, J=15.2,11.7 \mathrm{~Hz}, 1 \mathrm{H}), 6.56(\mathrm{~d}, J=$ $15.2 \mathrm{~Hz}, 1 \mathrm{H}), 6.38(\mathrm{~d}, J=11.7 \mathrm{~Hz}, 1 \mathrm{H}), 5.14(\mathrm{~s}, 1 \mathrm{H}), 4.99(\mathrm{~s}, 1 \mathrm{H}), 4.57(\mathrm{~s}, 1 \mathrm{H}), 4.59-4.43(\mathrm{~m}, 2 \mathrm{H}), 3.70(\mathrm{~s}$, 3H), $2.70(\mathrm{dd}, J=15.3,6.8 \mathrm{~Hz}, 1 \mathrm{H}), 2.52(\mathrm{dd}, J=15.3,6.3 \mathrm{~Hz}, 1 \mathrm{H}), 2.28(\mathrm{dtd}, J=12.3,7.8,4.1 \mathrm{~Hz}, 1 \mathrm{H}), 2.12$ $-1.85(\mathrm{~m}, 5 \mathrm{H}), 1.81(\mathrm{~s}, 3 \mathrm{H}), 1.65(\mathrm{dq}, J=11.2,7.9 \mathrm{~Hz}, 2 \mathrm{H}), 1.49-1.16(\mathrm{~m}, 4 \mathrm{H}), 0.89(\mathrm{t}, J=7.2 \mathrm{~Hz}, 3 \mathrm{H})$. ${ }^{13} \mathrm{C}\left\{{ }^{1} \mathrm{H}\right\}$ NMR $\left(75 \mathrm{MHz}, \mathrm{CDCl}_{3}\right) \delta 201.18,171.47,150.22,149.04,139.71,124.52,124.40,111.55,82.99$, 79.96, 76.88, 51.85, 40.37, 31.34, 30.22, 29.51, 22.64, 14.09, 13.69. HRMS (ESI) calcd for $\mathrm{C}_{20} \mathrm{H}_{31} \mathrm{O}_{5}[\mathrm{M}+\mathrm{H}]^{+}$: 351.2171, found 351.2175; calcd for $\mathrm{C}_{20} \mathrm{H}_{34} \mathrm{NO}_{5}\left[\mathrm{M}+\mathrm{NH}_{4}\right]^{+}: 368.2437$, found 368.2440.

\section{Compound 98}

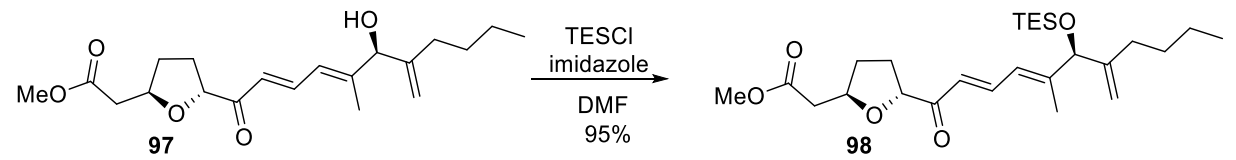

To a solution of compound 97 (1.13 g, $3.223 \mathrm{mmol}$, 1 equiv) in DMF (3 mL) at rt, was added imidazole (1.1 $\mathrm{g}, 16.15 \mathrm{mmol}, 5$ equiv), and TESCl $(1.6 \mathrm{~mL}, 9.69 \mathrm{mmol}, 3$ equiv). The reaction mixture was stirred $5 \mathrm{~h}$ at $\mathrm{rt}$ and was quenched with saturated $\mathrm{NH}_{4} \mathrm{Cl}$ and extracted with $\mathrm{Et}_{2} \mathrm{O}$ three times. Combined organic phases were washed wit brine three times, then dried over $\mathrm{MgSO}_{4}$, filtered, and concentrated. The residue was purified on a silica gel column chromatography (10\% AcOEt in petroleum ether) to provide compound 98 as a colorless oil (1.42g, 95\%). 
$[\alpha]^{20} \mathrm{D}=+17.7\left(c 1.13, \mathrm{CH}_{2} \mathrm{Cl}_{2}\right)$. IR (neat): $v=2955,1743,1682,1627,1586,1460,1437,1282,1200,1076$, 1005, 853, $726 \mathrm{~cm}^{-1}{ }^{1}{ }^{1} \mathrm{H}$ NMR (300 MHz, Chloroform- $\left.d\right) \delta 7.64(\mathrm{dd}, J=15.2,11.8 \mathrm{~Hz}, 1 \mathrm{H}), 6.53(\mathrm{~d}, J=15.2$ $\mathrm{Hz}, 1 \mathrm{H}), 6.34(\mathrm{~d}, J=11.8 \mathrm{~Hz}, 1 \mathrm{H}), 5.12(\mathrm{~s}, 1 \mathrm{H}), 4.89(\mathrm{~s}, 1 \mathrm{H}), 4.74-4.43(\mathrm{~m}, 2 \mathrm{H}), 4.49(\mathrm{~s}, 1 \mathrm{H}), 3.70(\mathrm{~s}, 3 \mathrm{H})$, $2.71(\mathrm{dd}, J=15.3,6.7 \mathrm{~Hz}, 1 \mathrm{H}), 2.53(\mathrm{dd}, J=15.3,6.4 \mathrm{~Hz}, 1 \mathrm{H}), 2.28(\mathrm{dtd}, J=12.3,7.8,4.0 \mathrm{~Hz}, 1 \mathrm{H}), 2.22-$ $2.06(\mathrm{~m}, 1 \mathrm{H}), 2.06-1.83(\mathrm{~m}, 2 \mathrm{H}), 1.84-1.57(\mathrm{~m}, 2 \mathrm{H}), 1.77(\mathrm{~s}, 3 \mathrm{H}), 1.44-1.17(\mathrm{~m}, 4 \mathrm{H}), 0.93(\mathrm{t}, \mathrm{J}=7.8 \mathrm{~Hz}$, $6 \mathrm{H}), 0.88(\mathrm{t}, J=7.0 \mathrm{~Hz}, 3 \mathrm{H}), 0.59(\mathrm{q}, J=7.9 \mathrm{~Hz}, 9 \mathrm{H}) .{ }^{13} \mathrm{C}\left\{{ }^{1} \mathrm{H}\right\}$ NMR $\left(75 \mathrm{MHz}, \mathrm{CDCl}_{3}\right) \delta 201.2,171.5,152.0$, 149.2, 140.3, 123.8, 123.8, 111.0, 83.0, 80.6, 76.9, 51.8, 40.4, 31.3, 30.4, 30.2, 29.5, 22.7, 14.1, 13.5, 6.9, 4.9. HRMS (ESI) calcd for $\mathrm{C}_{26} \mathrm{H}_{45} \mathrm{O}_{5} \mathrm{Si}[\mathrm{M}+\mathrm{H}]^{+}: 465.3036$, found 465.3031 ; calcd for $\mathrm{C}_{26} \mathrm{H}_{48} \mathrm{NO}_{5} \mathrm{Si}\left[\mathrm{M}+\mathrm{NH}_{4}\right]^{+}$: 482.3302, found 482.3303; calcd for $\mathrm{C}_{26} \mathrm{H}_{44} \mathrm{O}_{5} \mathrm{NaSi}[\mathrm{M}+\mathrm{Na}]^{+}:$487.2856, found 487.2848.

\section{Compound 99}

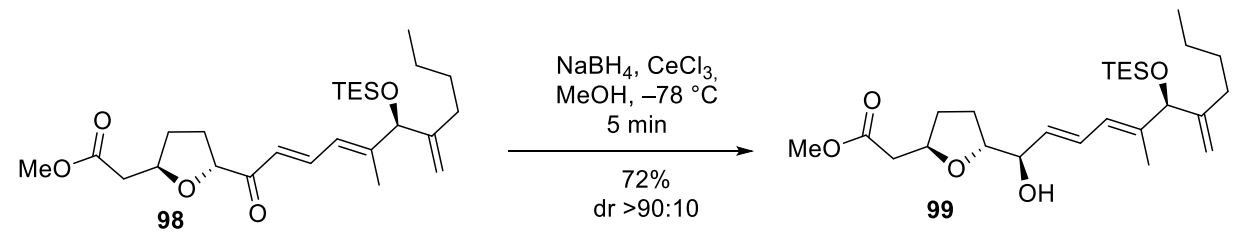

To a solution of dienone 98 in $\mathrm{MeOH}(20 \mathrm{~mL})$ at $-78{ }^{\circ} \mathrm{C}$ was added $\mathrm{CeCl}_{3}$ [the hydrate was flame-dried under vaccum] (690 mg, $2.80 \mathrm{mmol}, 1.0$ equiv) in $\mathrm{MeOH}(20 \mathrm{~mL})$. After $15 \mathrm{~min}$ at $-78{ }^{\circ} \mathrm{C}, \mathrm{NaBH}_{4}(43 \mathrm{mg}$, $1.12 \mathrm{mmol}, 0.4$ equiv) was added, and the reaction mixture was quenched after $5 \mathrm{~min}$ (a longer reaction time provided ester reduction side reaction) with saturated $\mathrm{NH}_{4} \mathrm{Cl}$. The reaction mixture was extracted with a petroleum ether: $\mathrm{Et}_{2} \mathrm{O}$ mixture $(50: 50, x 2)$, washed with brine, dried over $\mathrm{MgSO}_{4}$, filtered, and concentrated. The residue was purified on a silica gel chromatography to give alcohol 99 as a colorless oil $(0.86 \mathrm{~g}, 72 \%$, $\mathrm{dr}$ $\geq 90: 10$ as judged on ${ }^{1} \mathrm{H}$ NMR).

$[\alpha]^{20} \mathrm{D}=-6.1\left(c 1.97, \mathrm{CH}_{2} \mathrm{Cl}_{2}\right)$. IR (neat): $v=2955,1740,1461,1437,1414,1238,1202,1056,1016,1005$, 901, 856, 745, $725 \mathrm{~cm}^{-11} \mathrm{H}$ NMR (300 MHz, Chloroform- $d$ ) $\delta 6.55$ (dd, $\left.J=15.0,11.1 \mathrm{~Hz}, 1 \mathrm{H}\right), 6.09(\mathrm{~d}, J=11.0$ $\mathrm{Hz}, 1 \mathrm{H}), 5.60(\mathrm{dd}, J=15.0,6.6 \mathrm{~Hz}, 1 \mathrm{H}), 5.12(\mathrm{~s}, 1 \mathrm{H}), 4.50-4.30(\mathrm{~m}, 1 \mathrm{H}), 4.86(\mathrm{~s}, 1 \mathrm{H}), 4.42(\mathrm{~s}, 1 \mathrm{H}), 4.07-$ $3.88(\mathrm{~m}, 2 \mathrm{H}), 3.70(\mathrm{~s}, 3 \mathrm{H}), 2.67(\mathrm{dd}, J=15.3,6.9 \mathrm{~Hz}, 1 \mathrm{H}), 2.60$ (brs, $1 \mathrm{H}), 2.49$ (dd, $J=15.3,6.3 \mathrm{~Hz}, 1 \mathrm{H}), 2.24$ $-2.11(\mathrm{~m}, 1 \mathrm{H}), 2.07-1.65(\mathrm{~m}, 4 \mathrm{H}), 1.62(\mathrm{~s}, 3 \mathrm{H}), 1.48-1.11(\mathrm{~m}, 5 \mathrm{H}), 0.94(\mathrm{t}, J=7.9 \mathrm{~Hz}, 9 \mathrm{H}), 0.89(\mathrm{t}, J=7.1$ $\mathrm{Hz}, 3 \mathrm{H}), 0.58(\mathrm{q}, J=7.9 \mathrm{~Hz}, 6 \mathrm{H}) .{ }^{13} \mathrm{C}\left\{{ }^{1} \mathrm{H}\right\}$ NMR $\left(75 \mathrm{MHz}, \mathrm{CDCl}_{3}\right) \delta 171.6,149.8,140.0,130.4,129.0,124.7$, 109.9, 82.4, 80.5, 75.6, 75.5, 51.8, 40.5, 32.1, 30.9, 30.2, 28.0, 22.7, 14.1, 12.3, 6.9, 4.9. HRMS (ESI) calcd for $\mathrm{C}_{26} \mathrm{H}_{46} \mathrm{O} 5 \mathrm{NaSi}[\mathrm{M}+\mathrm{Na}]^{+}:$489.3012, found 489.3012 .

\section{Compound 100}

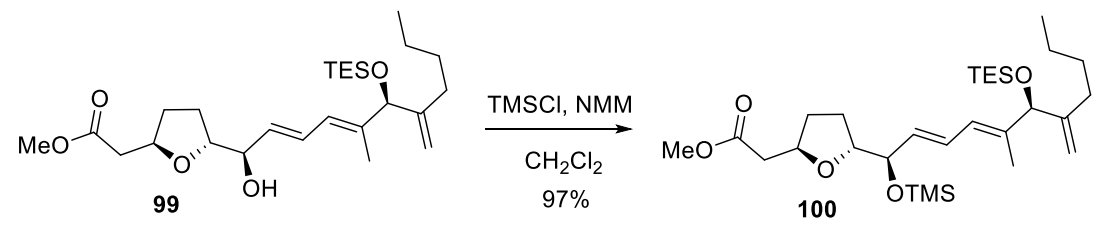

To a solution of alcohol 99 (840 mg, $1.8 \mathrm{mmol})$ in $\mathrm{CH}_{2} \mathrm{Cl}_{2}(20 \mathrm{~mL})$ at $0{ }^{\circ} \mathrm{C}$ was added $\mathrm{N}$-methyl morpholine ( $295 \mu \mathrm{L}, 2.7 \mathrm{mmol}, 1.5$ equiv) followed by TMSCl ( $345 \mu \mathrm{L}, 2.7 \mathrm{mmol}, 1.5$ equiv). The reaction mixture was stirred for $30 \mathrm{~min}$ at $\mathrm{rt}$ and was then quenched by water and diluted with pentane $(30 \mathrm{~mL})$. The two phases were separated, and the aqueous layer was extracted with $\mathrm{Et}_{2} \mathrm{O}(\mathrm{x} 2)$. The combined organic layers were washed with brine, dried over $\mathrm{MgSO}_{4}$, filtered, and concentrated. The residue was no further purified, and silyl ether 100 was collected as a colorless oil (937 mg, 97\%). 
$[\alpha]^{20} \mathrm{D}=-10.7\left(c 1.5, \mathrm{CH}_{2} \mathrm{Cl}_{2}\right) . \mathbf{I R}$ (neat): $v=2955,1743,1249,1065,1017,1005,900,841,746,725 \mathrm{~cm}^{-1} .{ }^{1} \mathbf{H}$ NMR ${ }^{1} \mathrm{H}$ NMR $(300 \mathrm{MHz}$, Chloroform- $d) \delta 6.44(\mathrm{dd}, J=15.2,11.0 \mathrm{~Hz}, 1 \mathrm{H}), 6.07(\mathrm{~d}, J=11.0 \mathrm{~Hz}, 1 \mathrm{H}), 5.64$ $(\mathrm{dd}, J=15.2,6.0 \mathrm{~Hz}, 1 \mathrm{H}), 5.12(\mathrm{~s}, 1 \mathrm{H}), 4.85(\mathrm{~s}, 1 \mathrm{H}), 4.41(\mathrm{~s}, 1 \mathrm{H}), 4.33$ (quint, $J=6.7 \mathrm{~Hz}, 2 \mathrm{H}), 4.15(\mathrm{t}, J=5.9$ $\mathrm{Hz}, 1 \mathrm{H}), 3.98(\mathrm{q}, J=6.7 \mathrm{~Hz}, 1 \mathrm{H}), 3.67(\mathrm{~s}, 3 \mathrm{H}), 2.63(\mathrm{dd}, J=15.0,6.7 \mathrm{~Hz}, 1 \mathrm{H}), 2.43(\mathrm{dd}, J=15.0,6.5 \mathrm{~Hz}, 1 \mathrm{H})$, $2.16-2.00(\mathrm{~m}, 1 \mathrm{H}), 1.99-1.64(\mathrm{~m}, 4 \mathrm{H}), 1.59(\mathrm{~s}, 3 \mathrm{H}), 1.55(\mathrm{dq}, J=12.4,8.5 \mathrm{~Hz}, 1 \mathrm{H}), 1.46-1.20(\mathrm{~m}, 4 \mathrm{H})$, $0.92(\mathrm{t}, J=7.9 \mathrm{~Hz}, 9 \mathrm{H}), 0.87(\mathrm{t}, J=7.0 \mathrm{~Hz}, 3 \mathrm{H}), 0.57(\mathrm{q}, J=7.9 \mathrm{~Hz}, 6 \mathrm{H}), 0.11(\mathrm{~s}, 9 \mathrm{H}) .{ }^{13} \mathrm{C}\left\{{ }^{1} \mathrm{H}\right\} \mathrm{NMR}(75 \mathrm{MHz}$, $\left.\mathrm{CDCl}_{3}\right) \delta 171.8,149.9,138.9,131.9,127.7,125.2,109.7,82.4,80.5,75.8,75.8,51.7,40.7,32.1,31.1,30.3$, 27.5, 22.7, 14.1, 12.1, 7.0, 5.0, 0.5. HRMS (ESI) calcd for $\mathrm{C}_{29} \mathrm{H}_{54} \mathrm{O}_{5} \mathrm{NaSi}_{2}\left[\mathrm{M}+\mathrm{Na}^{+}:\right.$561.3408, found 561.3404.

\section{Compound $77 \mathbf{b}$}

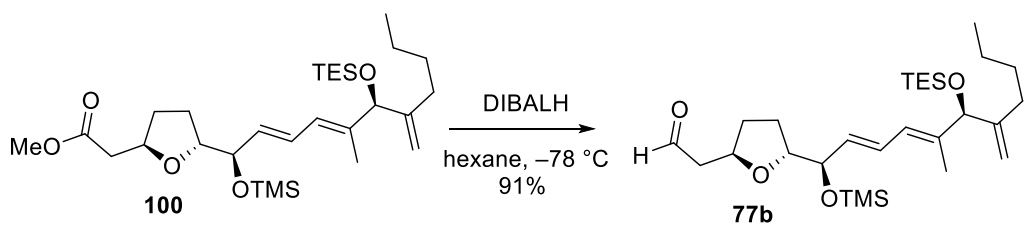

To a solution of methyl ester 100 (917 $\mathrm{mg}, 1.70 \mathrm{mmol}, 1$ equiv) in hexanes $(30 \mathrm{~mL})$ at $-78{ }^{\circ} \mathrm{C}$ was added dropwise DIBAL-H (1.70 mL, $1.875 \mathrm{mmol}, 1.1$ equiv, $1.1 \mathrm{M}$ in hexanes). After $1 \mathrm{~h}$ at $-78{ }^{\circ} \mathrm{C}$, the reaction mixture was poured into a $25 \%$ solution of potassium sodium tartrate $(30 \mathrm{~mL})$ under stirring. After 30 min stirring, the two layers were separated, the organic phase was extracted with $\mathrm{Et}_{2} \mathrm{O}(\mathrm{x} 3)$. The combined organic layers were washed with a $5 \% \mathrm{NaHCO}_{3}$ solution (x2). The ethereal solution was dried over $\mathrm{MgSO}_{4}$, filtered, and concentrated. The residue was purified on a silica gel column chromatography $\left(15-20 \% \mathrm{Et}_{2} \mathrm{O}\right.$ gradient in petroleum ether) to give aldehyde $\mathbf{7 7 b}$ as a colorless oil $(790.5 \mathrm{mg}, 91 \%)$.

$[\alpha]^{20} \mathrm{D}=-6.5\left(c 1.55, \mathrm{CH}_{2} \mathrm{Cl}_{2}\right)$. IR (neat): $v=2958,1728,1647,1461,1250,1073,1017,1005,903,841,746$, $725 \mathrm{~cm}^{-1} .{ }^{1} \mathrm{H}$ NMR $(300 \mathrm{MHz}$, Chloroform-d) $\delta 9.80(\mathrm{t}, J=1.9 \mathrm{~Hz}, 1 \mathrm{H}), 6.44(\mathrm{dd}, J=15.1,11.0 \mathrm{~Hz}, 1 \mathrm{H}), 6.07$ $(\mathrm{d}, J=10.9 \mathrm{~Hz}, 1 \mathrm{H}), 5.64(\mathrm{dd}, J=15.1,6.3 \mathrm{~Hz}, 1 \mathrm{H}), 5.12(\mathrm{~s}, 1 \mathrm{H}), 4.85(\mathrm{~s}, 1 \mathrm{H}), 4.41(\mathrm{~s}, 1 \mathrm{H}), 4.41-4.32(\mathrm{~m}$, $1 \mathrm{H}$ ), 4.14 (t, $J=5.7 \mathrm{~Hz}, 1 \mathrm{H}$ ), $3.99(\mathrm{q}, J=7.0 \mathrm{~Hz}, 1 \mathrm{H}$ ), 2.68 (ddd, $J=16.1,7.1,2.4 \mathrm{~Hz}, 1 \mathrm{H}$ ), 2.54 (ddd, $J=$ $16.1,5.4,1.7 \mathrm{~Hz}, 1 \mathrm{H}), 2.19-2.05(\mathrm{~m}, 1 \mathrm{H}), 2.03-1.69(\mathrm{~m}, 4 \mathrm{H}), 1.60(\mathrm{~s}, 3 \mathrm{H}), 1.53(\mathrm{dq}, J=12.0,8.8 \mathrm{~Hz}, 1 \mathrm{H})$, $1.46-1.16(\mathrm{~m}, 4 \mathrm{H}), 0.94(\mathrm{t}, J=7.9 \mathrm{~Hz}, 9 \mathrm{H}), 0.87(\mathrm{t}, J=7.0 \mathrm{~Hz}, 3 \mathrm{H}), 0.57(\mathrm{q}, J=8.0 \mathrm{~Hz}, 6 \mathrm{H}), 0.12(\mathrm{~s}, 9 \mathrm{H})$. ${ }^{13} \mathrm{C}\left\{{ }^{1} \mathrm{H}\right\}$ NMR $\left(75 \mathrm{MHz}, \mathrm{CDCl}_{3}\right) \delta 201.4,149.8,139.1,131.8,127.8,125.2,109.8,82.5,80.5,75.9,74.5,49.7$, $32.4,31.1,30.3,27.6,22.7,14.1,12.2,7.0,5.0,0.5$. HRMS (ESI) calcd for $\mathrm{C}_{29} \mathrm{H}_{56} \mathrm{O}_{5} \mathrm{NaSi}_{2}[\mathrm{M}+\mathrm{MeOH}+\mathrm{Na}]^{+}$: 563.3588, found 563.3572 .

\section{Compound 101}

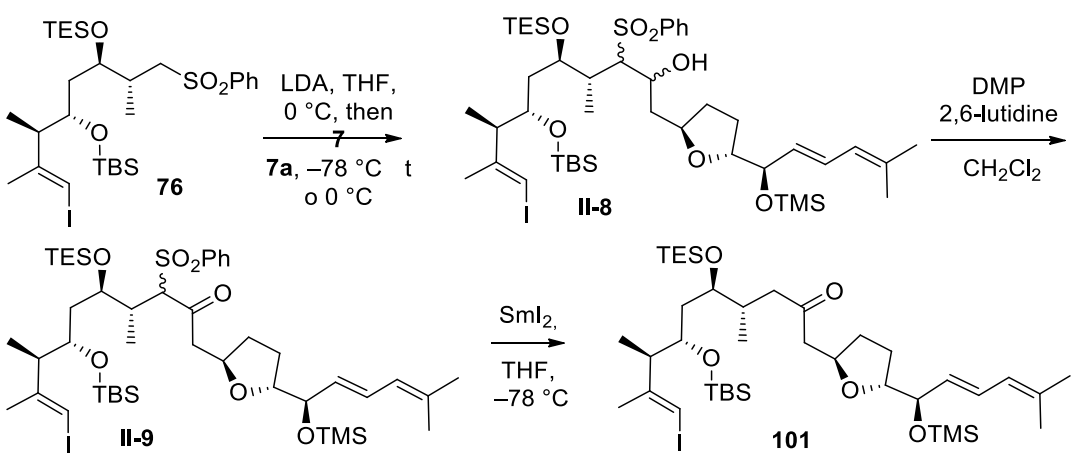

To a solution of sulfone 76 ( $714 \mathrm{mg}, 1.5 \mathrm{mmol}, 1.0$ equiv, dried by azeotropic distillation with toluene, three times) in THF ( $7 \mathrm{~mL}$ ) at $0^{\circ} \mathrm{C}$ was added dropwise LDA [2.52 mL, $1.26 \mathrm{mmol}, 1.2$ equiv, $0.5 \mathrm{M}$ in THF (prepared as followed: dropwise addition of $n$-BuLi $(3.125 \mathrm{~mL}, 5.0 \mathrm{mmol}, 1.6 \mathrm{M}$ in hexanes) onto diisopropylamine $(750$ 
$\mu \mathrm{L}, 5.35 \mathrm{mmol})$ in THF $(6.15 \mathrm{~mL})$ at $-78^{\circ} \mathrm{C}$, followed by slow warming to rt.)]. After $15 \mathrm{~min}$, the yellow reaction mixture was cooled to $-78^{\circ} \mathrm{C}$, and aldehyde $77 \mathrm{a}(373 \mathrm{mg}, 1.26 \mathrm{mmol}, 1.2$ equiv, dried by azeotropic distillation with toluene, three times) in THF $(3 \mathrm{~mL})$ was added. The reaction mixture was slowly warmed to $0{ }^{\circ} \mathrm{C}$, stirred for 30 min, quenched by saturated $\mathrm{NH}_{4} \mathrm{Cl}$, extracted with $\mathrm{Et}_{2} \mathrm{O}(\times 3)$, dried over $\mathrm{MgSO}_{4}$, filtered and concentrated. The residue was purified on a silica gel chromatography (8-15\% gradient $\mathrm{Et}_{2} \mathrm{O}$ in petroleum ether) to give, by order of elution, recovered sulfone $76(140.3 \mathrm{mg}, 20 \%)$ and sulfone adduct II-8 (618 mg, $60 \%, 75 \% \mathrm{brsm}$ ) as a complex mixture of four diastereomers. By consequence, its full characterization was postponed to the next 2 steps. HRMS (ESI) calcd for $\mathrm{C}_{45} \mathrm{H}_{81} \mathrm{O}_{7} \mathrm{Si}_{2} \mathrm{SINa}[\mathrm{M}+\mathrm{Na}]^{+}$: 999.3953, found 999.3954.

To a solution of sulfone adduct II-8 (600 mg, $0.744,1$ equiv), in a $\mathrm{CH}_{2} \mathrm{Cl}_{2} / 2,6$-lutidine mixture $(10 \mathrm{~mL} / 2 \mathrm{~mL})$ was added Dess-Martin periodinane (652 mg, $1.54 \mathrm{mmol}, 2.5$ equiv). After $1 \mathrm{~h}$ at $\mathrm{rt}$, the reaction mixture was quenched with saturated $\mathrm{NaHCO}_{3}$ and a solution of $10 \% \mathrm{Na}_{2} \mathrm{~S}_{2} \mathrm{O}_{3}$. After 15 min, the reaction mixture was extracted with $\mathrm{Et}_{2} \mathrm{O}(\mathrm{x} 3)$, washed with saturated $\mathrm{NaHCO}_{3}$, dried over $\mathrm{MgSO}_{4}$, filtered on a pad of silica gel (elution $\mathrm{Et}_{2} \mathrm{O}$ ) to give crude ketone II-9.

The residue containing crude ketone II-9 was then dissolved in THF $(10 \mathrm{~mL})$, cooled to $-78{ }^{\circ} \mathrm{C}$ and Sml 2 [20 mL, $2.0 \mathrm{mmol} \approx 0.1 \mathrm{M}$ in THF, (prepared as follows: addition of $\mathrm{CHI}_{3}(1.31 \mathrm{~g}, 3.33 \mathrm{mmol}$ ) to a suspension of Sm powder $(1.2 \mathrm{~g}, 8.0 \mathrm{mmol})$ in THF $(50 \mathrm{~mL})$, and stirring $18 \mathrm{~h}$ at rt] was added dropwise (until a blue/green color persists in the reaction mixture). The reaction mixture was then quenched with $\mathrm{NH}_{4} \mathrm{Cl}$, extracted with $\mathrm{Et}_{2} \mathrm{O}(\mathrm{x} 3)$, dried over $\mathrm{MgSO}_{4}$, filtered, and concentrated. The residue was purified on a silica gel column chromatography to give compound 101 (283 $\mathrm{mg}, 55 \%)$ as a colorless oil.

$[\alpha]^{20} \mathrm{D}=+10.1\left(\mathrm{c} 1.49, \mathrm{CHCl}_{3}\right)$. IR (neat): $v=2957,1713,1687,1461,1379,1250,1116,1068,1044,1044$, 1005, 959, 838, 775, 745, $724 \mathrm{~cm}^{-1}$. ${ }^{1} \mathrm{H}$ NMR $\left(300 \mathrm{MHz}, \mathrm{CDCl}_{3}\right) \delta 6.41$ (dd, $\left.J=15.2,11.1 \mathrm{~Hz}, 1 \mathrm{H}\right), 5.88$ (brs, $1 \mathrm{H}), 5.81(\mathrm{~d}, J=11.0 \mathrm{~Hz}, 1 \mathrm{H}), 5.50(\mathrm{dd}, J=15.2,6.2 \mathrm{~Hz}, 1 \mathrm{H}), 4.31(\mathrm{dq}, J=8.2,6.2 \mathrm{~Hz}, 1 \mathrm{H}), 4.12(\mathrm{t}, J=5.6$ $\mathrm{Hz}, 1 \mathrm{H}), 3.92(\mathrm{q}, J=6.7 \mathrm{~Hz}, 1 \mathrm{H}), 3.77(\mathrm{~m}, 1 \mathrm{H}), 3.63(\mathrm{~m}, 1 \mathrm{H}), 2.77(\mathrm{dd}, J=15.8,6.3 \mathrm{~Hz}, 1 \mathrm{H}), 2.48(\mathrm{dd}, J=$ 15.8, 6.6 Hz, 2H), $2.34-2.14(\mathrm{~m}, 2 \mathrm{H}), 2.23-1.99(\mathrm{~m}, 2 \mathrm{H}), 1.92-1.64(\mathrm{~m}, 2 \mathrm{H}), 1.82(\mathrm{~s}, 3 \mathrm{H}), 1.76(\mathrm{~s}, 3 \mathrm{H})$, $1.75(\mathrm{~s}, 3 \mathrm{H}), 1.53-1.38(\mathrm{~m}, 2 \mathrm{H}), 1.35-1.19(\mathrm{~m}, 1 \mathrm{H}), 1.06(\mathrm{~d}, J=7.0 \mathrm{~Hz}, 3 \mathrm{H}), 0.95(\mathrm{t}, J=7.9 \mathrm{~Hz}, 9 \mathrm{H}), 0.88$ $(\mathrm{s}, 9 \mathrm{H}), 0.86(\mathrm{~d}, J=6.5 \mathrm{~Hz}, 3 \mathrm{H}), 0.59(\mathrm{q}, J=7.7 \mathrm{~Hz}, 6 \mathrm{H}), 0.10(\mathrm{~s}, 9 \mathrm{H}), 0.05(\mathrm{~s}, 6 \mathrm{H}) .{ }^{13} \mathrm{C}\left\{{ }^{1} \mathrm{H}\right\} \mathbf{N M R}(75 \mathrm{MHz}$, $\left.\mathrm{CDCl}_{3}\right) \delta 208.6,150.0,135.4,129.8,127.9,124.8,82.3,77.4,75.8,75.5,72.4,71.5,49.6,47.6,45.7,37.2$, 33.3, 32.4, 27.6, 26.1, 26.0 (3C), 23.4, 18.4, 18.1, 15.3, 15.1, 7.1 (3C), 5.3 (3C), 0.6 (3C), -4.2, -4.5. HRMS (ESI) calcd for $\mathrm{C}_{39} \mathrm{H}_{75} \mathrm{O}_{5} \mathrm{Si}_{3} I \mathrm{Na}[\mathrm{M}+\mathrm{Na}]^{+}:$857.3865, found 857.3862.

\section{Compound 102}

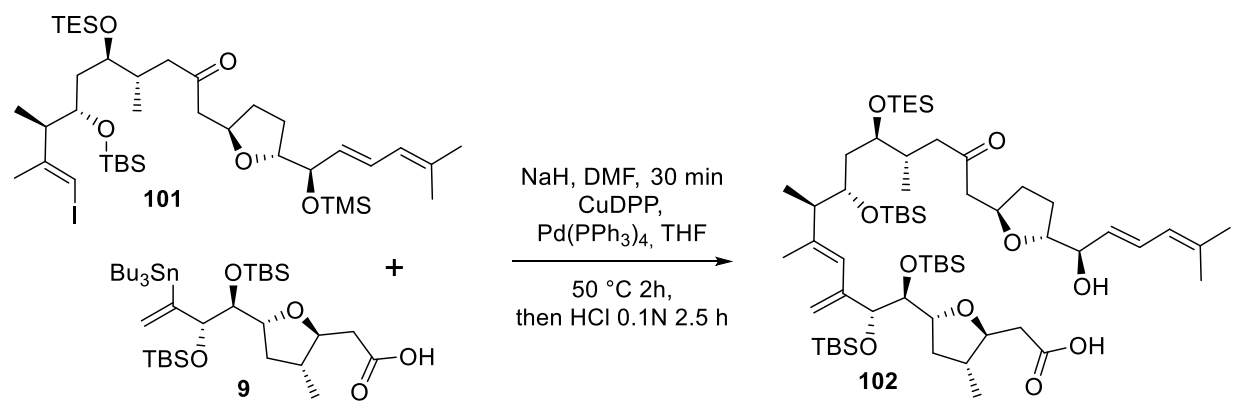

To a solution of carboxylic acid 9 (364 mg, $0.487 \mathrm{mmol}, 1.5$ equiv) in DMF (10 mL) was added $\mathrm{NaH}$ (20 $\mathrm{mg}, 0.487 \mathrm{mmol}, 1.5$ equiv, $60 \%$ in mineral oil) at rt. After $30 \mathrm{~min}$, a solution of vinyl iodide 101 (271 mg, $0.3245 \mathrm{mmol}, 1.0$ equiv) in THF $(2.5 \mathrm{~mL})$ was added followed by the simultaneous addition of copper (I) diphenylphosphinate (363 mg, $1.298 \mathrm{mmol}, 4$ equiv), $\mathrm{Pd}\left(\mathrm{PPh}_{3}\right)_{4}$ (168 mg, $0.163 \mathrm{mmol}, 0.5$ equiv). After $2 \mathrm{~h}$ of heating with an oil bath at $50^{\circ} \mathrm{C}$, the reaction mixture was quenched with $\mathrm{HCl} 0.1 \mathrm{~N}(10 \mathrm{~mL})$, and the reaction mixture was vigorously stirred for $2 \mathrm{~h}$ at $\mathrm{rt}$. The reaction mixture was filtered on a pad of celite and washed with AcOEt, extracted with AcOEt (x3), washed with brine (x2), dried over $\mathrm{MgSO}_{4}$, filtered, and 
concentrated. The residue was loaded on a silica gel column chromatography $(20-50 \%$ gradient AcOEt/petroleum ether) to give by order of elution, recovered TMS-cleaved compound derived from 101 (51.1 $\mathrm{mg}, 14 \%)$ and seco-acid $102(254 \mathrm{mg}, 72 \%)$ as a light yellow oil.

$[\alpha]^{20} \mathrm{D}=+22.7\left(\mathrm{c} \mathrm{1.98}, \mathrm{CHCl}_{3}\right)$. IR (neat): $v=3300(\mathrm{br}), 3500-2500(\mathrm{br}), 2956,1713,1535,1461,1253,1102$, 1072, 1047, 1005, 835, $776 \mathrm{~cm}^{-1}$. ${ }^{1} \mathbf{H}$ NMR $\left(300 \mathrm{MHz}, \mathrm{CDCl}_{3}\right) \delta 6.53(\mathrm{dd}, J=15.1,10.8 \mathrm{~Hz}, 1 \mathrm{H}), 5.83(\mathrm{~d}, J=$ $10.8 \mathrm{~Hz}, 1 \mathrm{H}), 5.67$ (brs, $1 \mathrm{H}), 5.46$ (dd, $J=15.1,6.7 \mathrm{~Hz}, 1 \mathrm{H}), 5.31$ (brs, $1 \mathrm{H}), 4.95$ (brs, $1 \mathrm{H}), 4.44-4.28(\mathrm{~m}$, $1 \mathrm{H}), 4.11$ (brs, $1 \mathrm{H}), 4.19-4.01(\mathrm{~m}, 1 \mathrm{H}), 3.97(\mathrm{t}, J=7.0 \mathrm{~Hz}, 2 \mathrm{H}), 3.92-3.81(\mathrm{~m}, 2 \mathrm{H}), 3.79-3.64(\mathrm{~m}, 2 \mathrm{H})$, $3.58(\mathrm{dd}, J=7.1,1.7 \mathrm{~Hz}, 1 \mathrm{H}), 2.76(\mathrm{dd}, J=16.3,5.6 \mathrm{~Hz}, 1 \mathrm{H}), 2.65(\mathrm{dd}, J=15.4,3.8 \mathrm{~Hz}, 1 \mathrm{H}), 2.58-2.42(\mathrm{~m}$, $2 \mathrm{H}), 2.41-2.29(\mathrm{~m}, 1 \mathrm{H}), 2.29-2.08(\mathrm{~m}, 4 \mathrm{H}), 2.04-1.87(\mathrm{~m}, 2 \mathrm{H}), 1.79(\mathrm{~s}, 6 \mathrm{H}), 1.78(\mathrm{~s}, 3 \mathrm{H}), 1.76-1.62(\mathrm{~m}$, $1 \mathrm{H}), 1.61-1.46(\mathrm{~m}, 2 \mathrm{H}), 1.46-1.21(\mathrm{~m}, 3 \mathrm{H}), 1.09(\mathrm{~d}, J=6.9 \mathrm{~Hz}, 3 \mathrm{H}), 1.03(\mathrm{~d}, J=7.3 \mathrm{~Hz}, 8 \mathrm{H}), 0.99(\mathrm{t}, J=$ $8.0 \mathrm{~Hz}, 9 \mathrm{H}), 0.85(\mathrm{~d}, J=6.0 \mathrm{~Hz}, 3 \mathrm{H}), 0.93(\mathrm{~s}, 9 \mathrm{H}), 0.92(\mathrm{~s}, 9 \mathrm{H}), 0.91(\mathrm{~s}, 9 \mathrm{H}), 0.89(\mathrm{~d}, J=6.3 \mathrm{~Hz}, 3 \mathrm{H}), 0.62$ $(\mathrm{q}, J=8.0 \mathrm{~Hz}, 6 \mathrm{H}), 0.10(\mathrm{~s}, 3 \mathrm{H}), 0.09(\mathrm{~s}, 6 \mathrm{H}), 0.07(\mathrm{~s}, 3 \mathrm{H}), 0.06(\mathrm{~s}, 3 \mathrm{H}), 0.03(\mathrm{~s}, 3 \mathrm{H}) .{ }^{13} \mathrm{C}\left\{{ }^{1} \mathrm{H}\right\} \mathrm{NMR}(75 \mathrm{MHz}$, $\left.\mathrm{CDCl}_{3}\right) \delta 208.7,175.4,145.6,141.4,136.5,129.4,128.1,125.5,124.6,114.8,82.3,80.3,80.1,79.1,78.7$, 75.5, 75.1, 72.9, 71.9, 49.1, 47.7, 45.4, 40.2, 38.7, 38.0, 37.6, 33.5, 32.3, 28.1, 26.2 (3C), 26.1 (4C), 26.1 (3C), 24.0, $18.5\left(2 \mathrm{Cq}, 1 \mathrm{CH}_{3}\right), 18.1,17.3,16.0,15.8,14.4,7.1,5.3,-4.2,-4.2,-4.3,-4.4,-4.6(2 \mathrm{C})$. HRMS (ESI) calcd for $\mathrm{C}_{59} \mathrm{H}_{112} \mathrm{O}_{10} \mathrm{Si}_{4} \mathrm{Na}[\mathrm{M}+\mathrm{Na}]^{+}:$1115.7230, found 1115.7231 .

\section{Compound 103}

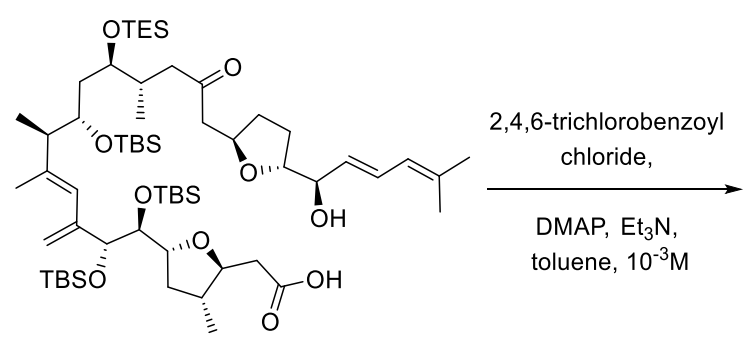

102

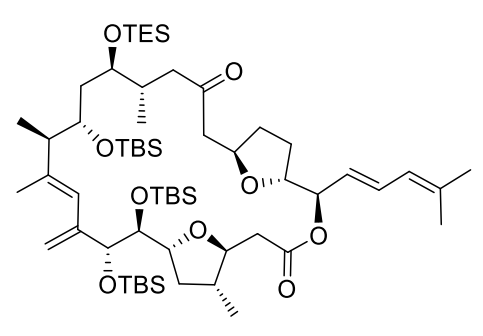

103

To a solution of 2,4,6-trichlorobenzoyl chloride (128 $\mu \mathrm{L}, 0.815 \mathrm{mmol}, 3$ equiv), DMAP (148 mg, $1.22 \mathrm{mmol}$, 3 equiv) and $\mathrm{Et}_{3} \mathrm{~N}(170 \mu \mathrm{L}, 1.22 \mathrm{mmol}, 6$ equiv) in toluene $(200 \mathrm{~mL})$ was added a solution of seco-acid 102 (223 mg, $0.204 \mathrm{mmol}, 1$ equiv) in toluene $\left(20 \mathrm{~mL}\right.$ ) via a syringe pump over $10 \mathrm{~h}$ at $50^{\circ} \mathrm{C}$ with the help of an oil bath, and the stirring was continued for $6 \mathrm{~h}$. The reaction mixture was quenched with water, and the organic layer was washed with $\mathrm{NH}_{4} \mathrm{Cl}$, dried over $\mathrm{MgSO}_{4}$, filtered, and concentrated. The residue was purified by silica gel chromatography (8-12\% gradient $\mathrm{Et}_{2} \mathrm{O}$ in petroleum ether) to give macrolactone 103 (89 mg, 41\%) as a colorless oil. The macrolactone exists as a mixture of at least two major conformers in a 3:1 ratio in solution.

$[\alpha]^{25} \mathrm{D}=+37.0\left(\mathrm{c} 0.27, \mathrm{CHCl}_{3}\right)$. IR (neat): $v=2958,1743,1720,1253,1127,1099,1069,1004,836,776 \mathrm{~cm}^{-}$ 1. ${ }^{1} \mathrm{H}$ NMR (400 MHz, $\mathrm{CDCl}_{3}$, mixture of 2 conformational isomers exists in about 3:1 ratio) $\delta 6.51$ (dd, $J=$ $14.8,11.1 \mathrm{~Hz}, 1 \mathrm{H}$ ), 6.04 (brs, $0.75 \mathrm{H}$, major conformer), 6.00 (brs, $0.25 \mathrm{H}$, minor conformer), 5.77 (d, $J=11.1$ $\mathrm{Hz}, 1 \mathrm{H}), 5.36(\mathrm{~m}, 1 \mathrm{H}), 5.24$ (brt, $J=8.0 \mathrm{~Hz}, 0.75 \mathrm{H}$, major conformer), 5.17 (t, $J=8.5 \mathrm{~Hz}, 0.25 \mathrm{H}$, minor conformer), 5.05 (brs, $0.75 \mathrm{H}$, major conformer), 4.92 (brs, $0.25 \mathrm{H}$, minor conformer), 4.86 (brs, $1 \mathrm{H}), 4.15-4.04$ $(\mathrm{m}, 1.75 \mathrm{H}, 1 \mathrm{H}+1$ major conformer), $4.00-3.90(\mathrm{~m}, 1.25 \mathrm{H}, 1 \mathrm{H}+1$ minor conformer), 3.90-3.77 $(\mathrm{m}, 2 \mathrm{H}), 3.65$ (brs, $0.25 \mathrm{H}$, minor conformer), $3.56(\mathrm{~m}, 1.75 \mathrm{H}, 1 \mathrm{H}+1$ major conformer), $3.47(\mathrm{~m}, 1 \mathrm{H}), 2.96(\mathrm{dd}, J=17.2,3.0$ $\mathrm{Hz}, 0.25 \mathrm{H}$, minor conformer), $2.87(\mathrm{~d}, \mathrm{~J}=16.4 \mathrm{~Hz}, 0.75 \mathrm{H}$, major conformer), $2.68-2.00(\mathrm{~m}, 11 \mathrm{H}), 1.99-$ $1.86(\mathrm{~m}, 1 \mathrm{H}), 1.76(\mathrm{~s}, 3 \mathrm{H}), 1.75(\mathrm{~s}, 6 \mathrm{H}), 1.61(\mathrm{~m}, 1 \mathrm{H}), 1.47-1.12(\mathrm{~m}, 3 \mathrm{H}), 1.08(\mathrm{~d}, J=6.9 \mathrm{~Hz}, 3 \mathrm{H}), 1.00(\mathrm{~d}$, $J=6.5 \mathrm{~Hz}, 3 \mathrm{H}), 0.96(\mathrm{t}, J=7.9 \mathrm{~Hz}, 9 \mathrm{H}), 0.89(\mathrm{~s}, 9 \mathrm{H}), 0.89(\mathrm{~s}, 9 \mathrm{H}), 0.87(\mathrm{~s}, 9 \mathrm{H}), 0.83(\mathrm{~d}, J=6.3 \mathrm{~Hz}, 2.25 \mathrm{H}$, major conformer), 0.79 (d, $J=6.8 \mathrm{~Hz}, 0.75 \mathrm{H}$, minor conformer), 0.59 (q, $J=7.9 \mathrm{~Hz}, 6 \mathrm{H}$ ), 0.09 (s, 3H), 0.07 $(\mathrm{s}, 3 \mathrm{H}), 0.05(\mathrm{~s}, 6 \mathrm{H}), 0.04(\mathrm{~s}, 3 \mathrm{H}),-0.01(\mathrm{~s}, 3 \mathrm{H}) .{ }^{13} \mathrm{C}\left\{{ }^{1} \mathrm{H}\right\} \mathrm{NMR}\left(101 \mathrm{MHz}, \mathrm{CDCl}_{3}\right.$, mixture of 2 conformational 
isomers exists in about 3:1 ratio) $\delta 207.0,206.7$ (2 conformers); 170.6, 169.3 (2 conformers); 147.2, 146.6 (2 conformers); 141.1; 138.0, 137.9 (2 conformers); 131.9, 131.9 (2 conformers); 128.9, 128.8 (2 conformers); 124.7; 124.3; 114.0, 113.3 (2 conformers); 83.2, 83.0 (2 conformers); 80.8; 79.6; 79.1, 78.8 (2 conformers); 77.4, 76.8 (2 conformers); 76.6, 75.8 (2 conformers); 74.3, 74.0 (2 conformers); 73.3, 72.9 (2 conformers); 72.7, 72.5 (2 conformers); 49.1, 48.1 (2 conformers); 46.9, 46.3 (2 conformers); 46.5; 38.9, 38.6 (2 conformers); 38.0, 37.9 (2 conformers); 37.7, 35.9 (2 conformers); 37.4; 33.4; 33.1, 32.4 (2 conformers); 28.7, 28.3 (2 conformers), 26.5 (3C); 26.2; 26.1 (6C); 18.7; 18.6; 18.6, 18.4; 18.2; 16.6, 16.4 (2 conformers); 16.0, 15.8 (2 conformers); 13.5; 7.1 (3C); $5.3(3 \mathrm{C})$; -4.2, -4.2 (2 conformers); $-4.4(2 \mathrm{C}) ;-4.6$; -4.6 , -4.7 (2 conformers); $-4.8,-4.9$ (2 conformers). HRMS (ESI) calcd for $\mathrm{C}_{59} \mathrm{H}_{110} \mathrm{O}_{9} \mathrm{Si}_{4} \mathrm{Na}[\mathrm{M}+\mathrm{Na}]^{+}: 1097.7125$, found 1097.7128.

\section{Compound 104}
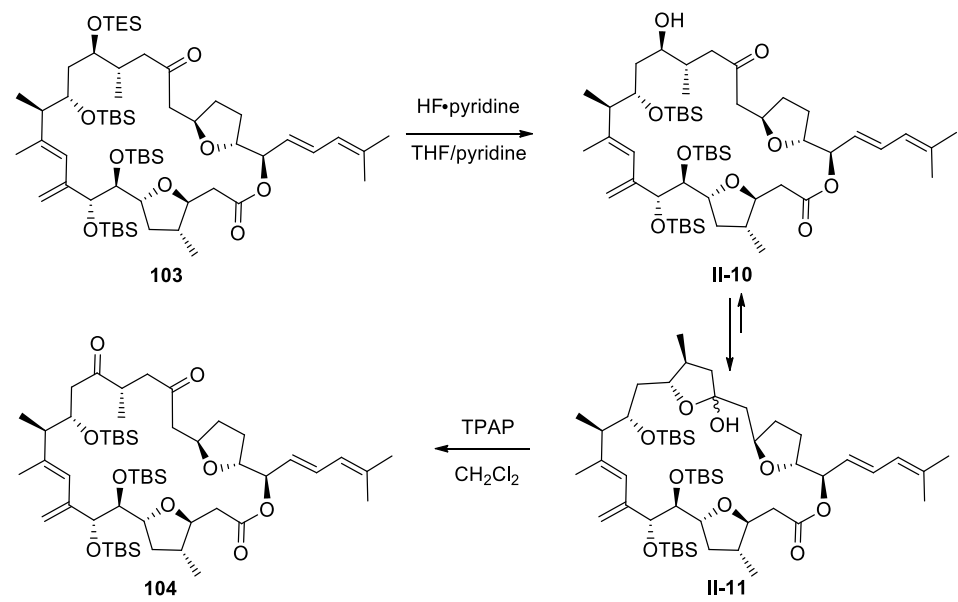

To a solution of macrolactone 103 (34 mg, $31.7 \mu \mathrm{mol}, 1$ equiv) in THF (600 $\mu \mathrm{L})$ in a plastic vial was added HF•pyridine [ $500 \mu \mathrm{L}, 2.2 \mathrm{mmol}, 70$ equiv, $4.5 \mathrm{M}$ in THF/pyridine (prepared from HF•pyridine complex: pyridine: THF, 0.5:1:2.5 mL)]. After $2 \mathrm{~h}$ at $\mathrm{rt}$, the reaction mixture was poured into an erlenmeyer containing saturated $\mathrm{NaHCO}_{3}$. The reaction mixture was then stirred for $5 \mathrm{~min}$ and was extracted with $\mathrm{Et}_{2} \mathrm{O}(\times 3)$, dried on $\mathrm{MgSO}_{4}$, filtered and concentrated. The residue was purified on a silica gel chromatography $\left(20 \% \mathrm{Et}_{2} \mathrm{O}\right.$ in petroleum ether) to afford macrolactone II-10 (29.1 mg, 96\%) as colorless oil.

The product is in fact a mixture of three compounds (hydroxyl ketone II-10 and two hemiketals II-11); each probably giving, in addition, a mixture of conformers, which makes this compound hardly characterizable by NMR. Nevertheless, the absence of a ketone absorption band at $\approx 1715 \mathrm{~cm}^{-1}$ indicates that the hemiketal is the major form.

IR (neat): $v=3400$ (br), 2957, 1738, 1472, 1461, 1361, 1252, 1096, 1077, 835, $775 \mathrm{~cm}^{-1}$. HRMS (ESI) calcd for $\mathrm{C}_{53} \mathrm{H}_{96} \mathrm{O}_{9} \mathrm{Si}_{3} \mathrm{Na}[\mathrm{M}+\mathrm{Na}]^{+}:$983.6260, found 983.6273 .

To a solution of a mixture of compound II-10/II-11 (14.5 mg, $14 \mu \mathrm{mol}, 1$ equiv) in $\mathrm{CH}_{2} \mathrm{Cl}_{2}$ was added TPAP (14.7 mg, $42 \mu \mathrm{mol}, 3$ equiv) in 3 portions over $20 \mathrm{~min}$. After $2.5 \mathrm{~h}$ at $\mathrm{rt}$, the reaction mixture was diluted with $\mathrm{Et}_{2} \mathrm{O}$ and filtered on Celite. After concentration, the residue was purified on a silica gel column ( $20 \% \mathrm{Et}_{2} \mathrm{O}$ in petroleum ether) to give diketone $104(10.8 \mathrm{mg}, 74 \%)$ as a colorless oil. The macrolactone exists as a mixture of more than two conformers into solution. As a consequence, characterization of the compound by ${ }^{13} \mathbf{C}\left\{{ }^{1} \mathrm{H}\right\}$ NMR was complex and we were not able to identify and attribute clearly all the peaks.

$[\alpha]^{20} \mathrm{D}=+8.6\left(c 0.7, \mathrm{CHCl}_{3}\right)$. IR (neat): $v=2956,1740,1706,1461,1253,1073,1036,836,777 \mathrm{~cm}^{-1} .{ }^{1} \mathbf{H}$ NMR $\left(300 \mathrm{MHz}, \mathrm{CDCl}_{3}\right) \delta 6.51(\mathrm{dd}, J=15.1,11.0 \mathrm{~Hz}, 1 \mathrm{H}), 5.98(\mathrm{brs}, 0.25 \mathrm{H}), 5.75(\mathrm{brd}, J=11.0 \mathrm{~Hz}, 1 \mathrm{H}), 5.65-$ $5.24(\mathrm{~m}, 1.75 \mathrm{H}), 5.22-4.87(\mathrm{~m}, 3 \mathrm{H}), 4.68-4.29(\mathrm{~m}, 1 \mathrm{H}), 4.33-4.14(\mathrm{~m}, 1 \mathrm{H}), 4.16-4.00(\mathrm{~m}, 2 \mathrm{H}), 4.01-$ 
$3.79(\mathrm{~m}, 1 \mathrm{H}), 3.79-3.62(\mathrm{~m}, 1 \mathrm{H}), 3.62-3.41(\mathrm{~m}, 1 \mathrm{H}), 3.10-2.79(\mathrm{~m}, 2 \mathrm{H}), 2.80-2.23(\mathrm{~m}, 6 \mathrm{H}), 2.20-1.83$ $(\mathrm{m}, 3 \mathrm{H}), 1.76$ (brs, 9H), $1.68-1.50(\mathrm{~m}, 3 \mathrm{H}), 1.39-1.04(\mathrm{~m}, 3 \mathrm{H}), 1.08(\mathrm{~d}, \mathrm{~J}=6.6 \mathrm{~Hz}, 3 \mathrm{H}), 1.03-0.67(\mathrm{~m}$, $33 \mathrm{H}), 0.15--0.05(\mathrm{~m}, 18 \mathrm{H})$. HRMS $(\mathrm{ESI})$ calcd for $\mathrm{C}_{59} \mathrm{H}_{110} \mathrm{O}_{9} \mathrm{Si}_{4} \mathrm{Na}[\mathrm{M}+\mathrm{Na}]^{+}: 981.6103$, found 981.6099 .

\section{$\underline{\text { Amphidinolide F (4) }}$}

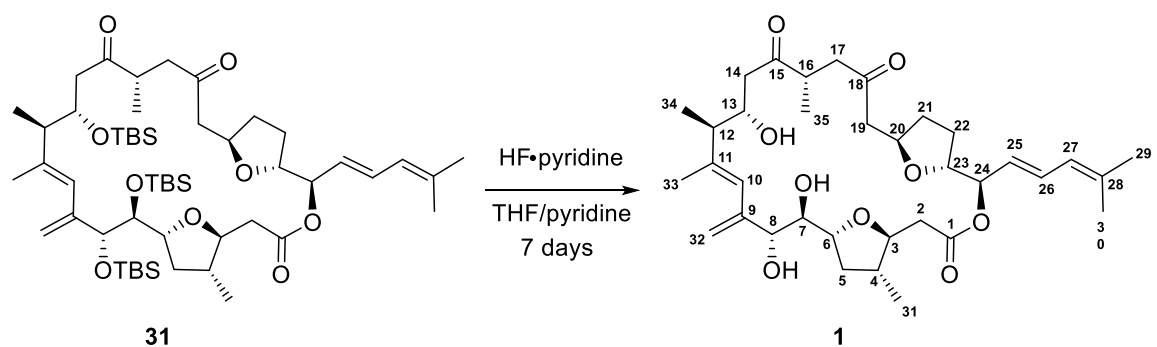

Compound $104(8.0 \mathrm{mg}, 8.37 \mu \mathrm{mol})$ was placed in a polypropylene vial and HF•pyridine in THF [2 $\mathrm{mL}$ (prepared from HF•pyridine complex: pyridine: THF, 1:2:1 mL)] was added. After 7 days at rt, the vial mixture was poured into saturated $\mathrm{NaHCO}_{3}$ and washed with AcOEt. The aqueous layer was extracted with AcOEt (x4), and the combined organic phases were dried over $\mathrm{MgSO}_{4}$, filtered and concentrated. The residue was purified on a silica gel column (20-30\% gradient acetone in hexanes) to give amphidinolide $\mathbf{F}(4)(1.4 \mathrm{mg}$, $27 \%)$ as a white waxy solid. Spectroscopic data agreed with other total syntheses ${ }^{[13,14]}$ and with the natural product. ${ }^{[15]}[\alpha]^{20} \mathrm{D}=-65.0\left(c 0.065, \mathrm{CHCl}_{3}\right)$. IR (neat): $v=3400(\mathrm{br}), 2921,1742,1710,1459,1378,1261,1092$, 1018, 910, 819, 800, $733 \mathrm{~cm}^{-1} .{ }^{1} \mathbf{H}$ NMR $\left(800 \mathrm{MHz}, \mathrm{CDCl}_{3}, 1.4 \mathrm{mg}\right.$ in $\left.220 \mu \mathrm{L}\right) \delta 6.52(\mathrm{dd}, J=15.0,11.1 \mathrm{~Hz}$, $\left.1 \mathrm{H}, \mathrm{H}_{26}\right), 6.00\left(\mathrm{~s}, 1 \mathrm{H}, \mathrm{H}_{10}\right), 5.77\left(\mathrm{~d}, J=11.1 \mathrm{~Hz}, 1 \mathrm{H}, \mathrm{H}_{27}\right), 5.35$ (dd, $\left.J=14.9,8.1 \mathrm{~Hz}, 1 \mathrm{H}, \mathrm{H}_{25}\right), 5.21$ (t, $J=8.2$

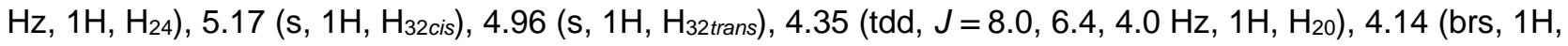
$\mathrm{H}_{8}$ ), 4.09 (q, $\left.J=7.3 \mathrm{~Hz}, 1 \mathrm{H}, \mathrm{H}_{23}\right), 3.97-3.87$ (brs, $\left.1 \mathrm{H}, \mathrm{OH}\right), 3.94$ (td, $\left.J=8.9,2.0 \mathrm{~Hz}, 1 \mathrm{H}, \mathrm{H}_{13}\right), 3.84(\mathrm{~m}, 1 \mathrm{H}$, $\mathrm{H}_{6}$ ), 3.81 (td, $\left.J=9.5,2.3 \mathrm{~Hz}, 2 \mathrm{H}, \mathrm{H}_{3}\right), 3.59-3.43$ (brs, $\left.1 \mathrm{H}, \mathrm{OH}\right), 3.55$ (brs, $\left.1 \mathrm{H}, \mathrm{H}_{7}\right), 3.13\left(\mathrm{~m}, 1 \mathrm{H}, \mathrm{H}_{16}\right), 3.06$ (dd, $J=17.6,8.9 \mathrm{~Hz}, 1 \mathrm{H}, \mathrm{H}_{17 \mathrm{a}}$ ), 2.75 (dd, $\left.J=15.3,9.2 \mathrm{~Hz}, 1 \mathrm{H}, \mathrm{H}_{14 \mathrm{a}}\right), 2.71$ (dd, $J=15.9,8.5 \mathrm{~Hz}, 1 \mathrm{H}, \mathrm{H}_{19 a}$ ), 2.54 (dd, $J=15.3,1.7 \mathrm{~Hz}, 1 \mathrm{H}, \mathrm{H}_{14 \mathrm{~b}}$ ), 2.53 (dd, $J=15.7,9.0 \mathrm{~Hz}, 1 \mathrm{H}, \mathrm{H}_{2 \mathrm{a}}$ ), 2.50 (dd, $J=15.9,3.1 \mathrm{~Hz}, 1 \mathrm{H}, \mathrm{H}_{19 b}$ ), 2.49 (dd, $J=15.7,2.4 \mathrm{~Hz}, 1 \mathrm{H}, \mathrm{H}_{2 b}$ ), 2.34 (dd, $J=17.6,4.2 \mathrm{~Hz}, 2 \mathrm{H}, \mathrm{H}_{17 \mathrm{~b}}$ ), 2.28 (quint, $J=7.3 \mathrm{~Hz}, 1 \mathrm{H}, \mathrm{H}_{12}$ ), 2.09 (dt, $J=12.1,6.0 \mathrm{~Hz}, 1 \mathrm{H}, \mathrm{H}_{5 \mathrm{a}}$ ), 2.08 (dq, $\left.J=12.1,6.0 \mathrm{~Hz}, 1 \mathrm{H}, \mathrm{H}_{21 \mathrm{a}}\right), 1.93$ (dtd, $J=11.7,7.8,3.8 \mathrm{~Hz}, 1 \mathrm{H}$, $\left.\mathrm{H}_{22 \mathrm{a}}\right), 1.81\left(\mathrm{~m}, 1 \mathrm{H}, \mathrm{H}_{4}\right), 1.77\left(\mathrm{~s}, 3 \mathrm{H}, \mathrm{H}_{29}\right), 1.75\left(\mathrm{~s}, 3 \mathrm{H}, \mathrm{H}_{30}\right), 1.72\left(\mathrm{~s}, 3 \mathrm{H}, \mathrm{H}_{33}\right), 1.70-1.59(\mathrm{~m}, 1 \mathrm{H}, \mathrm{OH}), 1.60$ (m, 1H, $\mathrm{H}_{22 \mathrm{~b}}$ ), 1.49 (dq, $J=12.2,8.6 \mathrm{~Hz}, 1 \mathrm{H}, \mathrm{H}_{21 \mathrm{a}}$ ), 1.46 (m, 1H, $\left.\mathrm{H}_{5 \mathrm{~b}}\right), 1.09$ (d, J = 7.2 Hz, 3H, H35), 1.04 (d, $J$ $\left.=6.9 \mathrm{~Hz}, 3 \mathrm{H}, \mathrm{H}_{34}\right), 1.00\left(\mathrm{~d}, J=6.5 \mathrm{~Hz}, 3 \mathrm{H}, \mathrm{H}_{31}\right) .{ }^{13} \mathrm{C}\left\{{ }^{1} \mathrm{H}\right\} \mathbf{N M R}\left(201 \mathrm{MHz}, \mathrm{CDCl}_{3}\right.$, reference solvent peak: 77.00 ppm, $1.4 \mathrm{mg}$ in $220 \mu \mathrm{L}) \delta 213.8\left(\mathrm{C}_{15}\right), 207.8\left(\mathrm{C}_{18}\right), 171.2\left(\mathrm{C}_{1}\right), 144.4\left(\mathrm{C}_{9}\right), 140.0\left(\mathrm{C}_{11}\right), 138.3\left(\mathrm{C}_{28}\right)$, $132.0\left(\mathrm{C}_{26}\right), 124.5\left(\mathrm{C}_{10}\right), 124.2\left(\mathrm{C}_{25}\right), 124.0\left(\mathrm{C}_{27}\right), 116.1\left(\mathrm{C}_{32}\right), 81.4\left(\mathrm{C}_{3}\right), 79.9\left(\mathrm{C}_{23}\right), 78.9\left(\mathrm{C}_{6}\right), 77.8\left(\mathrm{C}_{24}\right), 77.0$ $\left(\mathrm{C}_{8}\right), 76.4\left(\mathrm{C}_{7}\right), 75.0\left(\mathrm{C}_{20}\right), 70.6\left(\mathrm{C}_{13}\right), 49.3\left(\mathrm{C}_{12}\right), 48.5\left(\mathrm{C}_{19}\right), 46.0\left(\mathrm{C}_{17}\right), 45.5\left(\mathrm{C}_{14}\right), 42.7\left(\mathrm{C}_{16}\right), 39.8\left(\mathrm{C}_{4}\right), 38.7$ $\left(\mathrm{C}_{2}\right), 36.7\left(\mathrm{C}_{5}\right), 31.9\left(\mathrm{C}_{21}\right), 28.4\left(\mathrm{C}_{22}\right), 26.1\left(\mathrm{C}_{29}\right), 18.5\left(\mathrm{C}_{30}\right), 16.2\left(\mathrm{C}_{35}\right), 15.5\left(\mathrm{C}_{34}\right), 15.4\left(\mathrm{C}_{31}\right), 14.3\left(\mathrm{C}_{33}\right)$. HRMS (ESI) calcd for $\mathrm{C}_{35} \mathrm{H}_{56} \mathrm{O}_{9} \mathrm{NO}_{9}\left[\mathrm{M}+\mathrm{NH}_{4}\right]^{+}: 634.3955$, found 634.3946; $\mathrm{C}_{35} \mathrm{H}_{52} \mathrm{O}_{9} \mathrm{Na}[\mathrm{M}+\mathrm{Na}]^{+}: 639.3494$, found 639.3509 . 


\section{SUPPORTING INFORMATION}

Table S1. Comparison of ${ }^{1} \mathrm{H}$ NMR spectra[a] of our synthetic sample of amphidinolide $\mathrm{F}$ with the reported Carter synthetic sample ${ }^{[30]}$ Furstner synthetic sample ${ }^{[31]}$ and Kobayashi natural sample. ${ }^{[32]}$

\begin{tabular}{|c|c|c|c|c|c|c|c|c|c|c|c|c|c|}
\hline \multirow{2}{*}{$\begin{array}{l}\text { 을 } \\
: \frac{0}{0} \\
00 \\
00 \\
0\end{array}$} & \multicolumn{2}{|c|}{ Ferrié synthesis[b] } & \multicolumn{2}{|c|}{ Carter synthesis[c] } & \multicolumn{2}{|c|}{ Fürstner synthesis[d] } & \multicolumn{2}{|c|}{ Natural Sample[e] } & \multirow{2}{*}{$\begin{array}{l}\Delta \text { Nat. } \\
\text { sample } \\
\text { vs } \\
\text { Ferrié }\end{array}$} & \multirow{2}{*}{$\begin{array}{c}\Delta \text { Nat. } \\
\text { sample } \\
\text { vs Carter }\end{array}$} & \multirow{2}{*}{$\begin{array}{c}\Delta \text { Nat. } \\
\text { sample } \\
\text { vs } \\
\text { Fürstner }\end{array}$} & \multirow{2}{*}{$\begin{array}{c}\Delta \\
\text { Ferrié } \\
\text { vs } \\
\text { Carter }\end{array}$} & \multirow{2}{*}{$\begin{array}{c}\Delta \text { Ferrié } \\
\text { vs } \\
\text { Fürstne }\end{array}$} \\
\hline & $\begin{array}{c}\delta \\
(\mathrm{ppm})\end{array}$ & $\begin{array}{c}\text { multiplicity } \\
\mathrm{J}(\mathrm{Hz})\end{array}$ & $\begin{array}{c}\delta \\
(\mathrm{ppm})\end{array}$ & $\begin{array}{c}\text { multiplicity } \\
\mathrm{J}(\mathrm{Hz})\end{array}$ & $\begin{array}{c}\delta \\
(\mathrm{ppm})\end{array}$ & $\begin{array}{l}\text { multiplicity } \\
\mathrm{J}(\mathrm{Hz})\end{array}$ & $\begin{array}{c}\delta \\
(\mathrm{ppm})\end{array}$ & $\begin{array}{c}\text { multiplicity } \\
\mathrm{J}(\mathrm{Hz})\end{array}$ & & & & & \\
\hline \multicolumn{14}{|l|}{1} \\
\hline $2 a$ & 2.51 & dd $15.7,9.0$ & $2.50[f]$ & $\mathrm{m}$ & 2.51 & dd $15.8,9.0$ & 2.49 & $\mathrm{~m}$ & -0.02 & -0.01 & -0.02 & 0.01 & 0.00 \\
\hline $2 b$ & 2.47 & dd 15.7, 2.4 & $2.50[f]$ & $\mathrm{m}$ & 2.48 & dd 15.7, 3.6 & 2.49 & $\mathrm{~m}$ & 0.02 & -0.01 & 0.01 & -0.03 & -0.01 \\
\hline 3 & 3.79 & $\operatorname{td} 9.5,2.3$ & 3.80 & dt $9.5,2.6$ & 3.79 & td 9.3, 3.1 & 3.81 & $\operatorname{td} 6.8,2.4$ & 0.02 & 0.01 & 0.02 & -0.01 & 0.00 \\
\hline 4 & 1.79 & $\mathrm{~m}$ & 1.79 & $\mathrm{~m}$ & 1.79 & $\mathrm{~m}$ & 1.81 & $\mathrm{~m}$ & 0.02 & 0.02 & 0.02 & 0.00 & 0.00 \\
\hline $5 a$ & 2.07 & dt $12.1,6.0$ & 2.07 & $\mathrm{~m}$ & 2.08 & $\mathrm{~m}$ & 2.11 & $\mathrm{~m}$ & 0.04 & 0.04 & 0.03 & 0.00 & -0.01 \\
\hline $5 b$ & 1.44 & $\mathrm{~m}$ & $1.47[\mathrm{~g}]$ & $\mathrm{m}$ & 1.43 & $\mathrm{~m}$ & 1.39 & $\mathrm{~m}$ & -0.05 & -0.08 & -0.04 & -0.03 & 0.01 \\
\hline 6 & 3.82 & $\mathrm{~m}$ & 3.82 & $\mathrm{~m}$ & 3.79 & $\operatorname{td} 9.3,3.1$ & 3.78 & $\operatorname{td} 7.3,2.1$ & -0.04 & -0.04 & -0.01 & 0.00 & 0.03 \\
\hline 7 & 3.53 & brs & 3.52 & brs & 3.53 & dd $6.0,3.4$ & 3.53 & $\mathrm{~m}$ & 0.00 & 0.01 & 0.00 & 0.01 & 0.00 \\
\hline 8 & 4.12 & brs & 4.13 & brs & 4.12 & m & 4.05 & d 4.0 & -0.07 & -0.08 & -0.07 & -0.01 & 0.00 \\
\hline \multicolumn{14}{|l|}{9} \\
\hline 10 & 5.98 & $\mathrm{~s}$ & 5.98 & brs & 5.98 & $\mathrm{~s}$ & 5.98 & brs & 0.00 & 0.00 & 0.00 & 0.00 & 0.00 \\
\hline \multicolumn{14}{|l|}{11} \\
\hline 12 & 2.26 & quint 7.3 & 2.26 & $\mathrm{~m}$ & 2.25 & $\mathrm{dq}, 9.0,6.8$ & 2.25 & $\mathrm{~m}$ & -0.01 & -0.01 & 0.00 & 0.00 & 0.01 \\
\hline 13 & 3.92 & $\operatorname{td} 8.9,2.0$ & 3.93 & brt 9.0 & 3.92 & $\operatorname{td} 9.0,1.7$ & 3.93 & $\operatorname{td} 9.8,2.0$ & 0.01 & 0.00 & 0.01 & -0.01 & 0.00 \\
\hline $14 a$ & 2.73 & dd 15.3, 9.2 & 2.73 & dd 15.2, 9.1 & 2.73 & dd 15.2, 9.4 & 2.74 & dd 15.1, 9.3 & 0.01 & 0.01 & 0.01 & 0.00 & 0.00 \\
\hline $14 b$ & 2.52 & dd $15.3,1.7$ & $2.50[f]$ & $\mathrm{m}$ & 2.51 & dd $15.4,2.4$ & 2.51 & $\mathrm{~m}$ & -0.01 & 0.01 & 0.00 & 0.02 & 0.01 \\
\hline 15 & & & & & & & & & 0.00 & 0.00 & 0.00 & 0.00 & 0.00 \\
\hline 16 & 3.11 & $\mathrm{~m}$ & 3.11 & $\mathrm{~m}$ & 3.11 & $\mathrm{~m}$ & 3.15 & $\mathrm{~m}$ & 0.04 & 0.04 & 0.04 & 0.00 & 0.00 \\
\hline $17 a$ & 3.04 & dd 17.6, 8.9 & 3.04 & dd $17.5,8.9$ & 3.02 & dd $17.5,9.0$ & 3.04 & dd $17.1,9.3$ & & 0.00 & 0.02 & 0.00 & 0.02 \\
\hline $17 b$ & 2.32 & dd $17.6,4.2$ & 2.32 & $\mathrm{~m}$ & 2.30 & dd $17.5,4.1$ & 2.29 & $\mathrm{~m}$ & -0.03 & -0.03 & -0.01 & 0.00 & 0.02 \\
\hline \multicolumn{14}{|l|}{18} \\
\hline $19 a$ & 2.69 & dd $15.9,8.5$ & 2.70 & dd $15.8,8.4$ & 2.69 & dd $16.1,8.4$ & 2.73 & dd 16.6, 8.8 & 0.04 & 0.03 & 0.04 & -0.01 & 0.00 \\
\hline $19 b$ & 2.48 & dd $15.9,3.1$ & $2.50[f]$ & $\mathrm{m}$ & 2.46 & dd $15.8,3.0$ & 2.50 & $\mathrm{~m}$ & 0.02 & 0.00 & 0.04 & -0.02 & 0.02 \\
\hline 20 & 4.33 & $\operatorname{tdd} 8.0,6.4,4.0$ & 4.33 & $\mathrm{~m}$ & 4.33 & tdd $8.2,6.0,4.0$ & 4.36 & $\mathrm{~m}$ & 0.03 & 0.03 & 0.03 & 0.00 & 0.00 \\
\hline $21 a$ & 2.06 & $\mathrm{dq} 12.1,6.0$ & 2.07 & $\mathrm{~m}$ & 2.06 & $\mathrm{~m}$ & 2.08 & $\mathrm{~m}$ & 0.02 & 0.01 & 0.02 & -0.01 & 0.00 \\
\hline $21 b$ & 1.47 & $\mathrm{dq} 12.1,6.0$ & $1.47[\mathrm{~g}]$ & $\mathrm{m}$ & 1.48 & $\mathrm{~m}$ & 1.47 & $\mathrm{~m}$ & 0.00 & 0.00 & -0.01 & 0.00 & -0.01 \\
\hline $22 a$ & 1.91 & $\mathrm{dtd} 11.7,7.8,3.8$ & 1.92 & $\mathrm{~m}$ & 1.91 & dddd12.8,8.2,7.2,3.8 & 1.90 & $\mathrm{~m}$ & -0.01 & -0.02 & -0.01 & -0.01 & 0.00 \\
\hline $22 b$ & 1.58 & m & $1.34[\mathrm{~h}]$ & $\mathrm{m}$ & 1.58 & $\mathrm{~m}$ & 1.54 & $\mathrm{~m}$ & -0.04 & $0.20[\mathrm{~h}]$ & -0.04 & $0.24[\mathrm{~h}]$ & 0.00 \\
\hline 23 & 4.07 & $q 7.3$ & 4.07 & dd $14.9,7.3$ & 4.06 & q 7.7 & 4.08 & dd $14.8,7.8$ & 0.01 & 0.01 & 0.02 & 0.00 & 0.01 \\
\hline 24 & 5.19 & t 8.2 & 5.18 & $\mathrm{t} 8.2$ & 5.18 & t 8.2 & 5.17 & $t 7.8$ & -0.02 & -0.01 & -0.01 & 0.01 & 0.01 \\
\hline 25 & 5.33 & dd $14.9,8.1$ & 5.33 & dd $15.0,8.4$ & 5.31 & dd $15.1,8.6$ & 5.31 & dd $14.7,7.8$ & -0.02 & -0.02 & 0.00 & 0.00 & 0.02 \\
\hline 26 & 6.50 & dd 15.0, 11.1 & 6.51 & dd $14.9,11.0$ & 6.50 & dd $15.1,11.0$ & 6.50 & dd $14.7,11.2$ & 0.00 & -0.01 & 0.00 & -0.01 & 0.00 \\
\hline 27 & 5.75 & d 11.1 & 5.75 & brd 11.0 & 5.74 & d 11.1 & 5.76 & brd 11.2 & 0.01 & 0.01 & 0.02 & 0.00 & 0.01 \\
\hline \multicolumn{14}{|l|}{28} \\
\hline 29 & 1.75 & s & 1.75 & s & 1.74 & $\mathrm{~s}$ & 1.75 & s & 0.00 & 0.00 & 0.01 & 0.00 & 0.01 \\
\hline 30 & 1.73 & $\mathrm{~s}$ & 1.74 & $\mathrm{~s}$ & 1.73 & $\mathrm{~s}$ & 1.73 & $\mathrm{~s}$ & 0.00 & -0.01 & 0.00 & -0.01 & 0.00 \\
\hline 31 & 0.98 & d 6.5 & 0.98 & d 6.5 & 0.97 & d 6.6 & 1.00 & d 6.3 & 0.02 & 0.02 & 0.03 & 0.00 & 0.01 \\
\hline $32 a$ & 5.15 & s & 5.16 & d 1.3 & 5.15 & d 1.6 & 5.14 & brs & -0.01 & -0.02 & -0.01 & -0.01 & 0.00 \\
\hline $32 b$ & 4.94 & s & 4.94 & brs & 4.93 & $\mathrm{t} 1.4$ & 4.93 & brs & -0.01 & -0.01 & 0.00 & 0.00 & 0.01 \\
\hline 33 & 1.70 & s & 1.70 & $\mathrm{~s}$ & 1.69 & d 1.3 & 1.67 & $\mathrm{~s}$ & -0.03 & -0.03 & -0.02 & 0.00 & 0.01 \\
\hline 34 & 1.02 & d 6.9 & 1.02 & d 6.9 & 1.02 & d 7.0 & 1.03 & d 7.3 & 0.01 & 0.01 & 0.01 & 0.00 & 0.00 \\
\hline 35 & 1.07 & d 7.2 & 1.08 & d 7.2 & 1.07 & d 7.2 & 1.10 & d 6.8 & 0.03 & 0.02 & 0.03 & -0.01 & 0.00 \\
\hline
\end{tabular}

[a] solvent $\mathrm{CDCl}_{3}$. [b] Concentration: $1.4 \mathrm{mg}$ in $220 \mu \mathrm{L}, 800 \mathrm{Mhz}$. All chemical shift were corrected by -0.02 ppm compared to reported values. [c] Concentration: $1.4 \mathrm{mg}$ in $180 \mu \mathrm{L}, 800 \mathrm{MHz}$. All chemical shift were corrected by $-0.04 \mathrm{ppm}$ compared to reported values. Corresponding assignment was deduced from reported chemical shift, multiplicities, and by comparison with our own assignment. [d] Concentration: $5.5 \mathrm{mg}$ in $700 \mu \mathrm{L}, 600$ Mhz. [e] Unknow concentration, $500 \mathrm{Mhz}$. [f] reported as a massif: "2.49-2.58 (m, 4H)", median value was selected for all assigned protons. [g] reported as a massif: "1.47-1.54 (m, $2 \mathrm{H})$ “, median value was selected for all assigned protons. [h] Probable misassignment and/or report of $\mathrm{H}_{22 \mathrm{~b}}$ at $1.34 \mathrm{ppm}$. 


\section{SUPPORTING INFORMATION}

Table S2. Comparison of ${ }^{13} \mathbf{C}\left\{{ }^{1} \mathbf{H}\right\}$ NMR spectra ${ }^{[a]}$ of our synthetic sample of amphidinolide $\mathrm{F}$ with the reported Carter synthetic sample, ${ }^{[30]}$ Furstner synthetic sample ${ }^{[31]}$, and Kobayashi natural sample. ${ }^{[32]}$

\begin{tabular}{|c|c|c|c|c|c|c|c|c|c|}
\hline $\begin{array}{c}\text { C- } \\
\text { position s }\end{array}$ & $\begin{array}{c}\text { Ferrié } \\
\text { synthesis }{ }^{[b]}\end{array}$ & $\begin{array}{c}\text { Carter } \\
\text { synthesis }{ }^{[\mathrm{c}]}\end{array}$ & $\begin{array}{l}\text { Fürstner } \\
\text { synthesis }^{[d]}\end{array}$ & $\begin{array}{l}\text { Natural } \\
\text { sample }\end{array}$ & $\begin{array}{c}\Delta \text { nat. sample } \\
\text { vs Ferrié }\end{array}$ & $\begin{array}{c}\Delta \text { nat. sample } \\
\text { vs Carter }\end{array}$ & $\begin{array}{c}\Delta \text { nat. sample } \\
\text { vs Fürstner }\end{array}$ & $\begin{array}{l}\Delta \text { Ferrie } \\
\text { vs Carter vs }\end{array}$ & $\begin{array}{l}\Delta \text { Ferrié } \\
\text { vs Fürstner }\end{array}$ \\
\hline 1 & 171.20 & 171.22 & 171.2 & 171.16 & -0.04 & -0.06 & -0.04 & -0.02 & 0.00 \\
\hline 2 & 38.67 & 38.70 & 38.7 & 38.65 & -0.02 & -0.05 & -0.05 & -0.03 & -0.03 \\
\hline 3 & 81.42 & 81.45 & 81.5 & 81.26 & -0.16 & -0.19 & -0.24 & -0.03 & -0.08 \\
\hline 4 & 39.79 & 39.79 & 39.8 & 39.67 & -0.12 & -0.12 & -0.13 & 0.00 & -0.01 \\
\hline 5 & 36.74 & 36.77 & 36.7 & 36.81 & 0.07 & 0.04 & 0.11 & -0.03 & 0.04 \\
\hline 6 & 78.86 & 78.94 & 78.8 & 79.08 & 0.22 & 0.14 & 0.28 & -0.08 & 0.06 \\
\hline 7 & 76.44 & 76.51 & 76.4 & 76.71 & 0.27 & 0.20 & 0.31 & -0.07 & 0.04 \\
\hline 8 & 77.00 & $76.51^{[f]}$ & 77.0 & 76.71 & -0.29 & $0.20^{[f]}$ & -0.29 & $0.49^{[f]}$ & 0.00 \\
\hline 9 & 144.41 & 144.45 & 144.4 & 144.37 & -0.04 & -0.08 & -0.03 & -0.04 & 0.01 \\
\hline 10 & 124.54 & 124.52 & 124.6 & 124.62 & 0.08 & 0.10 & 0.02 & 0.02 & -0.06 \\
\hline 11 & 140.00 & 140.04 & 140.0 & 140.00 & 0.00 & -0.04 & 0.00 & -0.04 & 0.00 \\
\hline 12 & 49.34 & 49.38 & 49.3 & 49.46 & 0.12 & 0.08 & 0.16 & -0.04 & 0.04 \\
\hline 13 & 70.63 & 70.62 & 70.7 & 70.50 & -0.13 & -0.12 & -0.20 & 0.01 & -0.07 \\
\hline 14 & 45.50 & 45.55 & 45.5 & 45.65 & 0.15 & 0.10 & 0.15 & -0.05 & 0.00 \\
\hline 15 & 213.76 & 213.77 & 213.8 & 213.58 & -0.18 & -0.19 & -0.22 & -0.01 & -0.04 \\
\hline 16 & 42.71 & 42.79 & 42.7 & 42.93 & 0.22 & 0.14 & 0.23 & -0.08 & 0.01 \\
\hline 17 & 46.04 & 46.03 & 46.1 & 45.81 & -0.23 & -0.22 & -0.29 & 0.01 & -0.06 \\
\hline 18 & 207.82 & 207.77 & 207.9 & 207.47 & -0.35 & -0.30 & -0.43 & 0.05 & -0.08 \\
\hline 19 & 48.53 & 48.57 & 48.5 & 48.45 & -0.08 & -0.12 & -0.05 & -0.04 & 0.03 \\
\hline 20 & 74.96 & 75.01 & 75.0 & 74.82 & -0.14 & -0.19 & -0.18 & -0.05 & -0.04 \\
\hline 21 & 31.92 & 31.98 & 32.0 & 31.84 & -0.08 & -0.14 & -0.16 & -0.06 & -0.08 \\
\hline 22 & 28.38 & 28.43 & 28.4 & 28.46 & 0.08 & 0.03 & 0.06 & -0.05 & -0.02 \\
\hline 23 & 79.91 & 79.90 & 79.9 & 79.87 & -0.04 & -0.03 & -0.03 & 0.01 & 0.01 \\
\hline 24 & 77.76 & 77.84 & 77.7 & 77.93 & 0.17 & 0.09 & 0.23 & -0.08 & 0.06 \\
\hline 25 & 124.15 & 124.19 & 124.2 & 123.97 & -0.18 & -0.22 & -0.23 & -0.04 & -0.05 \\
\hline 26 & 132.02 & 132.06 & 132.0 & 132.09 & 0.07 & 0.03 & 0.09 & -0.04 & 0.02 \\
\hline 27 & 124.01 & 124.04 & 124.0 & 124.06 & 0.05 & 0.02 & 0.06 & -0.03 & 0.01 \\
\hline 28 & 138.25 & 138.29 & 138.3 & 138.25 & 0.00 & -0.04 & -0.05 & -0.04 & -0.05 \\
\hline 29 & 26.05 & 26.08 & 26.1 & 26.00 & -0.05 & -0.08 & -0.10 & -0.03 & -0.05 \\
\hline 30 & 18.48 & 18.51 & 18.5 & 18.43 & -0.05 & -0.08 & -0.07 & -0.03 & -0.02 \\
\hline 31 & 15.38 & 15.40 & 15.4 & 15.39 & 0.01 & -0.01 & -0.01 & -0.02 & -0.02 \\
\hline 32 & 116.10 & 116.11 & 116.1 & 116.16 & 0.06 & 0.05 & 0.06 & -0.01 & 0.00 \\
\hline 33 & 14.27 & $13.77^{[g]}$ & 14.3 & 13.94 & -0.33 & $0.17^{[9]}$ & -0.36 & $0.50^{[g]}$ & -0.03 \\
\hline 34 & 15.52 & 15.53 & 15.5 & 15.29 & -0.23 & -0.24 & -0.21 & -0.01 & 0.02 \\
\hline 35 & 16.20 & 16.24 & 16.2 & 16.20 & 0.00 & -0.04 & 0.00 & -0.04 & 0.00 \\
\hline
\end{tabular}

[a] solvent $\mathrm{CDCl}_{3}$, reference at $77.0 \mathrm{ppm}$ [b] Concentration: $1.4 \mathrm{mg}$ in $220 \mu \mathrm{L}, 200 \mathrm{Mhz}$ [c] Concentration: $1.4 \mathrm{mg}$ in $180 \mu \mathrm{L}, 175$ $\mathrm{MHz}$. The corresponding assignment was deduced from reported chemical shifts, multiplicities, and by comparison with our own assignment [d] Concentration: $5.5 \mathrm{mg}$ in $700 \mu \mathrm{L}, 150 \mathrm{Mhz}$ [e] Unknown concentration, $125 \mathrm{Mhz}$. [f] misassignment probably due to overlap of $\mathrm{C}_{8}$ with the solvent peak. [g] Probable confusion with a $\mathrm{CH}_{3}$ grease peak in the assignment of $\mathrm{C}_{33}$. 


\section{Compound 105}
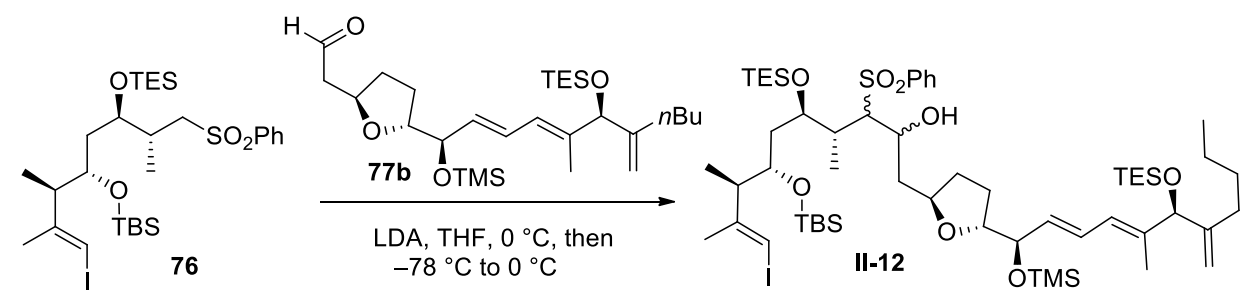

LDA, THF, $0^{\circ} \mathrm{C}$, then $-78^{\circ} \mathrm{C}$ to $0^{\circ} \mathrm{C}$

$\mid \begin{gathered}\text { DMP } \\ \text { 2,6-lutidine } \\ \mathrm{CH}_{2} \mathrm{Cl}_{2}\end{gathered}$

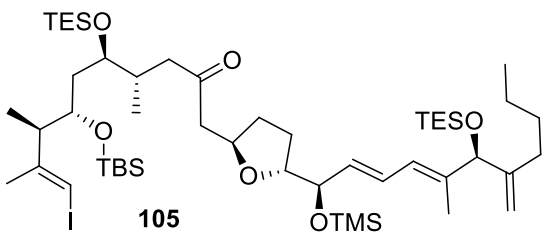

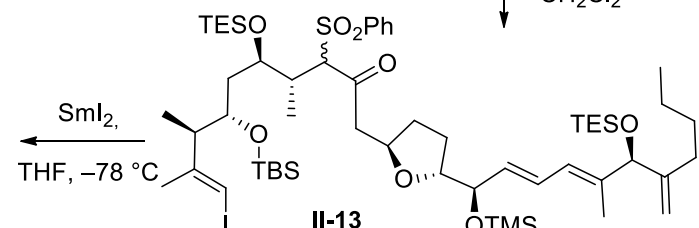

To a solution of sulfone $76(1.463 \mathrm{~g}, 2.15 \mathrm{mmol}, 1.4$ equiv, dried by azeotropic distillation with toluene, three times) in THF $(7 \mathrm{~mL})$ at $0{ }^{\circ} \mathrm{C}$ was added dropwise LDA [4.3 mL, $2.156 \mathrm{mmol}, 1.44$ equiv, $0.5 \mathrm{M}$ in THF (prepared as followed: dropwise addition of $n$-BuLi $(1.95 \mathrm{~mL}, 5.0 \mathrm{mmol}, 2.57 \mathrm{M}$ in hexanes) onto diisopropylamine $(750 \mu \mathrm{L}, 5.35 \mathrm{mmol})$ in THF $(7.325 \mathrm{~mL})$ at $-78{ }^{\circ} \mathrm{C}$, followed by slow warming to rt.)]. After $15 \mathrm{~min}$, the yellow reaction mixture was cooled to $-78^{\circ} \mathrm{C}$, and aldehyde $77 \mathrm{~b}(782 \mathrm{mg}, 1.54 \mathrm{mmol}, 1.2$ equiv, dried by azeotropic distillation with toluene, three times) in THF $(3 \mathrm{~mL})$ was added. The reaction mixture was slowly warmed to $0{ }^{\circ} \mathrm{C}$, stirred for 30 min, quenched by saturated $\mathrm{NH}_{4} \mathrm{Cl}$, extracted with $\mathrm{Et}_{2} \mathrm{O}(\mathrm{x} 3)$, dried over $\mathrm{MgSO}_{4}$, filtered, and concentrated. The residue was purified by silica gel chromatography (8-15\% gradient $\mathrm{Et}_{2} \mathrm{O}$ in petroleum ether) to give, by order of elution, recovered sulfone $76(1.02 \mathrm{~g})$ and sulfone adduct II-12 (892 mg, 49\%) as a complex mixture of four diastereomers. By consequence, its complete characterization was postponed to the following 2 steps.

To a solution of sulfone adduct II-12 (750 mg, $0.63 \mathrm{mmol}, 1$ equiv), in a $\mathrm{CH}_{2} \mathrm{Cl}_{2} / 2,6$-lutidine mixture (10 $\mathrm{mL} / 2 \mathrm{~mL}$ ) was added Dess-Martin periodinane (750 mg, $1.77 \mathrm{mmol}, 2.5$ equiv). After $1 \mathrm{~h}$ at $\mathrm{rt}$, the reaction mixture was quenched with saturated $\mathrm{NaHCO}_{3}$ and a solution of $10 \% \mathrm{Na}_{2} \mathrm{~S}_{2} \mathrm{O}_{3}$. After 15 min, the reaction mixture was extracted with $\mathrm{Et}_{2} \mathrm{O}(\mathrm{x} 3)$, washed with saturated $\mathrm{NaHCO}_{3}$, dried over $\mathrm{MgSO}_{4}$, filtered on a pad of silica gel (elution $\mathrm{Et}_{2} \mathrm{O}$ ) to give crude ketone II-13.

The residue containing crude ketone $\mathrm{Il}-13$ was then dissolved in THF $(10 \mathrm{~mL})$, cooled to $-78{ }^{\circ} \mathrm{C}$ and Sml 2 [20 mL, $2.0 \mathrm{mmol} \approx 0.1 \mathrm{M}$ in THF, (prepared as follows: addition of $\mathrm{CHI}_{3}(1.31 \mathrm{~g}, 3.33 \mathrm{mmol}$ ) to a suspension of Sm powder (1.2 g, $8.0 \mathrm{mmol})$ in THF $(50 \mathrm{~mL})$, and stirring $18 \mathrm{~h}$ at rt] was added dropwise (until a blue/green color persists in the reaction mixture). The reaction mixture was then quenched with $\mathrm{NH}_{4} \mathrm{Cl}$, extracted with $\mathrm{Et}_{2} \mathrm{O}(\mathrm{x} 3)$, dried over $\mathrm{MgSO}_{4}$, filtered, and concentrated. The residue was purified on a silica gel column chromatography to give compound 105 (137 $\mathrm{mg}, 21 \%)$ as a colorless oil.

$[\alpha]^{20} \mathrm{D}=+2.0\left(c\right.$ 1.0, $\left.\mathrm{CH}_{2} \mathrm{Cl}_{2}\right)$. IR (neat): $v=2957,1714,1461,1413,1379,1251,1069,1005,839,775$, $745,724 \mathrm{~cm}^{-1}$. ${ }^{1} \mathrm{H}$ NMR $(300 \mathrm{MHz}$, Chloroform- $d) \delta 6.43(\mathrm{t}, J=14.9,11.0 \mathrm{~Hz}, 1 \mathrm{H}), 6.06(\mathrm{~d}, J=11.0 \mathrm{~Hz}, 1 \mathrm{H})$, $5.89(\mathrm{~s}, 1 \mathrm{H}), 5.64(\mathrm{dd}, J=14.9,6.1 \mathrm{~Hz}, 1 \mathrm{H}), 5.12(\mathrm{~s}, 1 \mathrm{H}), 4.85(\mathrm{~s}, 1 \mathrm{H}), 4.40(\mathrm{~s}, 1 \mathrm{H}), 4.34(\mathrm{t}, J=6.8 \mathrm{~Hz}, 1 \mathrm{H})$, $4.14(\mathrm{t}, J=4.9 \mathrm{~Hz}, 1 \mathrm{H}), 3.95(\mathrm{q}, J=6.1 \mathrm{~Hz}, 1 \mathrm{H}), 3.83-3.75(\mathrm{~m}, 1 \mathrm{H}), 3.71-3.59(\mathrm{~m}, 1 \mathrm{H}), 2.78(\mathrm{dd}, J=15.6$, $6.0 \mathrm{~Hz}, 1 \mathrm{H}), 2.59-2.48(\mathrm{~m}, 1 \mathrm{H}), 2.47(\mathrm{dd}, J=15.6,6.6 \mathrm{~Hz}, 1 \mathrm{H}), 2.34-2.21(\mathrm{~m}, 2 \mathrm{H}), 2.21-2.15(\mathrm{~m}, 1 \mathrm{H})$, $2.15-2.01(\mathrm{~m}, 1 \mathrm{H}), 1.98-1.83(\mathrm{~m}, 3 \mathrm{H}), 1.83(\mathrm{~s}, 3 \mathrm{H}), 1.73(\mathrm{dd}, J=19.5,10.6 \mathrm{~Hz}, 2 \mathrm{H}), 1.59(\mathrm{~s}, 2 \mathrm{H}), 1.35$ (ddt, $J=31.8,13.0,7.2 \mathrm{~Hz}, 7 \mathrm{H}), 1.07(\mathrm{~d}, J=6.9 \mathrm{~Hz}, 2 \mathrm{H}), 0.96(\mathrm{t}, J=8.0 \mathrm{~Hz}, 9 \mathrm{H}), 0.92(\mathrm{t}, J=8.0 \mathrm{~Hz}, 9 \mathrm{H}$ ), $0.89(\mathrm{~s}, 9 \mathrm{H}), 0.89-0.83(\mathrm{~m}, 6 \mathrm{H}), 0.61(\mathrm{t}, J=8.0 \mathrm{~Hz}, 6 \mathrm{H}), 0.56(\mathrm{t}, J=8.0 \mathrm{~Hz}, 7 \mathrm{H}), 0.11(\mathrm{~s}, 9 \mathrm{H}), 0.06(\mathrm{~s}, 6 \mathrm{H})$. 
${ }^{13} \mathbf{C}\left\{{ }^{1} \mathrm{H}\right\}$ NMR $\left(75 \mathrm{MHz}, \mathrm{CDCl}_{3}\right) \delta 208.5,150.0,149.8,138.8,132.0,127.6,125.3,109.7,82.2,80.6,77.3$, 75.8, 75.5, 72.4, 71.5, 49.6, 47.6, 45.7, 37.2, 33.3, 32.3, 31.1, 30.3, 27.6, 26.0, 23.4, 22.7, 18.1, 15.3, 15.1, 14.1, 12.1, 7.1, 7.0, 5.3, 5.0, 0.5, -4.2, -4.5. HRMS (ESI) calcd for $\mathrm{C}_{51} \mathrm{H}_{99} \mathrm{INaO}_{6} \mathrm{Si}_{4}[\mathrm{M}+\mathrm{Na}]^{+}:$1069.5461, found 1069.5459 .

\section{Compound 106}
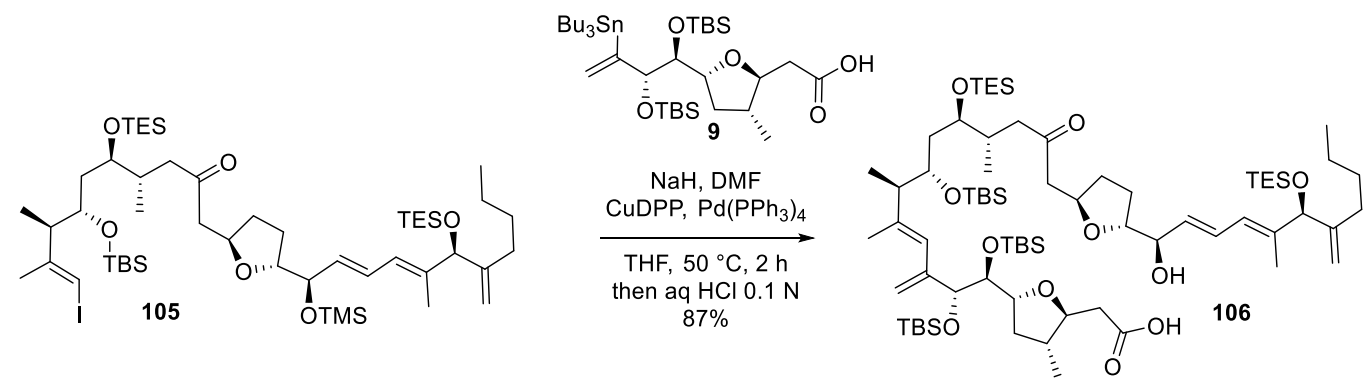

To a solution of carboxylic acid 9 (224 mg, $0.299 \mathrm{mmol}, 2.29$ equiv) in DMF ( $5 \mathrm{~mL}$ ) was added $\mathrm{NaH}$ (9.4 $\mathrm{mg}, 0.299 \mathrm{mmol}, 2.29$ equiv, $60 \%$ in mineral oil) at rt. After $30 \mathrm{~min}$, a solution of vinyl iodide 105 (137 mg, $0.131 \mathrm{mmol}, 1.0$ equiv) in THF $(1.75 \mathrm{~mL})$ was added followed by the simultaneous addition of copper (I) diphenylphosphinate (110 mg, $0.393 \mathrm{mmol}, 3.0$ equiv), $\mathrm{Pd}\left(\mathrm{PPh}_{3}\right)_{4}(68 \mathrm{mg}, 0.0655 \mathrm{mmol}, 0.5$ equiv). After $2 \mathrm{~h}$ of heating with an oil bath at $50^{\circ} \mathrm{C}$, the reaction mixture was quenched with $\mathrm{HCl} 0.1 \mathrm{~N}(5 \mathrm{~mL})$, and the reaction mixture was vigorously stirred for $2 \mathrm{~h}$ at rt. The reaction mixture was filtered on a pad of celite and washed with AcOEt, extracted with AcOEt (x3), washed with brine (x2), dried over $\mathrm{MgSO}_{4}$, filtered, and concentrated. The residue was loaded on a silica gel column chromatography (5\%-20\% gradient AcOEt/petroleum ether) to give seco-acid 106 (149.3 mg, $87 \%$ ) as a light yellow and viscous oil.

$[\alpha]^{20} \mathrm{D}=+20\left(c 1.1, \mathrm{CH}_{2} \mathrm{Cl}_{2}\right.$ ). IR (neat): $v=3300$ (br), 3500-2500 (br), 2956, 1717, 1505, 1460, 1392, 1367, 1251, 1215, 1120, 1074, 1005, 957, 836, $745 \mathrm{~cm}^{-1} .{ }^{1} \mathrm{H}$ NMR $(400 \mathrm{MHz}$, Chloroform-d) $\delta 9.10$ (brs, $1 \mathrm{H}), 6.53$ (dd, $J=15.1,10.9 \mathrm{~Hz}, 1 \mathrm{H}), 6.07$ (d, $J=10.9 \mathrm{~Hz}, 1 \mathrm{H}), 5.64$ (s, 1H), 5.55 (dd, $J=15.1,6.8 \mathrm{~Hz}, 1 \mathrm{H}$ ), 5.29 (s, $1 \mathrm{H}), 5.11(\mathrm{~s}, 1 \mathrm{H}), 4.91(\mathrm{~s}, 1 \mathrm{H}), 4.85(\mathrm{~s}, 1 \mathrm{H}), 4.40(\mathrm{~s}, 1 \mathrm{H}), 4.38-4.25(\mathrm{~m}, 1 \mathrm{H}), 4.08(\mathrm{~s}, 1 \mathrm{H}), 4.06-3.99(\mathrm{~m}$, $1 \mathrm{H}), 3.97(\mathrm{t}, J=7.0 \mathrm{~Hz}, 1 \mathrm{H}), 3.91-3.81(\mathrm{~m}, 2 \mathrm{H}), 3.71-3.62(\mathrm{~m}, 2 \mathrm{H}), 3.56(\mathrm{dd}, J=7.2,1.8 \mathrm{~Hz}, 1 \mathrm{H}), 2.74$ (dd, $J=16.3,6.1 \mathrm{~Hz}, 1 \mathrm{H}), 2.58(\mathrm{dd}, J=8.7,3.8 \mathrm{~Hz}, 1 \mathrm{H}), 2.53-2.40(\mathrm{~m}, 2 \mathrm{H}), 2.37-2.05(\mathrm{~m}, 7 \mathrm{H}), 2.01-$ $1.84(\mathrm{~m}, 2 \mathrm{H}), 1.83-1.70(\mathrm{~m}, 1 \mathrm{H}), 1.77(\mathrm{~s}, 3 \mathrm{H}), 1.69-1.61(\mathrm{~m}, 3 \mathrm{H}), 1.60(\mathrm{~s}, 3 \mathrm{H}), 1.58-1.45(\mathrm{~m}, 2 \mathrm{H}), 1.43-$ $1.20(\mathrm{~m}, 5 \mathrm{H}), 1.07(\mathrm{~d}, J=6.4 \mathrm{~Hz}, 3 \mathrm{H}), 1.00(\mathrm{~d}, J=6.5 \mathrm{~Hz}, 3 \mathrm{H}), 0.98-0.84(\mathrm{~m}, 51 \mathrm{H}), 0.60(\mathrm{q}, J=7.3 \mathrm{~Hz}, 6 \mathrm{H})$, $0.57(\mathrm{q}, J=7.3 \mathrm{~Hz}, 6 \mathrm{H}), 0.09(\mathrm{~s}, 3 \mathrm{H}), 0.08(\mathrm{~s}, 3 \mathrm{H}), 0.07(\mathrm{~s}, 3 \mathrm{H}), 0.05(\mathrm{~s}, 3 \mathrm{H}), 0.04(\mathrm{~s}, 3 \mathrm{H}), 0.01(\mathrm{~s}, 3 \mathrm{H}) .{ }^{13} \mathrm{C}\left\{{ }^{1} \mathrm{H}\right\}$ NMR $\left(101 \mathrm{MHz}, \mathrm{CDCl}_{3}\right) \delta 208.4,174.1,149.6,145.4,141.5,139.8,130.0,128.9,125.4,124.6,114.6,109.7$, $82.1,80.3,80.2,80.0,78.8,78.7,75.4,75.0,72.8,71.8,49.0,47.6,45.3,40.0,38.3,37.8,37.5,33.5,32.2$, $30.7,27.9,26.8,26.1,26.0,25.9,22.5,18.3,18.0,17.18,15.8,15.7,14.3,13.6,12.2,7.0,6.8,5.2,4.8,-4.3$, $-4.3,-4.5,-4.5,-4.7,-4.8$. HRMS (ESI) calcd for $\mathrm{C}_{71} \mathrm{H}_{136} \mathrm{O}_{11} \mathrm{Si}_{5} \mathrm{Na}[\mathrm{M}+\mathrm{Na}]^{+}:$1327.8817, found 1327.8827 .

\section{Compound 107}
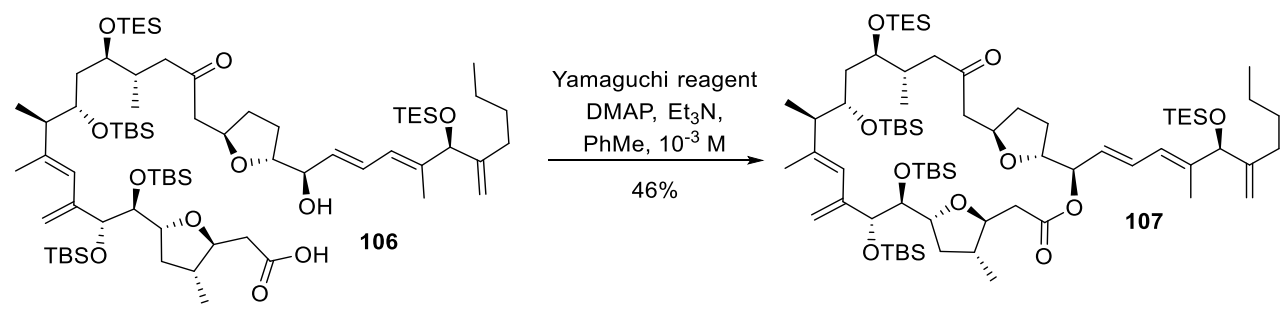

To a solution of 2,4,6-trichlorobenzoyl chloride ( $47 \mu \mathrm{L}, 0.303 \mathrm{mmol}, 3$ equiv), DMAP (36.6 mg, $1.22 \mathrm{mmol}$, 3 equiv) and $\mathrm{Et}_{3} \mathrm{~N}(84 \mu \mathrm{L}, 0.607 \mathrm{mmol}, 6$ equiv) in toluene $(100 \mathrm{~mL})$ was added a solution of seco-acid 106 
(132 $\mathrm{mg}, 0.101 \mathrm{mmol}, 1$ equiv) in toluene $(10 \mathrm{~mL})$ via a syringe pump over $10 \mathrm{~h}$ at $50{ }^{\circ} \mathrm{C}$ with the help of an oil bath, and the stirring was continued for $6 \mathrm{~h}$. The reaction mixture was quenched with water, and the organic layer was washed with $\mathrm{NH}_{4} \mathrm{Cl}$, dried over $\mathrm{MgSO}_{4}$, filtered, and concentrated. The residue was purified on a silica gel chromatography (8-15\% gradient $\mathrm{Et}_{2} \mathrm{O}$ in petroleum ether) to give macrolactone 107 (61.3 mg, 46\%) as a colorless oil.

$[\alpha]^{25} \mathrm{D}=+43.0\left(c 1.15, \mathrm{CH}_{2} \mathrm{Cl}_{2}\right)$. IR (neat) $: v=2957,1743,1721,1462,1251,1127,1102,1066,1004,835$, 774, 745, $726 \mathrm{~cm}^{-1} .{ }^{1} \mathrm{H}$ NMR (400 MHz, Chloroform- $\left.d\right) \delta 6.51(\mathrm{dd}, J=15.0,11.1 \mathrm{~Hz}, 1 \mathrm{H}), 6.06(\mathrm{~d}, J=11.1$ $\mathrm{Hz}, 1 \mathrm{H}$ ), 6.02 (brs, 1H), 5.49 (dd, $J=15.0,7.8 \mathrm{~Hz}, 1 \mathrm{H}), 5.27$ (t, $J=7.0 \mathrm{~Hz}, 1 \mathrm{H}), 5.09(\mathrm{~s}, 1 \mathrm{H}), 5.06(\mathrm{brs}, 1 \mathrm{H})$, 4.87 (brs, 1H), $4.85(\mathrm{~s}, 1 \mathrm{H}), 4.40(\mathrm{~s}, 1 \mathrm{H}), 4.13(\mathrm{~s}, 1 \mathrm{H}), 4.07$ (brs, $1 \mathrm{H}), 3.96(\mathrm{q}, J=7.5 \mathrm{~Hz}, 1 \mathrm{H}), 3.83(\mathrm{brs}, 2 \mathrm{H})$, $3.63-3.57(\mathrm{~m}, 1 \mathrm{H}), 3.56$ (dd, $J=8.5,1.7 \mathrm{~Hz}, 1 \mathrm{H}), 3.47$ (brs, $1 \mathrm{H}), 2.87$ (brd, $J=15.3 \mathrm{~Hz}, 1 \mathrm{H}), 2.61$ (brd, $J=$ $13.8 \mathrm{~Hz}, 1 \mathrm{H}), 2.52(\mathrm{brd}, J=13.8 \mathrm{~Hz}, 1 \mathrm{H}), 2.47-2.34(\mathrm{~m}, 2 \mathrm{H}), 2.32-2.23(\mathrm{~m}, 3 \mathrm{H}), 2.21-2.06(\mathrm{~m}, 3 \mathrm{H}), 2.01$ - $1.92(\mathrm{~m}, 1 \mathrm{H}), 1.88(\mathrm{dt}, J=15.8,7.8 \mathrm{~Hz}, 1 \mathrm{H}), 1.80$ (dt, $J=15.8,7.8 \mathrm{~Hz}, 1 \mathrm{H}), 1.77$ (brs, 3H), $1.69-1.61(\mathrm{~m}$, $1 \mathrm{H}), 1.60(\mathrm{~s}, 3 \mathrm{H}), 1.45-1.34(\mathrm{~m}, 5 \mathrm{H}), 1.29(\mathrm{dd}, J=9.3,5.0 \mathrm{~Hz}, 2 \mathrm{H}), 1.24-1.18(\mathrm{~m}, 1 \mathrm{H}), 1.08(\mathrm{~d}, J=7.0 \mathrm{~Hz}$, $3 \mathrm{H}), 1.01(\mathrm{~d}, J=5.8 \mathrm{~Hz}, 3 \mathrm{H}), 0.97(\mathrm{t}, J=8.0 \mathrm{~Hz}, 9 \mathrm{H}), 0.92(\mathrm{t}, J=8.1 \mathrm{~Hz}, 9 \mathrm{H}), 0.91-0.86(\mathrm{~m}, 30 \mathrm{H}), 0.85-$ $0.81(\mathrm{~m}, 3 \mathrm{H}), 0.59(\mathrm{q}, J=8.0 \mathrm{~Hz}, 6 \mathrm{H}), 0.57(\mathrm{q}, J=8.1 \mathrm{~Hz}, 6 \mathrm{H}), 0.09(\mathrm{~s}, 3 \mathrm{H}), 0.08(\mathrm{~s}, 3 \mathrm{H}), 0.06(\mathrm{~s}, 6 \mathrm{H}), 0.04$ $(\mathrm{s}, 3 \mathrm{H}),-0.01(\mathrm{~s}, 3 \mathrm{H}) .{ }^{13} \mathrm{C}\left\{{ }^{1} \mathrm{H}\right\}$ NMR $\left(101 \mathrm{MHz}, \mathrm{CDCl}_{3}\right) \delta 207.0,169.4,149.7,147.2,141.1,141.1,131.2$, 128.8, 126.8, 124.2, 114.1, 110.2, 83.0, 80.8, 80.5, 79.6, 78.9, 76.8, 75.7, 74.1, 73.0, 72.7, 49.1, 46.9, 46.5, 38.6, 38.0, 37.7, 37.4, 33.4, 32.4, 30.7, 30.2, 29.9, 28.4, 26.5 (1tBu), 26.1 (2tBu), 22.7, 18.7, 18.5, 18.2, 16.3, 15.9, 14.2, 13.5, 12.6, 7.1, 7.0, 5.3, 5.0, -4.2, -4.4 (2C), -4.5, -4.6, -4.8. HRMS (ESI) calcd for $\mathrm{C}_{71} \mathrm{H}_{134} \mathrm{O}_{10} \mathrm{Si}_{5} \mathrm{Na}$ $[\mathrm{M}+\mathrm{Na}]^{+}:$1309.8721, found 1309.8733 .

\section{Compound 108}
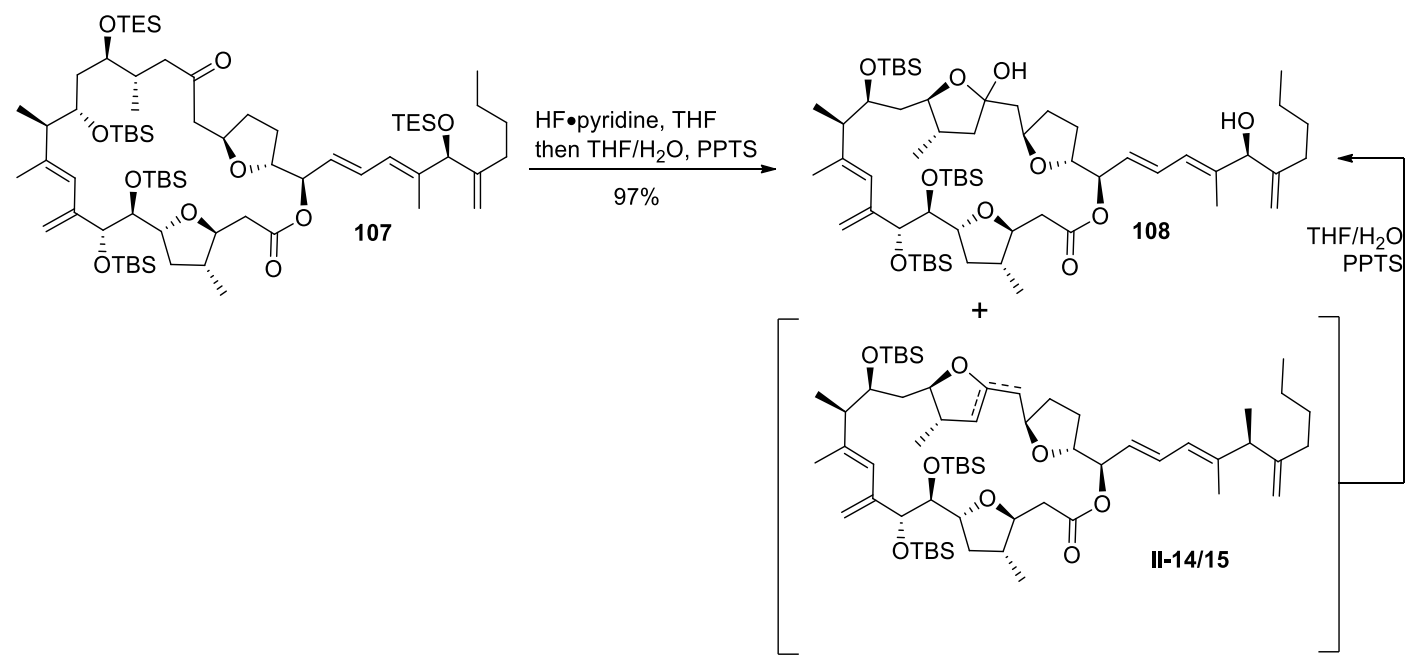

To a solution of macrolactone 107 (25.5 mg, $0.0198 \mathrm{mmol}, 1$ equiv) in THF (400 $\mu \mathrm{L})$ in a plastic vial was added HF•pyridine [330 $\mu \mathrm{L}, 1.485 \mathrm{mmol}, 75$ equiv, $4.5 \mathrm{M}$ in THF/pyridine (prepared from HF•pyridine complex: pyridine: THF, 0.5:1:2.5 mL)]. After $1 \mathrm{~h}$ at $\mathrm{rt}$, the reaction mixture was poured into an erlenmeyer containing saturated $\mathrm{NaHCO}_{3}$. The reaction mixture was then stirred for 5 min and was extracted with $\mathrm{Et}_{2} \mathrm{O}(\times 3)$, dried on $\mathrm{MgSO}_{4}$, filtered and concentrated. The residue was purified on a silica gel chromatography (30 to $50 \%$ $\mathrm{Et}_{2} \mathrm{O}$ in petroleum ether) to afford macrolactone $108(11.6 \mathrm{mg}, 55 \%)$ as colorless oil along with dehydrated products II-13/14 (8.5 mg, 43\%). IR (neat): $v=3400$ (br), 2957, 1739, 1472, 1461, 1253, 1109, 1077, 835, 776 $\mathrm{cm}^{-1}$. HRMS (ESI) calcd for $\mathrm{C}_{59} \mathrm{H}_{105} \mathrm{O}_{9} \mathrm{Si}_{3}[\mathrm{M}+\mathrm{H}]^{+}: 1041.7061$, found 1041.7645

Dehydrated compounds II-13/14 were converted in 108 as follows: II-14/15 were dissolved in THF (2 mL) and $\mathrm{H}_{2} \mathrm{O}(200 \mu \mathrm{L})$ followed by PPTS $(3 \mathrm{mg})$ was added. After $2 \mathrm{~h}$ at $\mathrm{rt}, \mathrm{Il-14} / \mathbf{1 5}$ was converted entirely into 
108. Aqueous $\mathrm{NaHCO}_{3}$ was added, and the reaction mixture was extracted with $\mathrm{Et}_{2} \mathrm{O}$. The ethereal layer was dried over $\mathrm{MgSO}_{4}$, filtered, concentrated, and the residue was purified on a silica gel chromatography (30 to $50 \% \mathrm{Et}_{2} \mathrm{O}$ in petroleum ether) to afford macrolactone 108 as a colorless oil ( $8.5 \mathrm{mg}, 42 \%$, combined fractions: $20.1 \mathrm{mg}, 97 \%)$.

Product 108 was a mixture of three compounds (hydroxylketone and two hemi-ketals), and each probably gives a mixture of conformers on NMR spectra. ${ }^{[33,34]}$ Consequently, this compound was hardly characterizable by this technique. Nevertheless, the absence of ketone absorption band at $\approx 1715 \mathrm{~cm}^{-1}$ indicates that the hemiketal is the major form.

IR (neat): $v=3400$ (br), 2957, 1737, 1472, 1461, 1253, 1109, 1078, 835, $776 \mathrm{~cm}^{-1}$. HRMS (ESI) calcd for $\mathrm{C}_{59} \mathrm{H}_{110} \mathrm{NO}_{10} \mathrm{Si}_{3}\left[\mathrm{M}+\mathrm{NH}_{4}\right]^{+}:$1076.7438, found 1076.7432 .

\section{Compound 110}

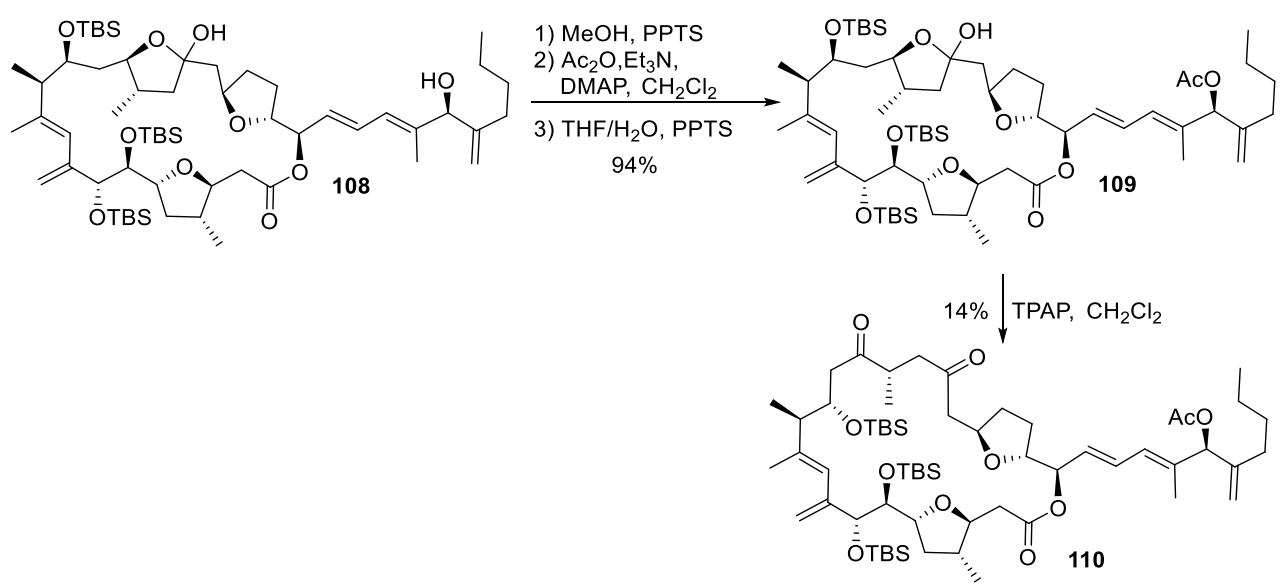

To a solution of compound 108 (9.0 mg, $8.51 \mu \mathrm{L}, 1$ equiv) in $\mathrm{MeOH}(3 \mathrm{~mL})$ was added PPTS (5 mg). After $30 \mathrm{~min}$ (which correspond to complete conversion on TLC) at rt, the reaction mixture was quenched with aqueous $\mathrm{NaHCO}_{3}$, extracted with $\mathrm{Et}_{2} \mathrm{O}$, dried over $\mathrm{MgSO}_{4}$, filtered, and concentrated. The residue was dissolved in $\mathrm{CH}_{2} \mathrm{Cl}_{2}(1 \mathrm{~mL})$, and $\mathrm{Et}_{3} \mathrm{~N}(100 \mu \mathrm{L}), \mathrm{Ac}_{2} \mathrm{O}(100 \mu \mathrm{L})$, and DMAP (2 mg, $16.5 \mathrm{mmol}$, 2 equiv) were added successively. After $30 \mathrm{~min}$ at $\mathrm{rt}$ (which correspond to complete conversion on TLC), the reaction mixture was quenched by $\mathrm{H}_{2} \mathrm{O}(1 \mathrm{~mL})$, and the reaction mixture was extracted thrice with $\mathrm{Et}_{2} \mathrm{O}$. Combined ethereal layers were washed with water, followed by $\mathrm{NaHCO}_{3}$, dried over $\mathrm{MgSO}_{4}$, filtered, and concentrated. The residue was then dissolved in THF $(3 \mathrm{~mL})$, and $\mathrm{H}_{2} \mathrm{O}(200 \mu \mathrm{L})$ \& PPTS $(5 \mathrm{mg})$ were added. After stirring $90 \mathrm{~min}$ at rt (which corresponds to complete conversion on TLC), the reaction mixture was quenched with aqueous $\mathrm{NaHCO}_{3}$, extracted with $\mathrm{Et}_{2} \mathrm{O}$, dried over $\mathrm{MgSO}_{4}$, filtered, and concentrated. The residue, which corresponds to 109 was used without further purification in the next step ( $8.8 \mathrm{mg}, 94 \%)$.

IR (neat): $v=3400$ (br), 2957, 1742, 1504, 1365, 1236, 1073, 1078, 835, $776 \mathrm{~cm}^{-1}$. HRMS (ESI) calcd for $\mathrm{C}_{61} \mathrm{H}_{112} \mathrm{NO}_{11} \mathrm{Si}_{3}[\mathrm{M}+\mathrm{H}]^{+}:$1118.7543, found 1118.7548 .

To a solution of a mixture of compound 109 (8.8 mg, $14 \mu \mathrm{mol}, 1$ equiv) in $\mathrm{CH}_{2} \mathrm{Cl}_{2}(1 \mathrm{~mL})$ was added TPAP (8.7 mg, $24.9 \mu \mathrm{mol}, 3$ equiv) in 3 portions over $20 \mathrm{~min}$. After $2 \mathrm{~h}$ at rt, additional TPAP $(8.7 \mathrm{mg}, 24.9 \mu \mathrm{mol}, 3$ equiv) was added, and the reaction was stirred $1 \mathrm{~h}$ more. Additional TPAP was added $(2.9 \mathrm{mg}, 8.3 \mu \mathrm{mol}, 1$ equiv), and the reaction mixture was stirred $2 \mathrm{~h}$ more. The reaction mixture was then diluted with $\mathrm{Et}_{2} \mathrm{O}$ and filtered on Celite. After concentration, the residue was purified on a silica gel column $\left(20-30 \% \mathrm{Et}_{2} \mathrm{O}\right.$ in petroleum ether) to give diketone $110(1.2 \mathrm{mg}, 14 \%)$ as a colorless oil.

The macrolactone exists as a mixture of many conformers into solution, leading to broaden and/or splitting signals. As a consequence characterization of the compound by ${ }^{13} \mathrm{C}\left\{{ }^{1} \mathrm{H}\right\}$ NMR was not possible with our 
routine NMR apparatus on the scale that we obtained 110. IR (neat): $\mathrm{V}=2957,1743,1647,1500,1365,1254$, 1232, 1100, 1035, 836, $777 \mathrm{~cm}^{-1}$. ${ }^{1} \mathrm{H}$ NMR $(400 \mathrm{MHz}$, Chloroform-d) $\delta 6.52$ (dd, J = 14.8, $11.2 \mathrm{~Hz}, 1 \mathrm{H}), 6.04$ $(\mathrm{d}, \mathrm{J}=11.2 \mathrm{~Hz}, 1 \mathrm{H}), 5.97-5.83(\mathrm{~m}, 1 \mathrm{H}), 5.66-5.56(\mathrm{~m}, 1 \mathrm{H}), 5.54(\mathrm{~s}, 1 \mathrm{H}), 5.41-5.27(\mathrm{~m}, 1 \mathrm{H}), 5.24-5.06$ $(\mathrm{m}, 1 \mathrm{H}), 5.04(\mathrm{~s}, 1 \mathrm{H}), 5.00-4.81(\mathrm{~m}, 3 \mathrm{H}), 4.33-4.11(\mathrm{~m}, 2 \mathrm{H}), 4.11-3.68(\mathrm{~m}, 4 \mathrm{H}), 3.66-3.42(\mathrm{~m}, 2 \mathrm{H}), 3.08$ - $2.92(\mathrm{~m}, 1 \mathrm{H}), 2.87(\mathrm{dd}, \mathrm{J}=18.0,5.5 \mathrm{~Hz}, 1 \mathrm{H}), 2.73(\mathrm{dd}, \mathrm{J}=14.7,9.8 \mathrm{~Hz}, 1 \mathrm{H}), 2.63-2.29(\mathrm{~m}, 4 \mathrm{H}), 2.08(\mathrm{~s}$, $3 \mathrm{H}), 2.05-1.97(\mathrm{~m}, 2 \mathrm{H}), 1.96-1.87(\mathrm{~m}, 2 \mathrm{H}), 1.87-1.74(\mathrm{~m}, 2 \mathrm{H}), 1.68(\mathrm{~s}, 3 \mathrm{H}), 1.66-1.50(\mathrm{~m}, 3 \mathrm{H}), 1.55-$ $1.48(\mathrm{~m}, 3 \mathrm{H}), 1.46-1.20(\mathrm{~m}, 5 \mathrm{H}), 1.15(\mathrm{~d}, \mathrm{~J}=7.3 \mathrm{~Hz}, 3 \mathrm{H}), 1.09(\mathrm{~d}, \mathrm{~J}=7.0 \mathrm{~Hz}, 3 \mathrm{H}), 1.03-0.98(\mathrm{~m}, 3 \mathrm{H}), 0.95$ $-0.81(\mathrm{~m}, 30 \mathrm{H}), 0.11--0.04(\mathrm{~m}, 18 \mathrm{H})$. HRMS (ESI) calcd for $\mathrm{C}_{61} \mathrm{H}_{110} \mathrm{NO}_{11} \mathrm{Si}_{3}\left[\mathrm{M}+\mathrm{NH}_{4}\right]^{+}: 1116.7009$, found 1116.7061; $\mathrm{C}_{61} \mathrm{H}_{106} \mathrm{NaO}_{11} \mathrm{Si}_{3}[\mathrm{M}+\mathrm{Na}]^{+}:$1121.6941, found 1121.7062 .

Amphidinolide C2 (2)
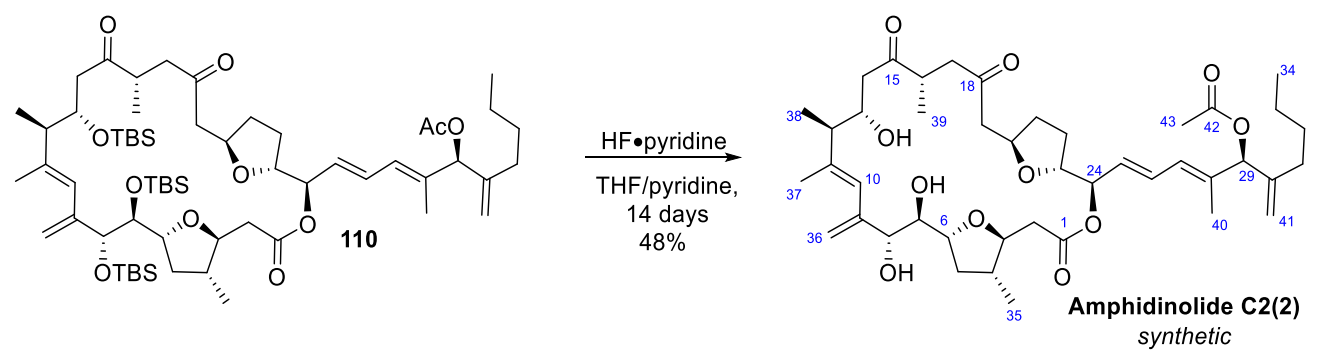

Compound $110(1.2 \mathrm{mg}, 1.09 \mu \mathrm{mol})$ was placed in a polypropylene vial, and HF•pyridine in THF [1 mL (prepared from HF•pyridine complex: pyridine: THF, 1:2:1 mL)] was added. After 7 days at $\mathrm{rt}$, the vial mixture was poured into saturated $\mathrm{NaHCO}_{3}$ and washed with AcOEt. The aqueous layer was extracted with $\mathrm{AcOEt}$ $(\mathrm{x} 4)$, and the combined organic phases were dried over $\mathrm{MgSO}_{4}$, filtered, and concentrated. The residue was purified on a silica gel column (20-30\% gradient acetone in hexanes) to give amphidinolide C2 (2) (0.4 mg, $48 \%)$ as a white waxy solid. Spectroscopic data were in agreement with the natural product. ${ }^{[15]}$

$[\alpha]^{20} \mathrm{D}=-90\left(c 0.022, \mathrm{CHCl}_{3}\right)$. IR (neat): $v=3358(\mathrm{br}), 2922,2850,1734,1716,1660,1633,1458,1377,1259$, 1236, 1091, 1022, $800 \mathrm{~cm}^{-1}$. ${ }^{1} \mathrm{H}$ NMR $\left(600 \mathrm{MHz}, \mathrm{C}_{6} \mathrm{D}_{6}\right) \delta 6.70(\mathrm{dd}, J=15.1,11.0 \mathrm{~Hz}, 1 \mathrm{H}, \mathrm{H}-26), 6.28(\mathrm{~s}, 1 \mathrm{H}$, $\mathrm{H}-10), 6.26(\mathrm{~d}, J=11.0 \mathrm{~Hz}, 1 \mathrm{H}, \mathrm{H}-27), 5.88(\mathrm{~s}, 1 \mathrm{H}, \mathrm{H}-29), 5.58$ (dd, $J=15.1,8.0 \mathrm{~Hz}, 1 \mathrm{H}, \mathrm{H}-25), 5.45(\mathrm{t}, J=$ $8.0 \mathrm{~Hz}, 1 \mathrm{H}, \mathrm{H}-24), 5.32$ (s, 1H, H-36a), 5.17 (s, 1H, H-41a), 5.02 (s, 1H, H-36b), 4.95 (s, 1H, H-41b), 4.46 (brs, $1 \mathrm{H}, \mathrm{H}-8), 4.25-4.19(\mathrm{~m}, 1 \mathrm{H}, \mathrm{H}-20), 4.16$ (brq, $J=7.0 \mathrm{~Hz}, 1 \mathrm{H}, \mathrm{H}-6), 4.07$ (t, $J=7.4 \mathrm{~Hz}, 1 \mathrm{H}, \mathrm{H}-13$ ), 3.82 (q, $J=7.4 \mathrm{~Hz}, 1 \mathrm{H}, \mathrm{H}-23$ ), $3.80(\mathrm{t}, J=5.2 \mathrm{~Hz}, 1 \mathrm{H}, \mathrm{H}-7), 3.75$ (td, $J=9.5,1.6 \mathrm{~Hz}, 1 \mathrm{H}, \mathrm{H}-3), 4.50-3.65(\mathrm{~m}, 3 \mathrm{H}$, $3 \mathrm{OH}$ ), $3.05(\mathrm{~m}, 1 \mathrm{H}, \mathrm{H}-16), 2.91$ (dd, $J=18.0,9.7 \mathrm{~Hz}, 1 \mathrm{H}, \mathrm{H}-17 \mathrm{a}), 2.90$ (dd, $J=15.7,8.5 \mathrm{~Hz}, 1 \mathrm{H}, \mathrm{H}-14 \mathrm{a}), 2.50$ (dd, $J=16.0,8.9 \mathrm{~Hz}, 1 \mathrm{H}, \mathrm{H}-19 \mathrm{a}), 2.46$ (dd, $J=14.6,9.9 \mathrm{~Hz}, 1 \mathrm{H}, \mathrm{H}-2 \mathrm{a}), 2.41$ (dd, $J=15.7,1.9 \mathrm{~Hz}, 1 \mathrm{H}, \mathrm{H}-$ 14b), 2.37 (dd, $J=18.0,3.5 \mathrm{~Hz}, 1 \mathrm{H}, \mathrm{H}-17 \mathrm{~b}$ ), 2.27 (dd, $J=14.7,1.9 \mathrm{~Hz}, 1 \mathrm{H}, \mathrm{H}-2 \mathrm{~b}$ ), 2.15 (quint, $J=7.3 \mathrm{~Hz}$, $1 \mathrm{H}, \mathrm{H}-12), 1.96(\mathrm{~m}, 1 \mathrm{H}, \mathrm{H}-31 \mathrm{a}), 1.96$ (dd, J = 16.0, $8.5 \mathrm{~Hz}, 1 \mathrm{H}, \mathrm{H}-19 \mathrm{~b}), 1.91(\mathrm{~m}, 1 \mathrm{H}, \mathrm{H}-31 \mathrm{~b}), 1.89(\mathrm{~m}, \mathrm{H}-5 \mathrm{a})$, 1.81 (s, 3H, H-37), 1.68 (s, 3H, H-43), $1.66(\mathrm{~s}, 3 \mathrm{H}, \mathrm{H}-40), 1.45(\mathrm{~m}, 1 \mathrm{H}, \mathrm{H}-4), 1.44(\mathrm{~m}, 1 \mathrm{H}, \mathrm{H}-5 \mathrm{~b}), 1.42-1.30$ (m, 2H, H-32), $1.39(\mathrm{~m}, 1 \mathrm{H}, \mathrm{H}-22 \mathrm{a}), 1.32(\mathrm{~m}, 1 \mathrm{H}, \mathrm{H}-22 \mathrm{~b}), 1.29(\mathrm{~m}, 1 \mathrm{H}, \mathrm{H}-21 \mathrm{a}) 1.21(\mathrm{~m}, 1 \mathrm{H}, \mathrm{H}-21 \mathrm{a}), 1.23-$ $1.16(\mathrm{~m}, 2 \mathrm{H}, \mathrm{H}-33), 1.06$ (d, J = 6.9 Hz, 3H, H-38), 0.97 (d, J=7.0 Hz, 3H, H-39), 0.83 (t, J = 7.4 Hz, 3H, H34), 0.67 (d, J = 6.2 Hz, 3H, H-35). ${ }^{13} \mathrm{C}\left\{{ }^{1} \mathrm{H}\right\}$ NMR (176 MHz, $\left.\mathrm{C}_{6} \mathrm{D}_{6}\right) \delta 214.7$ (C-15), 207.7 (C-18), 171.5 (C-1), 169.0 (C-42), 146.1 (C-9), 146.0 (C-30), 141.3 (C-11), 136.8 (C-28), 130.3 (C-25), 129.5 (C-26), 127.4 (C27), 125.2 (C-10), 114.7 (C-36), 111.5 (C-41), 82.0 (C-3), 80.8 (C-23), 80.3 (C-29), 79.2 (C-6), 77.9 (C-8), 77.4 (C-24), 76.6 (C-7), 75.7 (C-20), 71.3 (C-13), 48.7 (C-12), 48.4 (C-19), 47.6 (C-17), 46.6 (C-14), 42.2 (C16), 40.5 (C-4), 39.5 (C-2), 37.7 (C-5), 32.4 (C-21), 32.4 (C-31), 30.1 (C-32), 27.9 (C-22), 22.7 (C-33), 20.6 (C-43), 17.0 (C-37), 16.3 (C-39), 16.2 (C-38), 15.6 (C-35), 14.2 (C-34), 13.1 (C-40). HRMS (ESI) calcd for $\mathrm{C}_{43} \mathrm{H}_{68} \mathrm{NO}_{11}\left[\mathrm{M}+\mathrm{NH}_{4}\right]^{+}:$774.4787, found $774.4785 ; \mathrm{C}_{43} \mathrm{H}_{64} \mathrm{O}_{11} \mathrm{Na}[\mathrm{M}+\mathrm{Na}]^{+}:$779.4341, found 779.4342 .

Table S3. Comparison of ${ }^{1} \mathrm{H} N R^{[a]}$ and ${ }^{13} \mathbf{C}\left\{{ }^{1} \mathrm{H}\right\} \mathbf{N M R}^{[a]}$ spectra of synthetic and natural sample of amphidinolide $\mathrm{C}^{\left[{ }^{[35]}\right.}$ 


\begin{tabular}{|c|c|c|c|c|c|c|c|c|}
\hline \multirow[t]{2}{*}{ C-position } & \multicolumn{2}{|c|}{${ }^{1} \mathrm{H}$ NMR Synthetic Sample ${ }^{[b]}$} & \multicolumn{3}{|c|}{${ }^{1} \mathrm{H}$ NMR Natural Sample ${ }^{[c]}$} & \multirow{2}{*}{$\begin{array}{c}{ }^{13} \mathrm{C}\left\{{ }^{1} \mathrm{H}\right\} \text { NMR } \\
\text { Synthetic }{ }^{[d]} \\
\delta(\mathrm{ppm})\end{array}$} & \multicolumn{2}{|l|}{$\begin{array}{c}{ }^{13} \mathrm{C}\left\{{ }^{1} \mathrm{H}\right\} \text { NMR } \\
\text { Natural[ }\end{array}$} \\
\hline & $\delta(\mathrm{ppm})$ & Multiplicity J(Hz) & $\delta(\mathrm{ppm})$ & Multiplicity J(Hz) & $\Delta$ & & $\delta(\mathrm{ppm})$ & $\Delta$ \\
\hline 1 & & & & & & 171.53 & 171.13 & -0.40 \\
\hline $2 a$ & 2.46 & dd 14.6, 9.9 & $2.59^{[e]}$ & dd $15.7,9.5^{[e]}$ & 0.13 & & & 025 \\
\hline $2 b$ & 2.27 & dd $14.7,1.9$ & $2.35^{[\mathrm{e}]}$ & $\mathrm{m}^{[e]}$ & 0.08 & 39.48 & $39.13^{(10)}$ & -0.35 \\
\hline 3 & 3.75 & $\operatorname{td} 9.5,1.6$ & 3.89 & dt $9.4,2.5$ & 0.14 & 81.96 & 81.73 & -0.23 \\
\hline 4 & 1.45 & $\mathrm{~m}$ & 1.53 & $\mathrm{~m}$ & 0.08 & 40.51 & 40.12 & -0.39 \\
\hline $5 a$ & 1.89 & $\mathrm{~m}$ & 1.86 & $\mathrm{~m}$ & -0.03 & 3772 & 3706 & -0.66 \\
\hline $5 b$ & 1.44 & $\mathrm{~m}$ & 1.45 & $\mathrm{~m}$ & 0.01 & 37.12 & 37.00 & -0.66 \\
\hline 6 & 4.16 & brq 7.0 & 4.09 & $\mathrm{~m}$ & -0.07 & 79.23 & 79.28 & 0.05 \\
\hline 7 & 3.8 & t 5.2 & 3.62 & $\mathrm{t} 4.5$ & -0.18 & 76.21 & 76.64 & 0.43 \\
\hline 8 & 4.46 & brs & 4.32 & d 4.5 & -0.14 & 77.87 & 77.67 & -0.20 \\
\hline 9 & & & & & & 146.14 & $146.07^{[e]}$ & -0.07 \\
\hline 10 & 6.28 & s & 6.30 & s & 0.02 & 125.15 & 125.42 & 0.27 \\
\hline 11 & & & & & & 141.27 & $140.35^{[e]}$ & -0.92 \\
\hline 12 & 2.15 & quint 7.3 & 2.29 & $\mathrm{~m}$ & 0.14 & 48.71 & 49.38 & 0.67 \\
\hline 13 & 4.07 & $t 7.4$ & 4.10 & $\mathrm{~m}$ & 0.03 & 71.33 & 71.03 & -0.30 \\
\hline $14 a$ & 2.90 & dd $15.7,1.9$ & $2.75^{[\mathrm{e}]}$ & dd $15.7,9.5^{[\mathrm{e}]}$ & -0.15 & & & \\
\hline $14 b$ & 2.41 & dd $15.7,1.9$ & $2.43^{[e]}$ & $\mathrm{m}^{[\mathrm{e}]}$ & 0.02 & 46.55 & $45.83^{[e]}$ & -0.72 \\
\hline 15 & & & & & & 214.66 & 213.27 & -1.39 \\
\hline 16 & 3.05 & $\mathrm{~m}$ & 3.14 & $\mathrm{~m}$ & 0.09 & 42.16 & 42.57 & 0.41 \\
\hline $17 a$ & 2.91 & dd 18.0, 9.7 & $3.00^{[\mathrm{e}]}$ & $\mathrm{dd} 17.6,8.5^{[\mathrm{e}]}$ & 0.09 & & & \\
\hline $17 \mathrm{~b}$ & 2.37 & dd $18.0,3.5$ & $2.17^{[e]}$ & $\mathrm{m}^{[\mathrm{e}]}$ & -0.2 & 47.56 & 46.25 & -1.31 \\
\hline 18 & & & & & & 207.16 & 207.38 & 0.22 \\
\hline $19 a$ & 2.50 & dd 16.0, 8.9 & $2.50^{[\mathrm{e}]}$ & dd $14.9,8.2^{[\mathrm{e}]}$ & 0.00 & & & \\
\hline $19 b$ & 1.96 & dd $16.0,8.5$ & $2.17^{[\mathrm{e}]}$ & $\mathrm{m}^{[\mathrm{e}]}$ & 0.21 & 48.35 & $48.72^{[\mathrm{e}]}$ & 0.37 \\
\hline 20 & 4.23 & m & 4.30 & $\mathrm{~m}$ & 0.07 & 75.74 & 75.6 & -0.14 \\
\hline $21 a$ & 1.29 & $\mathrm{~m}$ & 1.13 & $\mathrm{~m}$ & -0.16 & & & \\
\hline $21 b$ & 1.21 & $\mathrm{~m}$ & 1.75 & $\mathrm{~m}$ & 0.54 & 32.41 & 32.18 & -0.23 \\
\hline $22 a$ & 1.39 & $\mathrm{~m}$ & 1.56 & $\mathrm{~m}$ & 0.17 & & & \\
\hline $22 b$ & 1.32 & $\mathrm{~m}$ & 1.36 & $\mathrm{~m}$ & 0.04 & 27.92 & 28.4 & 0.48 \\
\hline 23 & 3.82 & $q 7.4$ & 4.00 & $\mathrm{~m}$ & 0.18 & 80.75 & 80.02 & -0.73 \\
\hline 24 & 5.45 & t 8.0 & 5.47 & t 8.0 & 0.02 & 77.42 & 77.13 & -0.29 \\
\hline 25 & 5.58 & dd $15.1,8.0$ & 5.64 & dd $15.1,8.0$ & 0.06 & 129.53 & 130.46 & 0.93 \\
\hline 26 & 6.70 & dd $15.1,11.0$ & 6.74 & dd $151,10.9$ & 0.04 & 130.26 & 129.44 & -0.82 \\
\hline 27 & 6.26 & d 11.0 & 6.30 & d 10.9 & 0.04 & 127.4 & 127.46 & 0.06 \\
\hline 28 & & & & & & 136.83 & 136.67 & -0.16 \\
\hline 29 & 5.88 & $\mathrm{~s}$ & 5.89 & s & 0.01 & 80.34 & 80.34 & 0.00 \\
\hline 30 & & & & & & 145.95 & 146.15 & 0.20 \\
\hline $31 a$ & 1.96 & $\mathrm{~m}$ & 1.95 & $\mathrm{~m}$ & -0.01 & 3236 & 3238 & 002 \\
\hline $31 \mathrm{~b}$ & 1.91 & $\mathrm{~m}$ & 1.99 & $\mathrm{~m}$ & 0.08 & 32.36 & 32.38 & 0.02 \\
\hline 32 & $1.42-1.30$ & $\mathrm{~m}$ & 1.56 & $\mathrm{~m}$ & 0.2 & 30.14 & 30.1 & -0.04 \\
\hline 33 & $1.23-1.16$ & $\mathrm{~m}$ & 1.24 & $\mathrm{~m}$ & 0.04 & 22.72 & 22.64 & -0.08 \\
\hline 34 & 0.83 & d 7.4 & 0.86 & d 7.4 & 0.03 & 14.15 & 14.07 & -0.08 \\
\hline 35 & 0.67 & d 6.2 & 0.72 & d 6.4 & 0.05 & 15.58 & 15.71 & 0.13 \\
\hline $36 a$ & 5.32 & $\mathrm{~s}$ & 5.19 & s & -0.13 & & & \\
\hline $36 \mathrm{~b}$ & 5.02 & $\mathrm{~s}$ & 5.00 & $\mathrm{~s}$ & -0.02 & 114.71 & 115.27 & 0.56 \\
\hline 37 & 1.81 & $\mathrm{~s}$ & 1.77 & brs & -0.04 & 17.02 & 15.09 & -1.93 \\
\hline 38 & 1.06 & d 6.9 & 1.00 & d 7.0 & -0.06 & 16.17 & 15.39 & -0.78 \\
\hline 39 & 0.97 & d 7.0 & 1.00 & d 7.0 & 0.03 & 16.25 & 16.27 & 0.02 \\
\hline 40 & 1.66 & $\mathrm{~s}$ & 1.72 & $\mathrm{~s}$ & 0.06 & 13.09 & 13.06 & -0.03 \\
\hline $41 a$ & 5.17 & $\mathrm{~s}$ & 5.19 & $\mathrm{~s}$ & 0.02 & & & \\
\hline $41 \mathrm{~b}$ & 4.95 & $\mathrm{~s}$ & 4.97 & $\mathrm{~s}$ & 0.02 & 111.45 & 111.36 & -0.09 \\
\hline 42 & & & & & & 168.96 & 168.91 & -0.05 \\
\hline 43 & 1.68 & $\mathrm{~s}$ & 1.74 & $\mathrm{~s}$ & 0.06 & 20.63 & 20.59 & -0.04 \\
\hline
\end{tabular}

[a] solvent $\mathrm{C}_{6} \mathrm{D}_{6}$. [b] Concentration: $0.4 \mathrm{mg}$ in $200 \mu \mathrm{L} .600 \mathrm{Mhz}$. Reference solvent peak $=7.16 \mathrm{ppm}$ [c] Concentration: unknown. $600 \mathrm{MHz}$. Reference solvent peak = unknown. [d] Concentration: $0.4 \mathrm{mg}$ in $200 \mu \mathrm{L}$. $171 \mathrm{MHz}$ (700 Mhz). Reference solvent peak = $128.06 \mathrm{ppm}$. [e] Corrected values and attributions toward original report, according a personal communication with Takaaki Kubota. ${ }^{[35,36]}$ 


\section{III-References}

[1] G. H. L. Nefkens, J. W. J. F. Thuring, B. Zwanenburg, Synthesis 1997, 1997, 290-292.

[2] D. A. Evans, L. Kværnø, T. B. Dunn, A. Beauchemin, B. Raymer, J. A. Mulder, E. J. Olhava, M. Juhl, K. Kagechika, D. A. Favor, J. Am. Chem. Soc. 2008, 130, 16295-16309.

[3] C. R. Schmid, J. D. Bryant, Org. Synth. 1995, 72, 6-13.

[4] M. Skulski, D. L. Garmaise, A. F. McKay, Can. J. Chem. 1956, 34, 815-820.

[5] G. Jalce, X. Franck, B. Figadère, Eur. J. Org. Chem. 2009, 2009, 378-386.

[6] M. O. Albers, N. J. Coville, T. V. Ashworth, E. Singleton, H. E. Swanepoel, Journal of Organometallic Chemistry 1980, 199, 55-62.

[7] P. Fristrup, T. Jensen, J. Hoppe, P.-O. Norrby, Chem. Eur. J. 2006, 12, 5352-5360.

[8] W. Zhang, A. Basak, Y. Kosugi, Y. Hoshino, H. Yamamoto, Angew. Chem. Int. Ed. 2005, 44, 43894391.

[9] A. U. Barlan, W. Zhang, H. Yamamoto, Tetrahedron 2007, 63, 6075-6087.

[10] P. A. Wender, A. J. Schrier, J. Am. Chem. Soc. 2011, 133, 9228-9231.

[11] J. Boukouvalas, in Encyclopedia of Reagents for Organic Synthesis, John Wiley \& Sons, Ltd, 2001.

[12] S. G. Levy, V. Jacques, K. L. Zhou, S. Kalogeropoulos, K. Schumacher, J. C. Amedio, J. E. Scherer, S. R. Witowski, R. Lombardy, K. Koppetsch, Org. Process Res. Dev. 2009, 13, 535-542.

[13] S. Zhang, D. Zhang, L. S. Liebeskind, J. Org. Chem. 1997, 62, 2312-2313.

[14] I. Bitter, L. Töke, Z. Bende, E. Kárpáti-Ádám, R. Soós, Tetrahedron 1984, 40, 4501-4505.

[15] E. A. Shapiro, A. V. Kalinin, B. I. Ugrak, O. M. Nefedov, J. Chem. Soc., Perkin Trans. 2 1994, 709-713.

[16] G.-H. Fang, Z.-J. Yan, J. Yang, M.-Z. Deng, Synthesis 2006, 2006, 1148-1154.

[17] K. C. Nicolaou, A. D. Piscopio, P. Bertinato, T. K. Chakraborty, N. Minowa, K. Koide, Chem. Eur. J. 1995, 1, 318-333.

[18] E. J. Corey, H. A. Kirst, Tetrahedron Letters 1968, 9, 5041-5043.

[19] H. T. Dieck, H. Friedel, Journal of Organometallic Chemistry 1968, 14, 375-385.

[20] K. Hayashi, J. Iyoda, I. Shiihara, Journal of Organometallic Chemistry 1967, 10, 81-94.

[21] J. R. Struble, S. J. Lee, M. D. Burke, Tetrahedron 2010, 66, 4710-4718.

[22] E. J. Corey, R. H. Wollenberg, J. Org. Chem. 1975, 40, 3788-3789.

[23] T. Saito, H. Fuwa, M. Sasaki, Tetrahedron 2011, 67, 429-445.

[24] S. Itsuno, M. Nakano, K. Miyazaki, H. Masuda, K. Ito, A. Hirao, S. Nakahama, J. Chem. Soc., Perkin Trans. 1 1985, 0, 2039-2044.

[25] G. Jalce, M. Seck, X. Franck, R. Hocquemiller, B. Figadère, J. Org. Chem. 2004, 69, 3240-3241.

[26] A. Darwish, A. Lang, T. Kim, J. M. Chong, Org. Lett. 2008, 10, 861-864.

[27] H. Oda, T. Kobayashi, M. Kosugi, T. Migita, Tetrahedron 1995, 51, 695-702.

[28] C. Marti, E. M. Carreira, J. Am. Chem. Soc. 2005, 127, 11505-11515.

[29] N. Dussart, H. V. Trinh, D. Gueyrard, Org. Lett. 2016, 18, 4790-4793.

[30] S. Mahapatra, R. G. Carter, Angew. Chem. Int. Ed. 2012, 51, 7948-7951.

[31] G. Valot, C. S. Regens, D. P. O'Malley, E. Godineau, H. Takikawa, A. Fürstner, Angewandte Chemie 2013, 125, 9713-9717.

[32] J. Kobayashi, M. Tsuda, M. Ishibashi, H. Shigemori, T. Yamasu, H. Hirota, T. Sasaki, J. Antibiot. 1991, 44, 1259-1261.

[33] G. Valot, D. Mailhol, C. S. Regens, D. P. O'Malley, E. Godineau, H. Takikawa, P. Philipps, A. Fürstner, Chem. Eur. J. 2015, 21, 2398-2408.

[34] S. Mahapatra, R. G. Carter, J. Am. Chem. Soc. 2013, 135, 10792-10803.

[35] T. Kubota, Y. Sakuma, M. Tsuda, J. Kobayashi, Marine Drugs 2004, 2, 83-87.

[36] Kubota, T. Personal Communication. NMR Data in Marine Drugs ${ }^{8}{ }^{1} \mathrm{H}: 2.17(2 \mathrm{H}, \mathrm{m}, \mathrm{H}-2 / \mathrm{H}-17), 2.35(2 \mathrm{H}$, $m, H-14 / H-19), 2.50(1 H, d d, J=8.2,14.9 \mathrm{~Hz}, \mathrm{H}-2), 2.59(1 \mathrm{H}, d d, J=9.5,15.7 \mathrm{~Hz}, \mathrm{H}-14), 2.75(1 \mathrm{H}, d d$, $J=9.5,15.7 \mathrm{~Hz}, \mathrm{H}-19), 3.00(1 \mathrm{H}, d d, J=8.5,17.6 \mathrm{~Hz}, \mathrm{H}-2){ }^{13} \mathrm{C}: 39.13(\mathrm{C}-14), 45.83(\mathrm{C}-19), 48.72(\mathrm{C}-$ 2), 140.35 (C-9), 146.07 (C-11). Corrected NMR Data ${ }^{1} \mathrm{H}: 2.17$ (2H, $\left.m, \mathrm{H}-17 / \mathrm{H}-19\right), 2.35$ (1H, $\left.\mathrm{m}, \mathrm{H}-2\right)$, $2.43(1 \mathrm{H}, \mathrm{m}, \mathrm{H}-14), 2.50(1 \mathrm{H}, \mathrm{Dd}, \mathrm{J}=8.2,14.9 \mathrm{~Hz}, \mathrm{H}-19), 2.59(1 \mathrm{H}, \mathrm{dd}, \mathrm{J}=9.5,15.7 \mathrm{~Hz}, \mathrm{H}-2), 2.75$ $(1 \mathrm{H}, \mathrm{dd}, \mathrm{J}=9.5,15.7 \mathrm{~Hz}, \mathrm{H}-14), 3.00(1 \mathrm{H}, \mathrm{Dd}, \mathrm{J}=8.5,17.6 \mathrm{~Hz}, \mathrm{H}-17){ }^{13} \mathrm{C}: 39.13(\mathrm{C}-2), 45.83(\mathrm{C}-14)$, 48.72 (C-19), 140.35 (C-11), 146.07 (C-9).; 2021. 
SUPPORTING INFORMATION

IV-Copy of ${ }^{1} \mathrm{H}$ and ${ }^{13} \mathrm{C}\left\{{ }^{1} \mathrm{H}\right\}$ NMR spectra 


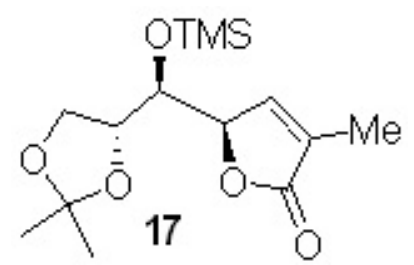

${ }^{1} \mathrm{H}$ NMR $300 \mathrm{MHz}, \mathrm{CDCl}_{3}$

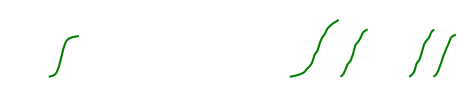




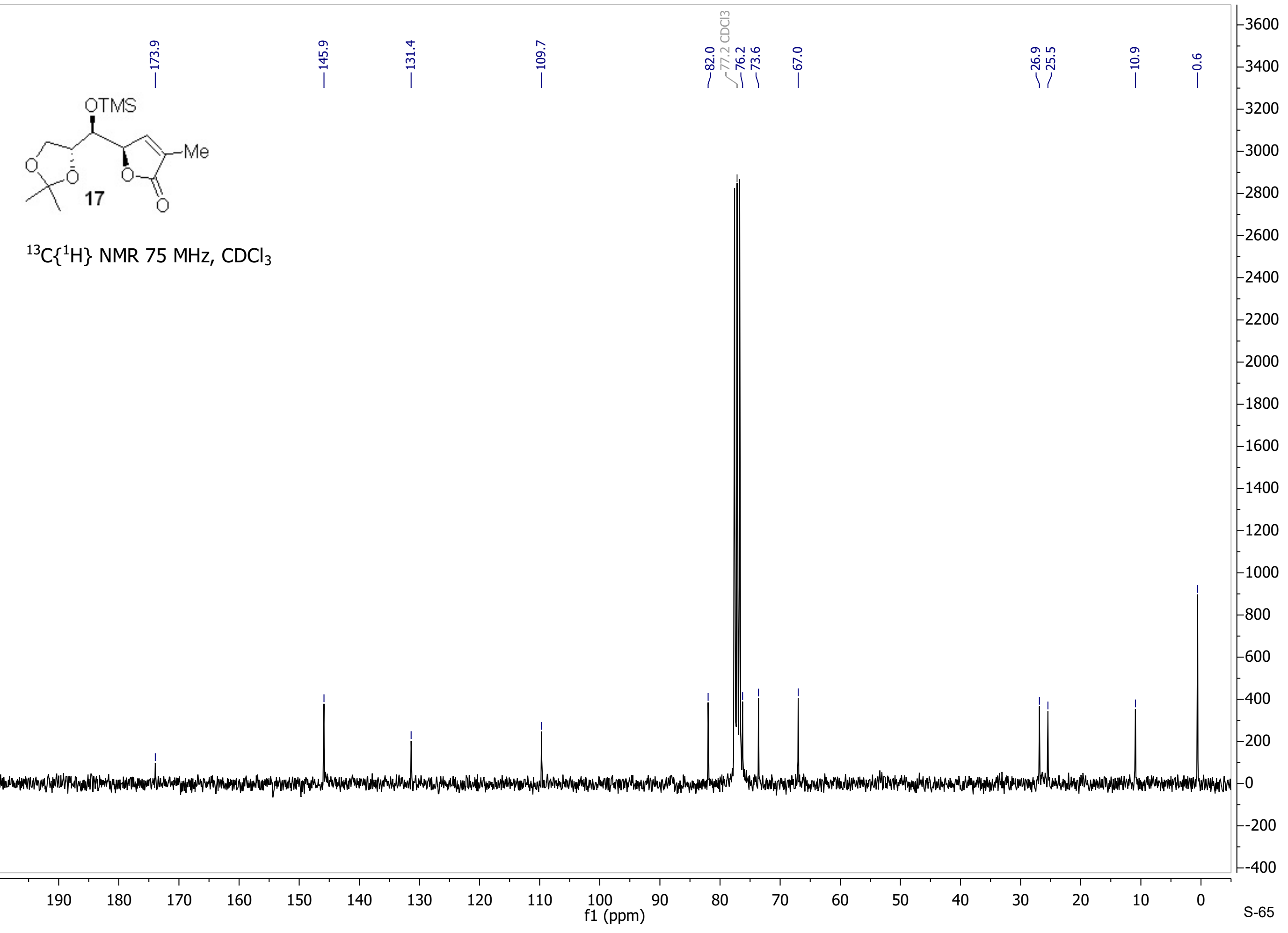




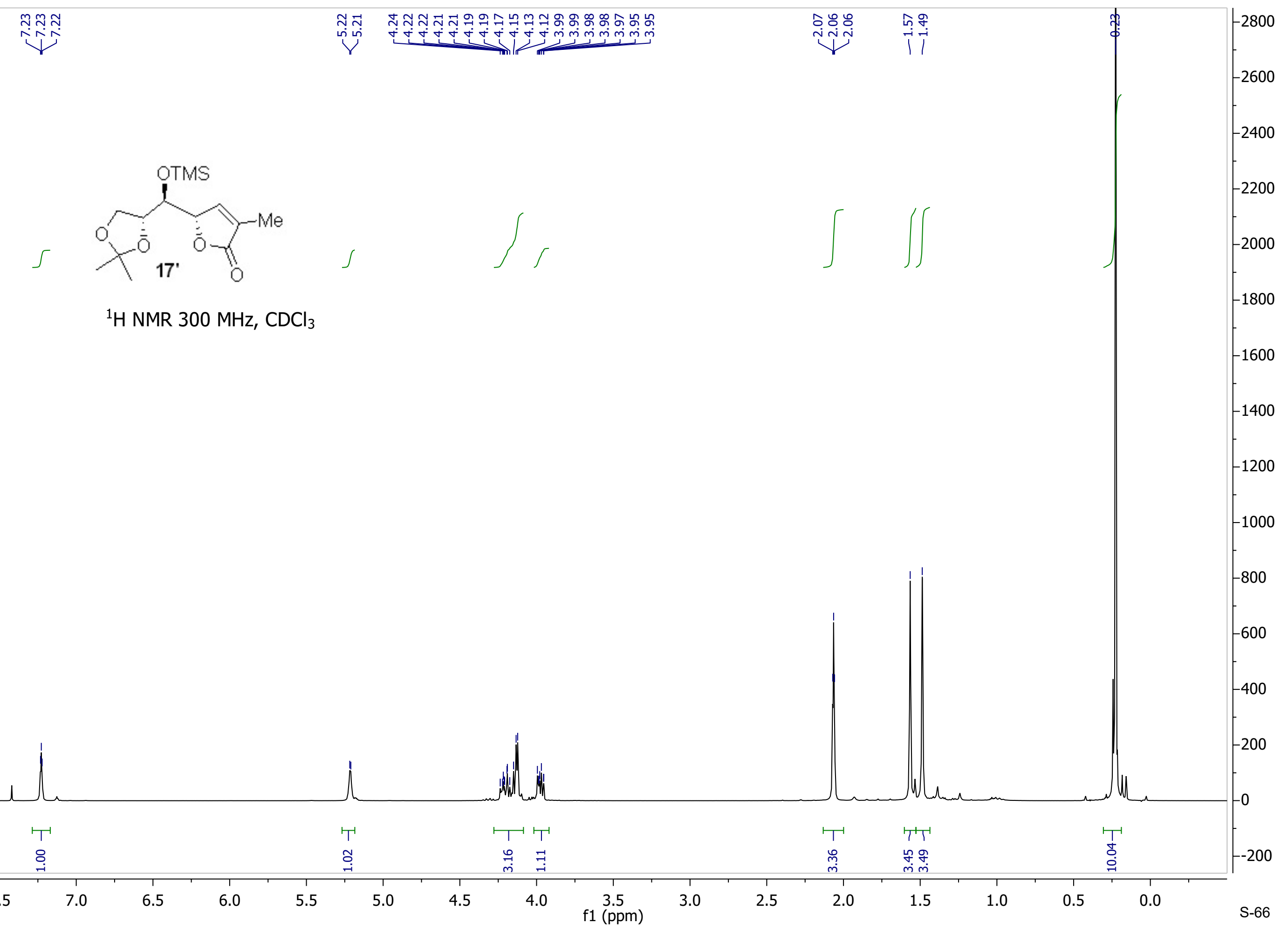




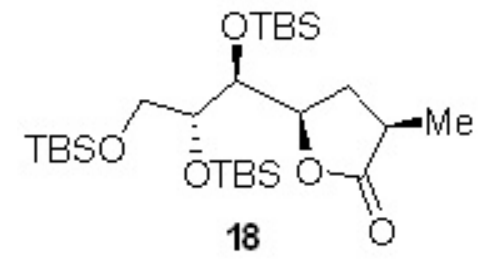

${ }^{13} \mathrm{C}\left\{{ }^{1} \mathrm{H}\right\}$ NMR $75 \mathrm{MHz}, \mathrm{CDCl}_{3}$ 


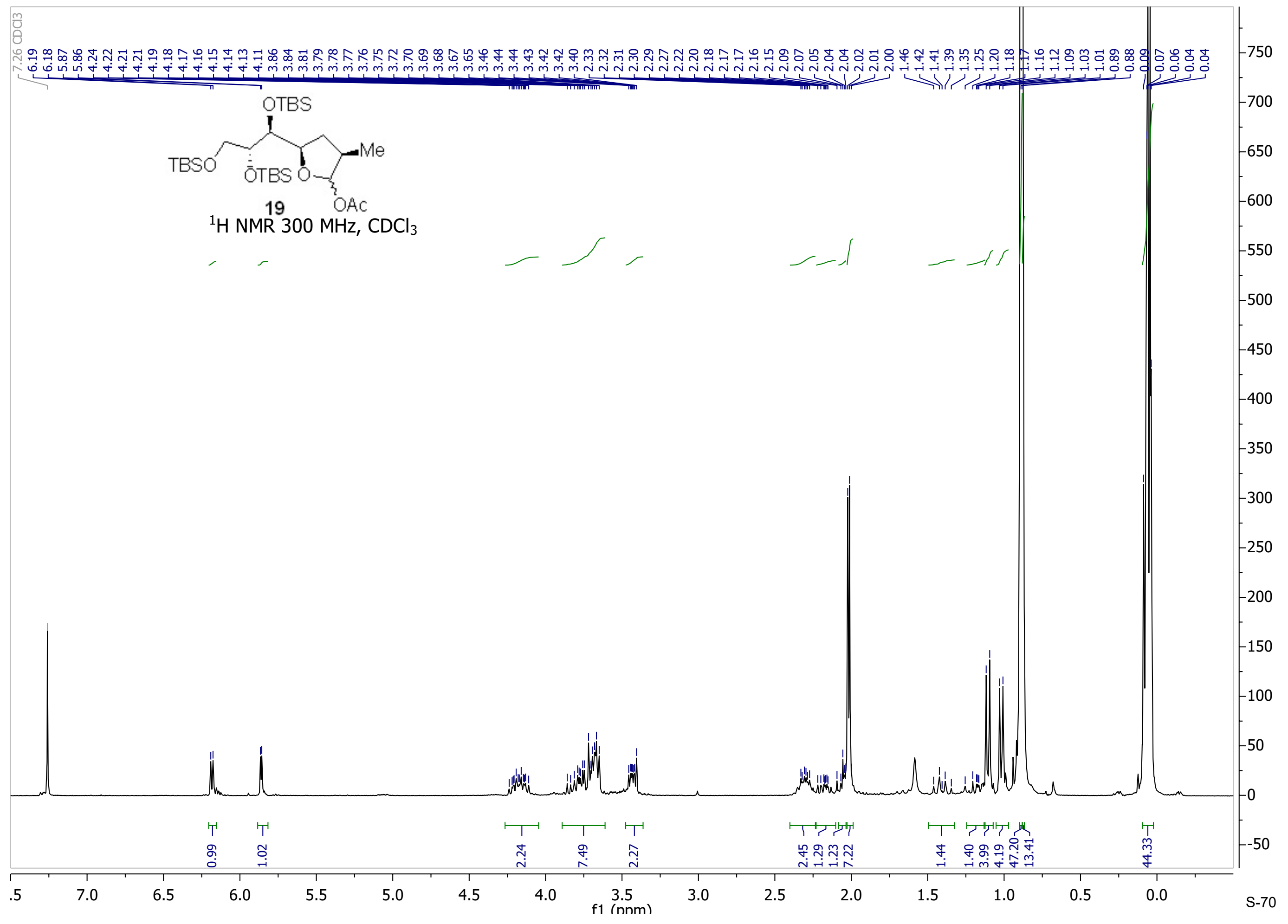




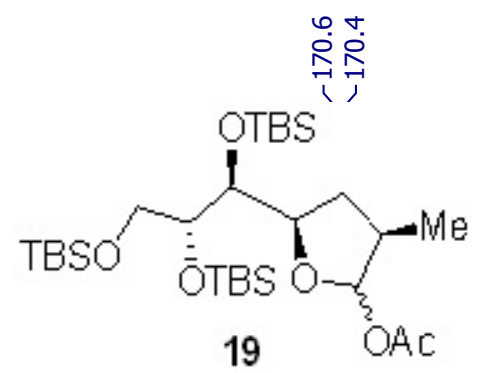

${ }^{13} \mathrm{C}\left\{{ }^{1} \mathrm{H}\right\}$ NMR $75 \mathrm{MHz}, \mathrm{CDCl}_{3}$ 


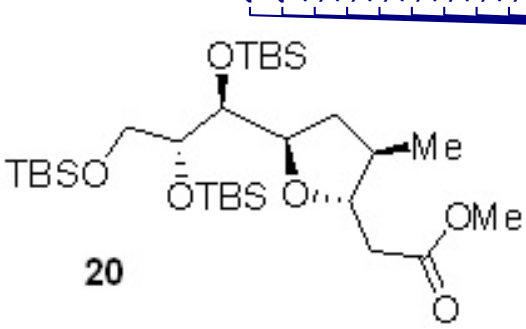

${ }^{1} \mathrm{H}$ NMR $300 \mathrm{MHz}, \mathrm{CDCl}_{3}$

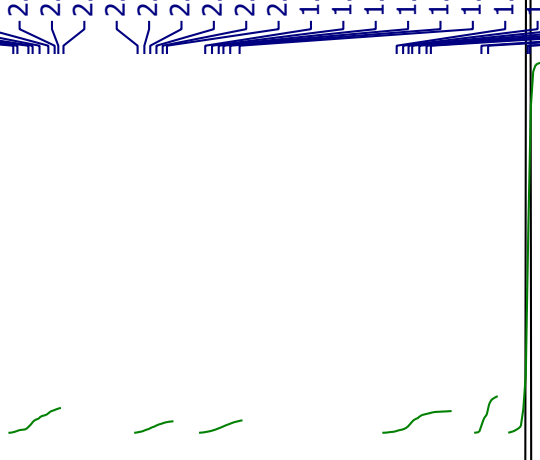

$\mid$

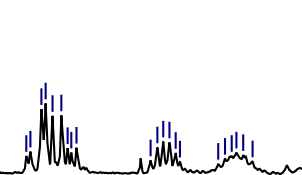

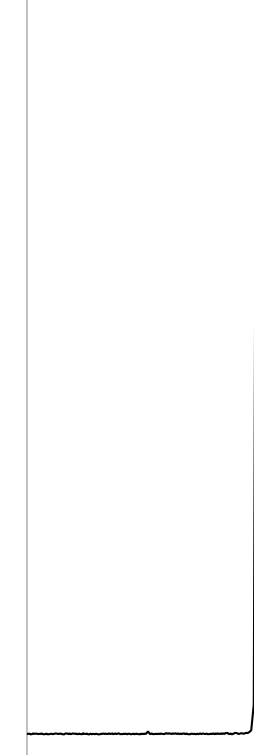

$\mapsto \mapsto \mapsto$

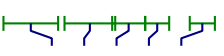

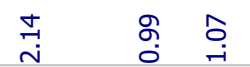

$\longmapsto$ †丁

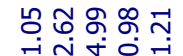

7.5

2.5

2.

$2.0 \quad 1.5$

$+$ 

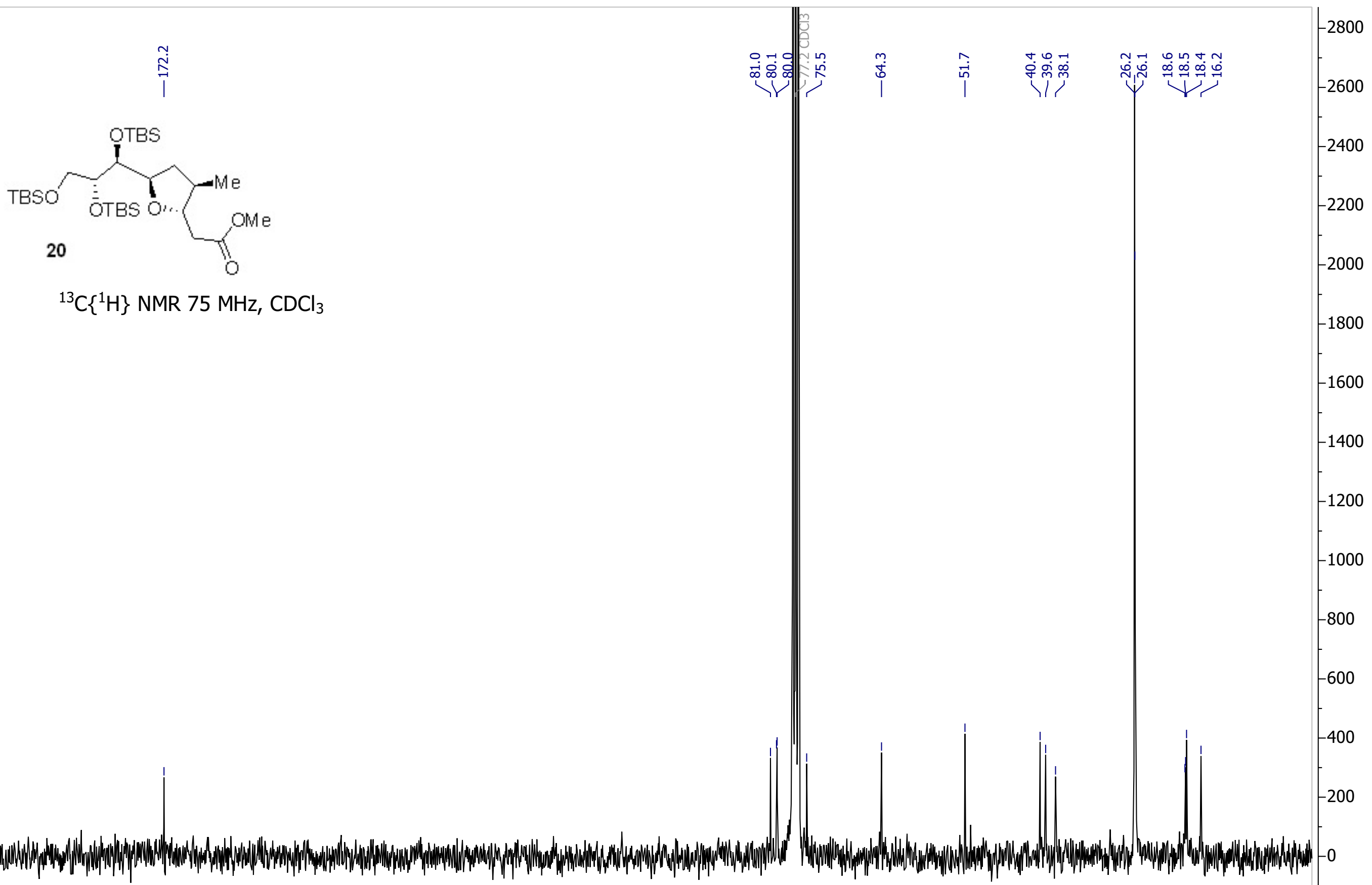

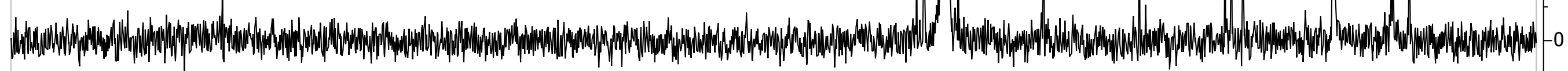

${ }^{13} \mathrm{C}\left\{{ }^{1} \mathrm{H}\right\}$ NMR $75 \mathrm{MHz}, \mathrm{CDCl}_{3}$

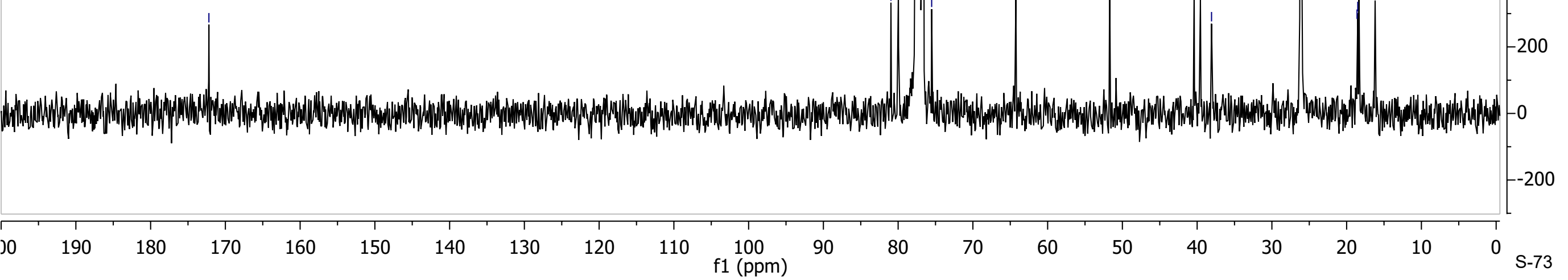


ஜ

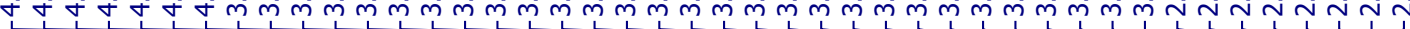

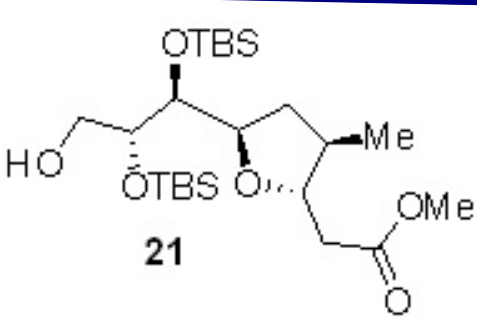

${ }^{1} \mathrm{H}$ NMR $300 \mathrm{MHz}, \mathrm{CDCl}_{3}$

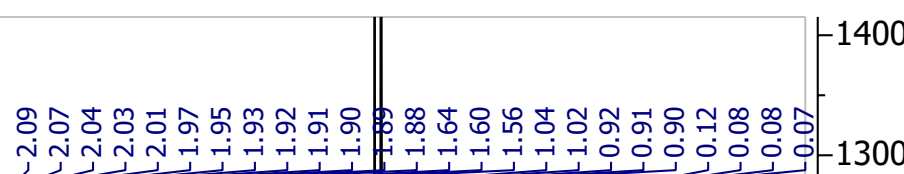

$-1400$
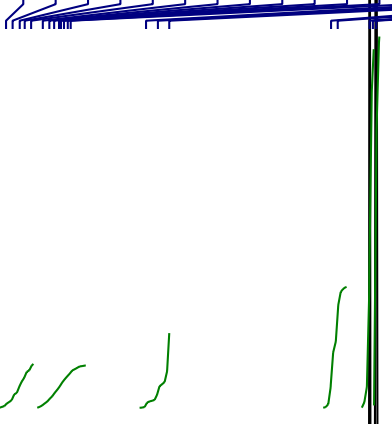

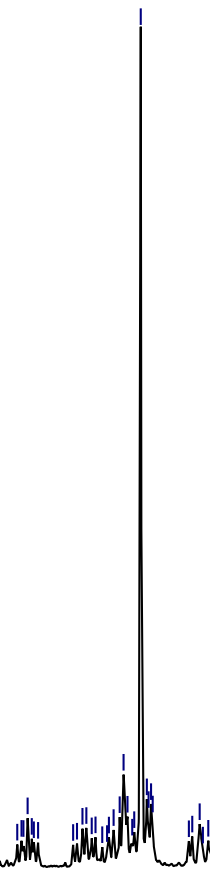

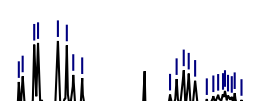




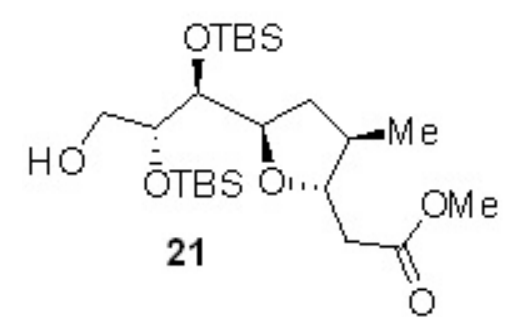

${ }^{13} \mathrm{C}\left\{{ }^{1} \mathrm{H}\right\}$ NMR $75 \mathrm{MHz} \mathrm{CDCl}_{3}$

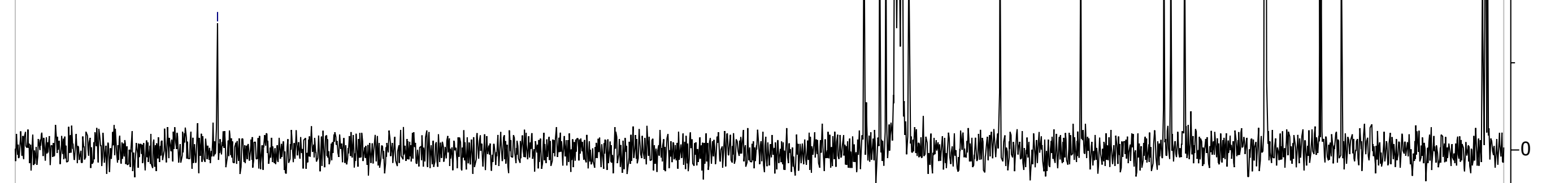




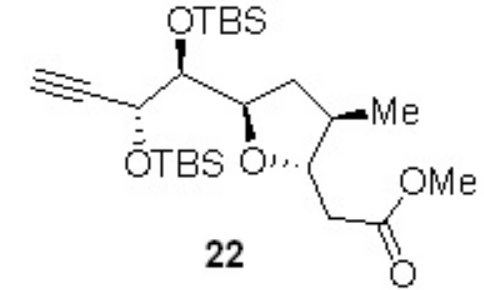

${ }^{1} \mathrm{H} \mathrm{NMR} 300 \mathrm{MHz}, \mathrm{CDCl}_{3}$

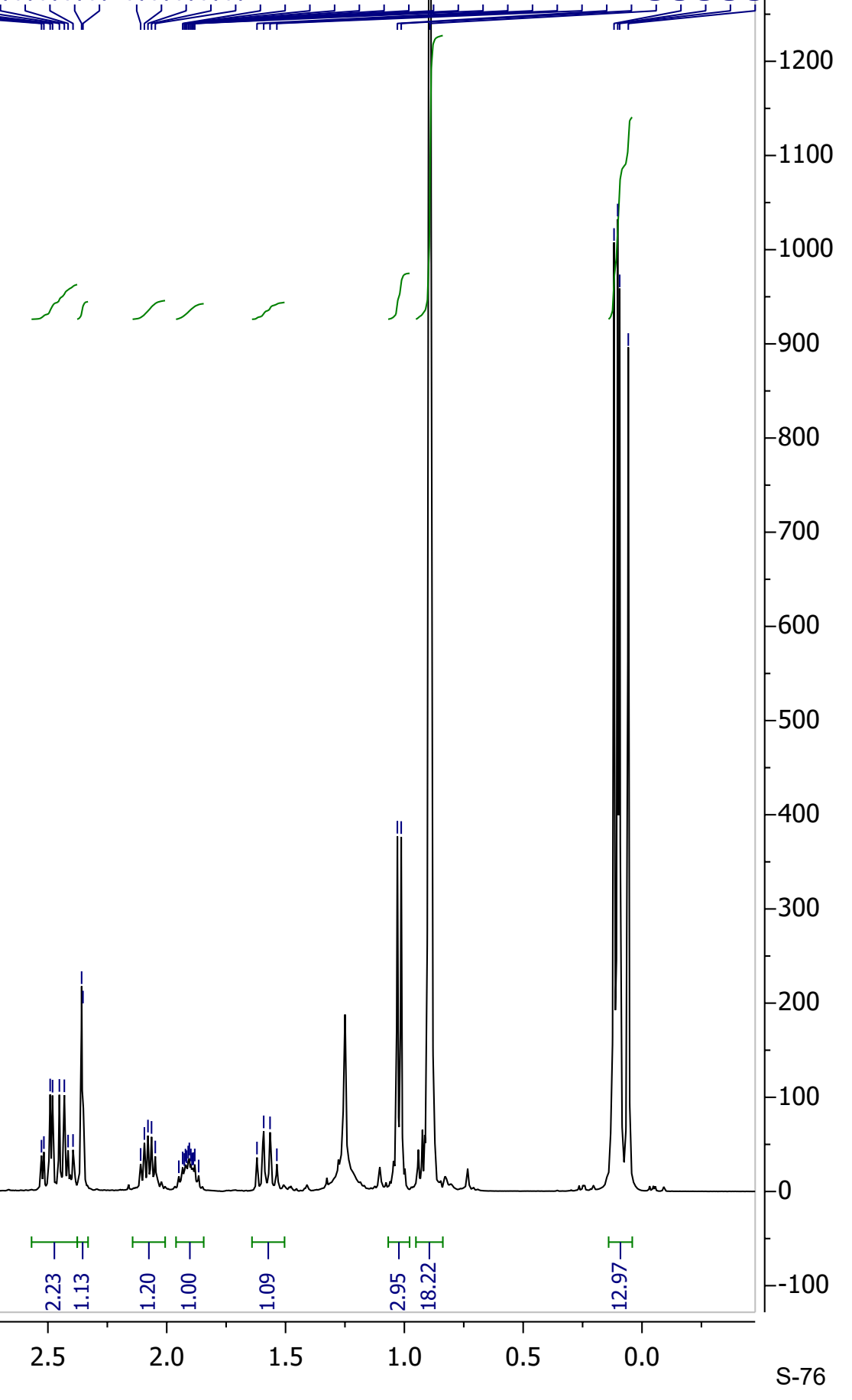



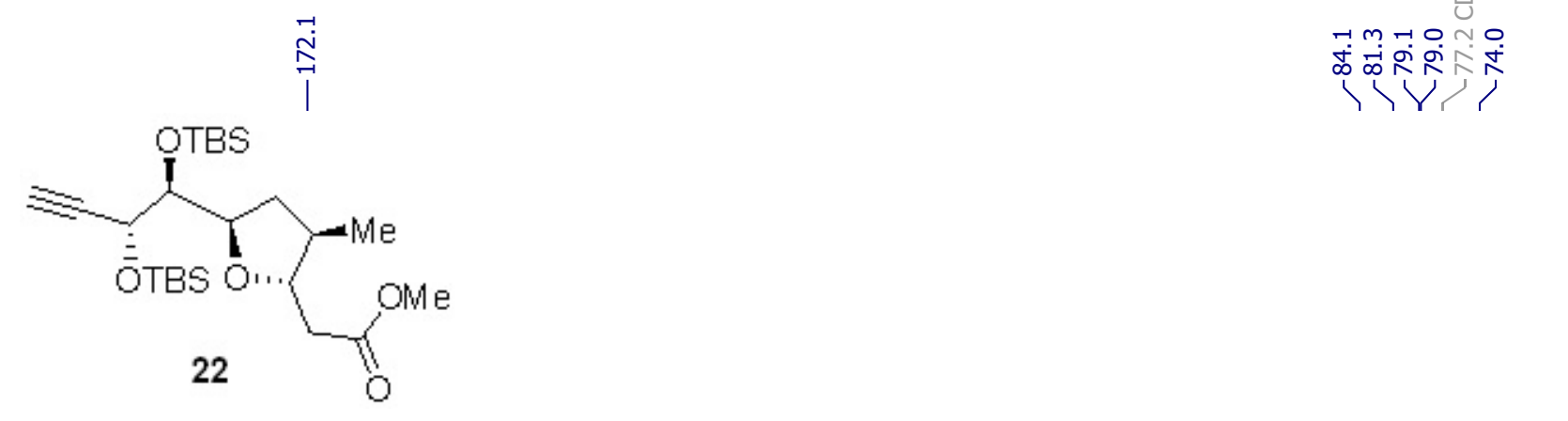

in

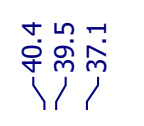

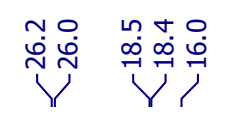

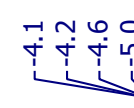

${ }^{13} \mathrm{C}\left\{{ }^{1} \mathrm{H}\right\}$ NMR $75 \mathrm{MHz}, \mathrm{CDCl}_{3}$ 


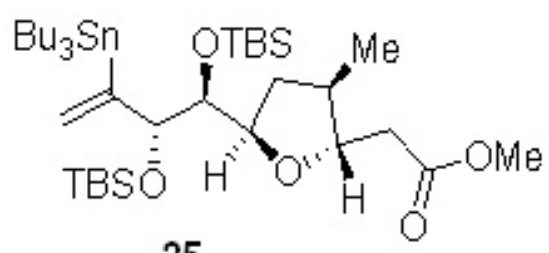

25

${ }^{1} \mathrm{H}$ NMR $300 \mathrm{MHz}, \mathrm{CDCl}_{3}$

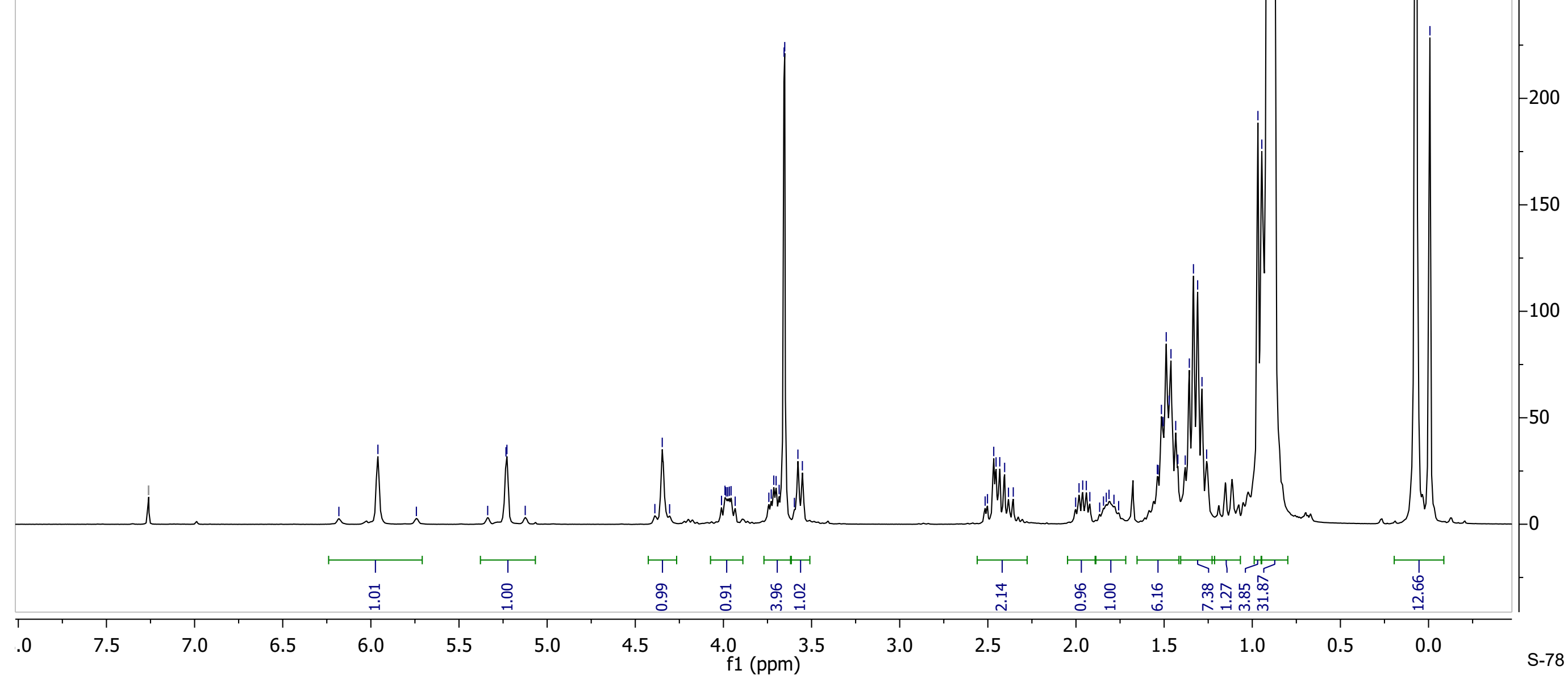




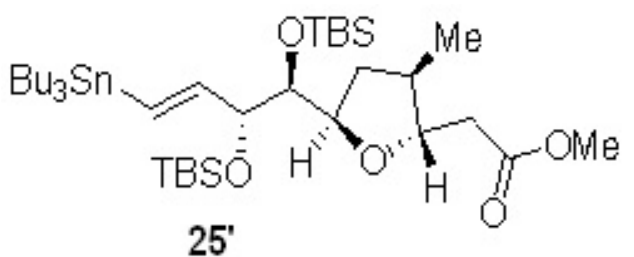

$25^{\prime}$

${ }^{1} \mathrm{H}$ NMR $300 \mathrm{MHz}, \mathrm{CDCl}_{3}$

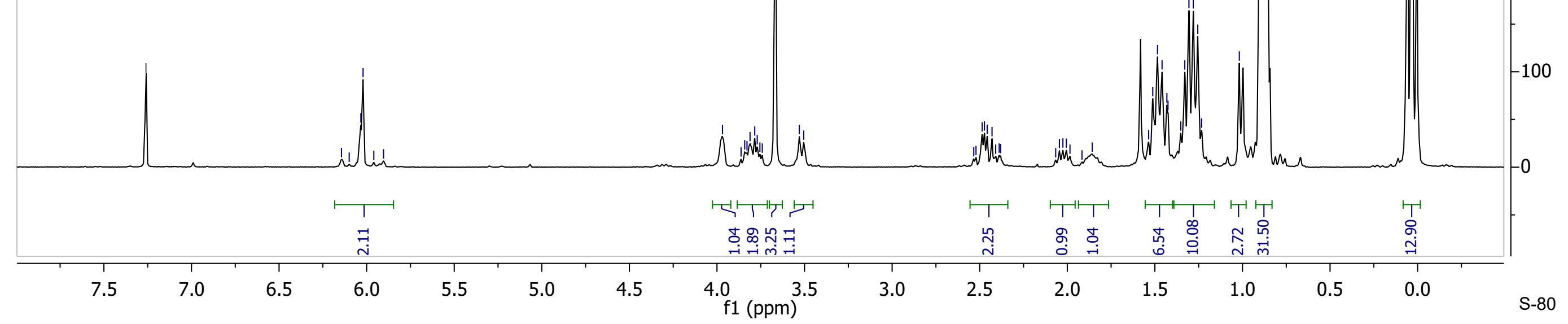




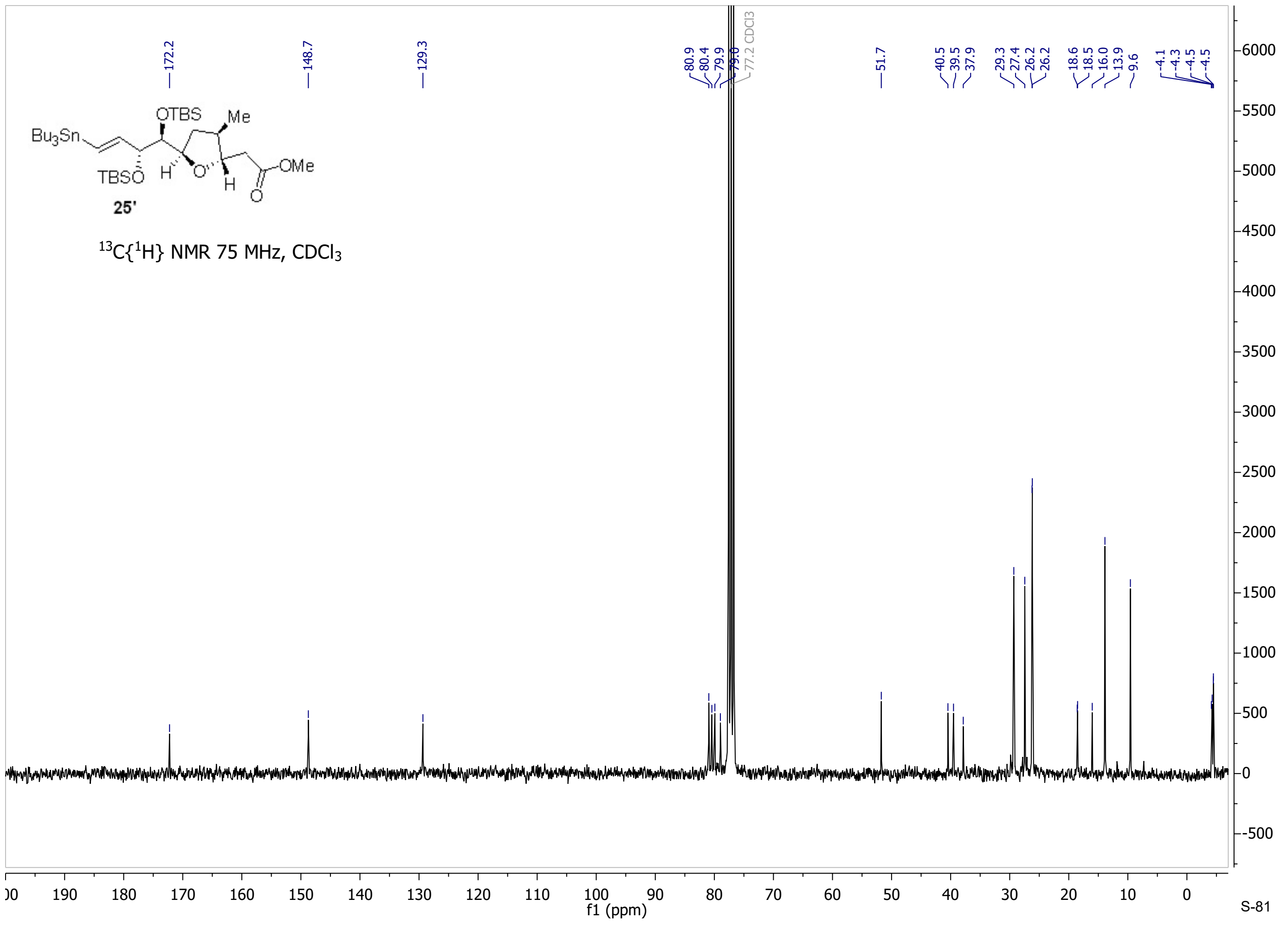




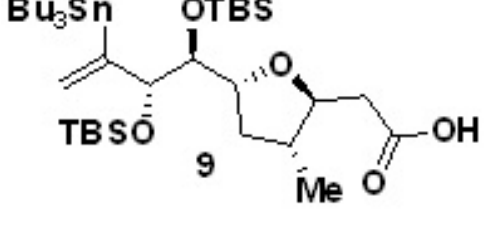

${ }^{1} \mathrm{H}$ NMR $300 \mathrm{MHz}, \mathrm{CDCl}_{3}$

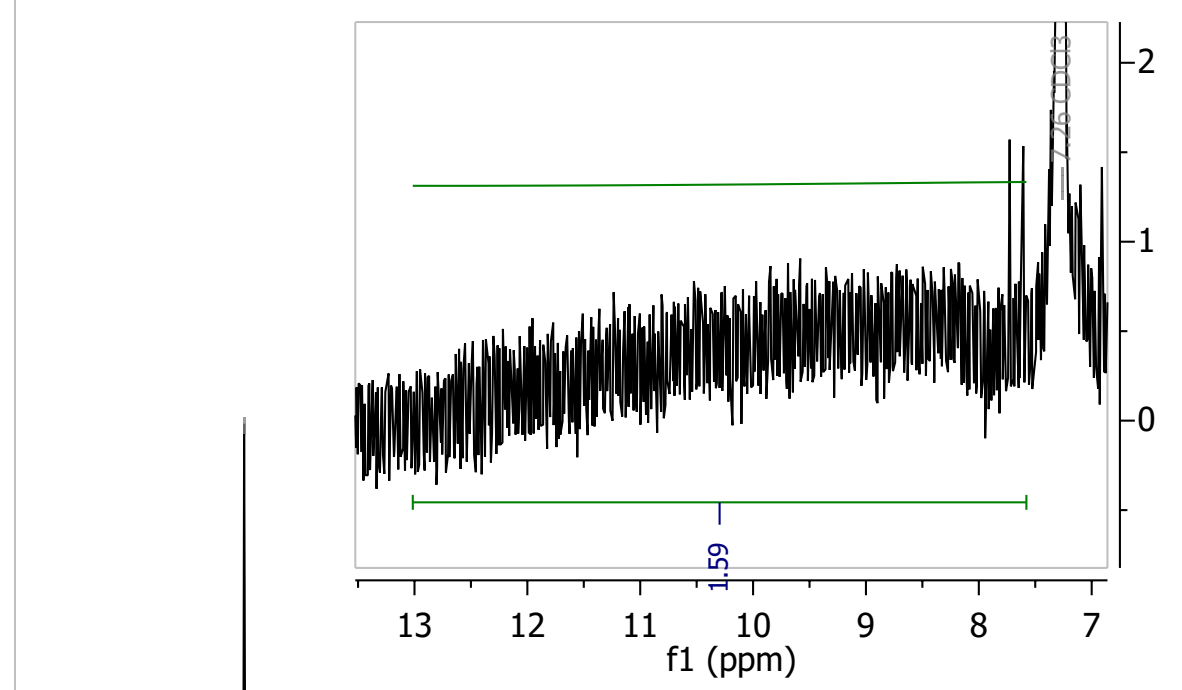

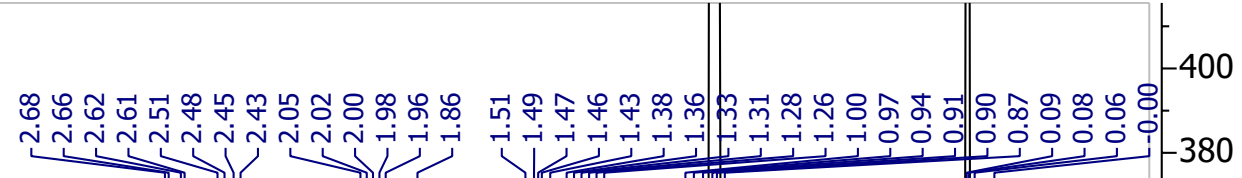

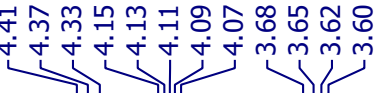




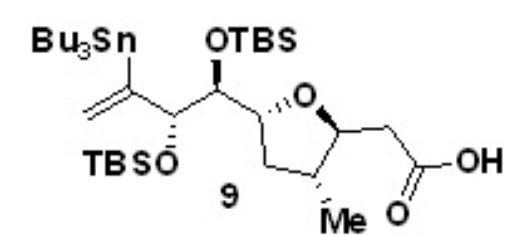

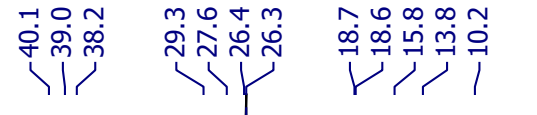

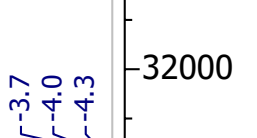

${ }^{13} \mathrm{C}\left\{{ }^{1} \mathrm{H}\right\}$ NMR $75 \mathrm{MHz}, \mathrm{CDCl}_{3}$ 
$\mathrm{Me} \int_{26}^{\mathrm{OH}}$

${ }^{1} \mathrm{H}$ NMR $300 \mathrm{MHz}, \mathrm{CDCl}_{3}$ 
Me $\int_{26}^{\mathrm{OH}}$

${ }^{13} \mathrm{C}\left\{{ }^{1} \mathrm{H}\right\}$ NMR $75 \mathrm{MHz}, \mathrm{CDCl}_{3}$
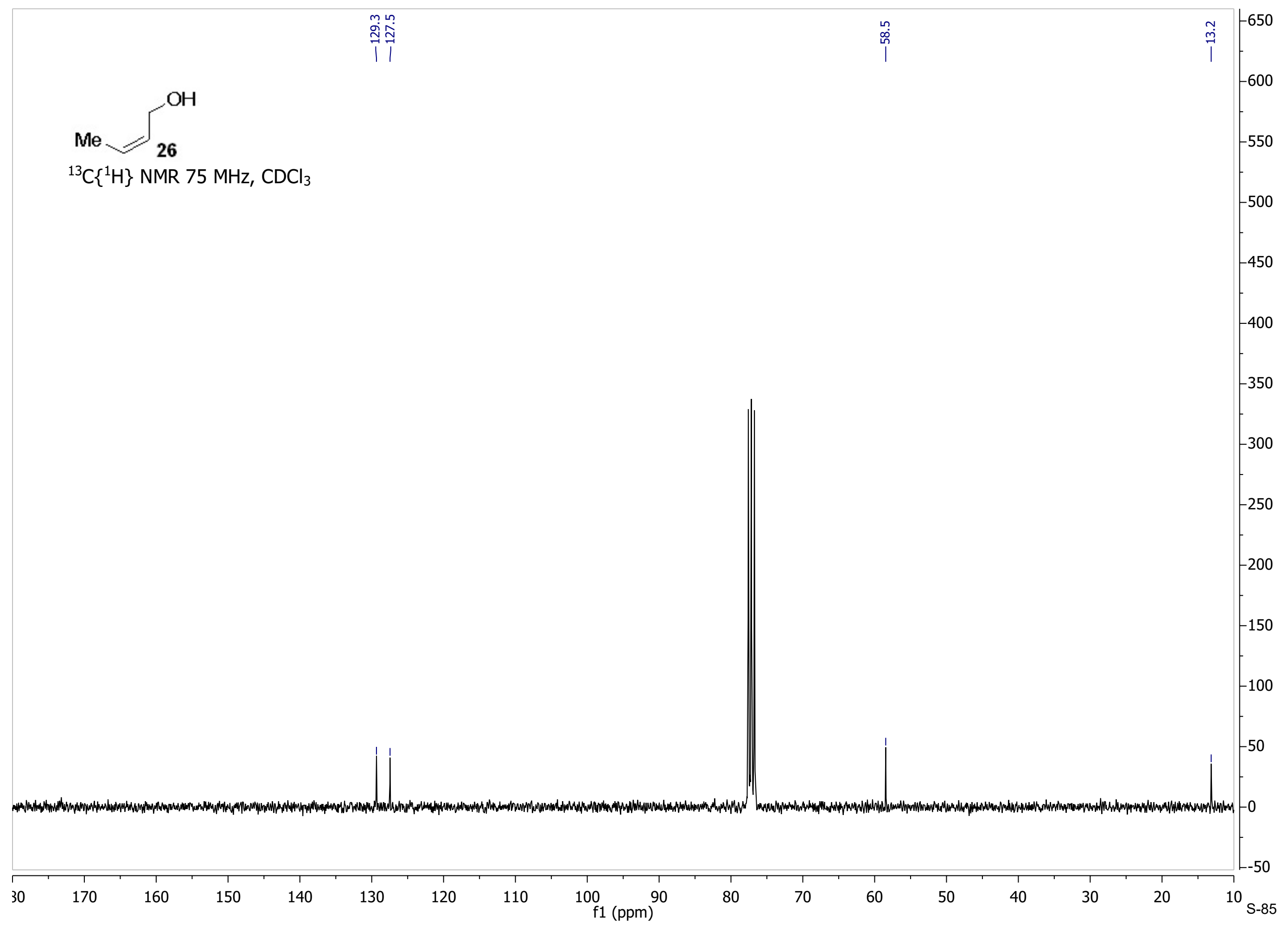


$$
M e \int_{i \overline{\bar{O}}}^{O H} 28
$$

${ }^{1} \mathrm{H}$ NMR $300 \mathrm{MHz}, \mathrm{CDCl}_{3}$

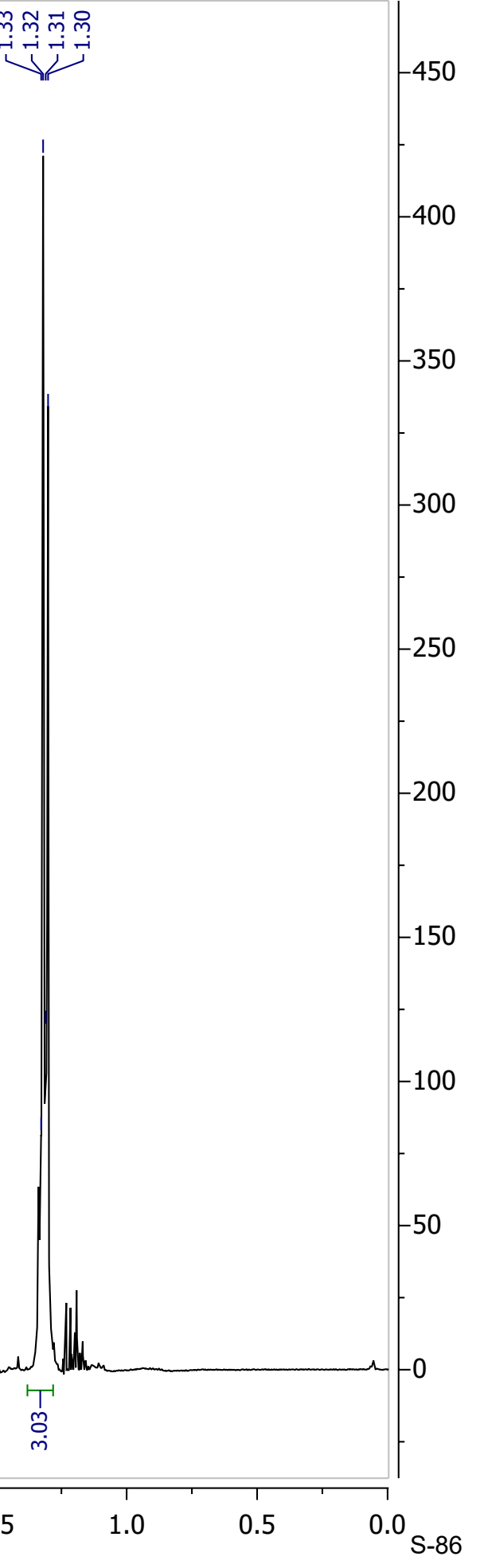


$\mathrm{Me} \int_{\overline{\bar{O}}}^{\mathrm{OH}} 28$

${ }^{13} \mathrm{C}\left\{{ }^{1} \mathrm{H}\right\}$ NMR $75 \mathrm{MHz}^{\mathrm{CDCl}} 3$ 


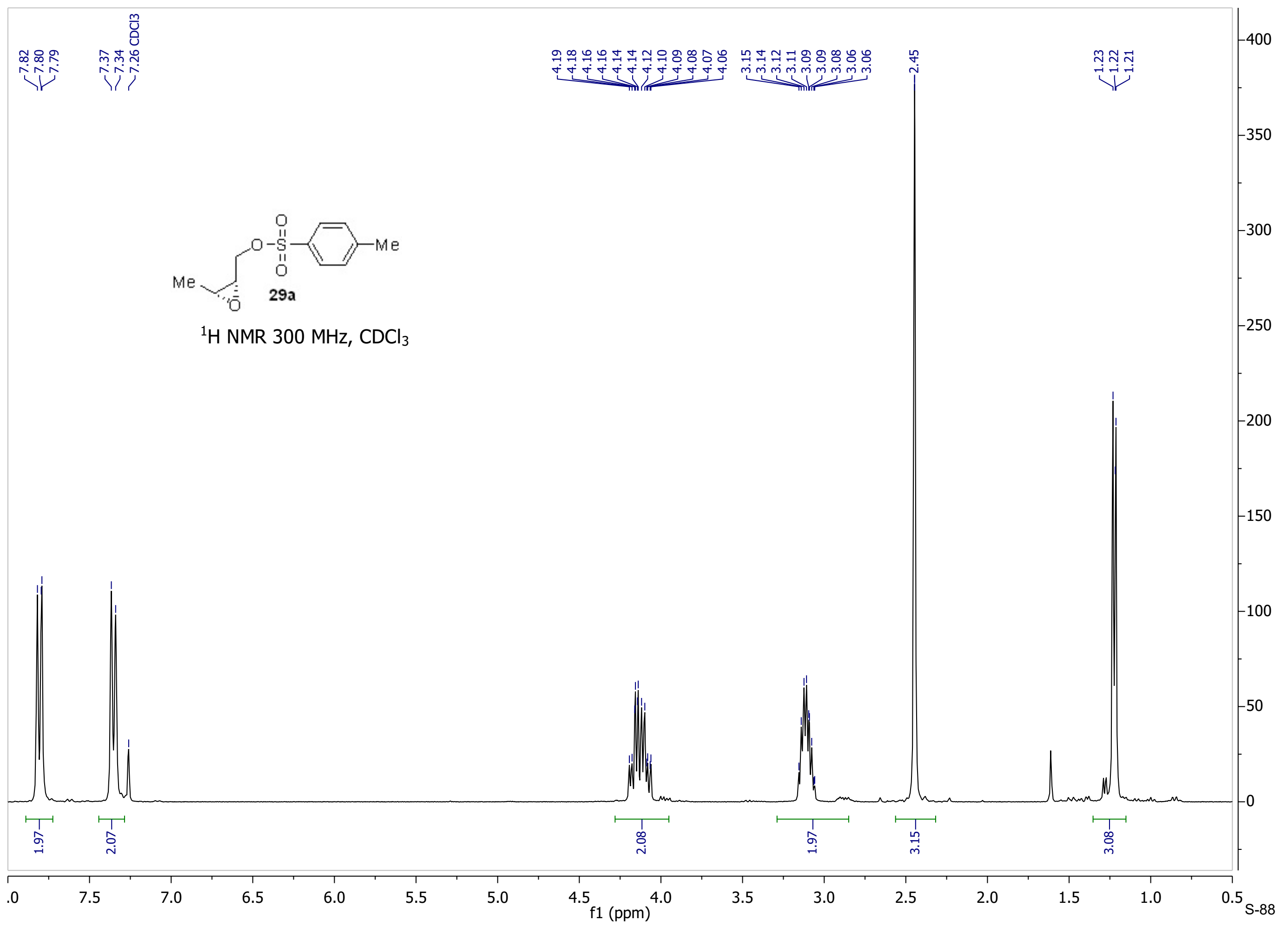




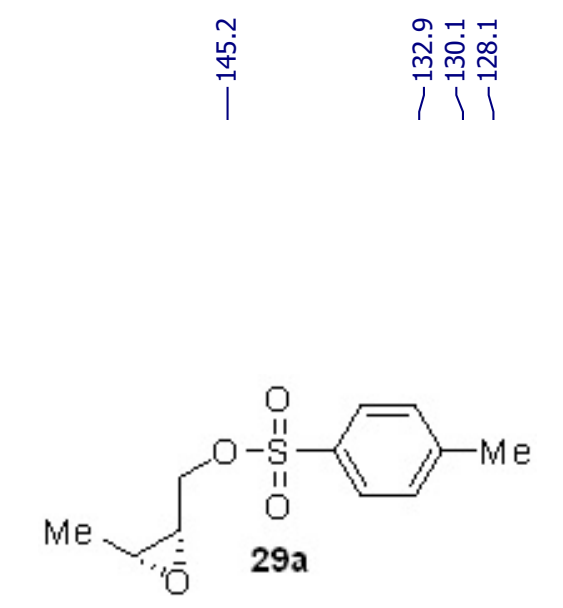

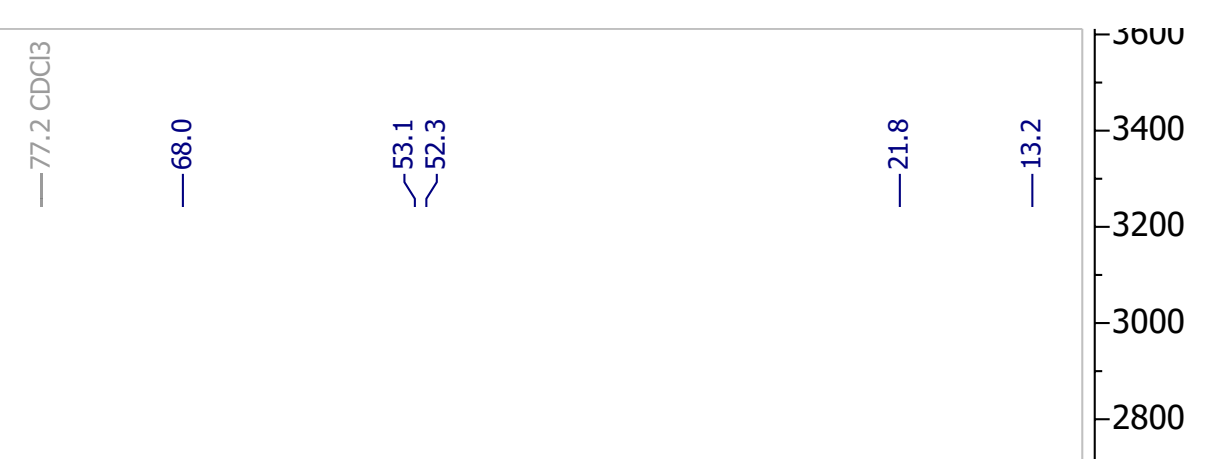

${ }^{13} \mathrm{C}\left\{{ }^{1} \mathrm{H}\right\}$ NMR $75 \mathrm{MHz}, \mathrm{CDCl}_{3}$ 

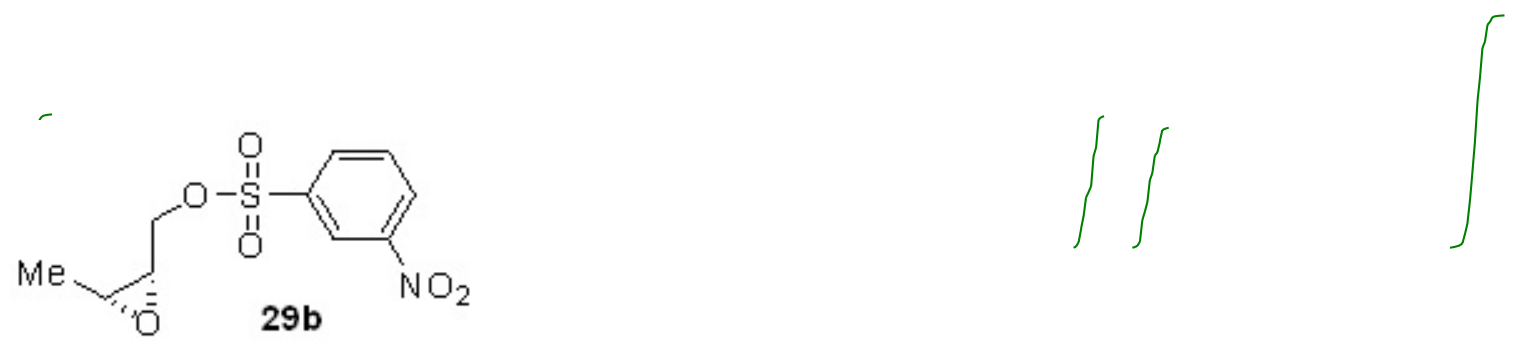

${ }^{1} \mathrm{H}$ NMR $300 \mathrm{MHz}, \mathrm{CDCl}_{3}$
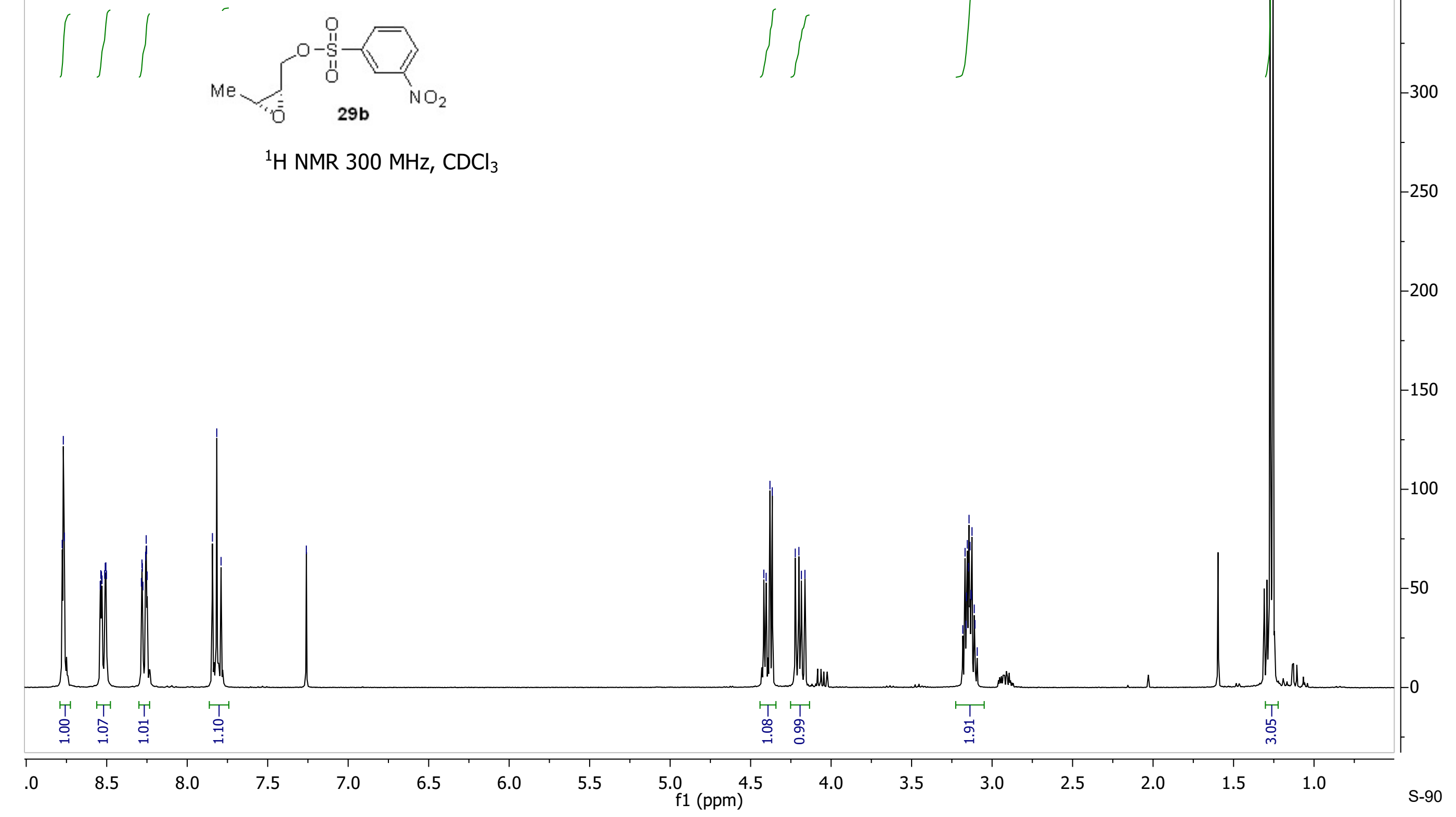


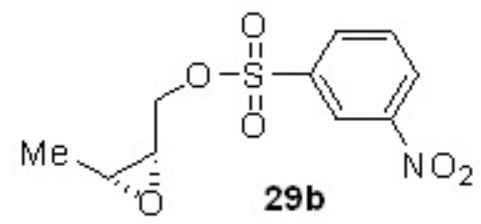

${ }^{13} \mathrm{C}\left\{{ }^{1} \mathrm{H}\right\}$ NMR $75 \mathrm{MHz}, \mathrm{CDCl}_{3}$ 


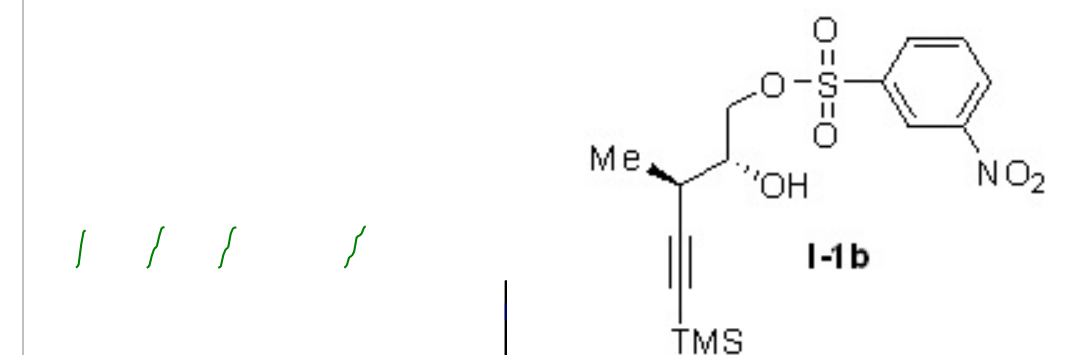

TMS

${ }^{1} \mathrm{H}$ NMR $300 \mathrm{MHz}, \mathrm{CDCl}_{3}$

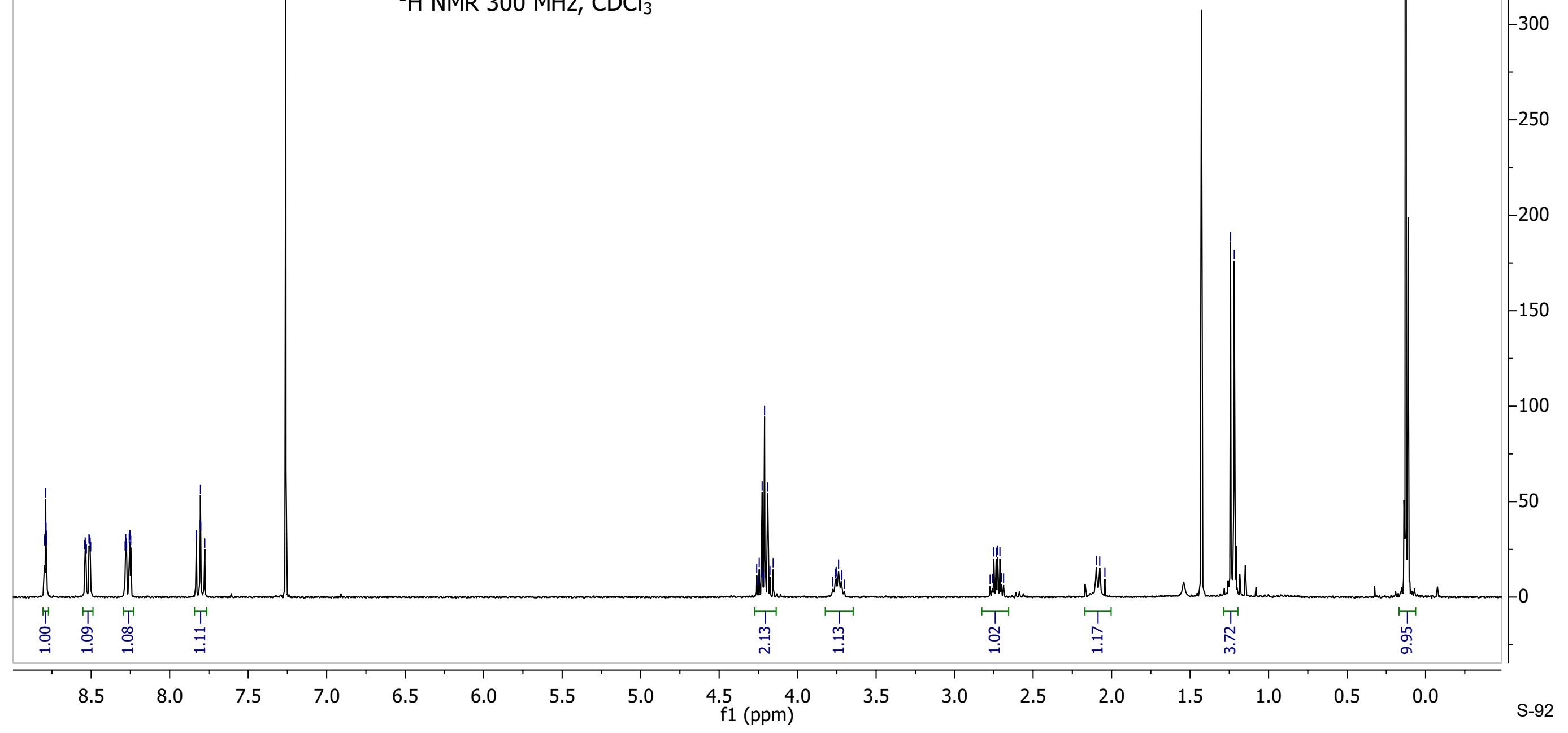




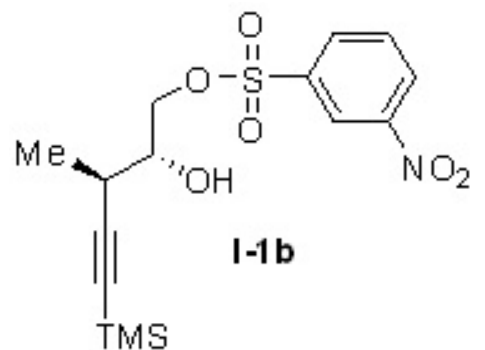

${ }^{13} \mathrm{C}\left\{{ }^{1} \mathrm{H}\right\}$ NMR $75 \mathrm{MHz}, \mathrm{CDCl}_{3}$ 


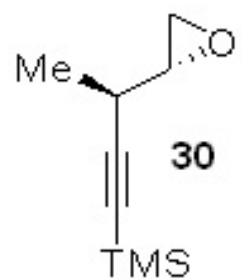

${ }^{1} \mathrm{H}$ NMR $300 \mathrm{MHz} \mathrm{CDCl}_{3}$

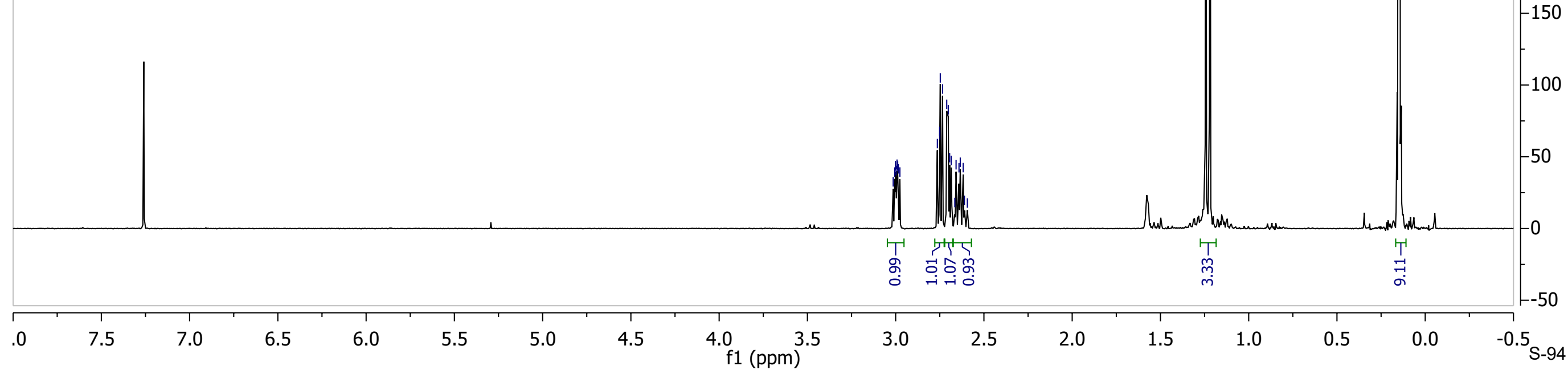



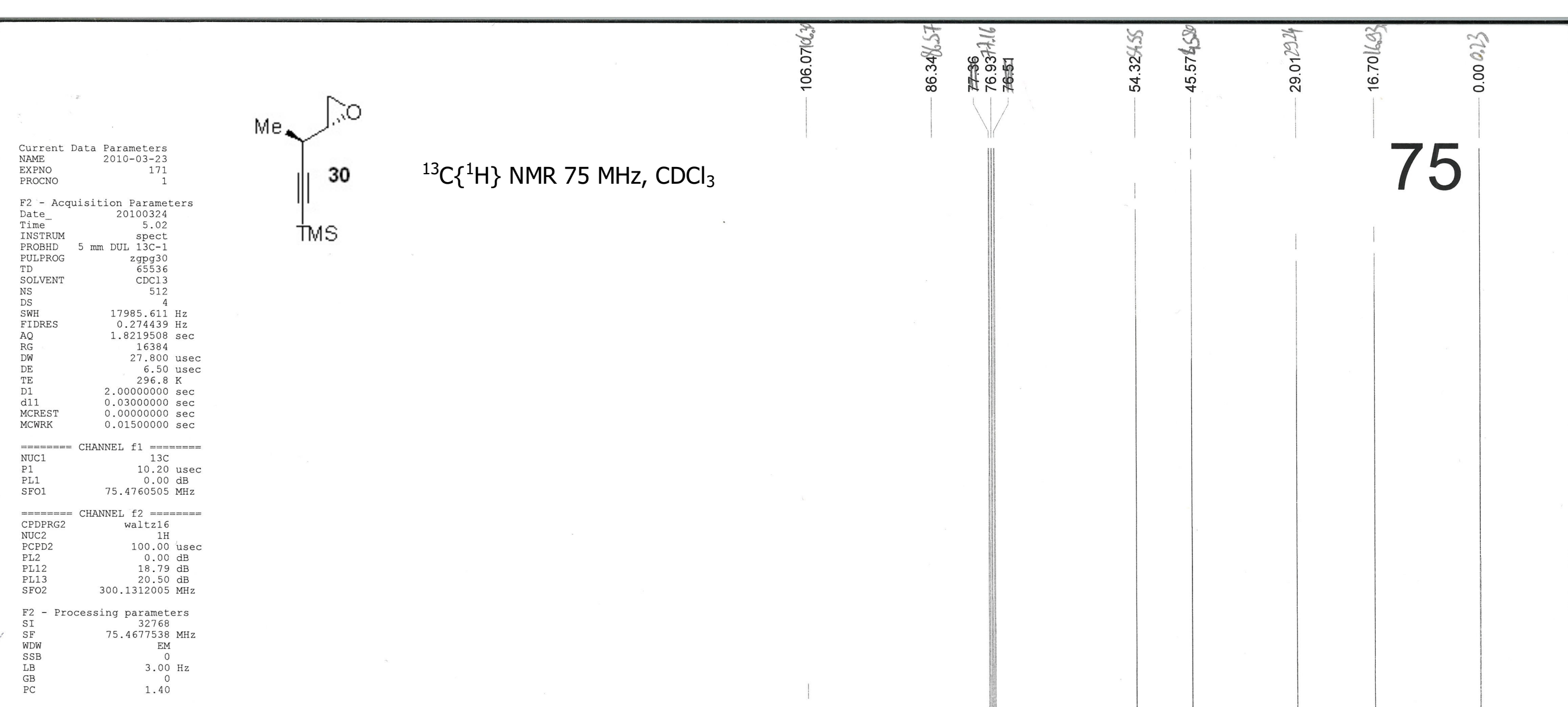

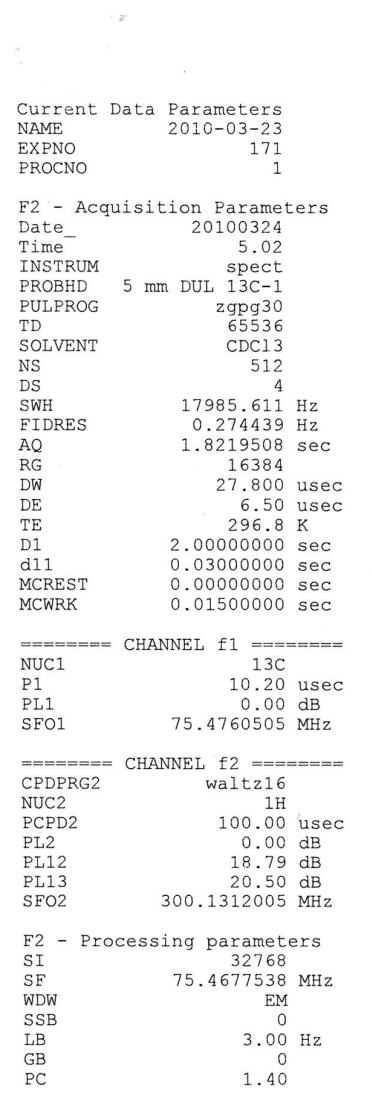

75

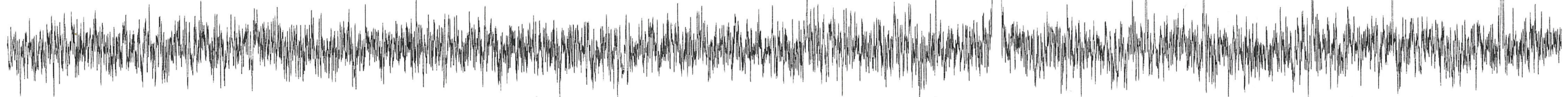


${ }^{1} \mathrm{H} \mathrm{NMR} 300 \mathrm{MHz}, \mathrm{CDCl}_{3}$

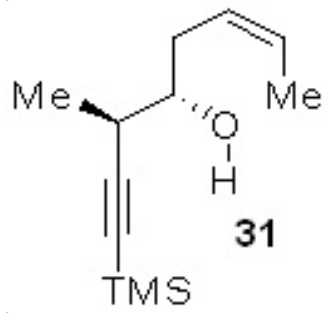

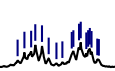
喓 


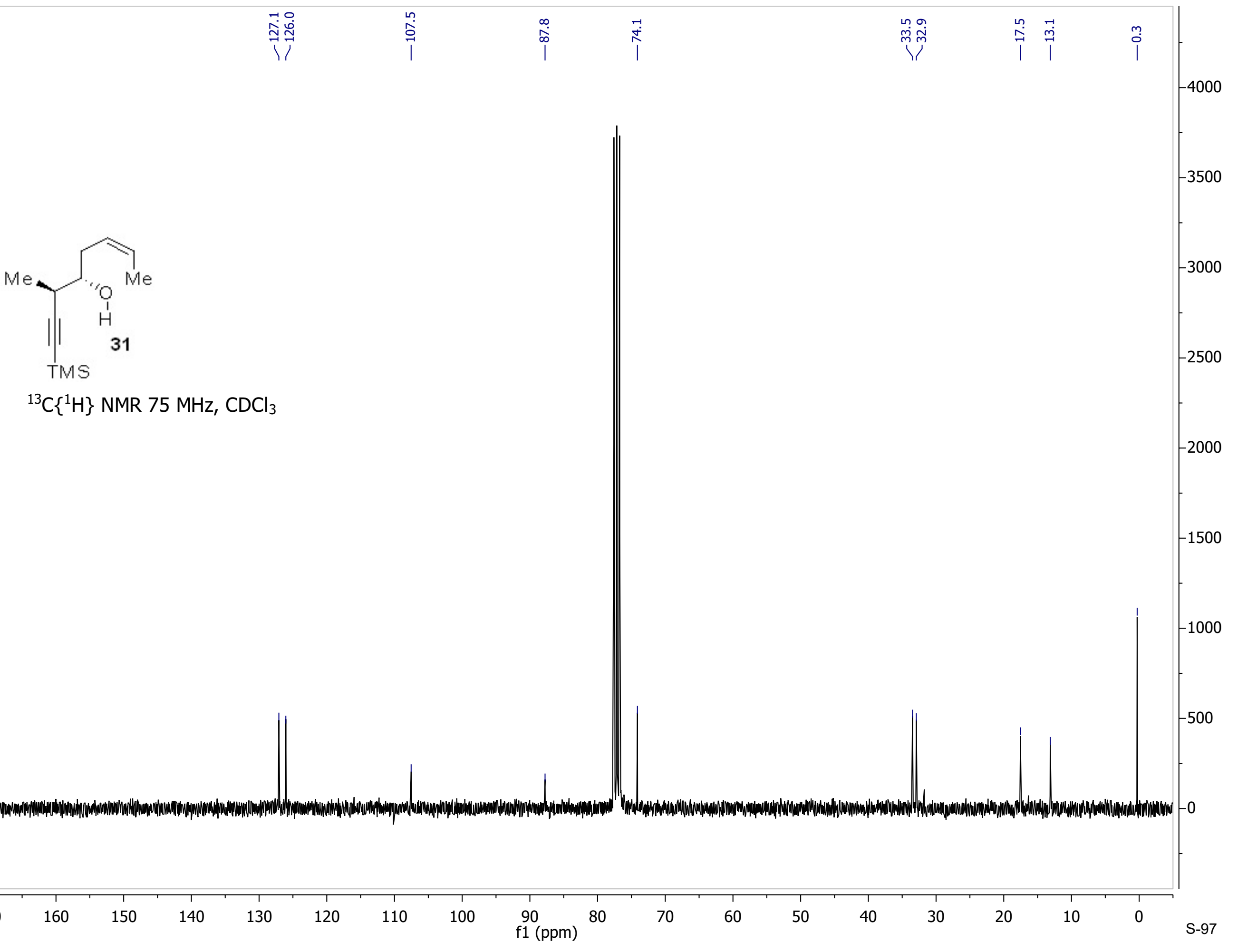




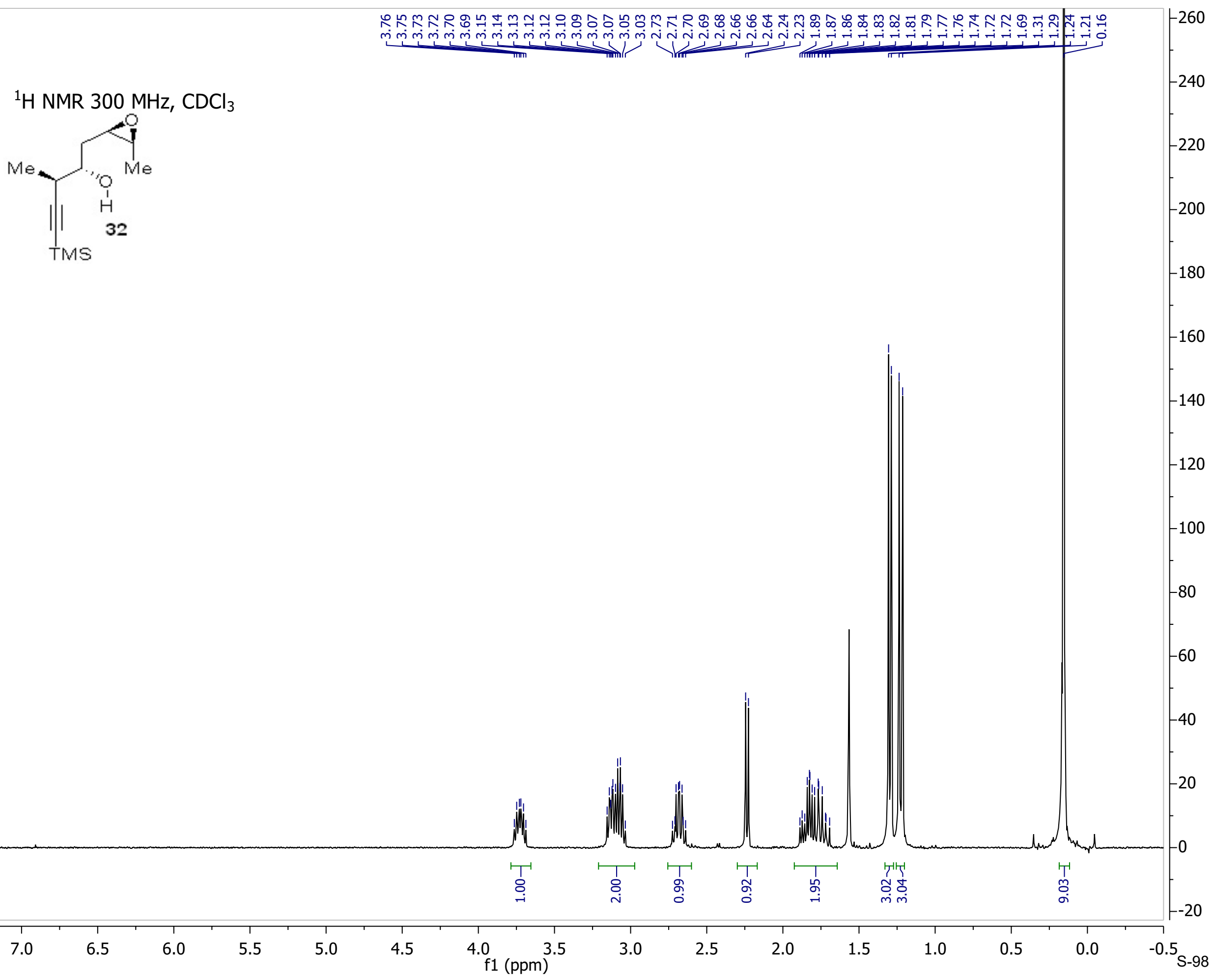




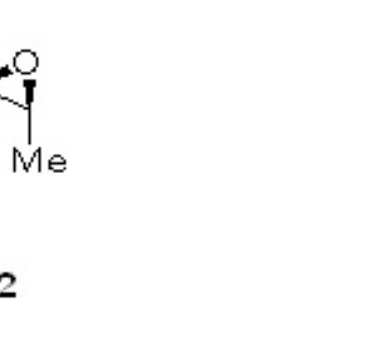

${ }^{13} \mathrm{C}\left\{{ }^{1} \mathrm{H}\right\}$ NMR $75 \mathrm{MHz}, \mathrm{CDCl}_{3}$

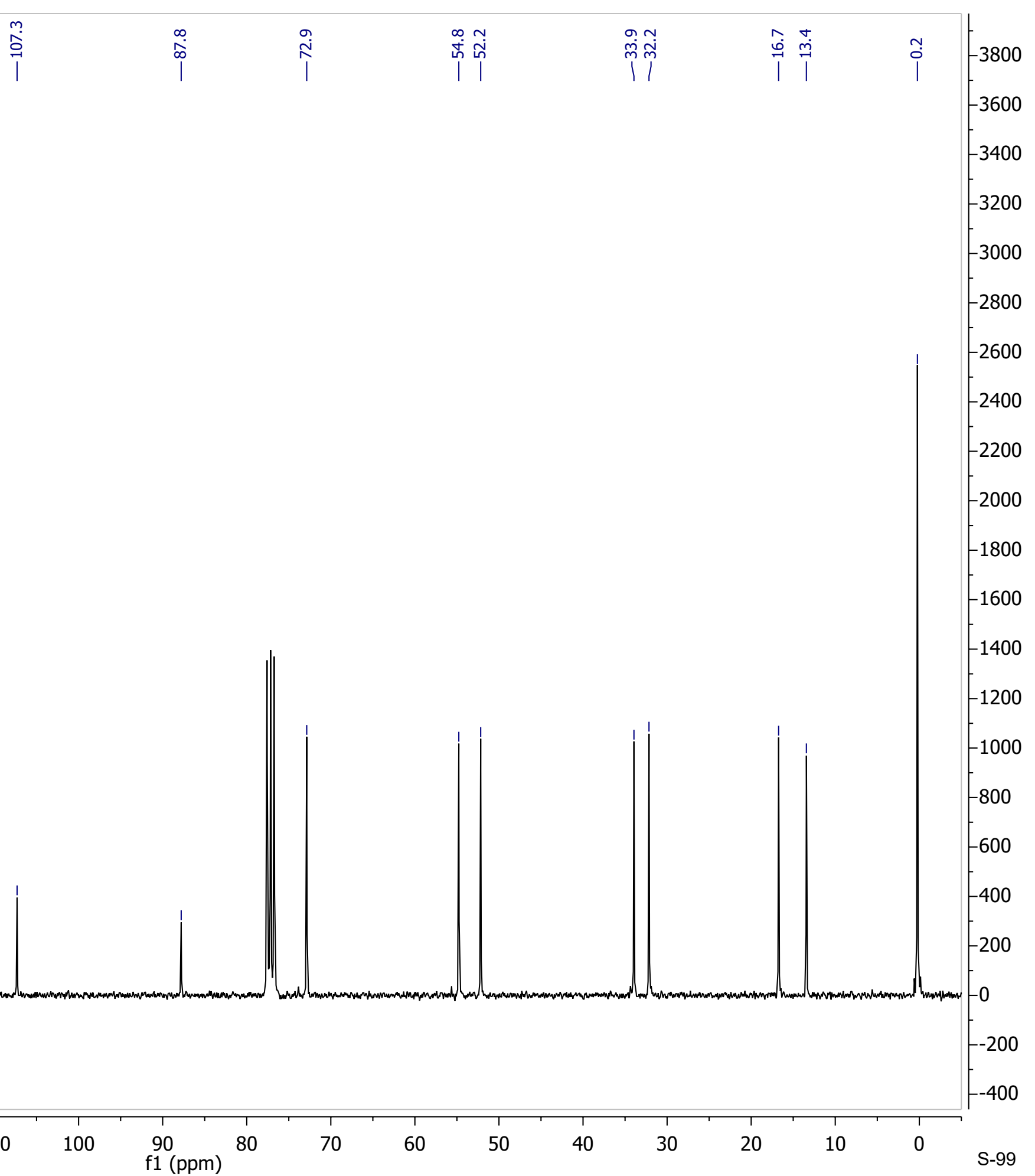




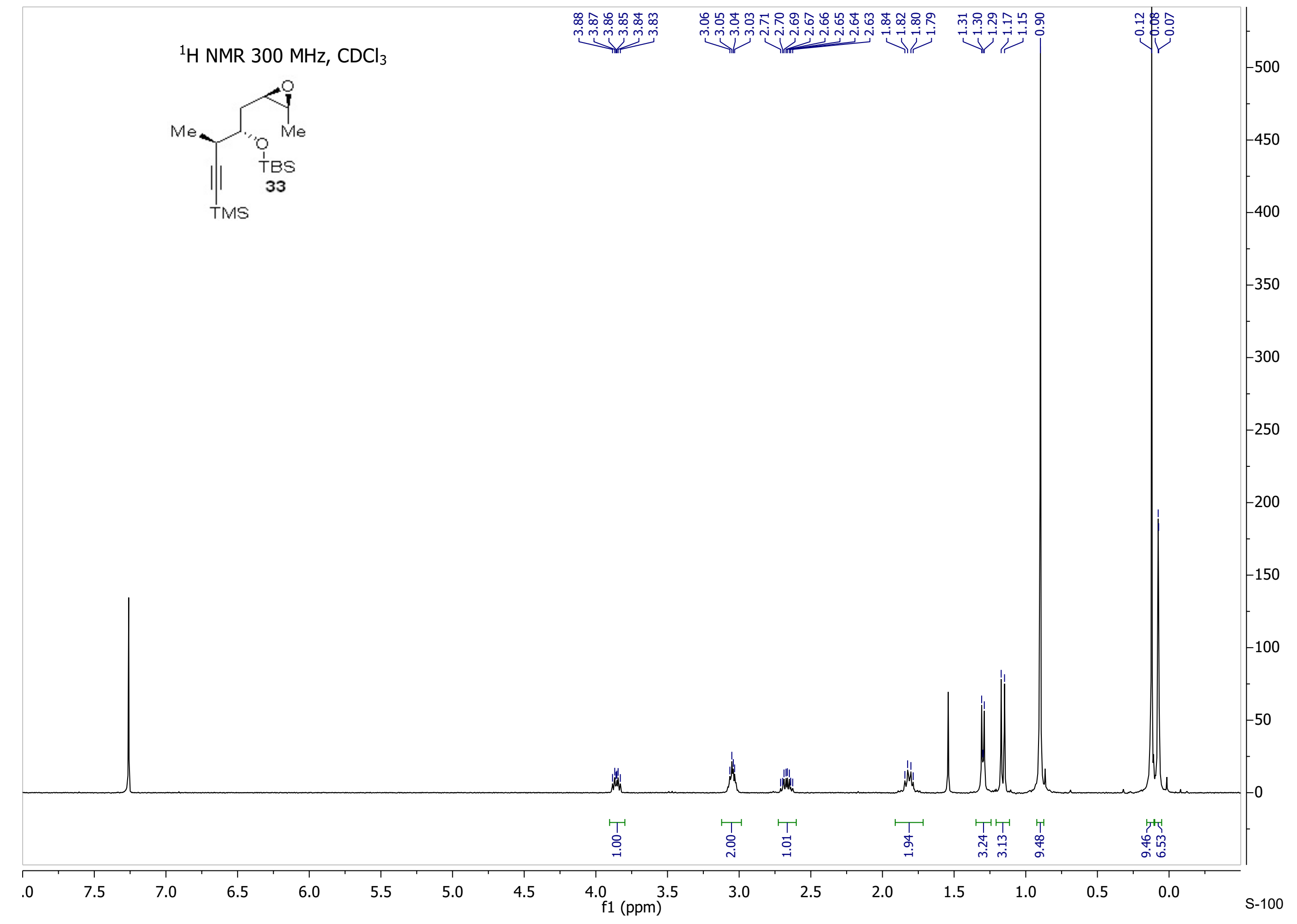




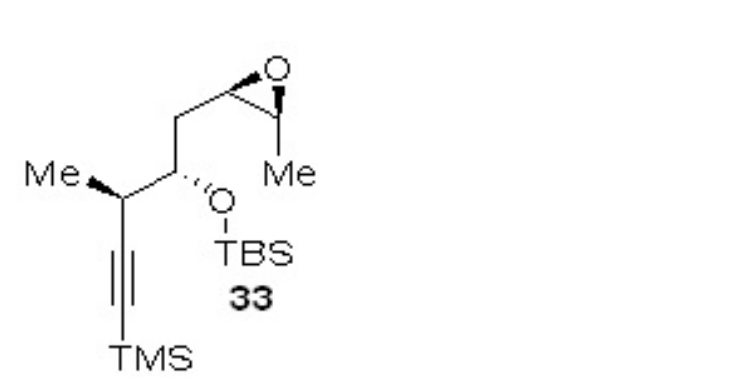

${ }^{13} \mathrm{C}\left\{{ }^{1} \mathrm{H}\right\}$ NMR $75 \mathrm{MHz}^{\mathrm{CDCl}} 3$ 


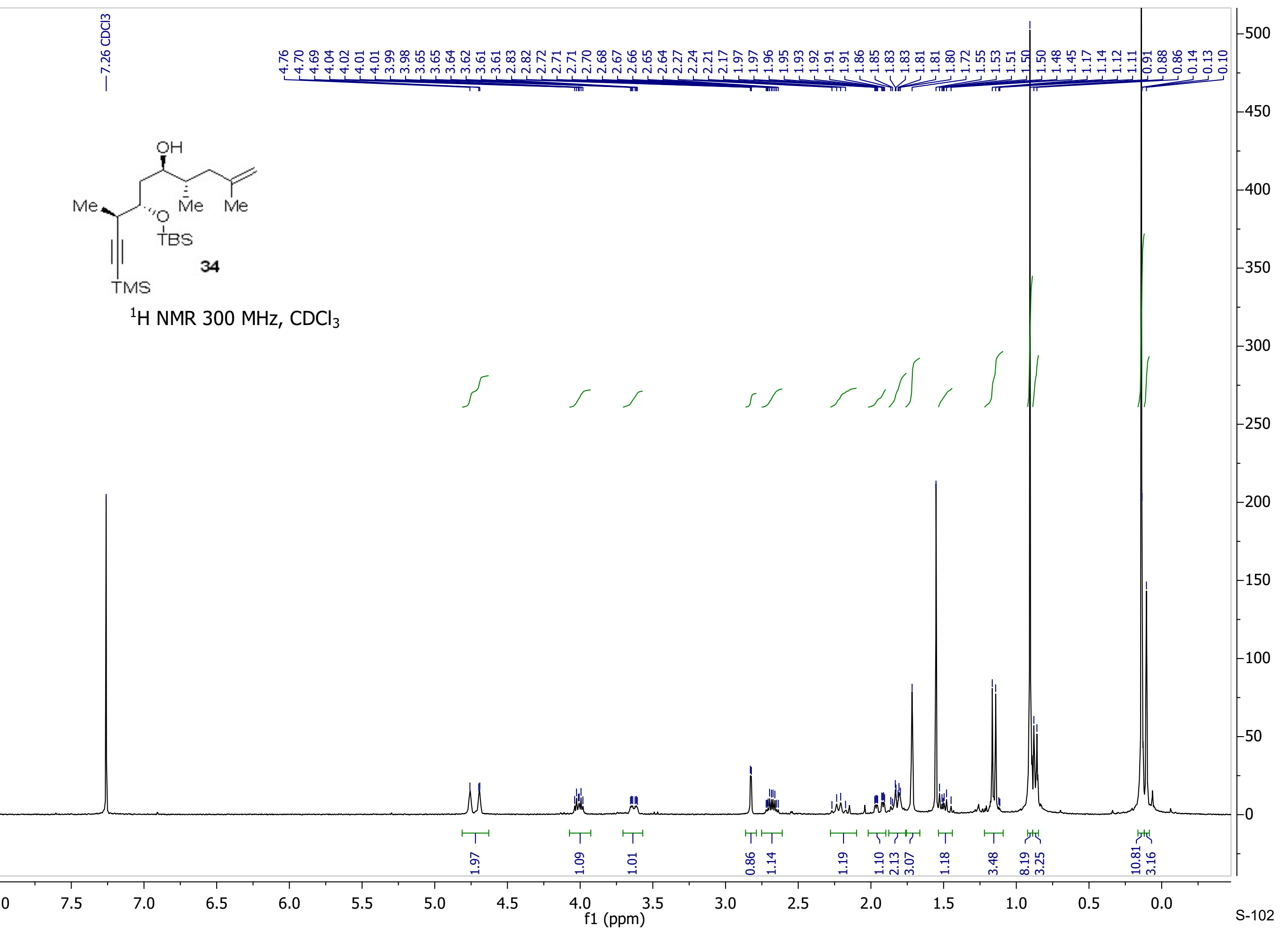




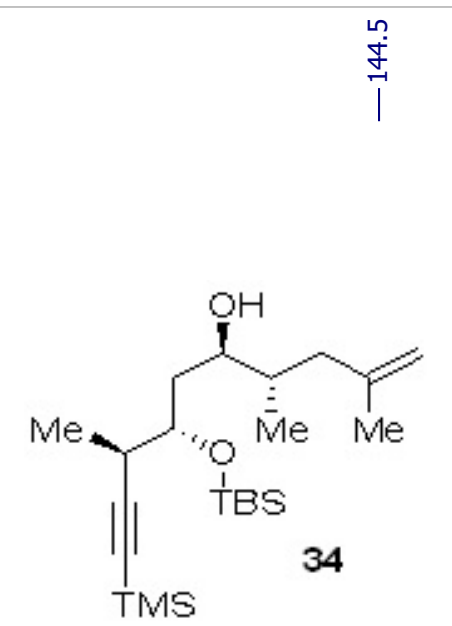

${ }^{13} \mathrm{C}\left\{{ }^{1} \mathrm{H}\right\}$ NMR $75 \mathrm{MHz}, \mathrm{CDCl}_{3}$

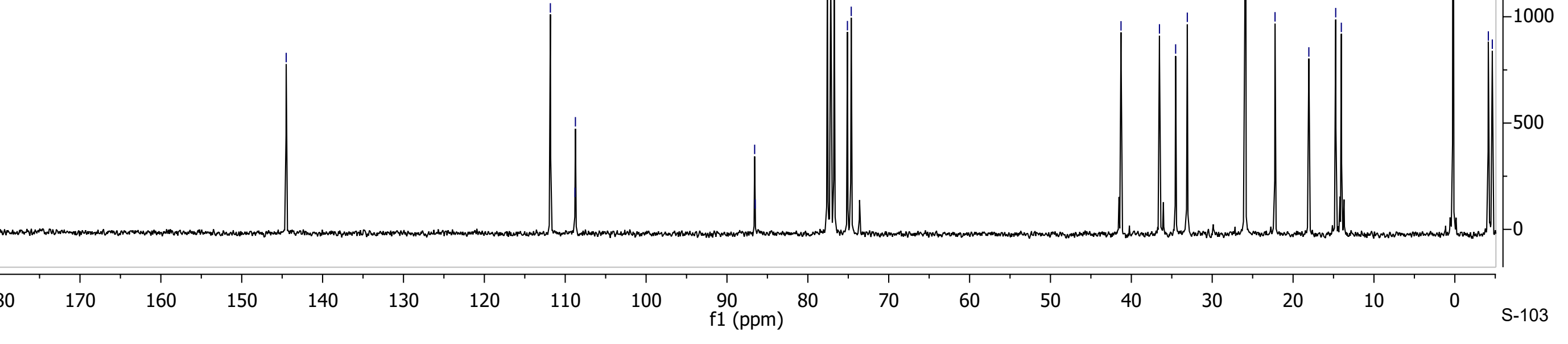




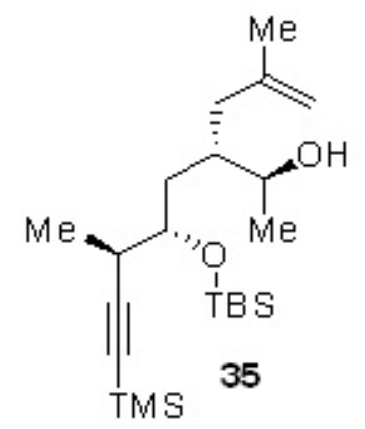

${ }^{1} \mathrm{H}$ NMR $300 \mathrm{MHz}, \mathrm{CDCl}_{3}$

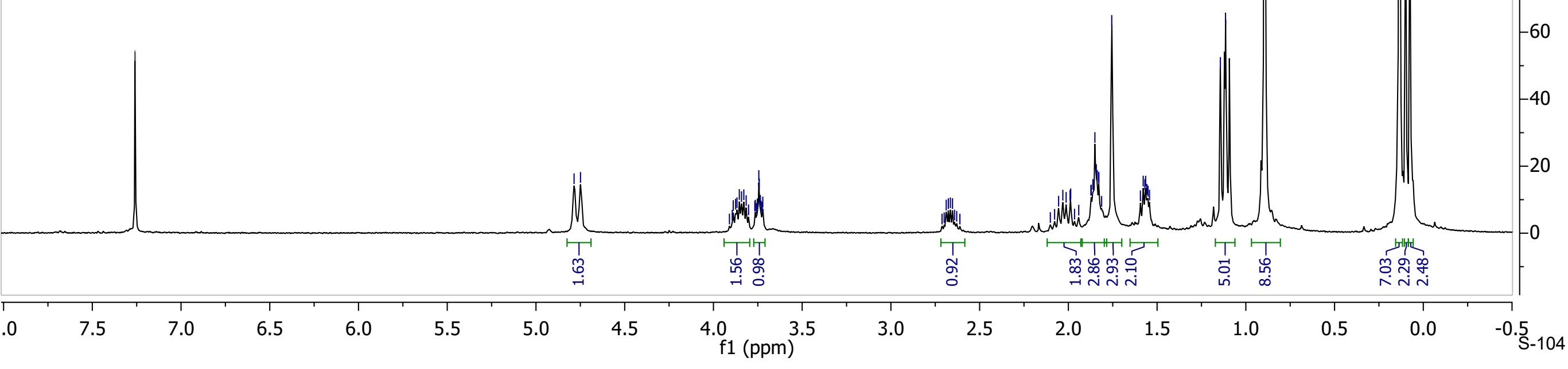




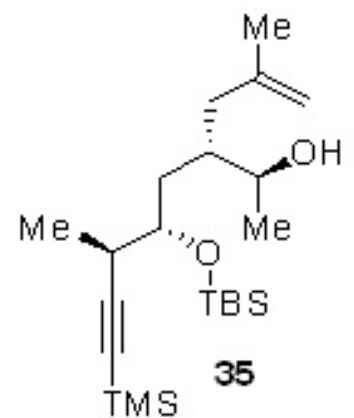

${ }^{13} \mathrm{C}\left\{{ }^{1} \mathrm{H}\right\}$ NMR $75 \mathrm{MHz}, \mathrm{CDCl}_{3}$

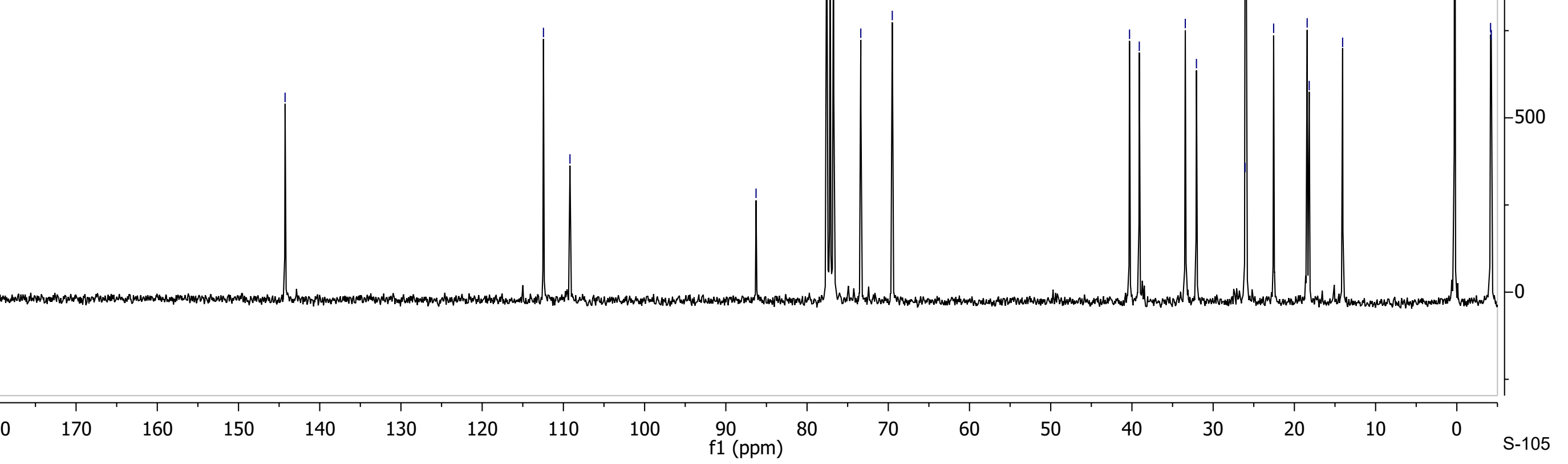




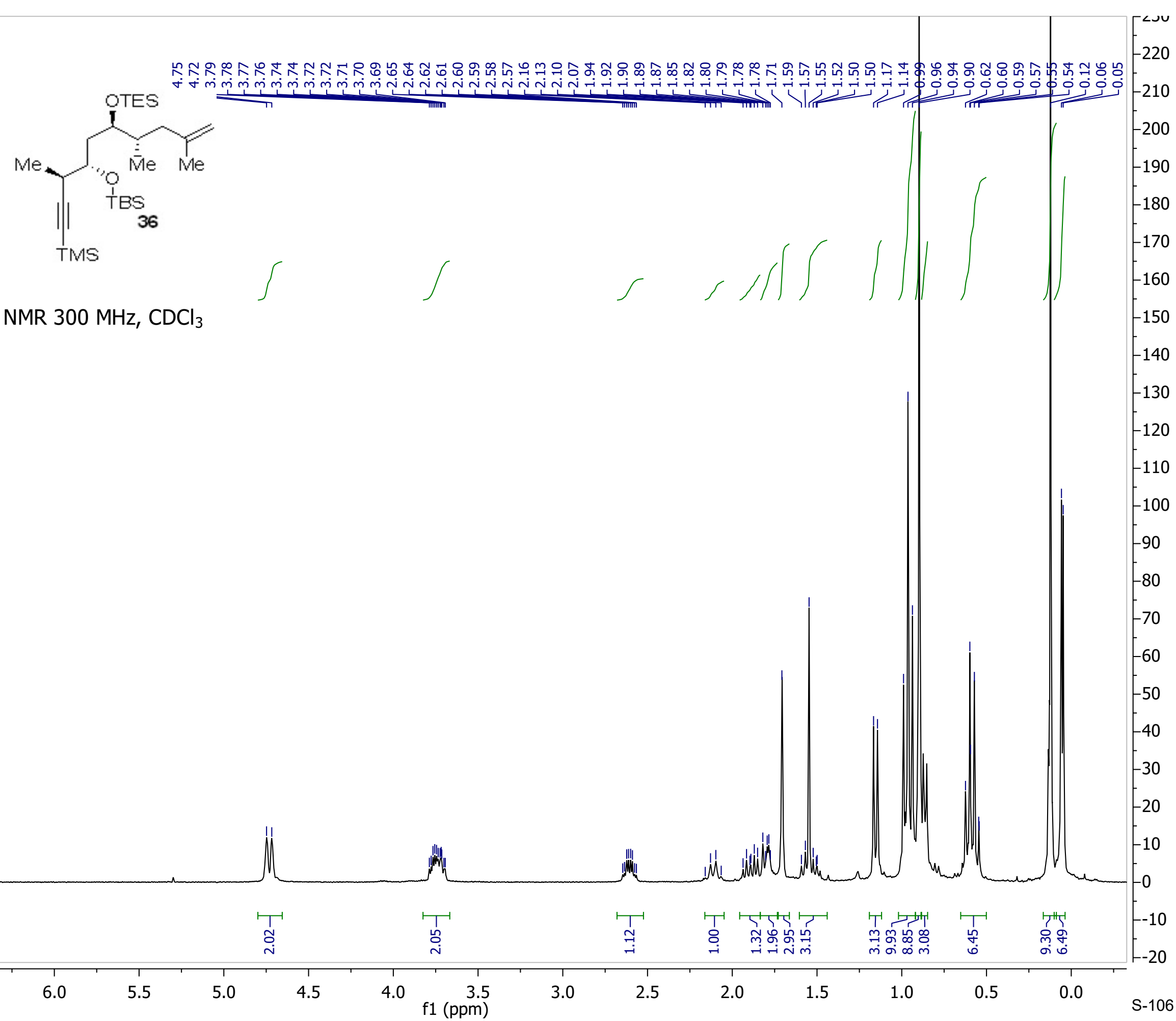




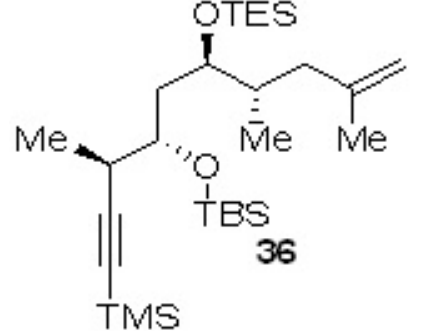

${ }^{13} \mathrm{C}\left\{{ }^{1} \mathrm{H}\right\}$ NMR $75 \mathrm{MHz}, \mathrm{CDCl}_{3}$ 


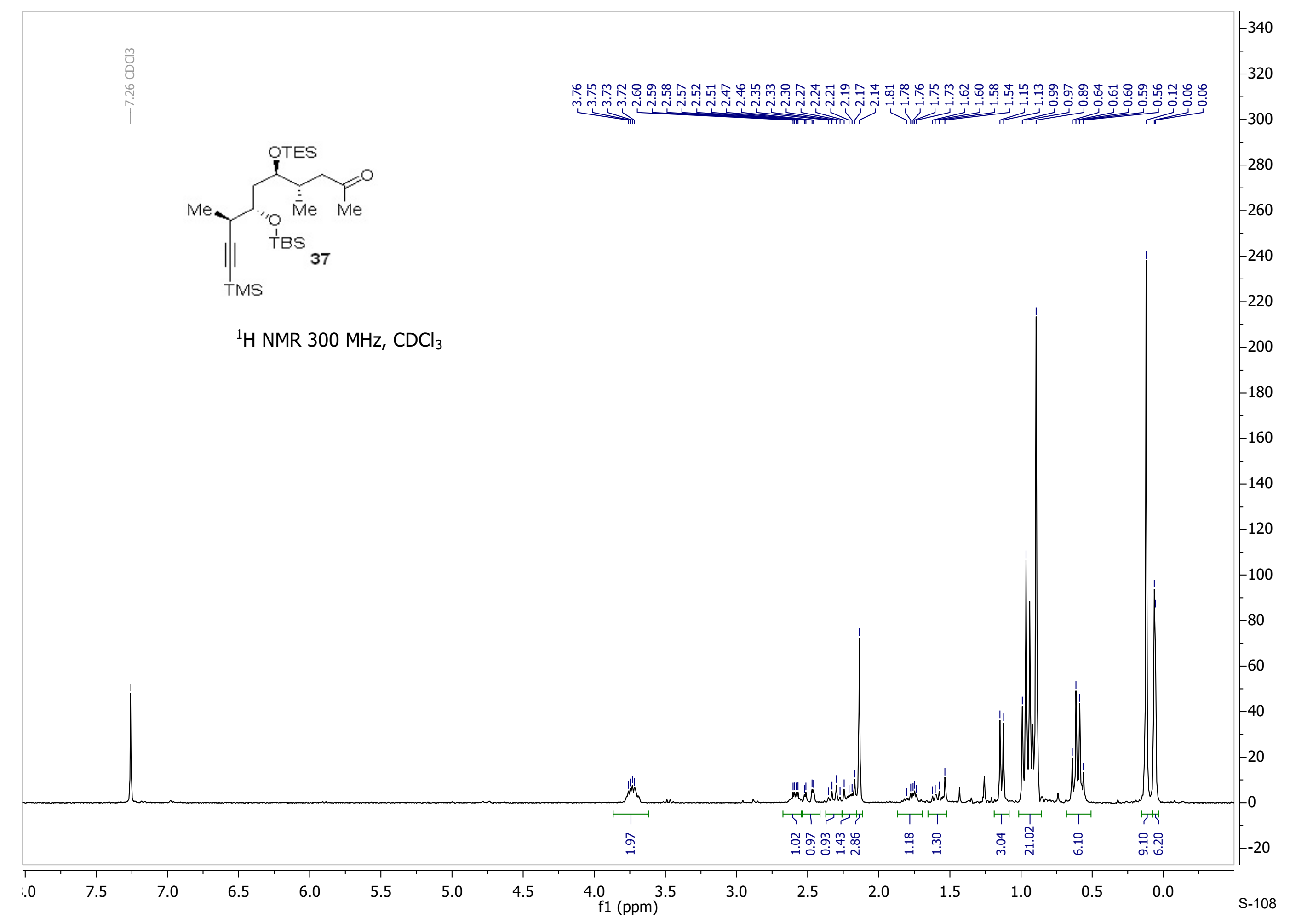




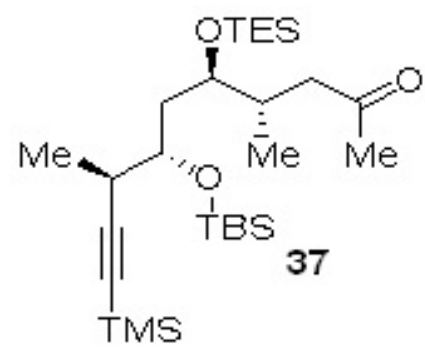

${ }^{13} \mathrm{C}\left\{{ }^{1} \mathrm{H}\right\}$ NMR $75 \mathrm{MHz}, \mathrm{CDCl}_{3}$ 


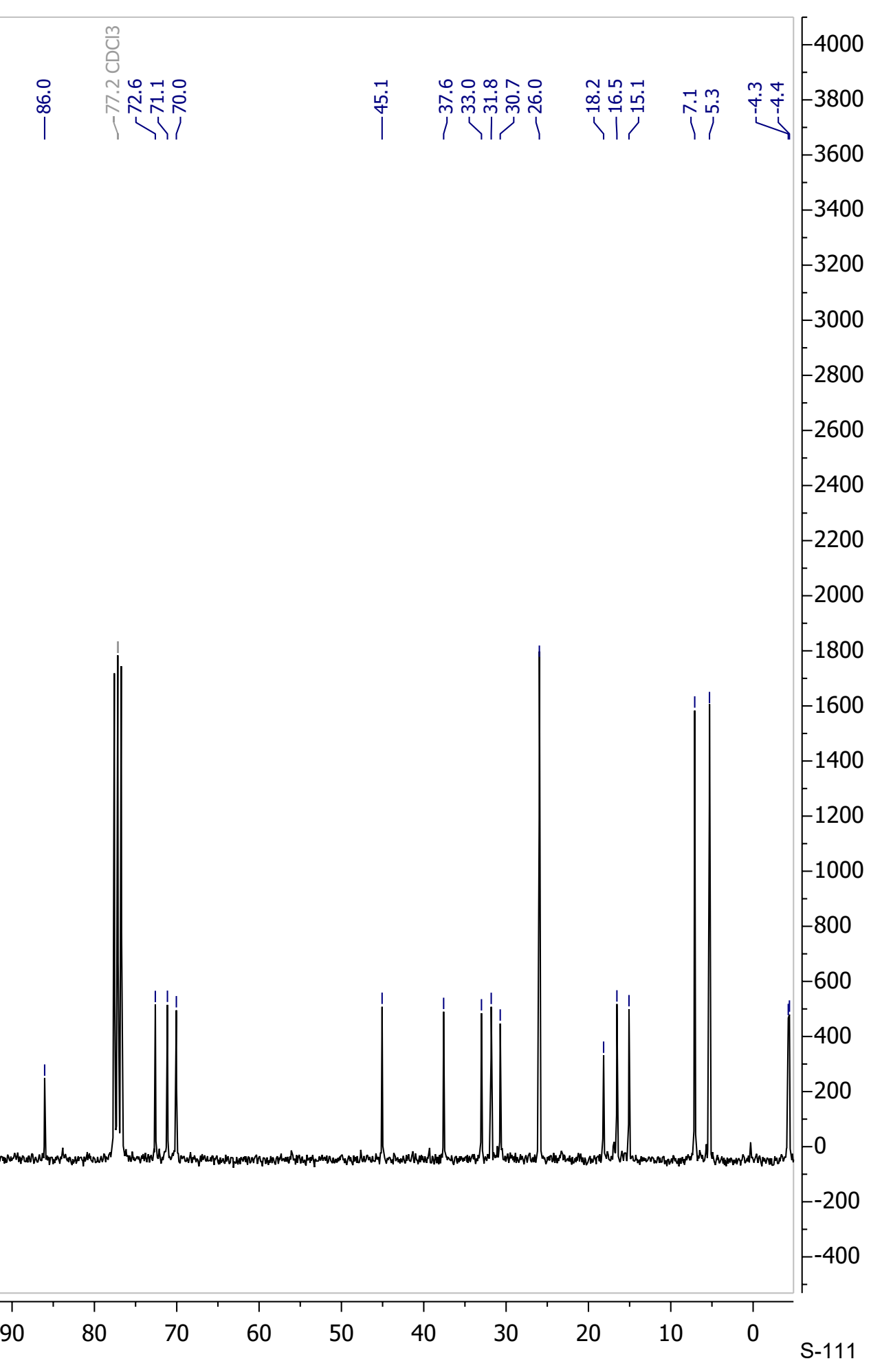




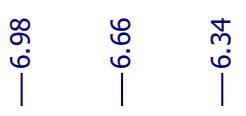

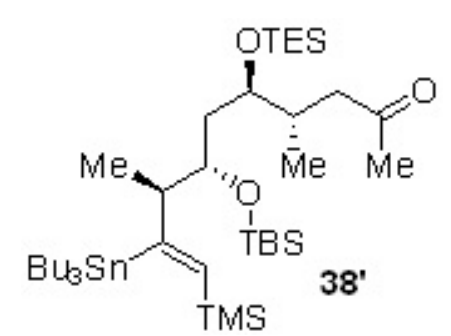

${ }^{1} \mathrm{H}$ NMR $300 \mathrm{MHz}, \mathrm{CDCl}_{3}$

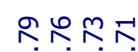

mingim

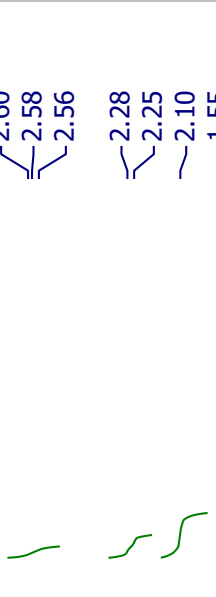

I'lu

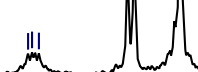

मा मा

t)

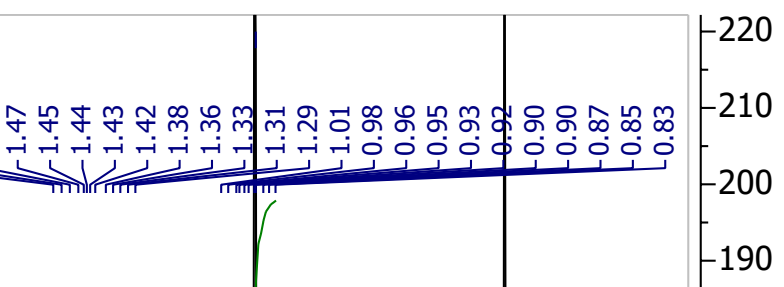

$-220$

210

$-200$ $-190$ $-180$ $-170$ $-160$ $-150$ $-140$ $-130$ $-120$ $-110$ $-100$ $-90$ $-80$ $-70$ 


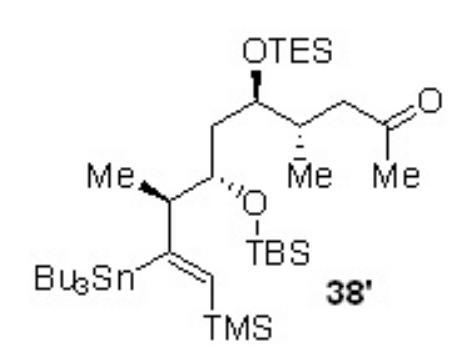

${ }^{13} \mathrm{C}\left\{{ }^{1} \mathrm{H}\right\}$ NMR $75 \mathrm{MHz}^{\mathrm{CDCl}} 3$ 
$-$<smiles>[M]C(CC(C)=O)C(CC(OC(C)=O)C(C)C(I)=CC(C)(C)C)OC(C)(C)C</smiles>

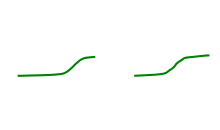

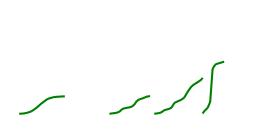

${ }^{1} \mathrm{H}$ NMR $300 \mathrm{MHz} \mathrm{CDCl}_{3}$

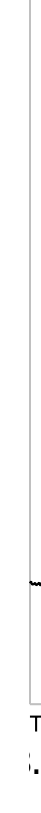




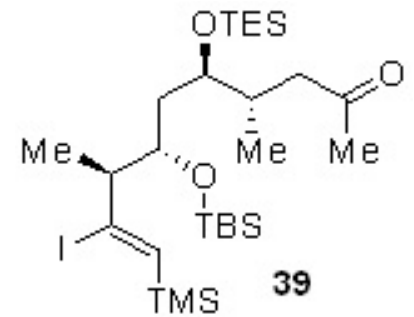

${ }^{13} \mathrm{C}\left\{{ }^{1} \mathrm{H}\right\}$ NMR $75 \mathrm{MHz}, \mathrm{CDCl}_{3}$ 


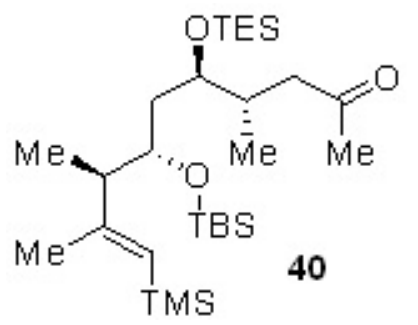

${ }^{1} \mathrm{H}$ NMR $300 \mathrm{MHz}, \mathrm{CDCl}_{3}$

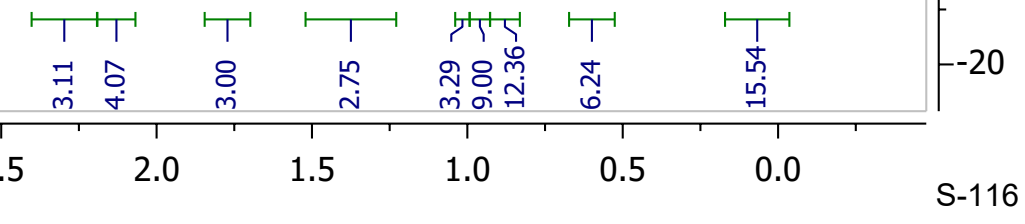




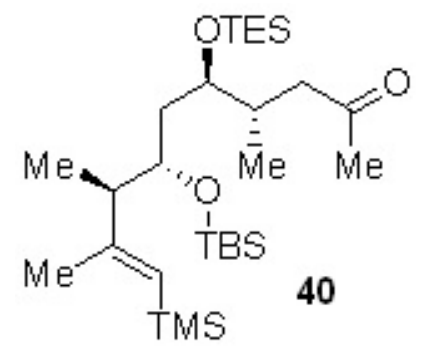

${ }^{13} \mathrm{C}\left\{{ }^{1} \mathrm{H}\right\}$ NMR $75 \mathrm{MHz}, \mathrm{CDCl}_{3}$

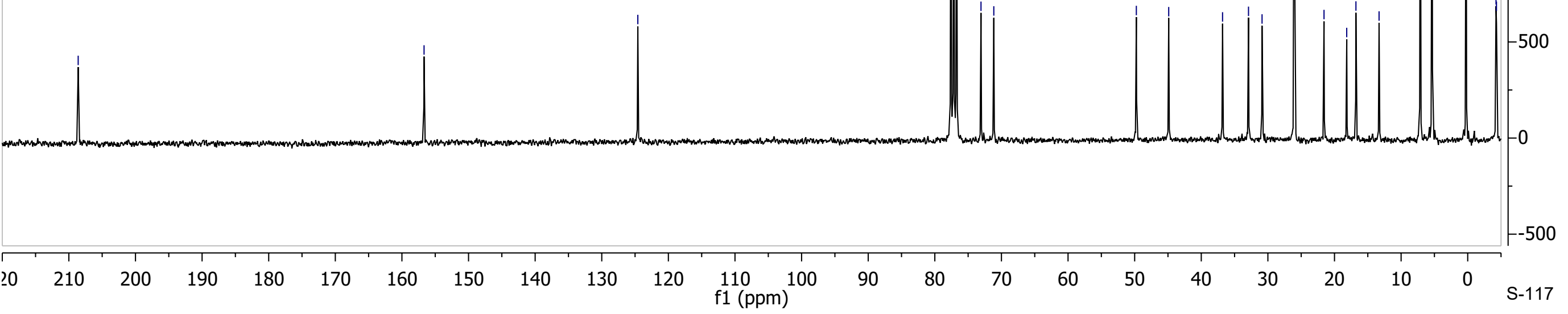




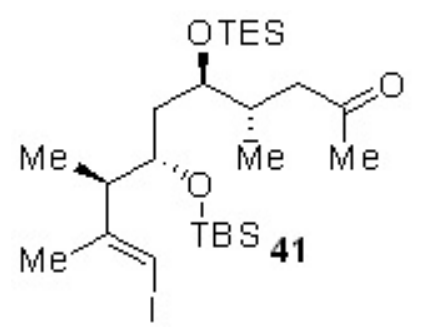

${ }^{13} \mathrm{C}\left\{{ }^{1} \mathrm{H}\right\}$ NMR $75 \mathrm{MHz}, \mathrm{CDCl}_{3}$ 

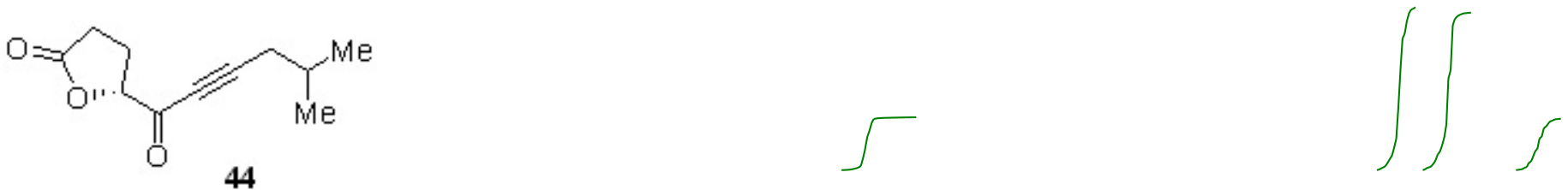

${ }^{1} \mathrm{HNMR}, 300 \mathrm{MHz}, \mathrm{CDCl}_{3}$

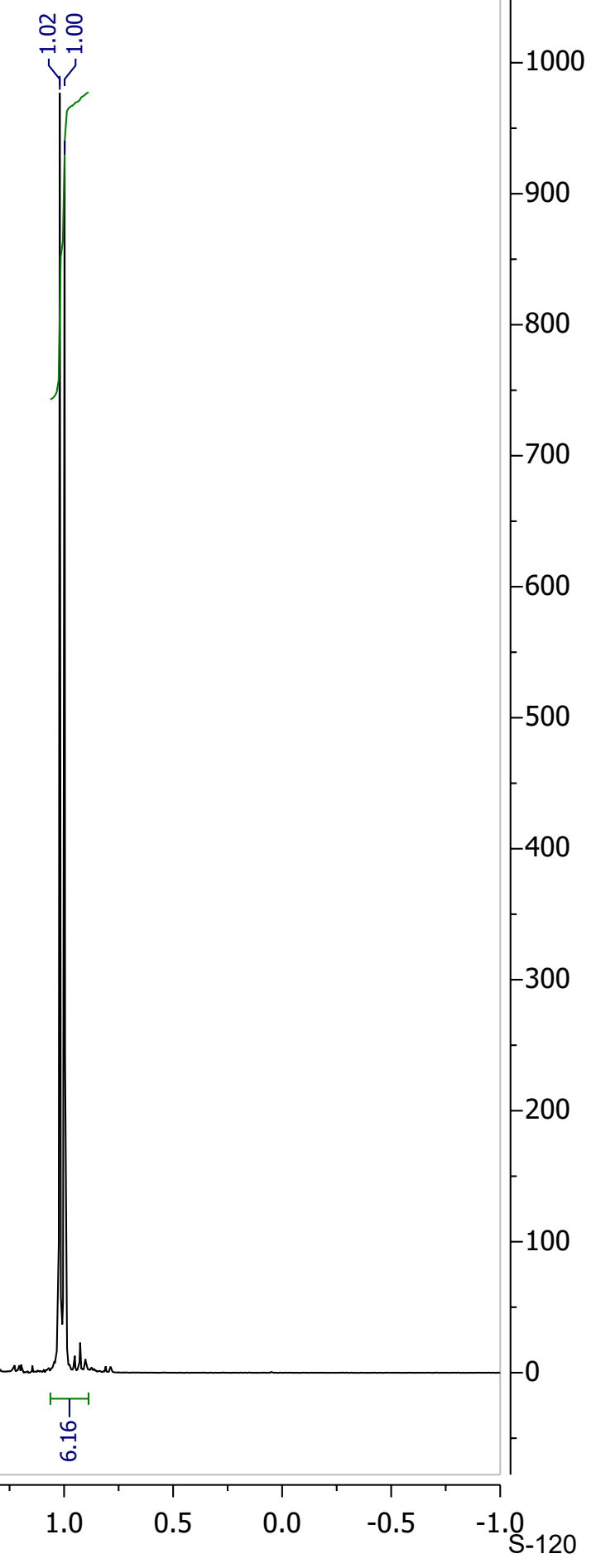




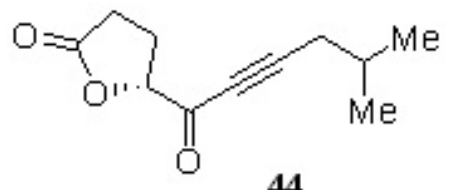

$\left.{ }^{13} \mathrm{C}_{\{}{ }^{1} \mathrm{H}\right\} \mathrm{NMR}, 75 \mathrm{MHz}, \mathrm{CDCl}_{3}$ 


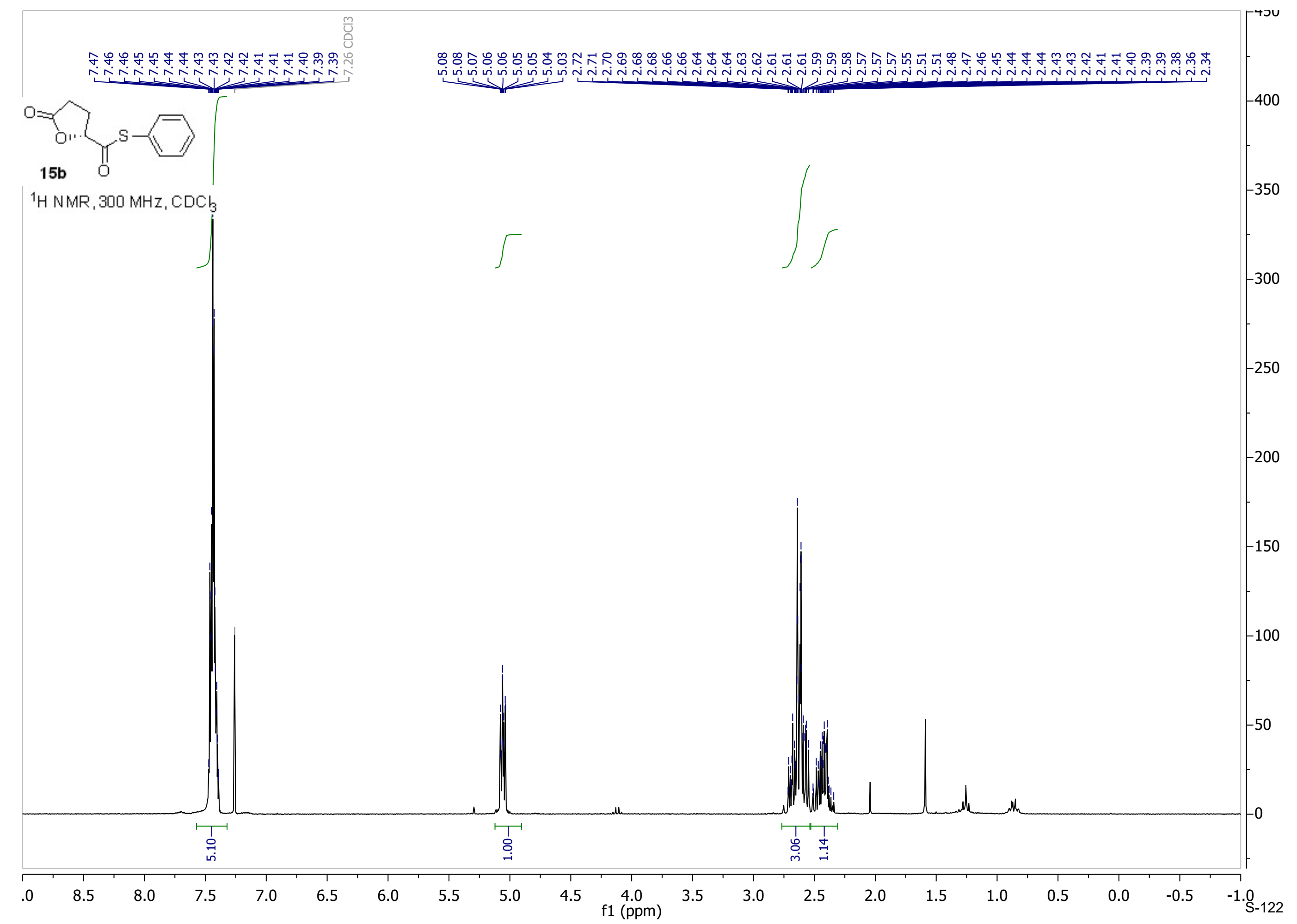




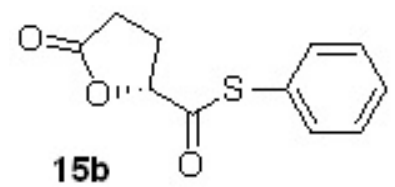

${ }^{13} \mathrm{C}\{\mathrm{H}\} \mathrm{NMR}, 75 \mathrm{MHz}, \mathrm{CDCl}_{3}$ 


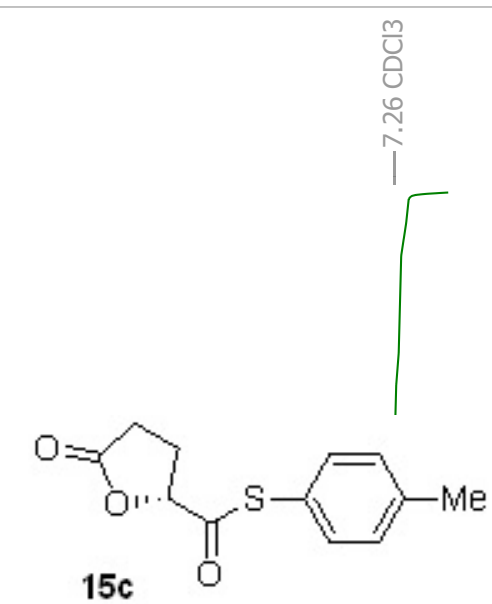

${ }^{1} \mathrm{HNMR}_{1}, 300 \mathrm{MHz}, \mathrm{CDCl}_{3}$ 


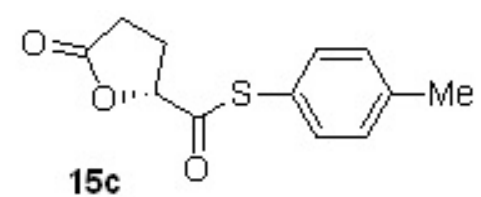

$\left.{ }^{13} \mathrm{C}\{\mathrm{H}\} \mathrm{NMR}, 75 \mathrm{MHz}, \mathrm{CDC}\right\}$
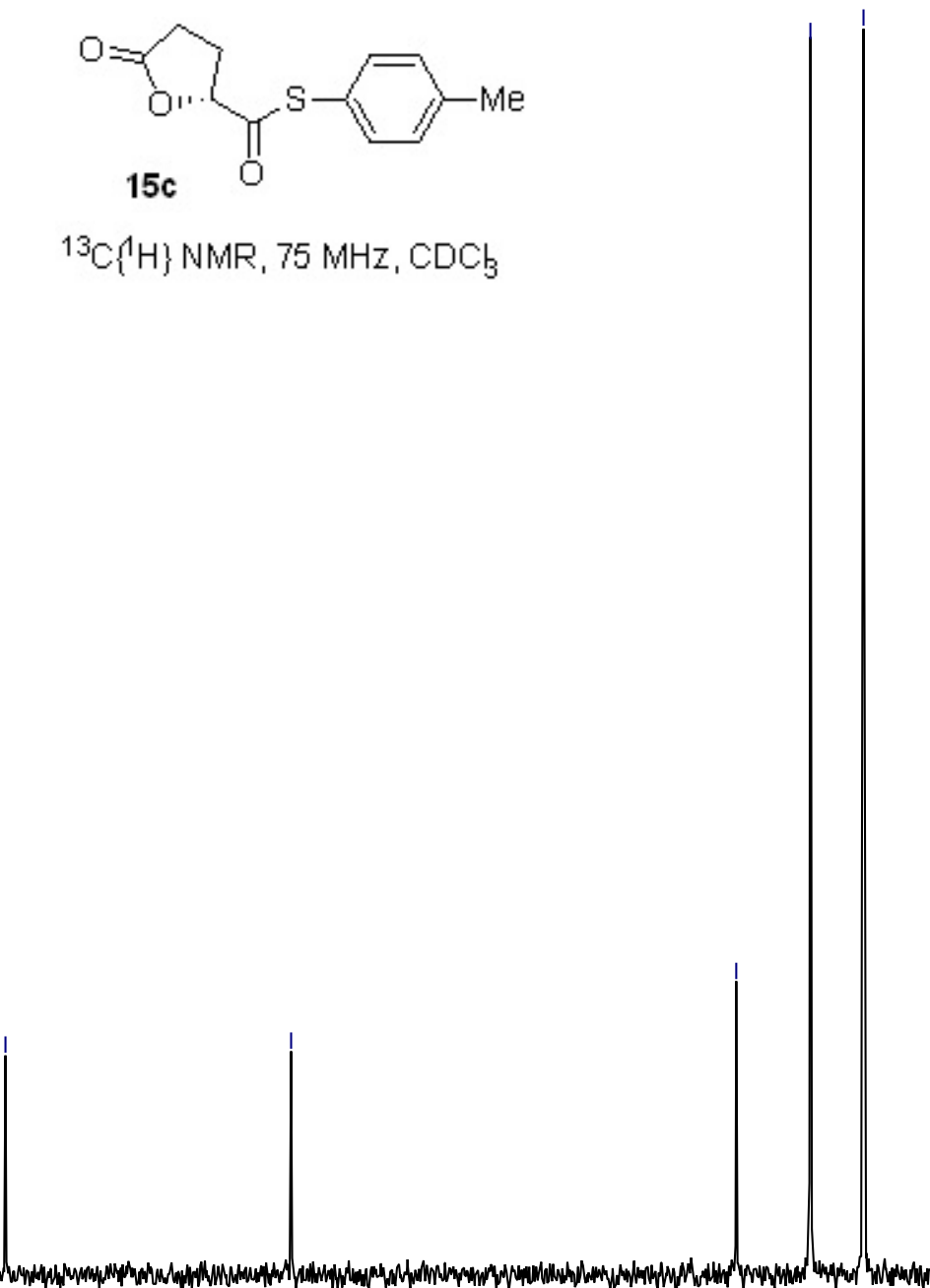

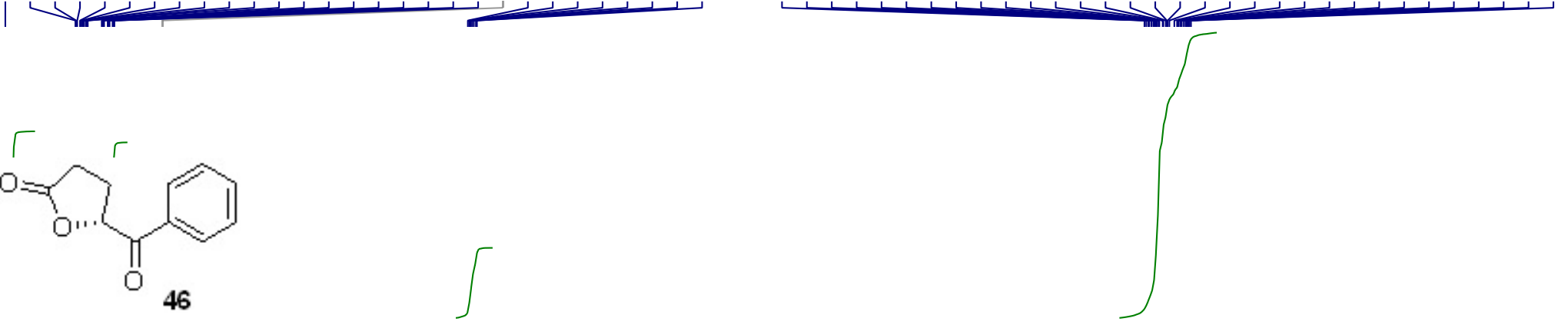

${ }^{1} \mathrm{HNMR}, 300 \mathrm{MHz}, \mathrm{CDCl}_{3}$

$\mapsto \mapsto$ ชั $8.0-7.5$ 


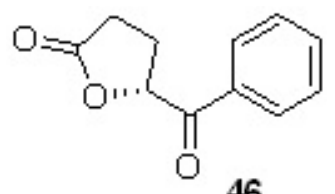

${ }^{13} \mathrm{C}\{\mathrm{H}\} \mathrm{NMR}, 75 \mathrm{MHz}, \mathrm{CDC}_{3}$ 


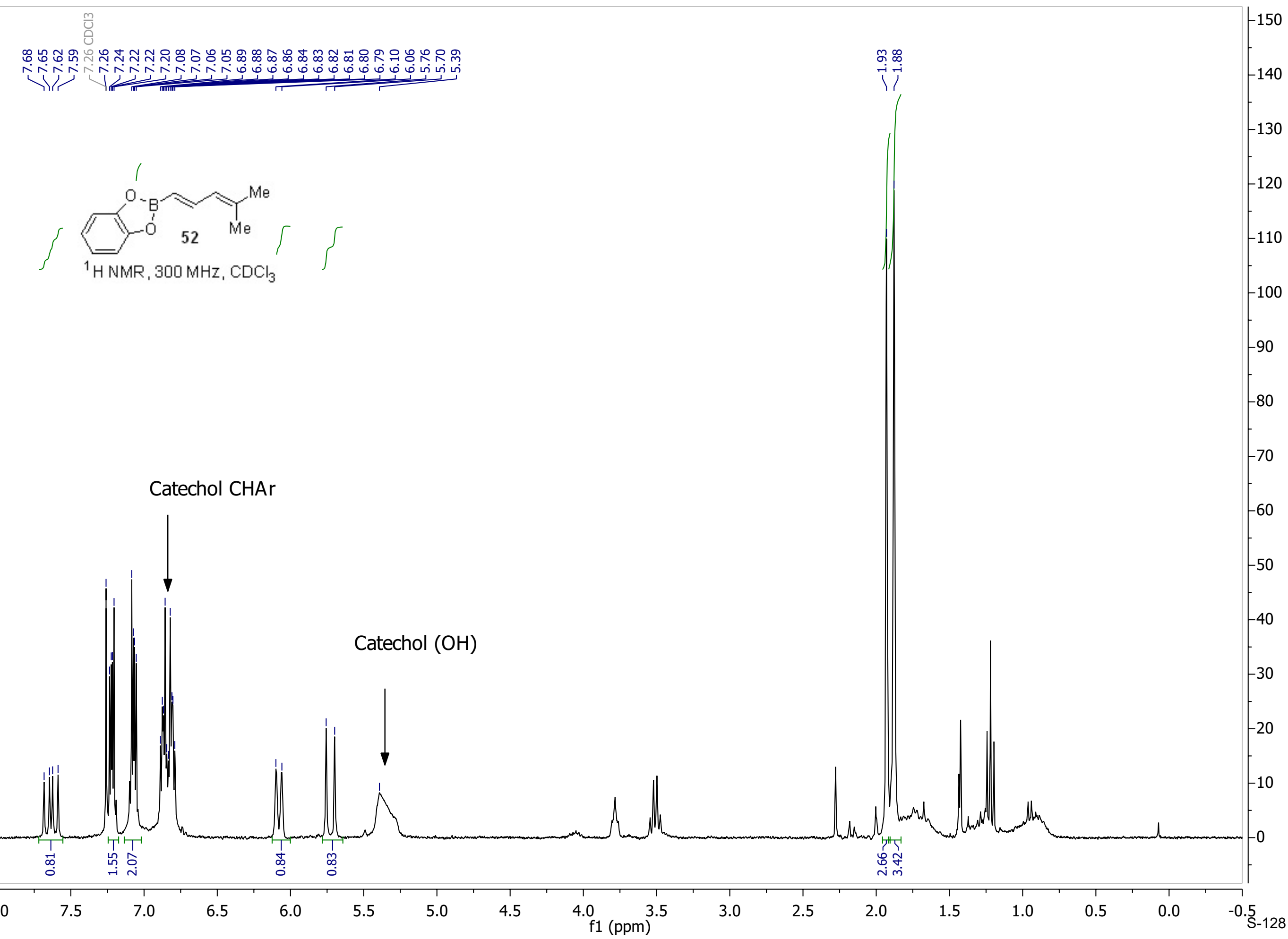




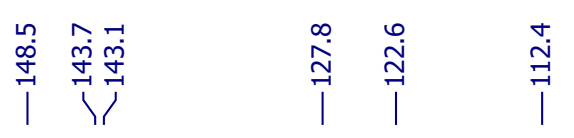

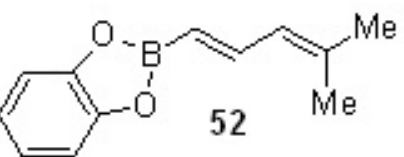

${ }^{13} \mathrm{C}\{\mathrm{H}\} \mathrm{NMR}, 75 \mathrm{MHz}, \mathrm{CDC} 3$

Catechol

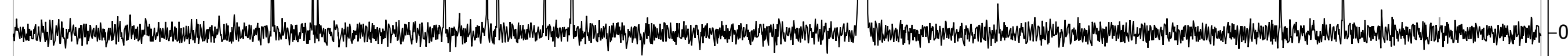




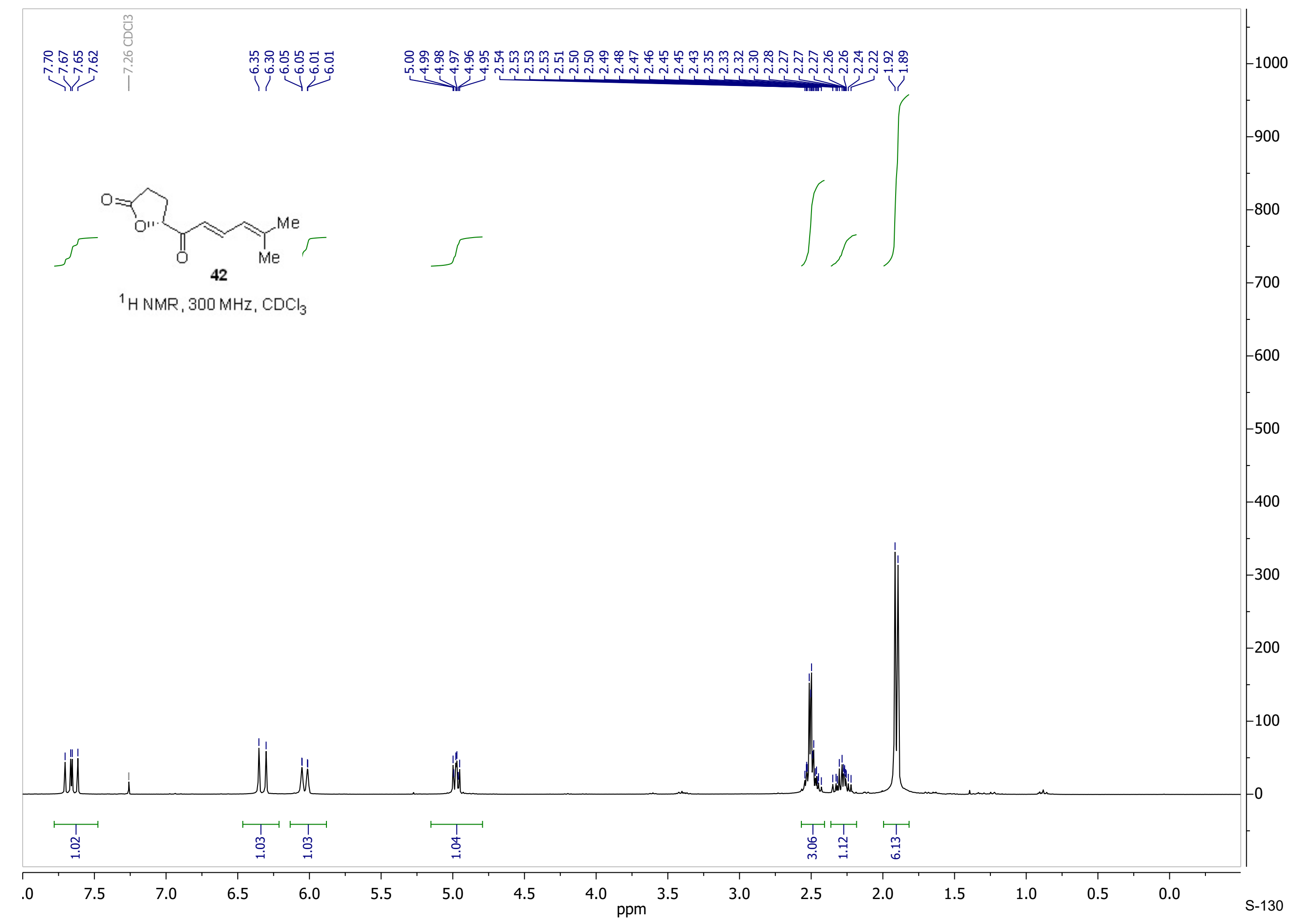




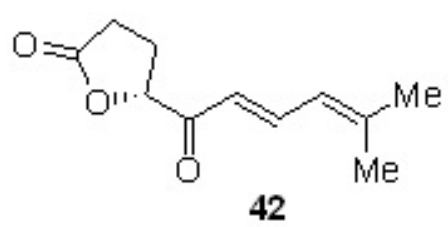

${ }^{13} \mathrm{C}\{\mathrm{H}\} \mathrm{NMR}, 75 \mathrm{MHz}_{1} \mathrm{CDC}_{3}$ 


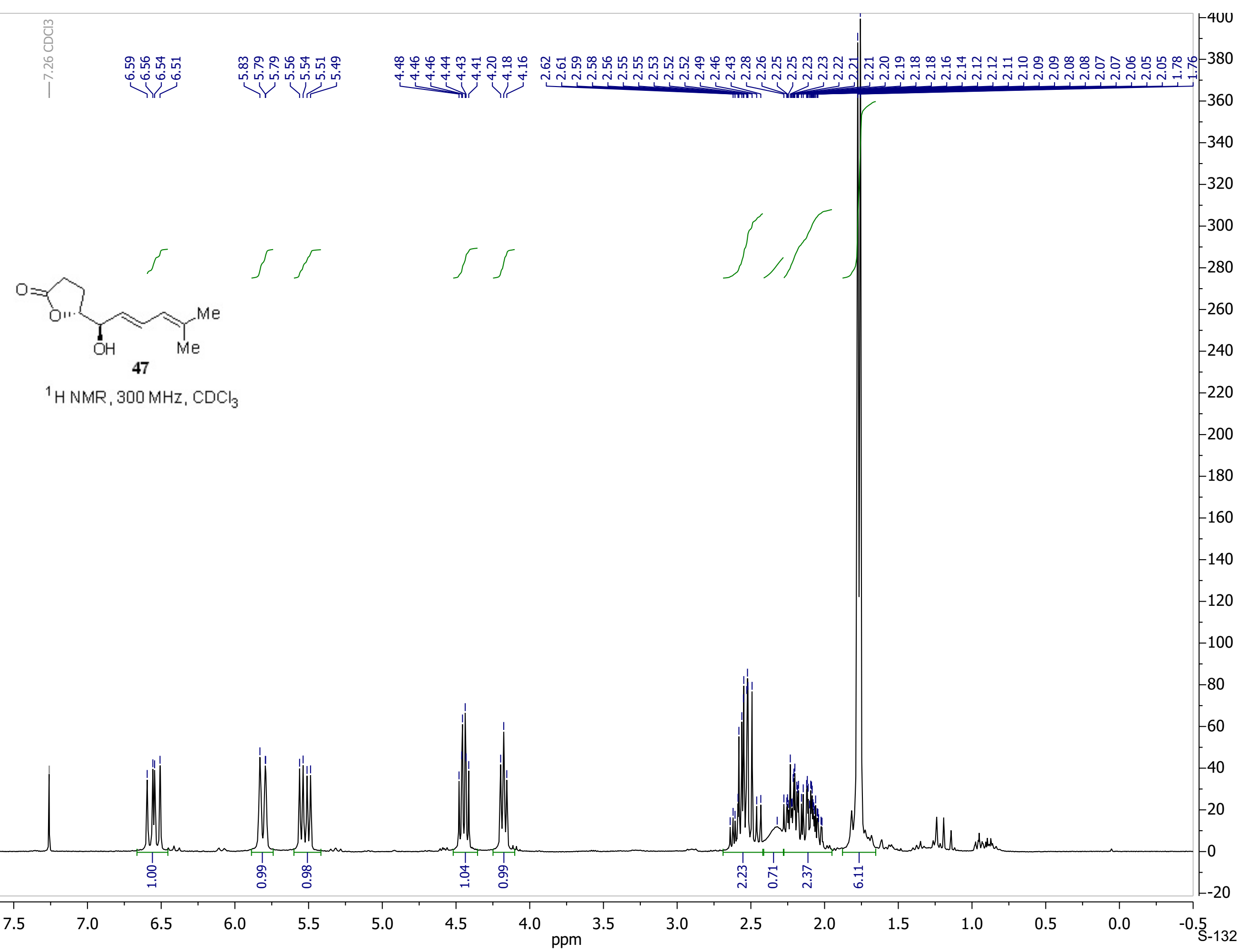




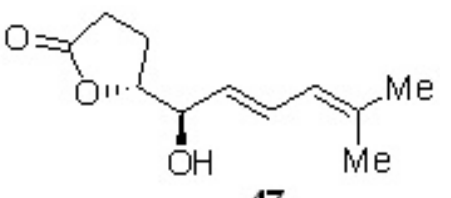

47

${ }^{13} \mathrm{C}\{\mathrm{H}\} \mathrm{NMR}, 75 \mathrm{MHz}, \mathrm{CDCl}$
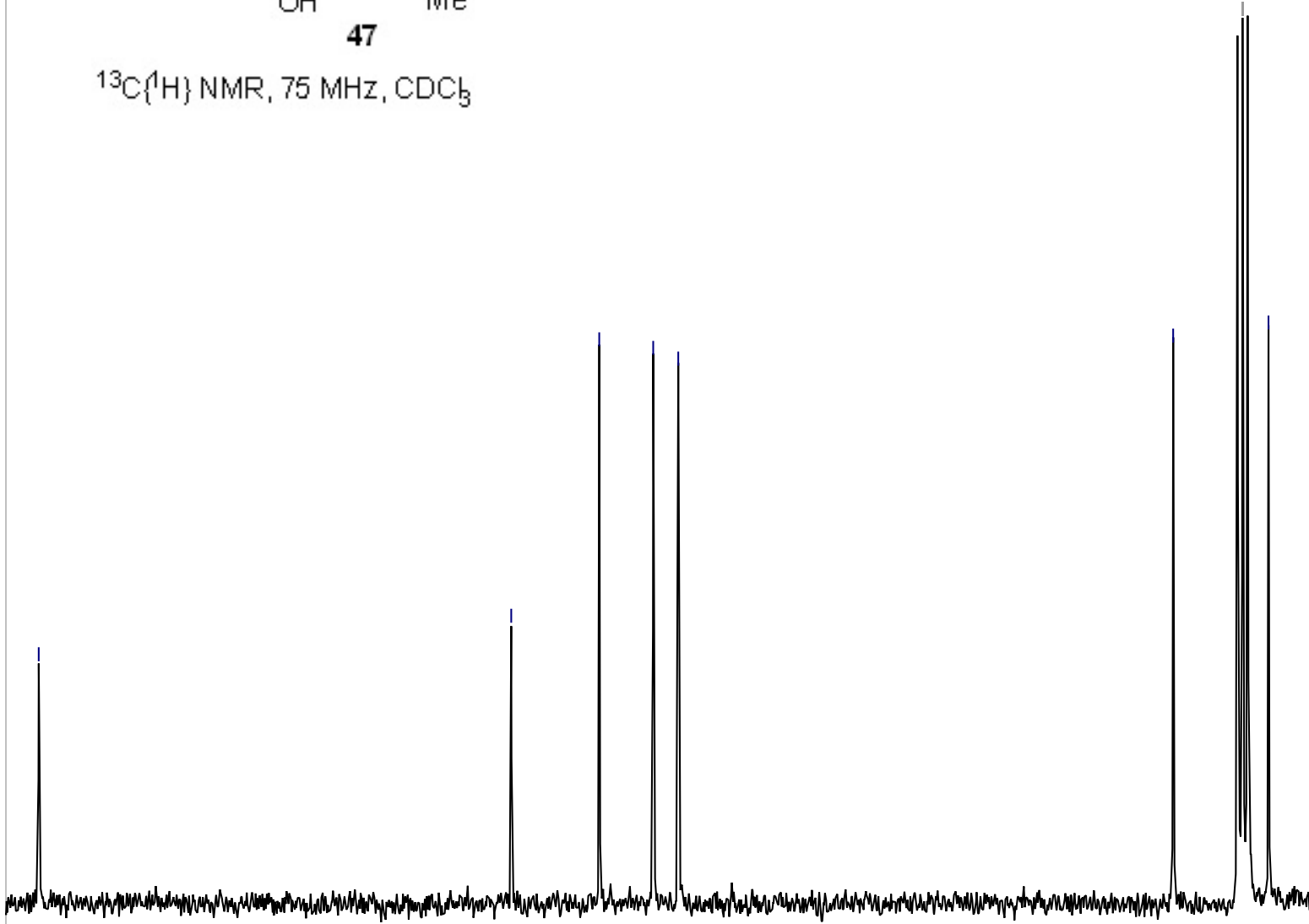


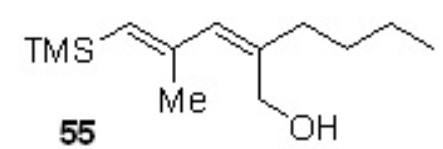

${ }^{1} \mathrm{HNMR}_{1}, 300 \mathrm{MHz}, \mathrm{CDCl}_{3}$

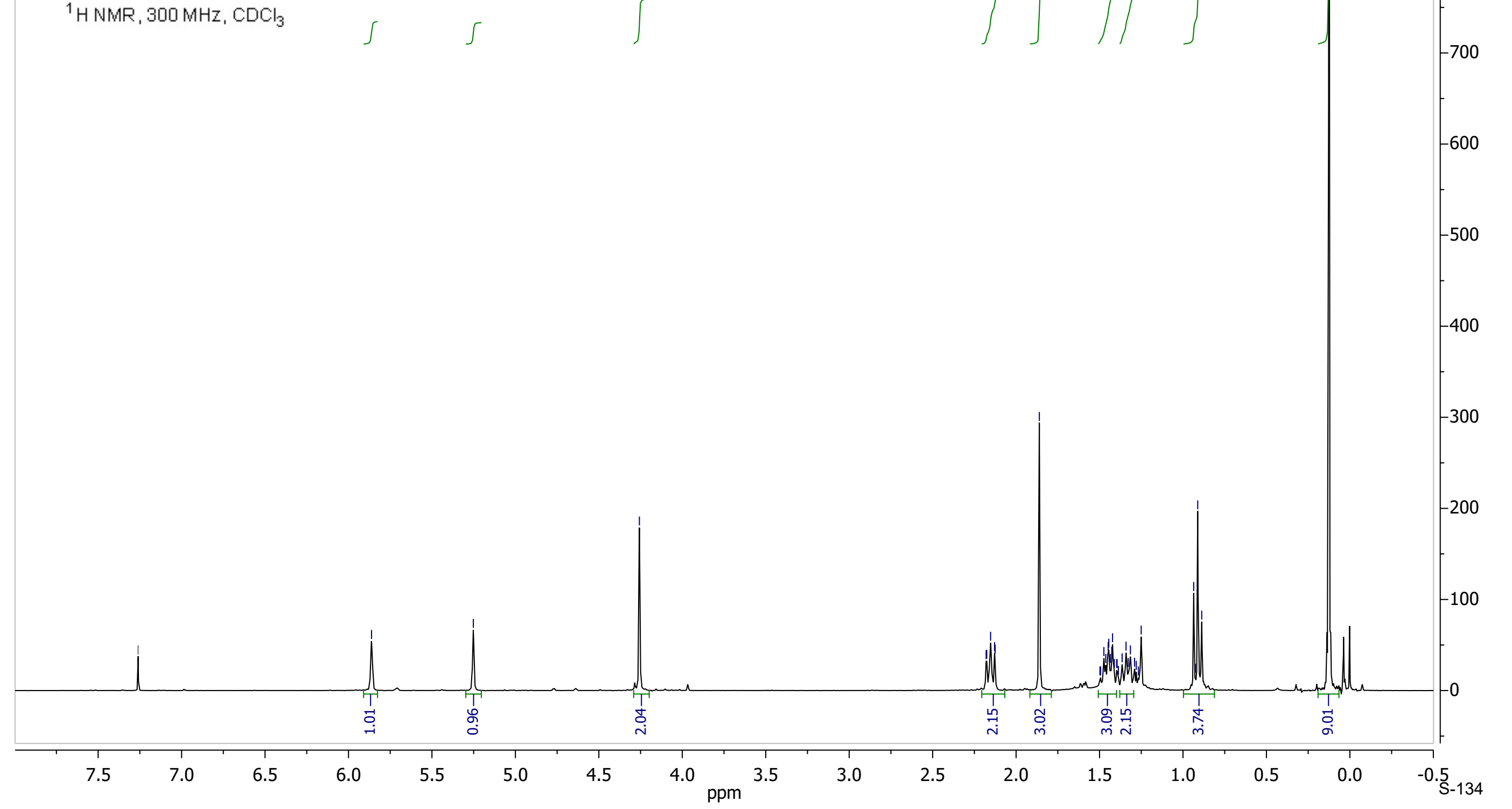




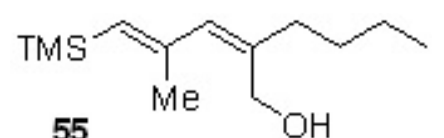

${ }^{13} \mathrm{C}\{\mathrm{H}\} \mathrm{NMR}, 75 \mathrm{MHz}, \mathrm{CDC}$ 


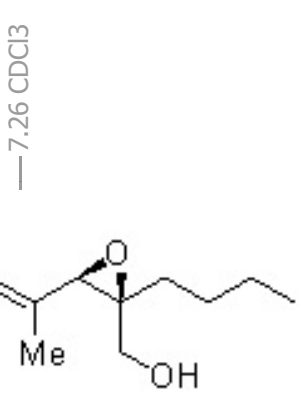

$\stackrel{\substack{g \\ \text { in }}}{i}$

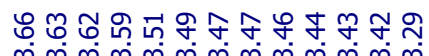
लूm

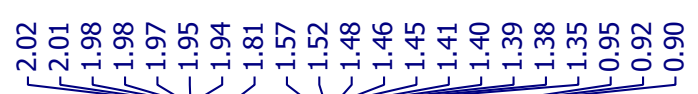

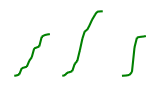

$\mathrm{HNMR}, 300 \mathrm{MHz}, \mathrm{CDCl}_{3}$
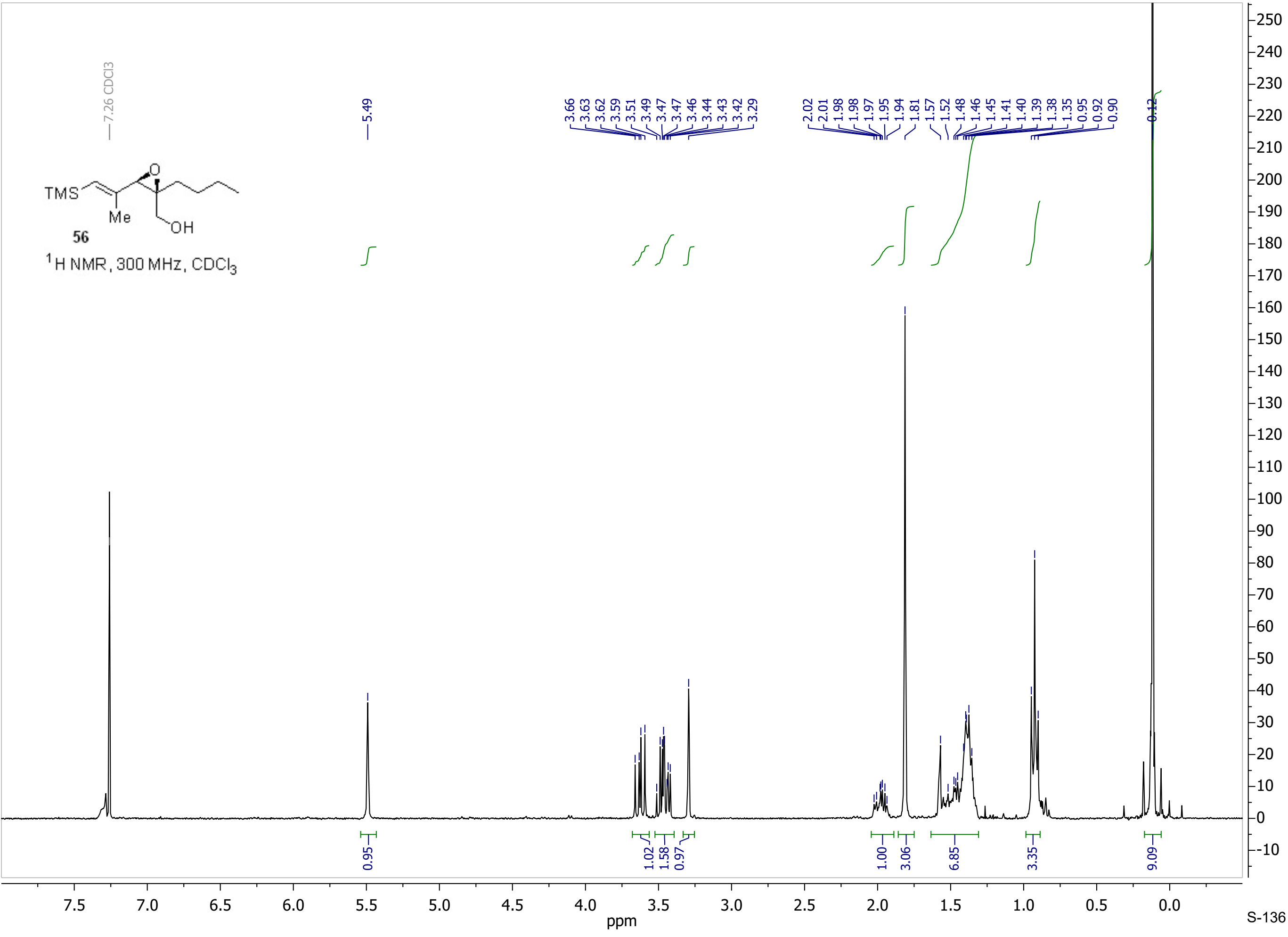

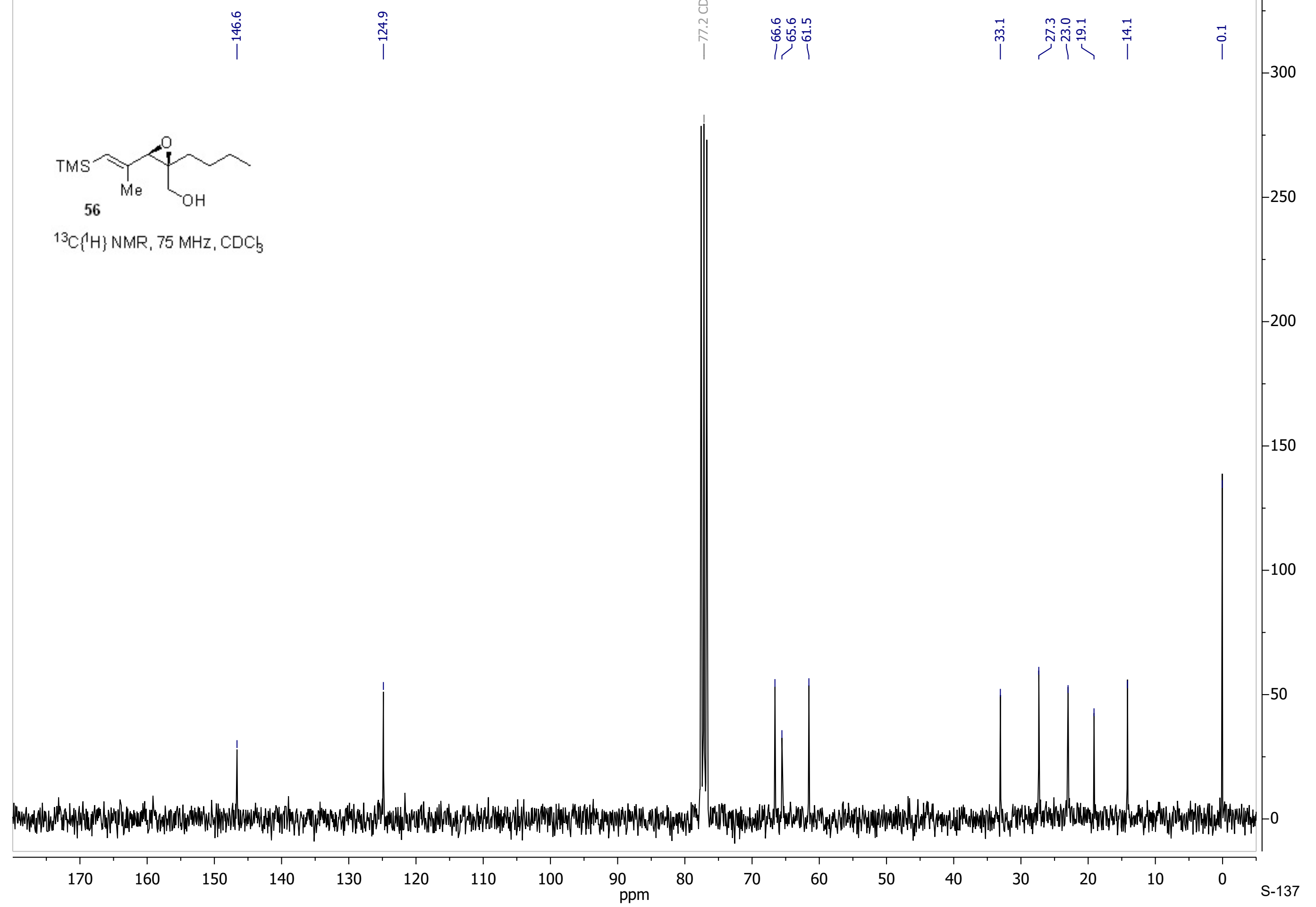

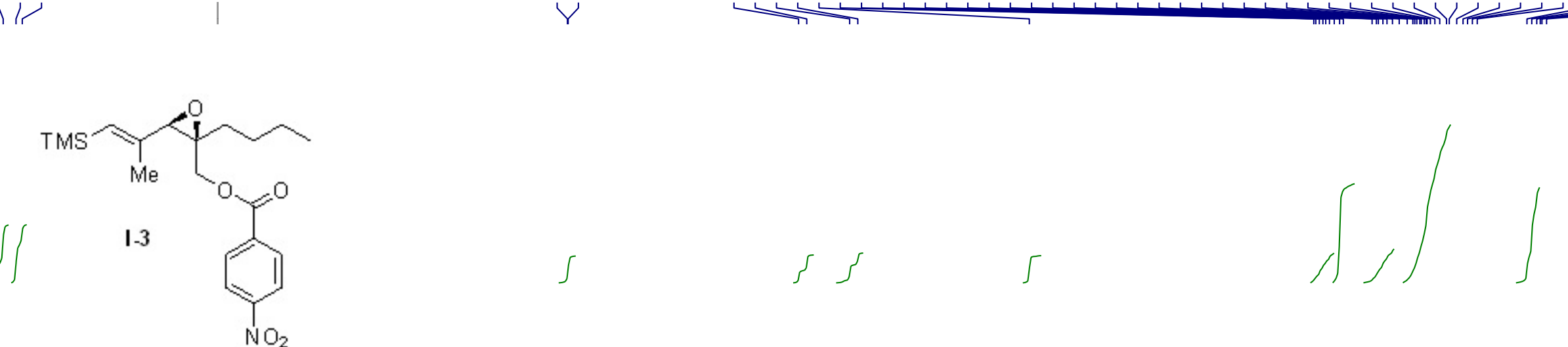

${ }^{1} \mathrm{HNMR}, 300 \mathrm{MHz}, \mathrm{CDCl}_{3}$
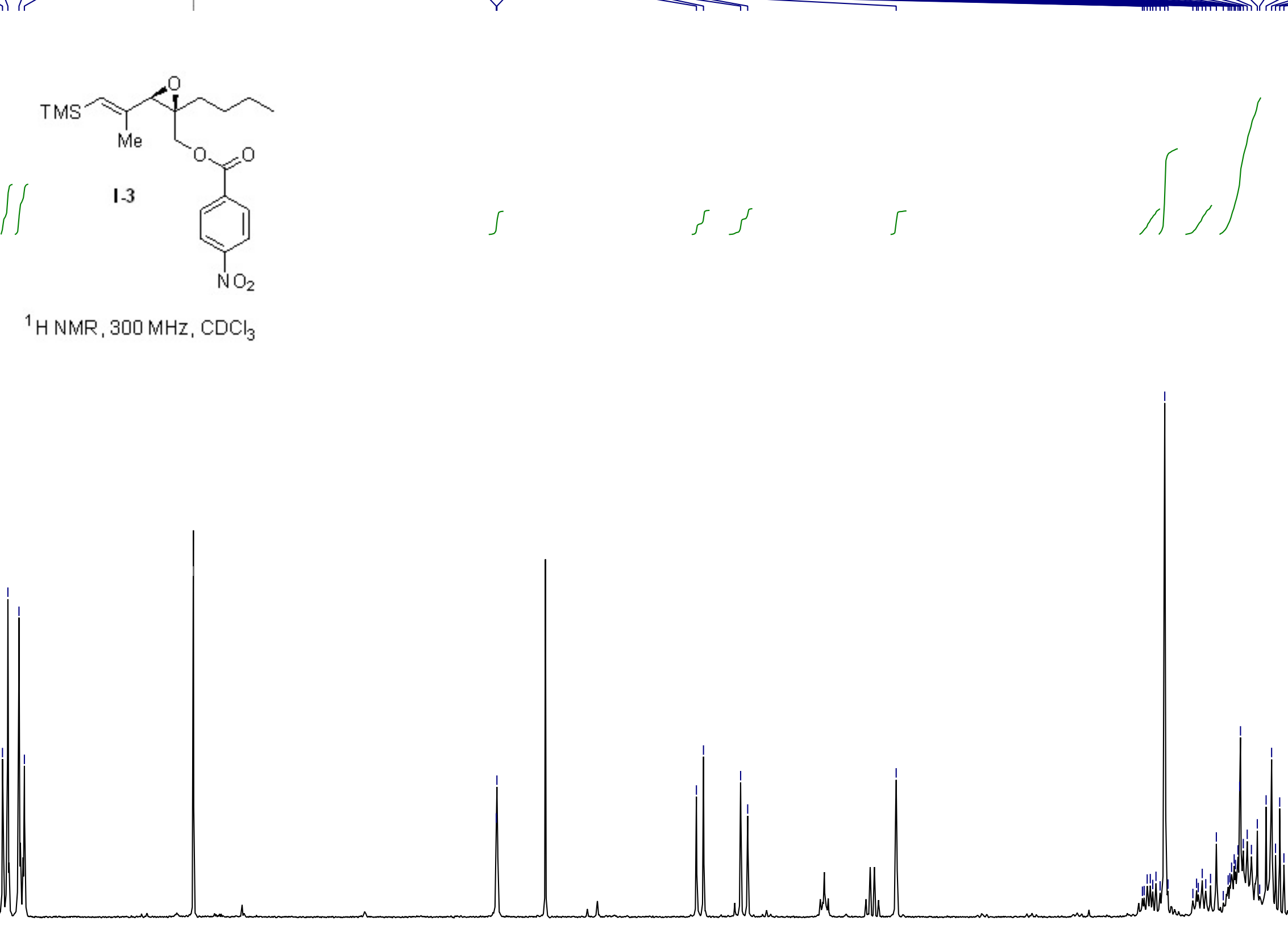

$$
\begin{aligned}
& \text {. }
\end{aligned}
$$

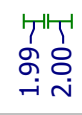
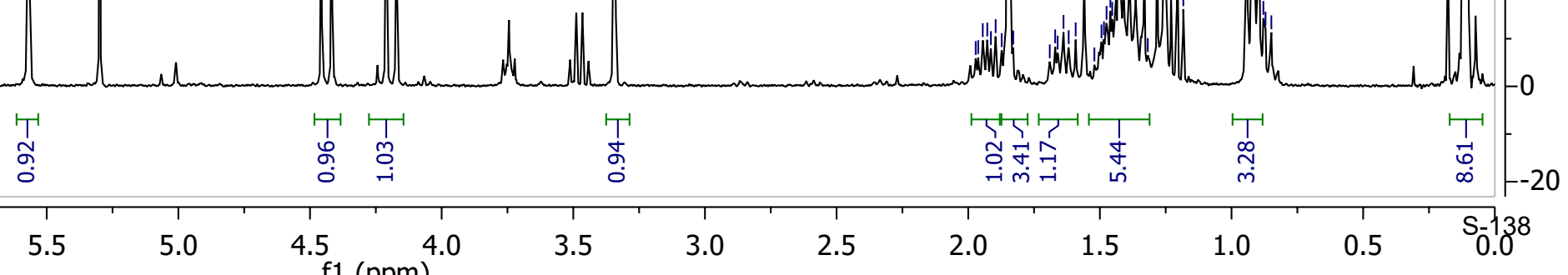


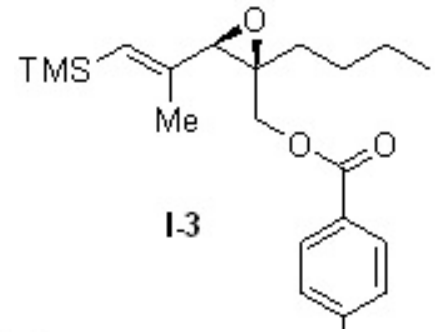

${ }^{13} \mathrm{C}\{\mathrm{H}\} \mathrm{NMR}, 75 \mathrm{MHz}, \mathrm{CDC} 3$
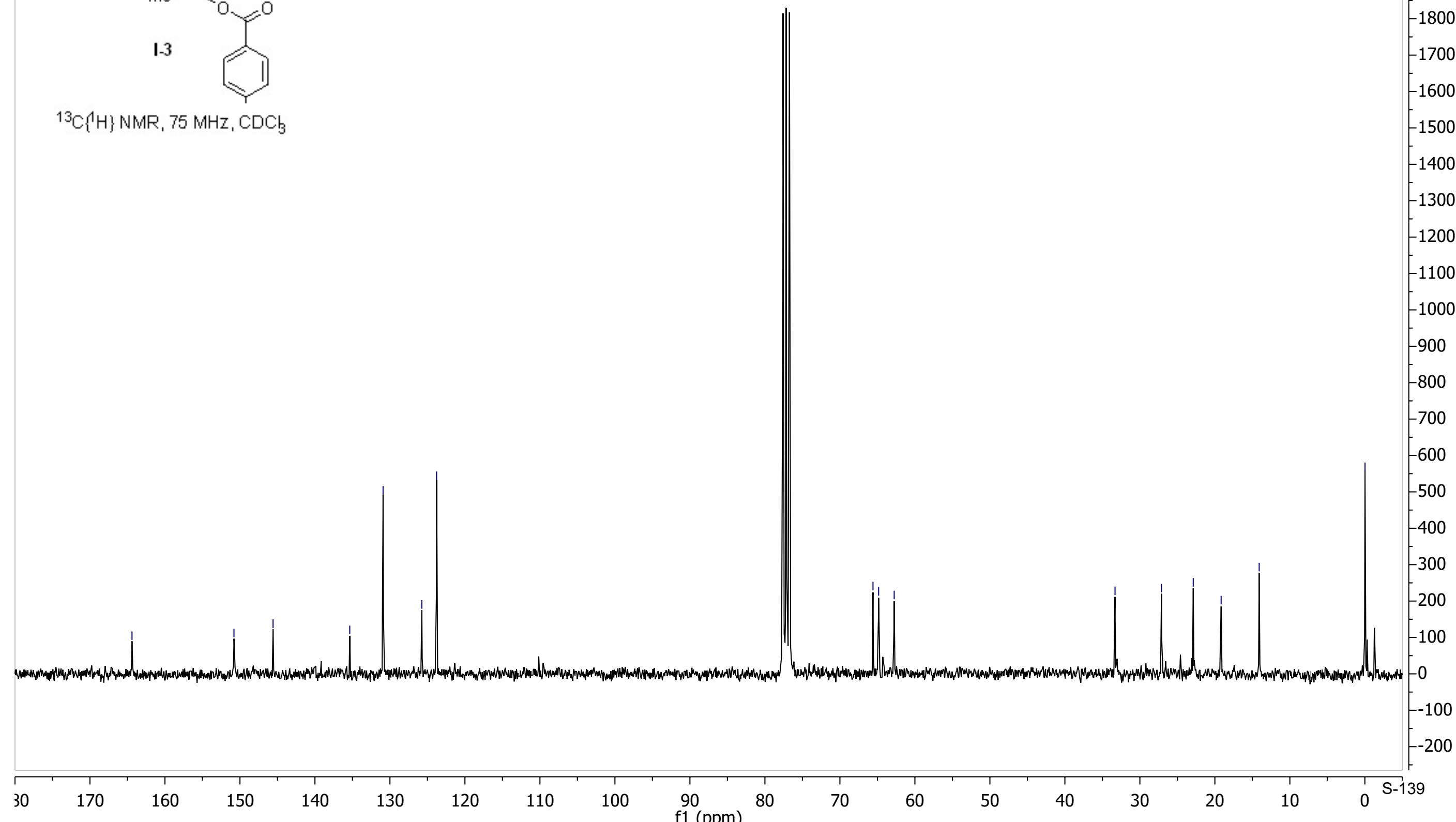


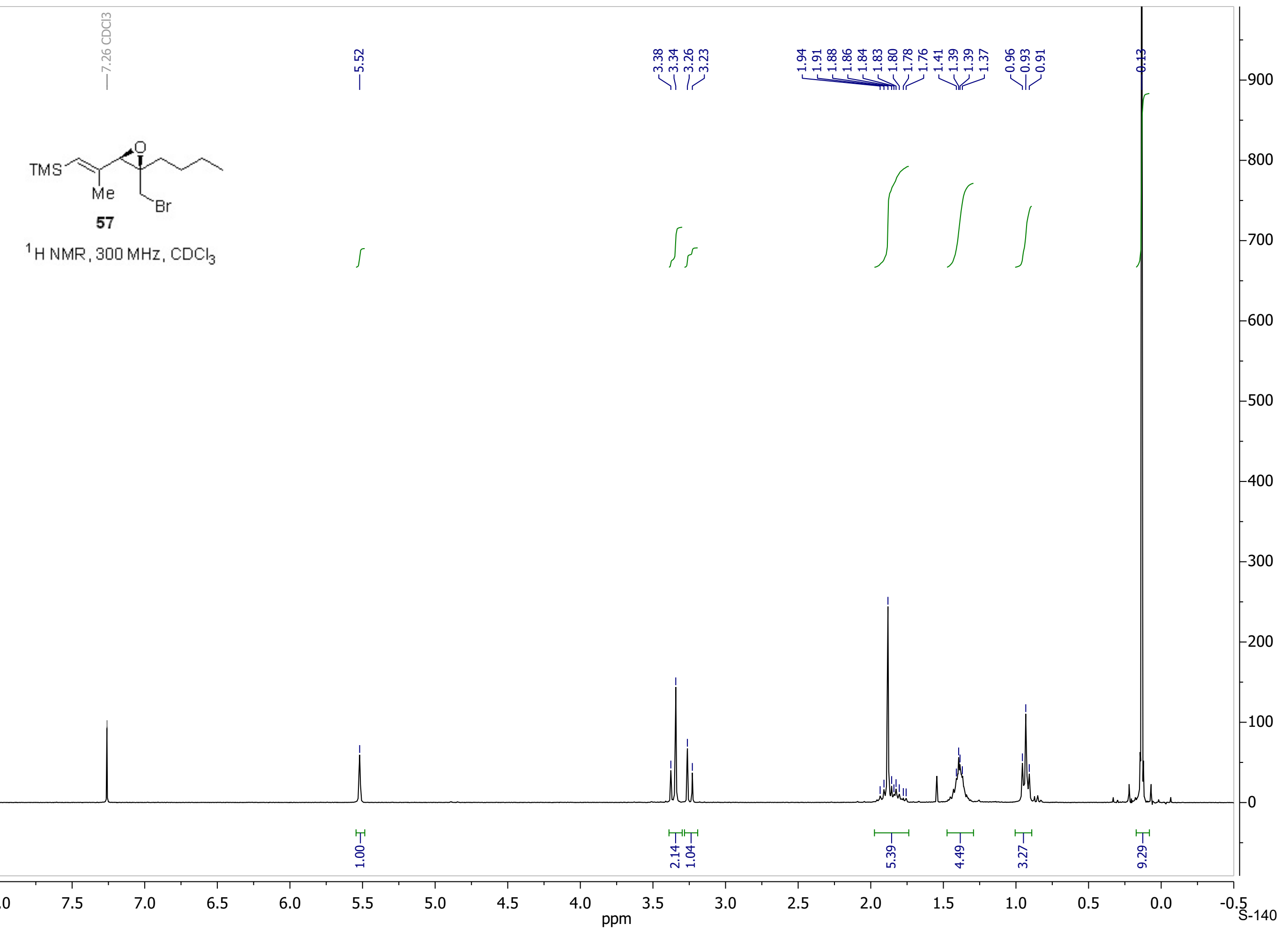




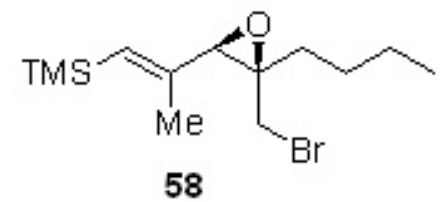

$\left.{ }^{13} \mathrm{C}\{\mathrm{H}\} \mathrm{NMR}, 75 \mathrm{MHz}, \mathrm{CDC}\right\}$ 

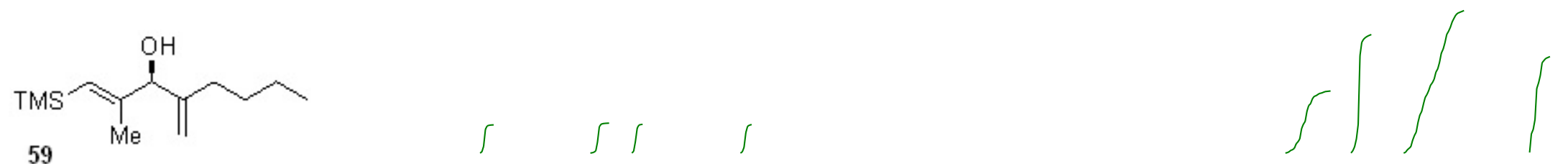

${ }^{1} \mathrm{HNMR}, 300 \mathrm{MHz}, \mathrm{CDCl}_{3}$ 


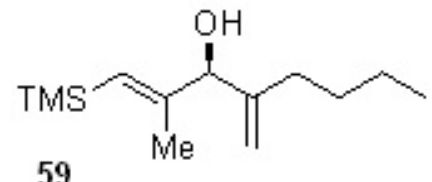

${ }^{13} \mathrm{C}\{\mathrm{H}\} \mathrm{NMR}, 75 \mathrm{MHz}, \mathrm{CDCl}$ 


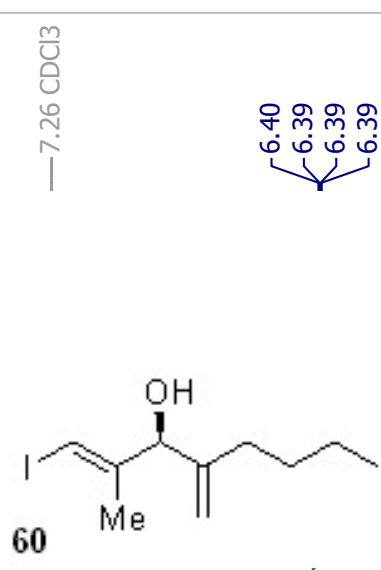

${ }^{1} \mathrm{HNMR}, 300 \mathrm{MHz}, \mathrm{CDCl}_{3}$

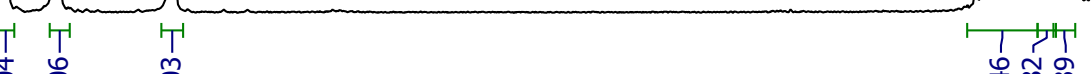




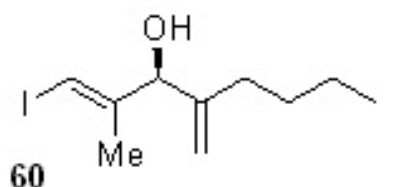

${ }^{13} \mathrm{C}\{\mathrm{H}\}$ NMR, $\left.75 \mathrm{MHz}, \mathrm{CDC}\right\}$ 
$\overbrace{M e}^{1} \overbrace{01}^{\mathrm{O}}$
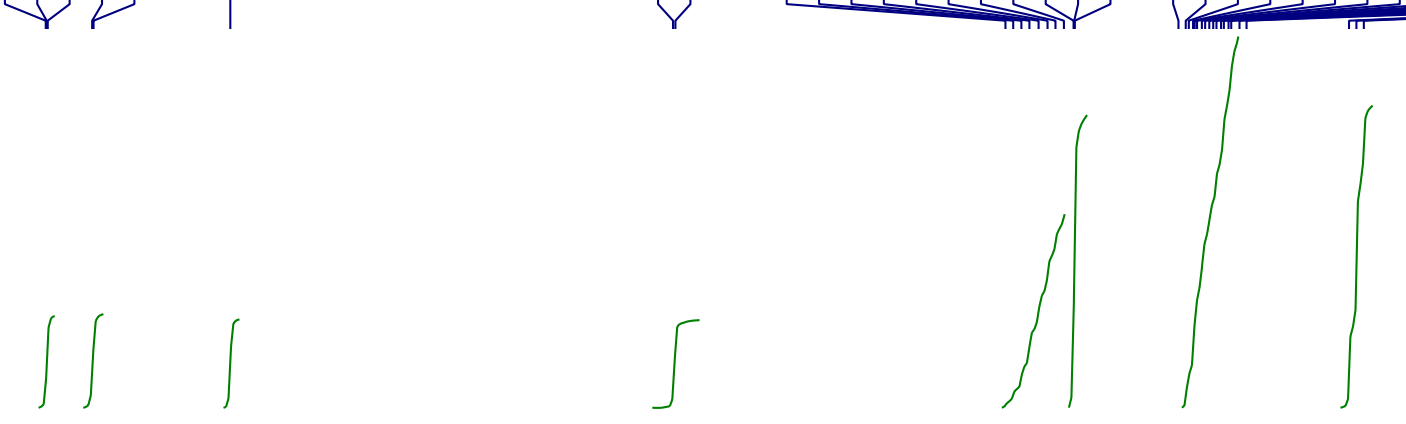

${ }^{1} \mathrm{HNMR}, 300 \mathrm{MHz}, \mathrm{CDCl}_{3}$

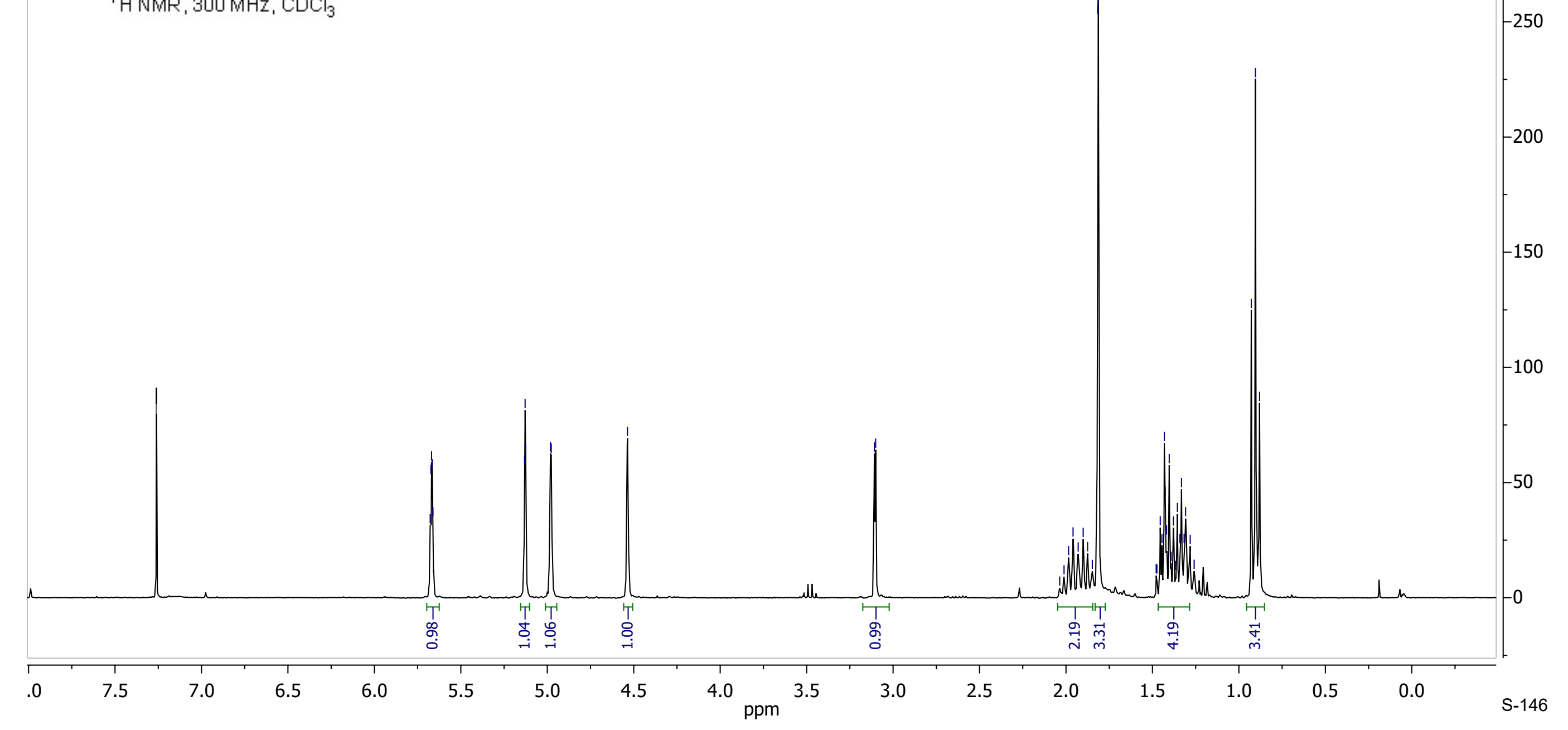




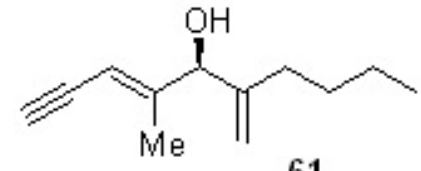

${ }^{13} \mathrm{C}_{\{}[\mathrm{H}\} \mathrm{NMR}, 75 \mathrm{MHz}, \mathrm{CDC} 3$

$\begin{array}{llllllll}60 & 150 & 140 & 130 & 120 & 110 & 100 & 90\end{array}$




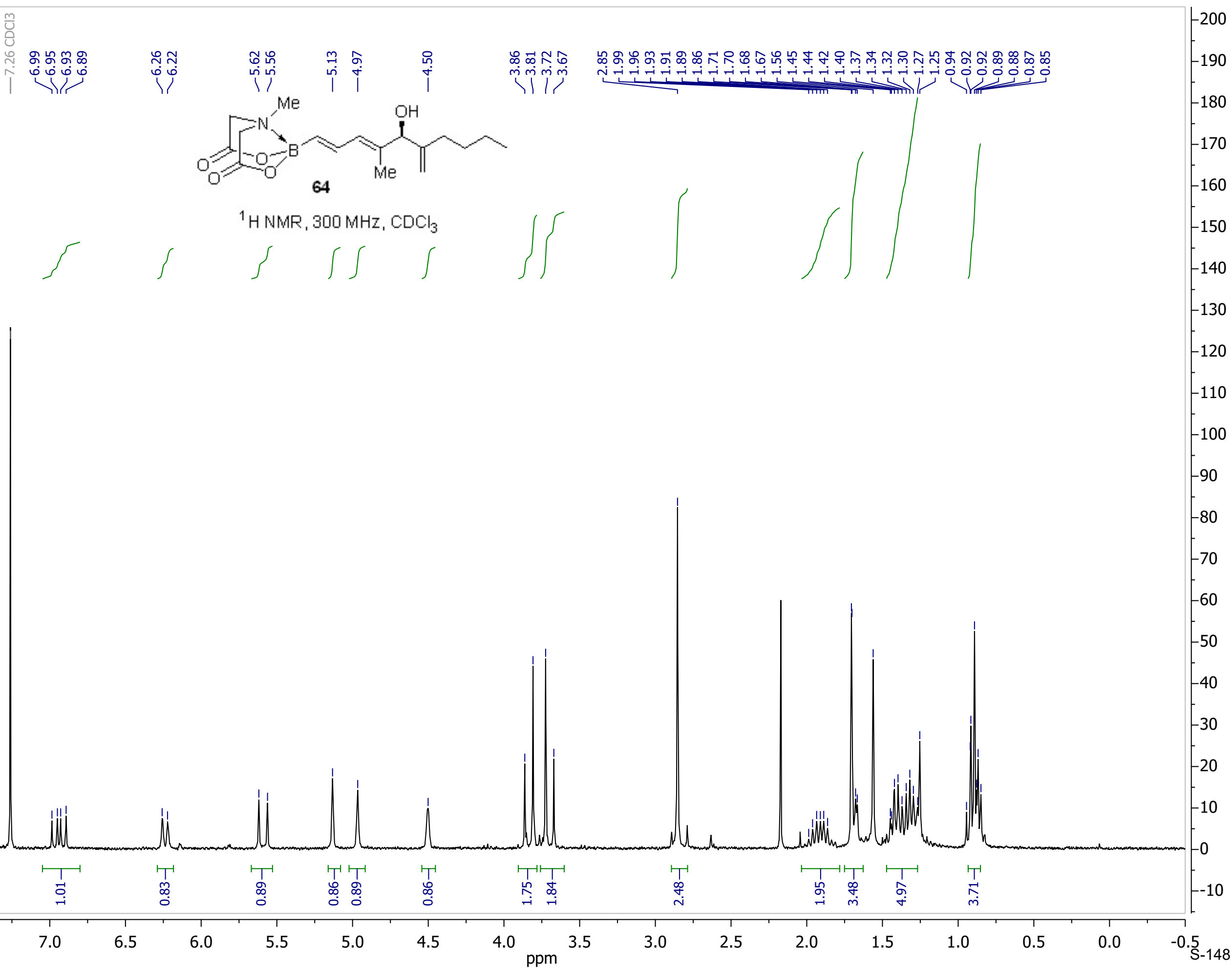




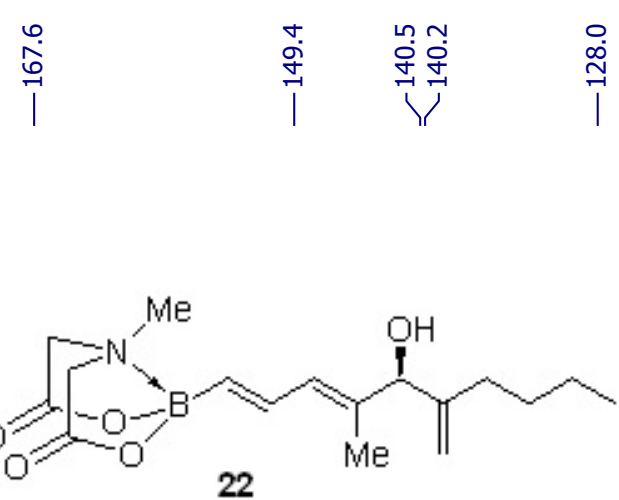




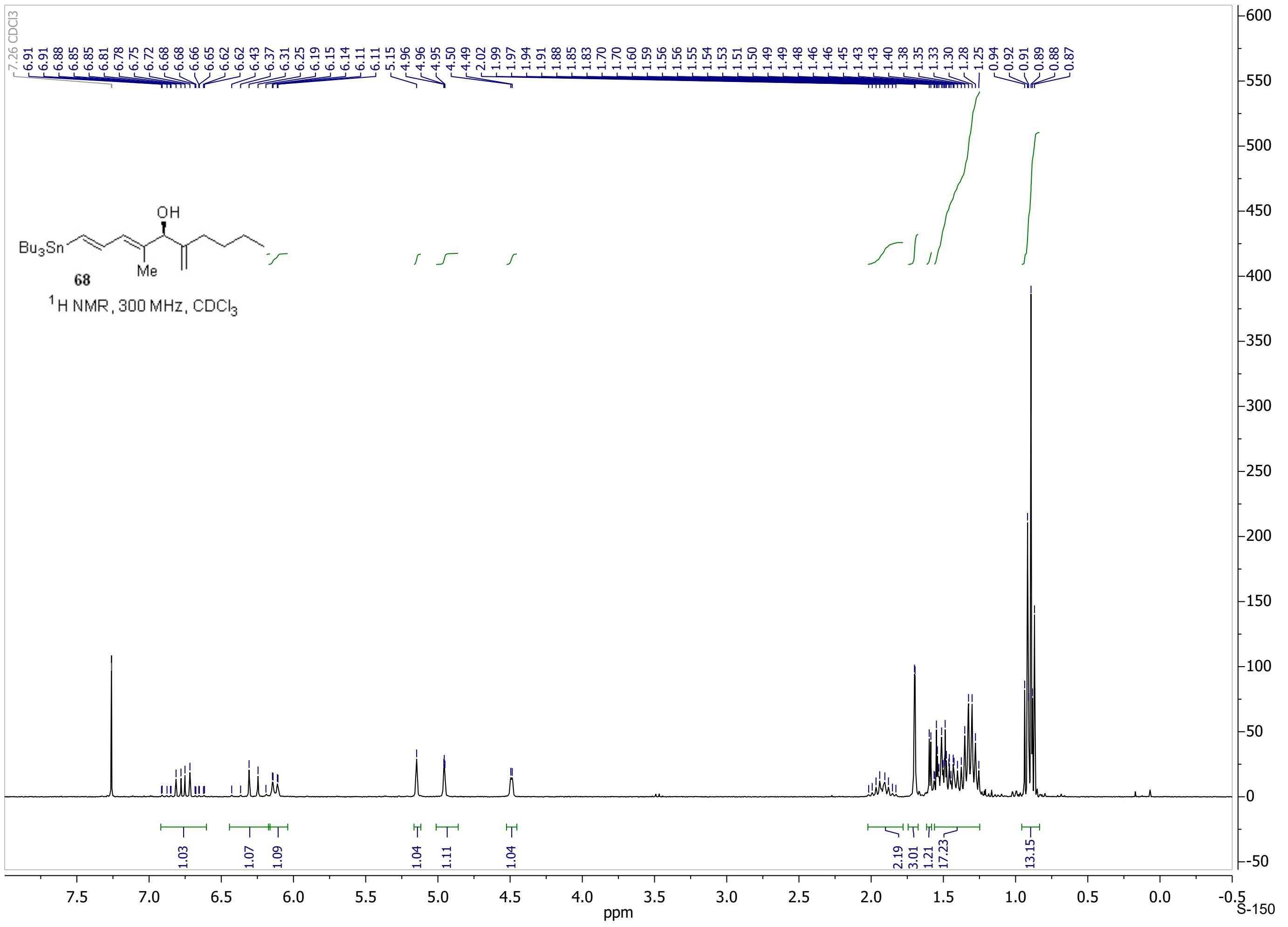




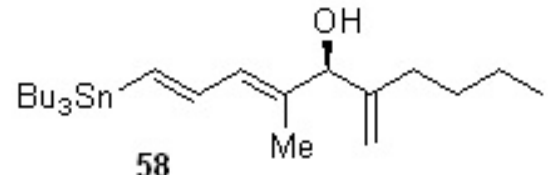

$\left.{ }^{13} \mathrm{C}\{\mathrm{H}\} \mathrm{NMR}, 75 \mathrm{MHz}, \mathrm{CDC}\right\}$ 


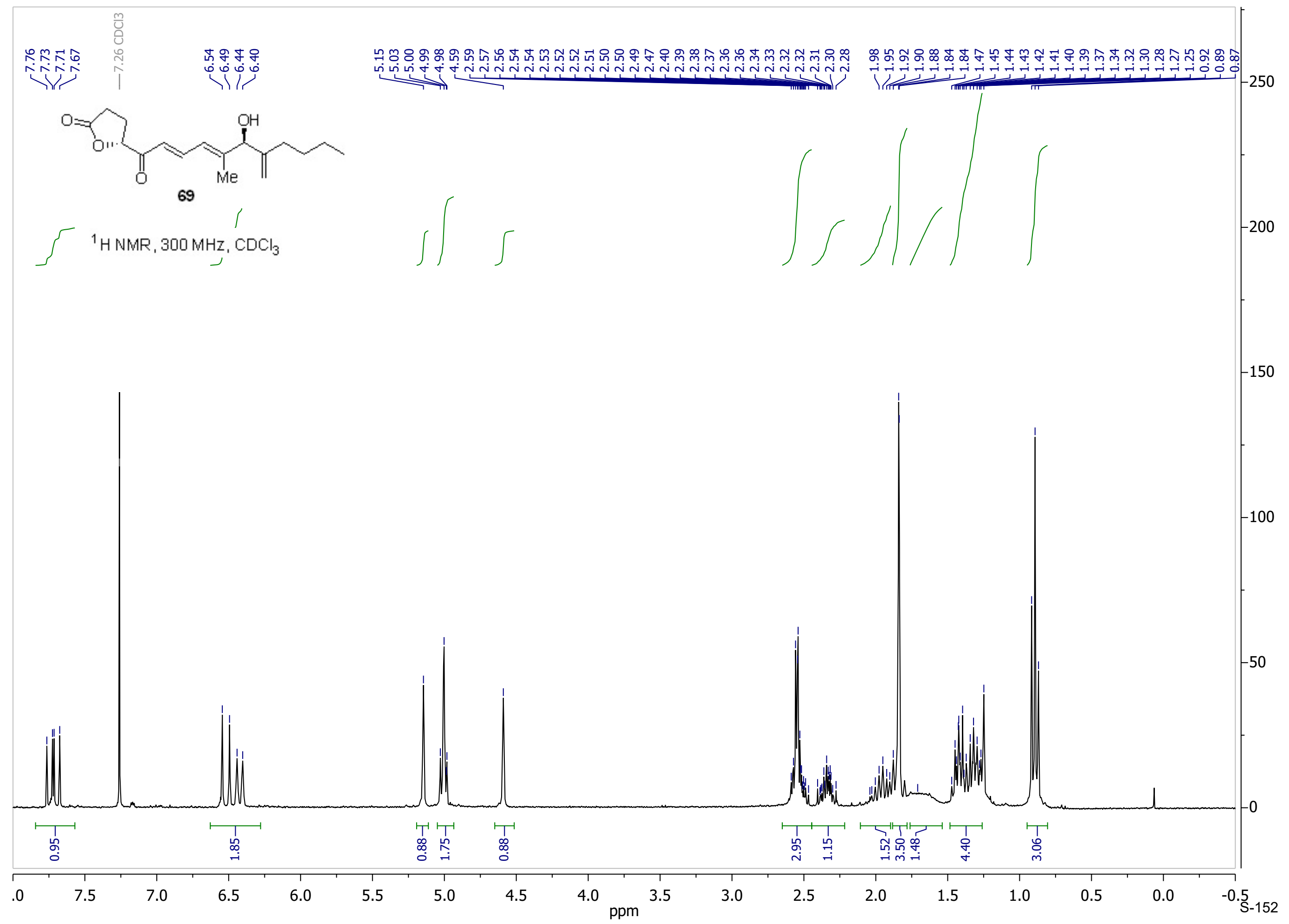




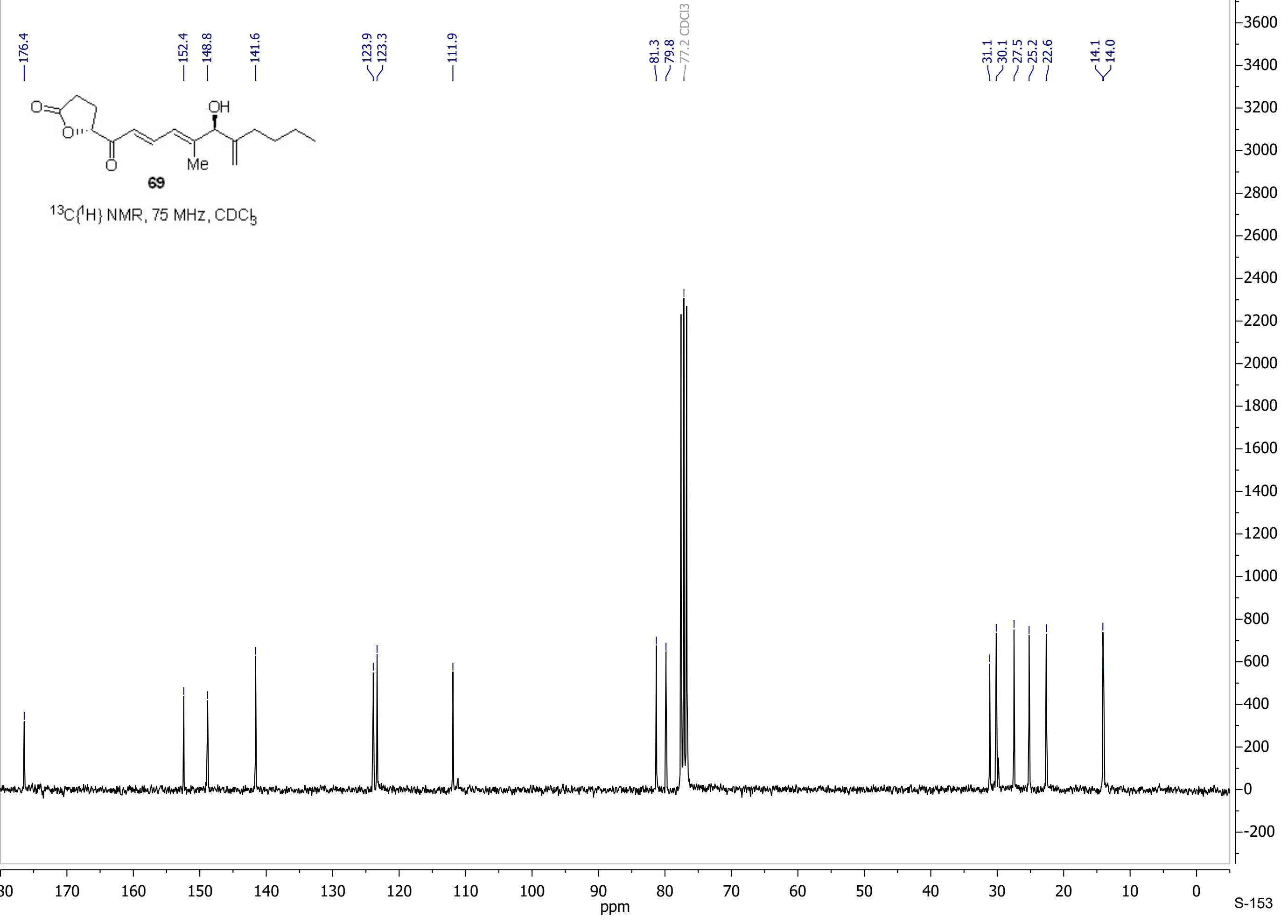




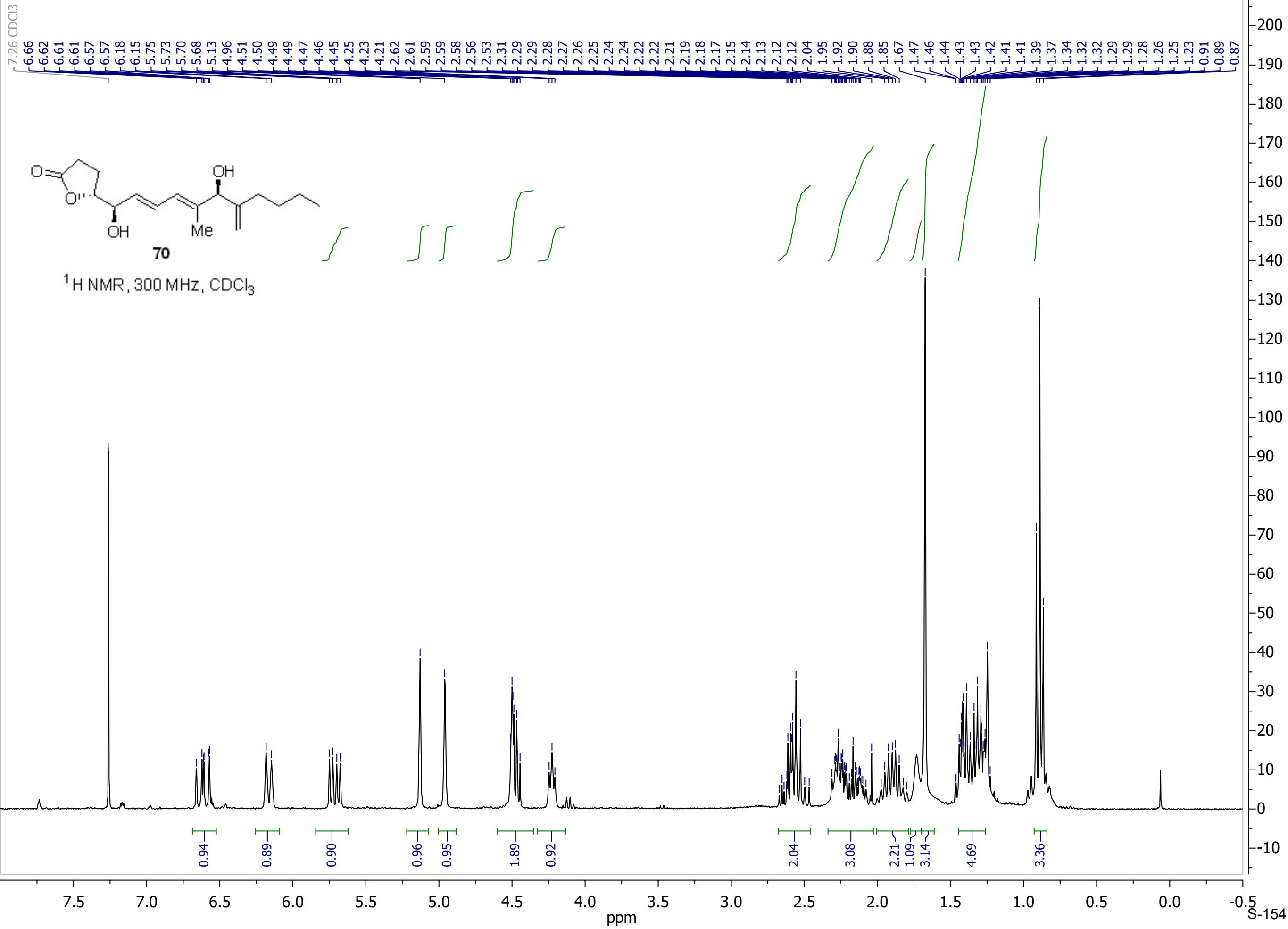




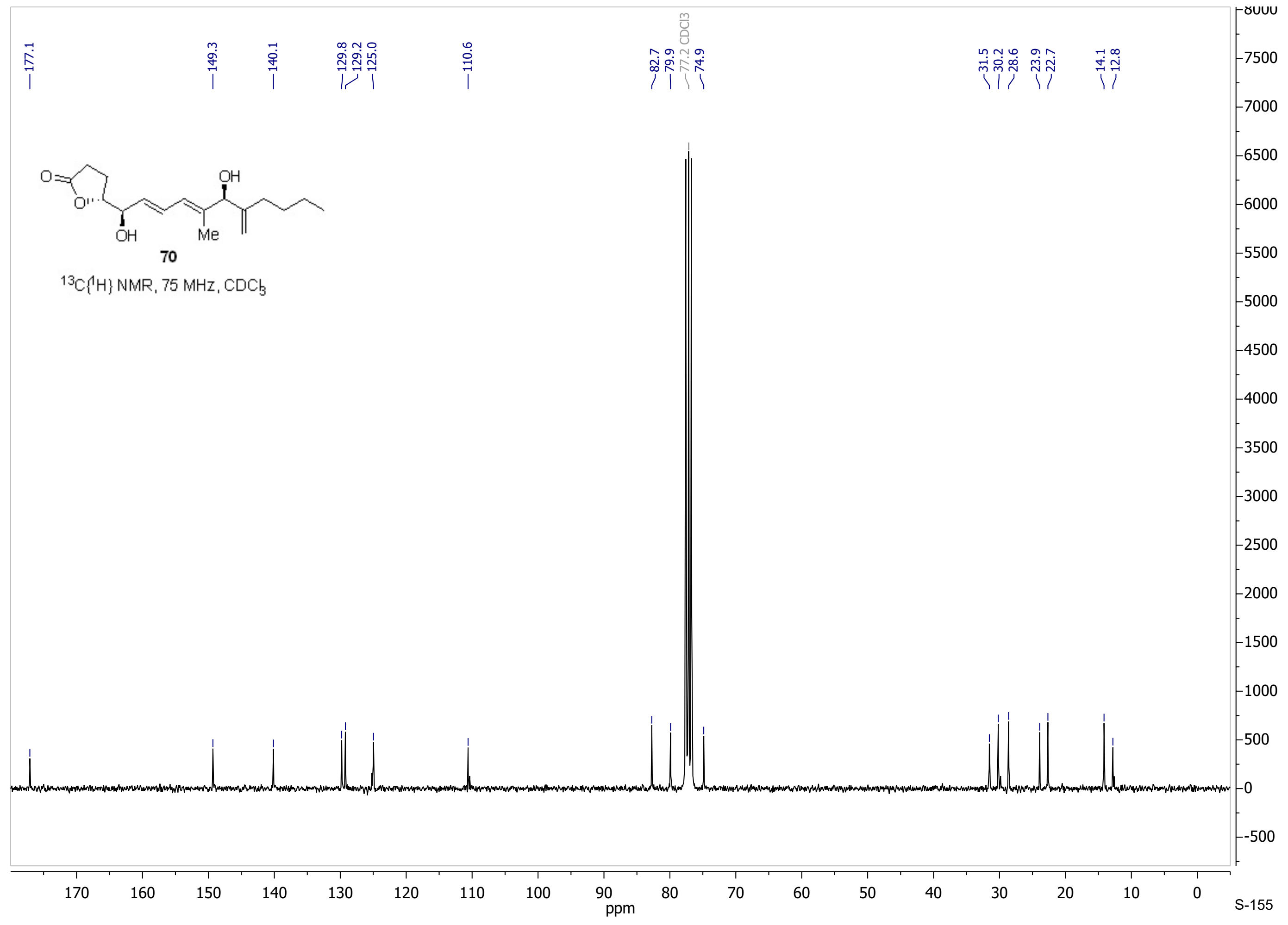




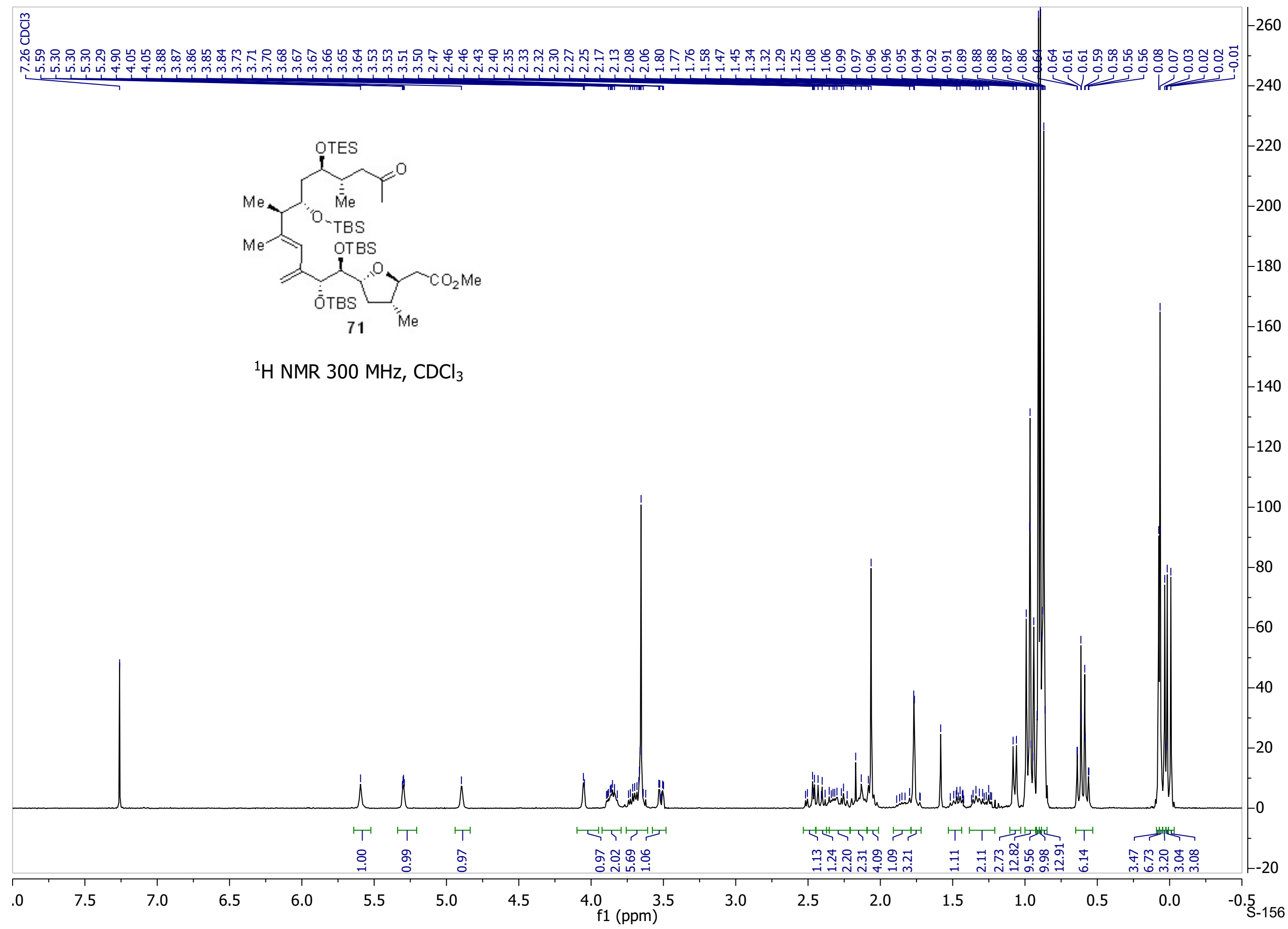




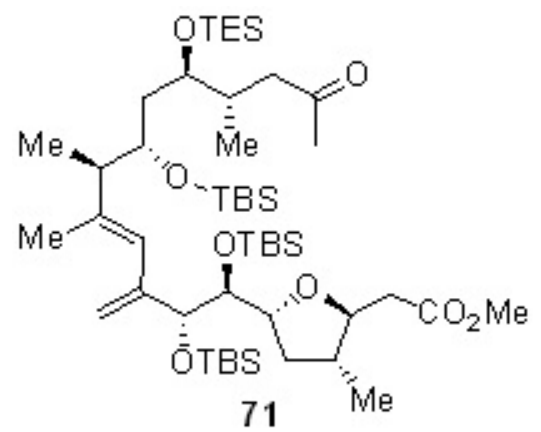

||

${ }^{13} \mathrm{C}\left\{{ }^{1} \mathrm{H}\right\}$ NMR $75 \mathrm{MHz}, \mathrm{CDCl}_{3}$

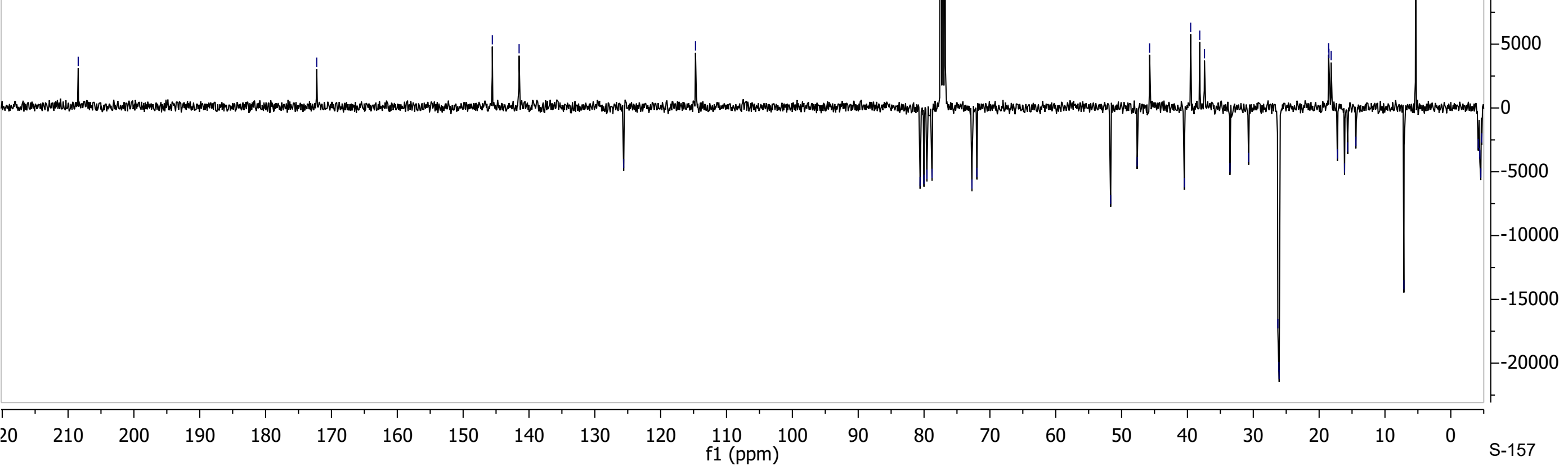




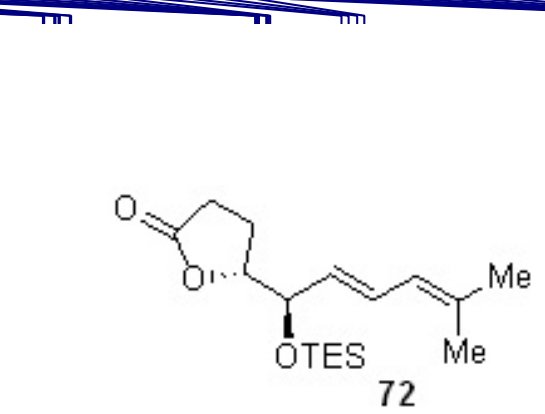

${ }^{1} \mathrm{H}$ NMR $300 \mathrm{MHz}, \mathrm{CDCl}_{3}$

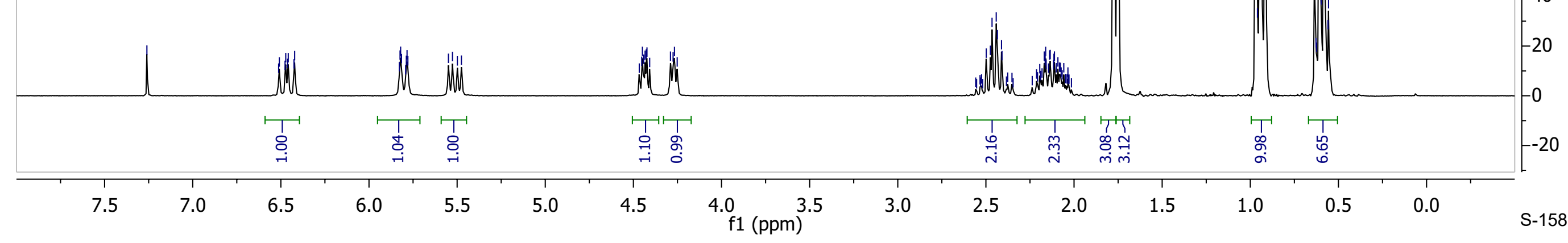




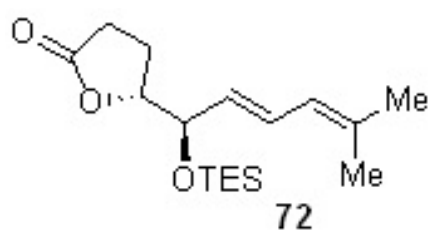

${ }^{13} \mathrm{C}\left\{{ }^{1} \mathrm{H}\right\}$ NMR $75 \mathrm{MHz}, \mathrm{CDCl}_{3}$

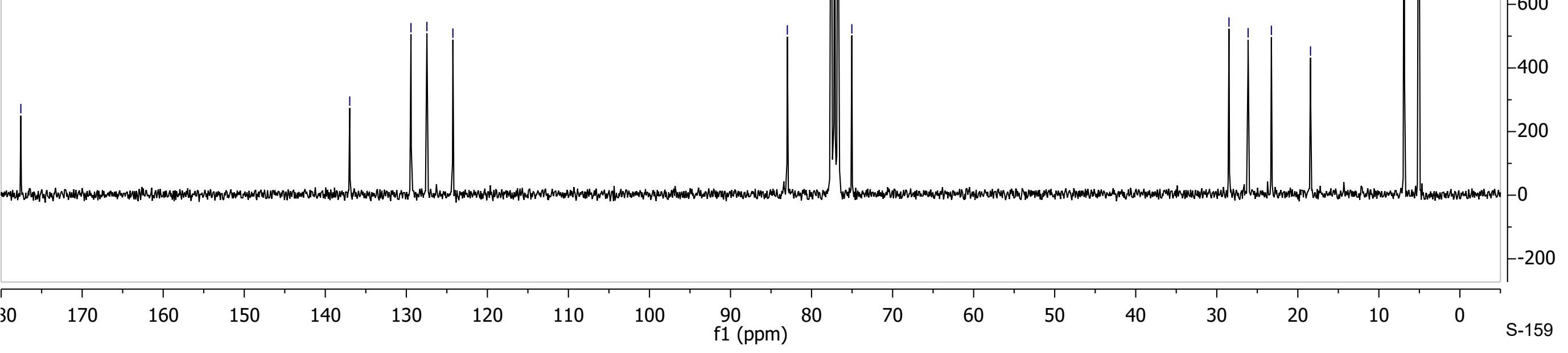



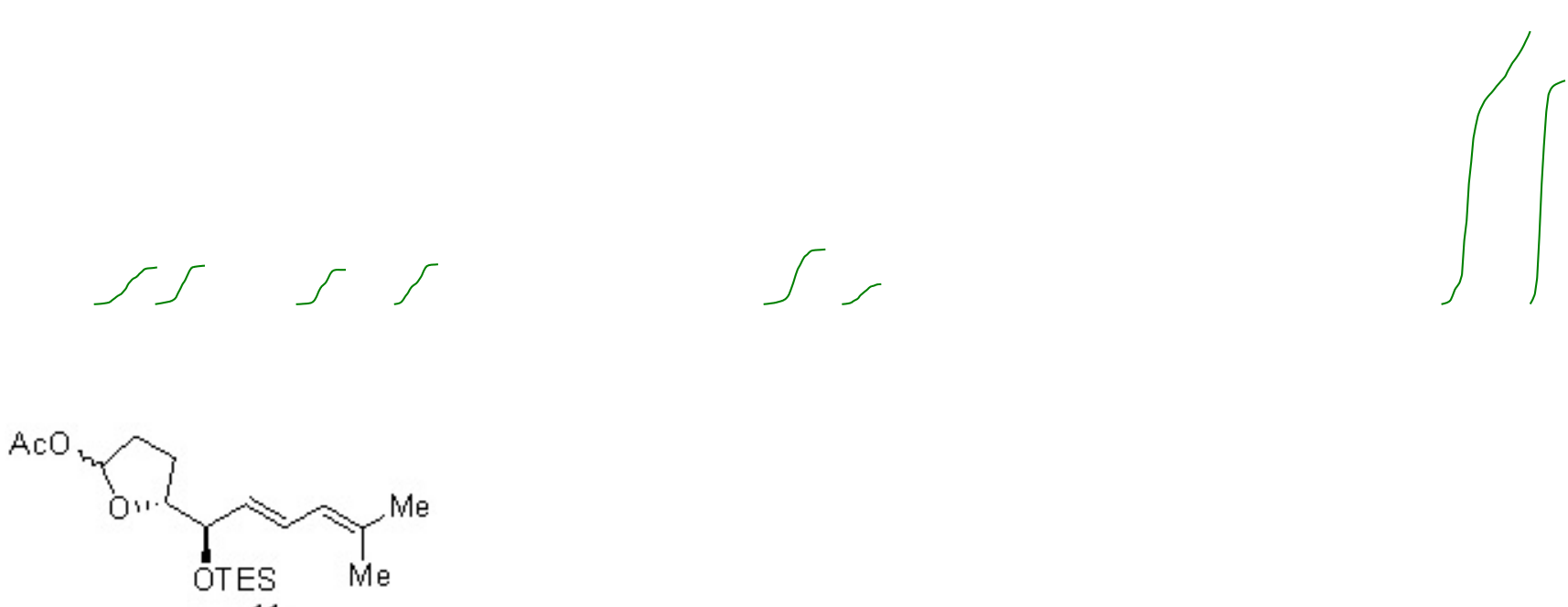

11a

${ }^{1} \mathrm{H}$ NMR $300 \mathrm{MHz}, \mathrm{CDCl}_{3}$

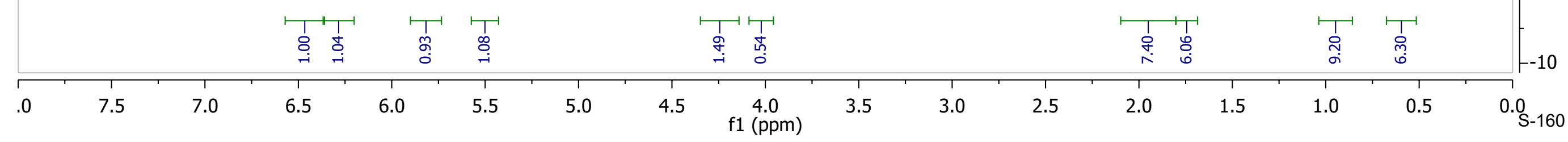




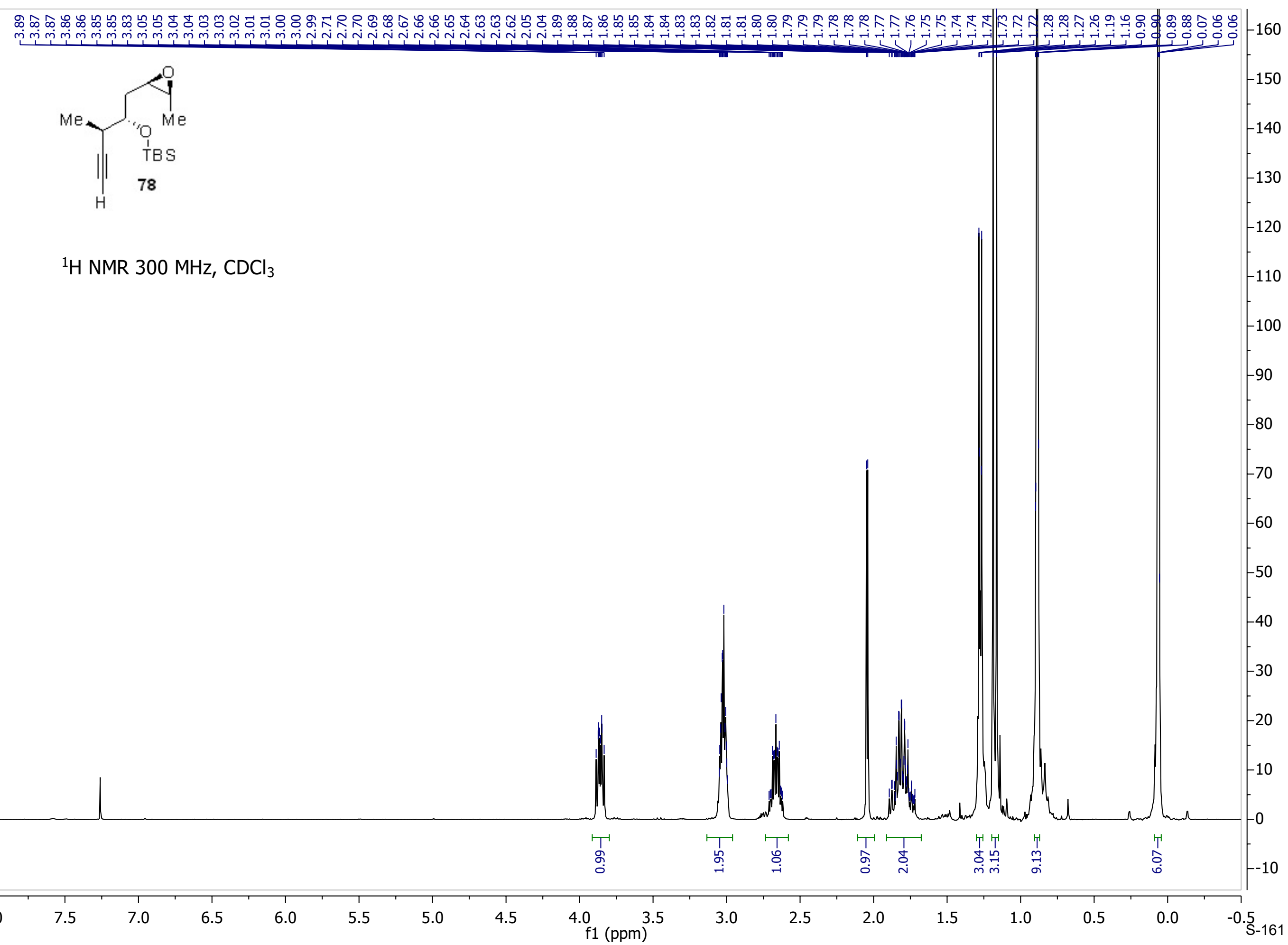




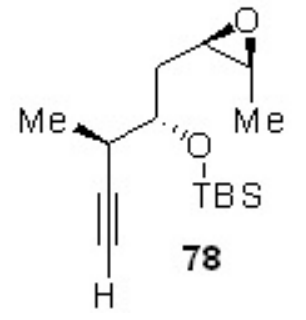

${ }^{13} \mathrm{C}\left\{{ }^{1} \mathrm{H}\right\} \mathrm{NMR} 75 \mathrm{MHz}, \mathrm{CDCl}_{3}$

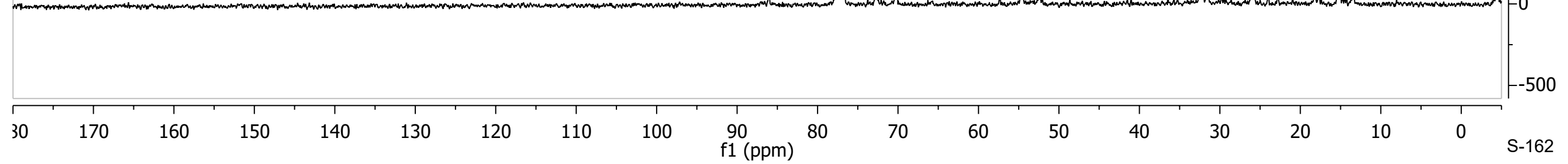



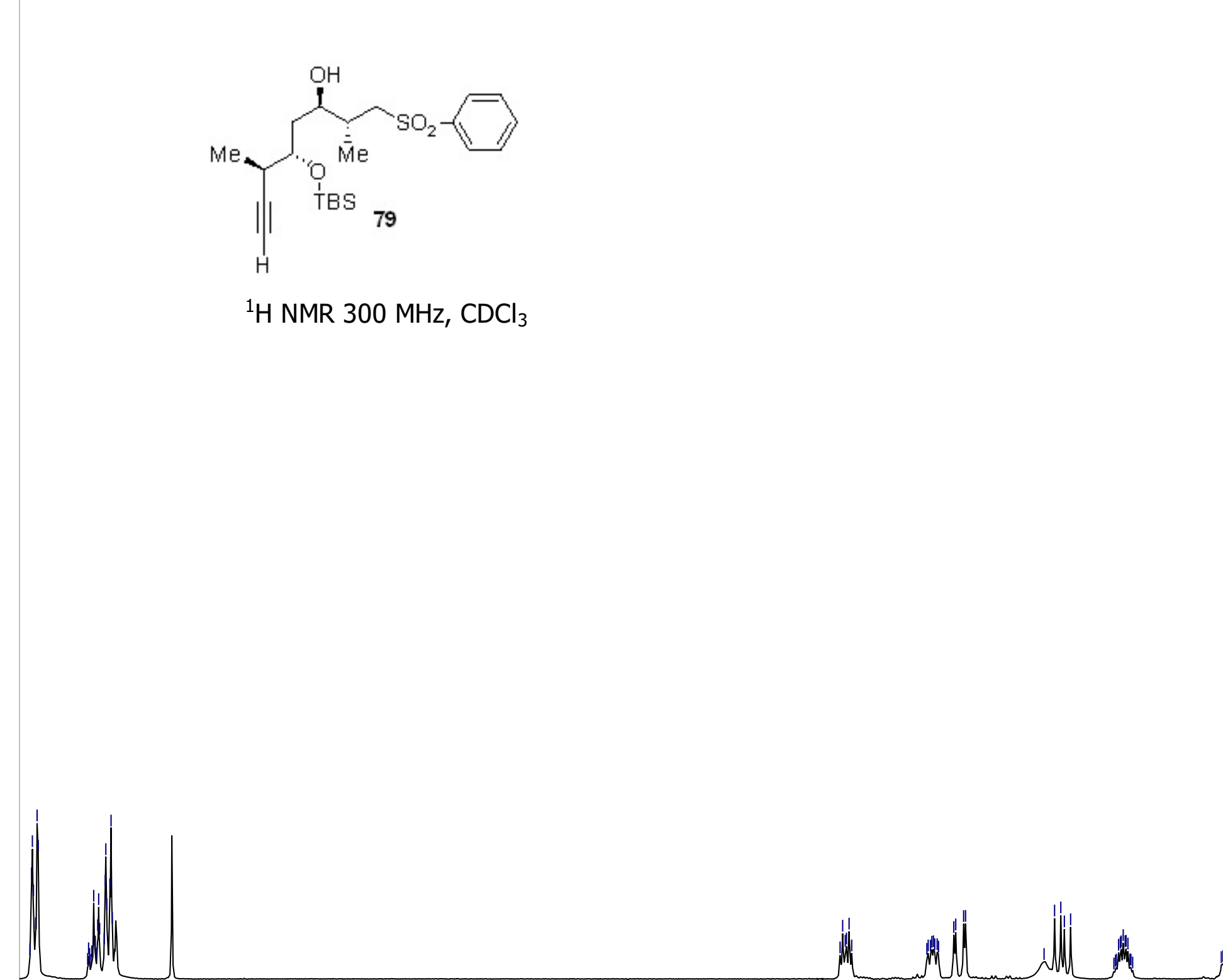

\section{${ }^{1} \mathrm{H}$ NMR $300 \mathrm{MHz}, \mathrm{CDCl}_{3}$ \\ ,}

.

T

సे ?ำ

7.5

7.0

6.5

$6.0 \quad 5.5$

$\begin{array}{lll}5.0 & 4.5 & 4.0 \\ & & f 1(\mathrm{ppm})\end{array}$

(2.0.0.




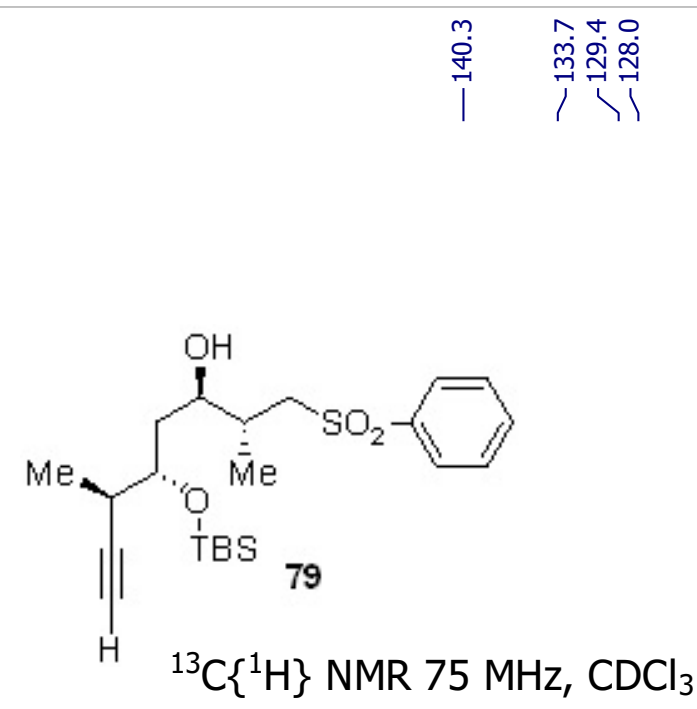

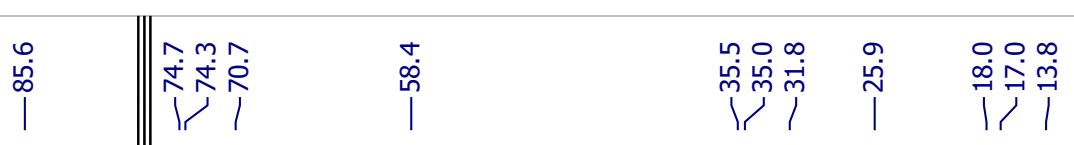

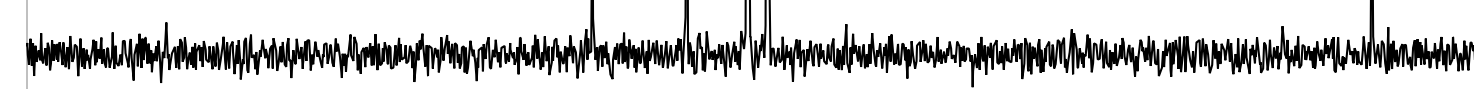

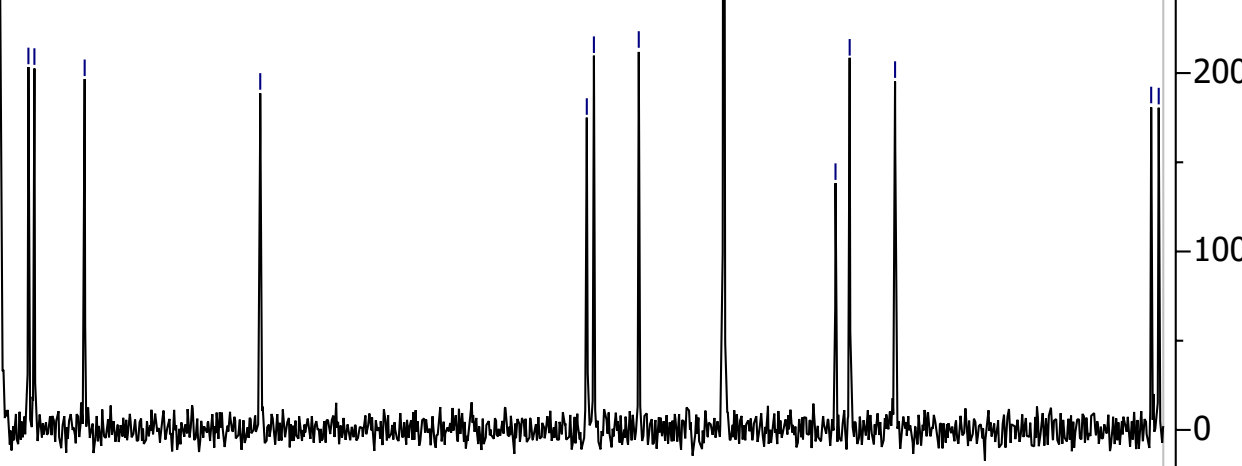




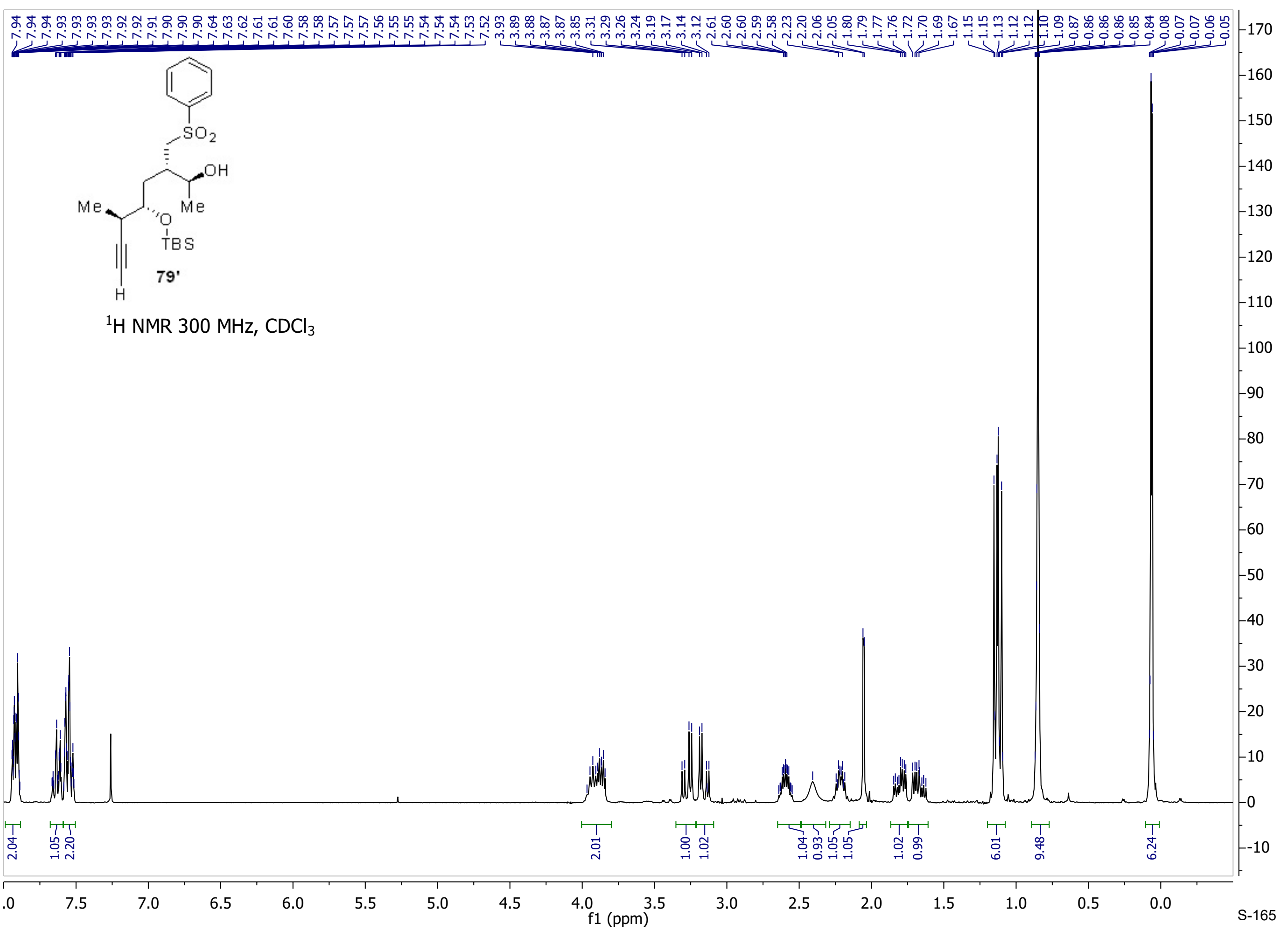




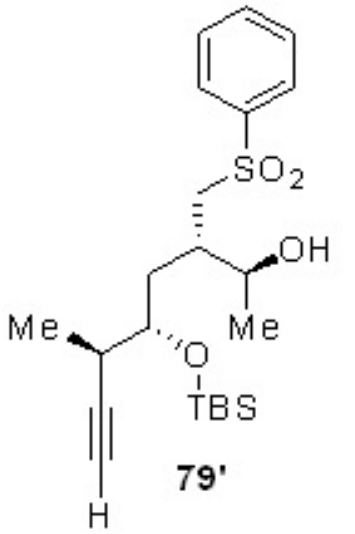

${ }^{13} \mathrm{C}\left\{{ }^{1} \mathrm{H}\right\}$ NMR $75 \mathrm{MHz}, \mathrm{CDCl}_{3}$ 


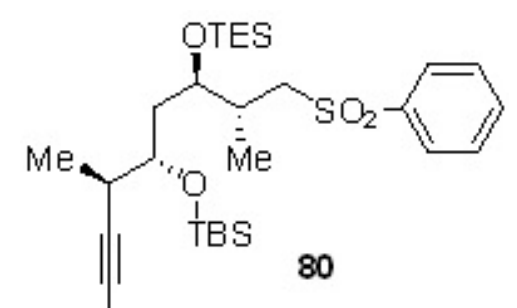

${ }^{1} \mathrm{H} \mathrm{NMR} 300 \mathrm{MHz}, \mathrm{CDCl}_{3}$
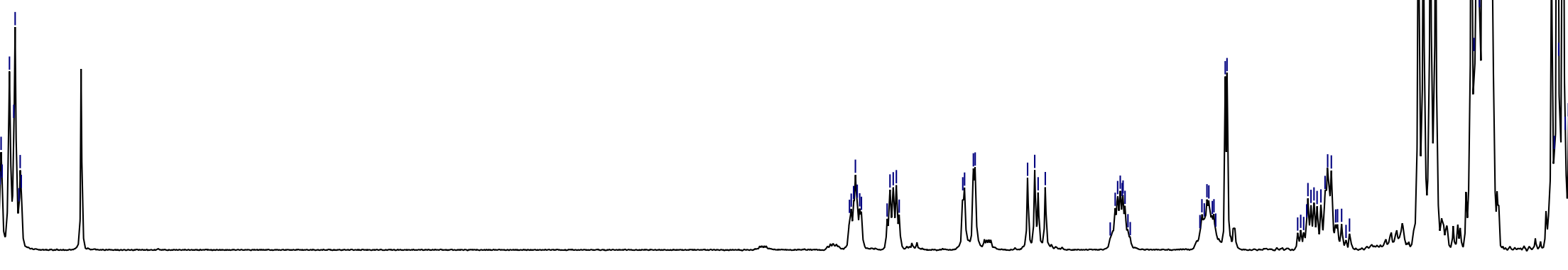
<smiles>CC(C)(C)C(=O)OCCC(=O)Oc1ccccc1</smiles> 

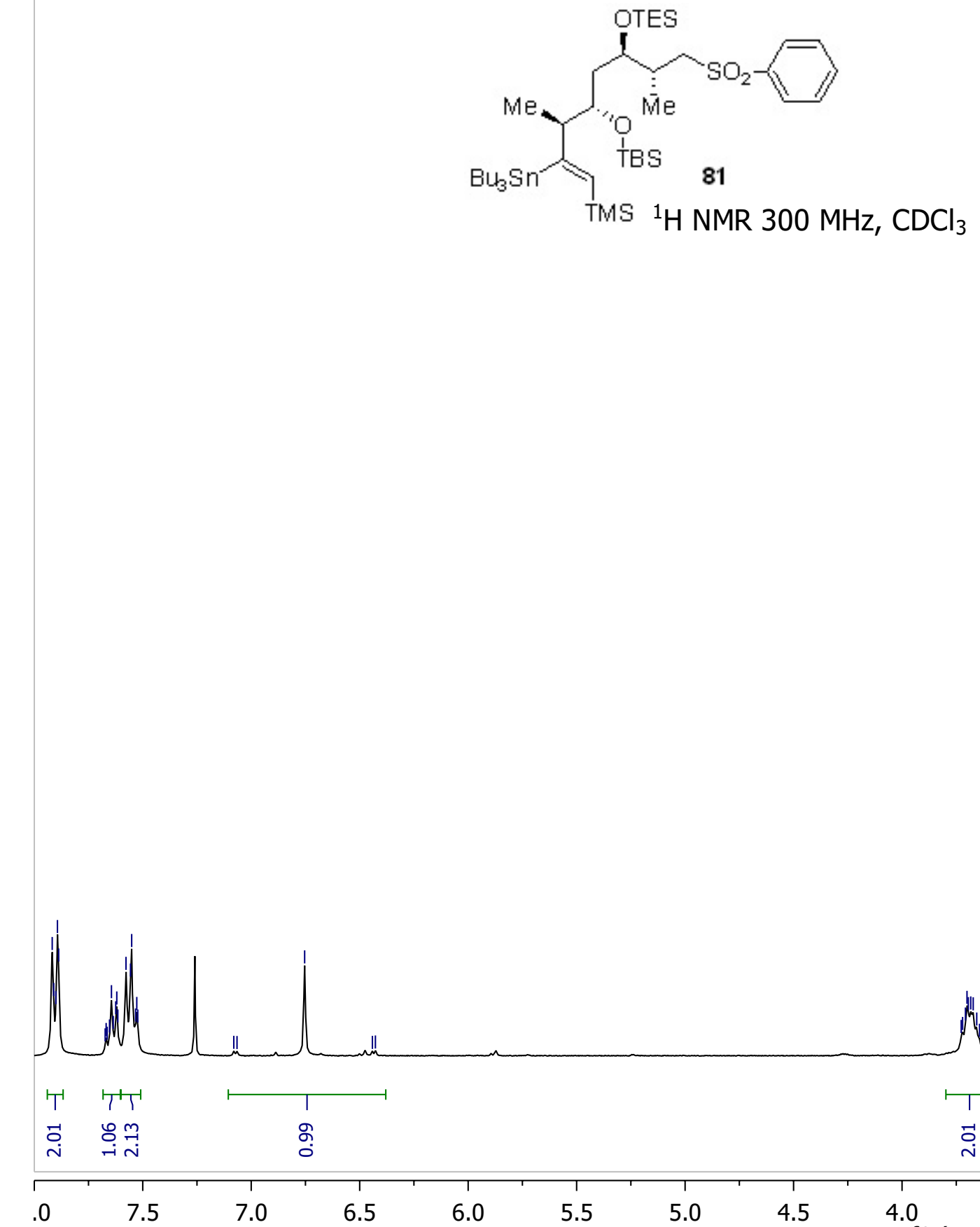

$\Lambda$ Min
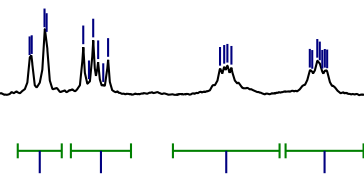

웅

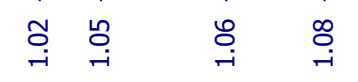

$\mathrm{f} 1(\mathrm{ppm})$

3.0

2.5

2.0

1.5

1.0

( 


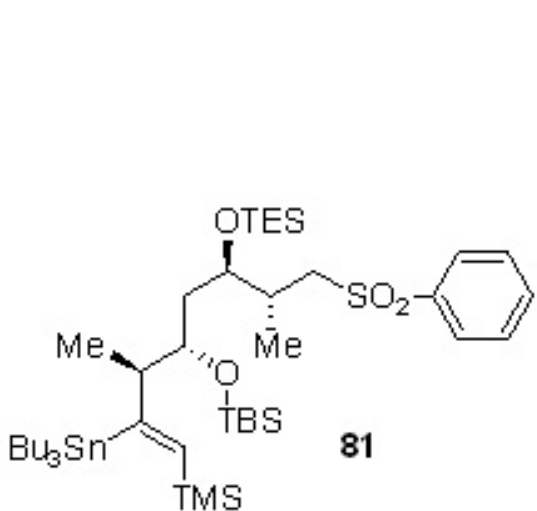

${ }^{13} \mathrm{C}\left\{{ }^{1} \mathrm{H}\right\}$ NMR $75 \mathrm{MHz}^{\mathrm{CDCl}} 3$ 


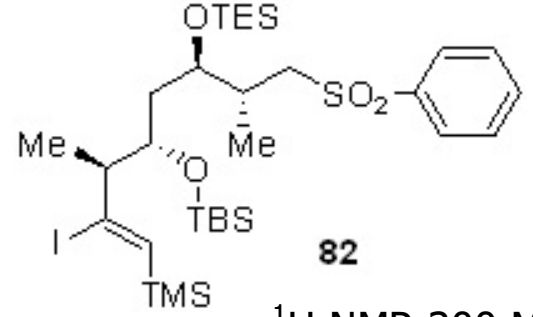

${ }^{1} \mathrm{H}$ NMR $300 \mathrm{MHz}, \mathrm{CDCl}_{3}$
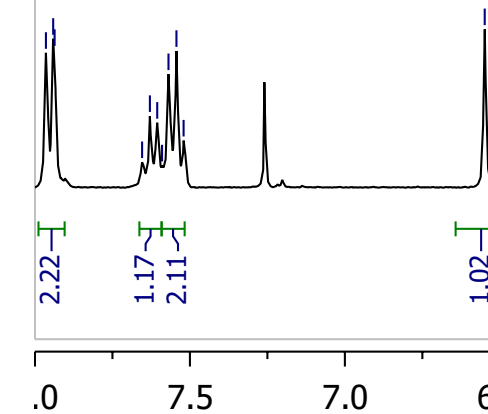$$
\text { กิ }
$$$$
\text { ำ }
$$
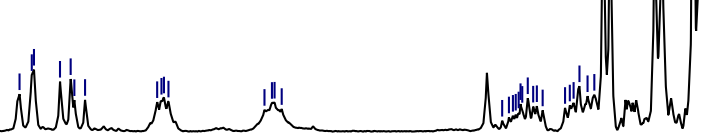

ri

2.5



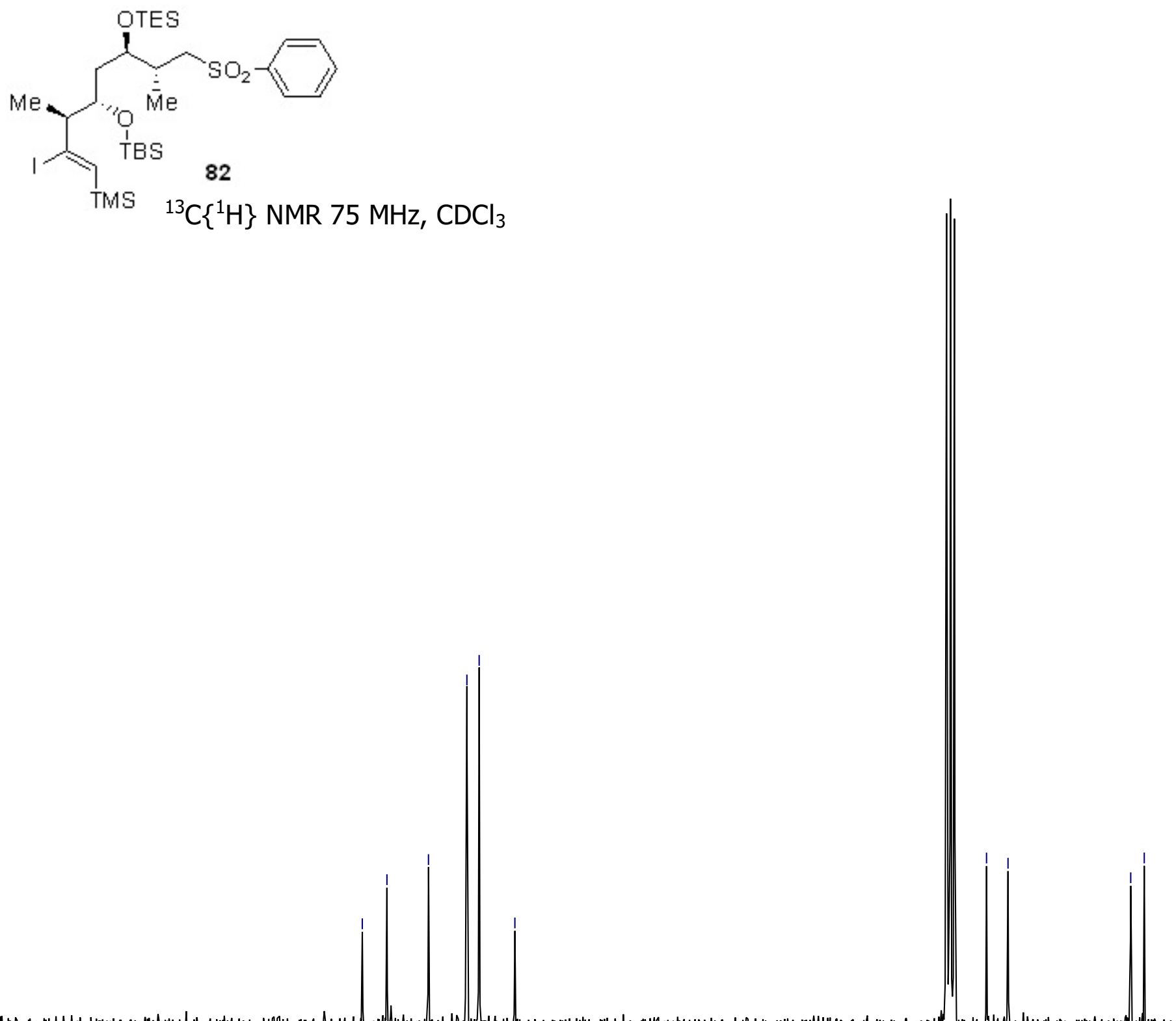


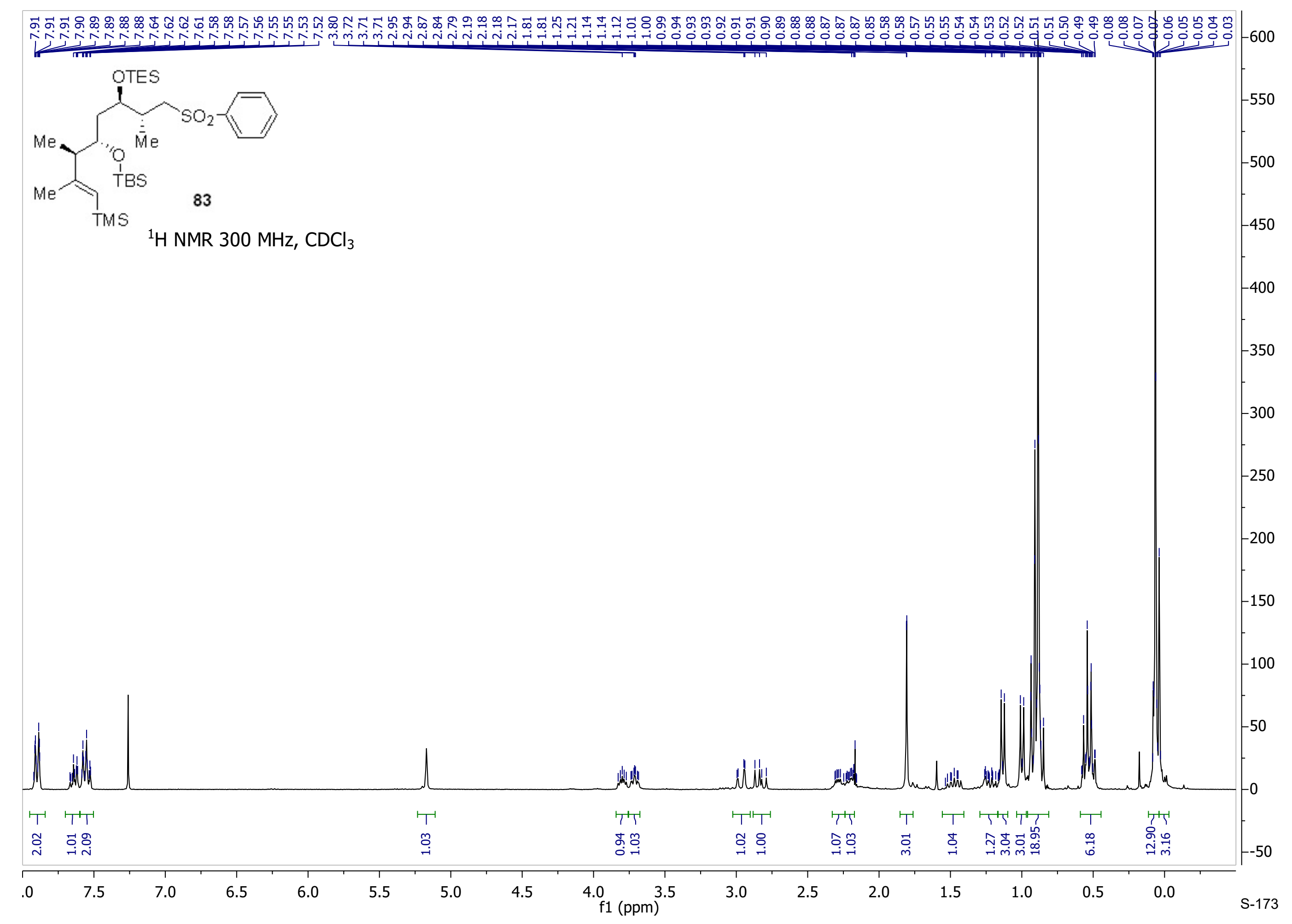




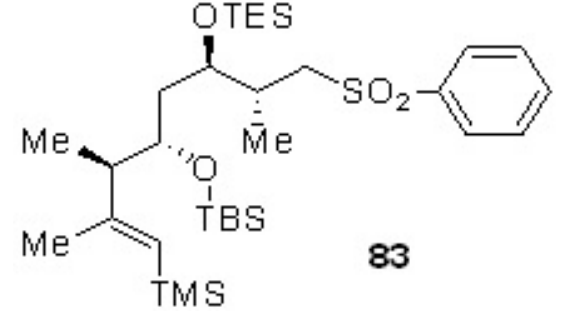

${ }^{13} \mathrm{C}\left\{{ }^{1} \mathrm{H}\right\}$ NMR $75 \mathrm{MHz}, \mathrm{CDCl}_{3}$ 


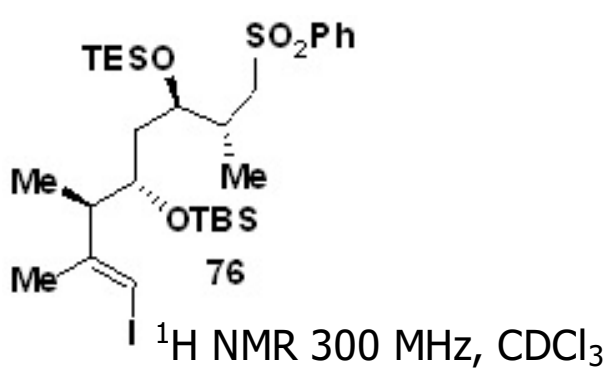

(1)
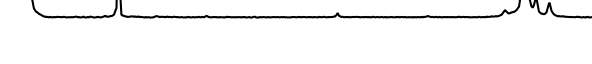

$1 \longmapsto \mapsto \longmapsto$
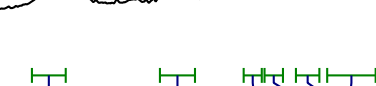

ஜू

$7.5 \quad 7.0$
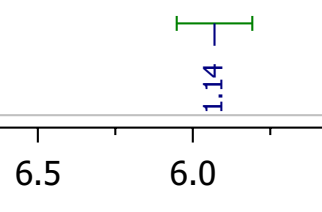

กั

f1 (ppm)

$\begin{array}{llll}3.0 & 2.5 & 2.0 & 1.5\end{array}$

\begin{tabular}{|c|}
\hline 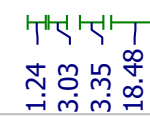 \\
\hline 1.0 \\
\hline
\end{tabular}




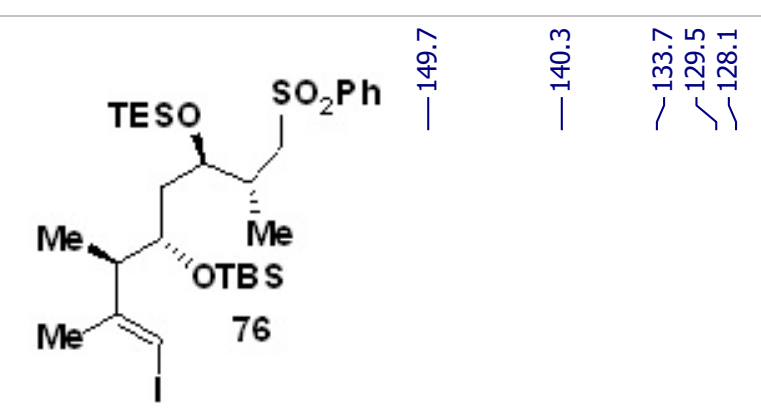

${ }^{13} \mathrm{C}\left\{{ }^{1} \mathrm{H}\right\}$ NMR $75 \mathrm{MHz}^{\mathrm{CDCl}} 3$

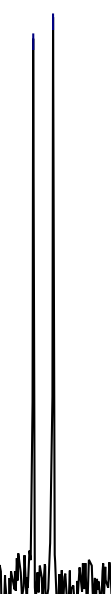


85

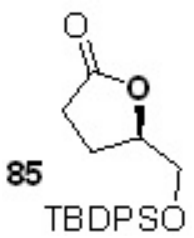

${ }^{1} \mathrm{H}$ NMR $300 \mathrm{MHz}, \mathrm{CDCl}_{3}$

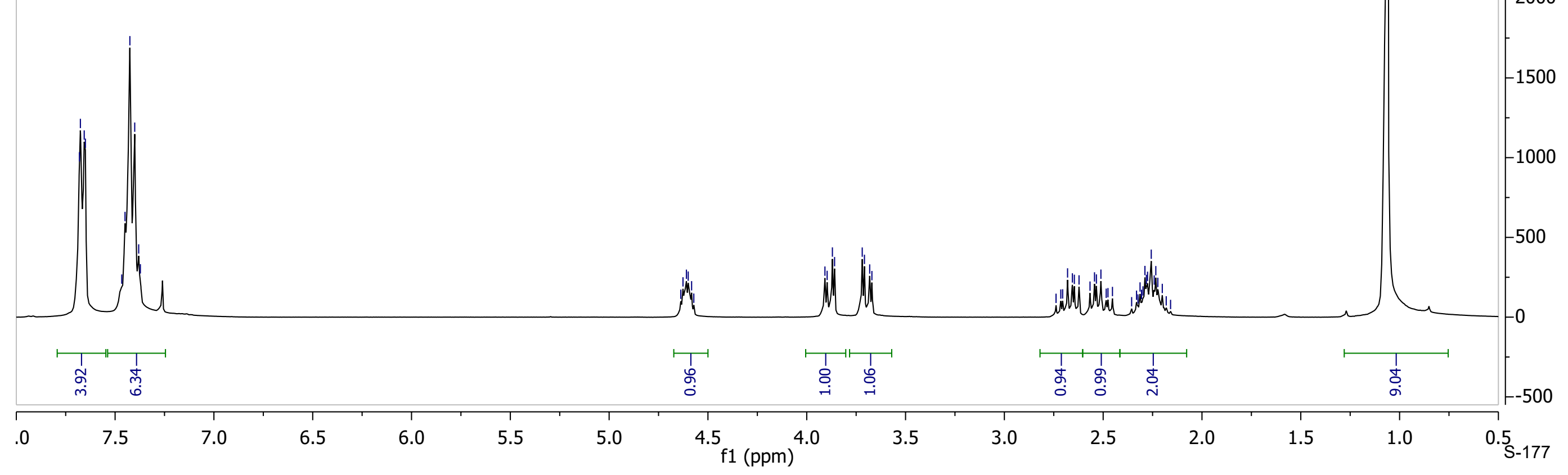




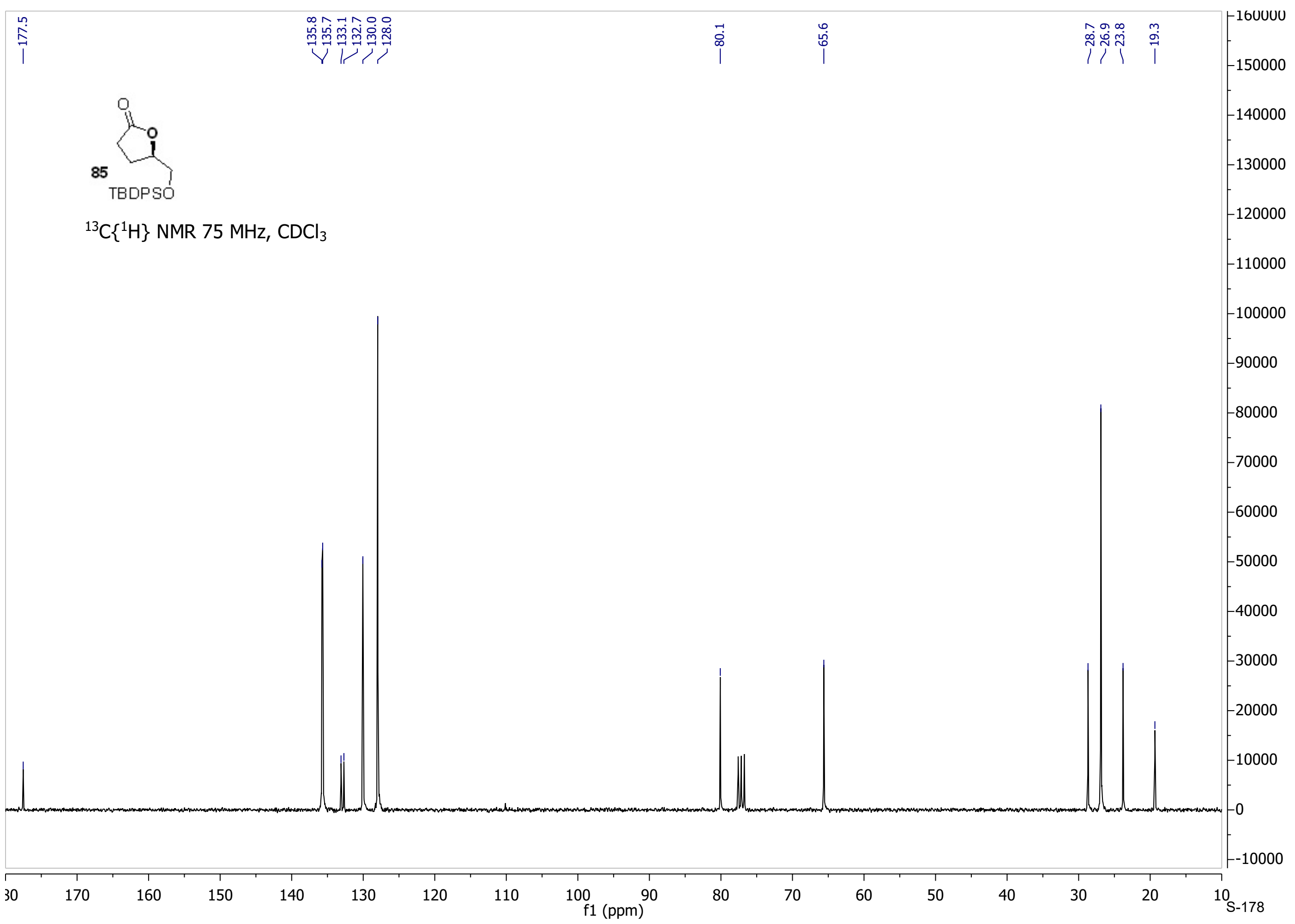




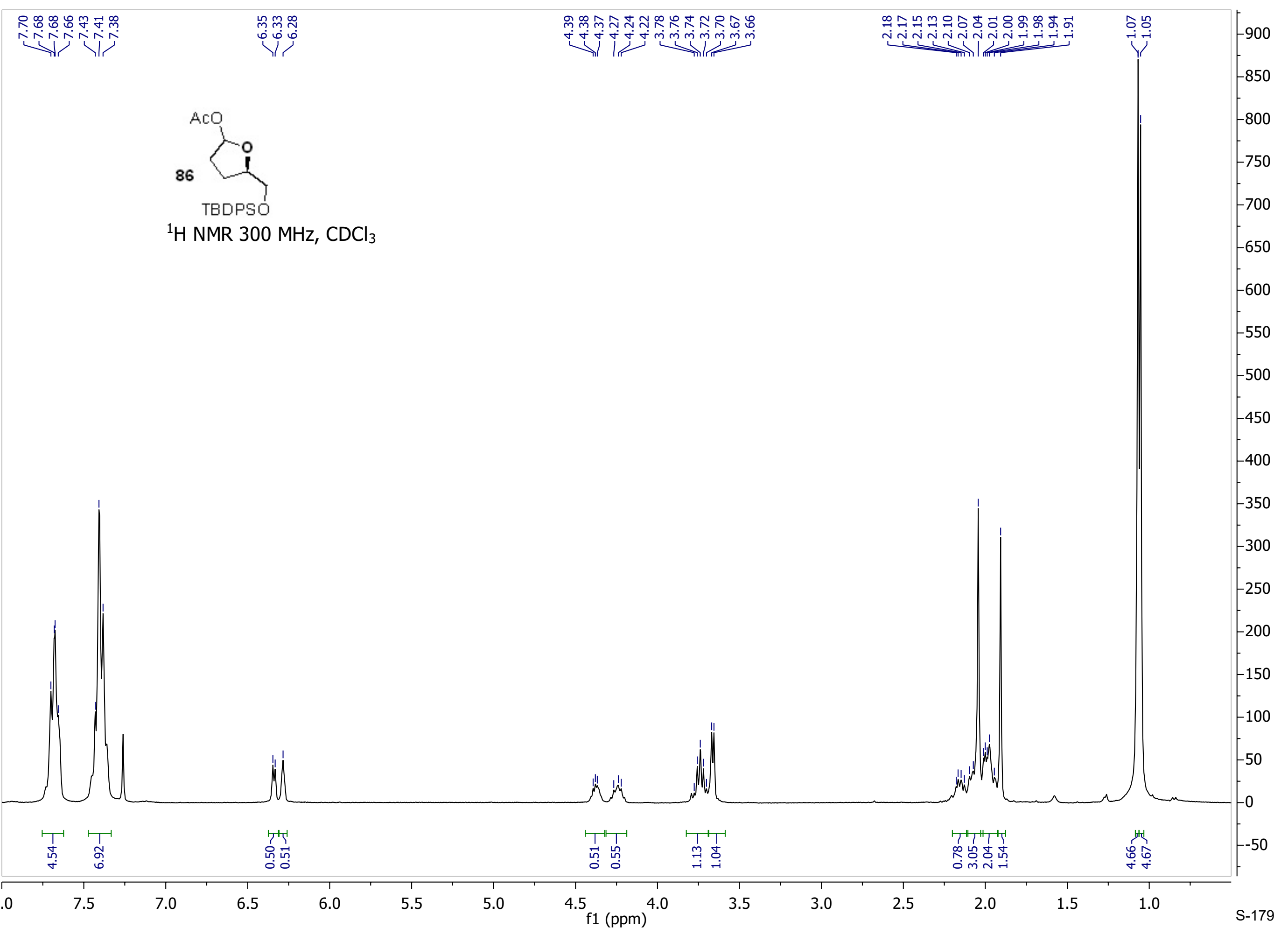


ACQ<smiles>CC1CCC2CCCC2O1</smiles>

TBDPSO

${ }^{13} \mathrm{C}\left\{{ }^{1} \mathrm{H}\right\}$ NMR $75 \mathrm{MHz}, \mathrm{CDCl}_{3}$ 


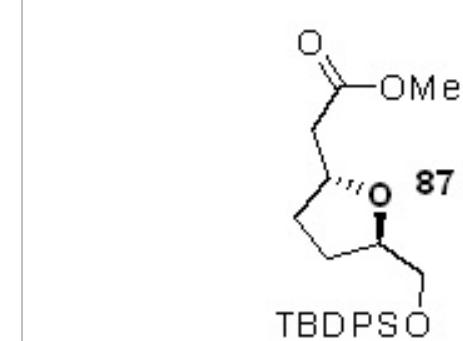

${ }^{1} \mathrm{H}$ NMR $300 \mathrm{MHz}, \mathrm{CDCl}_{3}$

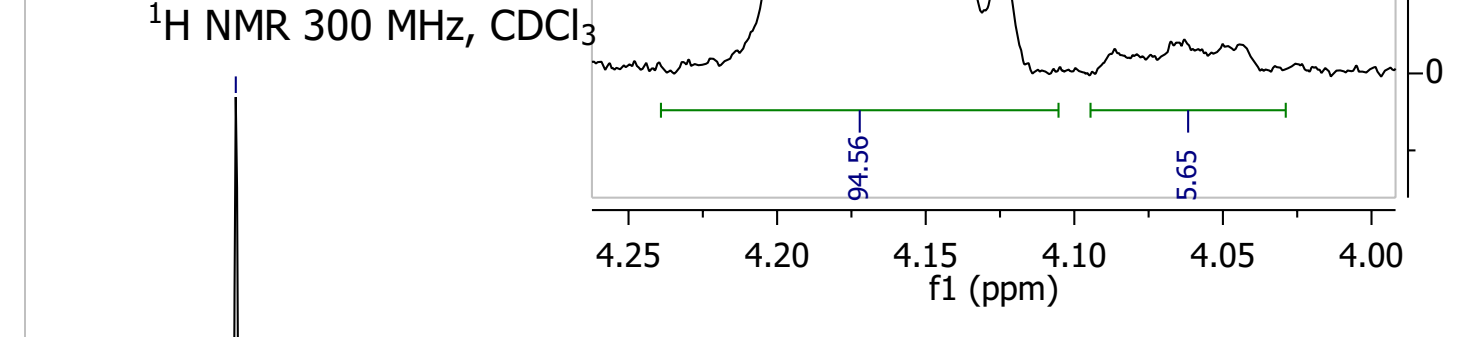

$-20$
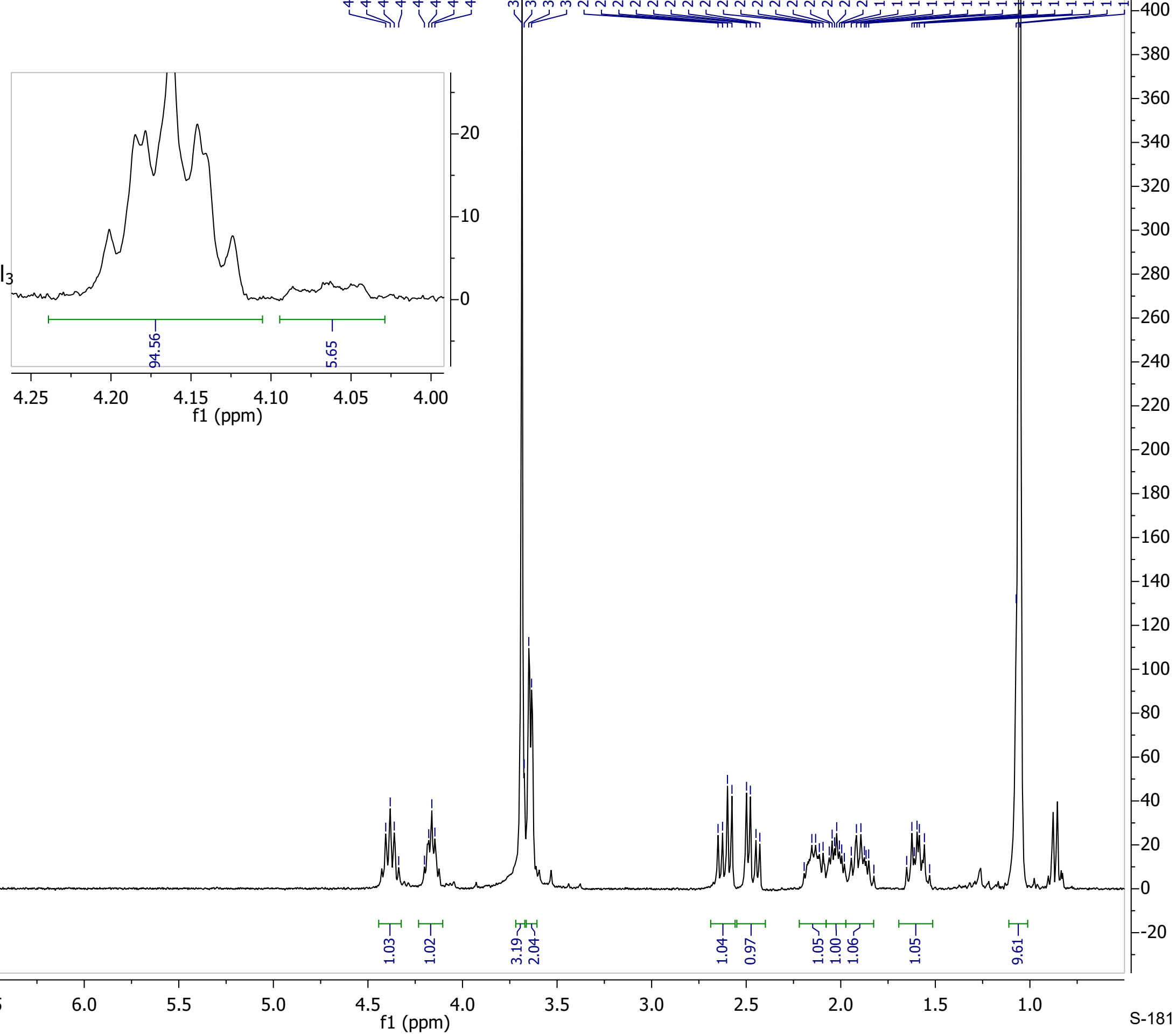

\begin{tabular}{l}
-380 \\
-360 \\
-340 \\
-320 \\
-300 \\
-280 \\
-260 \\
-240 \\
-220 \\
-200 \\
-180 \\
-160 \\
-140 \\
\hline-120 \\
-100 \\
-80 \\
-60 \\
-20 \\
-0 \\
\hline-20 \\
\hline-100 \\
\hline
\end{tabular}




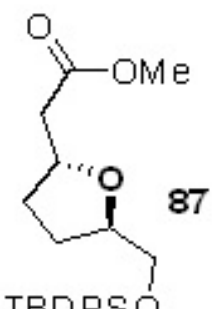

${ }^{13} \mathrm{C}\left\{{ }^{1} \mathrm{H}\right\}$ NMR $75 \mathrm{MHz}, \mathrm{CDCl}_{3}$ 


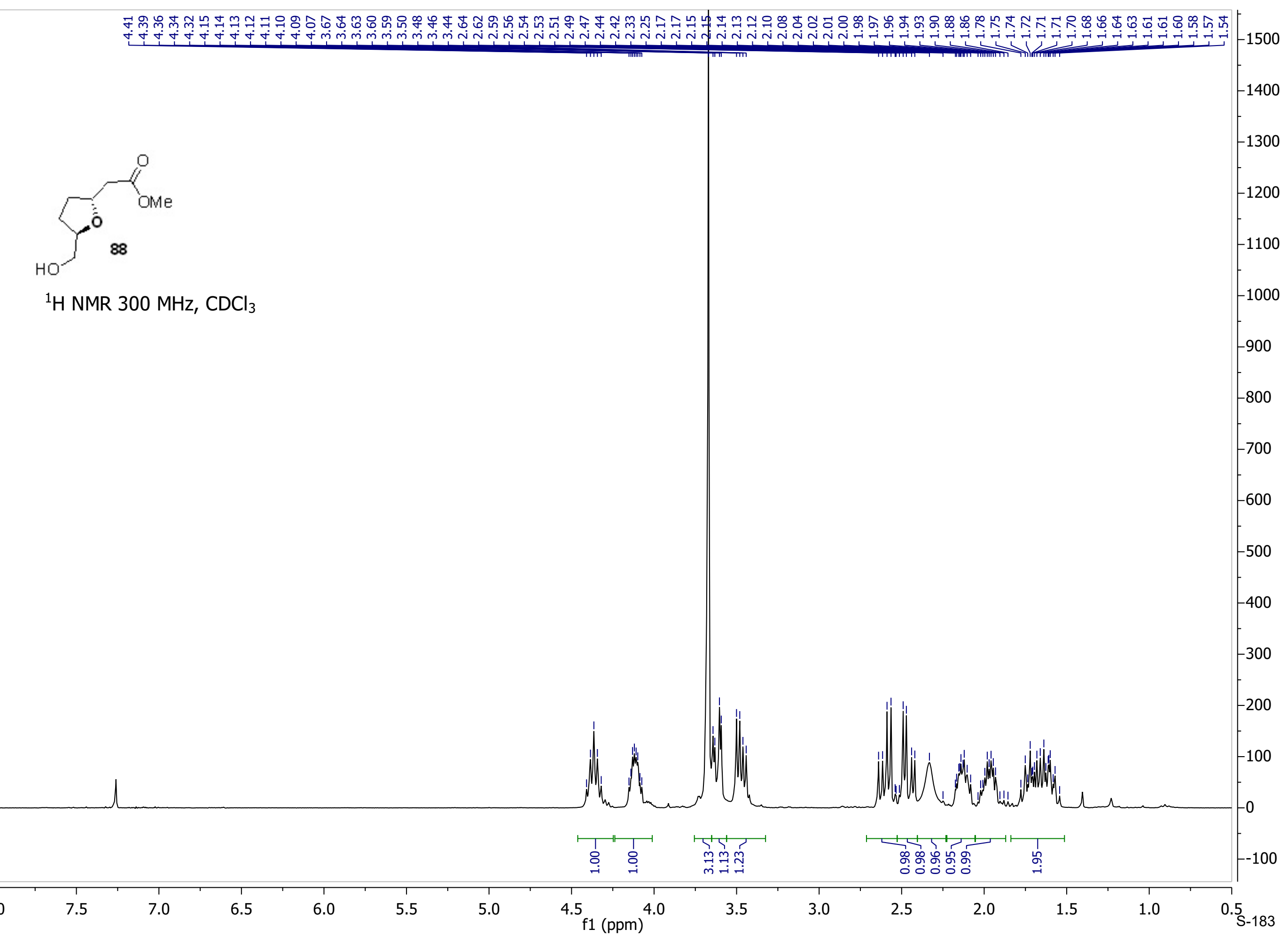




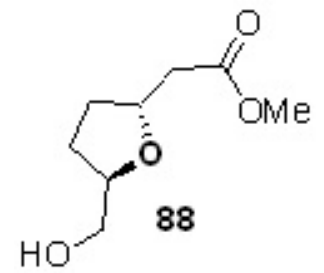

${ }^{13} \mathrm{C}\left\{{ }^{1} \mathrm{H}\right\}$ NMR $75 \mathrm{MHz}^{\mathrm{CDCl}} 3$

\section{0}


${ }^{1} \mathrm{H}$ NMR $300 \mathrm{MHz}, \mathrm{CDCl}_{3}$
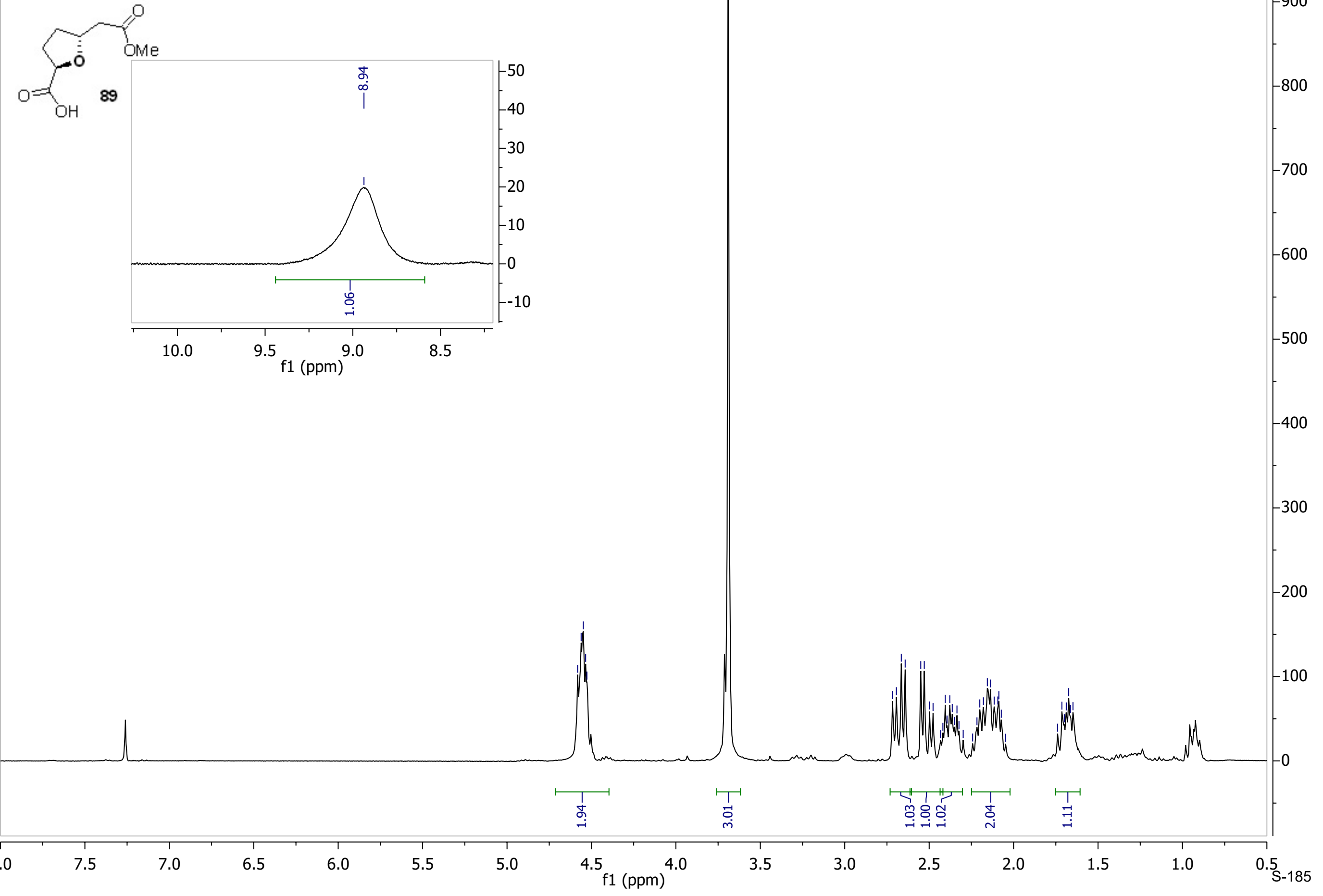
$\stackrel{m}{\stackrel{m}{5}}$

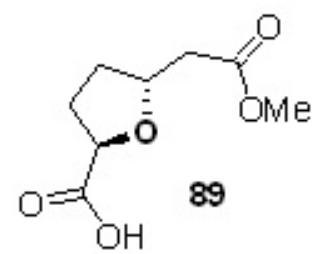

${ }^{13} \mathrm{C}\left\{{ }^{1} \mathrm{H}\right\}$ NMR $75 \mathrm{MHz}, \mathrm{CDCl}_{3}$

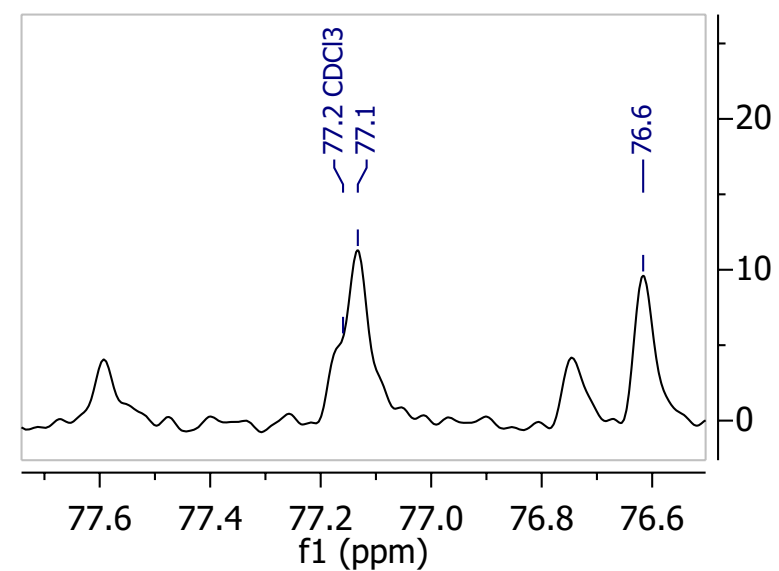

| 


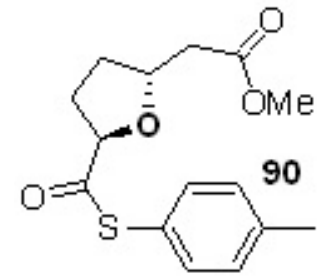

${ }^{1} \mathrm{H} \mathrm{NMR} 300 \mathrm{MHz}, \mathrm{CDCl}_{3}$

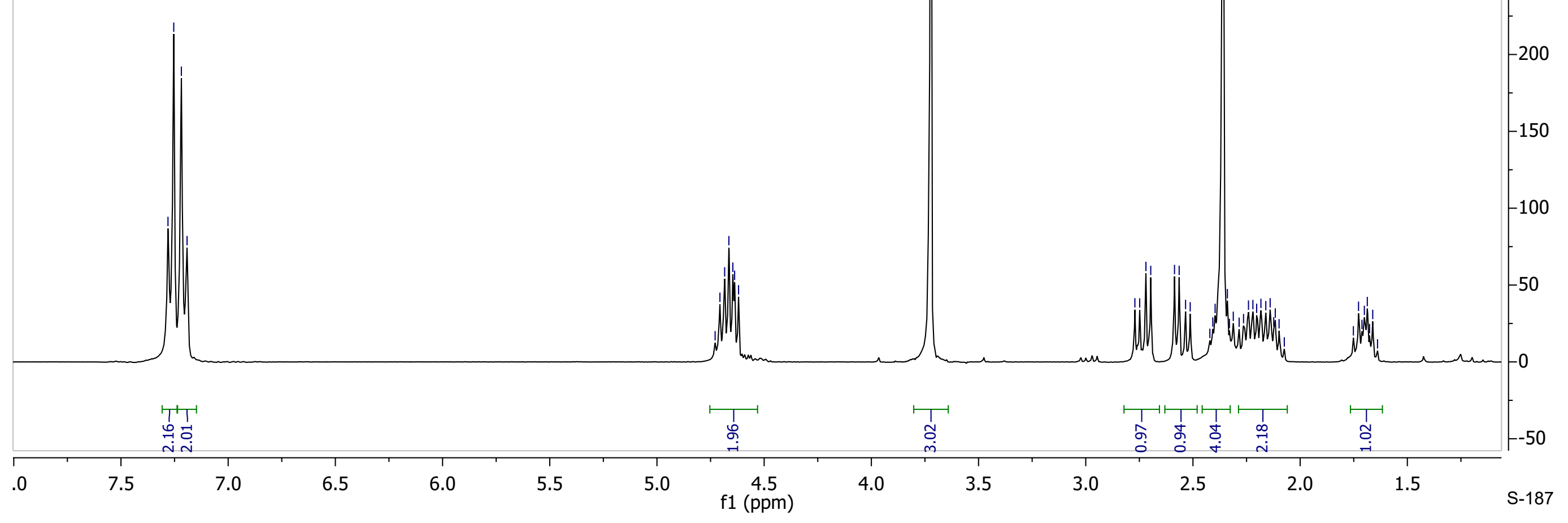




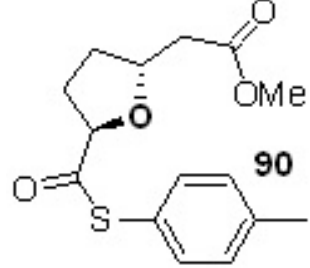

${ }^{13} \mathrm{C}\left\{{ }^{1} \mathrm{H}\right\}$ NMR $75 \mathrm{MHz}, \mathrm{CDCl}_{3}$

$\begin{array}{lllllllll}190 & 180 & 170 & 160 & 150 & 140 & 130 & 120 & 110\end{array}$




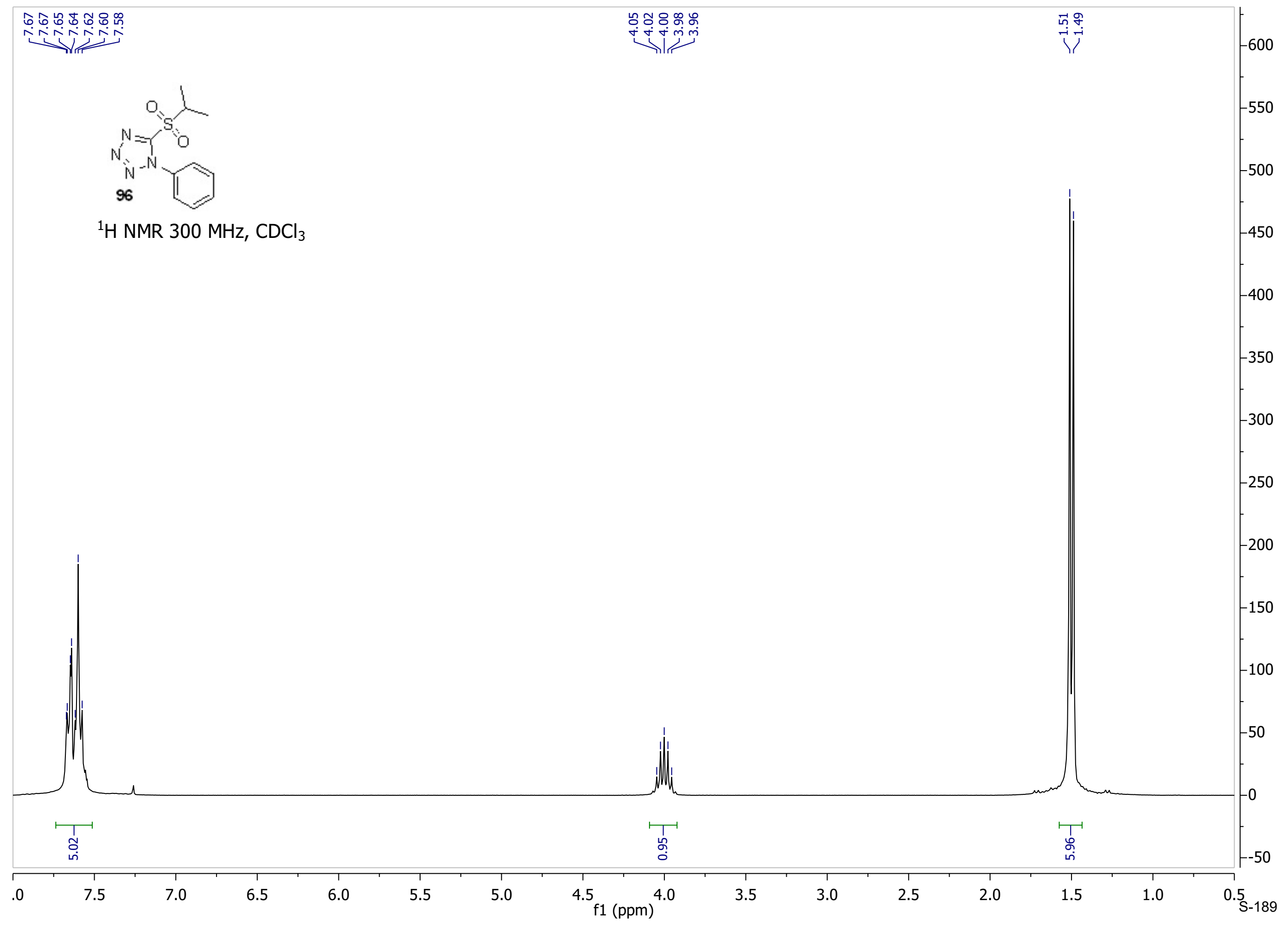




$$
\text { 㝵 }
$$

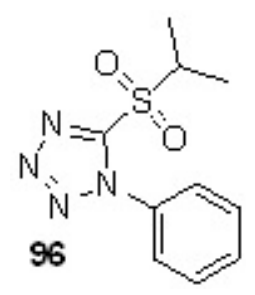

${ }^{13} \mathrm{C}\left\{{ }^{1} \mathrm{H}\right\}$ NMR $75 \mathrm{MHz}, \mathrm{CDCl}_{3}$ 
$\mathrm{SnBu}_{3}$

${ }^{1} \mathrm{H}$ NMR $300 \mathrm{MHz}, \mathrm{CDCl}_{3}$

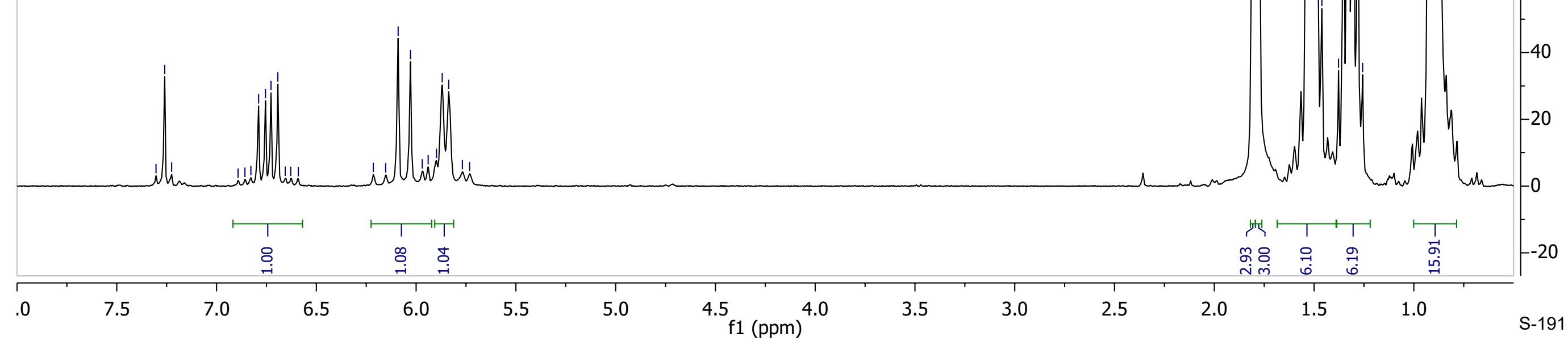




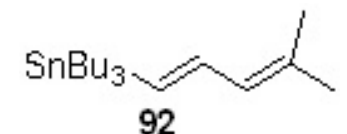
92

${ }^{13} \mathrm{C}\left\{{ }^{1} \mathrm{H}\right\}$ NMR $75 \mathrm{MHz}, \mathrm{CDCl}_{3}$

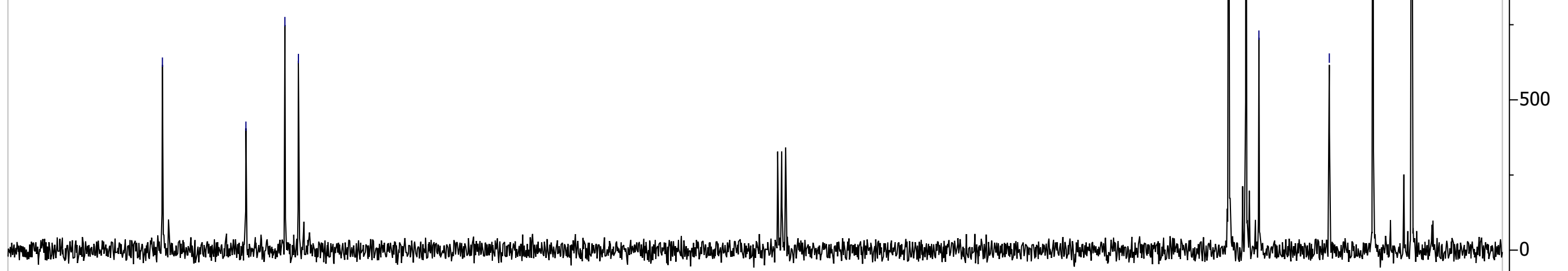

$$
30
$$




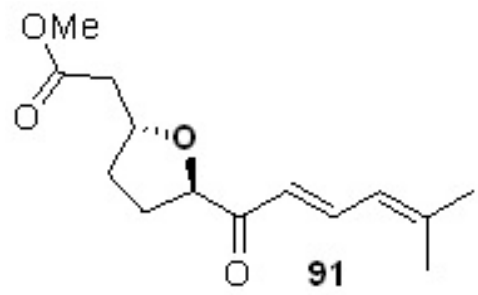

${ }^{1} \mathrm{H}$ NMR $300 \mathrm{MHz} \mathrm{CDCl}_{3}$

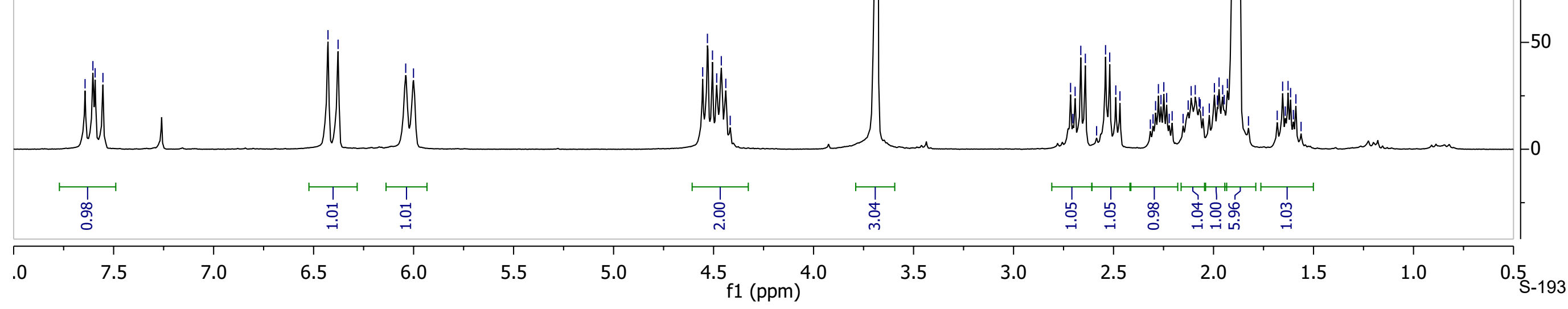




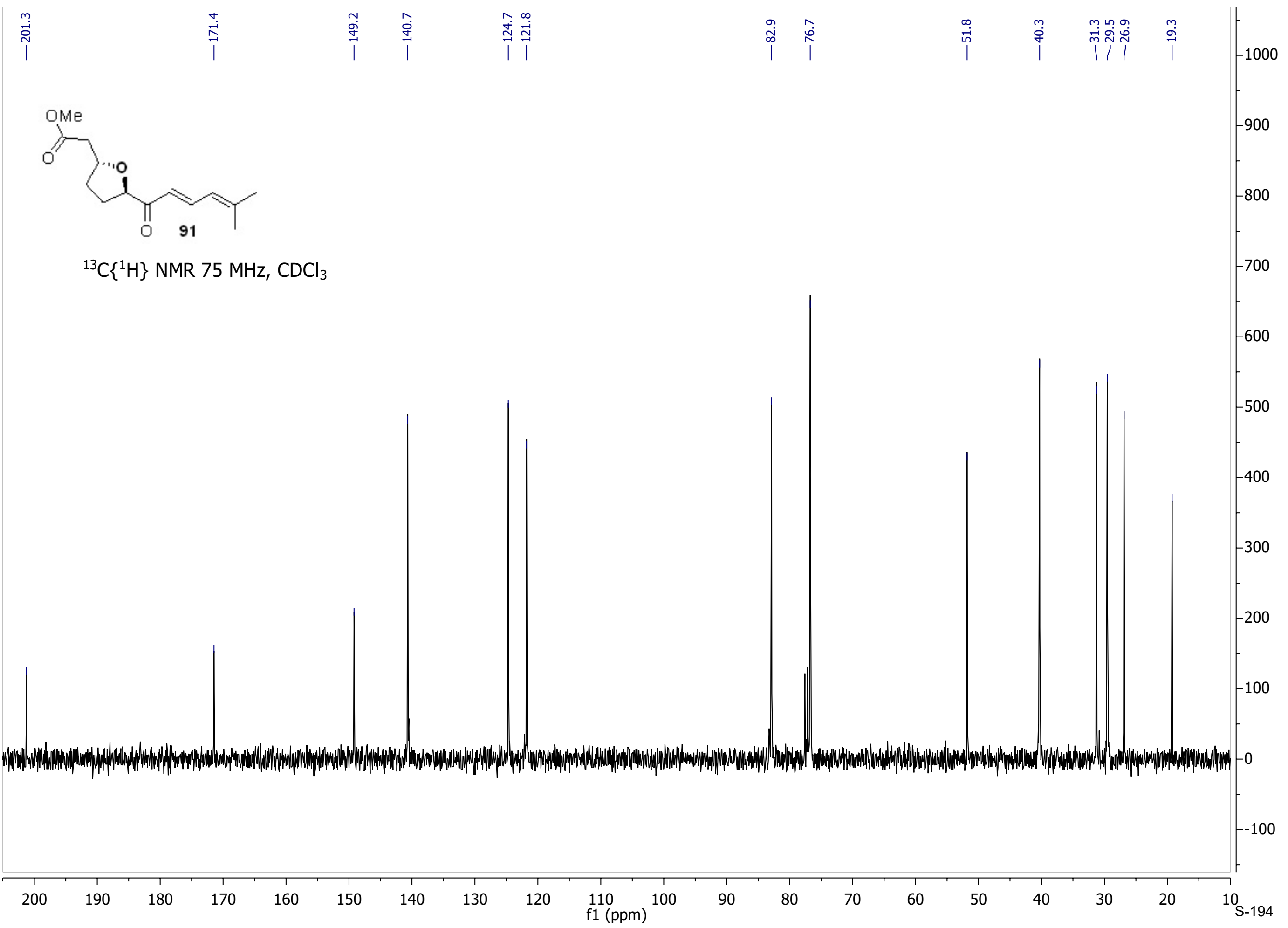




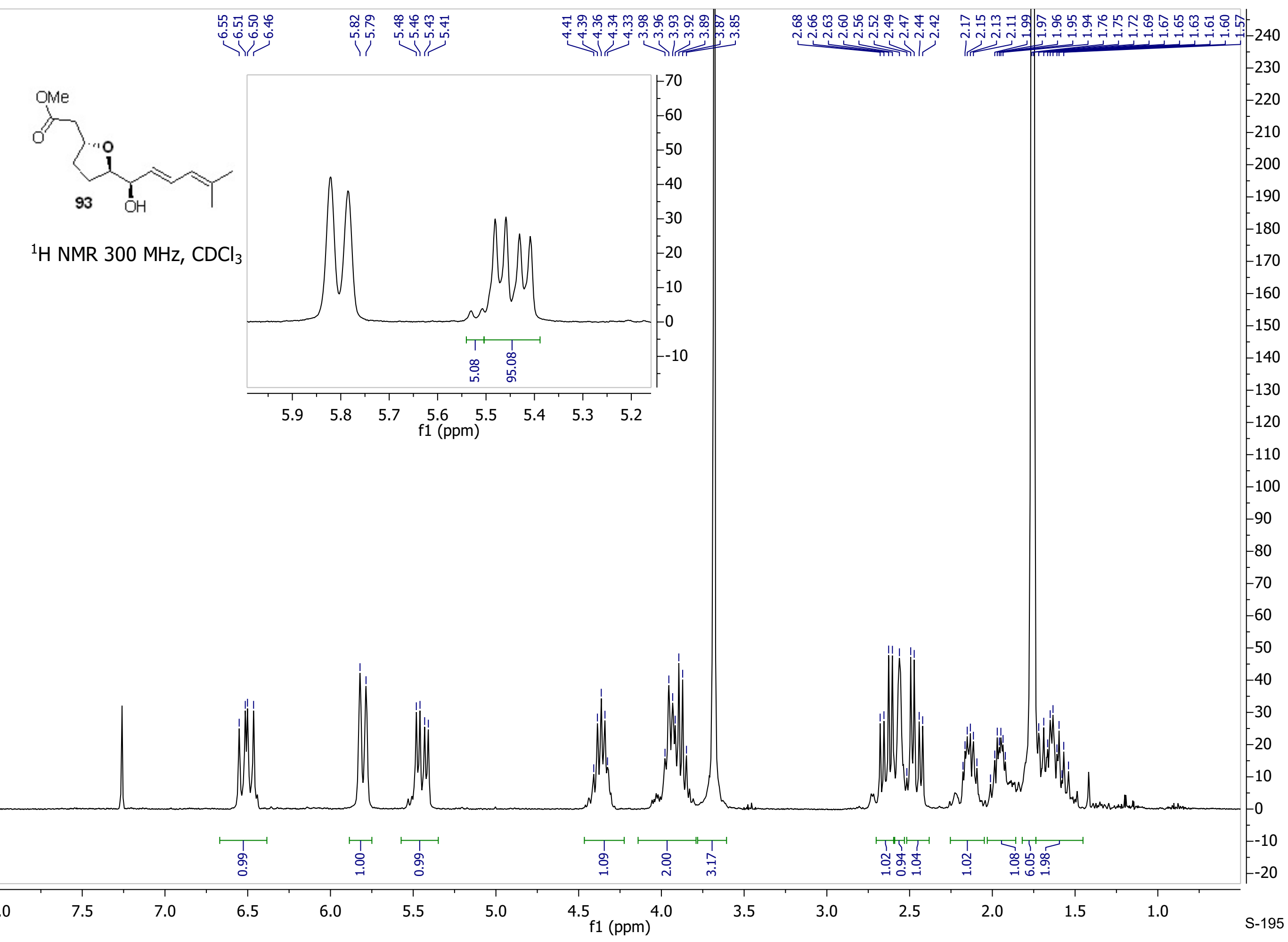


<smiles>CC(C)=CC=CC(O)C1CCCO1</smiles>

${ }^{13} \mathrm{C}\left\{{ }^{1} \mathrm{H}\right\}$ NMR $75 \mathrm{MHz}, \mathrm{CDCl}_{3}$

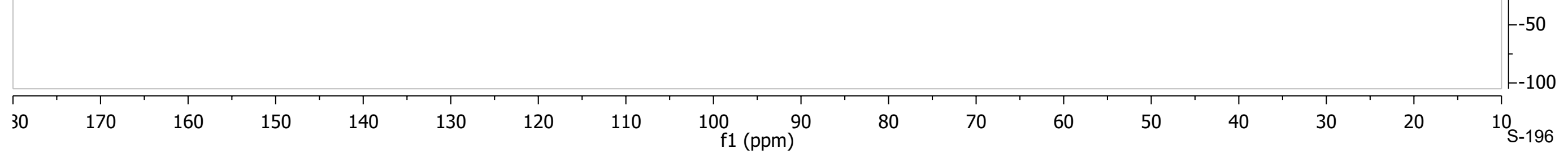


0

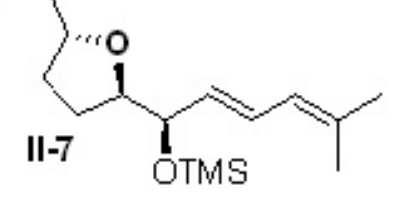

${ }^{1} \mathrm{H}$ NMR $300 \mathrm{MHz} \mathrm{CDCl}_{3}$

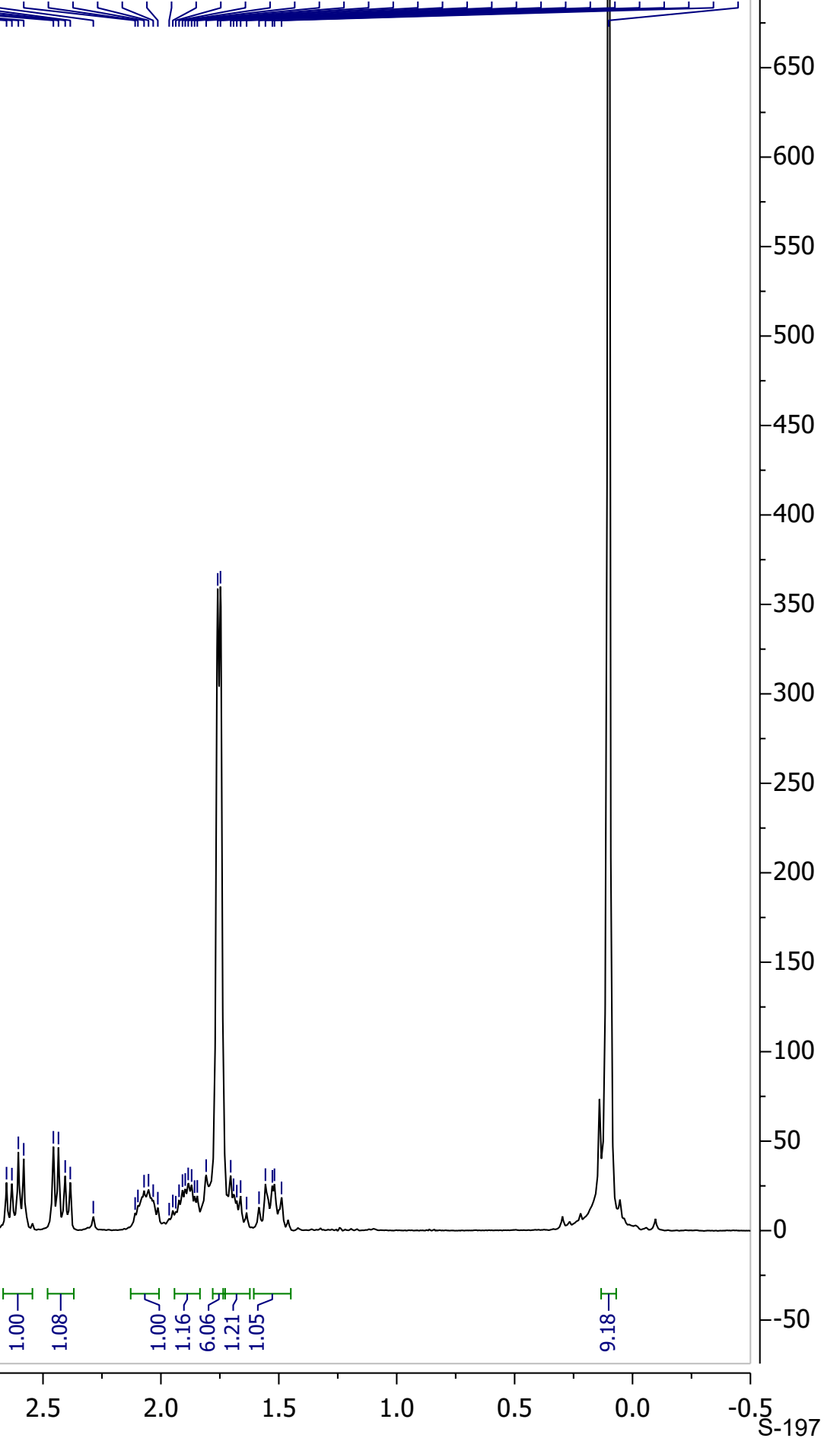




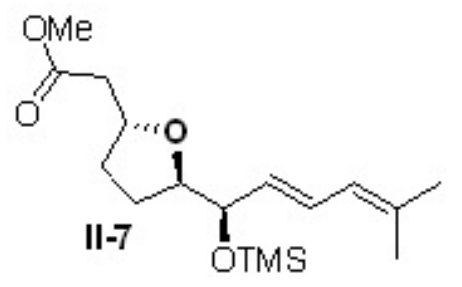

${ }^{13} \mathrm{C}\left\{{ }^{1} \mathrm{H}\right\}$ NMR $75 \mathrm{MHz} \mathrm{CDCl}_{3}$ 


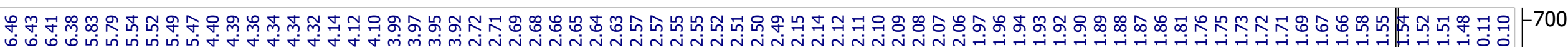
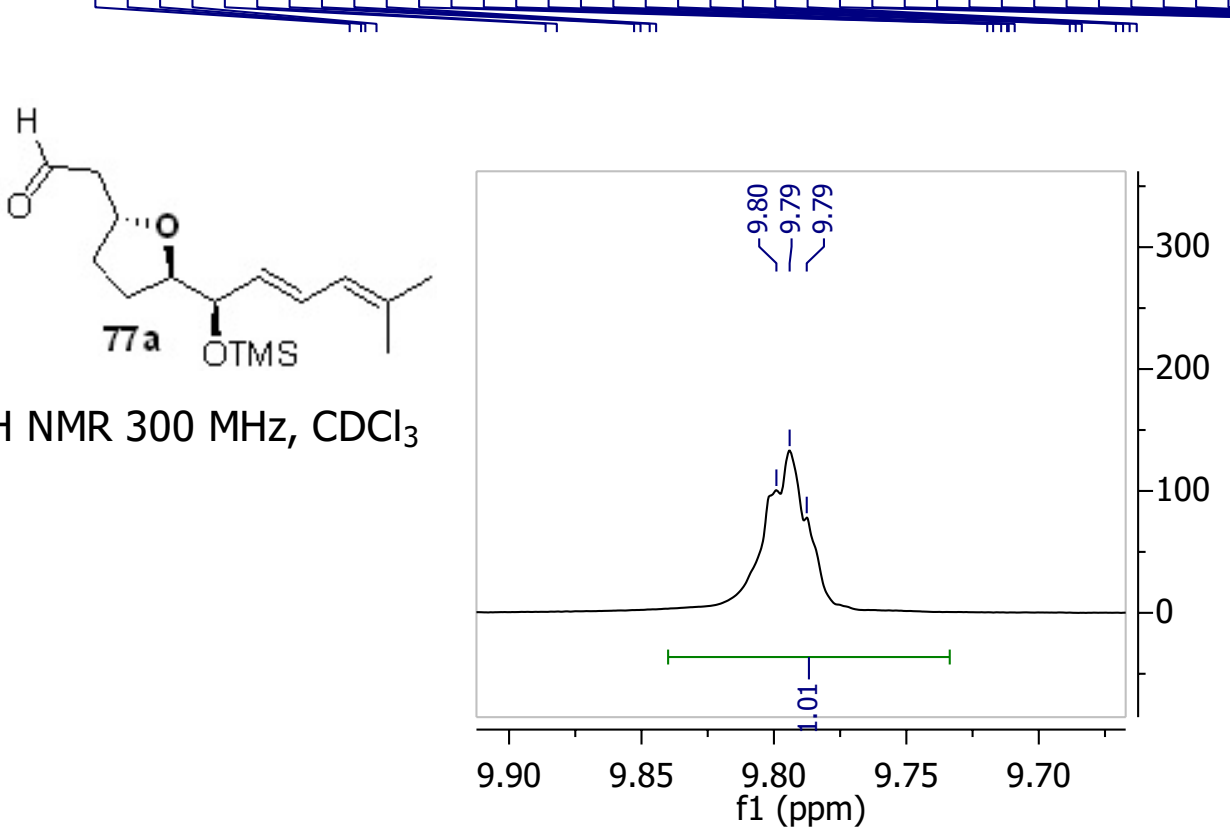

${ }^{1} \mathrm{H}$ NMR $300 \mathrm{MHz}, \mathrm{CDCl}_{3}$

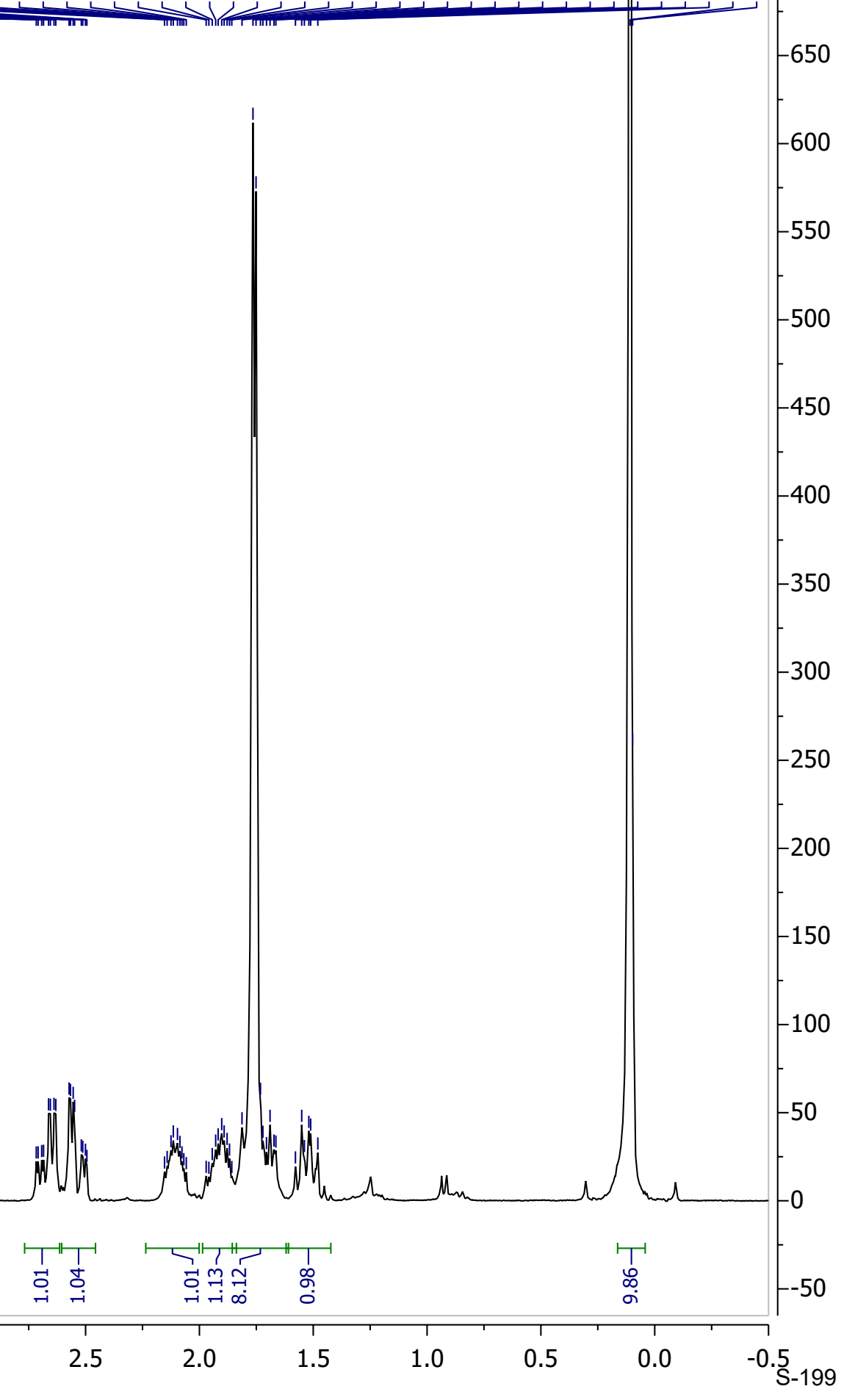


量

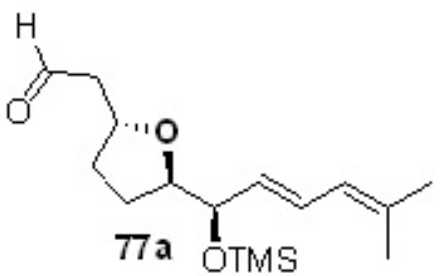

${ }^{13} \mathrm{C}\left\{{ }^{1} \mathrm{H}\right\}$ NMR $75 \mathrm{MHz}, \mathrm{CDCl}_{3}$ 


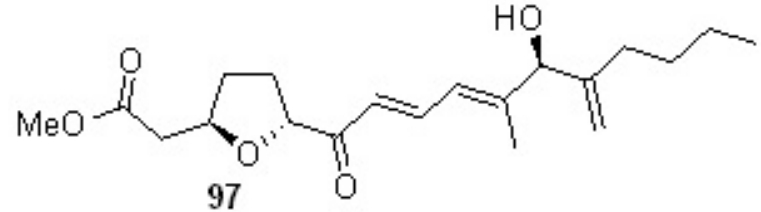

${ }^{1} \mathrm{H}$ NMR $300 \mathrm{MHz}, \mathrm{CDCl}_{3}$
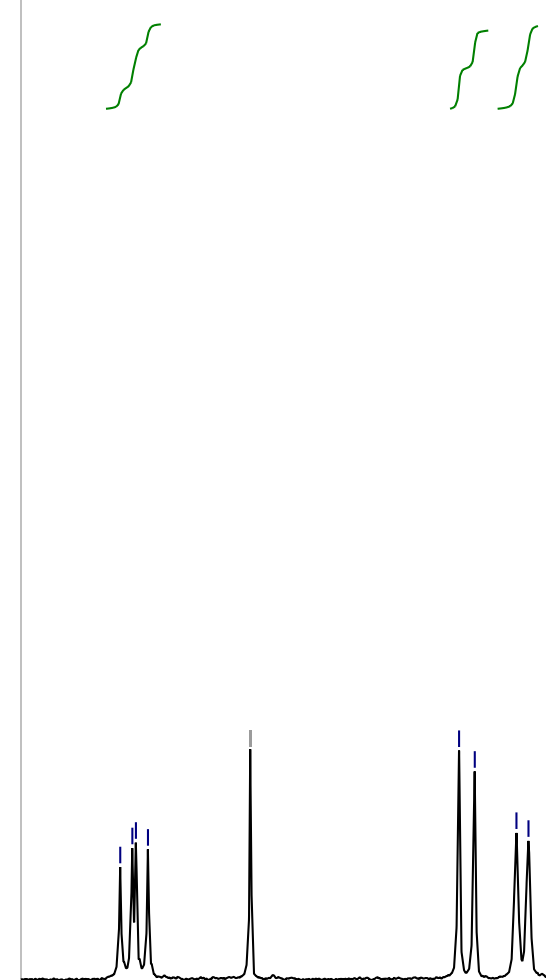

官

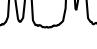

$T$
m.
ô
0
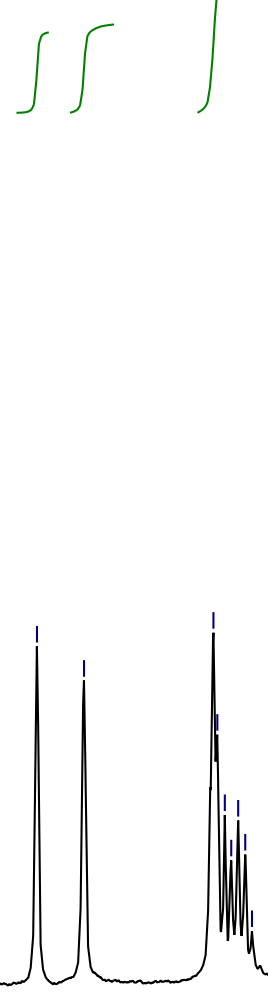

ำก เ
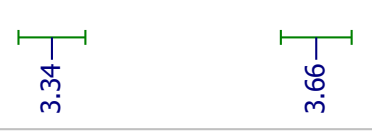

4.5

${ }_{\mathrm{f} 1(\mathrm{ppm})}^{3.5}$

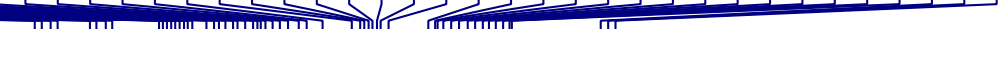

500

6.5

$\begin{array}{lll}6.0 & 5.5 & 5.0\end{array}$

4.5 


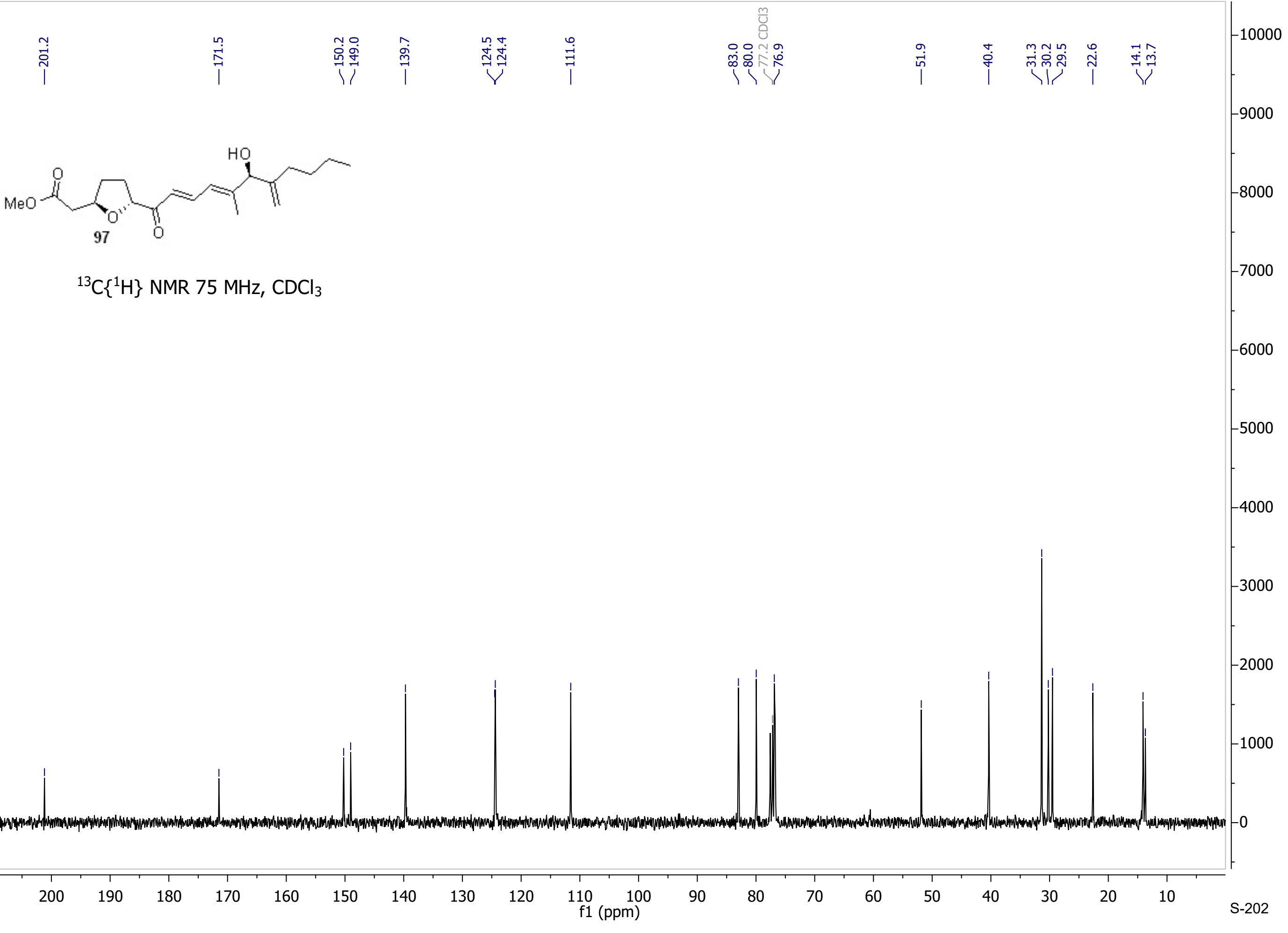




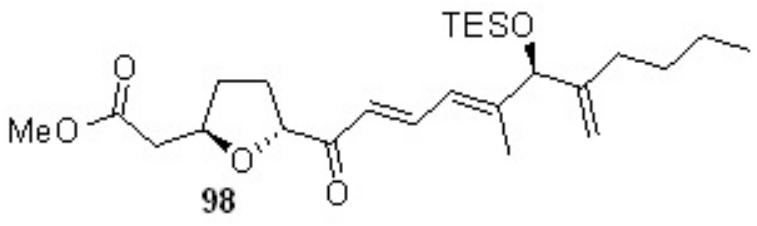

${ }^{1} \mathrm{H}$ NMR $300 \mathrm{MHz}, \mathrm{CDCl}_{3}$

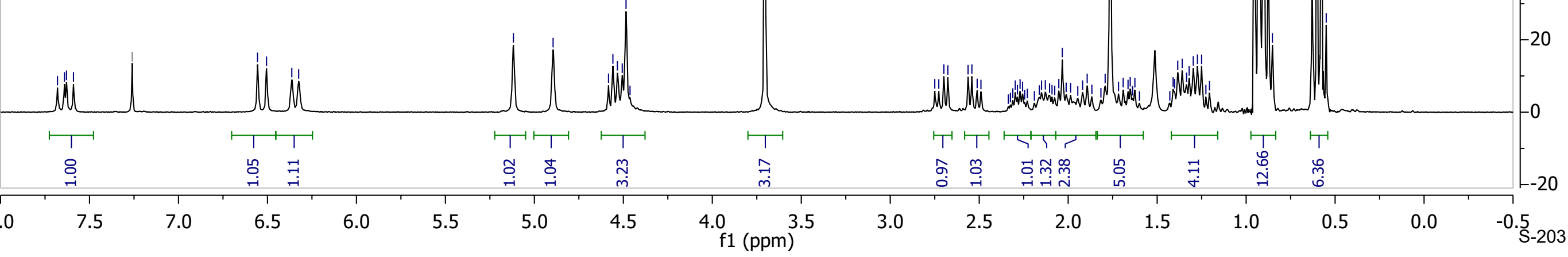




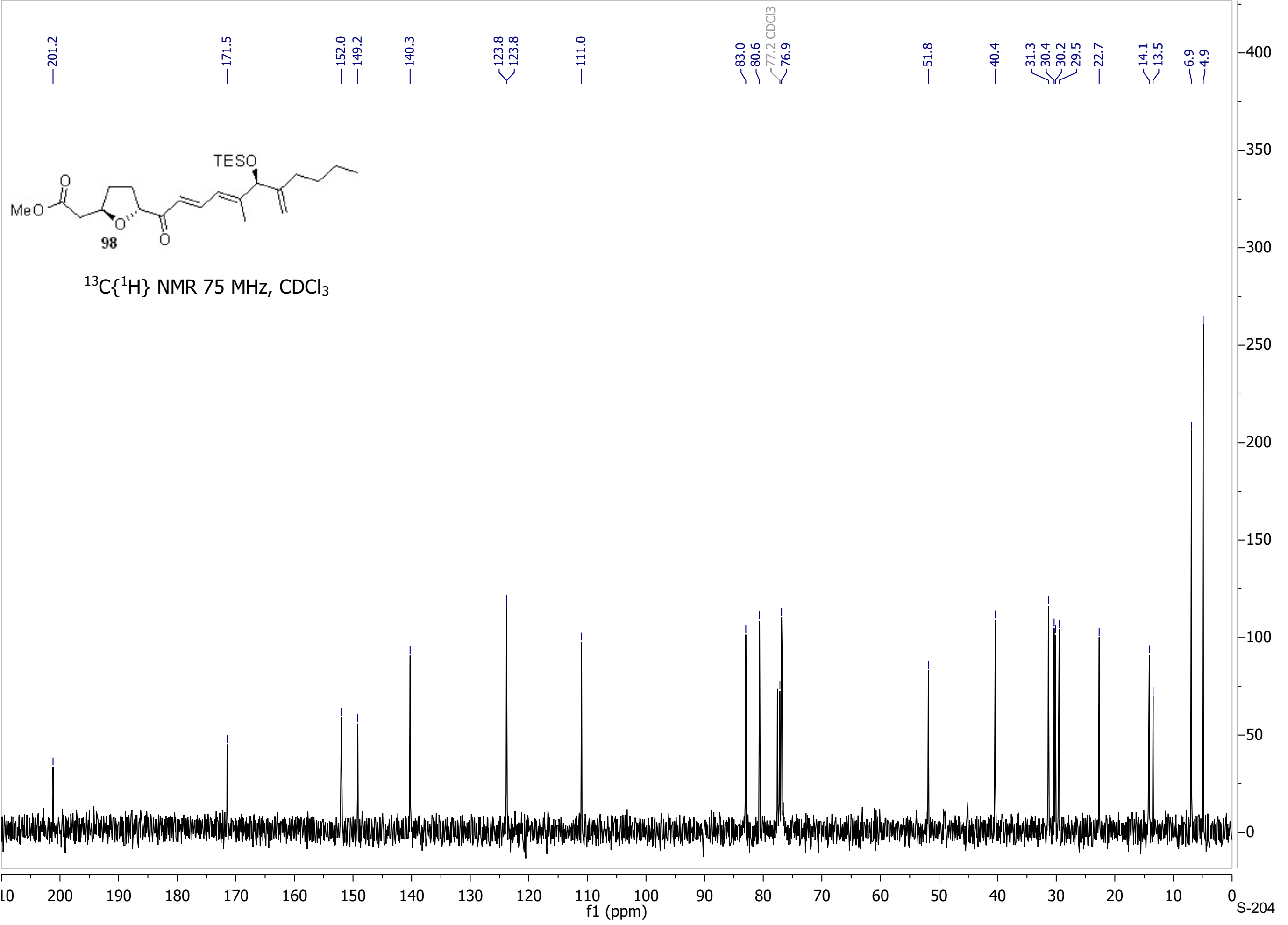



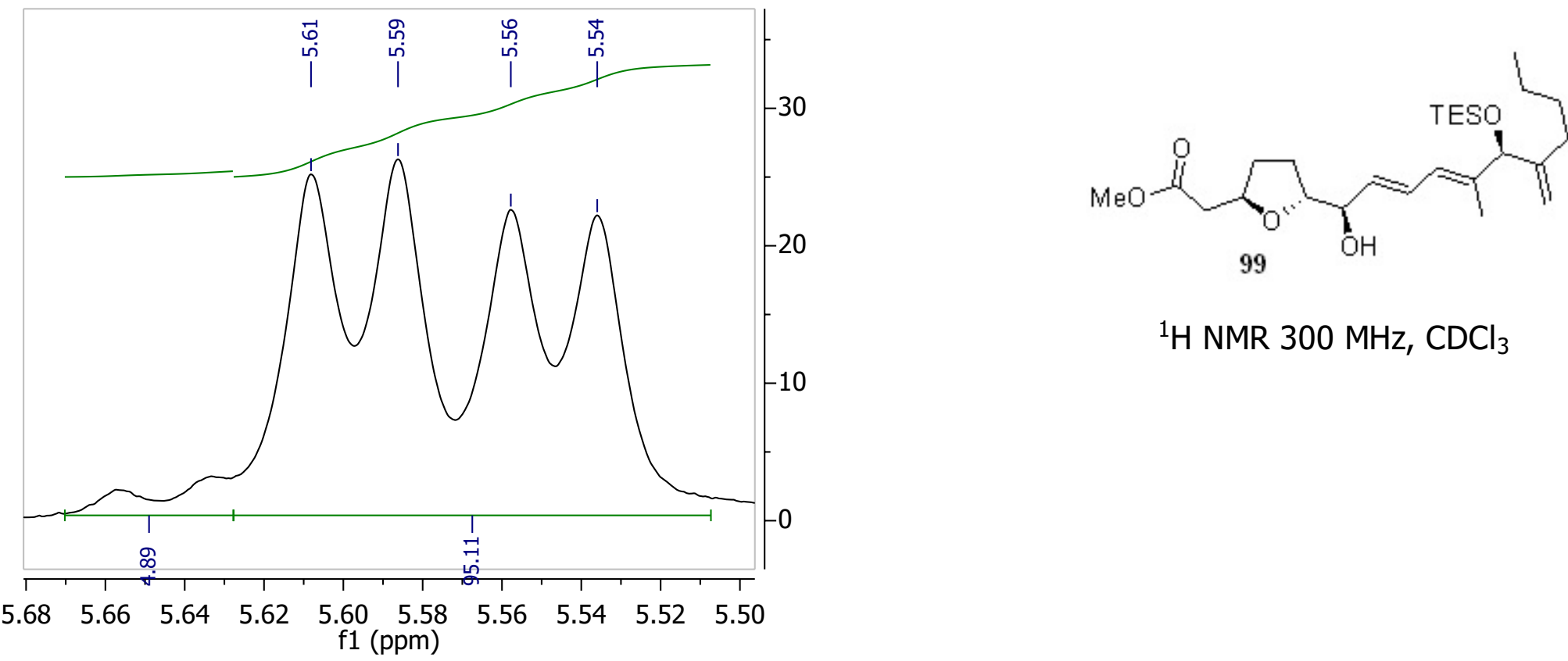

${ }^{1} \mathrm{H}$ NMR $300 \mathrm{MHz}, \mathrm{CDCl}_{3}$

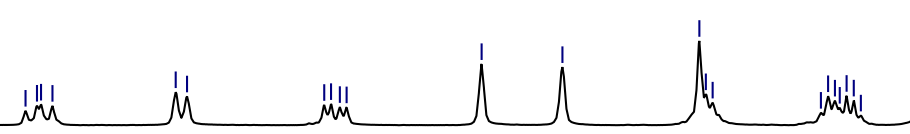

"Wh" 넨.

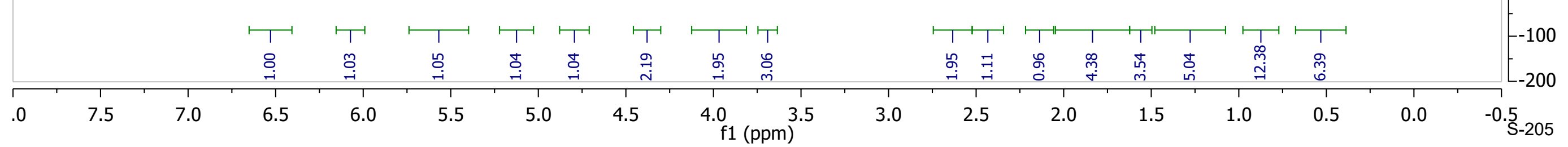




| ।

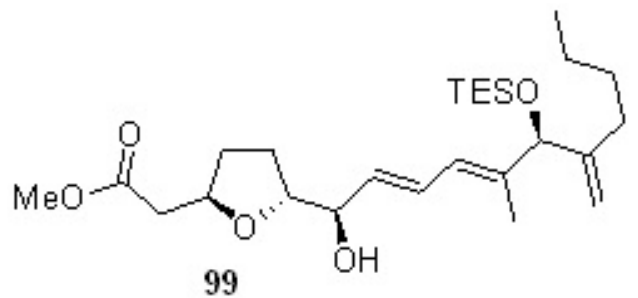

${ }^{13} \mathrm{C}\left\{{ }^{1} \mathrm{H}\right\}$ NMR $75 \mathrm{MHz}, \mathrm{CDCl}_{3}$ 
<smiles>C=C(CCCC)C(OCC)/C(C)=C/C=C/C(O[Si])C1CCC(CC(=O)OC)O1</smiles>

${ }^{1} \mathrm{H}$ NMR $300 \mathrm{MHz}, \mathrm{CDCl}_{3}$

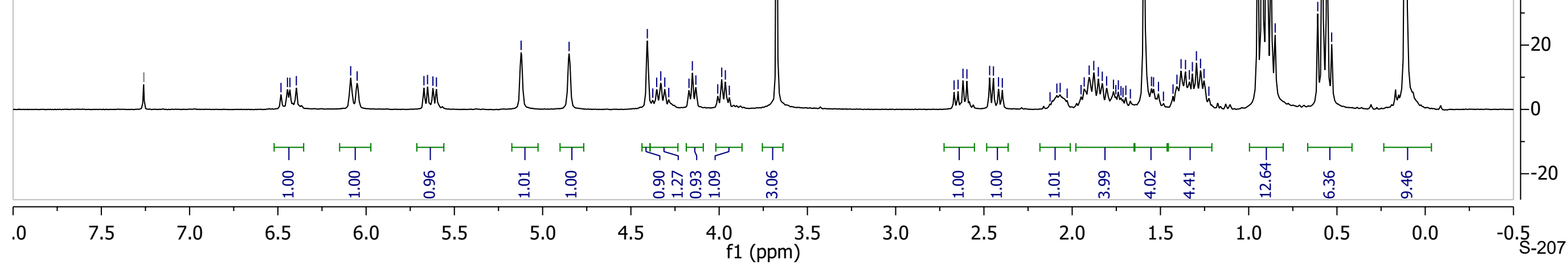




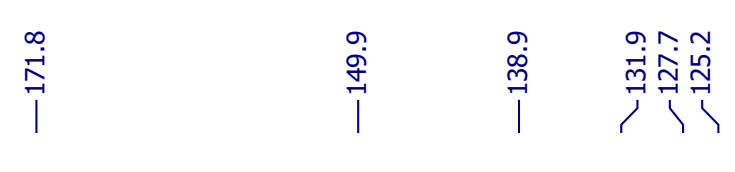

iे

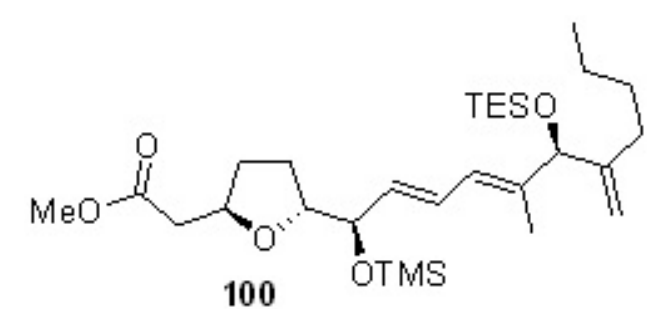

${ }^{13} \mathrm{C}\left\{{ }^{1} \mathrm{H}\right\} \mathrm{NMR} 75 \mathrm{MHz}, \mathrm{CDCl}{ }_{3}$ 

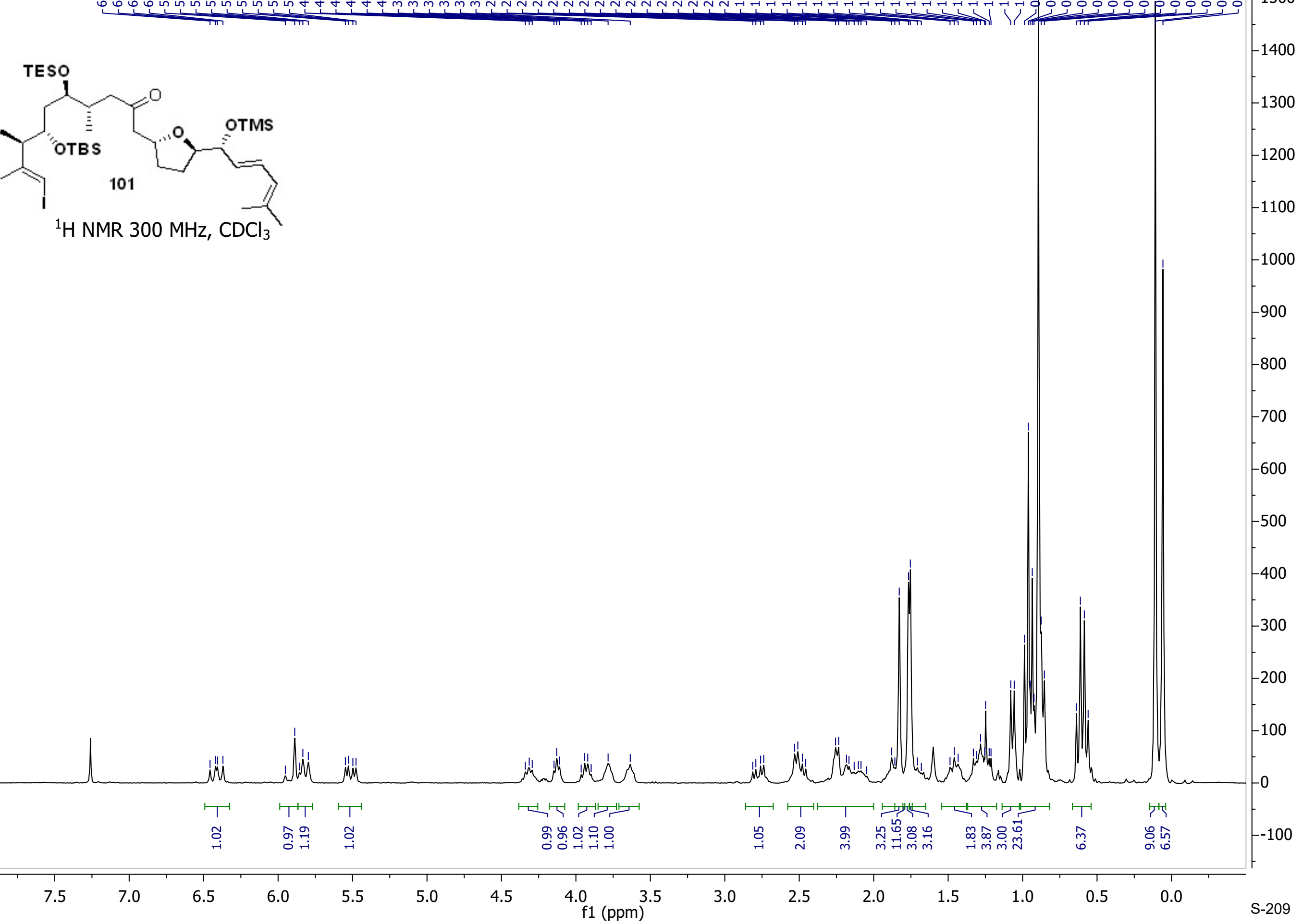

:




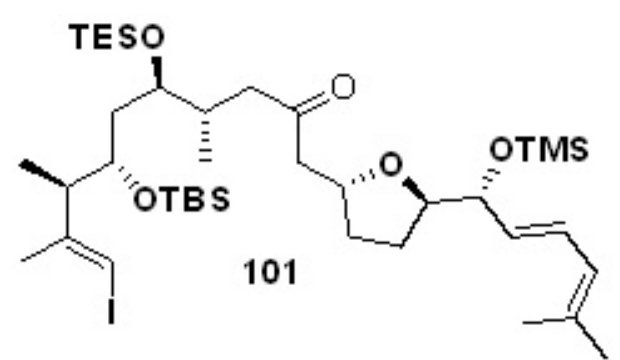

${ }^{13} \mathrm{C}\left\{{ }^{1} \mathrm{H}\right\}$ NMR $75 \mathrm{MHz}, \mathrm{CDCl}_{3}$ 


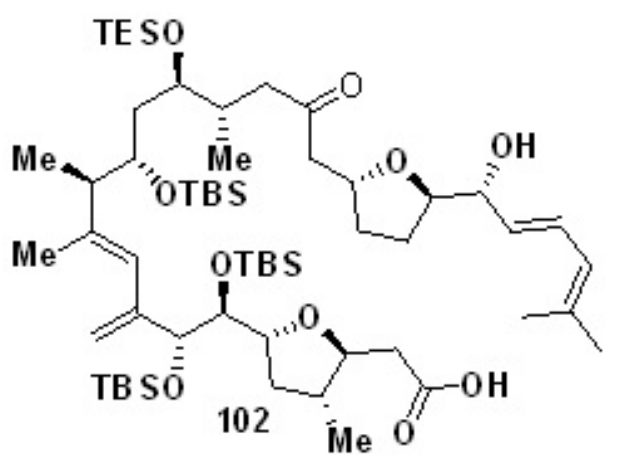

${ }^{1} \mathrm{H}$ NMR $300 \mathrm{MHz}, \mathrm{CDCl}_{3}$

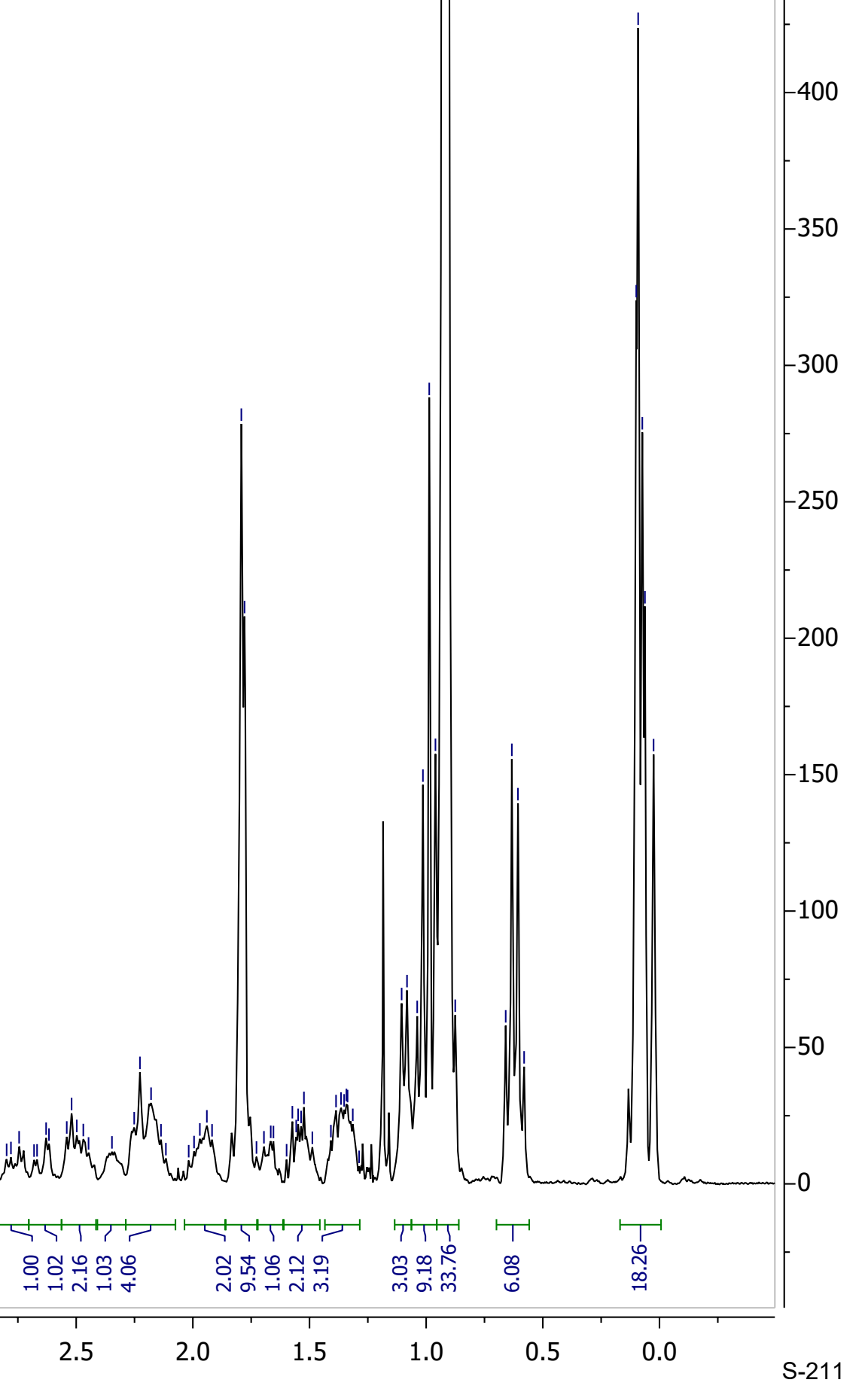




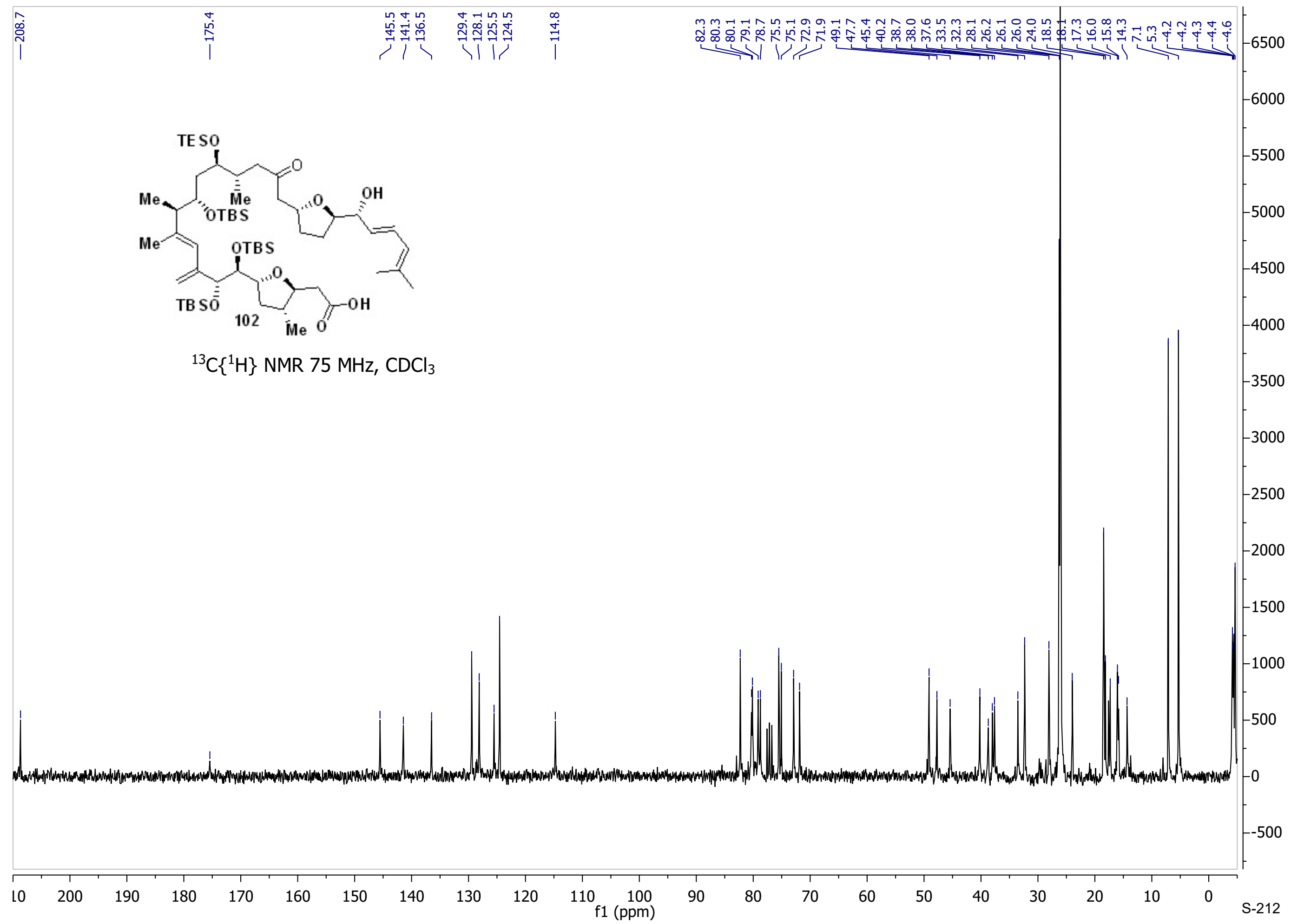




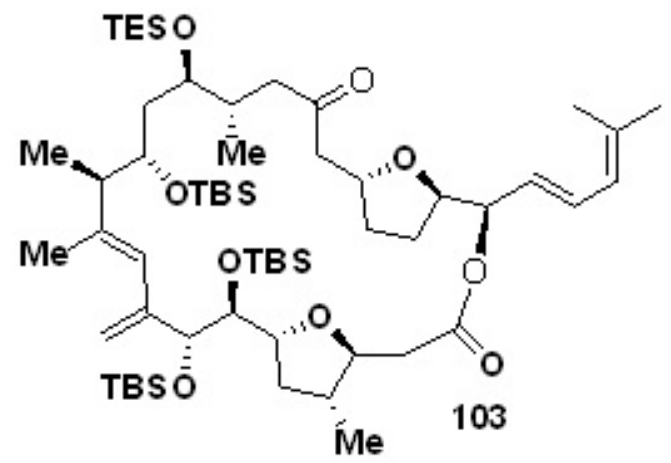

${ }^{1} \mathrm{H}$ NMR $400 \mathrm{MHz}, \mathrm{CDCl}_{3}$

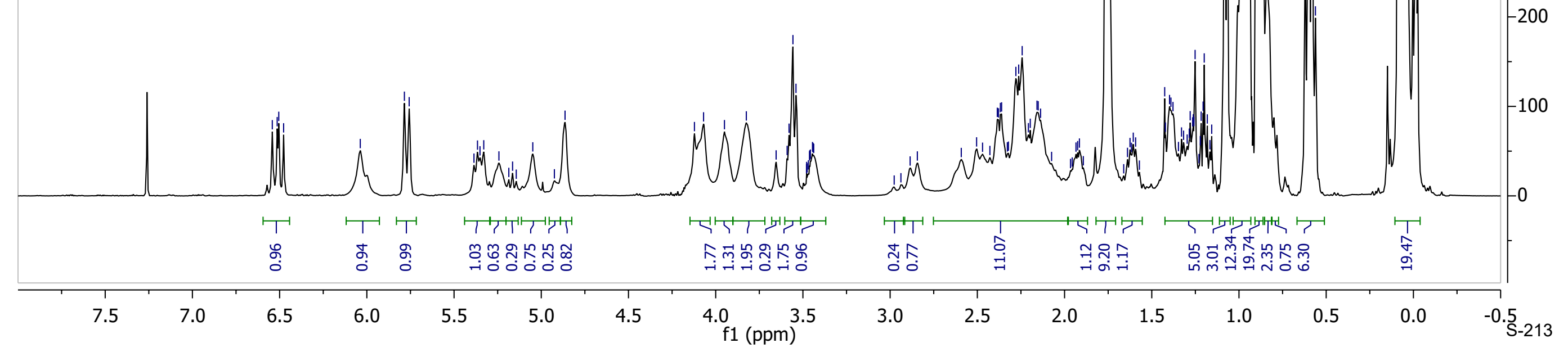




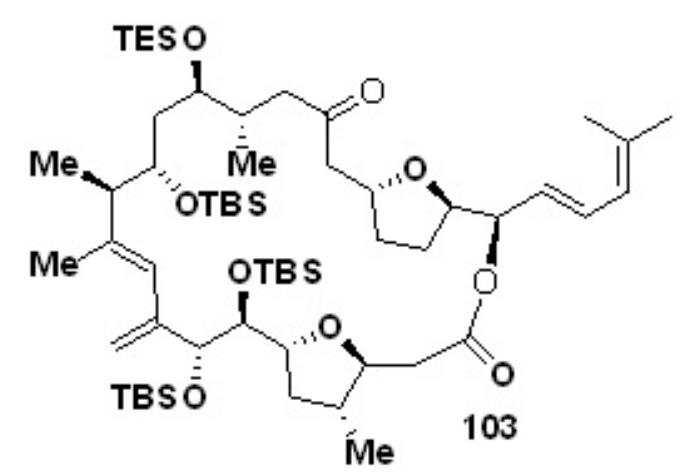

${ }^{13} \mathrm{C}\left\{{ }^{1} \mathrm{H}\right\} \mathrm{NMR} 100 \mathrm{MHz}, \mathrm{CDCl}_{3}$

$\begin{array}{llllllllllllllllllllllll}210 & 200 & 190 & 180 & 170 & 160 & 150 & 140 & 130 & 120 & 110 & \underset{\mathrm{f} 1}{(\mathrm{ppm})} & 90 & 80 & 70 & 60 & 50 & 40 & 30 & 20 & 10 & 0 & -10 & \mathrm{~S}-214\end{array}$




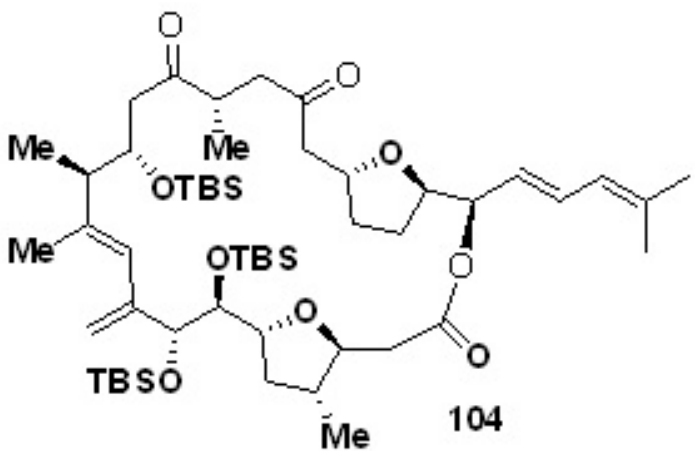

${ }^{1} \mathrm{H}$ NMR $400 \mathrm{MHz} \mathrm{CDCl}_{3}$

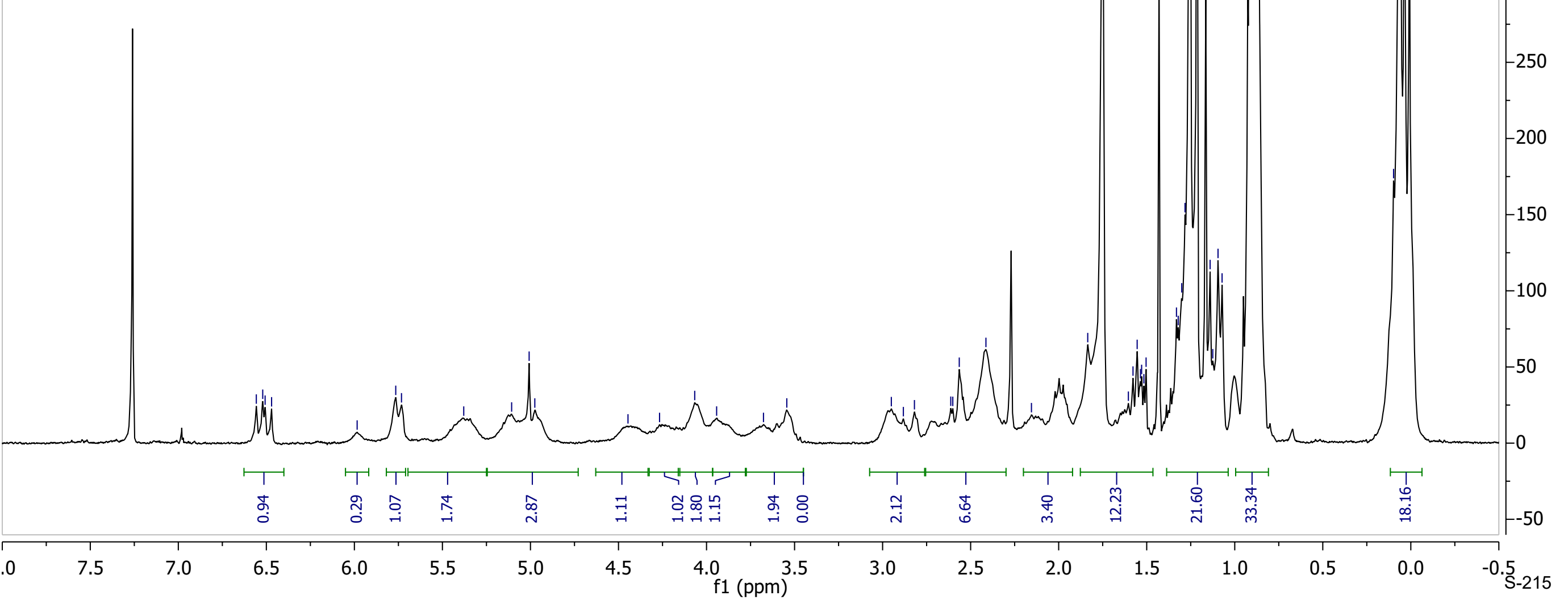




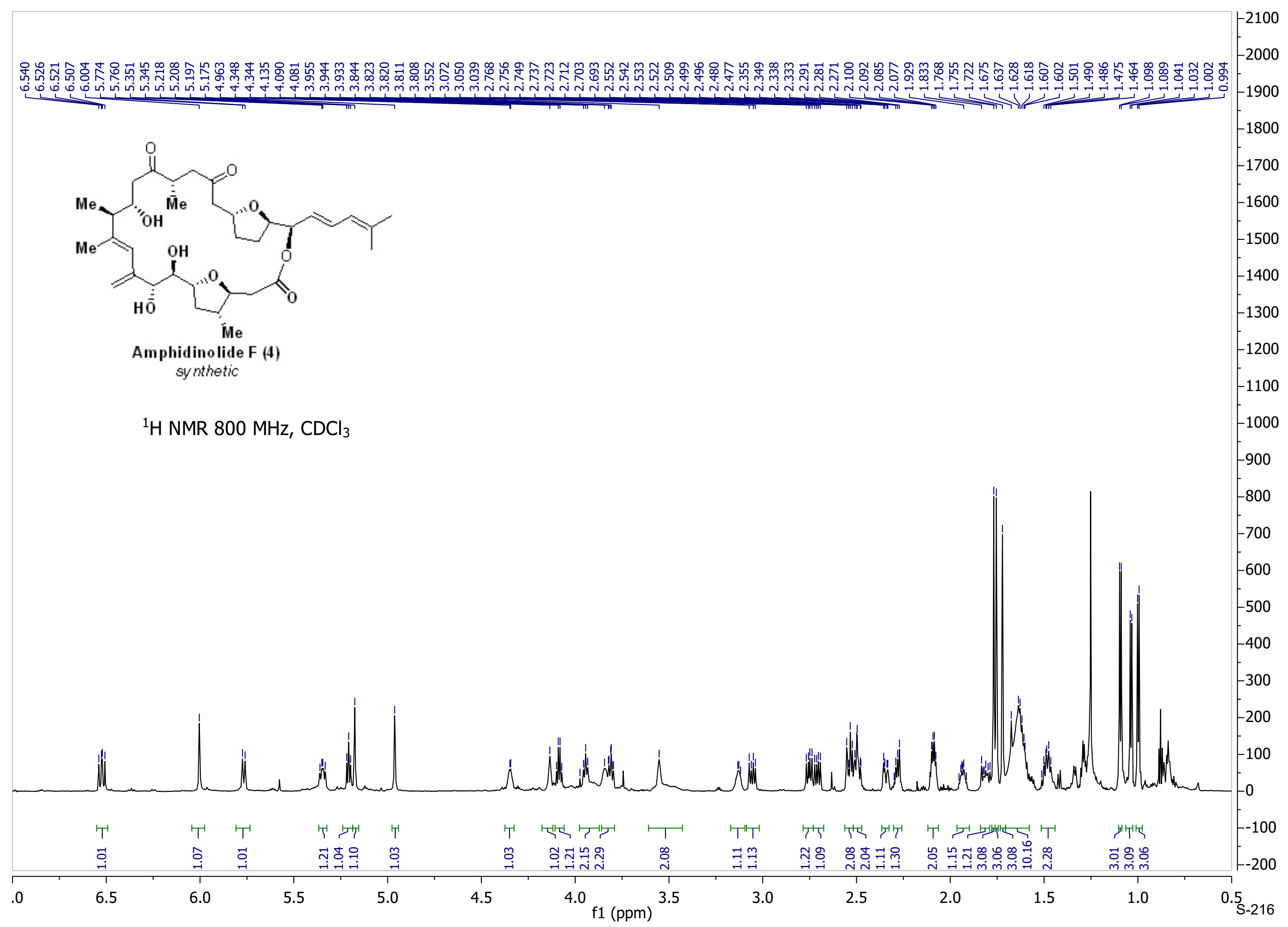



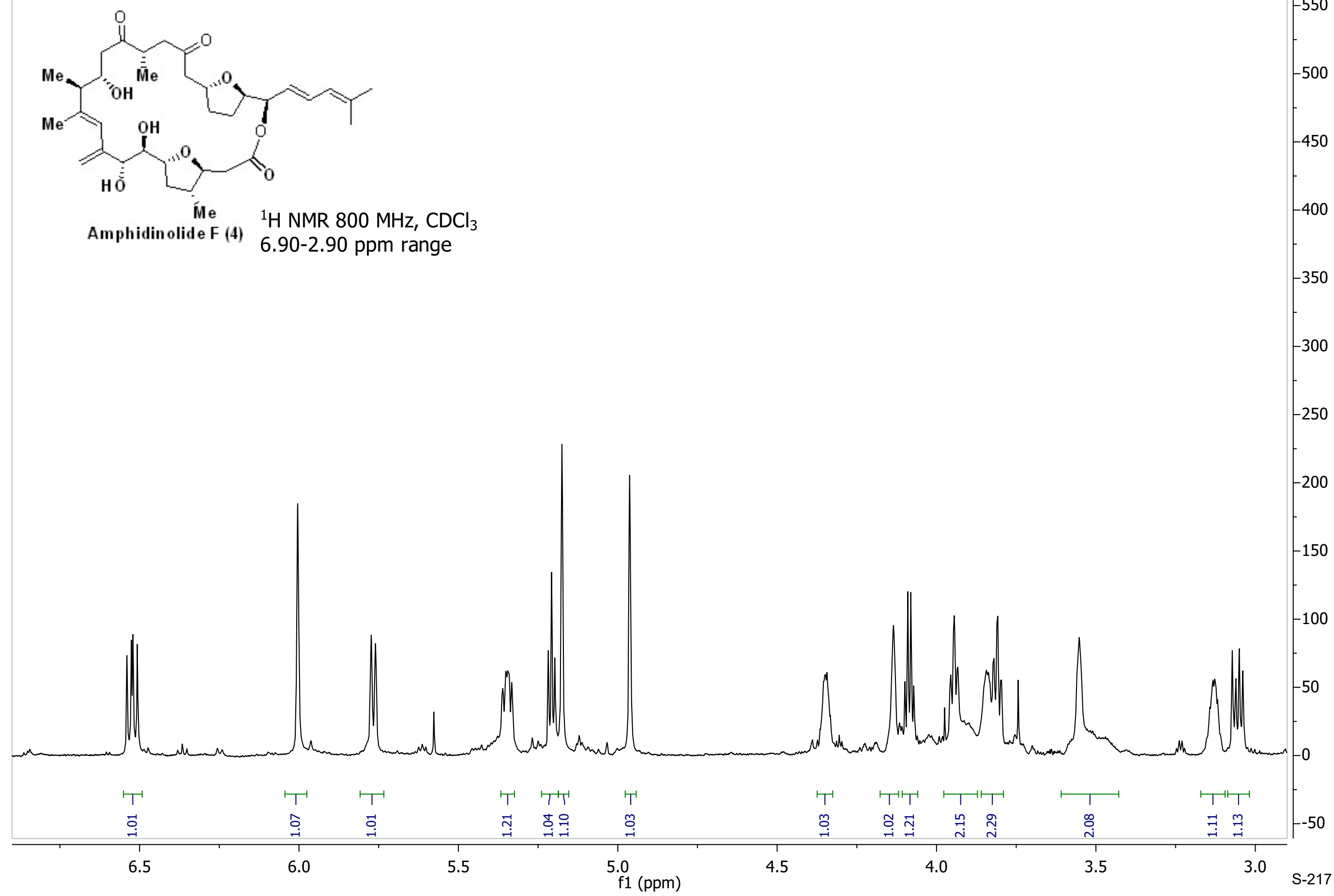


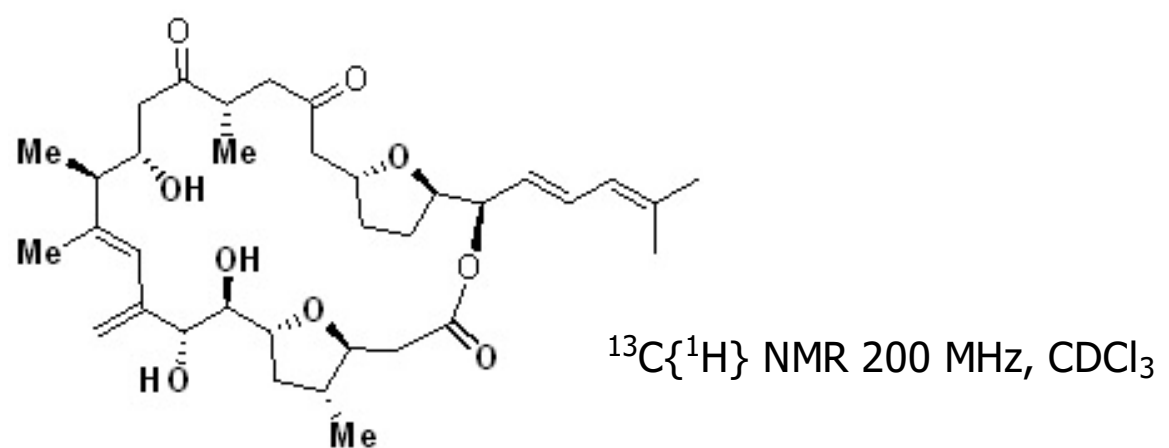

Amphidin olide F (4)

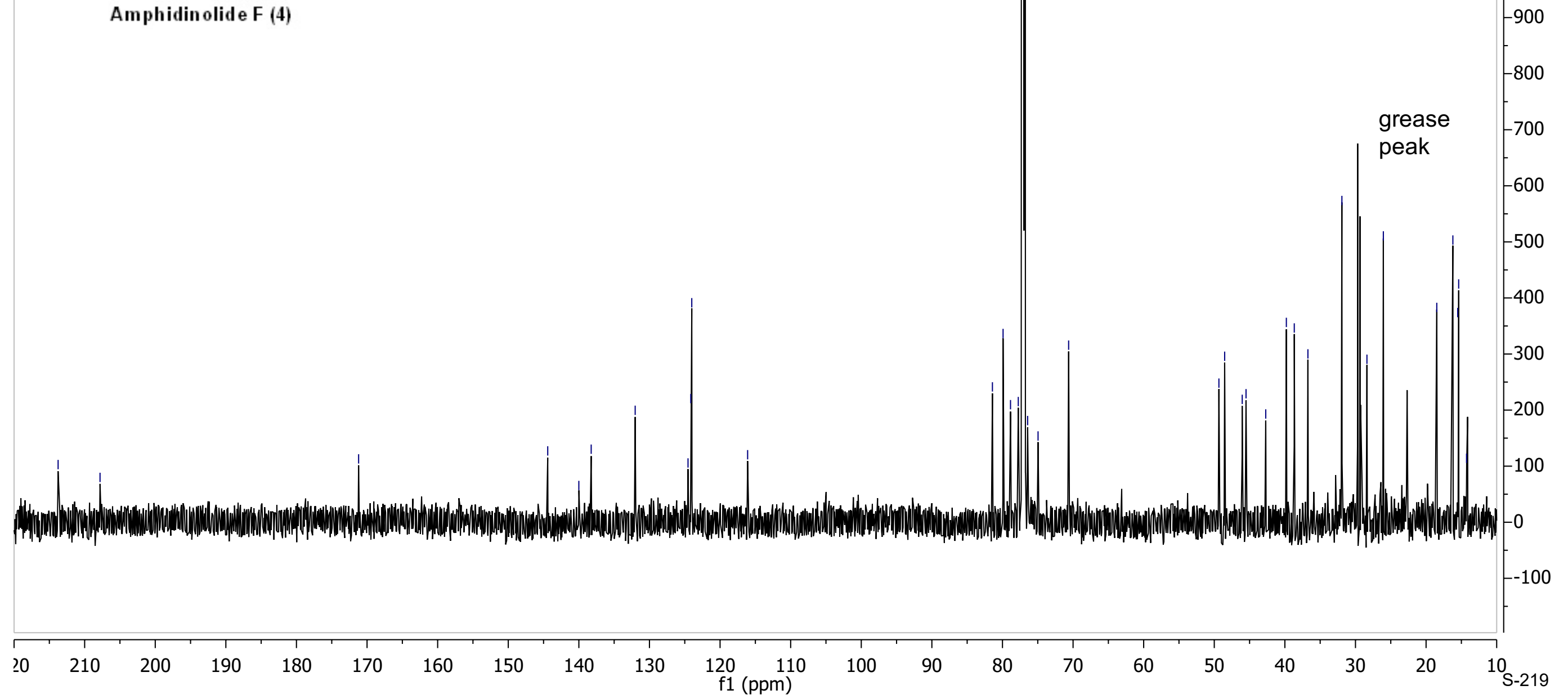




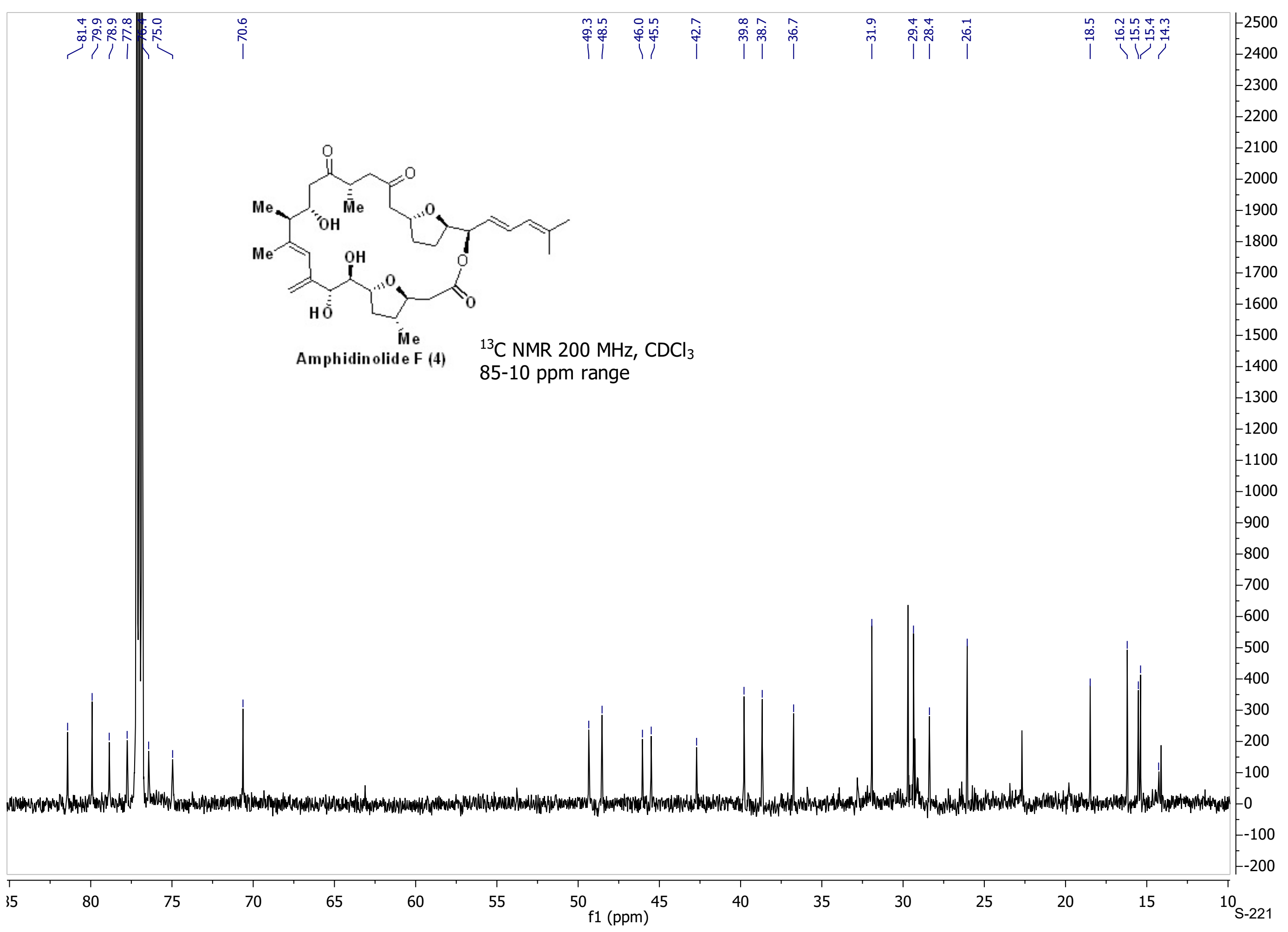



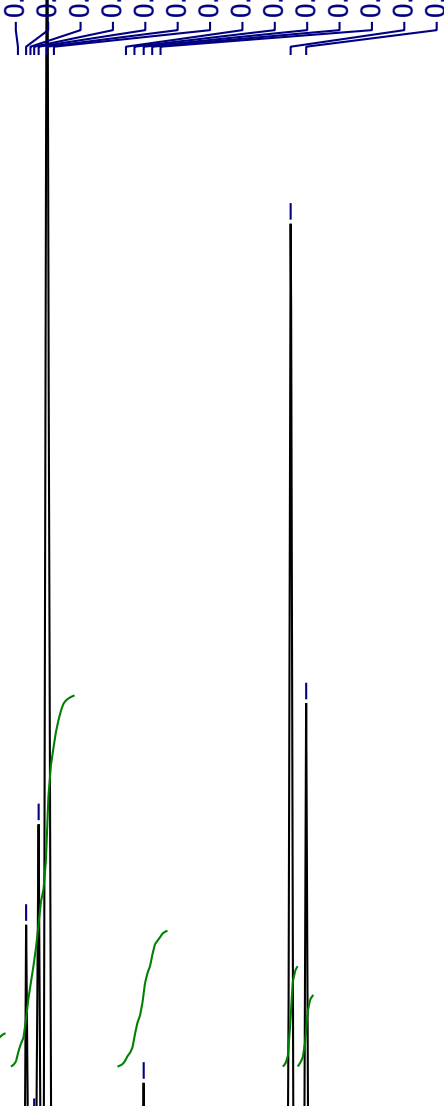

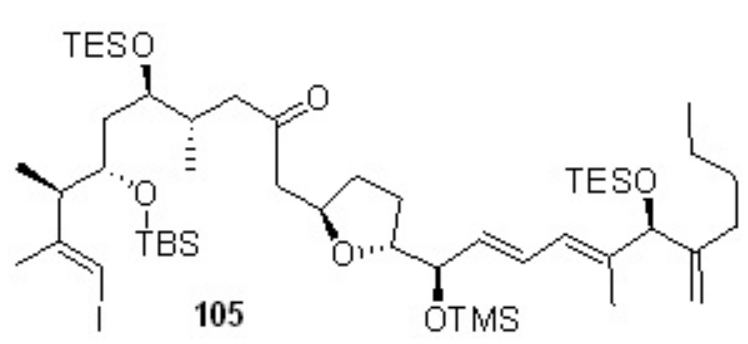

${ }^{1} \mathrm{H}$ NMR $300 \mathrm{MHz}, \mathrm{CDCl}_{3}$

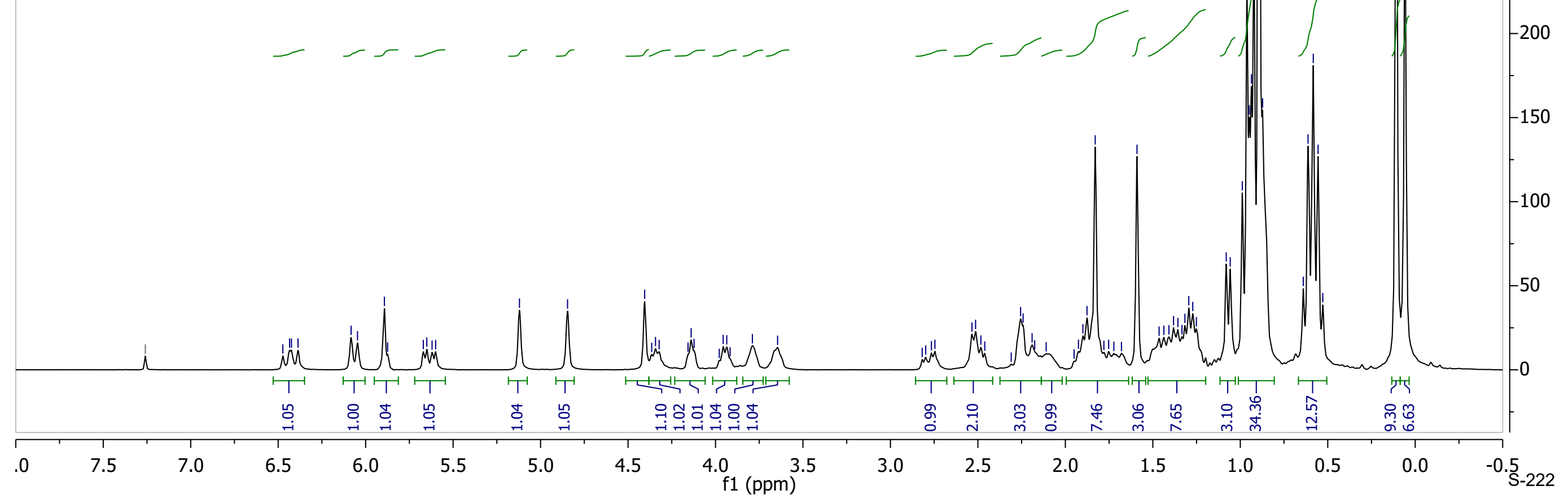




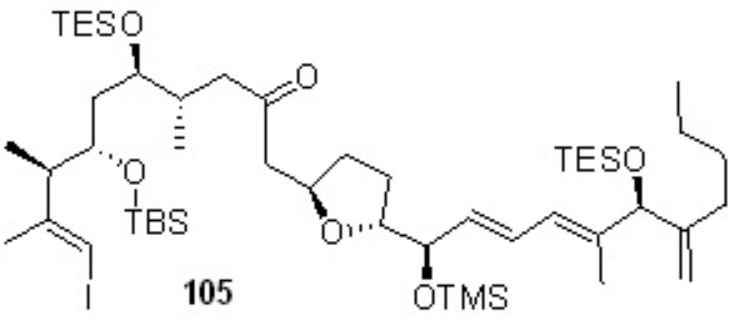

${ }^{13} \mathrm{C}\left\{{ }^{1} \mathrm{H}\right\}$ NMR $75 \mathrm{MHz}, \mathrm{CDCl}_{3}$ 

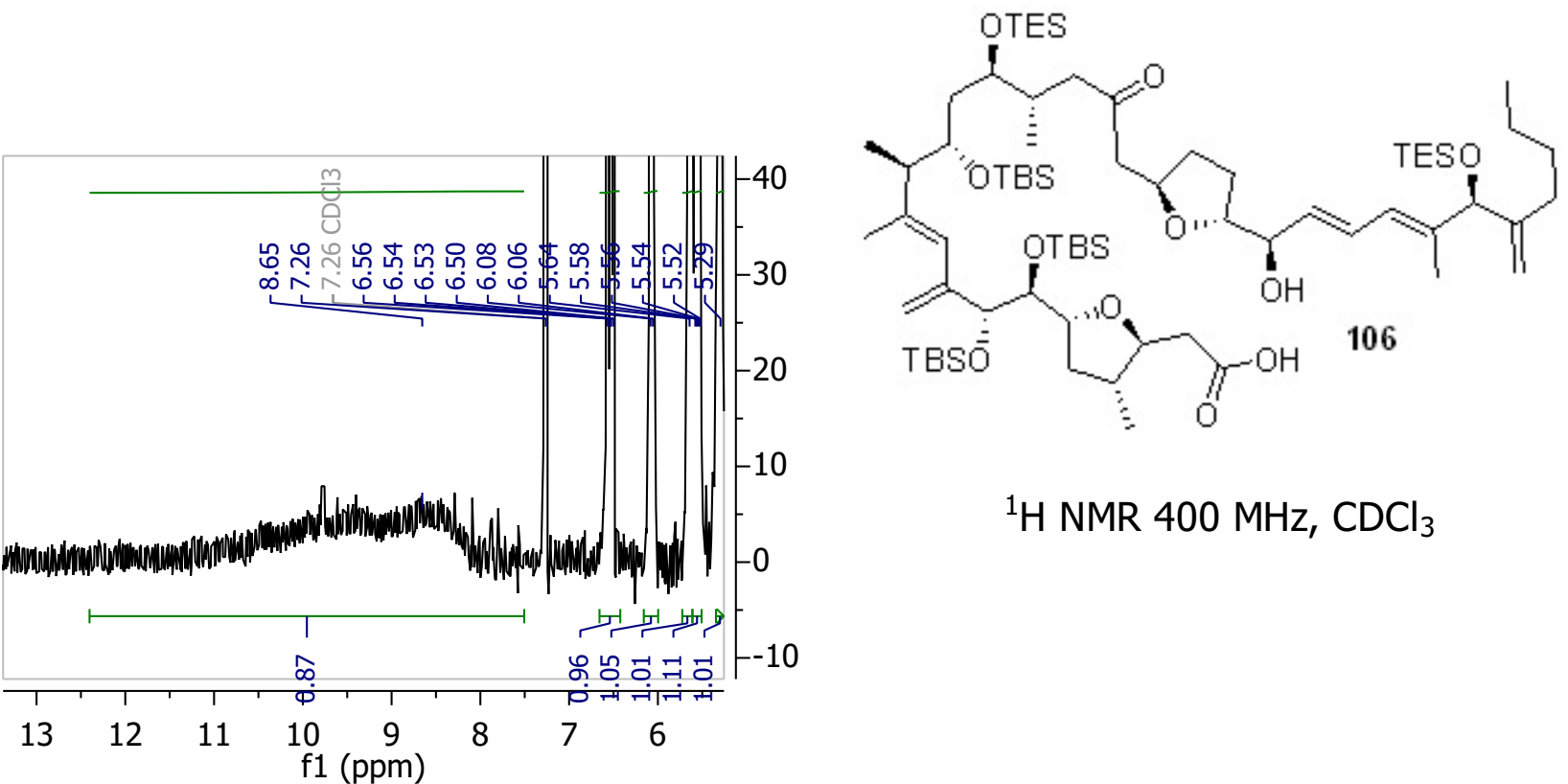

${ }^{1} \mathrm{H}$ NMR $400 \mathrm{MHz}, \mathrm{CDCl}_{3}$

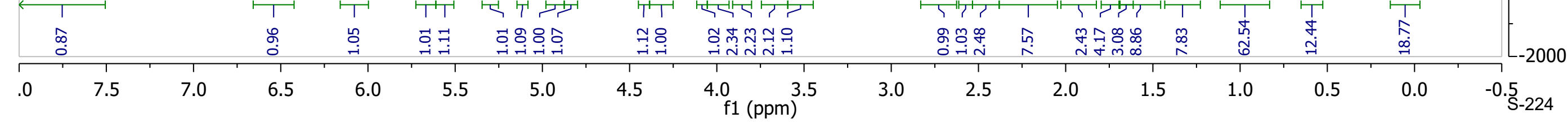




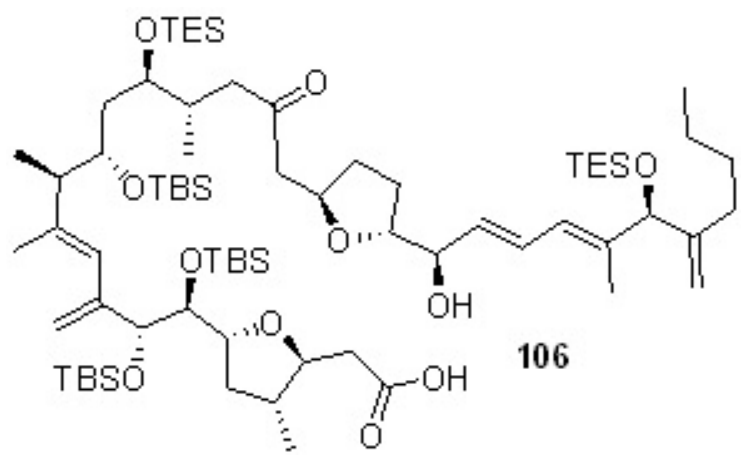

${ }^{13} \mathrm{C}\left\{{ }^{1} \mathrm{H}\right\}$ NMR $100 \mathrm{MHz}, \mathrm{CDCl}_{3}$ 


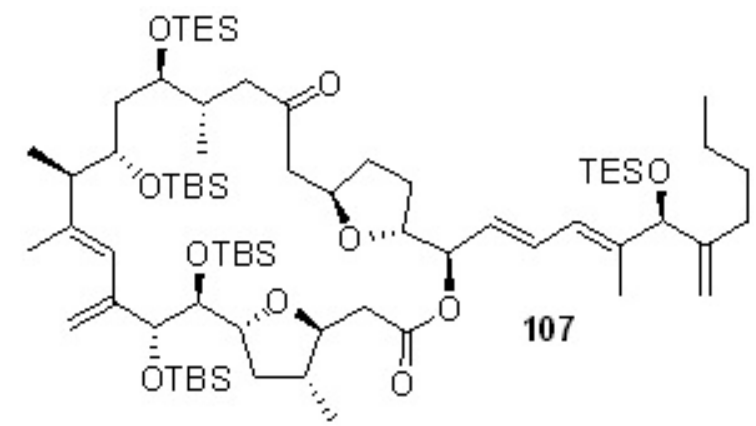

${ }^{1} \mathrm{H}$ NMR $400 \mathrm{MHz}, \mathrm{CDCl}_{3}$

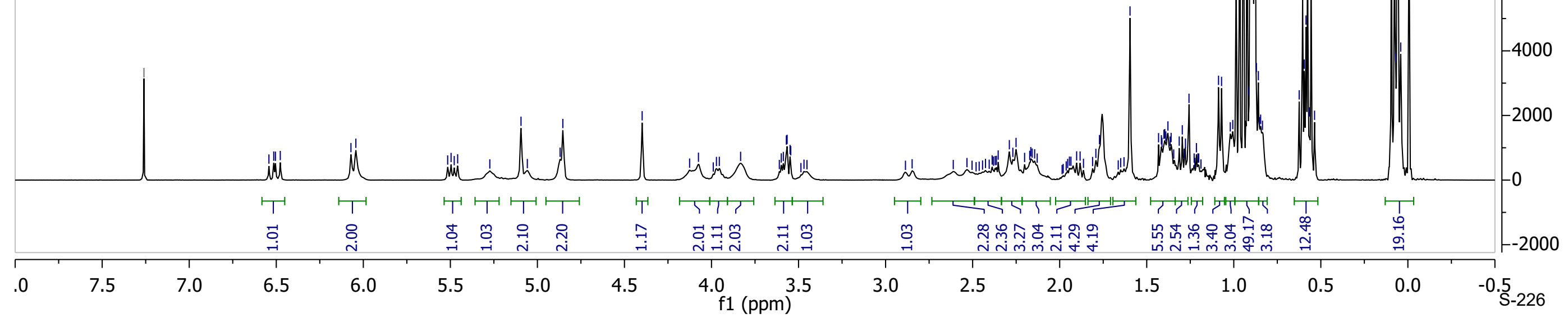




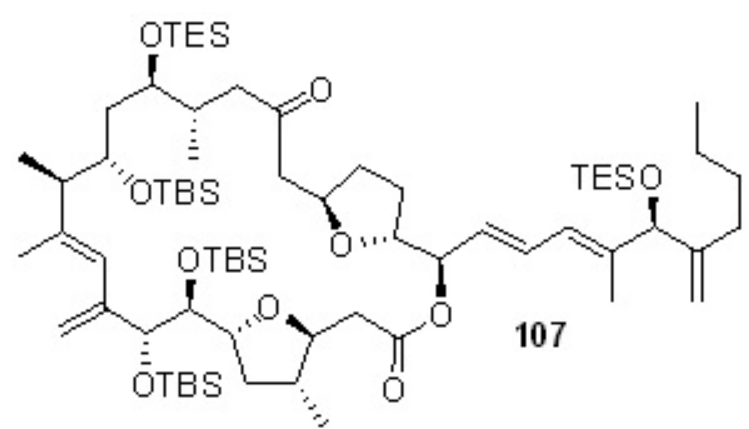

${ }^{13} \mathrm{C}\left\{{ }^{1} \mathrm{H}\right\}$ NMR $100 \mathrm{MHz}, \mathrm{CDCl}_{3}$ 


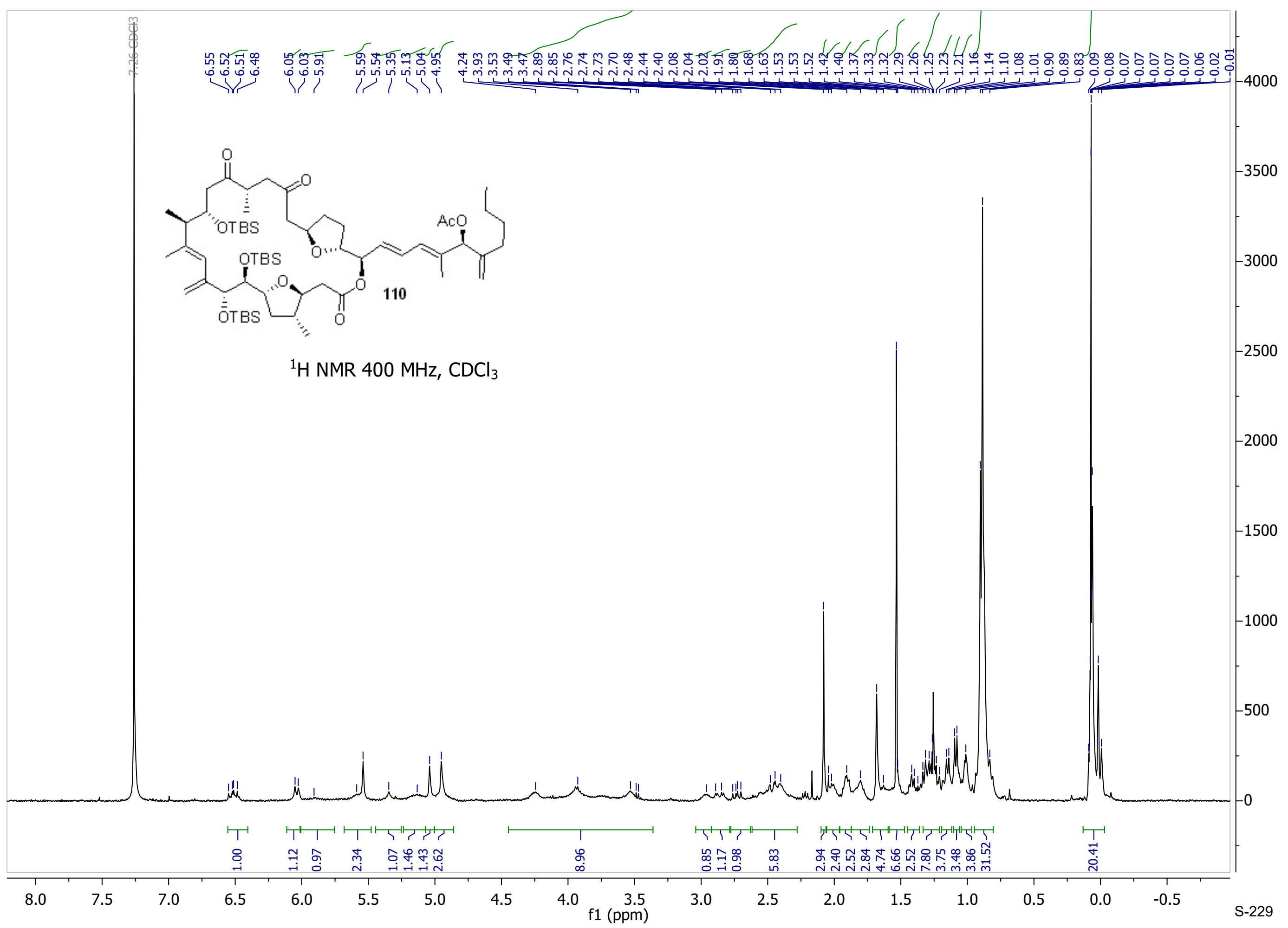




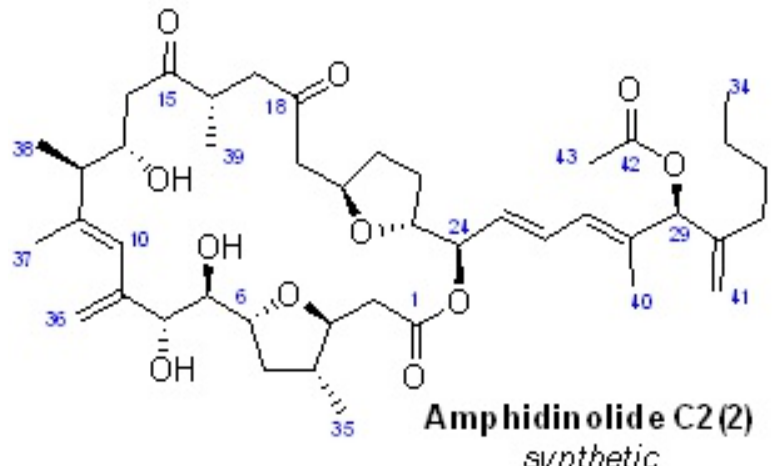

${ }^{1} \mathrm{H}$ NMR $600 \mathrm{MHz}-\mathrm{C}_{6} \mathrm{D}_{6}$ (solvant peak irradiated)

irradiated

solvant peak

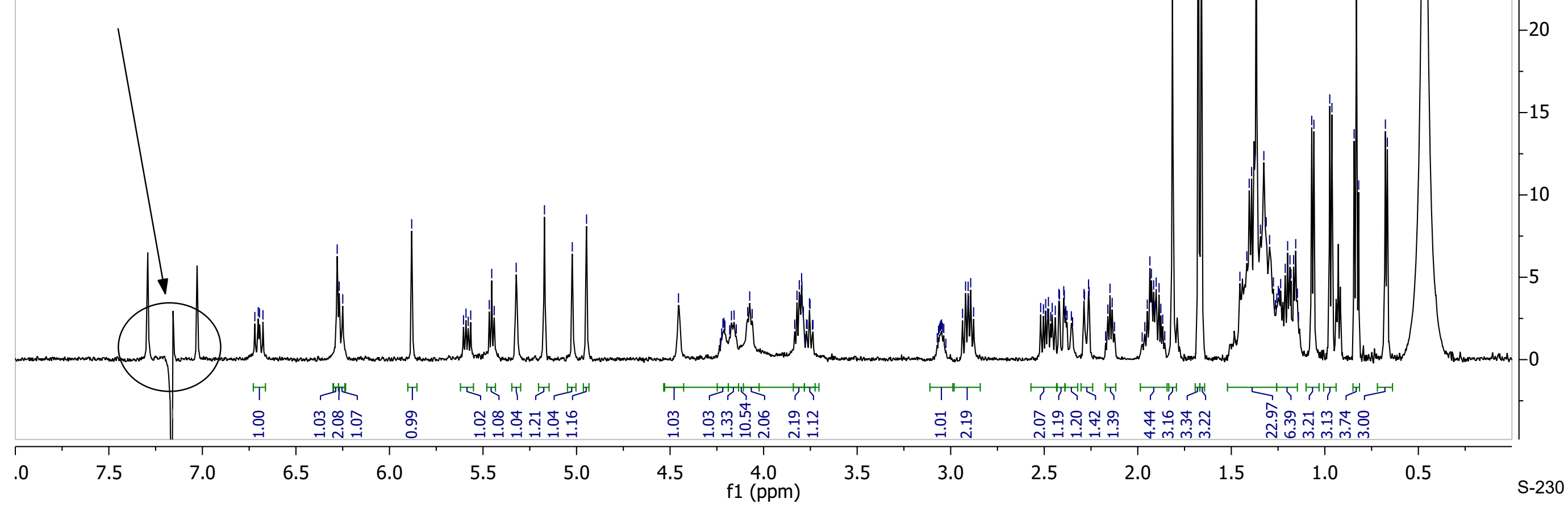



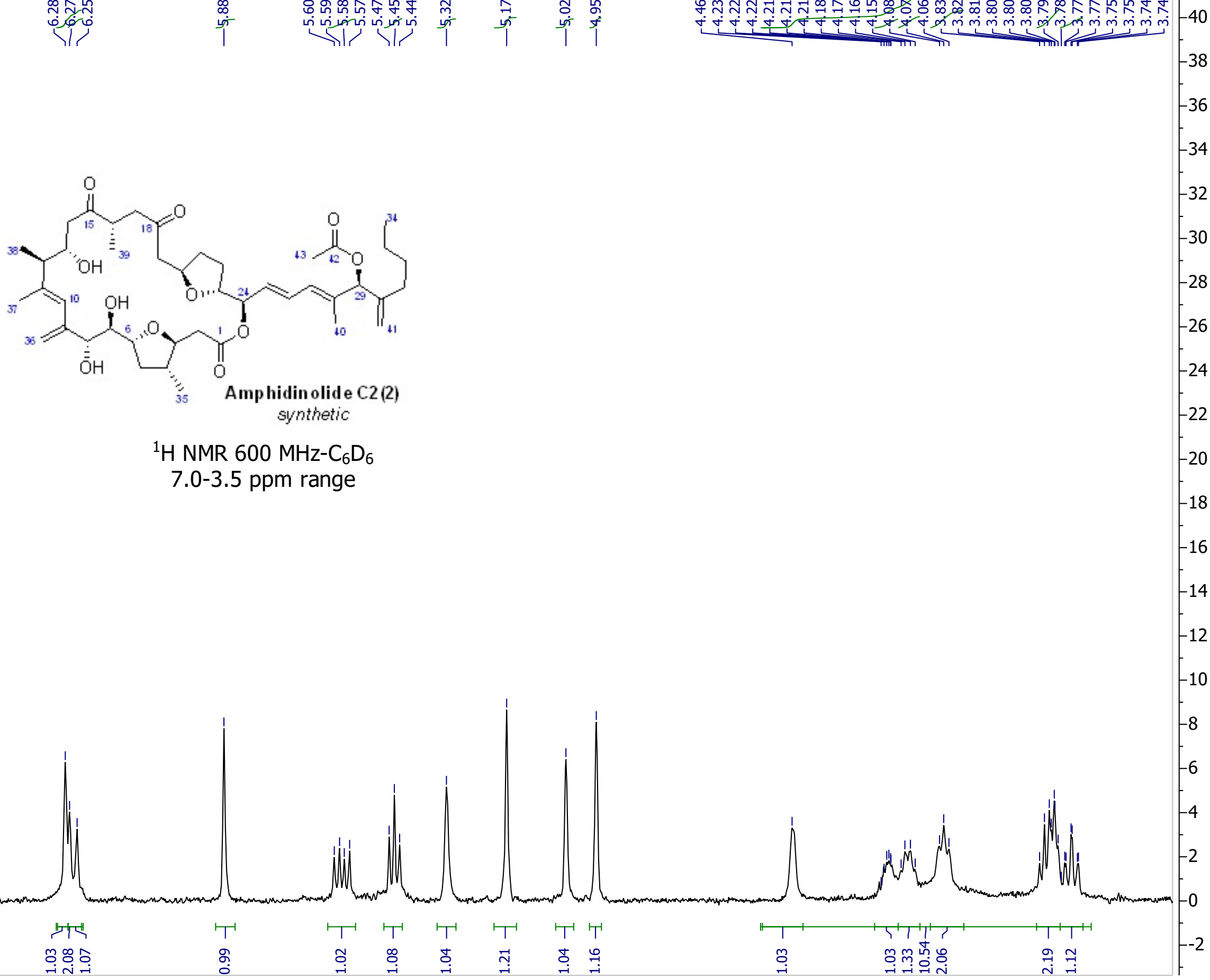

${ }^{1} \mathrm{H} \mathrm{NMR} 600 \mathrm{MHz}-\mathrm{C}_{6} \mathrm{D}_{6}$ 7.0-3.5 ppm range

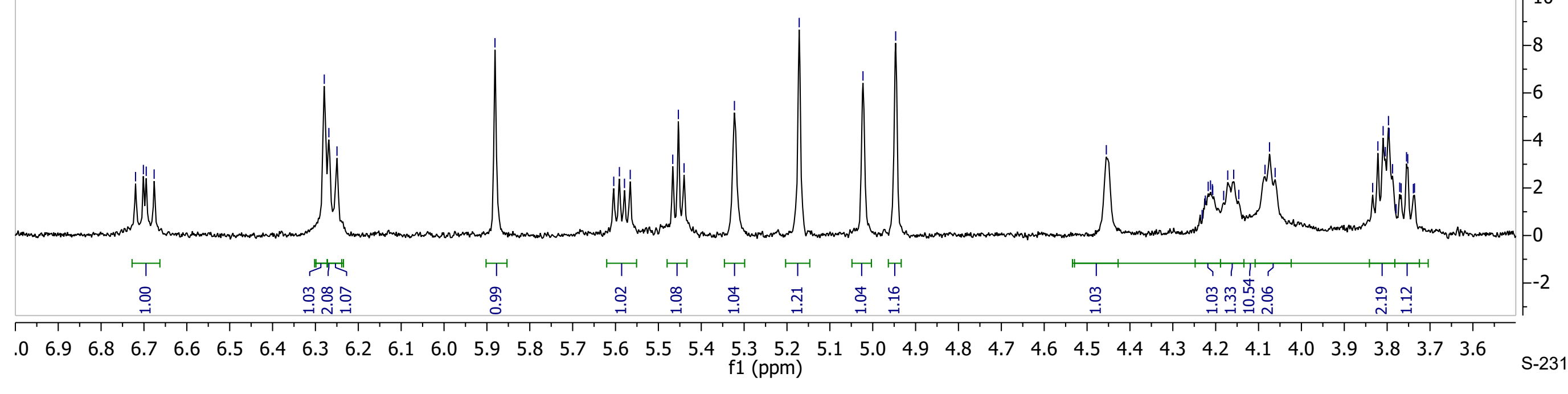



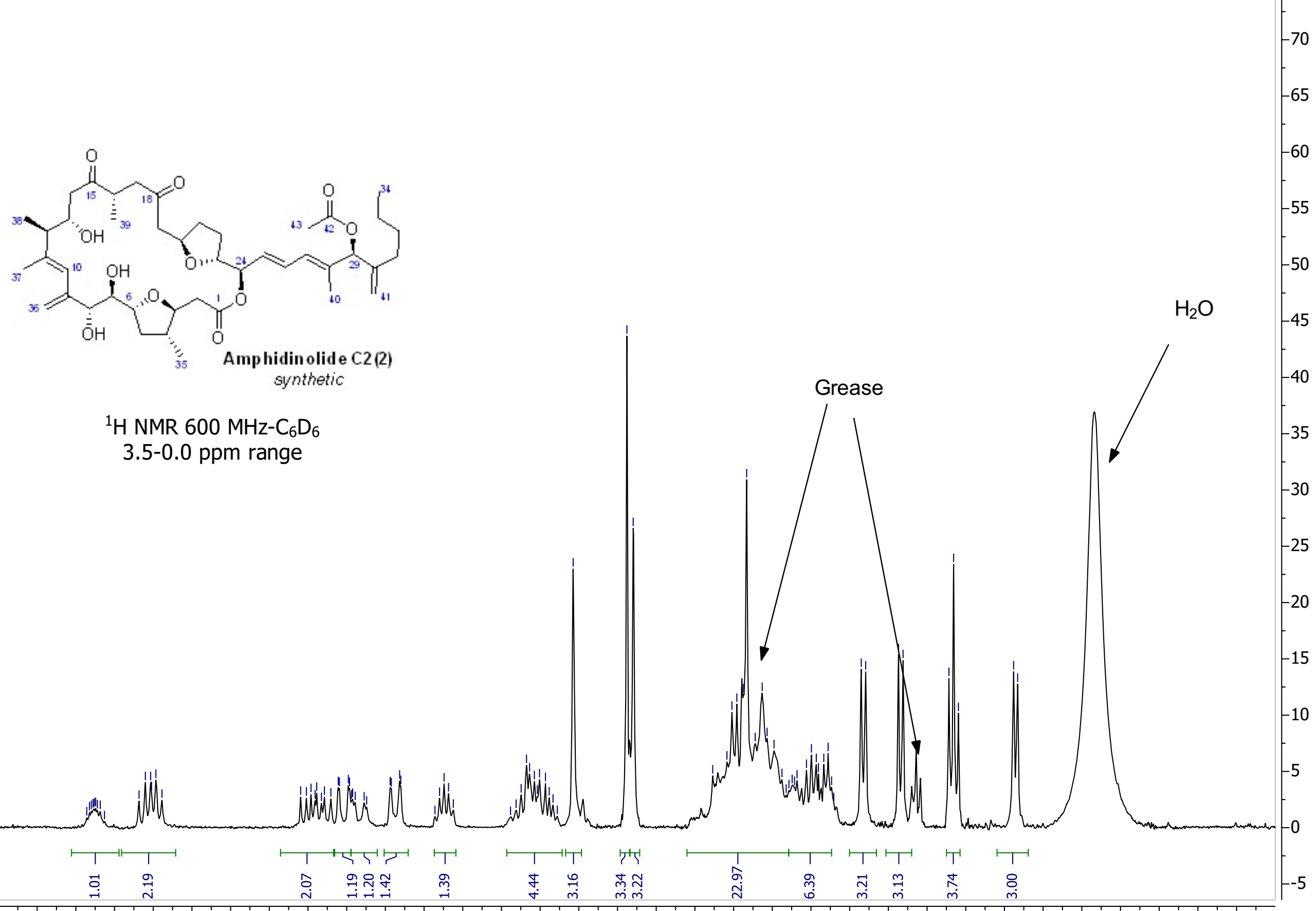

${ }^{1} \mathrm{H}$ NMR $600 \mathrm{MHz}-\mathrm{C}_{6} \mathrm{D}_{6}$ 3.5-0.0 ppm range

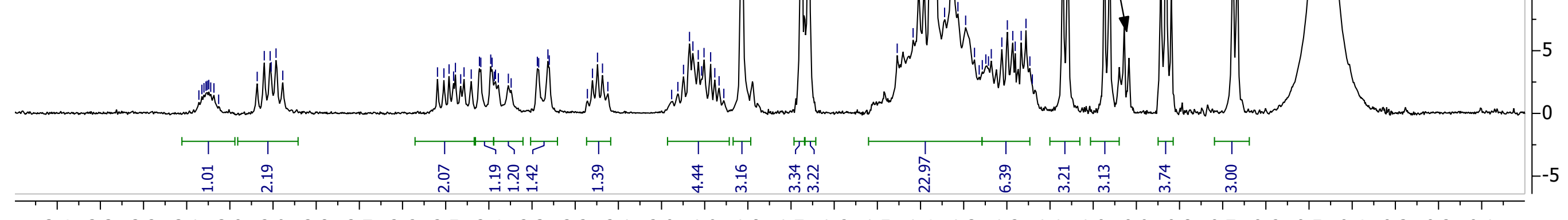

$\begin{array}{lllllllllllllllllllllllllllllllllll}3.4 & 3.3 & 3.2 & 3.1 & 3.0 & 2.9 & 2.8 & 2.7 & 2.6 & 2.5 & 2.4 & 2.3 & 2.2 & 2.1 & 2.0 & 1.9 & 1.8 & 1.7 & 1.6 & 1.5 & 1.4 & 1.3 & 1.2 & 1.1 & 1.0 & 0.9 & 0.8 & 0.7 & 0.6 & 0.5 & 0.4 & 0.3 & 0.2 & 0.1 & \mathrm{~S}-232\end{array}$ 


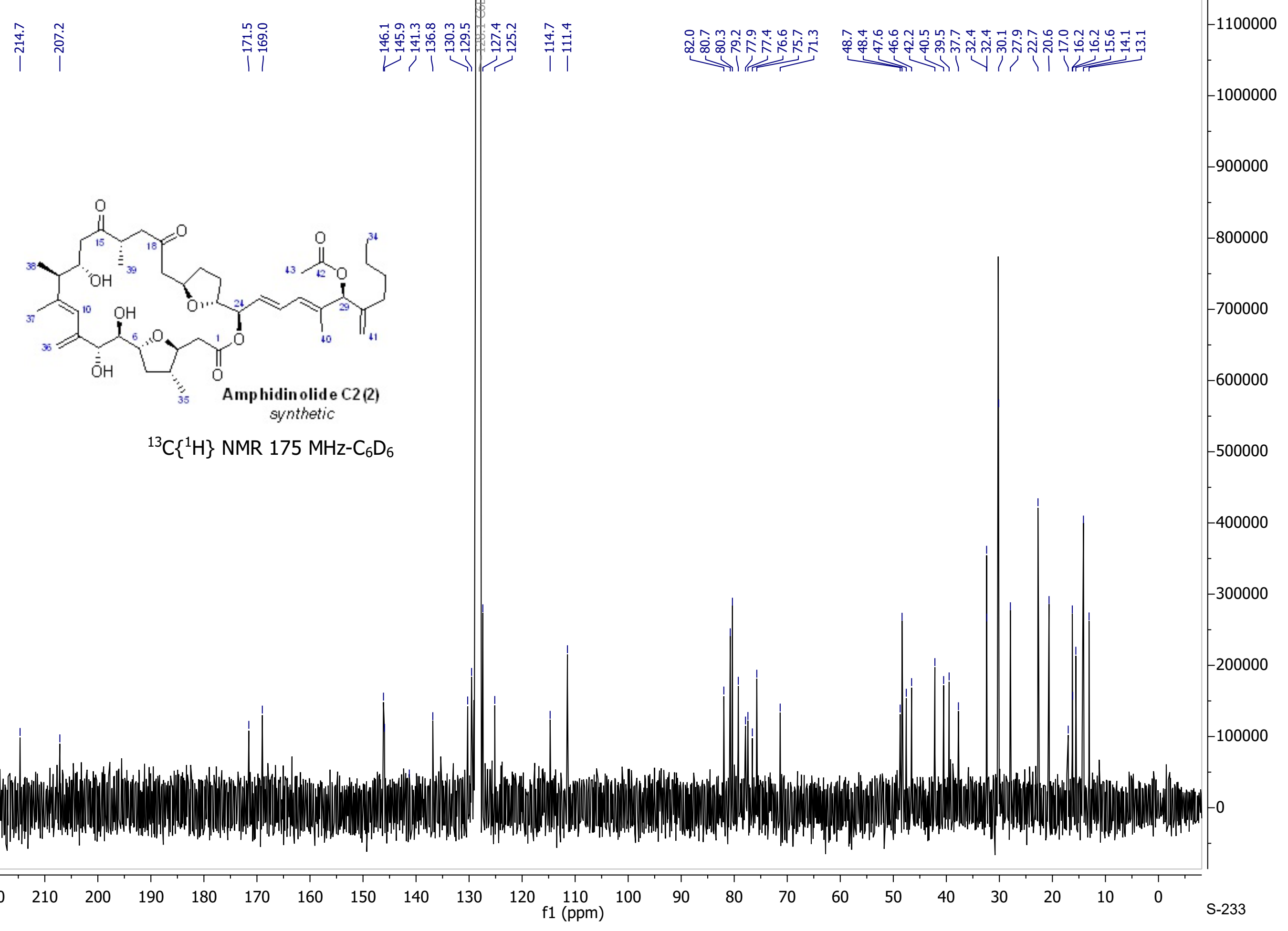




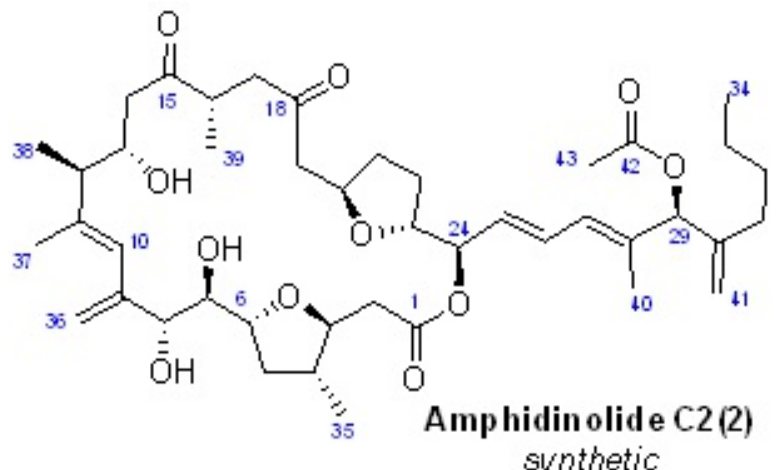

${ }^{13} \mathrm{C}\left\{{ }^{1} \mathrm{H}\right\}$ NMR $175 \mathrm{MHz}-\mathrm{C}_{6} \mathrm{D}_{6}$ 150-110 ppm range 


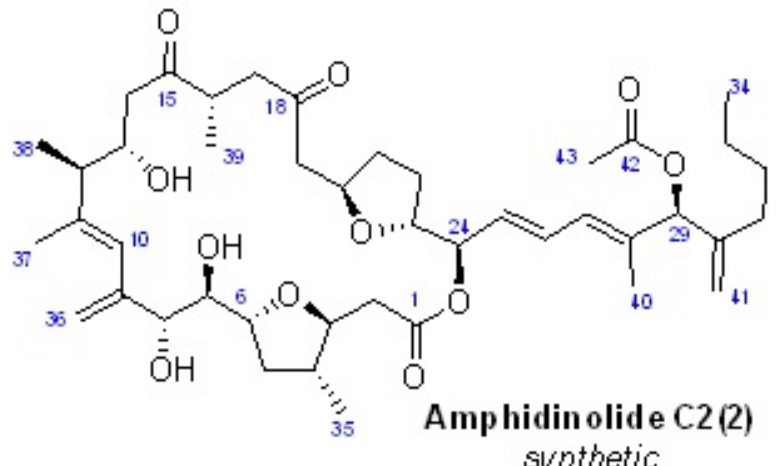

${ }^{13} \mathrm{C}\left\{{ }^{1} \mathrm{H}\right\}$ NMR $175 \mathrm{MHz}-\mathrm{C}_{6} \mathrm{D}_{6}$ 100-40 ppm range 

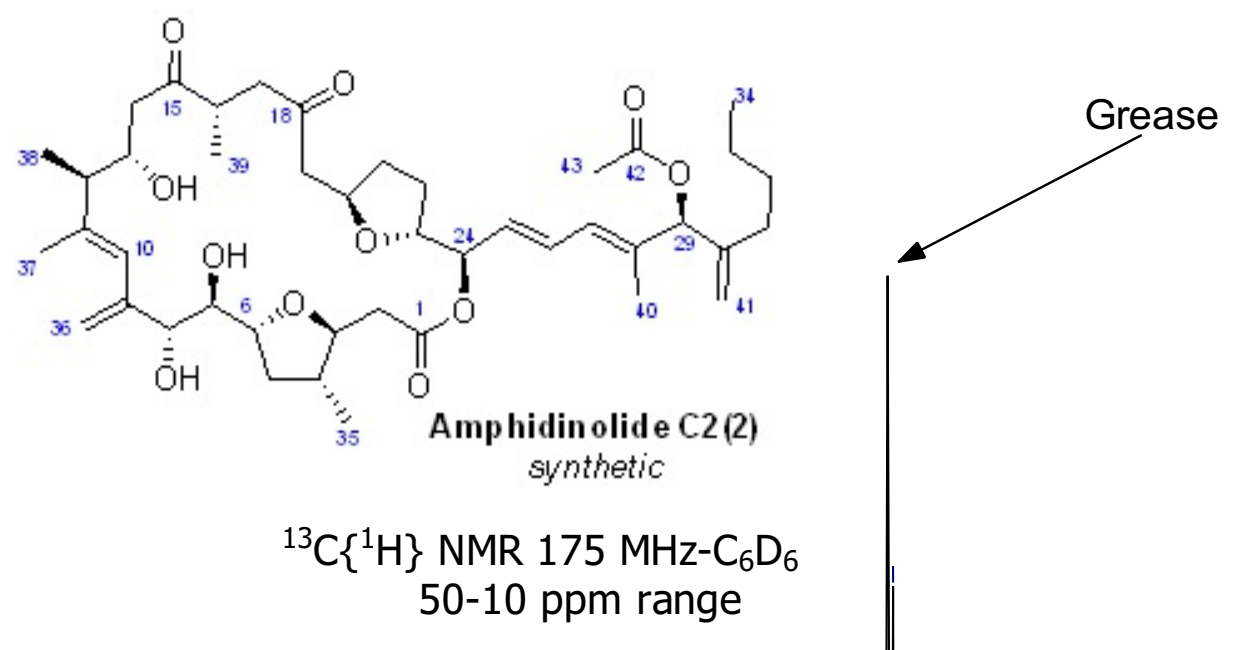

${ }^{13} \mathrm{C}\left\{{ }^{1} \mathrm{H}\right\}$ NMR $175 \mathrm{MHz}-\mathrm{C}_{6} \mathrm{D}_{6}$ 50-10 ppm range

4 


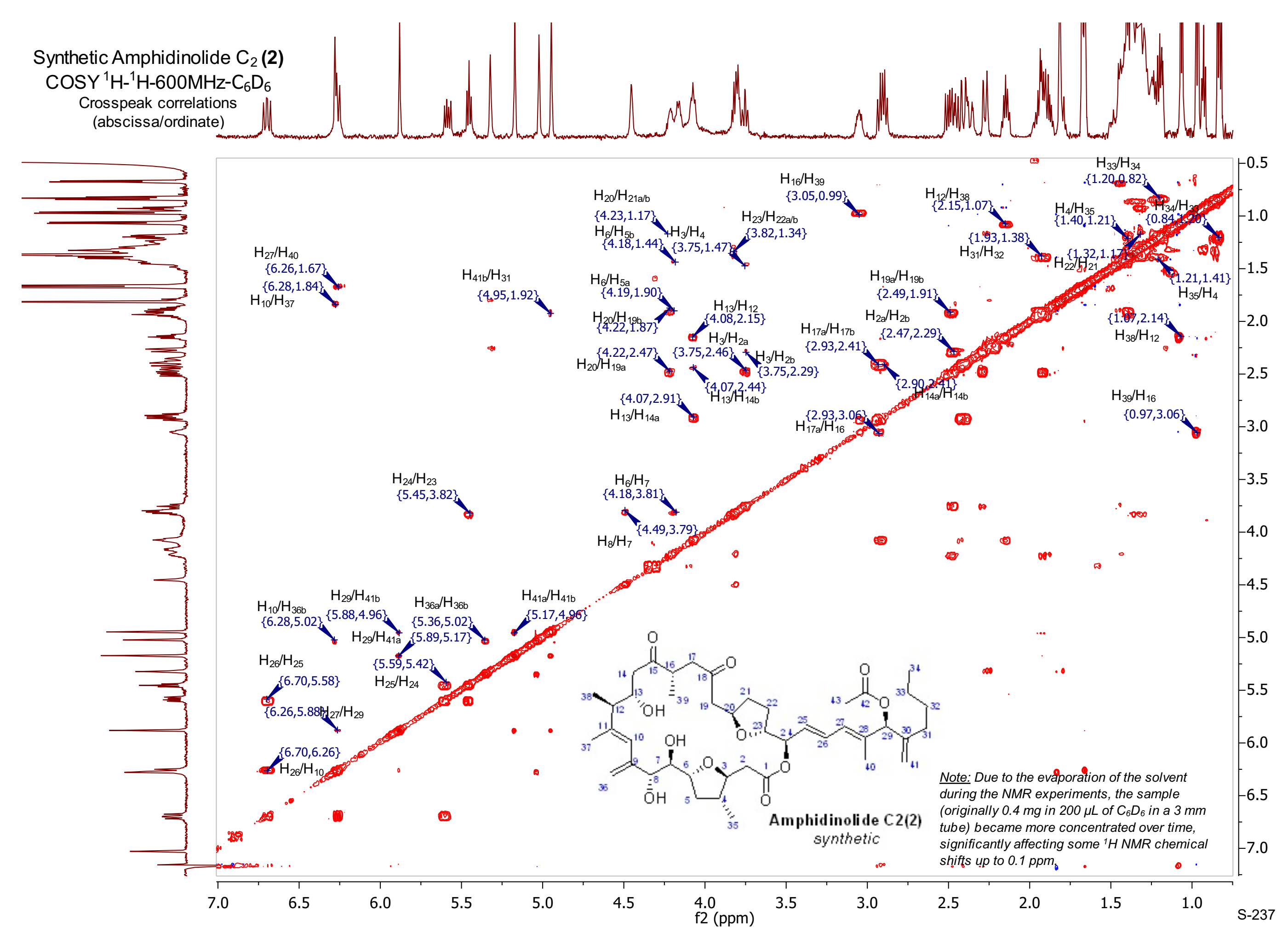


Synthetic Amphidinolide $\mathrm{C}_{2}$ (2)

$\left.\mathrm{HMQC}{ }^{1} \mathrm{H}^{13}{ }^{13} \mathrm{C}^{1} \mathrm{H}\right\}-600 \mathrm{MHz}-\mathrm{C}_{6} \mathrm{D}_{6}$

Crosspeak correlations (7.2-3.75; 150-60 ppm range)

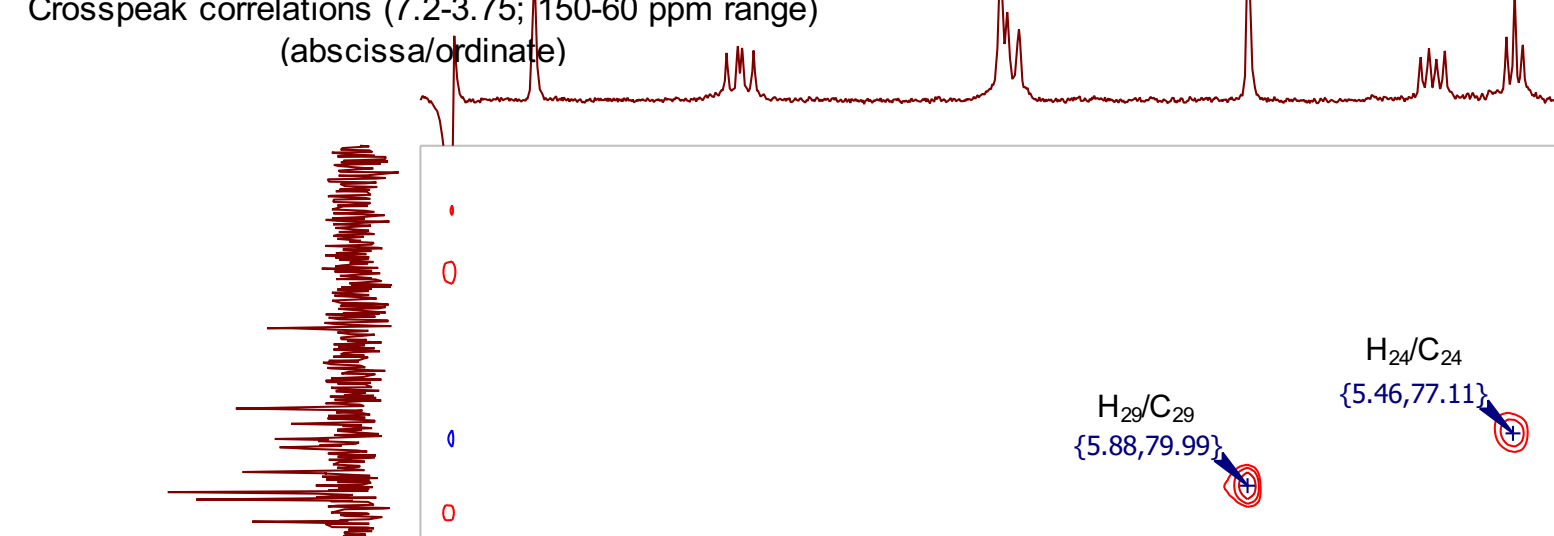

(abscissa/ordinate)
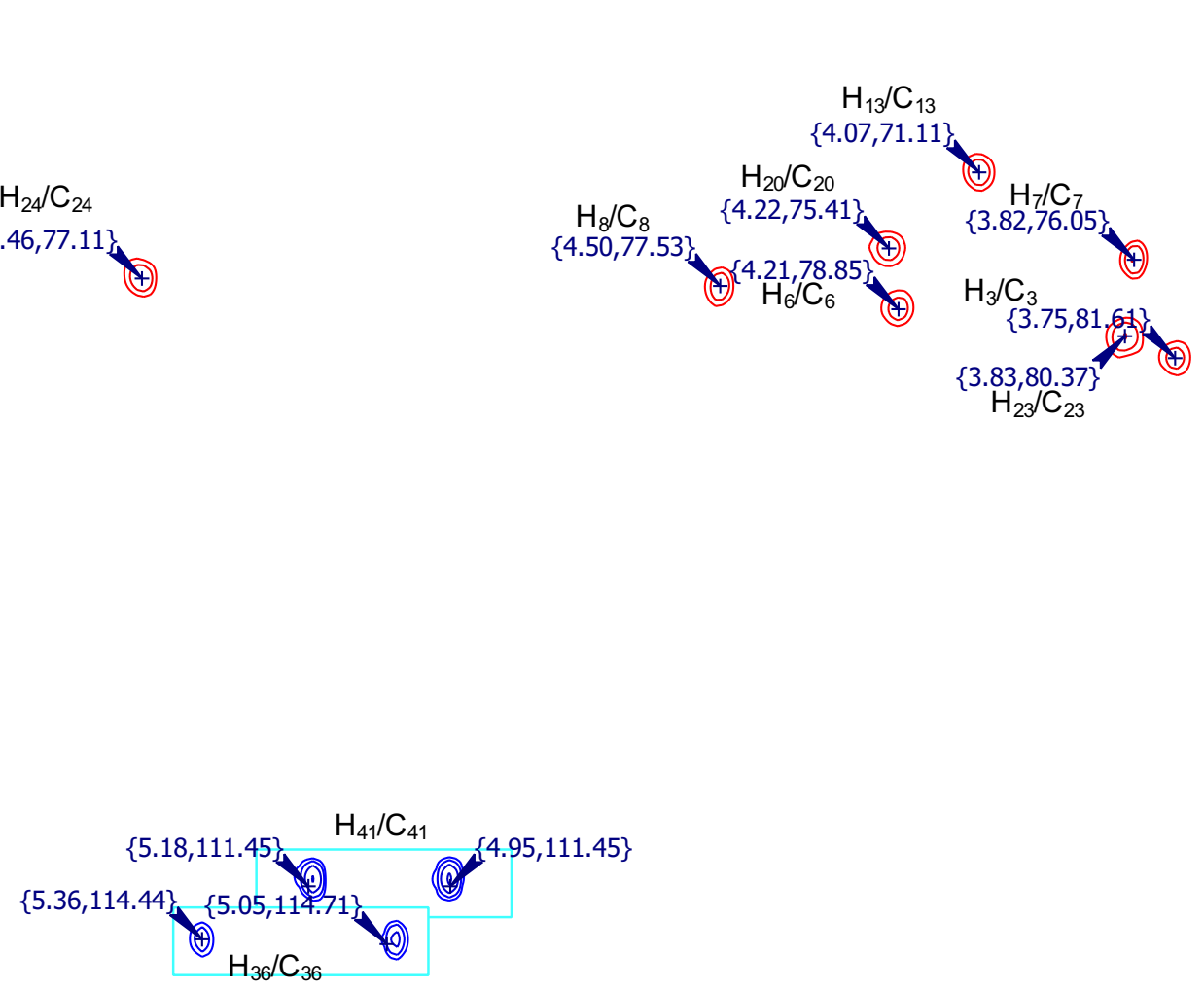

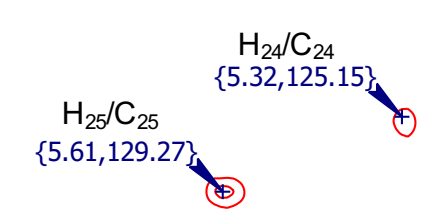

Note: Due to the evaporation of the solvent during the NMR experiments, the sample (originally $0.4 \mathrm{mg}$ in $200 \mu \mathrm{L}$ of $\mathrm{C}_{6} \mathrm{D}_{6}$ in a $3 \mathrm{~mm}$ tube) became more concentrated over time, significantly affecting some ${ }^{1} \mathrm{H}$ NMR chemical shifts up to $0.1 \mathrm{ppm}$.

\section{(n-}

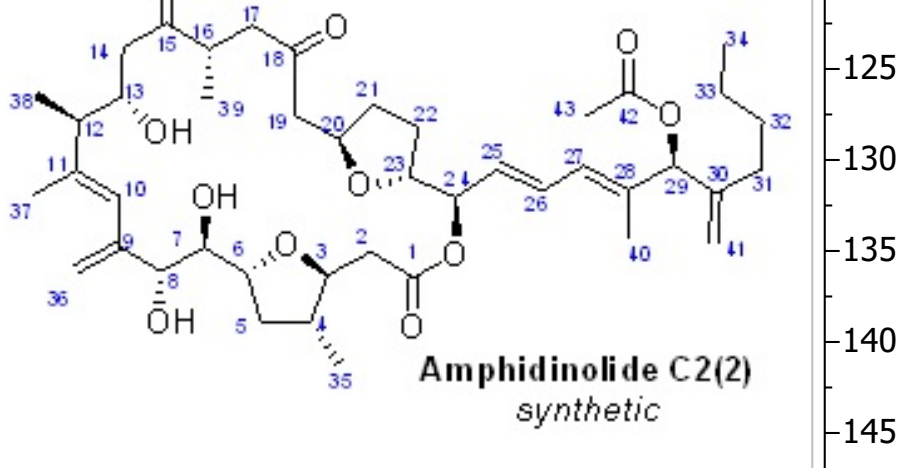


Synthetic Amphidinolide $\mathrm{C}_{2}$ (2)

$\mathrm{HMQC}{ }^{1} \mathrm{H}-{ }^{13} \mathrm{C}\left\{{ }^{1} \mathrm{H}\right\}-600 \mathrm{MHz}-\mathrm{C}_{6} \mathrm{D}_{6}$

Crosspeak correlations (3.1-0.45; 10-55 ppm range) (abscissa/ordinate) $M M$ MWWNM N Mh

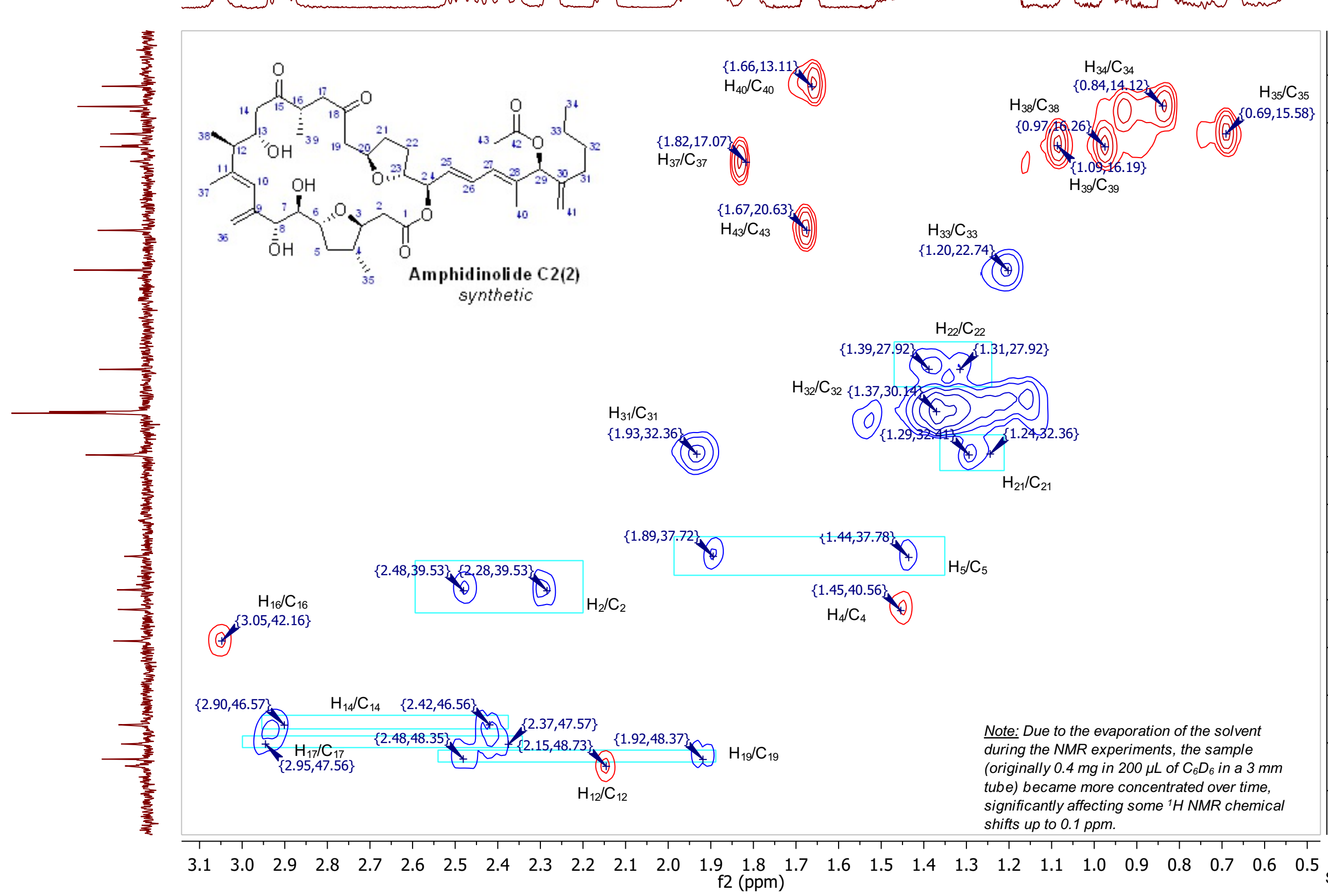

$\mathrm{H}_{40} / \mathrm{C}_{40}$ 
Synthetic Amphidinolide $\mathrm{C}_{2}$ (2)

$\mathrm{HMBC}{ }^{1} \mathrm{H}-{ }^{13} \mathrm{C}\left\{{ }^{1} \mathrm{H}\right\}-600 \mathrm{MHz}-\mathrm{C}_{6} \mathrm{D}_{6}$

Crosspeak correlations (6.9-4.95; 180-0 ppm range) (abscissa/ordinate)
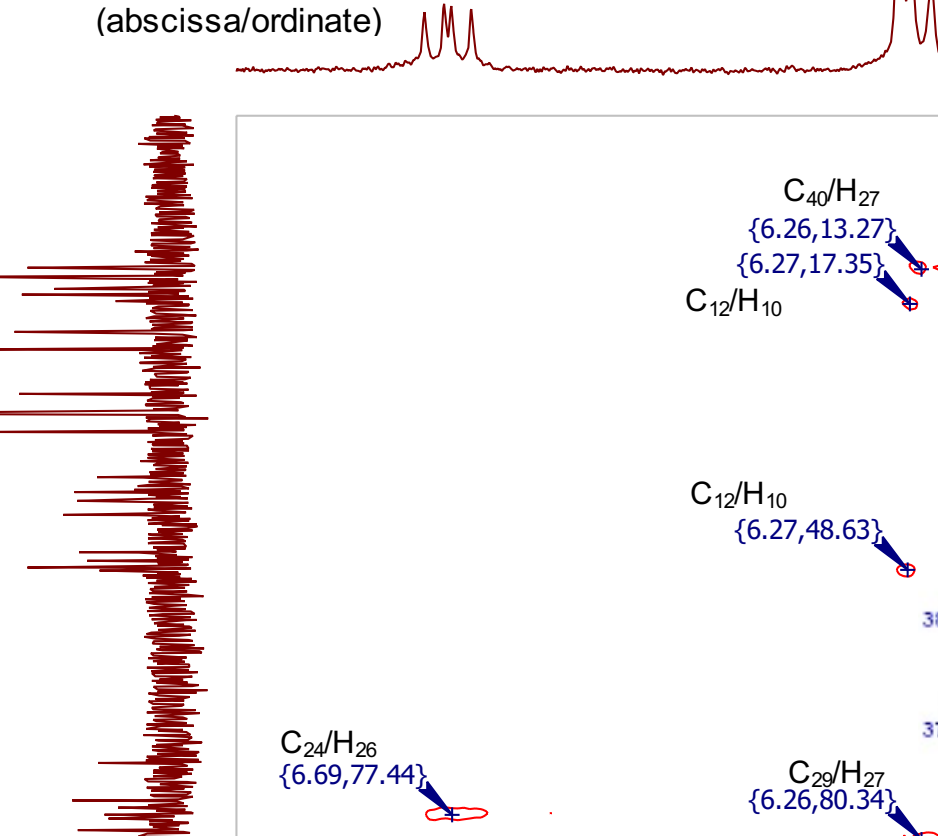

\section{$\mathrm{C}_{40} / \mathrm{H}_{29}$}

$\mathrm{C}_{12} / \mathrm{H}_{10}$

$\{6.26,13.27\}$

$\{5.88,13.16\}$

$\mathrm{C}_{31} / \mathrm{H}_{29}$

$\{5.88,32.41\}$

$\mathrm{C}_{12} / \mathrm{H}_{10}$
$\{6.27,48.63\}$

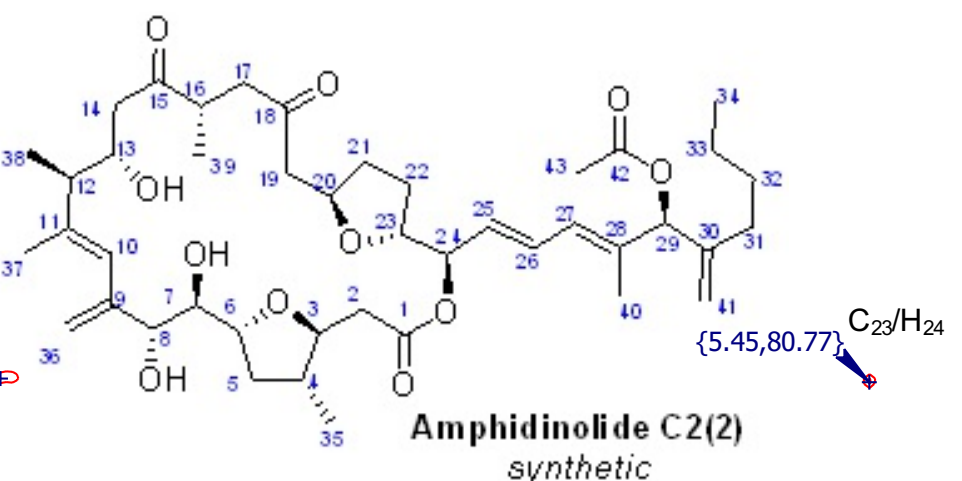

synthetic
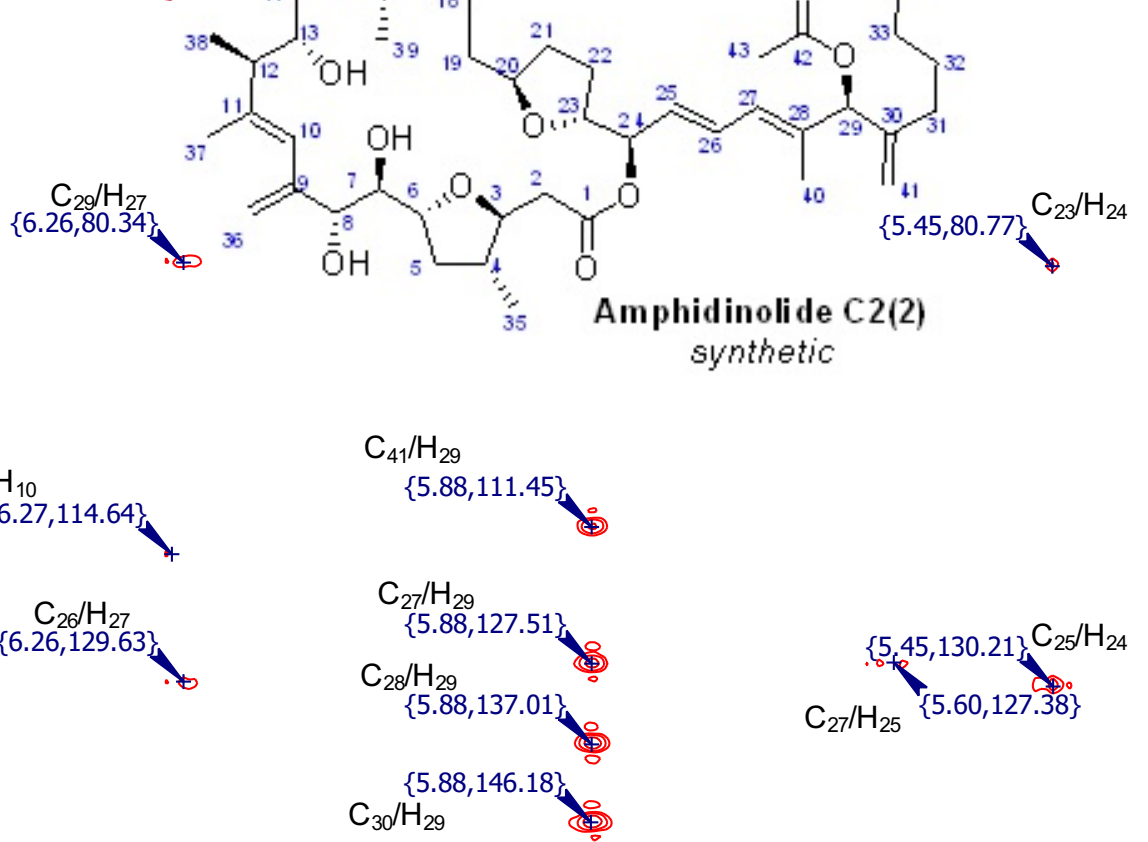

$\mathrm{C}_{41} / \mathrm{H}_{29}$

$\mathrm{C}_{36} / \mathrm{H}_{10}$

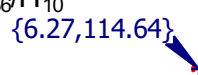

$\mathrm{C}_{27} / \mathrm{H}_{26}$

$\{6.69,127.40\}$

$\{6.69,136.7$

$\mathrm{C}_{26} / \mathrm{H}_{27}$
$\{6.26,129.63\}$

Note: Due to the evaporation of the solvent

during the NMR experiments, the sample

(originally $0.4 \mathrm{mg}$ in $200 \mu \mathrm{L}$ of $\mathrm{C}_{6} \mathrm{D}_{6}$ in a $3 \mathrm{~mm}$

tube) became more concentrated over time,

significantly affecting some ${ }^{1} \mathrm{H}$ NMR chemical

shifts up to $0.1 \mathrm{ppm}$.

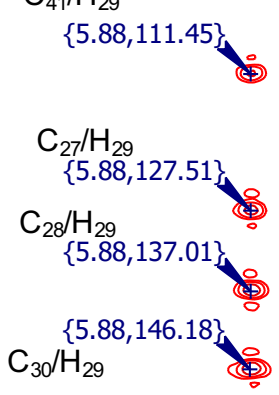

$\mathrm{C}_{42} / \mathrm{H}_{29}$

$\{5.88,169.03\}$

$\{5.47,171.81\} \quad \mathrm{C}_{1} / \mathrm{H}_{24}$

$-0$

$\mathrm{C}_{2 \mathrm{~g}} / \mathrm{H}_{41 \mathrm{a}}$

$\mathrm{C}_{29} / \mathrm{H}_{41 \mathrm{~b}}$

$\{4.95,80.46\}$ 
Synthetic Amphidinolide $\mathrm{C}_{2}$ (2)

$\mathrm{HMBC}{ }^{1} \mathrm{H}-{ }^{13} \mathrm{C}\left\{{ }^{1} \mathrm{H}\right\}-600 \mathrm{MHz}-\mathrm{C}_{6} \mathrm{D}_{6}$

Crosspeak correlations (3.2-0.5; 220-0 ppm range) (abscissa/ordinate)
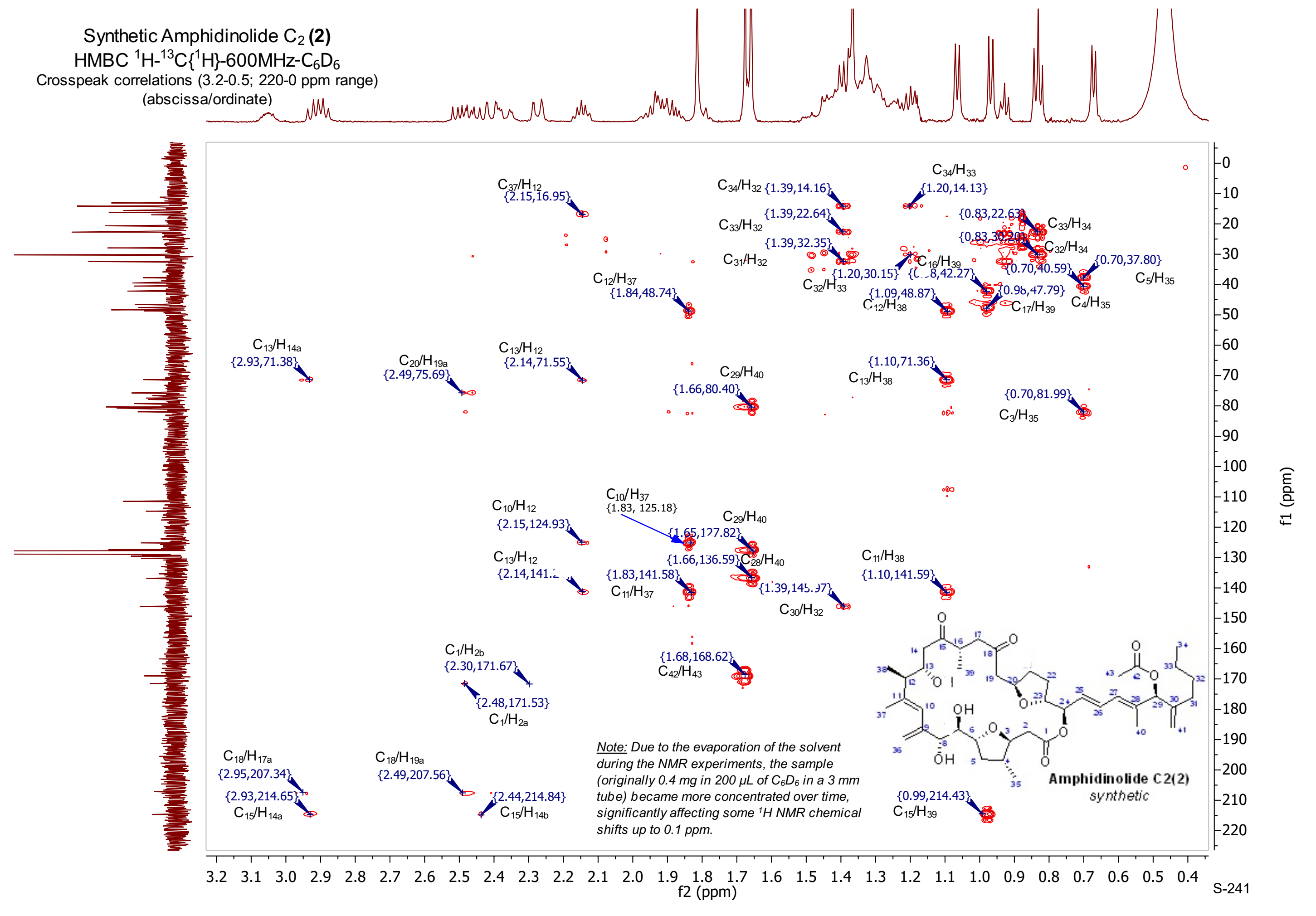
Synthetic Amphidinolide $\mathrm{C}_{2}$ (2) NOESY ${ }^{1} \mathrm{H}-{ }^{1} \mathrm{H}-600 \mathrm{MHz}-\mathrm{C}_{6} \mathrm{D}_{6}$

Selected crosspeak correlations

(6.7-3.75; 6.75-0.5 ppm/range)

(abscissa/ordinate)

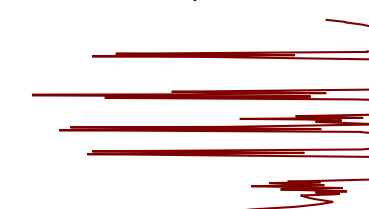

$=$

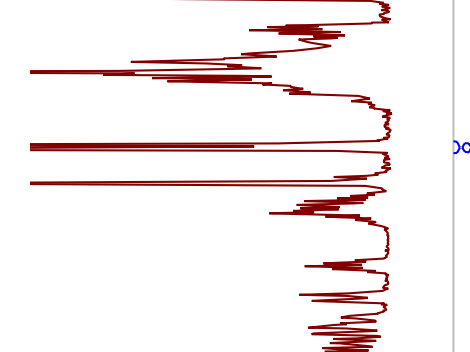

$\mathrm{H}_{10} / \mathrm{H}_{12}$ $\{6.28,2.1$

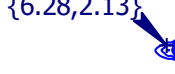

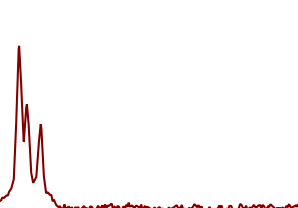
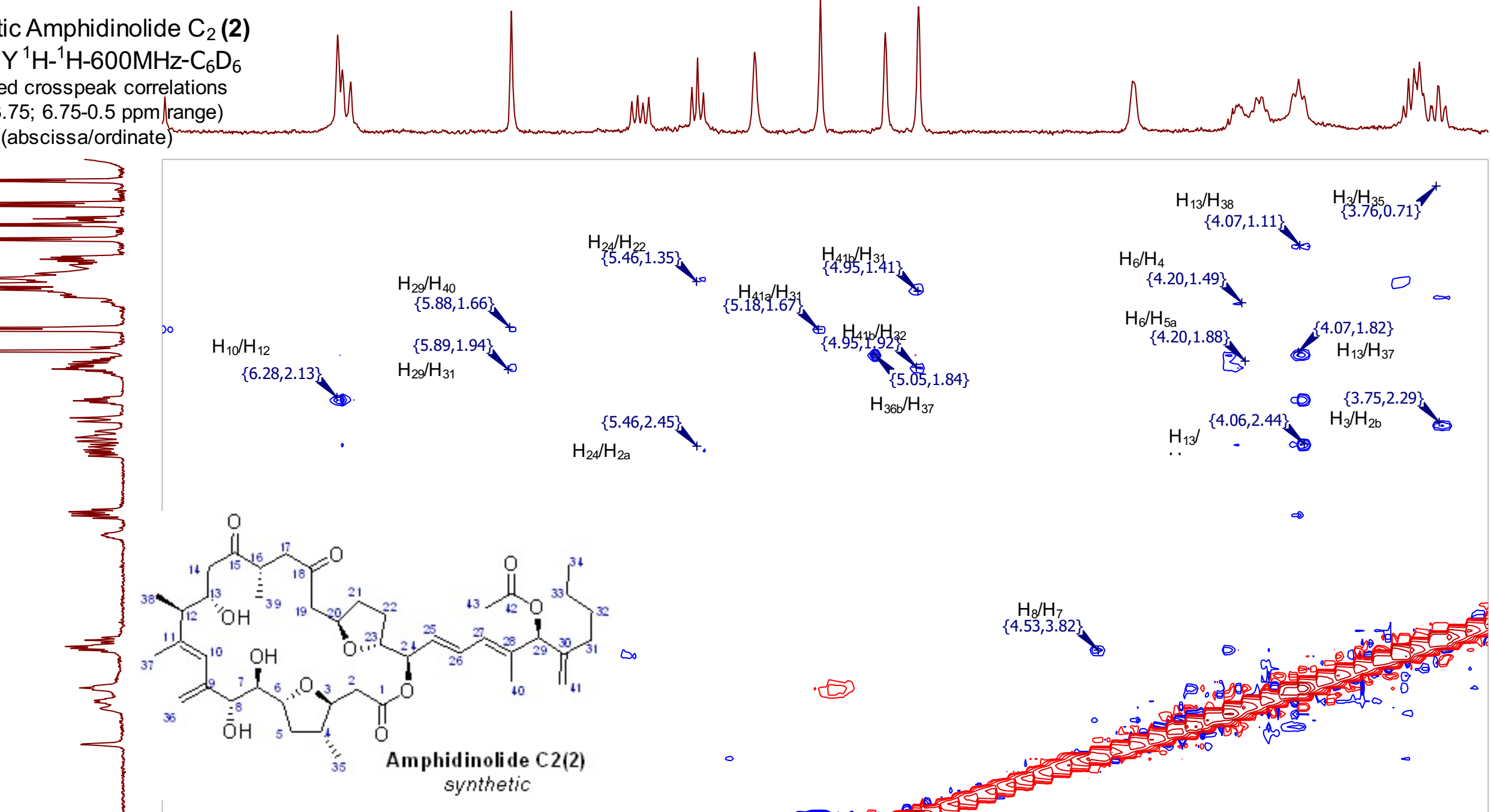

synthetic
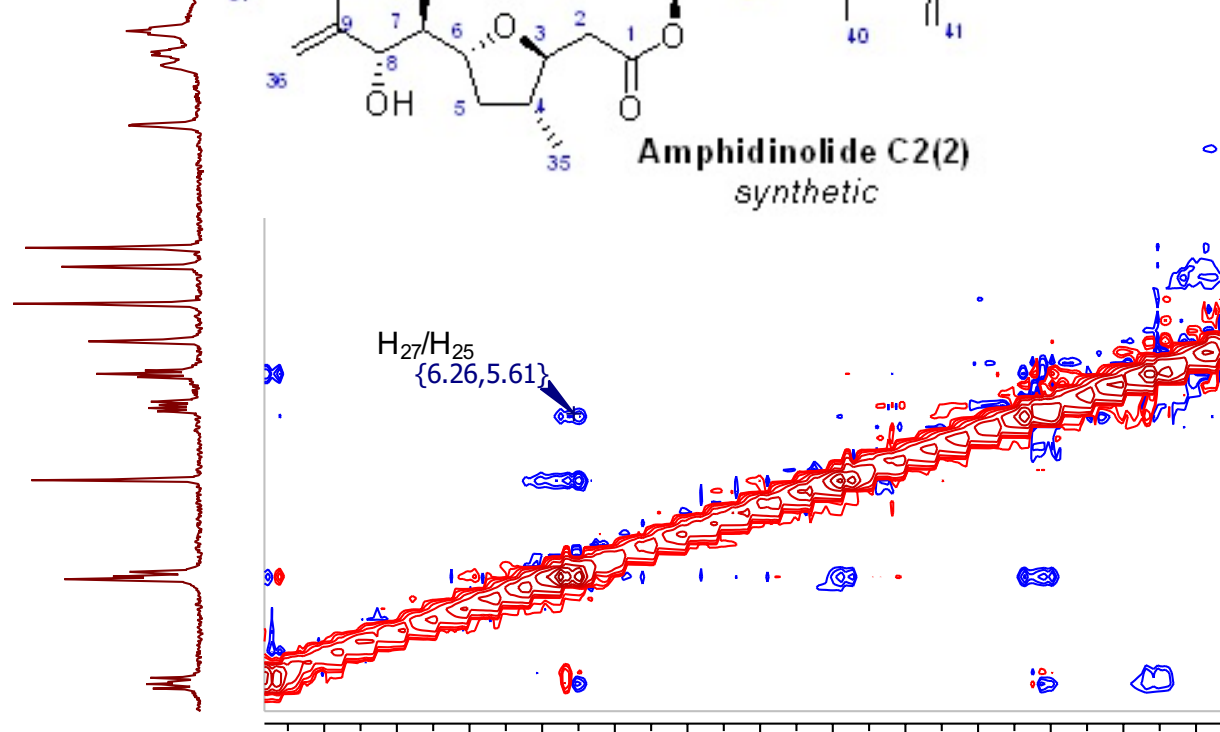

.
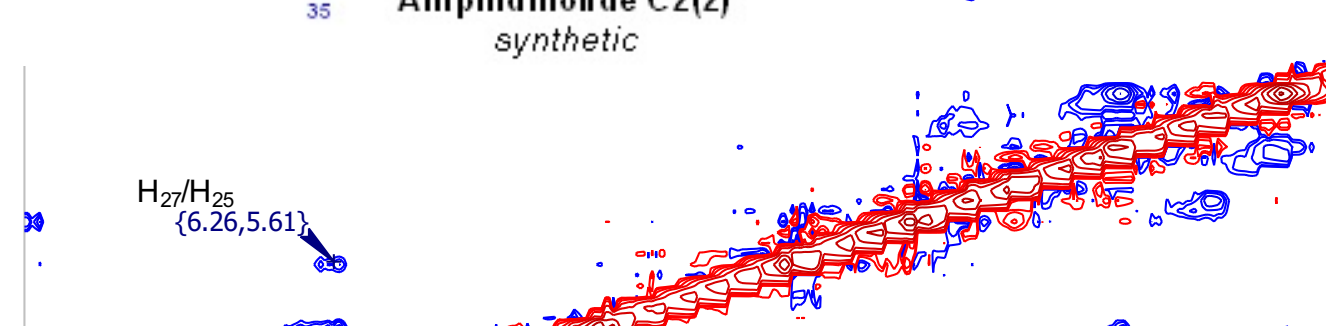

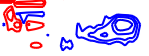
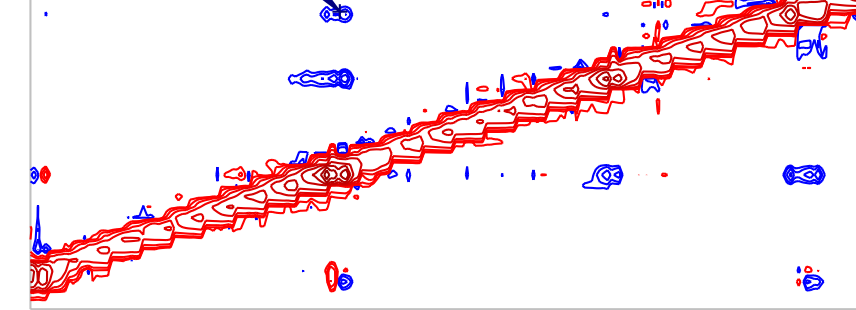

(c)

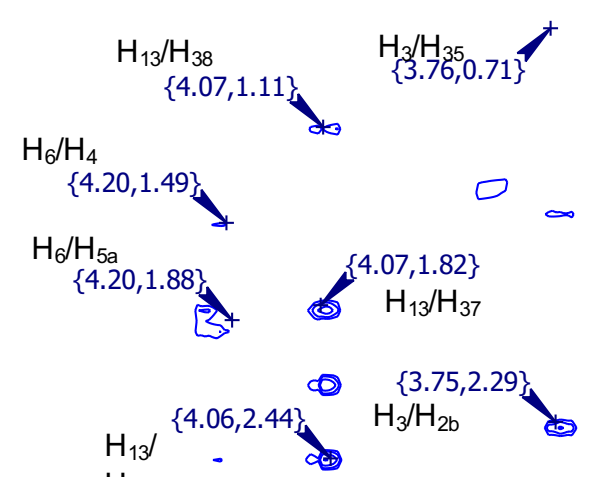


Synthetic Amphidinolide $\mathrm{C}_{2}$ (2)

NOESY ${ }^{1} \mathrm{H}-{ }^{1} \mathrm{H}-600 \mathrm{MHz}-\mathrm{C}_{6} \mathrm{D}_{6}$

Selected crosspeak correlations

(3.35-0; 6.75-0.5 ppm range)

(abscissa/ordinate)

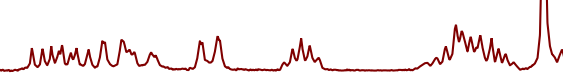
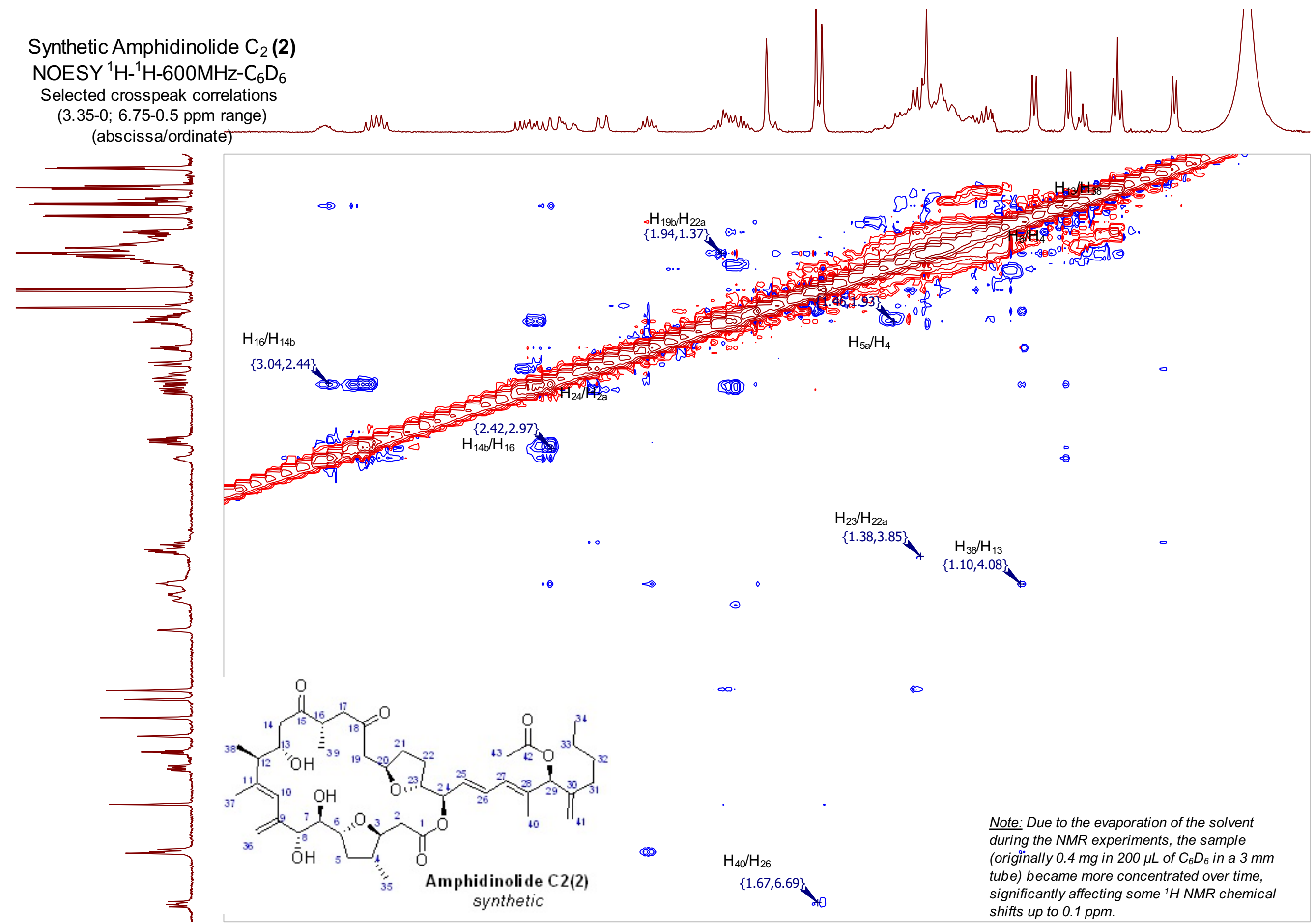

\section{$\mathrm{H}_{23} / \mathrm{H}_{22 \mathrm{a}}$}

$\{1.38,3.85\} \quad \mathrm{H}_{38} / \mathrm{H}_{13}$

$\{1.10,4.08\}$

E
$\stackrel{0}{0}$
$\square$

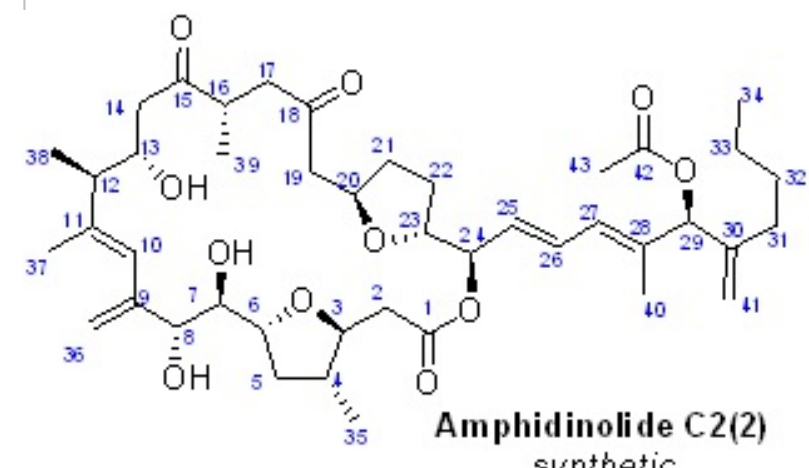

$\odot$

Note: Due to the evaporation of the solvent during the NMR experiments, the sample

(originally $0.4 \mathrm{mg}$ in $200 \mu \mathrm{L}$ of $C_{6} D_{6}$ in a $3 \mathrm{~mm}$ tube) became more concentrated over time, significantly affecting some ${ }^{1} \mathrm{H}$ NMR chemical shifts up to $0.1 \mathrm{ppm}$. 


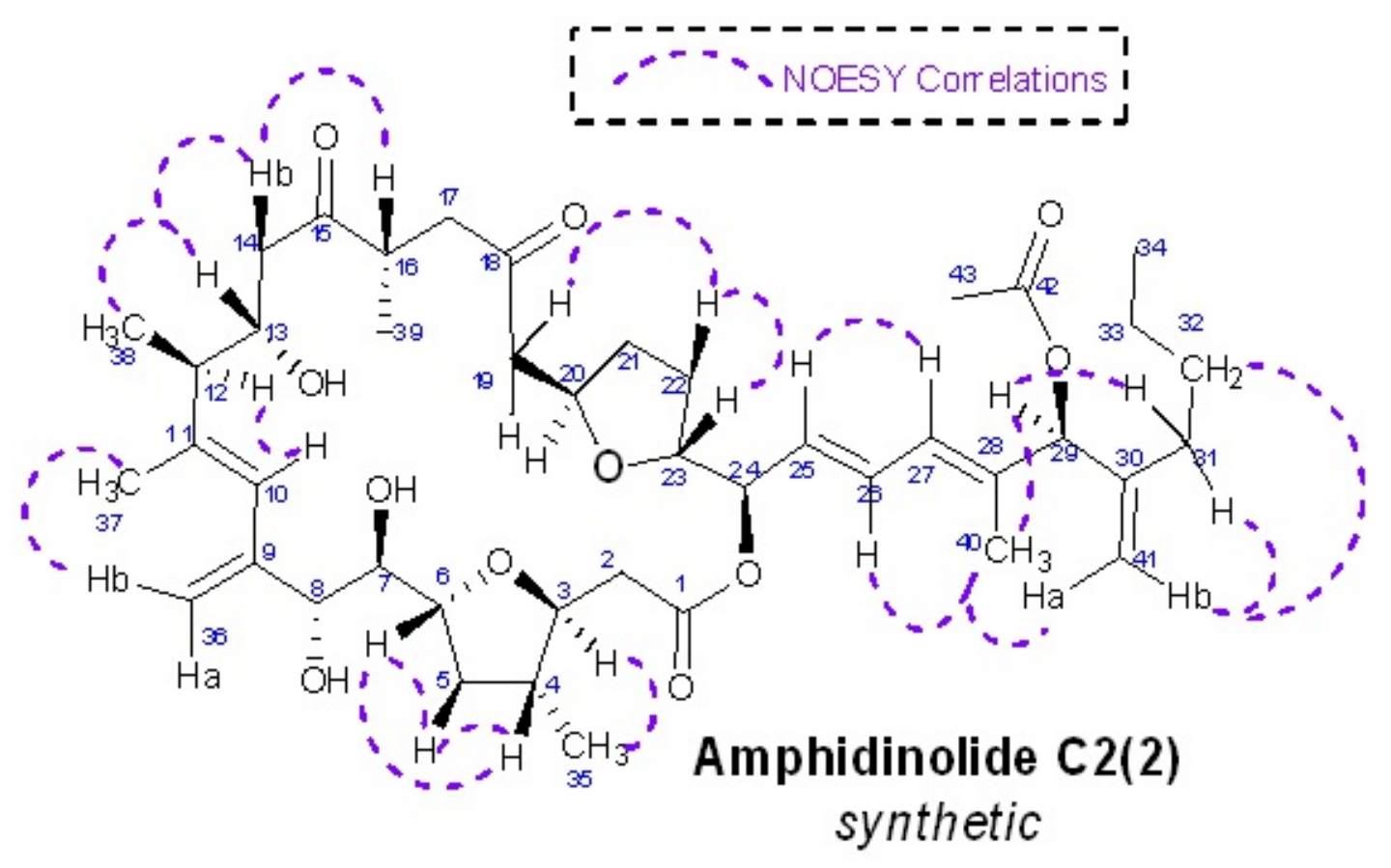

